\title{
Youth in India: Situation and Needs 2006-2007, Bihar
}

International Institute for Population Sciences (IIPS)

Population Council

Follow this and additional works at: https://knowledgecommons.popcouncil.org/departments_sbsr-pgy

Part of the Demography, Population, and Ecology Commons, Family, Life Course, and Society Commons, and the International Public Health Commons How does access to this work benefit you? Let us know!

\section{Recommended Citation}

International Institute for Population Sciences (IIPS) and Population Council. 2009. "Youth in India: Situation and Needs 2006-2007, Bihar," report. Mumbai: IIPS. 


\section{Youth in India: \\ Situation and Needs 2006-2007}

स्वास्थ्य एवं परिवार कल्याण मंत्रालय

निर्माण भवन, नई दिल्ली - 110011

GOVERNMENT OF INDIA

MINISTRY OF HEALTH \& FAMILY WELFARE

NIRMAN BHAWAN, NEW DELHI - 110011

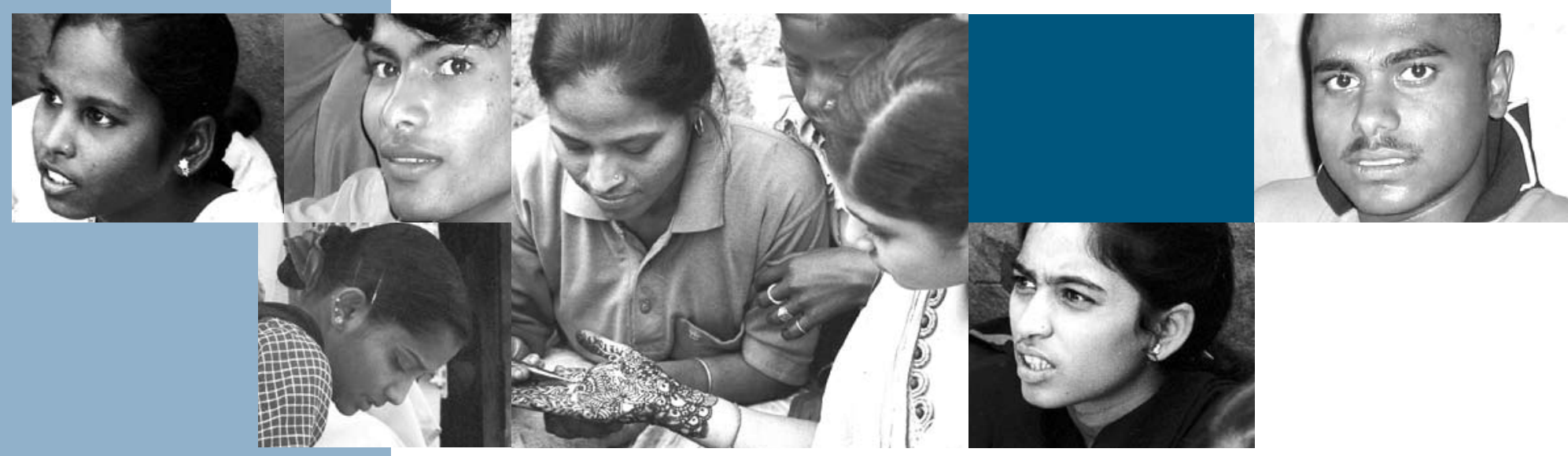

\section{BIHAR}

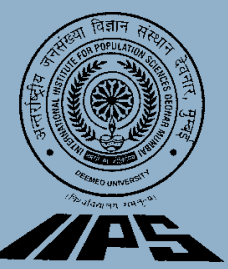

International Institute for Population Sciences, Mumbai

(1) Population Council

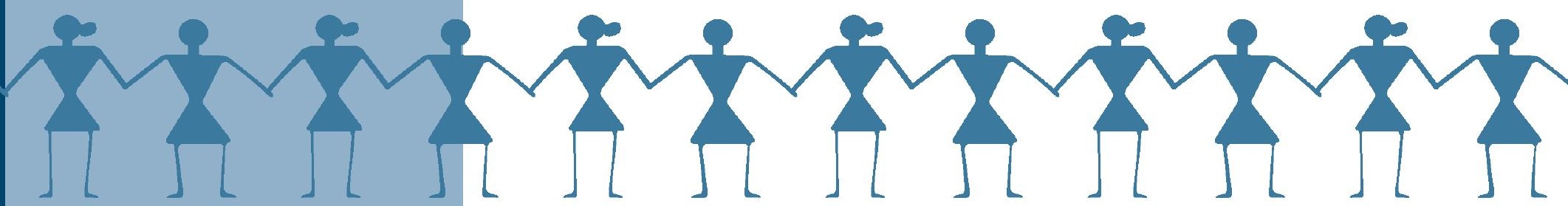


This report is the result of a sub-national study undertaken by the International Institute for Population Sciences, Mumbai and the Population Council, New Delhi, as part of a project to collect information on key transitions experienced by youth in India, including those related to education, work force participation, sexual activity, marriage, health and civic participation; the magnitude and patterns of young people's sexual and reproductive practices before, within and outside of marriage as well as related knowledge, decision-making and attitudes. The project was implemented in six states of India, namely, Andhra Pradesh, Bihar, Jharkhand, Maharashtra, Rajasthan and Tamil Nadu.

\section{For detailed reports please contact:}

International Institute for Population Sciences

Govandi Station Road, Deonar

Mumbai 400088

India

Phone: 022-42372400/42372518

email: iipsyouth@rediffmail.com

Website: http://www.iipsindia.org
Population Council

Zone 5-A, Ground Floor

India Habitat Centre

Lodi Road

New Delhi 110003

Phone: 011-2464 2901/02

email: info-india@popcouncil.org

Website: http://www.popcouncil.org/asia/india.html

The International Institute for Population Sciences (IIPS) is a deemed university under administrative control of Ministry of Health and Family Welfare, Government of India. The Institute engages in teaching and research in population sciences, and has been actively involved in building the capacity of Population Research Centres, and other state and central government offices that address population issues in the country and in the Asia-Pacific region. It has a proven record in conducting national- and sub-national-level studies in reproductive health, including the National Family Health Surveys and District Level Household and Facility Survey under the Reproductive and Child Health programme.

The Population Council is an international, non-profit, non-governmental organisation that seeks to improve the well-being and reproductive health of current and future generations around the world and to help achieve a humane, equitable and sustainable balance between people and resources. The Council conducts biomedical, social science and public health research, and helps build research capacities in developing countries.

Copyright @ 2009 International Institute for Population Sciences, Mumbai and Population Council, New Delhi

Suggested citation: International Institute for Population Sciences (IIPS) and Population Council. 2009.

Youth in India: Situation and Needs 2006-2007, Bihar. Mumbai: IIPS. 


\section{Youth in India: Situation and Needs 2006-2007}

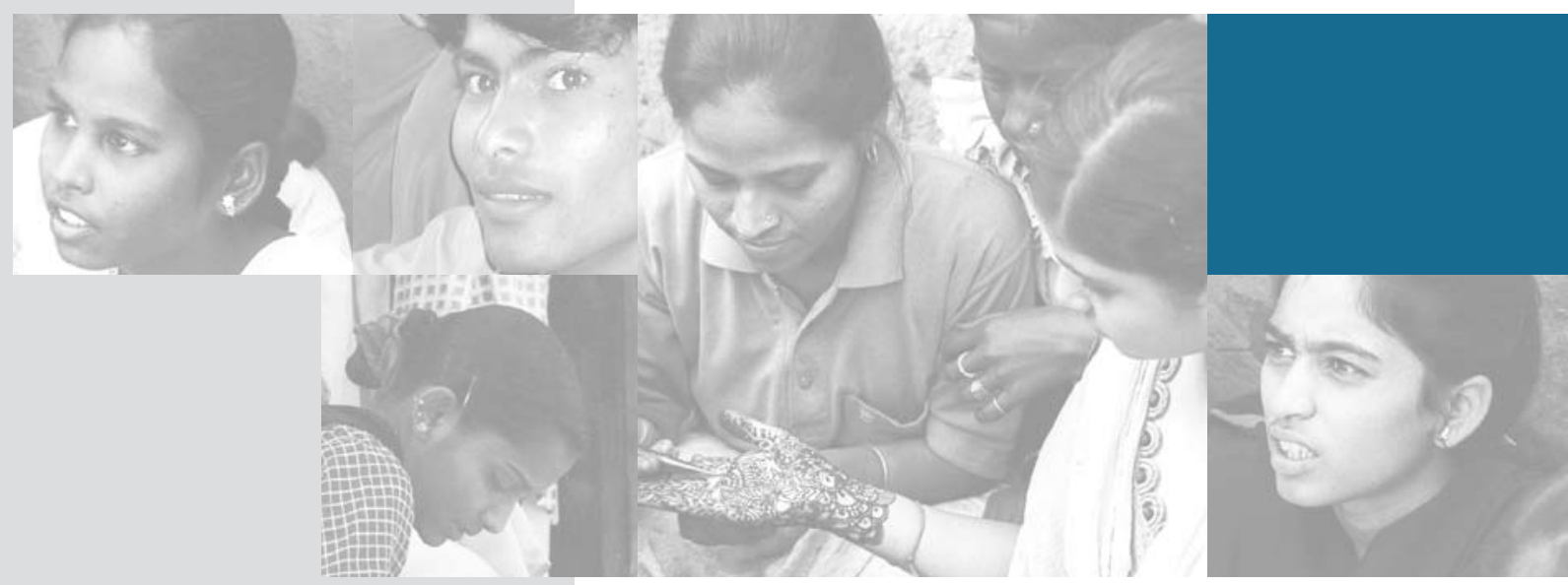

\section{BIHAR}

Usha Ram

S.K. Mohanty

Abhishek Singh

F. Ram

International Institute for Population Sciences, Mumbai

Rajib Acharya

Shireen J. Jejeebhoy

K.G. Santhya

Population Council, New Delhi

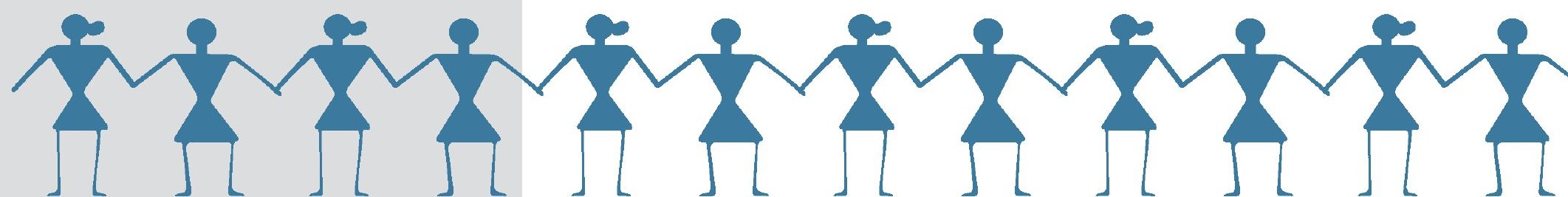




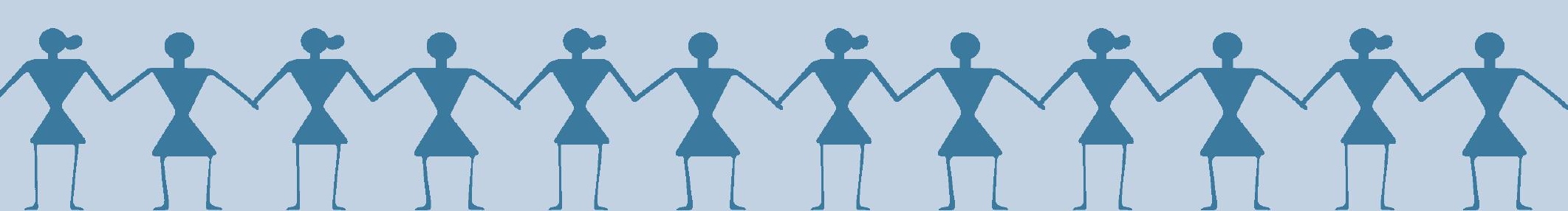




\section{Contents}

Page No.

Tables. viii

Figures xiii

Foreword. xvii

Acknowledgements ...xix

Executive summary xxii

Chapter 1: Introduction .........................................................................................................

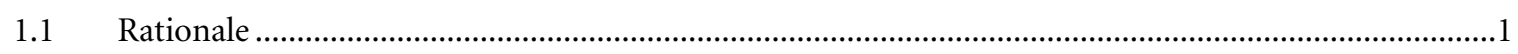

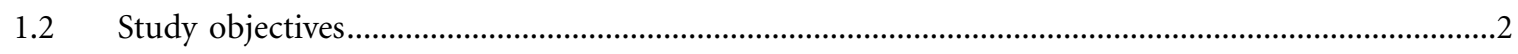

1.3 Bihar: Overview of demographic and socio-economic features .........................................................

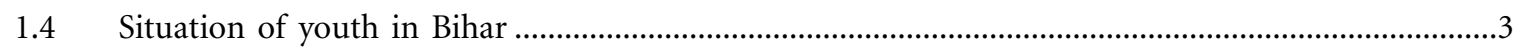

1.5 Youth-related policy and programme environment in Bihar ............................................................

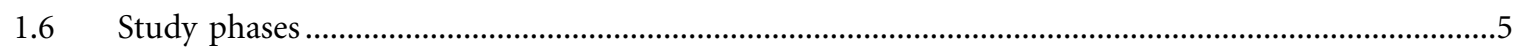

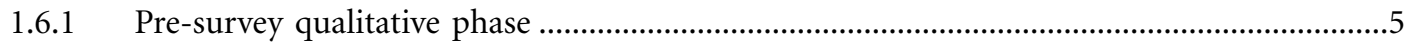

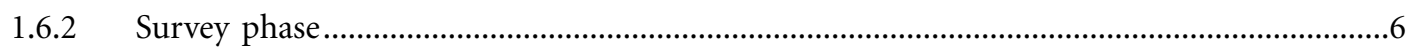

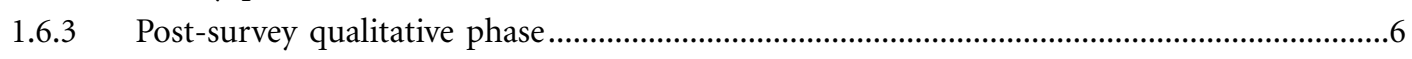

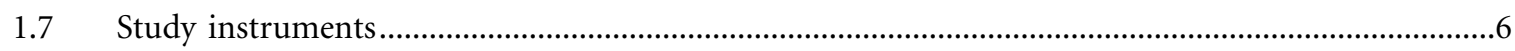

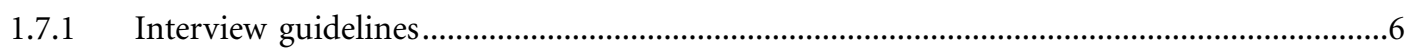

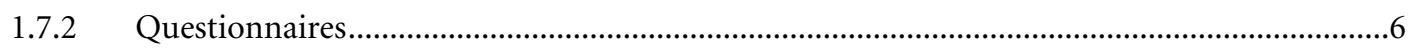

1.8 Study design and sample size estimation for individual interviews .................................................10

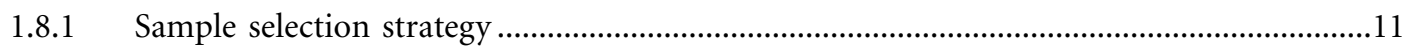

1.8.1.a Selection of households in rural areas ..................................................................11

1.8.1.b Selection of households in urban areas ....................................................................14

1.8.2 Selection of individual respondents within selected households............................................16

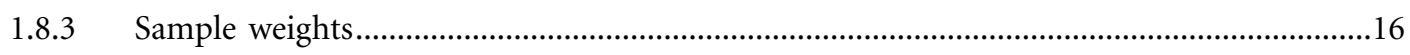

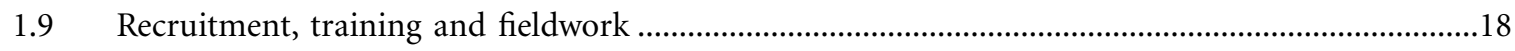

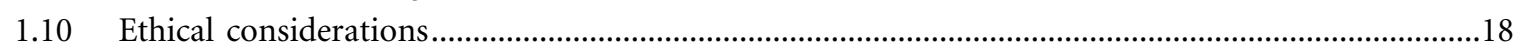

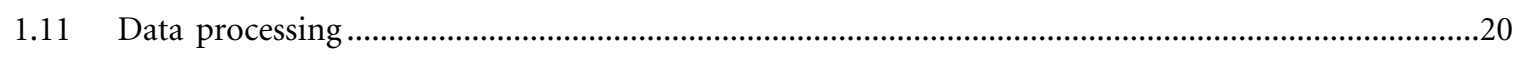

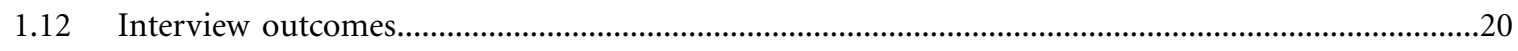

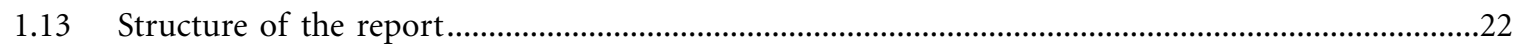


Page No.

Chapter 2: Profile of surveyed communities, households and respondents......................24

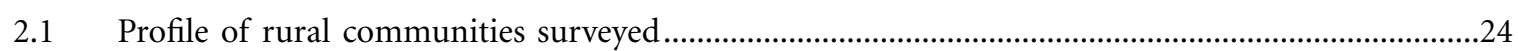

2.2 Profile of the household population: Age-sex distribution ...........................................................27

2.3 Profile of the household population: Marital status ....................................................................29

2.4 Profile of the household population: Educational attainment ......................................................31

2.5 Profile of the household population: Work participation .............................................................31

2.6 Socio-demographic characteristics of households and heads of households ...................................34

2.7 Profile of the household population: Housing characteristics ........................................................36

2.8 Profile of the household population: Ownership of agricultural land ............................................39

2.9 Profile of the household population: Overall economic status ........................................................39

2.10 Profile of surveyed youth: Background characteristics ....................................................................40

2.11 Profile of surveyed youth: Parental characteristics.....................................................................44

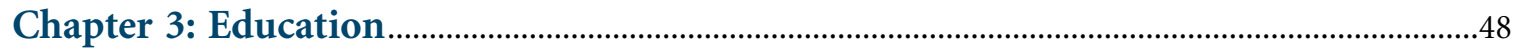

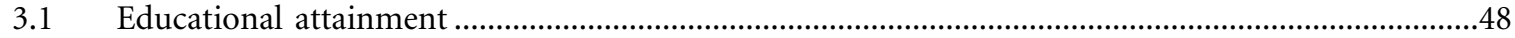

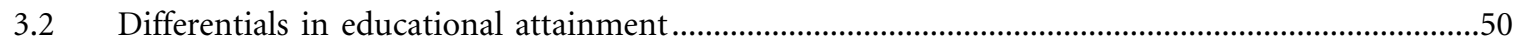

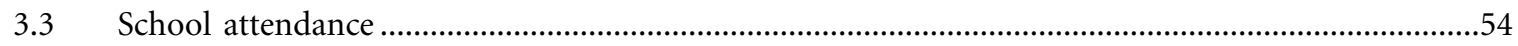

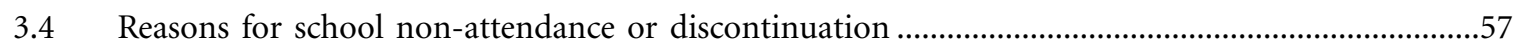

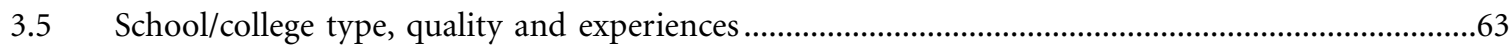

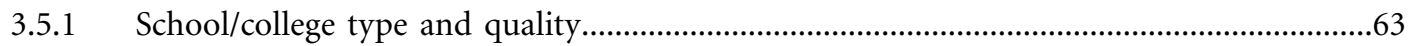

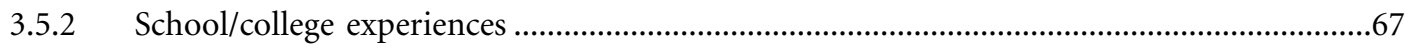

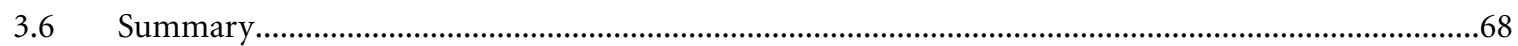

Chapter 4: Economic and non-economic activity....................................................................

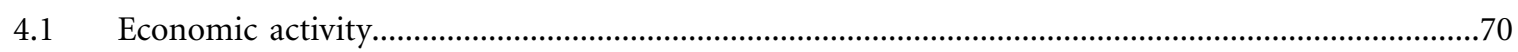

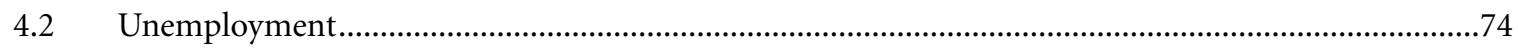

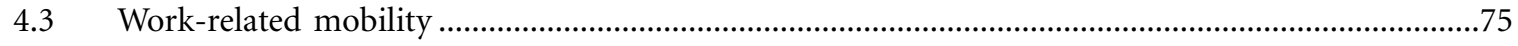

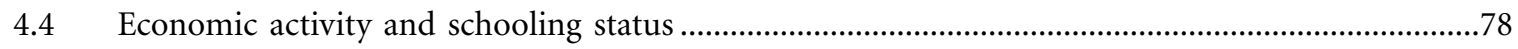

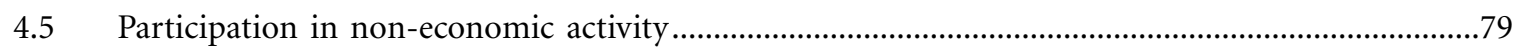

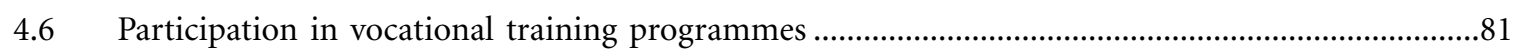

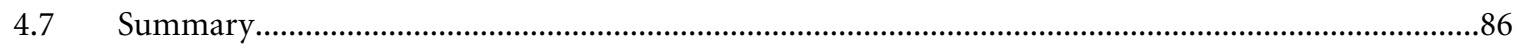

Chapter 5: Media exposure and access to pornographic materials......................................88

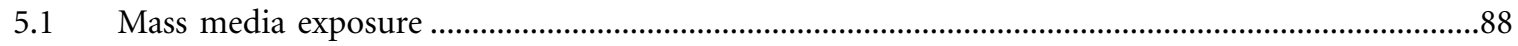

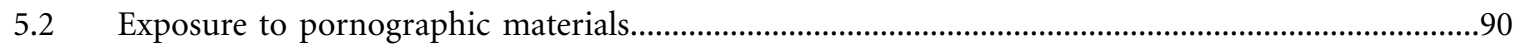

5.3 Youth perceptions about the influence of television and films on youth behaviours ....................93

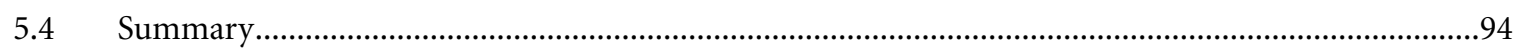




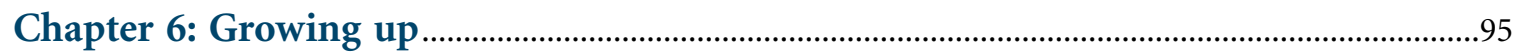

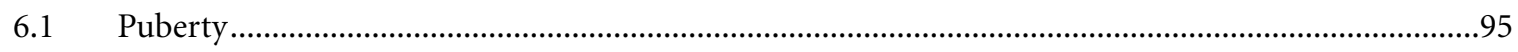

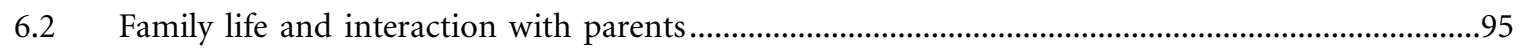

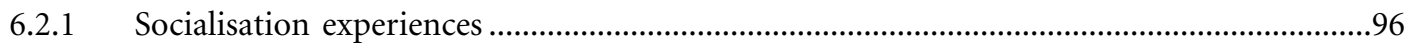

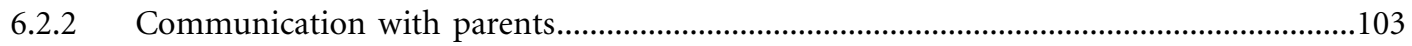

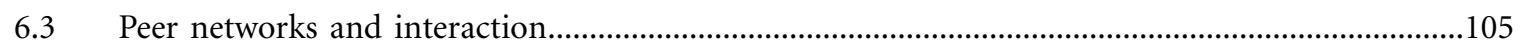

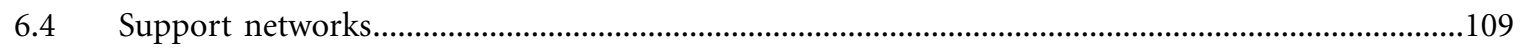

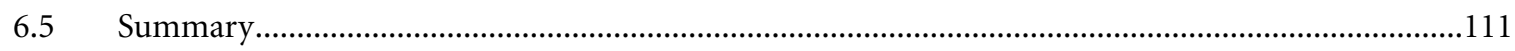

Chapter 7: Agency and gender role attitudes............................................................................113

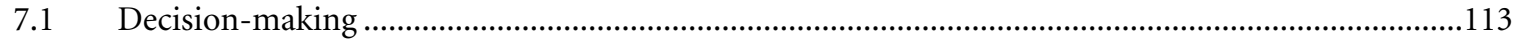

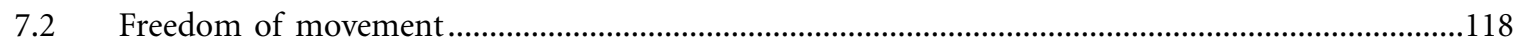

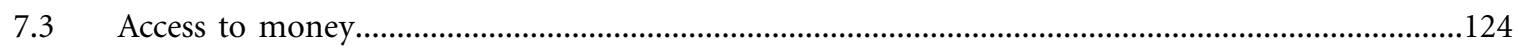

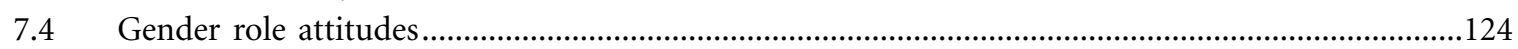

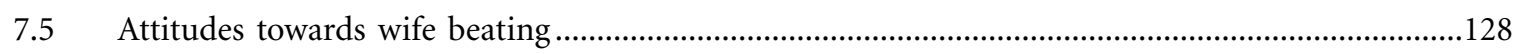

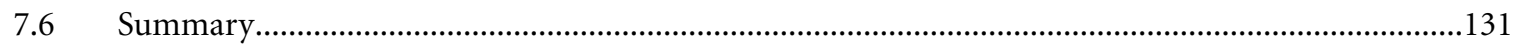

Chapter 8: Awareness of sexual and reproductive health matters.....................................132

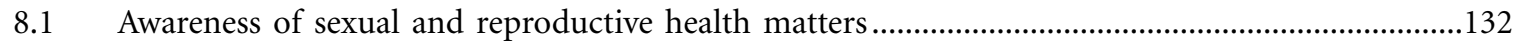

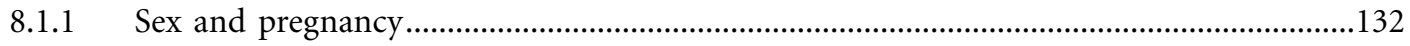

8.1.2 Socio-demographic differentials in awareness of sex- and pregnancy-related matters...135

8.1.3 Awareness of contraceptive methods .......................................................................138

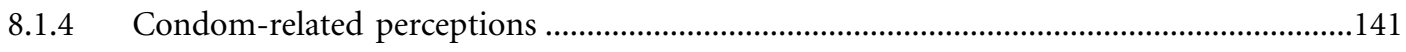

8.1.5 Awareness of contraception prior to marriage ..............................................................143

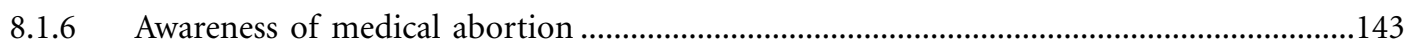

8.1.7 Awareness of sexually transmitted infections (STIs) and HIV/AIDS..............................144

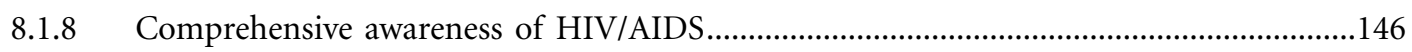

8.2 Knowledge of legal issues related to marriage and abortion ........................................................150

8.2.1 Knowledge of the legal minimum age at marriage.........................................................150

8.2.2 Awareness of the conditions under which abortion is legal...........................................151

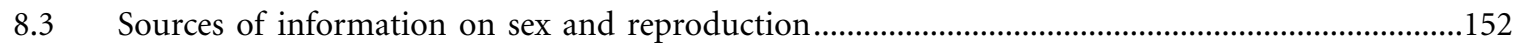

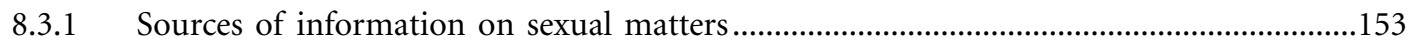

C.3.2 Current sources of information on contraception........................................................154

8.4 Perceptions and experience of family life or sex education.......................................................157

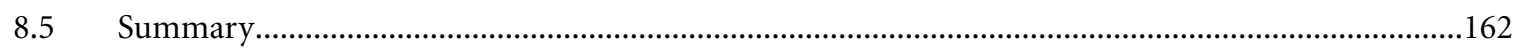


Page No.

\section{Chapter 9: Pre-marital romantic and sexual relationships}

9.1 Development of the questionnaire module on pre-marital romantic and sexual relationships

9.2 Attitudes toward pre-marital physical intimacy and sexual relations ..........................................165

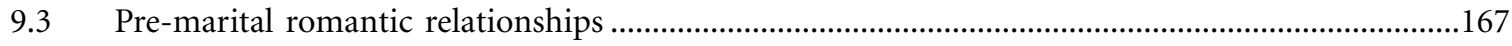

9.3.1 Prevalence of pre-marital romantic relationships ......................................................167

9.3.2 Characteristics of pre-marital romantic relationships .................................................172

9.3.3 Parental and peer awareness of romantic partnerships.................................................178

9.3.4 Marriage intentions and duration of pre-marital romantic relationships......................178

9.3.5 Pre-marital physical intimacy and sex with a romantic partner.....................................181

9.3.6 Characteristics of sexual experiences within pre-marital romantic relationships............181

9.4 Pre-marital sexual experiences within romantic and other relationships......................................184

9.4.1 Extent of pre-marital sexual experiences ........................................................................185

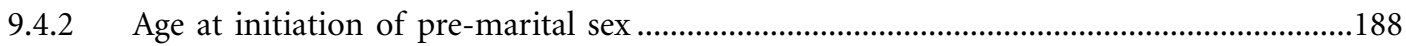

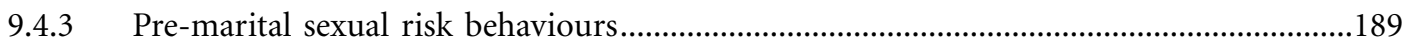

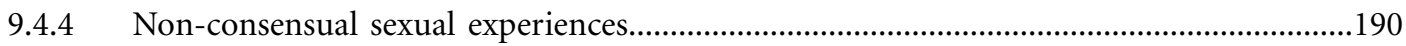

9.5 Triangulation of data on pre-marital sexual experiences among young people............................192

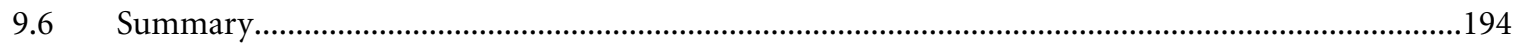

Chapter 10: Transitions to marriage and early married life ...................................................196

10.1 Young people's preferences regarding timing and type of marriage............................................196

10.2 Marriage planning and extent of youth involvement ................................................................198

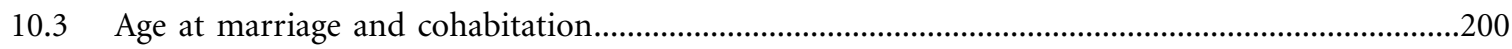

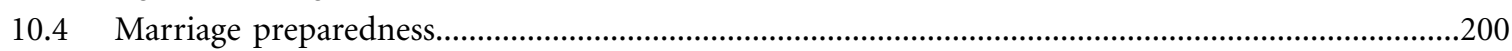

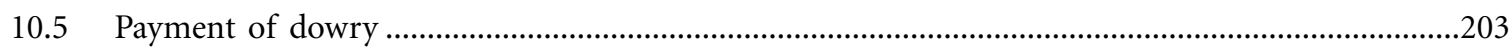

10.6 Early marital experiences: Spousal communication and interaction ..........................................204

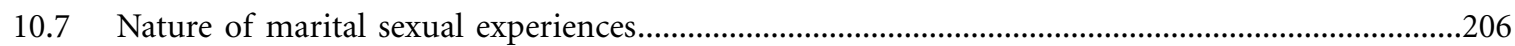

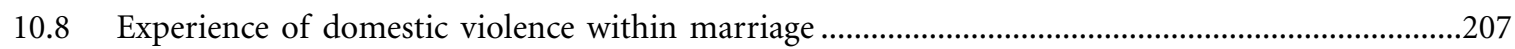

Extent and nature of extra-marital sexual relations ..............................................................209

10.10 Contraceptive practice within marriage: Lifetime, current and prior to first pregnancy .............210

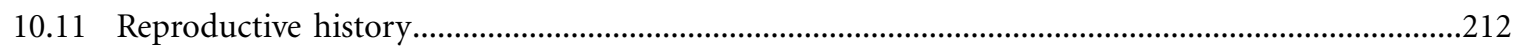

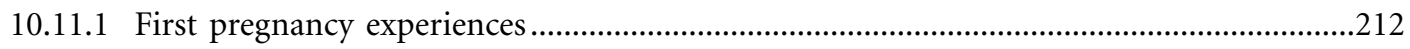

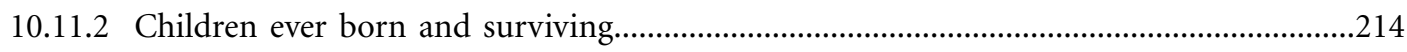

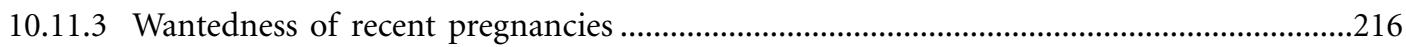

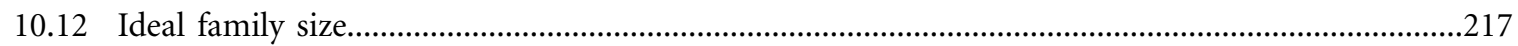

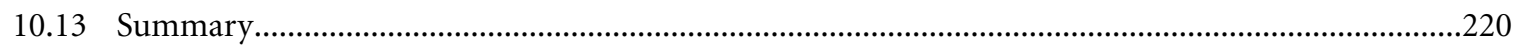




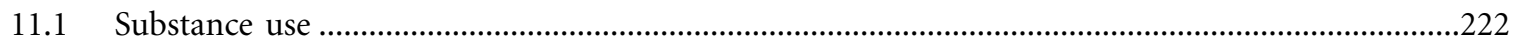

11.2 General and sexual and reproductive health problems ................................................................22

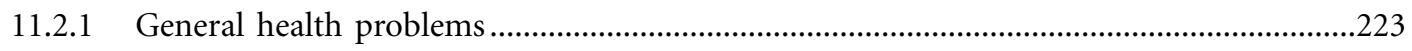

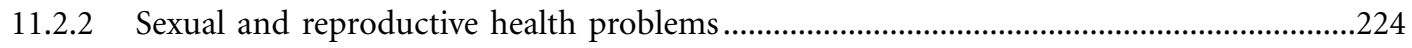

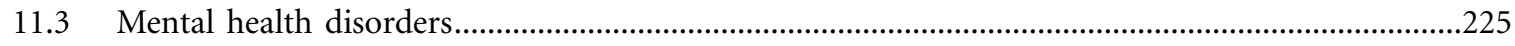

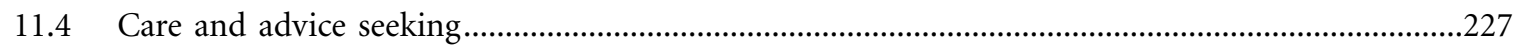

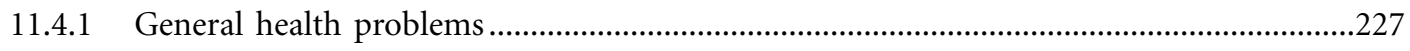

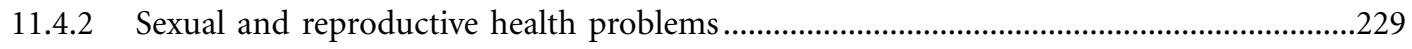

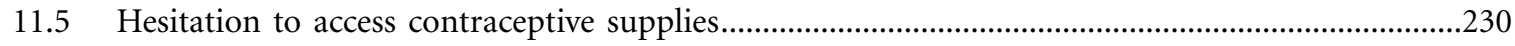

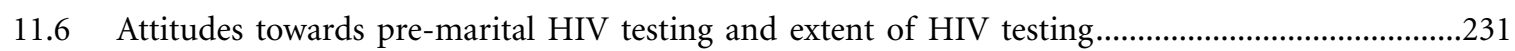

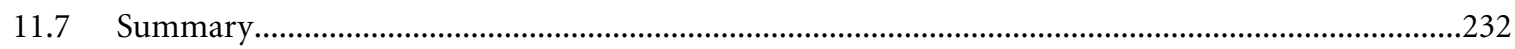

Chapter 12: Participation in civil society and political life ....................................................23

12.1 Awareness of and participation in government- and NGO-sponsored programmes ...................233

12.2 Participation in community- or panchayat-sponsored programmes ............................................237

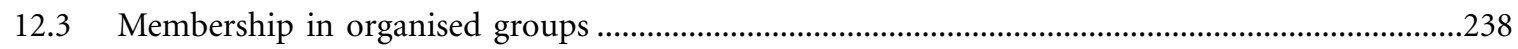

12.4 Perceptions about action taken by rural panchayats in addressing defiance of

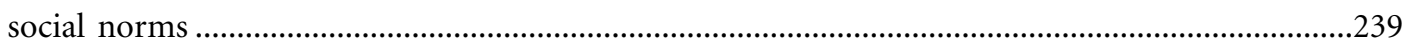

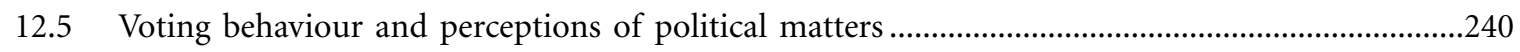

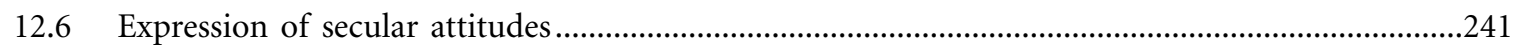

12.7 Physical fights in the village or urban neighbourhood ............................................................244

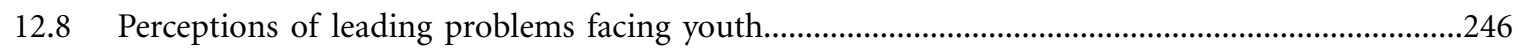

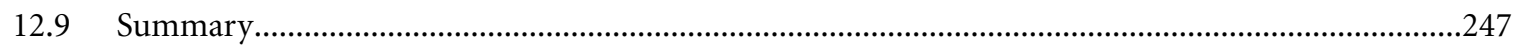

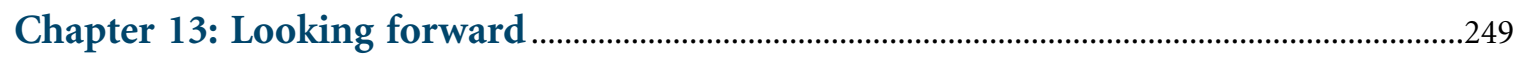

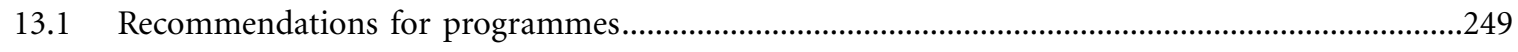

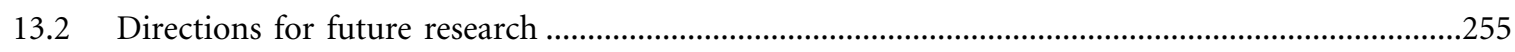

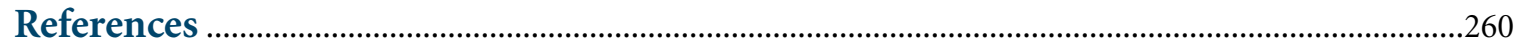

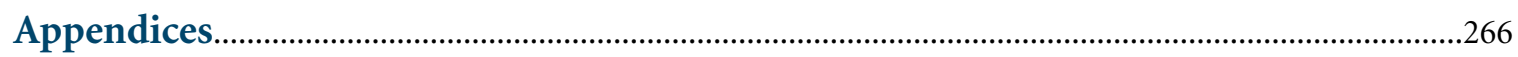

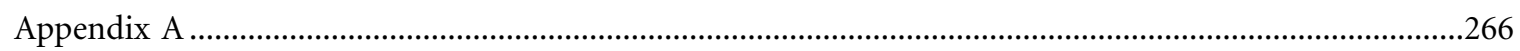

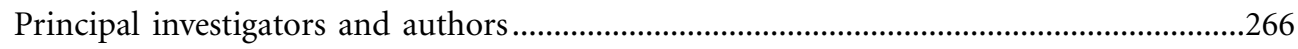

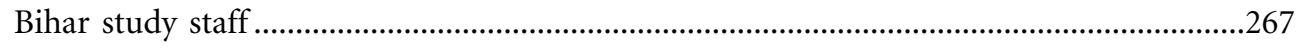

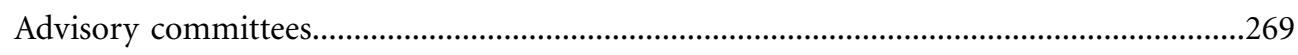

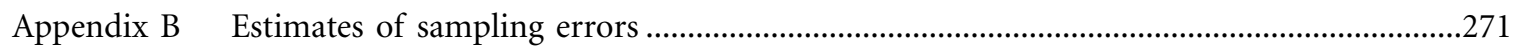

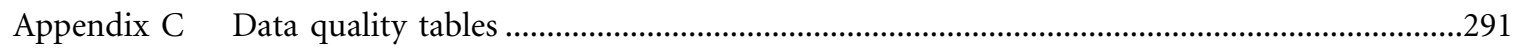




\section{Chapter 1: Introduction}

Table $1.1 \quad$ Sampling stratification scheme .............................................................................................14

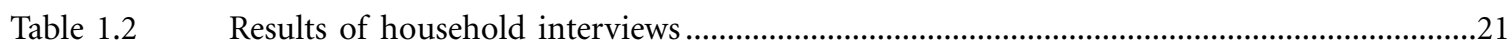

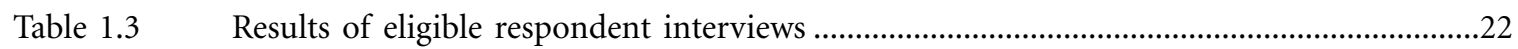

\section{Chapter 2: Profile of surveyed communities, households and respondents}

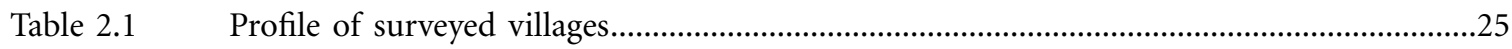

Table 2.2 Proximity of study residents to selected facilities.................................................................26

Table 2.3 Distribution of the surveyed population by age and sex ...................................................27

Table 2.4 Marital status of the surveyed population ……………………...............................................30

Table 2.5 Age at marriage of usual residents of households ..................................................................

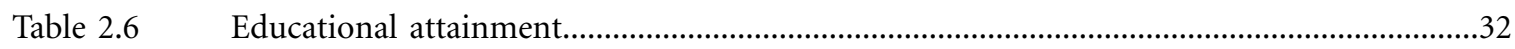

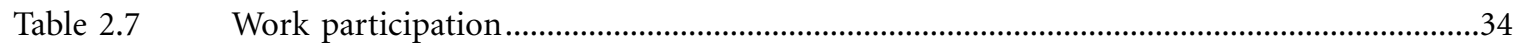

Table 2.8 Socio-demographic characteristics of households and heads of households ........................34

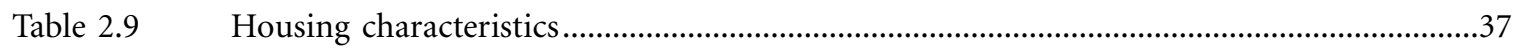

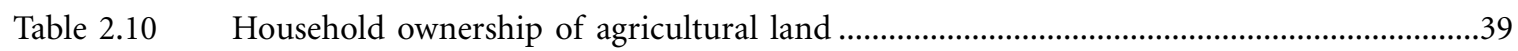

Table 2.11 Household assets and wealth status ..................................................................................

Table 2.12 Background characteristics of surveyed youth.....................................................................42

Table 2.13 Parental characteristics of surveyed youth ...........................................................................

\section{Chapter 3: Education}

Table 3.1 Educational attainment and current educational status ........................................................49

Table 3.2 Educational attainment of young men by selected background characteristics .................50

Table 3.3 Educational attainment of young women by selected background characteristics..............52

Table 3.4a Reasons for never attending school .....................................................................................58

Table 3.4b Reasons for school discontinuation by level of education ...............................................60

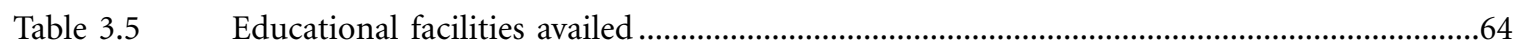

Table $3.6 \quad$ Schooling experiences ................................................................................................66 


\section{Chapter 4: Economic and non-economic activity}

Page No.

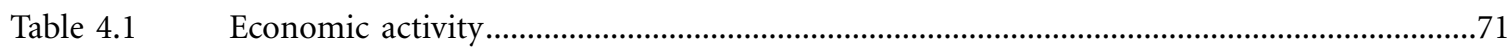

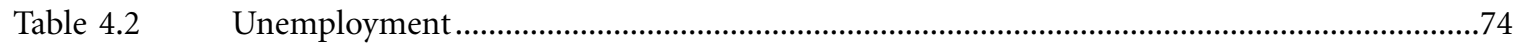

Table 4.3 Unemployment by selected background characteristics..................................................76

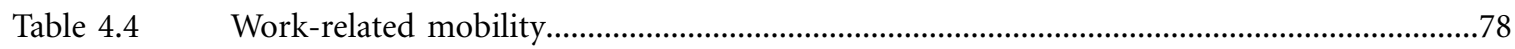

Table $4.5 \quad$ Participation in household chores..................................................................................82

Table 4.6 Participation in vocational training programmes.............................................................84

Table $4.7 \quad$ Willingness of youth to participate in vocational training programmes............................85

\section{Chapter 5: Media exposure and access to pornographic materials}

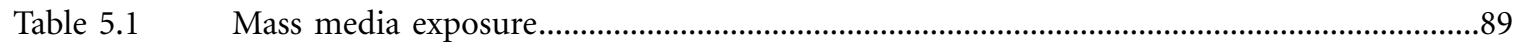

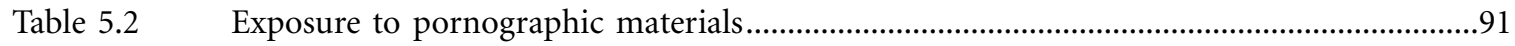

Table 5.3 Perceptions about the influence of television and films on youth behaviours...................94

\section{Chapter 6: Growing up}

Table 6.1a Age at puberty among young women ......................................................................96

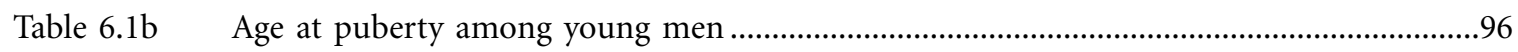

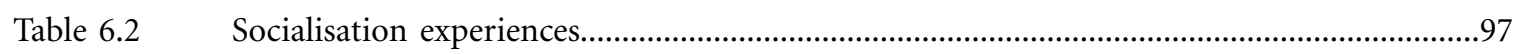

Table 6.3 Perceptions of parental reactions to selected activities ....................................................99

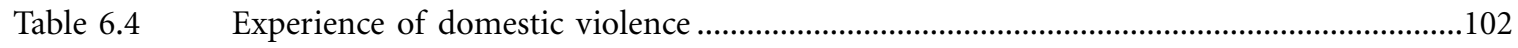

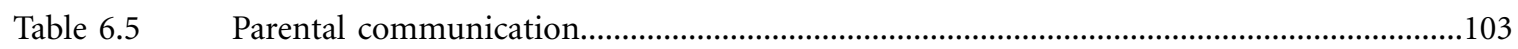

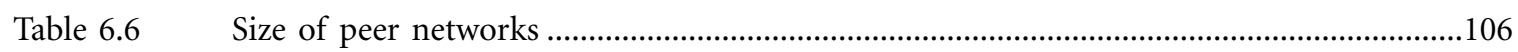

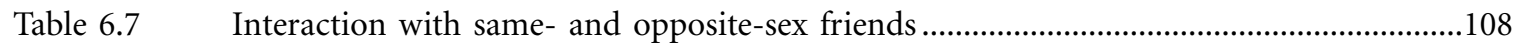

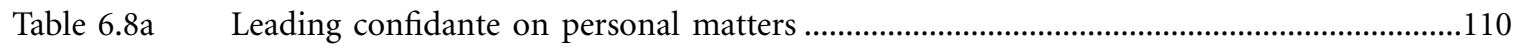

Table $6.8 \mathrm{~b} \quad$ Leading confidante on matters relating to the experience of teasing among young women ..................................................................................................... 111

\section{Chapter 7: Agency and gender role attitudes}

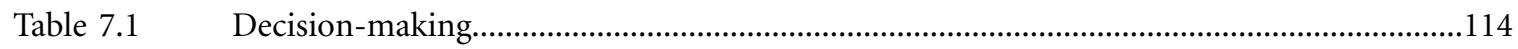

Table 7.2 Decision-making autonomy by selected background characteristics...............................116

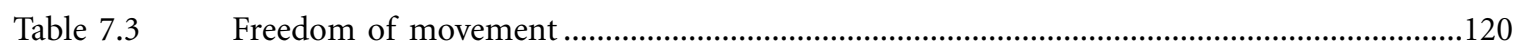

Table 7.4 Freedom of movement by selected background characteristics.......................................122

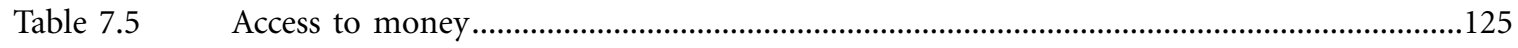

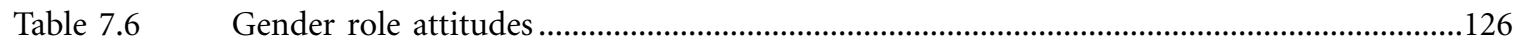

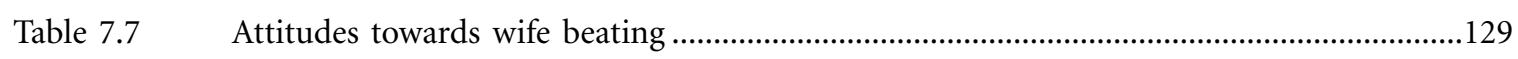


Page No.

\section{Chapter 8: Awareness of sexual and reproductive health matters}

Table 8.1 Awareness of sex- and pregnancy-related matters.

Table 8.2 Awareness of sex- and pregnancy-related matters by selected

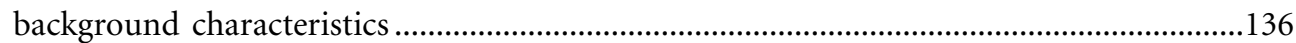

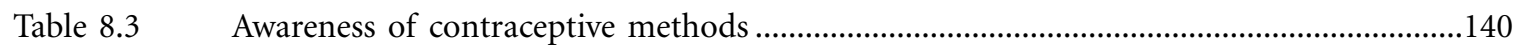

Table $8.4 \quad$ Perceptions of selected issues related to condom use .....................................................142

Table $8.5 \quad$ Awareness of contraception prior to marriage ...............................................................143

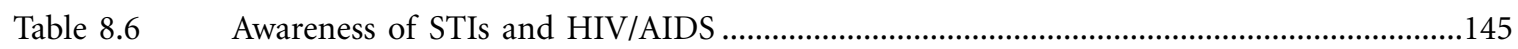

Table 8.7 Comprehensive knowledge of HIV/AIDS by selected background characteristics ...........147

Table $8.8 \quad$ Knowledge of the legal minimum age at marriage........................................................151

Table 8.9 Awareness of the conditions under which abortion is legal ...........................................153

Table 8.10 Sources of information on sexual matters before marriage...............................................155

Table $8.11 \quad$ Current sources of information on contraception ..........................................................156

Table $8.12 \quad$ Perceptions about family life or sex education..............................................................158

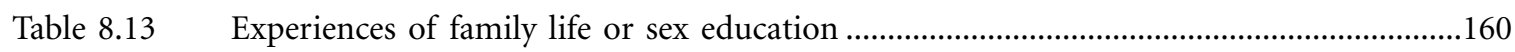

\section{Chapter 9: Pre-marital romantic and sexual relationships}

Table 9.1 Attitudes toward pre-marital physical intimacy and sexual relations ...............................166

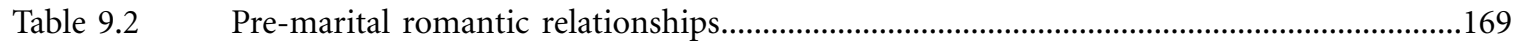

Table 9.3 Prevalence of pre-marital romantic relationships by selected

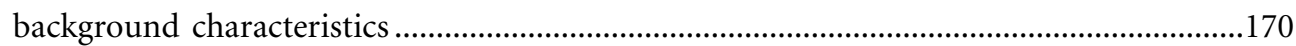

Table 9.4 Characteristics of pre-marital romantic relationships and partners.................................174

Table 9.5 Meeting places with pre-marital romantic partners........................................................177

Table 9.6 Peer and parental awareness of first pre-marital romantic relationship..........................179

Table 9.7 Marriage intentions and duration of pre-marital romantic relationships ........................180

Table 9.8 Physical intimacy and sexual experiences in pre-marital romantic relationships.............182

Table 9.9 Characteristics of sexual experiences within pre-marital romantic relationships .............184

Table 9.10 Overall pre-marital sexual experiences .............................................................................186

Table 9.11 Overall pre-marital sexual experiences by selected background characteristics................187

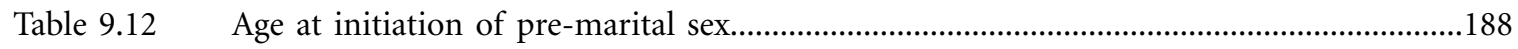

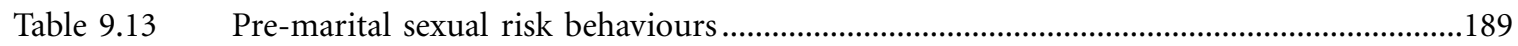

Table 9.14 Pre-marital non-consensual sexual experiences ................................................................191

Table 9.15 Levels of pre-marital romantic and sexual experiences by different reporting methods. 


\section{Chapter 10: Transitions to marriage and early married life}

Table 10.1 Preferences regarding timing and type of marriage $\quad$......................................................197

Table 10.2 Initiation of discussion on marriage and extent of youth involvement ...........................199

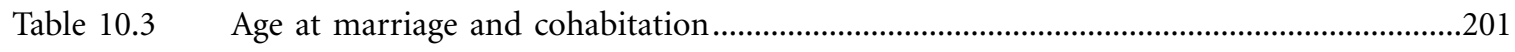

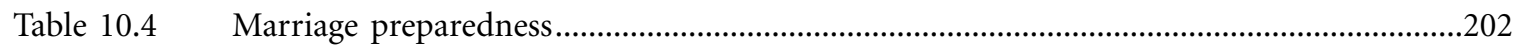

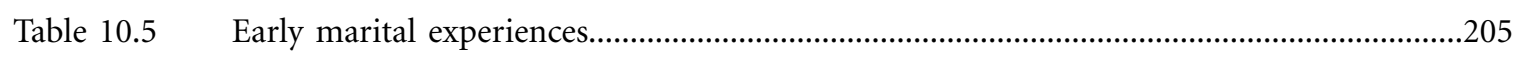

Table 10.6 Sexual experiences within marriage ..........................................................................206

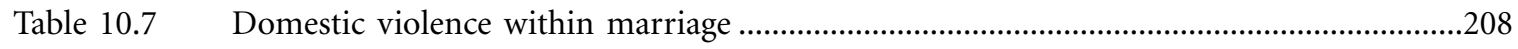

Table $10.8 \quad$ Extent of extra-marital sexual experiences..................................................................210

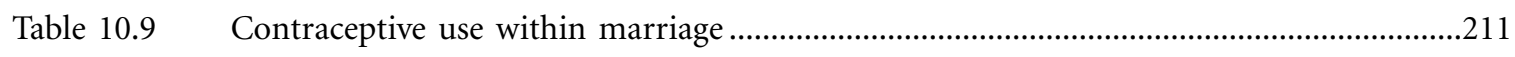

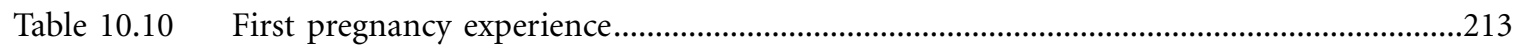

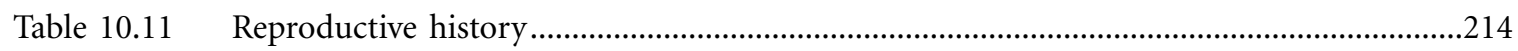

Table 10.12 Children ever born and surviving by selected background characteristics ......................215

Table 10.13 Wantedness of most recent pregnancy ...........................................................................216

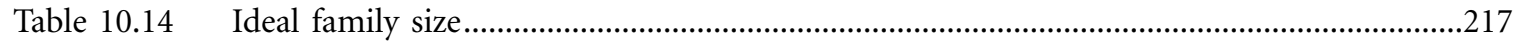

Table 10.15a Married young men's preferences for sons and daughters by selected

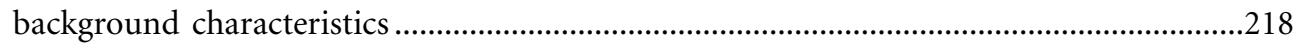

Table 10.15b Married young women's preferences for sons and daughters by selected

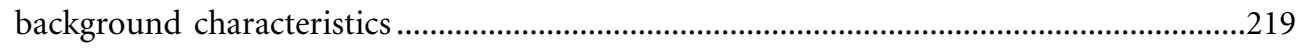

\section{Chapter 11: Health and health seeking behaviour}

Table 11.1 Substance use .223

Table $11.2 \quad$ Self-reported health problems .....................................................................................224

Table $11.3 \quad$ Reported symptoms or behaviours suggestive of mental health disorders.......................226

Table 11.4 Care and advice seeking for reported health problems ...................................................228

Table $11.5 \quad$ Hesitation to access contraceptive supplies ........................................................................230

Table 11.6 Attitudes towards pre-marital HIV testing and extent of HIV testing ............................231

\section{Chapter 12: Participation in civil society and political life}

Table 12.1 Awareness of and participation in government-and NGO-sponsored programmes ........234

Table 12.2 Participation in community-led programmes...............................................................237

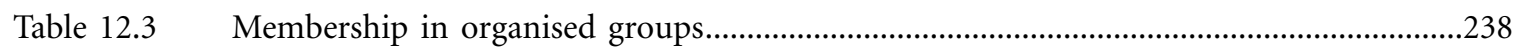

Table 12.4 Perceptions about actions taken by the panchayat in case of defiance of

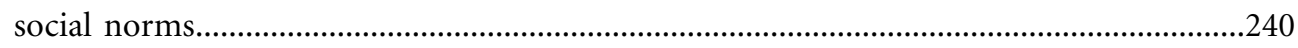

Table 12.5 Voting behaviour of eligible youth and perceptions about political matters ...................242

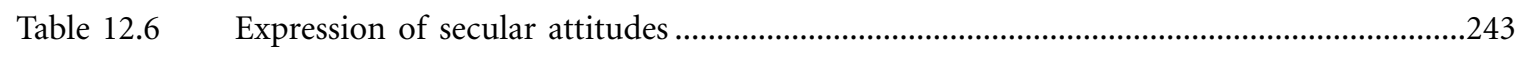

Table $12.7 \quad$ Physical fights in village/neighbourhood.....................................................................245

Table $12.8 \quad$ Perceptions about the leading problem facing youth ....................................................246 
Page No.

\section{Appendix B: Estimates of sampling errors}

Table B.1 List of selected variables for sampling errors, Bihar, 2007 ………………………............273

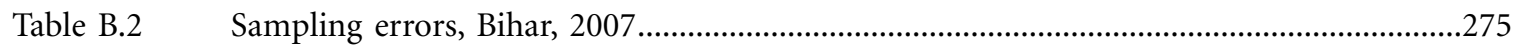

\section{Appendix C: Data quality tables}

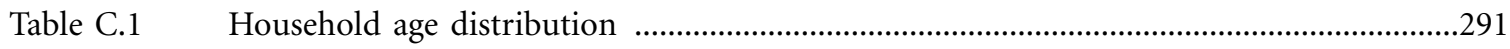

Table C.2 Single-year age distribution of eligible, selected and interviewed young men ..................292

Table C.3 Single-year age distribution of eligible, selected and interviewed young women ............293

Table C.4 Completeness of reporting .................................................................................................29 


\section{Figures}

Page No.

Figure 3.1 Percentage of youth who were in school at ages 12 and 15, according to residence, Bihar, 2007

Figure 3.2a Cumulative percentage of youth who had completed each year of education

(Classes 1 to 17 ), Bihar (combined), $2007 . .$.

Figure 3.2b Cumulative percentage of youth who had completed each year of education

(Classes 1 to 17), Bihar (urban), 2007

Figure 3.2c Cumulative percentage of youth who had completed each year of education

(Classes 1 to 17), Bihar (rural), 2007

Figure 3.3a Percentage of married youth who had discontinued schooling by class when discontinued and reasons for discontinuation, Bihar 2007.

Figure 3.3b Percentage of unmarried youth who had discontinued schooling by class when discontinued and reasons for discontinuation, Bihar, 2007.

Figure 4.1a Economic activity and schooling status among youth aged 15-24, by age, Bihar, 2007

Figure 4.1b Economic activity and schooling status among married men aged 15-29 and married women aged 15-24, by age, Bihar, 2007.

Figure 4.1c Economic activity and schooling status among unmarried men and women aged 15-24, by age, Bihar, 2007.

Figure 4.2 Percentage of youth who participated in domestic chores, according to residence, Bihar, 2007

Figure 4.3 Percentage of youth who ever attended a vocational training programme and percentage who were interested in participating in such programmes, according to residence, Bihar, 2007

Figure 5.1 Percentage of youth exposed to television, films, print media and the internet, Bihar, 2007

Figure 6.1 Percentage of youth reporting gendered socialisation experiences relative to an opposite-sex sibling/cousin, according to residence, Bihar, 2007

Figure 6.2 Percentage of youth reporting that their father and mother, respectively, would disapprove if they brought same- and opposite-sex friends home, according to residence, Bihar, 2007. 
Page No.

Figure 6.3a Percentage of youth who discussed various matters with their father, according to residence, Bihar, 2007.

Figure 6.3b Percentage of youth who discussed various matters with their mother, according to residence, Bihar, 2007.

Figure 6.4 Percentage of youth reporting at least one opposite-sex friend, according to residence, Bihar, 2007.

Figure 7.1 Percent distribution of youth by participation in decision-making on selected matters, Bihar, 2007

Figure 7.2 Percentage of youth allowed to visit selected places within and outside the village/neighbourhood unescorted, Bihar, 2007

Figure 7.3 Percentage of youth who expressed egalitarian gender role attitudes on selected issues, Bihar, 2007

Figure 7.4 Percentage of youth who believed wife beating is justified in selected situations, Bihar, 2007

Figure 8.1 Percentage of youth reporting awareness of selected sex- and pregnancy-related matters, according to residence, Bihar, 2007.

Figure 8.2 Percentage of youth who reported correct specific knowledge of oral pills and condoms, according to residence, Bihar, 2007.

Figure 8.3 Percent distribution of youth by awareness of medical abortion, according to residence, Bihar, 2007

Figure 8.4a Comprehensive knowledge of HIV/AIDS by educational level, Bihar, 2007 ....................149

Figure 8.4b Comprehensive knowledge of HIV/AIDS by wealth quintile, Bihar, 2007

Figure 8.5 Percentage of youth by awareness of HIV/AIDS, comprehensive knowledge about HIV/AIDS and awareness of STIs, Bihar, 2007

Figure 8.6 Percentage of youth who were aware of selected conditions under which abortion is legal, Bihar, 2007

Figure 8.7 Percentage of youth who received family life or sex education, according to residence, Bihar, 2007

Figure 8.8 Percentage of youth reporting knowledge of selected sexual and reproductive health matters according to whether they had or had not received family life or sex education, Bihar, 2007.

Figure 9.1 Percentage of youth who had made or received a "proposal" for romantic partnership formation and percentage who had an opposite-sex romantic partner, according to residence, Bihar, 2007 .168

Figure 9.2 Percentage of youth reporting experiences of physical intimacy and sex with a pre-marital romantic partner, Bihar, 2007

Figure 9.3 Percentage of youth reporting any pre-marital sexual experiences (in face-to-face interview or sealed envelope), according to residence, Bihar, 2007 
Figure 10.1 Percentage of youth reporting that their parents had ever sought their opinion on timing of marriage, according to residence, Bihar, 2007

Figure 10.2 Percent distribution of married youth by degree of acquaintance with future spouse before marriage, according to residence, Bihar, 2007

Figure 10.3 Percentage of married youth who reported receiving or giving dowry, according to residence, Bihar, 2007.

Figure 10.4 Percentage of married youth who reported spousal communication on selected topics, according to residence, Bihar, 2007

Figure 10.5 Percentage of married young women reporting experience of physical violence perpetrated by their husband and percentage of married young men reporting perpetration of physical violence against their wife, according to residence, Bihar, 2007

Figure 10.6 Percentage of married youth reporting lifetime and current use of contraceptive methods within marriage, Bihar, 2007.

Figure 11.1 Percentage of youth reporting symptoms/behaviours suggestive of mental health disorders in the month preceding the interview, according to residence, Bihar, 2007

Figure 12.1 Percentage of youth reporting awareness of and participation in government- and NGO-sponsored programmes in the three years preceding the interview, according to residence, Bihar, 2007.

Figure 12.2 Percentage of youth aged 20 or above who voted in the last election, according to residence, Bihar, 2007. 


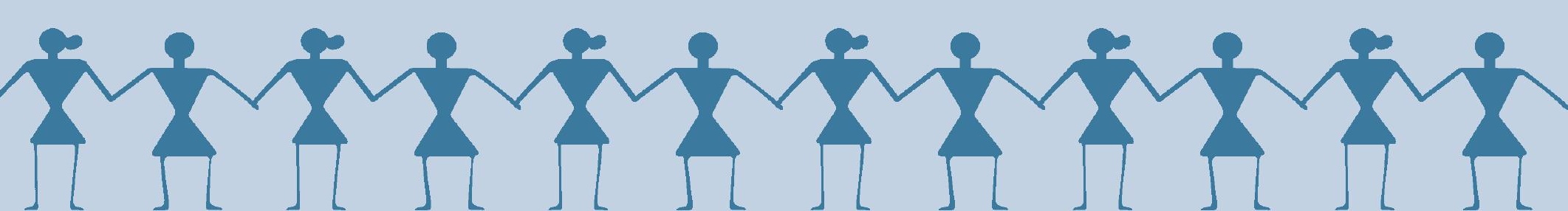




\section{Foreword}

The Government of India is committed to addressing the multiple needs of young people. The Eleventh Five Year Plan, the National Youth Policy, the National Population Policy 2000 and the National Rural Health Mission have all advocated special programmatic attention to addressing this population. National AIDS Control Programme, Reproductive and Child Health Programme and notably the National Adolescent Reproductive and Sexual Health Strategy provide the framework for a range of sexual and reproductive health services to be provided to youth.

Effective implementation of policies and programmes, however, has been difficult because of the lack of evidence on young people's situation and needs. The project Youth in India: Situation and Needs is intended to provide this evidence. Research has been conducted in a total of six states of India-Andhra Pradesh, Bihar, Jharkhand, Maharashtra, Rajasthan and Tamil Nadu. It provides a wealth of evidence on married and unmarried young women and young men from both rural and urban settings of each state. It covers almost every major dimension of youth life: education, work force participation, family life, sexual activity, marriage, health and civic participation. It provides state-level evidence on the magnitude and patterns of sexual and reproductive practices in and outside of marriage as well as related knowledge, decision-making and attitudes. Findings from the study provide important base-line indicators against which the long-term impact of programmes may be measured and will certainly go a long way in guiding policy, programmes and advocacy on youth issues.

This report focuses on findings from Bihar and is based on interviews with 8136 youth from all over the state. The report provides an enormous amount of information for the first time at the state level. The information will be useful to policy makers, programme implementers in government and non government sectors, rights activists and researchers alike who are committed to addressing the needs of Bihar's young generation. I appreciate the efforts put in by the International Institute for Population Sciences, Population Council and the technical advisory committee who guided the study.

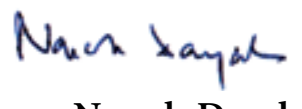

Naresh Dayal

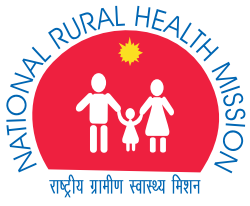




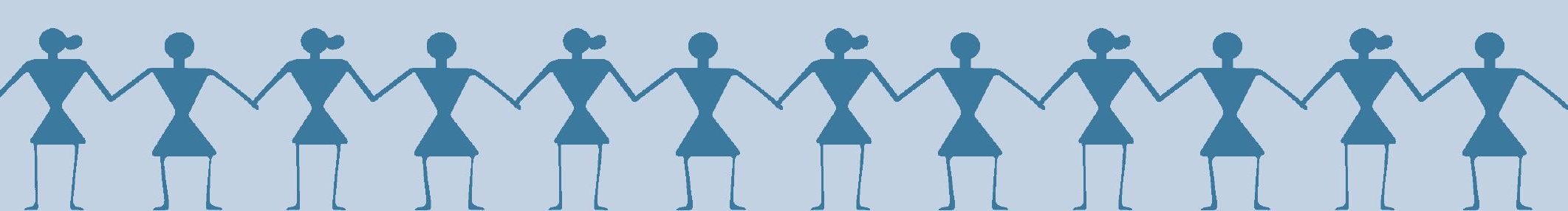




\section{Acknowledgements}

This report from the Youth in India: Situation and Needs study describes the transition to adulthood experienced by young men and women in Bihar. It covers multiple dimensions of their situation, ranging from education, work and marriage to sexual and reproductive health and behaviours. Evidence and recommendations contained in this report highlight, moreover, directions for programming and research that will enable youth in Bihar to make a successful transition to adulthood.

The Youth in India: Situation and Needs study has benefited immeasurably from the input of many. We are grateful to the Ministry of Health and Family Welfare, Government of India, for granting permission to conduct this study and to the Secretary, Shri Naresh Dayal, for his support throughout the project. We are also grateful to Shri G.C. Chaturvedi, Mission Director, National Rural Health Mission, Shrimati S. Jalaja, former Additional Secretary, Ministry of Health and Family Welfare, Shri V. K. Malhotra, Additional Director General, Dr. Rattan Chand, Chief Director, and Shri Rajesh Bhatia, Joint Director, Statistics Division, Ministry of Health and Family Welfare, Government of India for their support and guidance. We would also like to acknowledge the significant contribution of Shrimati S. Jalaja as chair of our Project Advisory Committee; and Shri S.K. Das, Director General, Central Statistical Organisation and former Additional Director General, Ministry of Health and Family Welfare, Government of India for support provided throughout the project.

We would also like to express our thanks to Shri C.K. Mishra, Principal Secretary Health and Shri Ravi Parmar, Mission Director, National Rural Health Mission, Government of Bihar for their support for the study in Bihar. We are thankful to the Director, State Institute of Health and Family Welfare (SIHFW), Patna for providing the logistic arrangement during the survey. The smooth functioning of fieldwork was due, in large part, to the support of the state health department, and we would like to acknowledge the contribution of all, including staff at the district headquarters level and primary health centre level.

The study received generous financial support from the John D. and Catherine T. MacArthur Foundation and the David and Lucile Packard Foundation; we are grateful for their financial support as well as for useful comments and suggestions provided by Lester Coutinho, Don Lauro and Lana Dakan of the Packard Foundation, and Poonam Muttreja and Dipa Nag Chowdhury of the MacArthur Foundation over the course of the project.

We would like to acknowledge the contribution of the late P.N. Mari Bhat, Director, International Institute for Population Sciences, during 2005-07. His input in the design and implementation of the study and in ensuring the progress of this challenging project contributed immensely to the quality of the study.

We are grateful to our Project Advisory Committee members for their input, which ensured that our study did indeed address all the key issues that require policy and programme attention. We appreciate their contribution both during meetings of the committee and in various one-on-one discussions during the course of the project. 
We acknowledge with gratitude the contribution of members of our Technical Advisory Committee. We were privileged that individuals with a wide range of expertise, from youth health and development to survey and qualitative approaches and ethics in research, agreed to serve as technical committee members. Our technical advisory group-Shalini Bharat, P.M. Kulkarni, Arvind Pandey, Pertti Pelto, T.K. Roy and Leela Visaria-supported the project from conceptualisation to completion. Their guidance at all stages of the study was central in enabling us to confront methodological, ethical and analytical challenges that arose over the course of the study and is gratefully acknowledged. We would like to record our deep appreciation, moreover, to P.M. Kulkarni for giving us so generously of his time in working through problems encountered during the design and implementation phases.

Several specialist group meetings were held over the course of the project that focused on study design, instrument development, qualitative component development and analysis, tabulation planning and report review;; several others who did not attend these meetings provided extensive comments on one or more of these issues. We are grateful to all these specialists-Dinesh Agarwal, Mallika Alexander, John Cleland, Nimesh Desai, Lalit Deshpande, Sudha Deshpande, Kamla Gupta, M.E. Khan, Sumati Kulkarni, Shiva Kumar, Cynthia Lloyd, S. Niranjan, Sulabha Parasuraman, Vikram Patel and Sunayana Walia-for their valuable contribution. John Cleland provided inputs at almost every phase of this study—design, instrument development and analysis—and his thought-provoking suggestions are gratefully acknowledged. In particular, we would like to acknowledge our external peer reviewers, P.M. Kulkarni and Leela Visaria, for their thoughtful and careful assessments of an earlier draft of this report.

Given that our study probed a number of highly sensitive matters, including young people's sexual and reproductive behaviours, it was an ethical imperative that those in need of information or services would be provided appropriate materials and referrals, respectively. We are grateful to those who provided services to our respondents in need and would like to acknowledge the TARSHI (New Delhi) whose materials (Neeli Kitab) were distributed to the young men and women during the survey.

The Office of the Registrar General, New Delhi and the State Census Office, Bihar generously provided the project with information and maps pertaining to the 2001 Census. Their role in enabling the study to implement its sample design is gratefully acknowledged.

We would like to express our appreciation and gratitude to the International Institute for Population Sciences and the Population Council and all their staff members who helped in small and big ways in making this report possible. T.K. Roy and the late P.N. Mari Bhat as Directors of the International Institute for Population Sciences, and G. Rama Rao and S. Lahiri as officiating Directors, oversaw the activities of the project from the Institute's perspective. Saroj Pachauri, Regional Director, South and East Asia Office, Population Council oversaw the project on behalf of the Population Council. Their insight and supervision are gratefully acknowledged.

A number of research officers, consultants and administrative staff members, both at the International Institute for Population Sciences, Mumbai, and at the Population Council, New Delhi, contributed to the smooth implementation of the study as well as the data management, analysis and report writing phases. We would like to thank those responsible for the administrative aspects of this project. At the International Institute for Population Sciences, we would like to acknowledge the Registrar, Accounts Officer, Computer Centre In-Charge and Library In-Charge; and Jeba Kumar, Pranita H. Dalvi, Usha D. Sonawane, Seema Jadhav and Avadesh Kumar who were responsible for the smooth functioning of the project. From the Population Council, we are grateful to Komal Saxena, M.A. Jose, Anil Paul and Ashutosh Mishra who ably managed the administrative, financial and IT aspects of the project. We are grateful to our Senior Research Officers, Research Officers and 
Assistant Research Officers for their excellent supervision of fieldwork and data management as well as for their support in analysing the data and preparing the tables for this report. Abhishekh Kumar, Lucky Singh, and Prashant Kumar Singh from the International Institute for Population Sciences, and Francis Zavier and Shilpi Rampal from the Population Council, did a painstaking job in ensuring the accuracy of data presented in the report and their contribution is gratefully acknowledged. Shanti Menon and Komal Saxena edited and ably managed the preparation of this report; we are grateful to them for their editorial contribution and meticulous attention to detail, which have made the report more readable and precise.

We would also like to acknowledge with thanks the contribution of our team of young and enthusiastic interviewers in eliciting information on difficult topics with sensitivity and skill. They were required to overcome discomfort when asking about intimate experiences and were required to record many disturbing experiences narrated by youth, which they did professionally and empathetically. It was due to their skill and ability to engage with youth in non-judgmental ways that this study was so well received by youth in Bihar.

We would also like to record our appreciation of the support and kindness of the people in the villages and urban neighbourhoods in which we conducted our study, and specifically panchayat members and community leaders. While initially sceptical about the study, community members opened their homes to us and acknowledged the importance of this study for the health and development of future generations. Indeed, despite the sensitive issues covered, not a single study community refused our field teams entry. The trust and support of the people are gratefully acknowledged.

Finally, and most importantly, we would like to thank the young women and men from Bihar who welcomed us, generously gave of their time and shared so many intimate details of their lives with us. We hope that the evidence generated in this report will be useful in influencing the design and content of programmes intended to meet their multiple needs and enable them to make a safe transition to adulthood.

Rajib Acharya

Shireen Jejeebhoy

K. G. Santhya

Population Council, New Delhi
Usha Ram

S. K. Mohanty

Abhishek Singh

F. Ram

International Institute for

Population Sciences, Mumbai 


\section{Executive summary}

The Youth in India: Situation and Needs Study (referred to as the Youth Study), implemented by the International Institute for Population Sciences, Mumbai and the Population Council, New Delhi is the first-ever sub-nationally representative study conducted to identify key transitions experienced by married and unmarried youth in India. Young people (aged 10-24) constituted almost 315 million and represented $31 \%$ of the Indian population in 2001. Not only does this cohort represent India's future in the socio-economic and political realms, but its experiences will largely determine India's achievement of its goal of population stabilisation and the extent to which the nation will be able to harness its demographic dividend. While today's youth are healthier, more urbanised and better educated than earlier generations, social and economic vulnerabilities persist. In the course of the transition to adulthood young people face significant risks related to sexual and reproductive health, and many lack the knowledge and power to make informed sexual and reproductive choices.

In recognition of the importance of investing in young people, several national policies and programmes formulated since 2000, including the National Population Policy 2000, the National Youth Policy 2003, the Tenth and Eleventh Five-Year Plans, the National Adolescent Reproductive and Sexual Health Strategy and the National Rural Health Mission, have underscored a commitment to addressing the multiple needs of this group in India. Effective implementation of both policies and programmes, however, has been handicapped by the lack of evidence on young people's situation and needs. Currently available evidence is limited, at best, and comes largely from small-scale and unrepresentative studies.

The Youth Study focused on married and unmarried young women and unmarried young men aged 15-24 and, because of the paucity of married young men in the younger ages, married men aged 15-29 in both rural and urban settings. It collected information pertaining to key transitions experienced by youth, including those related to education, work participation, sexual activity, marriage, health and civic participation; the magnitude and patterns of young people's sexual and reproductive practices within and outside of marriage as well as related knowledge, decision-making and attitudes.

The Youth Study comprised three phases, and included both a survey and qualitative data gathering exercises prior to and after the survey. The study was conducted in a phased manner in six states of India: Andhra Pradesh, Bihar, Jharkhand, Maharashtra, Rajasthan and Tamil Nadu.

This report focuses on findings from the survey conducted in Bihar. The survey was undertaken between January and August 2007. During the survey, 9,684 young people were contacted, of which a total of 8,136 married and unmarried young women and men were successfully interviewed. 


\section{Characteristics of the household population}

A total of 30,888 households were selected for interview. Among these, interviews were successfully completed in 28,205 sample households, and 156,197 individuals, who were usual residents in these households, were enumerated. The age distribution was typical of a high fertility population, with a larger proportion of the population in the younger age groups than in other age groups. Indeed, the virtually identical proportions of the population aged 0-4 observed in both the 2001 census and the Youth Study suggest unchanging levels of fertility. With regard to the youth population, the distribution suggests that at the time of the survey, $13 \%$ of the population was aged $10-14$ years, $9 \%$ was aged $15-19$ years and $7 \%$ was aged $20-24$ years. A total of $16.5 \%$ of the population was aged $15-24$ years.

Overall, the sex ratio of the de jure population of the state was 1,043 females per 1,000 males, considerably higher than that observed in the 2001 Census (919); a finding that may be attributed to increased employment-related out-migration of single young men in the state. The child sex ratio of the state stood at 935 females per 1,000 males aged 0-6, close to that reported in the 2001 Census (942).

The educational profile of the household population highlights low levels of educational attainment in the state: over half $(52 \%)$ of the population aged 6 years and above had no formal education. Notably, as many as $65 \%$ of females compared to $40 \%$ of males, and as many as $55 \%$ of the rural population compared to $30 \%$ of the urban population had never been to school. Findings also indicate that just $6 \%$ of the total population had received 12 or more years of education, thus reaffirming the low levels of educational attainment in the state.

Housing characteristics of the surveyed population underscore poor living conditions among the majority of the state's population. Overall, 53\% of all households lived in kachcha houses (constructed from mud, thatch or other low-quality materials), 25\% lived in semi-pucca houses (constructed using a mix of low- and high-quality materials) and $22 \%$ lived in pucca houses (constructed entirely from cement, masonry or other high-quality materials). Only $14 \%$ of households had electricity, including $71 \%$ of urban households and $9 \%$ of rural households. The vast majority of households (93\%) reported that their main source of drinking water was either piped water, water obtained from a hand-pump or a covered well. Access to a toilet facility of any kind was reported by just one-sixth of all households.

The distribution of households by wealth quintiles shows a stark rural-urban divide: two-thirds of urban households were in the wealthiest (fifth) quintile; in contrast, only one-sixth of rural households were in this quintile. Likewise, one-fifth of rural households were in the poorest (first) quintile of the index compared to only $3 \%$ of urban households.

\section{Situation of youth}

As mentioned earlier, a total of 8,136 youth were interviewed. Age profiles suggest that larger proportions of young men and women were concentrated in the 15-19 year age group than in the 20-24 year age group (60-62\% compared to 38-40\%). Moreover, the unmarried were clearly younger than the married. Religious distributions show that $86 \%$ of youth were Hindu and $14 \%$ were Muslim. Caste-wise distributions show that $14-20 \%$ of youth belonged to general castes, $20-21 \%$ to scheduled castes and $58-64 \%$ to other backward castes. About four in five youth reported that both parents were surviving. For those with just one parent surviving, this parent was more likely to be the mother (9-10\%) than the father $(5-6 \%)$. Finally, $1-2 \%$ reported that neither parent was alive. 


\section{Education}

While youth were better educated than the population at large in Bihar, schooling was far from universal among them. As many as one in six young men and half of young women had never attended school. Findings show, moreover, that young women in rural areas and married young women in general were particularly disadvantaged; over half of rural young women and almost two-thirds of married young women had never been to school. Leading reasons for never attending school among young men and women were economic (child required for work on the family farm/business or for outside wage earning work, or the family could not afford school-related expenses) and attitudes and perceptions (such as, that education was not necessary or that children were not interested). Housework-related reasons (required for care of siblings or housework) were additionally an important reason inhibiting young women from being enrolled in school.

Not only was school enrolment limited, but school completion rates were also low among young people. Findings show that of those who had completed Class 1, declines in class completion took place as early

as Class 3; only $95 \%$ and $93 \%$ of young men and women, respectively, who had completed one year of schooling had gone on to complete Class 3. Declines in class completion became progressively steeper as the level of schooling increased, with differences between young men and women widening as the level of schooling increased. For example, notable declines occurred between Classes 5 and 6, suggesting that many youth discontinued their education even without completing elementary education. Indeed, just $30 \%$ of young men and $13 \%$ of young women had completed high school in the state.

What is notable is that, at the time of interview, over half of unmarried young men and fewer than half of unmarried young women (and very few married) were still in school or college.

Economic issues and negative attitudes and perceptions about the importance or relevance of education were leading reasons for discontinuation, irrespective of the level at which schooling was discontinued. Among young women, such factors as school-related factors (academic failure, distance to school, poor school quality and infrastructure) and housework responsibilities were also leading factors behind discontinuation at all levels, and transition to adult roles became increasingly important reasons as the level at which schooling was discontinued increased. Of note, particularly, is that almost one in four and two in five married young women who discontinued schooling at primary and high school levels, respectively, reported doing so in order to marry.

The gender divide observed in school enrolment, attendance and reasons for discontinuation was evident even in the type of educational facility that youth attended. While young men by and large attended co-educational facilities at all levels of education, young women were less likely to attend a co-educational facility at higher levels of schooling. Similar gender differences were evident in terms of additional investment in schooling made, particularly in terms of private tuition; for example, more young men than women reported that they had taken private tuition, especially among those who were attending a school/college at the time of the interview.

The majority of youth at all levels attended government schools or colleges. Moreover, no apparent gender differences were observed in terms of the type of school—government or private-in which youth were enrolled.

Findings also show that amenities available within the educational facility attended differed vastly between youth who were still in school and those who had discontinued their education. For example, youth still studying were more likely to report the availability of water, toilets, playgrounds and libraries than were those 
who had discontinued. Schooling experiences also differed somewhat among those who had discontinued schooling and those who were studying at the time of the interview. While regular attendance did not differ much, youth who were continuing their education were considerably more likely to report private tuition, somewhat less likely to perceive academic workload to be heavy and more likely to have passed the last examination for which they had appeared.

\section{Work}

Work profiles suggest that three-quarters of young men and about one-half of young women had ever engaged in paid or unpaid work. Indeed, almost all married young men and two-thirds of unmarried young men had done so, compared with over half and about two-fifths of married and unmarried young women, respectively. Likewise, larger proportions of youth in rural than urban areas had ever worked. While the majority of youth were engaged in paid work, considerable proportions of young men (38\%) and women (25\%) reported unpaid work on the family farm or business. Economic activity was often initiated at an early age: over one in three young men and women reported initiating work as children (before age 15). Data on work participation in the 12 months prior to the interview indicate that the majority of young men (64\% of the unmarried and $97 \%$ of the married) and a substantial proportion of young women $(36 \%$ and $38 \%$, respectively) had engaged in paid or unpaid work at some point in the 12 months preceding the survey. The majority of young men (64\%) who worked in the year prior to the interview had done so for the major part (at least six months) of the year. In contrast among young women, over two-fifths had done so.

Findings also show substantial levels of unemployment among young men (22\%) and women (36\%). Unemployment tended to be considerably higher among unmarried than married young men; but roughly similar among married and unmarried young women. Findings also indicate that unemployment was particularly high among young women in urban areas, particularly the married. Unemployment was also exceptionally high among the educated and economically better off.

Youth were clearly interested in acquiring skills that would enable employment generation; two in three young men and four in five young women reported interest in vocational skills training. Although more unmarried than married youth expressed interest in attending vocational training programmes, it is notable that over half of married men and three-quarters of married women were interested in developing vocational skills. However, far fewer-just $14 \%$ of young men and $9 \%$ of young women-had attended even one vocational training programme.

\section{Media exposure}

Large proportions of youth were exposed to the media, typically newspapers, magazines or books ( $89 \%$ of young men and $81 \%$ of young women with five or more years of education), films ( $89 \%$ of all young men and $59 \%$ of all young women) and television ( $78 \%$ of all young men and $48 \%$ of all young women). Exposure to the internet was reported by many fewer (6\% of young men and $3 \%$ of young women with five or more years of education).

Findings also suggest that one in four young men and $4 \%$ of young women watched pornographic films, and of these, about two-fifths reported having viewed such films sometimes or frequently. One in five young men and $6 \%$ of young women accessed pornographic books and magazines, and of these, one-third of young men and half of young women reported that they accessed these materials sometimes or frequently. Of those exposed to the internet, one in three young men and one in ten young women had accessed pornographic 
materials on the internet. Finally, between half and three-fifths of young men and women acknowledged the influence that media have on youth behaviours.

\section{Socialisation experiences and communication with parents}

Findings suggest the gendered nature of socialisation of youth. For example, responses from both young men and women indicate that unequal gender norms regarding freedom of movement prevailed in most study households, with three-fifths of young men acknowledging that they had more freedom to go out than their sisters or female cousins did, and almost four-fifths of young women agreeing that they had less freedom to go out than their brothers or male cousins. Moreover, although most young men's responses to questions related to housework expectations suggest a gender equal socialisation experience, it is notable that about three-quarters of young women reported that they were expected to do more housework than their brothers or male cousins. Likewise, while parents appeared to control both young men's and women's social interactions, particularly those involving members of the opposite sex, young women were more likely than young men to experience such restrictions. For example, $79-80 \%$ of young men and $97 \%$ of young women reported expecting parental disapproval if they brought an opposite-sex friend to their home.

Nonetheless, it is notable that while young women were brought up with more restrictions, parents appeared to place considerable restrictions on young men's interactions as well, including with same-sex peers.

Findings regarding communication with parents on issues relevant to youth-such as school performance, friendships, being teased or bullied, physical maturation, romantic relationships and reproductive processes-reiterate those from other studies, showing that such communication was far from universal. Moreover, sensitive topics—such as romantic relationships, reproduction and contraception, among all youth, and even adolescent body change issues among young men-were rarely discussed with either parent.

That parent-child communication was restricted was also evident from responses to questions probing the most likely confidante on a range of topics from taking a job to boy-girl relationships. While parents were mentioned as leading confidantes on non-sensitive topics such as taking a job, they were rarely cited as leading confidantes on more sensitive matters. While young women identified their mothers as the most likely confidante on such matters as menstrual problems and experience of teasing, young men rarely identified a parent as a leading confidante on matters relating to nocturnal emission or swapnadosh. And neither young men nor women identified a parent as a leading confidante on boy-girl matters.

Young people's family lives were marked by violence, both experienced and witnessed. One in five youth had observed their fathers beating their mothers. Many respondents reported experiencing beating by a parent during adolescence; over half of young men and $11 \%$ of young women reported such experiences.

\section{Peer networks and interaction}

Growing up was associated with close peer networks. Almost all youth reported having same-sex friends. Opposite-sex peer networks were less common but nonetheless reported by $16 \%$ of young men and $5 \%$ of young women. Interaction with friends tended to be restricted to activities such as chatting and engaging in sports, although young men did tend to report studying and going on picnics or to films. Indeed, findings suggest that youth derived an important measure of support from their peer networks on sensitive matters: friends were by far the leading confidante on boy-girl relationships for both young men and women and on nocturnal emission for young men. 


\section{Agency and gender roles}

Findings clearly highlight young women's extremely limited agency. For example, just one in four young women reported independent decision-making on all three issues explored in the survey, namely, decisions on choice of friends, spending money and purchase of clothes. Likewise, freedom of movement even within the village or neighbourhood was not universal among young women; only about two in three young women had the freedom to visit locations within their own village or neighbourhood unescorted. Moreover, just one in ten young women reported having the freedom to visit at least one place outside the village or neighbourhood and a health facility unescorted. Access to and control over financial resources tended to be limited among young women; just over two in five reported some savings and one in twenty owned a bank or post office savings account. Of those who owned an account, about two-fifths operated it themselves.

Also notable from the findings is the striking gender divide in all these dimensions of young people's agency. Young women were far more disadvantaged than young men in terms of decision-making autonomy and mobility. Likewise, although young women were more likely than young men to have money saved ( $44 \%$ and $20 \%$, respectively), they were less likely than young men to own a bank or post office savings account (5\% and $11 \%$ ) and to operate these accounts themselves (38\% and $77 \%$ of those who had an account).

While young men were clearly not as disadvantaged as young women, findings indicate that many young men were unable to exercise agency in their everyday lives. For example, only $46 \%$ of young men reported independent decision-making on all three issues explored in the survey. Similarly, young men's mobility was far from universal; for example, just $48-56 \%$ unmarried young men were allowed to visit a place of entertainment or to attend a programme conducted outside their village or neighbourhood unescorted, and two in three were allowed to visit a health facility unescorted.

Although over two-fifths of young men and about three-fifths of young women justified wife beating in at least one situation, relatively large proportions of youth espoused egalitarian gender role attitudes on other issues explored. Even so, it is notable that young men were consistently more likely than young women to report unequal gender role attitudes on these issues.

\section{Awareness of sexual and reproductive health matters}

Findings underscore young people's extremely limited awareness of most sexual and reproductive matters, ranging from how pregnancy occurs to contraception, HIV and safe sex practices. For example, just 27-33\% of youth were even aware that a woman can get pregnant at first sex. Just $87 \%$ of young men and $47 \%$ of young women had ever heard of HIV/AIDS and many fewer-11-12\% of youth-reported awareness of STIs other than HIV/AIDS. Even on topics about which young people were generally aware, awareness was far from universal. For example, only $72 \%$ of young men and 58\% of young women knew that 18 years was the legal minimum age at marriage for females. Findings also show that misconceptions about sexual and reproductive health topics were common. For example, two in five youth believed that a woman has to bleed at first sexual intercourse. Likewise, 5-9\% of youth reported that condoms can disappear into the woman's body and $62-66 \%$ of youth reported that they were unsure about the issue.

Findings, moreover, show that in-depth understanding was limited even on topics about which young people were generally aware. For example, while $93-99 \%$ reported awareness of at least one contraceptive method, in-depth awareness of condoms and oral contraceptives, the methods most familiar to youth, was reported by just $62 \%$ and $26 \%$ of young men and $30 \%$ and $48 \%$ of young women, respectively. Likewise, while $87 \%$ of young men and $47 \%$ of young women had heard of HIV, only $28 \%$ of young men and $15 \%$ of young women were fully aware of HIV and its transmission routes. 
Not surprisingly, youth reported few reliable sources of information about sexual matters or contraception. Indeed, $16 \%$ of young men and $44 \%$ of young women reported that they had never received any information on sexual matters (prior to marriage among the married). Friends and the media were leading sources of information on both issues for both young men and women. Neither of these is necessarily a reliable source of information. For young women, in addition, family members were a leading source of information; they were rarely cited as a source of information by young men. Fewer than $6 \%$ of unmarried and hardly any married youth cited teachers as sources of information on sexual matters or contraception. Health care providers were important sources of information on contraception just for married young men; they were far less likely to have provided information to the unmarried and even to married young women. In short, health care providers, teachers and family members - often assumed to be a more reliable source of information than peers or the media-were infrequently and inconsistently cited as sources of information on sensitive topics such as sexual matters and contraception by young people.

Few youth had attended family life or sex education programmes either in or outside the school setting — just 7\% of young men and 3\% of young women. Despite this, youth were overwhelmingly in favour of the provision of family life or sex education to young people; while young men preferred to receive this education from a professional (health care provider, teacher and so on), young women preferred to obtain this education from parents or siblings. Findings suggest, moreover, that youth who had undergone family life or sex education were indeed more knowledgeable about sexual and reproductive matters than those not exposed to this education.

\section{Pre-marital romantic relations}

Findings confirm that despite norms prohibiting pre-marital opposite-sex mixing, opportunities do exist for the formation of pre-marital romantic relations. Indeed, significant minorities of young men and women had received or made a "proposal" for a romantic relationship (20\% of young men and $12 \%$ of young women), and had been involved in a romantic partnership (17\% of young men and $5 \%$ of young women). Patterns of pre-marital romantic partnerships suggest that where partnerships occurred, they were initiated at an early age and were usually hidden from parents but not from peers. Notable disparities in expectations of a longer-term commitment emerged that show that young women were considerably more likely than young men to have expected a romantic relationship to lead to marriage. However, the experiences of the married suggest a disconnect between intentions and reality: while $47 \%$ and $84 \%$ of married young men and women, respectively, reported the intention to marry their pre-marital partner, just $4 \%$ of young men and $38 \%$ of young women had done so.

There was a clear progression in reported physical intimacy and sexual experience with romantic partners. For example, while $87 \%$ of young men had held hands with a romantic partner, two-fifths had engaged in sexual relations. Among young women, while $70 \%$ had held hands with a romantic partner, almost one-third had engaged in sexual relations with this partner. Partner communication and negotiation regarding safe sex were rare, and the vast majority had engaged in unprotected sex. Over one in five young women who had engaged in sexual relations with a romantic partner reported that their opposite-sex romantic partner had forced them to engage in sex the first time.

\section{Pre-marital sexual experiences in romantic and other relationships}

One in seven (14\%) young men and 3\% of young women reported the experience of pre-marital sex within romantic and/or other partnerships. In general, first pre-marital sex took place earlier among young men 
than young women, and among rural than urban youth. Moreover, initiation into pre-marital sexual activity increased as young people transitioned from early to late adolescence and further as they transitioned into young adulthood.

While sex with a romantic partner characterised pre-marital experiences for many of the sexually experienced, findings suggest that young men, but not young women, also engaged in sex in other contexts; other partners reported by young men included, mainly, sex workers, married women and casual partners. Many of the pre-marital sexual experiences reported by youth were risky, for example, one-fifth of young men and onequarter of young women reporting pre-marital sex had engaged in sex with more than one partner. Moreover, consistent condom use was extremely limited-only $6 \%$ of young men and $2 \%$ of young women reported condom use in all pre-marital encounters reported.

We acknowledge that youth, especially young women, may not report sexual experience in a survey situation. Hence, the Youth Study supplemented a series of direct questions with an opportunity to report sexual experience in an anonymous format. In total, direct questioning supplemented by self-reporting in an anonymous format provided considerably higher estimates of sexual experience among young men than did face-to-face questioning alone or anonymous third-party reporting of peer behaviours among young men. However among young women, anonymous third-party reporting provided slightly higher estimates than self-reports.

\section{Transition to marriage and early married life}

Findings indicate that while most young men preferred to marry in young adulthood, almost one-quarter of young women preferred to marry before 18 years and as many as three-fifths preferred to marry before age 20 , indicating an adherence to social norms around early marriage even by youth. Reiterating the fact that early marriage continues to characterise the lives of many young women and to a certain extent the lives of young men as well, findings show that as many as $46 \%$ of young women aged 20-24 years were married before age $15,77 \%$ before age 18 and $87 \%$ before age 20 . Even though early marriage was less prevalent among young men, $13 \%$ of those aged $20-24$ years were married before age 18 and $31 \%$ before age 20 .

Not only did marriage occur at young ages but it was also often arranged without the participation of the young people themselves, particularly young women. Almost all youth reported arranged marriages. As many as one in ten young men and over two in five young women reported that their parents did not seek their approval while determining their marriage partners. Hence, not surprisingly, reported pre-marital acquaintance was extremely limited. Just $4-7 \%$ of youth reported that they had ever had a chance to meet and interact with their spouse-to-be alone prior to marriage. Over $90 \%$ of married youth reported that they had met their spouses for the first time on the wedding day. Compounding the lack of pre-marital acquaintance was the lack of awareness of what to expect of married life, reported by over three-fifths of young men and four-fifths of young women.

Dowry characterised the marriages of two-thirds of young men and women, in spite of the existence of laws against dowry. Findings also show that families of urban youth appeared no less likely to conform to traditional practices, such as payment of dowry, than their rural counterparts.

Reports of marital life suggest that although spousal communication was reported on several issues, it was far from universal, and that marital life was marked by violence for large proportions of women. For example, couple communication on most topics was reported by over three in four young men and women, yet communication on contraceptive use was reported by somewhat fewer ( $72 \%$ of young women and $38 \%$ 
of young men), clearly undermining married young people's ability to adopt protective actions. Physical violence and forced sex within marriage were reported by considerable proportions of youth. For example, $30 \%$ of young women reported that they had ever faced violence perpetrated by the husband and a similar percentage of young men reported perpetrating violence on their wives. Recent violence was likewise reported by one-quarter of young women and one-fifth of young men. Sexual violence was widespread. Indeed, $49 \%$ of young women reported that their first sexual experience within marriage had been forced. Overall, over half of young women (54\%) reported ever being forced to engage in sex with their husbands; as many as one in four young men reported forcing their wives to engage in sex. Recent sexual violence was reported by one in four young women and one in ten young men.

While the Youth Study did not explore extra-marital sexual experiences in detail, the available data indicate that $4 \%$ of young men reported an extra-marital sexual encounter. In contrast, hardly any young women reported an extra-marital sexual encounter.

\section{Contraceptive practice and pregnancy experience}

Contraceptive use at any time within marriage was limited, reported by $23 \%$ of young men and $20 \%$ of young women. Moreover, just $12 \%$ of young men and women reported current use of contraception. Among contraceptive methods currently used, condoms and female sterilisation were most likely to be reported. Few young people practised contraception to delay the first birth-just $8 \%$ of young men and $4 \%$ of young women. Not surprisingly, pregnancy typically occurred within a year of marriage for almost two-fifths of young women and half of young men who reported that they or their wives had been pregnant at least once. Moreover, large proportions of youth reported experiencing unintended pregnancy. For example, of those women who were not pregnant at the time of the interview and of those men whose wives were not pregnant at the time of the interview, $33 \%$ of young women and $23 \%$ of young men reported that the last pregnancy was mistimed or unwanted.

Circumstances of the first birth suggest that institutional delivery and skilled attendance at delivery were extremely limited: only $23-25 \%$ of first births were delivered institutionally and $35-42 \%$ reported delivery by a skilled attendant.

Findings also show that although most youth wanted one child of each sex, son preference was evident. Over one-third of young men and over two-fifths of young women preferred to have more sons than daughters. In contrast, just $2-4 \%$ preferred to have more daughters than sons.

\section{Substance use}

Findings show that substantial proportions of young men reported the consumption of tobacco and alcohol; two-fifths of young men reported tobacco consumption and one-sixth reported alcohol consumption. Drug use was reported by less than $2 \%$ of young men. Few young women reported that they consumed any of these substances.

\section{Health seeking behaviour}

Although youth is a generally healthy period of life, significant minorities reported experiencing general, mental, and sexual and reproductive health problems in the period preceding the interview. Over one-quarter of youth had experienced high fever, and $8 \%$ of young men and $22 \%$ of young women reported 
the experience of symptoms of genital infection in the three months preceding the interview. Moreover, $11 \%$ of young women reported menstrual problems; at the same time, one-fifth of young men reported anxiety about nocturnal emission. Finally, responses indicative of mental disorders were reported by $16 \%$ of young men and $9 \%$ of young women.

As far as care seeking for general and sexual and reproductive health problems was concerned, patterns varied by type of problem. While the large majority of those experiencing high fever sought care, many fewer sought care for sexual and reproductive health problems. Findings also show that care seeking for health problems tended to be more limited among young women than men, irrespective of the type of problem experienced. Of those who sought treatment, the majority sought advice or treatment from a private facility or provider, irrespective of the type of problem. However, it is notable that over one-quarter of youth who sought care for genital infections or menstrual problems used home remedies or the services of traditional or untrained providers. In the case of anxiety about nocturnal emission, the majority of young men preferred to seek advice from peers.

Findings suggest that youth were uncomfortable about seeking sexual and reproductive health services. For example, many youth-more women than men—reported that they would indeed find it difficult to approach a health care provider or a pharmacy/medical shop for contraceptives.

Finally, small minorities (1-2\%) reported that they had undergone HIV testing. Youth were, however, overwhelmingly in favour of pre-marital HIV testing.

\section{Participation in civil society and political life}

Findings highlight extremely limited participation by youth in civil society. Although a number of programmes are held to build youth skills, very few youth (8-15\%) reported familiarity with either government or NGO-sponsored programmes organised at the community level in which youth could participate. Even fewer youth-7\% of young men and $2 \%$ of young women-reported participating in any such programme. Almost one-quarter of young men $(23 \%)$ and $4 \%$ of young women reported that they had participated in community-sponsored programmes such as cleanliness drives, celebration of festivals and national days, and so on. Finally, just $8 \%$ of young men and $2 \%$ of young women reported membership in organised groups.

Participation in political processes was also far from universal. Among those eligible to vote, $65 \%$ of young men and $51 \%$ of young women had cast their votes in the most recent election. Four-fifths of youth perceived that one could vote freely and without fear and pressure. However, large proportions of youth, particularly young men- $69 \%$ of young men and $35 \%$ of young women—reported disillusionment with the commitment of political parties to work for change at the community level.

Expressions of secular attitudes varied. Over $90 \%$ of young men and over $80 \%$ of young women reported that they mixed freely with individuals of different religions and castes. However, only $64 \%$ of young men and $38 \%$ of young women would eat together with a person of a different caste or religion, just $35 \%$ of young men and $46 \%$ of young women would talk to a person who has had an inter-caste marriage and only $32 \%$ of young men and $42 \%$ of young women agreed that it was best to tolerate rather than punish someone who had shown disrespect towards their religion.

Considerable proportions of young men and women acknowledged that physical fights among young men and also among young women did occur in their villages or urban neighbourhoods; one in seven young men and $4 \%$ of young women reported that they had been involved in a physical fight in the year preceding the interview. 
The four leading problems facing youth expressed by both young men and women were unemployment, poverty, lack of amenities and lack of educational opportunities. However, young people's perceptions of the leading problems facing youth varied enormously by sex. Among young men, the majority reported difficulty in finding employment as the leading problem, followed by concerns about poverty, lack of educational opportunities and lack of amenities or infrastructure. In contrast, the leading problem expressed by young women was lack of amenities and infrastructure, and to a lesser extent, poverty, lack of opportunities for education and difficulty in finding employment.

\section{Recommendations for programmes}

Findings presented above underscore the fact that youth face numerous challenges while making the transition to adulthood. These challenges call for multiple areas for programme intervention at the youth, family and service delivery levels. Key recommendations emerging from the present study are outlined below.

\section{Strengthen efforts to achieve universal school enrolment and at least primary school completion}

Youth Study findings that primary school enrolment is far from universal even among the youth cohort call for concerted efforts to achieve universal enrolment of children in school. Moreover, findings suggesting substantial declines in school attendance even at the primary level and relatively low rates of primary school completion emphasize in no uncertain terms that rigorous efforts are needed in order that the state meets the Millennium Development Goal of ensuring universal primary school completion.

While achieving universal enrolment and primary school attainment are key shorter-term goals, the importance of high school education in enabling youth to make a successful transition to adulthood underscores the need, at the same time, for efforts to overcome barriers to high school completion.

A number of factors have been identified in the Youth Study that inhibit school enrolment and primary school completion; leading among these are economic reasons, attitudes and perceptions of youth and their parents, as well as housework responsibilities among young women. Multiple activities are needed to address these barriers. Efforts must be made, for example, to address the economic pressures that dissuade parents from enrolling their children in school in the first place or from keeping them in school once enrolled. Conditional grants and targeted subsidies that encourage school enrolment and completion among disadvantaged groups need to be considered. At the same time, activities directed at parents are needed that promote positive attitudes towards education and school completion, raise aspirations for the education of their children and encourage greater parental involvement in their children's education.

Activities must also address school-level barriers, notably, distance to school, poor infrastructure and poor quality of education, significant motivating factors behind discontinuation particularly among young women. The state government has launched some schemes to address some of these barriers (see for example the bicycle scheme for girls); it is important that the effectiveness of such schemes is evaluated and promising lessons are assimilated and scaled up.

There is also a need to incorporate livelihood skills building models within the school setting that provide opportunities for those in school to gain market-driven job skills and also expand young people's aspirations regarding their education and careers. Moreover, investments are needed that focus on providing better training and ensuring accountability for teachers and thereby improving the quality of the schooling experience. Finally, given the large proportions reporting that schooling had been interrupted because they were required for 
work on the family farm or business or for housework, and given the reality of young people's lives and the economic pressures on families, efforts need to be made to adjust school timings, including establishment of evening schools to enable children to accommodate work on the family farm or business without sacrificing their education.

Findings indicating transition to adult roles, particularly early marriage, as an important reason for school discontinuation—even as early as primary school—among girls, emphasise the fact that programmatic commitments outside the education sector are also critical to the achievement of universal school enrolment and completion. Specifically required are programmes that seek to critically examine norms and practices surrounding marriage and to eliminate the practice of early marriage. Explorations of subsidies and cash transfers that link school retention and delayed marriage among girls are needed.

The stark gender divide and rural-urban divide observed in school enrolment and attendance call for efforts that specifically target female children and rural children in general. Moreover, findings suggest that married young women remain considerably disadvantaged. Interventions are needed that give married young women a second chance to obtain a basic education.

\section{Invest in promoting youth employment}

Findings of the Youth Study that considerable proportions of youth had initiated work in childhood reiterate the recommendation highlighted above regarding the need to provide conditional grants and targeted subsidies to disadvantaged groups, which would encourage parents to opt for schooling over work for their children.

Findings have pointed to the effective unemployability of youth. For example, few youth had completed primary or high school and even fewer had attended a single vocational training programme. Moreover, considerable proportions of youth, particularly the educated, were unemployed. Clearly, the state must strengthen significantly its investments in programmes to enable youth to make successful transitions into work roles, including provision of soft loans for youth to set up their own enterprises. At the same time, efforts are needed that evaluate existing programmes, upscale successful models and raise awareness among youth about their availability. While enhancing employability will depend to a considerable degree on improvements in educational attainment discussed above, it will also require greater investment in enabling youth to acquire vocational skills. Formal mechanisms must be developed that provide opportunities to youth to acquire vocational skills for which there is an established market demand, and that link eligible youth to market opportunities. These efforts, through various livelihood schemes, must promote self-employment and entrepreneurship among young people.

Findings also suggest the need for a special focus on young women. Significant proportions of young women currently engaged in economic activities had done so only part-time and worked largely in agricultural activities. In addition, many young women were seeking employment at the time of the interview. These findings highlight the need for specially targeted programmes for young women.

\section{Promote youth agency and gender equitable norms among youth}

Findings presented in this report highlight the persistence of gender double standards and extremely limited agency of young women. Stark gender differences were evident in school enrolment, attendance and completion, participation in the labour market, exposure to mass media, parental control of adolescent's mobility and interactions with peers, exercise of choice in matters affecting young people's lives, freedom of movement 
and access to resources. Gender equitable norms were not universally expressed; young women were more likely to express equitable gender role attitudes than young men, but were also more likely than young men to justify wife beating. These findings call for multi-pronged interventions to promote gender equitable norms and practices that are directed at young women, young men, their families, communities, and educational, labour and health systems.

A programme priority is to promote life skills education programmes for young women, both unmarried and married, that will not only raise their awareness of new ideas and the world around them but also enable them to put information into practice, encourage them to question gender stereotypes, develop self-esteem and strengthen their abilities in problems-solving, decision-making, communication and inter-personal relations and negotiations. Safe spaces should be identified in which young women can build social networks and find social support among peers.

Interventions intended to build life skills must also be inclusive of young men. Indeed, findings indicate that inegalitarian gender role attitudes were expressed by many young men. Moreover, while young men were clearly not as disadvantaged as young women, findings indicate that many young men were not able to exercise agency in their everyday lives. These findings call for life skills education programmes for young men that promote new concepts of masculinity and femininity among youth and at the same time, promote messages that build egalitarian relations between women and men.

Promoting gender equitable norms and practices requires active engagement with the community. It is essential that programmes for youth work with key community members, such as parents, political and religious leaders in the community, to critically examine prevailing gender norms and forces that perpetuate such norms.

An increasing number of intervention models to build agency and promote egalitarian gender role attitudes among young people have been tested in India. These models should be reviewed and replicated or scaled up as appropriate.

\section{Provide opportunities for formal saving, especially for young women}

Findings suggest that while young women were more likely than young men to report savings, few youth, irrespective of sex, owned a savings account. Among those who did own an account, young women were far less likely than young men to operate the account independently. Programmes are needed that inculcate a savings orientation among both young men and young women, that offer savings products that are attractive and appropriate to the small and erratic savings patterns of young people and that enable young women in particular to overcome obstacles related to owning and controlling savings products.

\section{Promote youth participation in civil society and political processes and reinforce secular attitudes}

Findings suggest that for many youth, opportunities to engage in civic and political life are limited and secular attitudes are not uniformly expressed. Programmes are needed-at the school, college and community levels, through national service programmes, sports and other non-formal mechanisms-that encourage civic participation, incorporate value building components and reinforce secular attitudes and values that espouse responsible citizenship. 


\section{Provide family life or sex education for those in school and out of school}

Youth Study findings provide considerable evidence suggesting that family life or sex education is urgently needed among youth, both for those in school and those who have discontinued their education. Findings demonstrate extremely limited understanding of sexual and reproductive matters among young people, including the married. Misconceptions abound on most topics: sex and pregnancy, contraceptive methods including condoms, STIs and HIV/AIDS and the conditions under which abortion is legally available or restricted. And knowledge of STIs is far more limited than knowledge of HIV/AIDS. Where awareness of sexual and reproductive health matters exists, it is typically superficial.

Youth themselves have called for family life or sex education. Findings highlight that large proportions recognised the need for information on these issues, and indicated a preference for receiving this education from teachers, health care providers or parents. However, few young people had been exposed to family life or sex education. Substantial proportions of married young women and young men reported entering marriage completely unaware of what it entailed. At the same time, substantial minorities of young people had engaged in sexual risk taking.

There is clearly a pressing rationale for school-based family life or sex education for those in school and community-based expert-led education for those out of school. These programmes should be age-appropriate and provide information on sexual and reproductive matters and sexual and reproductive rights; about pregnancy as well as the causes, transmission routes and prevention of infection. However, they should be designed not only to raise awareness among youth but also to enable young people to correctly understand and assess the risks they face and to adopt appropriate protective actions.

In view of the finding that the media are a major source of sexual and reproductive health information for youth, efforts must be made, at the same time, for communications initiatives that inform while entertaining youth about sexual and reproductive matters.

In addition, special attention needs to be paid to the training of trainers. Considerable proportions of youth who reported having received formal family life or sex education reported feeling uncomfortable or embarrassed in the course of family life or sex education. These findings raise questions about the extent to which they were indeed able to participate freely and clarify their doubts and at the same time, about the ability of trainers to connect with youth to whom they provided this education. Such findings clearly highlight the need for improving the quality of training imparted to trainers. It is important that teachers, health care providers and other experts undergo training that enables them to overcome their reluctance about communicating with youth on sensitive sexual and reproductive matters, that dispels their misconceptions on these matters, and that enhances their technical knowledge on sexual and reproductive issues.

\section{Ensure that the transition to sexual life is safe and wanted}

While for the vast majority of young women and men sexual activity is initiated within the context of marriage, findings show that significant minorities of youth, particularly young men had engaged in sex before marriage. As documented in this report, many youth had initiated sexual activity uninformed, which reiterates the need for providing family life or sex education to young people. Moreover, findings that for many, pre-marital sexual experiences were unsafe or unwanted calls for programmes that focus on building sexual and reproductive health awareness among young people as well as developing their skills in negotiating safe sex and communicating with their partners. At the same time, programmes must make available appropriate 
family planning and infection prevention services for both married and unmarried young men and women in a manner acceptable to them.

\section{Intensify efforts to eliminate the practice of early marriage}

Findings indicate an adherence, even among youth, to the traditional social norms around child marriage and the practice of early marriage not only among young women but also, to a lesser extent, among young men in the state. These findings call for measures that go beyond information campaigns to address the social norms and economic factors driving early marriage and ensure the stricter enforcement of existing laws prohibiting early marriage in the state.

There is clearly a need for an intensified, multi-pronged approach to eliminate the practice of early marriage. Strategies are needed that mobilise communities to help parents resist pressures that foster the practice of early marriage. Strategies intended to evolve new norms and new practices should both actively engage influential persons in the community including religious and political leaders as well as implement campaigns highlighting the adverse consequences of early marriage, and how it is a violation of the rights of the child. Community mobilization efforts must involve youth themselves, their families, as well as influential persons in the community, including religious and political leaders.

Equally important is to ensure greater commitment on the part of law enforcement agencies to enforce existing laws on the minimum age at marriage and the registration of marriages, and to levy penalties for violators. Allowing anonymous reporting, working with the police and others to make clear that the practice of early marriage is not a minor violation, and making guidelines for penalties clear and transparent are some possible steps.

Efforts to delay marriage also require providing girls with meaningful and viable alternatives to early marriage. Advising families to send their daughters to school when schools are too far away, the classroom is hostile to girls, or education is of poor quality, will not work. Working with the education sector to make schooling for girls more accessible, and to make classrooms more gender-sensitive and responsive to the needs of young girls and the concerns of their parents is important. At the same time, it is necessary to make efforts to provide livelihoods training, within or outside the educational system.

Findings that marriages were often arranged without the participation of young people themselves and that few young people had an opportunity to meet their spouse-to-be prior to the wedding day call for actions to encourage parents to involve children in marriage-related decisions and enable them to interact with their prospective spouses prior to the wedding day. Parents must also be made aware of the physical and mental health dangers of early marriage and the adverse experiences of many young women (and some young men) who were married early or who were unprepared for marriage.

\section{Enable married young women to exercise greater control over their lives}

Findings on the multiple vulnerabilities faced by married young women underscore the need for programmes that support them, acknowledging that their situation and needs may differ from those of married adults. Married young women are notably isolated, have little decision-making authority and have few sources of support. They have limited communication with their husbands, and notable proportions have suffered physical and sexual violence perpetrated by their husbands. 
Efforts are needed that address the health and empowerment needs of married young women and enable them to have greater control over resources. Also needed are efforts to break down the social isolation of newly married young women, encourage couple communication and build negotiation and conflict management skills early in marriage. Intervention models that have attempted to address these needs exist in India; these should be reviewed and up-scaled as appropriate so that married young women have the opportunity to exercise control over their lives.

\section{Support newly-weds to postpone the first pregnancy and promote pregnancy-related care among those who become pregnant}

Findings show that the social pressure to bear children as soon as possible following marriage persists. Contraceptives were rarely used to postpone the first pregnancy and although the desire to have delayed the first pregnancy was expressed by large proportions of young men and women, many young women experienced their first pregnancy soon after marriage. It would appear that numerous forces work against delaying the first pregnancy-young people's lack of awareness of appropriate methods of contraception and access to supplies, their limited skills in countering social expectations and negotiating pregnancy postponement, overwhelming pressure from the family and community to bear children as soon as possible after marriage, and lack of attention from health care providers.

Programmes are needed that inform youth about their pregnancy postponement options and enable them to access appropriate contraception. At the same time, providers must be trained and charged with the responsibility of reaching married young women and men-including those who have not yet experienced pregnancy - with information regarding contraception and other reproductive health matters as well as contraceptive supplies. The limited mobility of married young women to seek health care underscores the need for health workers to seek these women—particularly those newly married and first time pregnant—in their homes.

Findings also underscore that access to maternal health services was limited, even at the time of the first-and often the most risky - pregnancy. Just one in four first births took place in a health facility; moreover, skilled attendance at first delivery was reported by just one in three young women and two in five young men. These findings highlight that reproductive and child health programmes in the state need to lay emphasis on increasing demand as well as improving the availability of such services to young people.

\section{Create a supportive family environment}

Findings highlight the limited interaction and social distance between parents and young people while growing up and the gendered nature of socialisation experiences. Efforts must be made to create a supportive environment for young people. While evidence on models that are effective in bridging the distance between parents and children or enabling parents to adopt gender-egalitarian socialisation practices is not currently available, findings presented in this report call for programmes that address parental inhibitions about discussing sexual matters with their children, encourage greater openness and interaction between parents and children, and enable the adoption of gender-egalitarian child-rearing practices. 


\section{Reorient service provision to address the unique needs of unmarried and married young women and men}

Although the RCH Programme has advocated special services for youth, including the unmarried, these services have not reached youth. Few youth were aware of sources of sexual and reproductive health information or contraceptive supplies, few had sought care for symptoms of STI or gynaecological problems, and most of those who had sought care for the latter preferred private to public sector facilities. Moreover, findings suggest that many youth, including the married, would indeed find it difficult to seek appropriate care for sexual and reproductive matters.

The disconnect between the public health sector and youth underscores the need to sensitise health care providers about the special needs, heterogeneity and vulnerability of unmarried and married young women and men, and to orient them to the need for developing appropriate strategies to reach these diverse groups, including young newly-weds. Programmes must be inclusive of unmarried young people and recognise their need and right to sexual and reproductive health and related information and services. Counselling and contraceptive services must be made available to unmarried young people in a non-threatening, nonjudgmental and confidential environment. Indeed, these findings call for the implementation of strategies outlined under the National Rural Health Mission's RCH Programme.

Findings that very few youth sought care for health problems and that those who sought care preferred to seek care from the private sector and traditional providers rather than the public sector, suggest the need to explore the feasibility of implementing various demand side financing strategies, for example, health insurance, competitive voucher schemes and community financing schemes, in enabling youth to obtain quality care from a wider array of providers.

In addition, there is a great need for mental health issues to be addressed. Symptoms suggestive of mental health disorders were evident among sizeable proportions of youth. Efforts are needed to screen young people for mental health disorders when they avail of other primary health services, including, for example, sexual and reproductive health services, and to refer youth with such symptoms to appropriate health facilitates and providers.

\section{Directions for future research}

Findings presented in this report provide a broad picture of youth in Bihar. At the same time, findings have raised a number of issues that require further investigation, particularly with regard to the determinants and consequences of youth behaviours and practices during the transition to adulthood. While the Youth Study is indeed a rich source of data that will enable investigators to fill many of the information gaps identified, there are several gaps in knowledge that will require additional research efforts.

Youth Study findings highlight the need for further research in terms of formative research that explores in greater depth factors impeding successful transitions to adulthood, including enrolment in school and completion of at least a primary education, entry into the labour force, initiation of sexual activity, and marriage and parenthood. Research is also needed that explores the role of peers, socialisation practices, access to information and access to services in young people's lives, and the ways in which these may contribute to or impede young people's ability to make successful transitions. A general research recommendation is the urgent need for prospective or panel study designs that follow a cohort of adolescents at regular intervals up to age 24. Prospective study designs would enable researchers to take a life course approach, identify, with compelling data, the factors responsible for healthy transitions to adulthood and point to the ways in which the situation and experiences of youth in adolescence influence their life courses at later ages. 
Operations research is also needed. While there are a number of interventions intended to address the needs of youth-for example, addressing the needs of married girls, changing norms of masculinity and femininity, encouraging education for girls, developing market-based vocational skills and providing family life and sex education-few of these have been rigorously evaluated. Urgently needed, therefore, are rigorously designed and tested intervention models that not only pay attention to the content and delivery of the intervention but also measure effectiveness and acceptability-in short, that will enable a shift from the implementation of promising to best practices in addressing young people's needs. In order to inform the field, multiple inputs are required. Ultimately, research is needed that monitors the scaling up of successful interventions in terms of their impact on young people's lives.

In brief, the Youth Study has documented, for the first time, the multi-faceted situation of youth in Bihar. The study alerts us to the many challenges confronting youth and their ability to make a successful transition to adulthood. It emphasises the heterogeneity of youth, not only in terms of their situation but also with regard to their stated needs and preferred mechanisms to address these needs. Programmes must recognise the heterogeneity of young people and interventions and delivery mechanisms should be appropriately tailored to meet their needs. Evidence presented here provides not only a blue-print for the programming needs of youth in Bihar but also a base-line by which to measure the impact of programmes intended to address youth needs. 


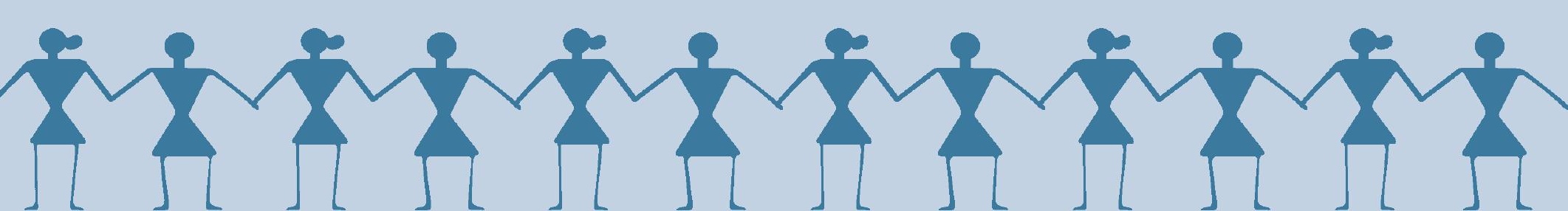


Chapter 1

Introduction

\subsection{Rationale}

The Youth in India: Situation and Needs Study (referred to as the Youth Study) is the first-ever sub-nationally representative study conducted to identify key transitions experienced by married and unmarried youth in India. There is a strong rationale for the Youth Study. Young people (aged 10-24) constitute almost 315 million and represent $31 \%$ of the Indian population (Office of the Registrar General and Census Commissioner, 2001a). Numbers are projected to increase and peak at around 358 million in 2011 before stabilising at around 336 million by 2026 (Office of the Registrar General and Census Commissioner, 2006). Not only does this cohort represent India's future in the socio-economic and political realms, but its experiences will largely determine India's achievement of its goal of population stabilisation articulated in the National Population Policy 2000 (MOHFW, 2000) and the extent to which the nation will be able to harness its demographic dividend. In addition, it is clear that the realisation of the Millennium Development Goals (UNDP, 2000) depends, to a considerable extent, upon the situation of young people. While today's youth are healthier, more urbanised and better educated than earlier generations, social vulnerabilities persist and transitions to adulthood are too frequently marked by early entry into the labour force, abrupt and premature exit from school, early marriage and strongly held gender norms. In the course of the transition to adulthood, moreover, young people face significant risks related to sexual and reproductive health, and many lack the knowledge and power to make informed sexual and reproductive choices (for a review, see Jejeebhoy and Sebastian, 2003).

In recognition of the importance of investing in young people, several national policies formulated since 2000 have underscored a commitment to addressing the multiple needs of this group in India. The National Population Policy 2000 recognised, for the first time, that adolescents constitute an under-served group with special sexual and reproductive health needs, and advocates special programmatic attention to addressing this population (MOHFW, 2000). The National Youth Policy 2003 focuses on the needs of those aged 13-35, but recognises adolescents (aged 13-19) as a special group requiring a different approach from that appropriate for young adults (aged 20-35), and promotes strategies to meet youth needs in areas including education, training and employment, health, recreation and sports, and good citizenship (Ministry of Youth Affairs and Sports, 2003). Also notable is the commitment to addressing the needs of adolescents and young people articulated in the Tenth and Eleventh Five-Year Plans (Planning Commission, 2002; 2006). In addition, the National Adolescent Reproductive and Sexual Health Strategy that provides the framework for the adolescent sexual and reproductive health services proposed in the Reproductive and Child Health (RCH) Programme II (MOHFW, 2006). The National Rural Health Mission (2005-12) has incorporated adolescent health services as part of its service guarantees in health sub-centres, primary health centres and schools (MOHFW, 2005).

Effective implementation of both policies and programmes, however, has been handicapped by a lack of evidence on young people's situation and needs. Currently available evidence is limited, at best, and comes largely from small scale and unrepresentative studies. The most recent National Family Health Survey (NFHS-3) obtained, for the first time, valuable data on unmarried young women and men (IIPS and Macro International, 
2007a). Even so, the information that it provides on young people's various transitions remains limited and the small sample sizes obtained in most states preclude the possibility of in-depth analysis and of obtaining state-representative estimates of behaviours and practices among different sub-groups of young people.

\subsection{Study objectives}

The objectives of the Youth Study were to identify key transitions experienced by youth, including those pertaining to education, work force participation, sexual activity, marriage, health and civic participation; provide state-level evidence on the magnitude and patterns of young people's sexual and reproductive practices in and outside of marriage as well as related knowledge, decision-making and attitudes; and, finally, identify key factors underlying young people's sexual and reproductive health knowledge, attitudes and life choices. Findings from the study are expected to guide policy, programmes and advocacy on youth issues, enable programmes and policies to recognise the heterogeneity of youth in India, and provide important base-line indicators against which the long-term impact of programmes may be measured.

The Youth Study focused on married and unmarried young women and unmarried young men aged 15-24 and, because of the paucity of married young men in the younger ages, married men aged 15-29 in both rural and urban settings. The study was conducted in a phased manner in six states of India: Andhra Pradesh, Bihar, Jharkhand, Maharashtra, Rajasthan and Tamil Nadu. This report focuses on findings from Bihar.

Funding for the Youth Study was provided by the David and Lucile Packard Foundation and the John D. and Catherine T. MacArthur Foundation. The Youth Study was conducted jointly by the International Institute for Population Sciences, Mumbai (IIPS) and the Population Council, New Delhi. The design and implementation of this study were guided by the Project Advisory Committee, headed by the Secretary, Ministry of Health and Family Welfare (MOHFW).

\subsection{Bihar: Overview of demographic and socio-economic features}

The state of Bihar, situated in the eastern part of India, is India's twelfth largest state in terms of geographical area. The state covers a total of 94,163 square kilometres and contains 38 districts divided into two geographic regions, namely, North Bihar Plain and South Bihar Plain and nine administrative divisions, namely, Patna, Magadh, Saran, Tirhut, Darbhanga, Koshi, Purnea, Bhagalpur and Munger (www.bstdc.bih.nic.in/factfile.htm).

Bihar, with a population of 83 million in 2001, ranks third in terms of total population among states in India (Office of the Registrar General and Census Commissioner, 2001b). The state's population is projected to have reached 93.6 million by 2008 (Office of the Registrar General and Census Commissioner, 2006). The sex ratio in Bihar (919 females per 1,000 males) is lower than in India as a whole (933). Population density in the state was 881 persons per square kilometre in 2001. The distribution of the population by religion indicates that $83 \%$ of the state's population was Hindu and $16.5 \%$ was Muslim (Office of the Registrar General and Census Commissioner, 2001c). Scheduled castes (SC) and scheduled tribes (ST) constituted 16\% and 1\%, respectively, of the state's total population (Office of the Registrar General and Census Commissioner, 2001b).

The state is characterised by a large rural population; just 11\% of the state's population live in urban areas. It has witnessed large-scale migration both within the state as well as to other states. Indeed, according to the 2001 Census, the state ranks second in terms of the number of inter-state migrants (Office of the Registrar General and Census Commissioner, 2001d). 
Economically, Bihar is one of the least developed states in the country. Its contribution to the national Gross Domestic Product (GDP) was 2.5\% in 2006-2007 (Ministry of Statistics and Programme Implementation, 2008). Its per capita income of Rs. 7,875 in 2005-2006 was the lowest among states in India and well below the national average of Rs. 25,716 (Ministry of Finance, 2008). Bihar's Gross State Domestic Product (GSDP) at current prices stood at Rs. 94,251 crores in 2006-07 (Ministry of Statistics and Programme Implementation, 2008); at constant prices (1999-00), the GSDP hardly increased (by 0.07\%) between 2004-05 and 2005-06, which may be attributed to annual fluctuations in the contributions of the primary sector and the poor performance of secondary and tertiary sectors (Department of Finance, Government of Bihar, 2008). Moreover, poverty levels remain high in the state. As of 2004-05, two-fifths of the state's population (based on the Uniform Recall Period consumption distribution) was estimated to live below the poverty line, with significant differences between those residing in urban (34.6\%) and rural (42.1\%) areas (Planning Commission, 2007). Unemployment rates are relatively low; in $2004-05,1.9 \%$ and $7.0 \%$ of rural and urban males, respectively, were unemployed for a major part of the year, as measured by the usual principal status definition. The corresponding figures for females were $0.3 \%$ and $5.7 \%$, respectively (NSSO, 2006).

Bihar lags behind the rest of India on many social indicators as well. For example, the state has the lowest literacy rate among Indian states; the overall literacy rate was $47 \%$ in 2001, ranging from $33 \%$ among females to $60 \%$ among males. Indeed, literacy rates were considerably lower than the national average of $54 \%$ for females and 75\% for males (Office of the Registrar General and Census Commissioner, 2001b).

The state's performance in the health sector is also notably poor. Life expectancy among males was one year lower in Bihar ${ }^{1}$ than in India in general: 62 and 63 years, respecti vely during 2002-06. For females, life expectancy was four years lower in Bihar than in India in general: 60 and 64 years, respectively (Office of the Registrar General and Census Commissioner, 2008). The state's infant mortality rate (62 per 1,000 live births) is higher than the national average (57) (IIPS and Macro International, 2008). Fertility rates are also considerably higher in Bihar than in India on average; indeed, the total fertility in the state (4.0) is the highest of any state in India. The contraceptive prevalence rate is, correspondingly, much lower and a much larger proportion of women in Bihar report an unmet need for contraception than in India on the whole. Indeed, use of contraceptive methods is as low as $34 \%$, while at the same time, as many as one in four (23\%) had reported an unmet need for contraception (IIPS and Macro International, 2008). This compares with a contraceptive prevalence rate of $56 \%$ and unmet need of $13 \%$ nationally (IIPS and Macro International, 2007a).

\subsection{Situation of youth in Bihar}

Young people aged 10-24 constitute a total of 24 million, and account for $29 \%$ of the state's population. The youth population, that is, those aged 15-24 numbered 13.1 million in 2001, accounting for $16 \%$ of the state's population (Office of the Registrar General and Census Commissioner, 2001a). The share of the youth population as a proportion of the total population of Bihar is projected to increase to $22 \%$ by 2011 , before beginning to decline (Office of the Registrar General and Census Commissioner, 2006).

Educational attainment levels among youth are considerably lower in the state than in India overall, and gender differences in enrolment are substantially wider. Among young people (aged 10-24), just $68 \%$ of men and $47 \%$ of women were literate in 2001 in Bihar compared to $85 \%$ and $71 \%$, respectively in India. (Office of the Registrar General and Census Commissioner, 2001e). Data on gross enrolment ratios also highlight the

\footnotetext{
${ }^{1}$ Including Jharkhand, individual estimates are not available.
} 
state's poor performance in the educational field. Even among children aged 6-11 years, the gross enrolment ratio was $84 \%$ in 2004-05, the corresponding figures for $11-14$ year-olds and $14-16$ year-olds were $32 \%$ and $22 \%$, respectively. The Gender Parity Index (GPI) at the elementary, secondary and higher secondary levels of education indicates limited access to educational opportunities for girls at all levels of schooling in the state; the GPI score ranged from 0.73 at the elementary level to 0.48 at the secondary and higher secondary level in 2004-05 (Ministry of Human Resource Development, 2007).

Bihar has few employment opportunities for youth, and as elsewhere in the country, unemployment rates were much higher among young people than among the general population in the state (as discussed in Section 1.3). For example, among the population aged 15-29, unemployment rates, as measured in terms of principal usual status, were $5.3 \%$ and $17.8 \%$ among young men in rural and urban settings, respectively, during 2004-05. The corresponding rates among young women were $1.3 \%$ and $30.9 \%$, respectively (NSSO, 2006).

Available evidence on the sexual and reproductive health profile of young people in Bihar highlights their vulnerability. Marriage continues to take place before the legal minimum age for both young women and men; as recently as $2005-06,69 \%$ of women aged $20-24$ were married by age 18 and $43 \%$ of men aged 25-29 were married by age 21 . Moreover, one in four 15-19 year-old girls have begun childbearing (IIPS and Macro International, 2008).

While it is clear that sexual relations are initiated early and within the context of marriage for large proportions of young women and men in Bihar, recent surveys and small-scale studies report that sexual risk taking before or within marriage is not unheard of among young people, particularly among young men in the state. For example, the National Family Health Survey 2005-06 reports that $11 \%$ and 1\%, respectively, of unmarried young men and women had experienced sex (IIPS and Macro International, 2008). Likewise, the recent National Behavioural Surveillance Survey reports that some 5\% and $0.6 \%$ of young men and women aged 15-24 had engaged in sexual intercourse with a non-regular partner in the 12 months preceding the survey (National Institute of Medical Statistics and NACO, 2008). Similarly, a recent small-scale study focusing on students in Class IX and XI in Patna reports that $10 \%$ of male students and $1 \%$ female students had experienced pre-marital sex (Shekhar, Ghosh and Panda, 2007).

Despite the early onset of sexual relations within or before marriage among significant proportions of youth, available evidence suggests that young people's knowledge of sexual and reproductive health matters tends to be limited. For example, data from NFHS-3 indicate that only $14 \%$ of young women and $27 \%$ of young men had comprehensive knowledge about HIV/AIDS. Some $60 \%$ of young women and $77 \%$ of young men were aware of a source for condoms (IIPS and Macro International, 2008).

\subsection{Youth-related policy and programme environment in Bihar}

The Bihar state government has articulated its commitment to promoting adolescent health in the state's Population Policy and its Reproductive and Child Health Programme-II. The Population Policy 2000 notes that the sexual and reproductive health needs of adolescents have remained unaddressed and calls for programmes to encourage delayed marriage and childbearing, to inform adolescents and young people about sexual and reproductive health matters and to make reproductive health and nutritional services accessible and affordable to them (Department of Health and Family Welfare, Government of Bihar, 2000). The Programme Implementation Plan of the Reproductive and Child Health Programme-II has proposed a number of activities to improve reproductive health and nutrition of adolescents. These include workshops to sensitise health service providers and other gatekeepers to the health needs of adolescents and behaviour 
change communication campaigns for adolescents that raise awareness about safe reproductive health practices, family planning, good nutritional practices and the importance of micronutrients. Also proposed are RTI/STI services for adolescents through an expanded network of health facilities and frontline health workers and distribution of micronutrient supplements to adolescents through health and education networks. The formation of partnership with NGOs and civil society networks to promote adolescent reproductive health is also envisaged (Department of Health and Family Welfare, Government of Bihar, n.d.). Additionally, programmes to raise adolescents' and young people's awareness about HIV/AIDS/STIs have also been introduced in the state, for example, the School AIDS Education Programme launched in 2001 (Bihar State AIDS Control Society, 2004).

In the field of education, the government has introduced a number of schemes to promote girls' schooling, including the Mukhyamantri Balika Poshak Yojana that provides financial assistance to girls in Classes VI-VIII to procure school uniforms, the Mukhyamantri Balika Cycle Yojana that provides bicycles to girls in Class IX, free distribution of textbooks among all girl students of Standard I-VIII and constitution of girls' groups (Meena Manch and Meena Rangmanch) in secondary schools that help in building awareness on issues related to education, health and nutrition, and water and sanitation at the community level (Department of Finance, 2008).

In addition, a number of non-governmental organisations (NGOs), including Bihar-based and national NGOs, implement programmes intended to meet the needs of young people in the state. These programmes have focused on providing sexual and reproductive health related information, counselling and services, including condoms, to young people; providing opportunities to build young people's livelihood skills; and mobilising communities to support young people's access to information and services.

\subsection{Study phases}

The Youth Study comprised three phases and included both a survey and qualitative data gathering exercises.

\subsubsection{Pre-survey qualitative phase}

As the Youth Study was one of the first of its kind in India, precedents did not exist for youth terminologies, particularly in reference to sensitive issues (romantic relationships, sexual experience and so on), and youth perceptions or youth willingness to share their experiences with study teams. In order to better understand these matters and to inform the design of the survey instrument, focus group discussions were conducted with married and unmarried young women and men, and key informant interviews conducted with teachers, health care providers, and community and youth leaders, in the first phase of the Youth Study. This phase also offered us an opportunity to explore community reactions to the kinds of issues to be raised in the survey.

In the course of this pre-survey qualitative phase, we also conducted in-depth interviews with parents of youth to collect parental perspectives on young people's situation and needs. In each site, eight categories of parents were selected (mothers and fathers of married and unmarried young men and women, respectively). The discussion focused on the life experiences of the child of interest.

The pre-survey qualitative phase was undertaken during May-July 2005 and covered at least one urban area and one rural area of six geographically diverse districts of the state. In total, 18 focus group discussions 
were held with young people; 39 key informant interviews were held with community leaders, health care providers, teachers and youth leaders; and 72 in-depth interviews were held with mothers and fathers.

\subsubsection{Survey phase}

Fieldwork was undertaken between January and August 2007. A total of 8,136 married and unmarried young women and men were interviewed during this phase.

\subsubsection{Post-survey qualitative phase}

In order to better understand the sexual and reproductive experiences of youth and the factors inhibiting and facilitating safe transitions into these behaviours, in-depth interviews were conducted with consenting survey respondents who reported certain experiences in the course of the survey interview. These experiences included, notably, having an opposite-sex romantic partner; having sexual relations with an opposite-sex romantic partner; experiencing same-sex, forced or exchange sexual relations; and among young men, engaging in relations with sex workers or married women. Among the married, in addition, experiences included exercising choice in spouse selection and practising contraception to delay the first pregnancy.

At the conclusion of the survey interview, interviewers sought the consent of respondents for an in-depth interview. Those who consented were then approached by a trained investigator who conducted the interview in the form of an unstructured conversation. In-depth interviews therefore took place at around the same time as did the survey. A total of 55 in-depth interviews were completed-33 and 22 among rural and urban respondents, respectively.

Findings from the survey are presented in this report. ${ }^{2}$

\subsection{Study instruments}

\subsubsection{Interview guidelines}

For the pre-survey qualitative phase, three sets of guidelines were prepared for focus group discussions, key informant interviews and in-depth interviews, respectively. These guidelines were appropriately modified for each youth group (married and unmarried young women and men) and parent group (mothers and fathers of married and unmarried young women and men). As mentioned above, specific guidelines were not prepared for the post-survey in-depth interviews with youth reporting selected behaviours; instead, interviewers were trained to steer the interview to focus on the experience of interest, and obtain information on the circumstances surrounding the experience and the respondent's own perceptions about the experience.

\subsubsection{Questionnaires}

A total of six questionnaires were developed for the study: a community questionnaire; a household questionnaire, administered in each selected household; and four individual questionnaires, one each for married young men, married young women, unmarried young men and unmarried young women.

\footnotetext{
${ }^{2}$ Separate reports, drawn from in-depth interviews with parents and youth, respectively, will discuss parental perspectives on young people's experience of growing up and provide insights on the sexual and reproductive experiences of youth, as well as factors inhibiting and facilitating safe transitions into these behaviours.
} 
The community questionnaire was administered in each village selected for the survey. This questionnaire collected information on different aspects of village life, including the village population, numbers engaged in agriculture, and the availability of various facilities and infrastructure in and around the village. Team supervisors administered the questionnaire to one or more individuals from each village who were well-informed about the village.

The household questionnaire listed all usual residents of the selected households and collected basic information on each listed household member, including his or her age, sex, marital status, relationship to the head of the household, education and current activity status. Information was also obtained on the religion and caste of the head of the household as well as on ownership of the residential structure and agricultural land, number of rooms in the residence, and such amenities available as type of toilet facility, main source of lighting, main type of cooking fuel and main source of drinking water. The survey also inquired about ownership of 17 consumer durables. Finally, information was sought on the marriage of any usual resident of the household in the three years preceding the interview as well as the sex and age of the person at the time of marriage.

The development of individual questionnaires was informed by other survey instruments, notably the World Health Organisation core questionnaire for youth surveys (Cleland, 2001) and a recent survey conducted in Pune district on the formation of partnerships among youth (Alexander et al., 2003). Other instruments consulted included surveys of youth conducted in India (Andrew, Patel and Ramakrishna, 2003; IIPS and Population Council, 2002; Sebastian, Grant and Mensch, 2003), Pakistan (Sathar et al., 2003), the Philippines (DRDF and UPPI, 2002), Vietnam (Mensch, Anh and Clark, 2000) and sub-Saharan Africa (Guttmacher Institute, 2004a; 2004b; 2004c). Finally, our survey instrument drew upon the questionnaire used in the NFHS-3 (IIPS and Macro International, 2007b).

The development of individual questionnaires was also informed by insights obtained in the pre-survey qualitative phase. Once the pre-survey qualitative phase was completed in all six states, the data generated were analysed to identify the kinds of issues that would be explored in the survey, ways of presenting sensitive issues, and terminologies to be used that would be comprehensible and acceptable to youth. The survey instrument was finalised after extensive pre-testing in several states.

Individual questionnaires were employed to interview eligible youth who usually resided in the selected households. Currently married young men and women aged 15-29 and 15-24, respectively, as well as unmarried young men and women aged 15-24, were eligible for interview. Widowed and divorced individuals were excluded from the survey. Keeping in mind the sensitive nature of the questions, the questionnaire was divided into several sections and arranged in such a way that the most sensitive questions were administered towards the middle of the interview. This strategy of asking a series of non-sensitive questions in the early part of the interview served two purposes: it enabled the interviewer and respondent to build rapport before sensitive questions were posed; and it permitted the investigator to maintain privacy for sensitive questions, as interested bystanders would usually depart while questions in the early sections were posed.

The individual questionnaires collected information on the following topics:

Background characteristics: Questions were asked regarding age, education and schooling, quality of school or college attended, work patterns including housework and paid employment, vocational training, short-term migration and characteristics of parents.

Additionally, a Life Event Calendar (LEC), adapted from that used in a nationally representative survey of adolescents and youth in Pakistan (Sathar et al., 2003) was administered to obtain information on education, 
work, living arrangements, marriage and family building (for married respondents), starting from the age of 12 years. This system of recording life events is considered one of the most effective approaches to minimise recall error.

Media exposure: Respondents were asked whether they were exposed to newspapers, television or the internet, and whether they watched pornographic films or read pornographic magazines. They were also asked about their views on the influence of films and television on their own life as well as young people's lives in general.

Puberty: In order to assess the age at which respondents experienced puberty, respondents were asked to report their age at key signs of maturation. Young women were questioned about their first menstruation while young men were asked about the onset of voice change and growth of pubic hair.

Parental interaction/relationship: Detailed questions were asked on the extent of parent-child communication on everyday activities as well as sexual and reproductive issues. Questions were also asked that assessed the extent to which a respondent had witnessed parental violence or been the victim of violence perpetrated by a parent while growing up.

Communication, mobility and decision-making: This section collected information on the person in whom youth were most likely to confide on matters related to getting a job, growing up, boy-girl relationships and personal problems. Detailed questions were also asked on decision-making and, for all groups except married males, mobility.

Gender and self-efficacy: In order to evaluate the respondent's gender role attitudes and level of self-efficacy, questions were asked to probe opinions on a range of gender-related issues such as the importance of boys' vis-à-vis girls' education, housework and freedom of movement.

Awareness of sexual and reproductive matters: This section probed young people's awareness about sexual relations, pregnancy, contraceptive methods, HIV/AIDS and sexually transmitted infection (STI) as well as the legal minimum age at marriage and conditions under which abortion is legally permitted. This section also probed young people's sources of information on sexual matters and contraception, the extent to which they had obtained formal sex or family life education, and their experiences and perceptions about this education.

Connectedness and friendship: Questions relating to connectedness and friendship explored respondents' friendship networks among those of the same sex and activities in which they participated with their friends. This was followed in a gradual fashion by questions on interaction with the opposite sex, whether or not the respondent had exchanged a "proposal" of romantic partnership with someone of the opposite sex and whether the respondent had ever met someone of the opposite sex secretly in a number of likely places.

Pre-marital romantic heterosexual relationships: This was a highly sensitive section, conducted only if complete privacy was assured. The section started by probing the pre-marital romantic and sexual experiences of up to five of the respondent's best friends. This technique, known as anonymous third-party reporting (developed by Rossier, 2003), was used to assess the extent to which youth were more likely to report the romantic and sexual relationships of their peers than of themselves. Respondents were then asked about their own experiences of pre-marital romantic partnership and, if reported, detailed questions were asked on the nature of such relationships with the first partner and the last or most recent partner (if more than one partner was reported). Questions were designed to gradually probe sensitive behaviours, for example, starting with whether the respondent had ever held hands with a romantic opposite-sex partner, and continuing with 
questions on hugging, kissing and finally having sex with the partner. We believe this gradual progression of questions was more culturally appropriate than a single question on pre-marital sex and provided insights into the range of behaviours youth experienced. If sex with a pre-marital romantic partner was reported, a host of questions followed that probed the consensuality of first sex with this partner, condom use, frequency of such relations and experience of pre-marital pregnancy. Questions were also asked about the characteristics of the romantic partner and parental awareness of and reactions to the romantic relationship.

Marriage process: In this section questions covered marriage planning, dowry, the participation of the respondent in decision-making related to marriage and the respondent's feelings about his or her own marriage. This section was administered, suitably modified, to both married and unmarried respondents.

Married life: Married respondents were asked detailed questions on married life. These included the nature of marriage (love or arranged), acquaintance with spouse before marriage and age at cohabitation. Questions about the marital relationship were also covered, including spousal communication and joint decision-making, the nature of the first sexual experience with spouse, experience of forced sex within marriage, inter-spousal violence, pregnancy experiences and outcomes, and contraceptive practice.

Same-sex, paid and forced sexual experiences: This was a second highly sensitive section in which respondents were asked a series of questions on their personal experience of several types of sexual encounters, for example, paid or exchange sex, forced sex perpetrated on the respondent and casual sex. In the case of male respondents, additional questions were asked about sex with a same-sex partner, relations with sex workers and married women (other than their wife for married males) and whether they had ever perpetrated forced sex. All married respondents were also asked about the experience of extra-marital sexual relations. Respondents who reported any of these experiences were probed about their age at their first experience of such a sexual encounter and the extent to which they had used condoms in these encounters.

Attitudes: This section probed respondents' views on pre-marital physical intimacy and wife beating.

Health and health seeking: This section collected information on respondents' experience of common health problems, specifically high fever and injury, as well as symptoms of genital infections in the three months preceding the survey. In addition, respondents were asked whether they had sought treatment for these health issues and, if so, from what source. Respondents' mental health in the last one month was assessed using the 12-item General Health Questionnaire, developed for use in field conditions (Goldberg, 1992).

Substance use and violence: A series of questions were asked about consumption of tobacco products, alcohol or drugs. In each case, questions were asked about use and frequency of use of such substances by family members and by the respondents themselves. Additional questions sought respondents' assessments of the frequency with which young people in their neighbourhoods engaged in violence (fights or beatings) and their own participation in such violence.

Programmes and participation: The final section of the questionnaire collected information on programmes available to young people in the village or neighbourhood in which they resided, and the extent to which youth participated in such programmes. In addition, rural respondents were asked about the role of panchayats in decisions affecting young people's lives. All respondents were asked about their participation in community activities, opinions about political processes, secular attitudes and participation in recent elections. Finally, respondents were asked to identify the most important problem facing youth in their village or neighbourhood. 
Sealed envelope response: However carefully designed and culturally sensitive the survey questions may have been, the possibility that young people would deliberately withhold information about their sexual experiences in a face-to-face interview could not be discounted. Drawing from other research in the field, an anonymous reporting method was included in our survey to obtain responses to a single question: Have you ever had sex with anyone [for the unmarried]/Did you ever have sex with anyone before marriage [for the married]? Interviewers first explained the technique to respondents, noting in particular its confidential nature. The interviewer then gave each respondent a blank card and asked him or her to simply mark a " $\checkmark$ " or an " $X$ " on the card to indicate that s/he had or had not experienced pre-marital sex. Once marked, the respondent placed the card inside an envelope provided by the interviewer; the envelope was sealed by the respondent and returned to the interviewer. Unique identification numbers linked the individual's questionnaire with his or her responses in the sealed envelope. Envelopes were opened only at the central office at the time of data entry.

Draft tools were extensively reviewed at meetings of the study's Technical Advisory Committee and were then translated into four languages (Hindi, Marathi, Tamil and Telegu), extensively pre-tested and finalised after appropriate modification. Copies of all these instruments are provided in the CD enclosed with this report.

\subsection{Study design and sample size estimation for individual interviews}

The Youth Survey was designed to provide estimates for the state as a whole, as well as for urban and rural areas for each of the four categories of respondents, namely married and unmarried young women and men, separately. The study was not designed to provide estimates at district or sub-district levels.

While arriving at sample size estimates, on the basis of the scarce available evidence, the following assumptions were made:

- $10 \%$ of unmarried young women would report the experience of pre-marital sexual relations;

- Among married men, 20\% would report unsafe sexual relations (multiple partner sex or non-use of condoms, unintended pregnancy or experience of STI symptoms);

- The coefficient of variation was set at $10 \%$ (equivalent to fixing the absolute error at $20 \%$ of the true value and $95 \%$ confidence interval);

- The non-response rate for the individual interviews was assumed to be $25-30 \%$;

- Design effect was assumed to be in the range of 1.5 to 2 .

The chances of finding an unmarried young man were greater than the chances of finding a married young man in a given household, and conversely, the chances of finding a married young woman were greater than the chances of finding an unmarried young woman. As a result, in the case of the male sample, our strategy was to estimate the number of households required to obtain the target number of married young men aged 15-29 in the male primary sampling units (PSUs), that is, the harder to reach group of males. Similarly, in female PSUs, the strategy was to identify the total number of households required based on the target number of unmarried young women aged 15-24, again, the harder to reach group of females.

Following from the assumptions described above, and in consultation with the study's Technical Advisory Committee, the required sample of each sub-group of youth was determined at 1,000 married young men, 
1,250 unmarried young men, 1,250 married young women and 1,750 unmarried young women each for urban and rural areas, that is, a total sample size of 5,250 in each area. ${ }^{3}$ However, our early experience suggested that because of the considerable mobility of youth, there was likely to be a shortfall in achieving these numbers. Hence in Bihar, Rajasthan and Andhra Pradesh, we revised the sample size to 1,200 married young men, 1,800 unmarried young men, 1,500 married young women and 2,100 unmarried young women, that is, a total sample size of 6,600 in rural and urban areas, respectively. In Bihar, moreover, because the relative proportion of unmarried young men in rural areas was lower than that observed in other states, we were further required to inflate the sample of married young men in rural areas to 1620 in order to obtain a minimum of 1,800 unmarried men. Hence, the targeted sample size for rural Bihar was 1,620 married young men, 1,800 unmarried young men, 1,500 married young women and 2,100 unmarried young women, that is a total sample size of 7020. In order to achieve the above-mentioned number of individual interviews, an estimated 31,391 households required to be covered in Bihar.

We further determined that a total of 300 PSUs-villages in rural areas and Census Enumeration Blocks (CEBs) in the urban areas-would be visited in order to conduct interviews in the required number of households. Thus, the average number of household interviews to be conducted in each rural PSU was calculated to be 191 among female PSUs and 66 among male PSUs. Corresponding averages for each urban PSU were 93 and 68, respectively.

\subsubsection{Sample selection strategy}

The study treated rural and urban areas of each state as independent sampling domains and, therefore, drew sample areas independently for each of these two domains. In order to avoid potential risks associated with interviewing both women and men from the same PSU, we decided to conduct interviews in separate PSUs for female and male respondents, that is, interviews with young women in 150 PSUs and young men in the remaining 150 (referred to as female and male PSUs, respectively). These 150 PSUs were further divided equally into rural and urban areas, that is, 75 for rural respondents and 75 for urban respondents. Within each sampling domain, a systematic, multi-stage sampling design was adopted. Sample selection procedures differed somewhat in rural and urban areas, as described below.

\subsection{1.a Selection of households in rural areas}

In rural areas, the 2001 Census list of villages served as the sampling frame for the selection of villages. This list was stratified using four variables, namely, region, village size, proportion of the population belonging to scheduled castes and scheduled tribes and female literacy. At the first level of stratification, the state of

${ }^{3}$ In estimating the number of households required, the study used the age-sex-marital status distributions observed in rural and urban areas, respectively, in the 2001 Census. The following formula was used to estimate sample size:

$$
\text { Coefficient of Variation } \begin{aligned}
(c v) & =\sqrt{\frac{q}{n p}} \\
n & =\frac{q}{c v^{2} p}
\end{aligned}
$$

In order to obtain the actual number of respondents, the above numbers were multiplied by the design effect and a factor ' $K$ ' $(1+$ the non-response rate). 
Bihar was stratified into 6 contiguous geographical regions, with districts (as defined in the 2001 census) classified into these regions as follows:

Region I : Paschim Champaran, Purbi Champaran, Gopalganj, Siwan, Saran

Region II : Sheohar, Sitamarhi, Madhubani, Supaul, Saharsha, Darbhanga, Muzaffarpur, Vaishali, Samastipur

Region III : Araria, Kishanganj, Purnea, Katihar, Madhepura

Region IV : Patna, Bhojpur, Buxar, Kaimur (Bhabua), Rohtas

Region V : Khagaria, Bhagalpur, Banka, Munger

Region VI : Begusarai, Lakhisarai, Sheikhpura, Nalanda, Jehanabad, Aurangabad, Gaya, Nawada, Jamui

In each region, villages were further stratified by village size, and except in regions IV and V, further by the percentage of the population belonging to scheduled castes or scheduled tribes. Table 1.1 gives detailed information on the stratification scheme in rural areas along with the population in each stratum. The last level of stratification was implicit for all strata, consisting of an ordering of villages within each stratum by level of female literacy, ordered alternatively in increasing and decreasing level of female literacy (obtained from the 2001 Census Village Directory).

The sample in rural areas was selected in two stages. At the first stage of selection, villages were selected systematically from the stratified list arranged as described above, with selection probability proportional to size (PPS). The 150 PSUs thus selected were then ordered by district and taluka codes and numbered from 1 to 150. Odd-numbered PSUs were designated for interviews with young men and even numbered PSUs for interviews with young women. In the case of male PSUs, selected PSUs containing fewer than 75 households were then linked to one or more adjoining villages so that the PSU had approximately 75 households. In the case of female PSUs, selected PSUs containing fewer than 200 households were linked to one or more adjoining villages so that the PSU had approximately 200 households. Those containing more than 300 and fewer than 601 households were segmented into two approximately equal parts, and one was chosen randomly for the survey. In the case of even larger villages, that is, those containing more than 600 households, segments of 150-200 households were made and numbered in a clockwise manner. Two segments were then selected using probability proportional to size.

The rural domain sampling fraction for a particular category, that is, the probability of selecting an eligible respondent of a particular category in rural Bihar $\left(f^{R}\right)$, was computed as:

$$
f^{R}=\frac{n^{R}}{N^{R}}
$$

where

$$
\begin{aligned}
& \mathrm{n}^{\mathrm{R}}=\begin{array}{l}
\text { number of eligible respondents in a particular category to be interviewed (target number of } \\
\text { interviews as described before), and }
\end{array} \\
& \mathrm{N}^{\mathrm{R}}=\text { projected rural population of eligible respondents in the state as of April 1, } 2006 .
\end{aligned}
$$


The probability of selecting a PSU from rural Bihar $\left(f_{1}^{R}\right)$ was computed as:

$$
f_{1}^{R}=\frac{a \times v_{i}}{\sum v_{i}}
$$

where

$$
\begin{aligned}
& \mathrm{a}=\text { number of PSUs selected from rural areas for the particular category, } \\
& \mathrm{v}_{\mathrm{i}}=\text { population of the } \mathrm{i}^{\text {th }} \text { PSU, and } \\
& \sum_{\mathrm{i}}=\text { total rural population of the state. }
\end{aligned}
$$

A complete mapping and household listing operation was carried out in each selected PSU (or in selected segments or linked villages as appropriate). This list of households provided the necessary frame for selecting households at the second stage. Mapping and listing were conducted by teams, each comprising one mapper and one lister. Households to be interviewed were selected with equal probability from the list using systematic sampling.

The probability of selecting a household from a selected rural PSU $\left(f_{2}^{R}\right)$ was calculated as:

$$
f_{2}^{R}=\frac{f^{R}}{f_{1}^{R}}
$$

No replacement for selected households was allowed even if a selected household could not be contacted after several attempts.

All the sampling fractions $\left(f^{R}, f_{1}^{R}, f_{2}^{R}\right)$ described above were computed separately for male and female PSUs on the basis of the target sample of married males and unmarried females, respectively.

Because we expected more unmarried than married males in our age groups, we needed to visit fewer households to obtain the required number of unmarried compared to married males. Likewise, because we expected more married than unmarried females, we needed to visit fewer households to obtain the required number of married compared to unmarried females. Appropriate intervals were computed to operationalise each of these selection processes. 
Table 1.1: Sampling stratification scheme

Details of the stratification used for sampling, Bihar (rural), 2007

\begin{tabular}{|c|c|c|c|c|}
\hline \multirow{2}{*}{$\begin{array}{l}\text { Stratum } \\
\text { number }\end{array}$} & \multicolumn{3}{|c|}{ Stratification variables } & \multirow{2}{*}{$\begin{array}{c}\text { Total } \\
\text { population }\end{array}$} \\
\hline & Region & $\begin{array}{l}\text { Village size (number of } \\
\text { residential households) }\end{array}$ & $\begin{array}{c}\text { Percent of SC/ST } \\
\text { population }\end{array}$ & \\
\hline 1 & 1 & $\leq 370$ & $\leq 12$ & 282,757 \\
\hline 2 & 1 & $\leq 370$ & $>12$ & $2,633,325$ \\
\hline 3 & 1 & $>370 \& 600 \leq$ & NA & $2,831,441$ \\
\hline 4 & 1 & $>600$ & $\leq 12$ & $2,875,471$ \\
\hline 5 & 1 & $>600$ & $>12$ & $2,673,551$ \\
\hline 6 & 2 & $\leq 450$ & $\leq 16$ & $3,148,570$ \\
\hline 7 & 2 & $\leq 450$ & $>16$ & $3,047,436$ \\
\hline 8 & 2 & $>450 \& 660 \leq$ & NA & $3,113,120$ \\
\hline 9 & 2 & $>660 \& 1320 \leq$ & $\leq 15$ & $3,261,086$ \\
\hline 10 & 2 & $>660 \& 1320 \leq$ & $>15$ & $2,945,500$ \\
\hline 11 & 2 & $>1320$ & $\leq 14$ & $3,211,296$ \\
\hline 12 & 2 & $>1320$ & $>14$ & $2,903,983$ \\
\hline 13 & 3 & $\leq 500$ & NA & $2,939,823$ \\
\hline 14 & 3 & $>500$ & $\leq 14$ & $3,103,101$ \\
\hline 15 & 3 & $>500$ & $>14$ & $3,038,315$ \\
\hline 16 & 4 & $\leq 240$ & NA & $3,074,828$ \\
\hline 17 & 4 & $>240 \& 550 \leq$ & NA & $3,124,459$ \\
\hline 18 & 4 & $>550$ & NA & $2,934,524$ \\
\hline 19 & 5 & $\leq 650$ & NA & $2,752,000$ \\
\hline 20 & 5 & $>650$ & NA & $2,691,038$ \\
\hline 21 & 6 & $\leq 170$ & NA & $2,860,221$ \\
\hline 22 & 6 & $>170 \& 500 \leq$ & $\leq 23$ & $2,894,045$ \\
\hline 23 & 6 & $>170 \& 500 \leq$ & $>23$ & $2,883,091$ \\
\hline 24 & 6 & $>500$ & $\leq 18$ & $2,901,637$ \\
\hline 25 & 6 & $>500$ & $>18$ & $2,733,687$ \\
\hline Total & NA & NA & NA & $70,858,305$ \\
\hline
\end{tabular}

Note: The level of female literacy (2001 Census) was used for implicit stratification. Villages with less than 50 households in the 2001 Census were excluded from the sampling frame. NA: Not applicable. SC: Scheduled caste. ST: Scheduled tribe. ${ }^{1} 2001$ Census population.

\subsection{1.b Selection of households in urban areas}

In selecting the urban sample, the 2001 Census list of wards (each consisting of several CEBs of 100-200 households) provided the sampling frame. For operational convenience, the Youth Study first determined male PSUs (equivalent to a CEB) and followed this with the selection of female PSUs (another CEB) in CEBs adjacent to male CEBs. As a result, half the total required number of PSUs was first selected. 
In urban areas, the 2001 Census list of wards was first arranged by district, and within each district by level of female literacy. The sample was then selected in three stages. At the first stage of selection, 75 wards were selected systematically with probability proportional to size. At the second stage, within each selected ward, CEBs were arranged by their administrative number and one CEB (designated as a male PSU) was selected using probability proportional to size. For each selected male CEB, an adjacent CEB was chosen to represent the female PSU in the same ward.

The urban domain sampling fraction for a particular category, that is, the probability of selecting an eligible respondent of a particular category in urban $\operatorname{Bihar}\left(f^{U}\right)$, was computed as:

where

$$
f^{U}=\frac{n^{U}}{N^{U}}
$$

$$
\begin{aligned}
& \mathrm{n}^{\mathrm{U}}=\text { number of eligible respondents in a particular category to be interviewed in urban areas (target } \\
& \text { number of interviews as described before), and } \\
& \mathrm{N}^{\mathrm{U}}=\text { projected urban population of eligible respondents in the state as of April 1, } 2006 .
\end{aligned}
$$

The probability of selecting a ward (or section) from urban Bihar $\left(f_{1}^{U}\right)$ was computed as:

where

$$
f_{1}^{U}=\frac{a \times w_{i}}{\sum w_{i}}
$$

$$
\begin{aligned}
& \mathrm{a}=\text { number of wards selected from urban areas for the particular category, } \\
& \mathrm{w}_{\mathrm{i}}=\text { population of } \mathrm{i}^{\text {th }} \text { ward, and } \\
& \sum \mathrm{w}_{\mathrm{i}}=\text { total urban population of the state. }
\end{aligned}
$$

The probability of selecting a CEB from a selected ward $\left(f_{2}^{U}\right)$ was computed as:

where

$$
f_{2}^{U}=\frac{c_{i}}{\sum c_{i}}
$$

$$
\begin{aligned}
& \mathrm{c}_{\mathrm{i}}=\text { population of } \mathrm{i}^{\text {th }} \text { CEB from a selected ward, and } \\
& \sum \mathrm{c}_{\mathrm{i}}=\text { total population of the selected ward. }
\end{aligned}
$$

A complete mapping and household listing operation was carried out in each selected PSU and the resulting list provided the necessary frame for selecting households at the third stage. Households to be interviewed were selected with equal probability from the list using systematic sampling. In some CEBs the number of households listed was smaller than the minimum expected number of households, and in such cases, a part of an adjacent CEB was listed.

The probability of selecting a household from a selected urban PSU $\left(f_{3}^{U}\right)$ was calculated as:

$$
f_{3}^{U}=\frac{f^{U}}{f_{1}^{U} \times f_{2}^{U}}
$$

As in the case of rural areas, (a) no replacement of selected households was allowed under any circumstances; (b) all sampling fractions $\left(f^{U}, f_{1}^{U}, f_{2}^{U}, f_{3}^{U}\right)$ were computed separately for male and female PSUs on the basis 
of the target sample of married males and unmarried females, respectively; and (c) appropriate intervals were computed to enable us to select fewer households for the interview of unmarried compared to married males and married compared to unmarried females.

\subsubsection{Selection of individual respondents within selected households}

In each PSU, households to be interviewed were selected by systematic sampling. The value of the interval (between one selected household and the next) was determined in advance to ensure a self-weighing design. As mentioned earlier, fewer households needed to be selected in order to obtain our sample of unmarried males and married females. Hence, further intervals were computed, using the target sample for unmarried males and married females.

Within each selected household, no more than one married and one unmarried respondent was interviewed, resulting in a maximum of two interviews from any household. In case more than one respondent from a single category was found in the household, one respondent was selected randomly using the Kish table. ${ }^{4}$ No replacement of the respondent thus selected was allowed.

\subsubsection{Sample weights}

In Bihar, the sample was weighted at the level of the sampling domain, that is, urban and rural males and females, respectively, making for a total of four sampling domains. In order to consider differential nonresponse rates in different geographical areas, non-response rates were calculated in smaller sub-domains of 2-3 PSUs within each domain. If $\mathrm{W}_{\mathrm{Di}}$ is the design weight for the $\mathrm{i}^{\text {th }}$ domain $(\mathrm{i}=1 \ldots 4)$ and $\mathrm{R}_{\mathrm{Hij}}$ is the response rate for households in the $\mathrm{j}^{\text {th }}$ sub-domain within the $\mathrm{i}^{\text {th }}$ domain, then the household weight for the $\mathrm{j}^{\text {th }}$ sub-domain within the $\mathrm{i}^{\text {th }}$ domain $\left(\mathrm{W}_{\mathrm{Hij}}\right)$ was calculated as follows:

$$
W_{H i j}=\frac{W_{D i}}{R_{H i j}}
$$

where $\mathrm{W}_{\mathrm{Di}}$ was calculated as the inverse of the probability of selecting an eligible married male in urban and rural male domains, respectively; and similarly, of selecting an eligible unmarried female in urban and rural female domains.

Weights were also calculated for eligible married males and unmarried females, denoted by $\mathrm{W}_{\text {Eij }}$ and calculated as follows:

$$
W_{E i j}=\frac{W_{D i}}{R_{H i j} \times R_{E i j} \times K_{i j}}
$$

where

$$
\begin{aligned}
\mathrm{R}_{\mathrm{Eij}}= & \text { response rate for married males or unmarried females in the } \mathrm{j}^{\text {th }} \text { sub-domain within the } \mathrm{i}^{\text {th }} \\
& \text { domain, and } \\
\mathrm{K}_{\mathrm{ij}}= & \text { probability that a married male or an unmarried female is selected by the Kish table procedure } \\
& \text { in the } \mathrm{j}^{\text {th }} \text { sub-domain within the } \mathrm{i}^{\text {th }} \text { domain. }
\end{aligned}
$$

\footnotetext{
${ }^{4}$ The probability of selection of individuals in rural areas is $\left(f^{R} / K_{i}^{R}\right)$ and in urban areas $\left(f^{U} / K_{i}^{U}\right)$, where $K_{i}^{R}$, and $K_{i}^{U}$ denote the number of individuals of the specified category (married and unmarried males and females, respectively) in the $i^{\text {th }}$ selected household in rural and urban areas, respectively.
} 
The design weight described above was also used in the case of unmarried males and married females in each domain. Also, since the survey did not attempt to interview an unmarried male or a married female in all selected households, an additional interval needed to be incorporated in the weight calculation. Hence, weights for eligible unmarried males and married females, denoted by $\mathrm{W}_{\mathrm{Eij}}$ were calculated using the following equation:

$$
W_{E i j}=\frac{W_{D i}}{R_{H i j} \times R_{E i j} \times K_{i j}} \times I_{i}
$$

where $\mathrm{I}_{\mathrm{i}}$ is the interval at which selected households were assigned for the interview of a married female (in female PSUs) or an unmarried male (in male PSUs) in the $i^{\text {th }}$ domain.

The weights were then normalised so that the total number of cases was unchanged after weighting. Hence, the normalised weights for households and eligible respondents were:

$$
\begin{aligned}
W_{H i j}^{\prime} & =\frac{\sum n_{i j}}{\sum W_{H i j} \times n_{i j}} \times W_{H i j} \\
W_{E i j}^{\prime} & =\frac{\sum n_{i j}}{\sum W_{E i j} \times n_{i j}} \times W_{E i j}
\end{aligned}
$$

where $n_{i j}$ refers to the number of completed interviews in the $j^{\text {th }}$ sub-domain within the $\mathrm{i}^{\text {th }}$ domain.

In order to provide estimates for all young males or females (married and unmarried), multiplication factors were computed for married and unmarried males and females (four categories) in urban and rural areas, which, when multiplied with existing individual weights, provided the combined weights for the male and female samples, respectively. For example, the multiplication factor for the male sample $\left(\mathrm{M}_{\mathrm{k}}^{\mathrm{l}}\right)$ was computed as follows:

$$
M_{k}^{l}=\frac{\frac{p_{k}^{l}}{p^{l}}}{\frac{s_{k}^{l}}{s^{l}}}
$$

where

$$
\begin{aligned}
\mathrm{p}_{\mathrm{k}}^{\mathrm{l}}= & \text { number of eligible male respondents of category } \mathrm{k} \text { (married or unmarried) in the } \mathrm{l}^{\text {th }} \text { area } \\
& \text { (urban or rural), } \\
\mathrm{p}^{1}= & \text { number of eligible male respondents in the } \mathrm{l}^{\text {th }} \text { area (urban or rural), } \\
s_{\mathrm{k}}^{1}= & \text { number of completed interviews with male respondents from category } \mathrm{k} \text { (married or unmarried) } \\
& \text { in the } \mathrm{l}^{\text {th }} \text { area (urban or rural), and } \\
\mathrm{s}^{1}= & \text { number of completed interviews with male respondents in the } \mathrm{l}^{\text {th }} \text { area (urban or rural). }
\end{aligned}
$$

Similar fractions were computed for the female sample. 


\subsection{Recruitment, training and fieldwork}

As the survey in Bihar followed that conducted in Jharkhand, available interviewers who had conducted the survey in Jharkhand were also responsible for conducting fieldwork in Bihar. As mentioned in the Jharkhand report, some 80 young men and women had undergone interviewer training prior to the Jharkhand survey and on the basis of their performance, 58 youth were recruited as field investigators. Prior to the initiation of fieldwork in Bihar, a refresher training lasting one week was conducted for 36 young men and women and another 9 received the full three week training two months later. Training of interviewers was conducted jointly by principal investigators from IIPS and the Population Council.

As mentioned in the Jharkhand report, initial training for field investigators for the main survey lasted three weeks. It included lectures and interactive sessions on a range of issues such as the sexual and reproductive health situation of youth in India, an overview of gender issues, ethical issues in research, violence against women and mental health as well as detailed explanations of sex and contraception. Efforts were also made to enable trainees to overcome their own inhibitions about discussing sexual and reproductive health matters. They were provided opportunities to ask questions via an anonymous drop-box; questions were then answered in the course of training. Trainees were familiarised with each module of the questionnaire, complicated concepts and questions and their underlying rationale. Role-plays and mock interviews were conducted in reference to each module. Towards the end of the training programme, field practice sessions were organised in which trainees were taken to a village and an urban slum setting and asked to conduct interviews. . The training team monitored each trainee's progress on a regular basis and selected as interviewers only those trainees who demonstrated full understanding of the questionnaire as well as the ability to ask questions appropriately and record responses accurately. During the refresher training prior to the initiation of the survey in Bihar, the focus was on content of the questionnaire and included role-plays, mock interviews and field practice sessions.

In addition, over 20 individuals underwent training for mapping and house-listing exercises in the course of a four-day training, during which trainees were familiarised with house-listing procedures in both classroom and field situations. All the trainees were then recruited for mapping and house-listing exercises.

Interviewers were divided into eight teams, four each to interview young women and men, respectively. Female interviewers interviewed young women and male interviewers interviewed young men. Each team comprised one field editor to take care of field editing, back-checks and quality control of interviews; and one supervisor, responsible for the overall management of fieldwork and team-related logistics as well as assisting in field editing and back-checking. Interviewer and supervisor/editor manuals were prepared, translated into Hindi and provided to each team member as appropriate. These manuals clarified the meaning and appropriate coding of every question in the questionnaire.

Research officers were deputed to oversee fieldwork and ensure that correct survey procedures were followed and data quality was maintained. Principal investigators from IIPS and the Population Council made monthly or bi-monthly visits to monitor and supervise data collection operations. Each team filled quality control sheets regularly, giving the team, research officers and coordinators a quick view of the quality of ongoing fieldwork. These control sheets were designed to provide information on response rates in each PSU covered, track sensitive issue reporting and interviewer performance.

\subsection{Ethical considerations}

As this was the first such study of its kind in India, in which sensitive sexual and reproductive experiences were sought in a survey situation, it was unclear how youth respondents and community members would 
react. At the same time, it was clear that if youth participated in the interviews, its content was likely to prompt questions and problems for which support would be requested. A number of ethical issues arose which influenced the design and implementation of the Youth Study.

First, to address our concern that if interviews with young women and men were conducted in the same PSU, it could lead to teasing, harassment, harm to girls' reputations and even violence, we decided that the study would be undertaken in one set of PSUs for young men and in a completely different set for young women. Likewise, we also ensured that two unmarried brothers or sisters, two married brothers or sisters or two sisters- or brothers-in-law would not be interviewed from the same household in case such a practice caused conflict within the family. Hence, just one individual from any category was selected for interview in each household. In case both a married and an unmarried individual were selected from a particular household, interviews were conducted separately but simultaneously.

Second, youth themselves contributed-albeit indirectly-to the development of the questionnaire. In the course of our pre-survey qualitative phase, youth and key informants informed our study teams of various youth behaviours; youth described the ways in which they referred to various sensitive behaviours. In order to minimise discomfort during questioning, the scenarios and terminologies described by youth themselves were adapted for use in the most sensitive parts of our questionnaires.

Third, interviewers underwent extensive training in ethical issues. Emphasis was laid on explaining the content of the questionnaire, respondents' right to refuse to participate or answer any question, and informed consent. At the same time, we trained interviewers on how to ask sensitive questions regarding sexual experience, domestic violence and forced sex in particular, in empathetic and non-judgemental ways and emphasised the importance of offering to refer those in need to appropriate nearby organisations.

Fourth, before entering a PSU, teams were instructed to apprise community leaders of the study and seek their support for its implementation in the community. This step ensured that community support was forthcoming and enabled team members to build rapport within the community easily. We note that despite the sensitive nature of the questions, not a single PSU in Bihar refused permission to Youth Study teams on the grounds of study content.

Fifth, even though consent was sought from each individual to be interviewed, in the case of unmarried youth aged 15-17, consent was also sought from a parent or guardian.

Sixth, all questionnaires were entirely anonymous and names were never recorded. In order to preserve the confidentiality of the respondent or the parent/guardian, signature on the consent form was optional; however, the interviewer was required to sign that she or he had explained the contents of the consent form to the respondent or parent. Consent forms were detached and stored separately from questionnaires.

Seventh, every effort was made to maintain privacy in the course of the interview. Interviewers were permitted to skip to relatively non-sensitive sections in case the interview was observed by parents or other family members. If possible, particularly in the case of young men, interviews were held outside the home-often in a nearby field-in order to ensure privacy. Each team was trained to assign one interviewer to conduct parallel discussion sessions with bystanders, thereby providing privacy to the interview. This proved particularly useful in the case of interviews with young women. Finally, interviewers were instructed that if privacy could not be ensured, the interview must be terminated without asking sensitive questions. Due to these strategies, few interviews had to be terminated for want of privacy and in no case was a young respondent's privacy breached. 
Eighth, the study team realised that this was perhaps one of the first opportunities many youth would have to discuss intimate matters and that respondents might request information on sexual and reproductive issues or seek counselling or treatment of a health problem. In each state, therefore, the study team approached non-governmental organisations (NGOs) that conducted youth- or health-related activities at the district level and sought their consent for referring any youth in need to their organisation. Many NGOs agreed, and youth (and some adults) in need were later referred to these organisations, along with an indication that the individual had been part of the Youth Study. At the same time, research officers and team members themselves built rapport with public health authorities and referred to their facilities those who preferred to seek public services, again, along with the information that the individual had been part of the Youth Study.

Finally, many youth were in need of information on sexual and reproductive health matters. On occasion, interviewers themselves responded to their questions. In addition, easy to read booklets (for example, the Neeli Kitab prepared by TARSHI) were distributed to youth who requested them. In total, some 300 booklets were distributed.

\subsection{Data processing}

All completed questionnaires were sent to project offices at IIPS, Mumbai and the Population Council, New Delhi for editing and data processing. Completed questionnaires were rechecked and further edited in the office for omissions and consistency. Responses to open-ended questions were scrutinised and common responses were provided codes. For entering the edited data, a special software package was developed using CSPro 3.0. Data were entered twice by different entry operators to minimise entry problems. The raw data were validated and cleaned to remove possible inconsistencies. The analysis of data was carried out using SPSS 14.0.

\subsection{Interview outcomes}

Table 1.2 provides the outcome of household interviews by type of PSU (male or female) and residence. In all, of the 30,888 households selected for interview, $7 \%$ could not be contacted because the house could not be located or was vacant or because the entire household was absent over an extended period of time. In total, however, the response to the household questionnaire was high: $98 \%$ and $99 \%$ in male and female PSUs, respectively. A total of 10,887 and 17,318 interviews were completed in urban and rural areas, respectively. Response rates in urban and rural areas were almost identical. We note that just two rural households and less than $1 \%$ of urban households selected refused to be interviewed.

Table 1.3 presents similar findings with regard to interviews with eligible respondents. In Bihar, 8,136 interviews were completed: 1,115 with married young men, 1,492 with unmarried young men, 2,341 with married young women and 3,188 with unmarried young women. Response rates for individual interviews were in the range of $78-90 \%$; the response rate was the lowest among married young men $(78 \%)$ and the highest among unmarried young women (90\%). Response rates did not vary much by residence or marital status, except that among young women, response rates for unmarried respondents were higher than those for married respondents. The main reason for non-response was that the respondent was not at home, ranging from $8-16 \%$ among unmarried respondents to $17-19 \%$ among married respondents. The high level of nonresponse for married and unmarried young men may be attributed to work-related temporary migration, and for married young women to their relatively frequent movement to their natal homes, particularly for delivery, a finding also noted in other studies of married youth (see for example, Santhya et al., 2008). We attribute the low refusal rates to efforts described earlier that were implemented for ethical reasons, which, at the same time, enabled the development of considerable rapport and trust between study communities and our interview teams. 
Table 1.2: Results of household interviews

Percent distribution of surveyed households by results of interviews, according to residence (unweighted), Bihar, 2007

\begin{tabular}{|c|c|c|c|c|c|c|}
\hline \multirow[t]{2}{*}{ Results of interviews } & \multicolumn{2}{|c|}{ All PSUs } & \multicolumn{2}{|c|}{ Male PSUs } & \multicolumn{2}{|c|}{ Female PSUs } \\
\hline & Percent & Number & Percent & Number & Percent & Number \\
\hline \multicolumn{7}{|c|}{ Combined } \\
\hline a. Interview completed & 91.3 & 28,205 & 91.4 & 8,950 & 91.3 & 19,255 \\
\hline $\begin{array}{l}\text { b. No respondent or no competent } \\
\text { respondent at home at the time of visit }\end{array}$ & 0.7 & 207 & 0.8 & 78 & 0.6 & 129 \\
\hline $\begin{array}{l}\text { c. Entire household absent for extended } \\
\text { period of time }\end{array}$ & 3.4 & 1,062 & 3.7 & 363 & 3.3 & 699 \\
\hline d. Refused & 0.2 & 77 & 0.5 & 48 & 0.1 & 29 \\
\hline e. Dwelling vacant/destroyed/not found & 3.2 & 990 & 2.9 & 285 & 3.3 & 705 \\
\hline f. Address not a dwelling & 0.8 & 251 & 0.5 & 48 & 1.0 & 203 \\
\hline g. Other & 0.3 & 96 & 0.3 & 25 & 0.3 & 71 \\
\hline Total households selected & 100.0 & 30,888 & 100.0 & 9,797 & 100.0 & 21,091 \\
\hline Response rate (HRR) & 98.7 & & 98.3 & & 98.9 & \\
\hline \multicolumn{7}{|c|}{ Urban } \\
\hline a. Interview completed & 91.1 & 10,887 & 90.2 & 4,558 & 91.8 & 6,329 \\
\hline $\begin{array}{l}\text { b. No respondent or no competent } \\
\text { respondent at home at the time of visit }\end{array}$ & 0.9 & 104 & 0.9 & 46 & 0.8 & 58 \\
\hline $\begin{array}{l}\text { c. Entire household absent for extended } \\
\text { period of time }\end{array}$ & 3.2 & 384 & 3.5 & 176 & 3.0 & 208 \\
\hline d. Refused & 0.6 & 75 & 0.9 & 47 & 0.4 & 28 \\
\hline e. Dwelling vacant/destroyed/not found & 3.3 & 393 & 3.5 & 178 & 3.1 & 215 \\
\hline f. Address not a dwelling & 0.7 & 79 & 0.7 & 33 & 0.7 & 46 \\
\hline g. Other & 0.2 & 23 & 0.3 & 13 & 0.1 & 10 \\
\hline Total households selected & 100.0 & 11,945 & 100.0 & 5,051 & 100.0 & 6,894 \\
\hline Response rate (HRR) & 98.2 & & 97.7 & & 98.6 & \\
\hline \multicolumn{7}{|c|}{ Rural } \\
\hline a. Interview completed & 91.4 & 17,318 & 92.5 & 4,392 & 91.0 & 12,926 \\
\hline $\begin{array}{l}\text { b. No respondent or no competent } \\
\text { respondent at home at the time of visit }\end{array}$ & 0.5 & 103 & 0.7 & 32 & 0.5 & 71 \\
\hline $\begin{array}{l}\text { c. Entire household absent for extended } \\
\text { period of time }\end{array}$ & 3.6 & 678 & 3.9 & 187 & 3.5 & 491 \\
\hline d. Refused & 0.0 & 2 & 0.0 & 1 & 0.0 & 1 \\
\hline e. Dwelling vacant/destroyed/not found & 3.2 & 597 & 2.3 & 107 & 3.5 & 490 \\
\hline f. Address not a dwelling & 0.9 & 172 & 0.3 & 15 & 1.1 & 157 \\
\hline g. Other & 0.4 & 73 & 0.3 & 12 & 0.4 & 61 \\
\hline Total households selected & 100.0 & 18,943 & 100.0 & 4,746 & 100.0 & 14,197 \\
\hline Response rate (HRR) & 99.0 & & 98.9 & & 99.0 & \\
\hline
\end{tabular}

Note: The household response rate (HRR) was calculated as: $H R R=(a / a+b+d+g)^{*} 100$. PSU: Primary sampling unit. 
Table 1.3: Results of eligible respondent interviews

Percent distribution of eligible respondents by results of interviews, according to residence (unweighted), Bihar, 2007

\begin{tabular}{|c|c|c|c|c|c|c|c|c|c|c|c|c|}
\hline \multirow[t]{3}{*}{ Results of interviews } & \multicolumn{4}{|c|}{ Combined } & \multicolumn{4}{|c|}{ Urban } & \multicolumn{4}{|c|}{ Rural } \\
\hline & \multicolumn{2}{|c|}{ Married } & \multicolumn{2}{|c|}{ Unmarried } & \multicolumn{2}{|c|}{ Married } & \multicolumn{2}{|c|}{ Unmarried } & \multicolumn{2}{|c|}{ Married } & \multicolumn{2}{|c|}{ Unmarried } \\
\hline & Percent & Number & Percent & Number & Percent & Number & Percent & Number & Percent & Number & Percent & Number \\
\hline \multicolumn{13}{|c|}{ Men (15-24) } \\
\hline a. Interview completed & 77.9 & 1,115 & 80.3 & 1,492 & 77.6 & 547 & 82.8 & 833 & 78.2 & 568 & 77.3 & 659 \\
\hline b. Interview partially completed & 0.1 & 2 & 0.1 & 2 & 0.1 & 1 & 0.1 & 1 & 0.1 & 1 & 0.1 & 1 \\
\hline c. Respondent not at home & 19.1 & 274 & 15.6 & 289 & 17.7 & 125 & 12.9 & 130 & 20.5 & 149 & 18.7 & 159 \\
\hline d. Respondent refused & 1.2 & 17 & 0.6 & 12 & 1.8 & 13 & 0.7 & 7 & 0.6 & 4 & 0.6 & 5 \\
\hline e. Respondent's parent refused & 0.8 & 11 & 1.1 & 21 & 1.1 & 8 & 1.6 & 16 & 0.4 & 3 & 0.6 & 5 \\
\hline f. Respondent incapacitated & 0.4 & 6 & 1.9 & 36 & 0.7 & 5 & 1.5 & 15 & 0.1 & 1 & 2.5 & 21 \\
\hline g. No reason given & 0.4 & 6 & 0.3 & 6 & 0.9 & 6 & 0.4 & 4 & 0.0 & 0 & 0.2 & 2 \\
\hline Total selected & 100.0 & 1,431 & 100.0 & 1,858 & 100.0 & 705 & 100.0 & 1,006 & 100.0 & 726 & 100.0 & 852 \\
\hline Response rate (IRR) & 78.0 & & 80.4 & & 77.7 & & 82.8 & & 78.3 & & 77.3 & \\
\hline \multicolumn{13}{|c|}{ Women (15-24) } \\
\hline a. Interview completed & 82.0 & 2,341 & 90.1 & 3,188 & 81.1 & 1,136 & 88.8 & 1,445 & 82.9 & 1,205 & 91.2 & 1,743 \\
\hline b. Interview partially completed & 0.3 & 8 & 0.3 & 11 & 0.4 & 5 & 0.4 & 6 & 0.2 & 3 & 0.3 & 5 \\
\hline c. Respondent not at home & 16.8 & 479 & 7.8 & 277 & 17.4 & 244 & 8.7 & 142 & 16.2 & 235 & 7.1 & 135 \\
\hline d. Respondent refused & 0.4 & 11 & 0.3 & 11 & 0.4 & 5 & 0.4 & 7 & 0.4 & 6 & 0.2 & 4 \\
\hline e. Respondent's parent refused & 0.2 & 6 & 0.6 & 21 & 0.4 & 6 & 1.0 & 16 & 0.0 & 0 & 0.3 & 5 \\
\hline f. Respondent incapacitated & 0.3 & 8 & 0.8 & 29 & 0.2 & 3 & 0.6 & 9 & 0.3 & 5 & 1.0 & 20 \\
\hline g. No reason given & 0.1 & 2 & 0.1 & 3 & 0.1 & 2 & 0.2 & 3 & 0.0 & 0 & 0.0 & 0 \\
\hline Total selected & 100.0 & 2,855 & 100.0 & 3,540 & 100.0 & 1,401 & 100.0 & 1,628 & 100.0 & 1,454 & 100.0 & 1,912 \\
\hline Response rate (IRR) & 81.9 & & 90.1 & & 81.1 & & 88.8 & & 82.9 & & 91.1 & \\
\hline
\end{tabular}

Note: The individual response rate (IRR) was calculated as: $I R R=(a / a+b+c+d+e+f+g)^{*} 100$.

\subsection{Structure of the report}

This report is structured as follows. Chapter 2 provides a socio-demographic profile of the surveyed population and respondents, and for those living in rural areas, of facilities available to the rural population. Chapters 3,4 and 5 discuss young people's educational attainment patterns, economic and non-economic activity experiences and media exposure, respectively. Chapter 6 discusses growing up issues, including young people's relationships with parents and peers. Chapters 7 and 8 focus, respectively, on young people's autonomy and gender role attitudes, and awareness of sexual and reproductive health matters. Chapter 9 describes the formation of pre-marital romantic relations and pre-marital sexual experience with romantic and non-romantic partners. Chapter 10 discusses the transition to marriage and experiences in early married life. Chapter 11 presents information on health and health seeking behaviours and substance use. Chapter 12 focuses on civic and political participation and related attitudes. A summary of each chapter (3-12) is provided at its conclusion. Finally, Chapter 13 offers recommendations for programmes and research.

In view of the heterogeneity of youth by sex, marital status and rural-urban residence, in each chapter, tables are presented that describe findings, separately, on the situation of married and unmarried young men and women residing in urban and rural areas, respectively. In order to provide information on all youth in Bihar, we provide findings for all young men and women aged 15-24. We excluded married young men aged 25-29 in order to enable comparison. 
Introduction

All means, medians and percentages indicated in tables have been weighted using normalised weights for the total population. However, in order to show the total number of youth interviewed, unweighted numbers of respondents (Ns) are provided in each table. Because numbers are unweighted and percentages are weighted, we caution readers against deriving numbers based on percentages provided in tables. 


\section{Profile of surveyed communities, households and respondents}

This chapter presents a summary of the community-level characteristics of the rural areas surveyed as well as household- and respondent-level profiles of the surveyed population. First, using data drawn from the community questionnaire, it describes the rural communities in which the survey was undertaken in terms of village size, agricultural land holding and access to facilities more generally available in urban settings. Thereafter, drawing on data from the household questionnaire, the chapter profiles surveyed households in terms of socio-demographic and housing characteristics, agricultural land holding and economic status. Comparisons are drawn throughout between the distribution of the population as recorded in the present survey and that reported by the 2001 Census of India (Office of the Registrar General and Census Commissioner, 2001b) as well as the most recent NFHS (IIPS and Macro International, 2008). Finally, we present the socio-demographic characteristics of youth respondents and their parents, drawn from individual questionnaires.

\subsection{Profile of rural communities surveyed}

This section provides a profile of the rural PSUs (150 selected villages and 7 link villages) in which the survey was conducted. It should be noted that as sampling of rural PSUs was conducted with the probability of selection proportional to size, the proportion of large villages in the Youth Study sample is likely to have been greater than the proportion of such villages in Bihar as a whole. However, because the selection of villages was made from a list of villages stratified by size, this effect of using the probability proportional to size sampling technique on village size distribution is likely to be small.

As indicated in Table 2.1, the vast majority of the villages surveyed were of either medium size $(1,000-4,999$ persons) or large size (5,000 or more persons); $46 \%$ each fell into these two categories. Just $8 \%$ of the villages were small in size (less than 1,000 persons). The majority (70\%) of villages surveyed contained less than 500 hectares of agricultural land. Nonetheless, most of the agricultural land was irrigated: for example, more than half of all agricultural land was irrigated in over three-quarters of the surveyed villages. Indeed, in half of the surveyed villages, three-quarters or more of the agricultural land was irrigated.

Table 2.2 presents data regarding access to a variety of facilities among the rural population surveyed. Findings show that the median distance to the nearest town was 10 kilometres from the village of residence. Over half of the rural population (53\%) reported having an all-weather road in their village. Almost one-fifth (18\%) of the rural population had a bank located in their village and over two-fifths (42\%) had a post office.

Primary schools were not available in all villages: only $94 \%$ of the rural population had access to a primary school in their village of residence. Middle, secondary and higher secondary schools were progressively less likely to be available, and of particular note is that just $14 \%$ and $3 \%$ of the rural population resided in a village containing a secondary and a higher secondary school, respectively. The median distance to the nearest secondary school was 3 kilometres and to a higher secondary school, 10 kilometres. Colleges and technical institutions were rarely available at the village level; just $1-4 \%$ of the population had such a facility 
Table 2.1: Profile of surveyed villages

\section{Percentage of surveyed villages and residents by village size and agricultural land holding, Bihar (rural), 2007}

\begin{tabular}{|c|c|c|c|c|}
\hline \multirow[t]{2}{*}{ Village characteristics } & \multicolumn{2}{|c|}{ Villages } & \multicolumn{2}{|c|}{ Residents } \\
\hline & Percent & Number & Percent & Number \\
\hline \multicolumn{5}{|c|}{ Current population (no. of persons) } \\
\hline Less than 1,000 & 8.3 & 13 & 5.3 & 4,885 \\
\hline $1,000-4,999$ & 45.9 & 72 & 46.8 & 43,837 \\
\hline $5,000-9,999$ & 25.5 & 40 & 26.8 & 25,730 \\
\hline 10,000 or more & 20.4 & 32 & 21.2 & 19,868 \\
\hline \multicolumn{5}{|c|}{ Size of agricultural land (hectares) } \\
\hline Less than 500 & 70.1 & 110 & 68.9 & 60,562 \\
\hline 500-999 & 12.1 & 19 & 12.3 & 12,548 \\
\hline $1,000-4,999$ & 5.1 & 8 & 5.1 & 4,847 \\
\hline 5,000 and more & 0.6 & 1 & 0.8 & 406 \\
\hline \multicolumn{5}{|c|}{ Proportion of irrigated agricultural land owned } \\
\hline Less than $25 \%$ & 8.3 & 13 & 8.2 & 10,091 \\
\hline $25-49 \%$ & 10.2 & 16 & 9.6 & 9,123 \\
\hline $50-74 \%$ & 26.1 & 41 & 27.5 & 27,081 \\
\hline $75 \%$ or more & 50.3 & 79 & 49.4 & 41,269 \\
\hline Total & 100.0 & 157 & 100.0 & 94,320 \\
\hline
\end{tabular}

Note: All Ns are unweighted. Column totals may not equal 100\% or the total number due to missing cases or "don't know" responses.

within the village and the median distance to the nearest college was 15 kilometres and the nearest technical institution, 30 kilometres.

While anganwadis were easily accessible, access to other health facilities was very limited; $94 \%$ of the population surveyed had an anganwadi in the village of residence. In contrast, just 38\% had a sub-centre within the village. The median distance to the nearest sub-centre was 2 kilometres. As in the case of education, higher-level facilities were even less accessible: only $10 \%$ of the population resided in a village containing a primary health centre. Median distances to the nearest primary health centre, community health centre, and government hospital were as much as 5, 10 and 21 kilometres, respectively, highlighting that access to government health facilities-even primary health centres-remains difficult in the rural areas of Bihar. Moreover, even private clinics or hospitals (including those practising Indian systems of medicine and homoeopathy) were relatively inaccessible to the rural population; only $16 \%$ and $4 \%$ of rural residents had access to a private clinic and hospital, respectively, within the village and median distances to a private clinic and hospital were 6 and 14 kilometres, respectively.

The availability of civic organisations and entertainment facilities was also assessed and findings again indicate limited access to such facilities. Just $11 \%$ of the population resided in villages containing a club or mandal. While half (52\%) lived in villages containing a community hall, fewer than $10 \%$ of rural population lived in villages containing a cinema theatre $(5 \%)$, drama theatre $(8 \%)$ or video parlour (9\%). Distances to the nearest such facilities were also considerable; for example, the nearest cinema theatre was an average of 10 kilometres from the village. Playgrounds and sports clubs were available in the village of residence to $31 \%$ and $8 \%$ of the population, respectively; the average distance to the nearest sports club was 12 kilometres. 
Table 2.2: Proximity of study residents to selected facilities

Percentage of residents covered by the survey by distance from the nearest facility/service, Bihar (rural), 2007

\begin{tabular}{|c|c|c|c|c|c|c|c|}
\hline \multirow[t]{2}{*}{ Nearest facility/service } & \multicolumn{6}{|c|}{$\%$ of residents } & \multirow{2}{*}{$\begin{array}{c}\text { Median } \\
\text { distance to } \\
\text { nearest facility/ } \\
\text { service }(\mathbf{k m})\end{array}$} \\
\hline & $\begin{array}{l}\text { Within } \\
\text { village }\end{array}$ & $\begin{array}{l}<2 \\
\mathrm{~km}\end{array}$ & $\begin{array}{l}2-5 \\
\text { km }\end{array}$ & $\begin{array}{l}6-9 \\
\mathrm{~km}\end{array}$ & $\begin{array}{c}10-19 \\
\text { km }\end{array}$ & $\begin{array}{l}20 \mathrm{~km} \\
\text { or more }\end{array}$ & \\
\hline Town & NA & 1.9 & 17.8 & 20.9 & 28.2 & 29.9 & 10.0 \\
\hline District headquarters & NA & 0.0 & 2.8 & 5.4 & 17.9 & 73.9 & 28.0 \\
\hline Railway station & 3.2 & 1.2 & 19.2 & 21.1 & 27.3 & 28.1 & 10.0 \\
\hline Transport service to other places & 19.1 & 8.2 & 41.8 & 17.7 & 6.3 & 6.8 & 3.0 \\
\hline All-weather road & 52.6 & 5.5 & 22.6 & 11.9 & 4.4 & 3.0 & $\mathrm{NC}$ \\
\hline Post office & 41.5 & 14.4 & 39.0 & 2.9 & 1.9 & 0.3 & 1.0 \\
\hline Bank & 17.6 & 8.8 & 51.3 & 15.2 & 6.1 & 0.9 & 3.0 \\
\hline \multicolumn{8}{|l|}{ Educational facilities } \\
\hline Primary school & 94.3 & 2.7 & 3.0 & 0.0 & 0.0 & 0.0 & NC \\
\hline Middle school & 59.3 & 7.6 & 31.8 & 1.3 & 0.0 & 0.0 & $\mathrm{NC}$ \\
\hline Secondary school & 13.7 & 6.3 & 56.8 & 14.5 & 8.2 & 0.6 & 3.0 \\
\hline Higher secondary school & 3.2 & 0.8 & 22.9 & 21.3 & 27.0 & 24.9 & 10.0 \\
\hline College & 3.7 & 0.0 & 9.7 & 15.5 & 32.4 & 38.7 & 15.0 \\
\hline Technical school/college & 0.7 & 0.4 & 1.3 & 8.0 & 15.6 & 71.8 & 30.0 \\
\hline Ashram school & 1.2 & 0.7 & 9.5 & 7.7 & 9.7 & 21.8 & 15.0 \\
\hline Madarsa & 32.8 & 5.5 & 31.6 & 16.7 & 8.1 & 4.0 & 3.0 \\
\hline Any of the above & 95.6 & 2.0 & 2.4 & 0.0 & 0.0 & 0.0 & $\mathrm{NC}$ \\
\hline \multicolumn{8}{|l|}{ Health facilities } \\
\hline ICDS (anganwadi) & 93.5 & 0.9 & 3.8 & 0.7 & 0.0 & 0.0 & $\mathrm{NC}$ \\
\hline Sub-centre & 37.7 & 9.1 & 37.6 & 8.5 & 3.9 & 1.3 & 2.0 \\
\hline Primary health centre & 9.6 & 4.5 & 38.2 & 29.5 & 14.9 & 3.4 & 5.0 \\
\hline Community health centre & 1.3 & 1.1 & 21.9 & 14.0 & 27.6 & 20.5 & 10.0 \\
\hline Government dispensary & 2.3 & 0.8 & 11.4 & 8.1 & 12.7 & 27.0 & 14.0 \\
\hline Government hospital & 2.7 & 0.8 & 7.7 & 8.4 & 22.7 & 57.7 & 21.0 \\
\hline Private clinic, including ISMH & 15.8 & 3.7 & 27.8 & 16.9 & 16.0 & 19.2 & 6.0 \\
\hline Private hospital & 3.9 & 0.0 & 15.5 & 12.8 & 29.1 & 38.8 & 14.0 \\
\hline Any of the above & 95.5 & 1.2 & 3.2 & 0.0 & 0.0 & 0.0 & NC \\
\hline Club/Mandal & 10.7 & NA & NA & NA & NA & NA & NA \\
\hline \multicolumn{8}{|l|}{ Entertainment/sports facilities } \\
\hline Community hall & 51.7 & 3.9 & 29.1 & 5.5 & 3.0 & 5.3 & $\mathrm{NC}$ \\
\hline Playground & 30.9 & 5.2 & 40.0 & 10.9 & 7.3 & 5.0 & 3.0 \\
\hline Sports club & 8.3 & 0.0 & 18.5 & 12.3 & 20.3 & 34.5 & 12.0 \\
\hline Video parlour & 9.0 & 0.4 & 20.2 & 19.5 & 22.0 & 13.1 & 8.0 \\
\hline Cinema theatre & 4.6 & 1.3 & 17.8 & 21.3 & 31.7 & 23.4 & 10.0 \\
\hline Drama theatre & 7.8 & 7.2 & 7.8 & 13.4 & 24.4 & 39.4 & 12.0 \\
\hline Any of the above & 64.7 & 2.2 & 27.8 & 3.9 & 0.6 & 0.7 & $\mathrm{NC}$ \\
\hline
\end{tabular}

Note: ICDS: Integrated Child Development Services. ISMH: Indian systems of medicine and homoeopathy. NA: Not applicable. NC: Median cannot be calculated. 


\subsection{Profile of the household population: Age-sex distribution}

Age and sex distributions play an important role in the study of demographic processes. Details of the age and sex distribution of the de jure population in the survey area are presented in Table 2.3. Corresponding distributions from the 2001 Census are provided to enable comparison.

The age distribution was typical of a high fertility population, with a larger proportion of the population in the younger age groups than other age groups. Indeed, the virtually identical proportions of the population aged 0-4 recorded in 2001 and 2006 suggest unchanging levels of fertility, In comparison, total fertility rates recorded in NFHS-3 suggest some increase in fertility (3.7 and 4.0 in 1998-99 and 2005-06, respectively (IIPS and Macro International 2008).

With regard to the youth population, the distribution suggests that at the time of the survey, $13 \%$ of the population was aged $10-14$ years, $9 \%$ was aged $15-19$ years and $7 \%$ was aged $20-24$ years. A total of $16.5 \%$ of the population was aged 15-24 years, similar to that observed in the 2001 Census (16.3\%) (Office of the Registrar General and Census Commissioner, 2001b).

Table 2.3: Distribution of the surveyed population by age and sex

Percent distribution of the surveyed population by age and sex, according to residence, Bihar, 2007, and population distribution as reported in the 2001 Census for Bihar

\begin{tabular}{|c|c|c|c|c|c|c|}
\hline \multirow[t]{2}{*}{ Age (years) (\%) } & \multicolumn{3}{|c|}{ Youth Study, 2007} & \multicolumn{3}{|c|}{ Census, 2001} \\
\hline & Total & Male & Female & Total & Male & Female \\
\hline \multicolumn{7}{|c|}{ Combined } \\
\hline Below 1 & 2.7 & 2.8 & 2.6 & 1.7 & 1.7 & 1.7 \\
\hline $1-4$ & 11.7 & 12.4 & 11.1 & 11.6 & 11.3 & 11.9 \\
\hline $5-9$ & 15.9 & 16.7 & 15.2 & 15.4 & 15.5 & 15.3 \\
\hline $10-14$ & 13.2 & 14.3 & 12.1 & 13.3 & 13.8 & 12.8 \\
\hline $15-19$ & 9.3 & 8.6 & 9.9 & 8.7 & 9.3 & 8.0 \\
\hline $20-24$ & 7.2 & 5.6 & 8.7 & 7.6 & 7.4 & 7.9 \\
\hline $25-29$ & 6.3 & 5.5 & 7.0 & 7.1 & 6.7 & 7.6 \\
\hline $30-34$ & 5.9 & 5.2 & 6.6 & 6.7 & 6.3 & 7.1 \\
\hline $35-39$ & 5.8 & 6.0 & 5.6 & 6.1 & 6.0 & 6.3 \\
\hline $40-44$ & 4.6 & 4.5 & 4.7 & 5.0 & 5.1 & 4.9 \\
\hline $45-49$ & 4.3 & 4.3 & 4.3 & 4.2 & 4.1 & 4.2 \\
\hline $50-54$ & 3.4 & 3.6 & 3.2 & 3.3 & 3.6 & 2.9 \\
\hline $55-59$ & 2.7 & 2.7 & 2.8 & 2.5 & 2.3 & 2.7 \\
\hline $60-64$ & 2.8 & 3.0 & 2.6 & 2.5 & 2.6 & 2.5 \\
\hline $65-69$ & 1.9 & 2.0 & 1.7 & 1.6 & 1.6 & 1.7 \\
\hline $70-74$ & 1.3 & 1.6 & 1.0 & 1.2 & 1.3 & 1.2 \\
\hline 75 and above & 1.0 & 1.2 & 0.9 & 1.2 & 1.3 & 1.2 \\
\hline Age not stated & 0.0 & 0.0 & 0.0 & 0.2 & 0.2 & 0.2 \\
\hline Number & 156,197 & 78,553 & 77,639 & $82,998,509$ & $43,243,795$ & $39,754,714$ \\
\hline Median age (years) & 18.0 & 16.0 & 19.0 & 19.5 & 19.1 & 20.2 \\
\hline Sex ratio, all ages ${ }^{1}$ & 1,043 & NA & NA & 919 & NA & NA \\
\hline Sex ratio, age $0-6$ years ${ }^{1}$ & 935 & NA & NA & 942 & NA & NA \\
\hline
\end{tabular}

Cont'd on next page.. 
Table 2.3: (Cont'd)

\begin{tabular}{|c|c|c|c|c|c|c|}
\hline \multirow[t]{2}{*}{ Age (years) (\%) } & \multicolumn{3}{|c|}{ Youth Study, 2007} & \multicolumn{3}{|c|}{ Census, 2001} \\
\hline & Total & Male & Female & Total & Male & Female \\
\hline \multicolumn{7}{|c|}{ Urban } \\
\hline Below 1 & 1.9 & 2.0 & 1.8 & 1.2 & 1.2 & 1.3 \\
\hline $1-4$ & 8.8 & 8.8 & 8.9 & 9.4 & 9.0 & 9.8 \\
\hline $5-9$ & 12.6 & 13.0 & 12.1 & 13.2 & 12.9 & 13.4 \\
\hline $10-14$ & 12.7 & 12.9 & 12.5 & 13.6 & 13.6 & 13.7 \\
\hline $15-19$ & 11.3 & 11.2 & 11.3 & 10.7 & 11.2 & 10.1 \\
\hline $20-24$ & 9.1 & 8.5 & 9.7 & 8.8 & 9.2 & 8.3 \\
\hline $25-29$ & 7.2 & 7.0 & 7.4 & 7.5 & 7.4 & 7.7 \\
\hline $30-34$ & 6.7 & 6.5 & 7.0 & 6.8 & 6.5 & 7.1 \\
\hline $35-39$ & 6.5 & 6.8 & 6.3 & 6.5 & 6.3 & 6.8 \\
\hline $40-44$ & 5.2 & 5.2 & 5.1 & 5.4 & 5.5 & 5.2 \\
\hline $45-49$ & 4.5 & 4.5 & 4.5 & 4.6 & 4.7 & 4.5 \\
\hline $50-54$ & 3.6 & 3.7 & 3.6 & 3.4 & 3.8 & 3.1 \\
\hline $55-59$ & 2.9 & 2.9 & 2.9 & 2.5 & 2.5 & 2.6 \\
\hline $60-64$ & 2.7 & 2.7 & 2.8 & 2.3 & 2.3 & 2.3 \\
\hline $65-69$ & 1.9 & 2.0 & 1.8 & 1.6 & 1.5 & 1.6 \\
\hline $70-74$ & 1.2 & 1.3 & 1.1 & 1.1 & 1.2 & 1.1 \\
\hline 75 and above & 1.2 & 1.2 & 1.1 & 1.2 & 1.1 & 1.2 \\
\hline Age not stated & 0.0 & 0.0 & 0.0 & 0.2 & 0.2 & 0.1 \\
\hline Number & 61,877 & 32,141 & 29,735 & $8,681,800$ & $4,648,799$ & $4,033,001$ \\
\hline Median age (years) & 20.7 & 20.0 & 21.0 & 21.0 & 21.1 & 21.0 \\
\hline Sex ratio, all ages ${ }^{1}$ & 926 & NA & NA & 868 & NA & NA \\
\hline Sex ratio, age $0-6$ years ${ }^{1}$ & 901 & NA & NA & 924 & NA & NA \\
\hline \multicolumn{7}{|c|}{ Rural } \\
\hline Below 1 & 2.8 & 2.8 & 2.7 & 1.7 & 1.7 & 1.7 \\
\hline $1-4$ & 12.0 & 12.7 & 11.3 & 11.9 & 11.6 & 12.1 \\
\hline $5-9$ & 16.2 & 17.0 & 15.4 & 15.7 & 15.8 & 15.6 \\
\hline $10-14$ & 13.3 & 14.5 & 12.1 & 13.3 & 13.8 & 12.7 \\
\hline $15-19$ & 9.1 & 8.4 & 9.7 & 8.4 & 9.1 & 7.7 \\
\hline $20-24$ & 7.0 & 5.3 & 8.7 & 7.5 & 7.1 & 7.9 \\
\hline $25-29$ & 6.2 & 5.3 & 7.0 & 7.1 & 6.6 & 7.6 \\
\hline $30-34$ & 5.8 & 5.1 & 6.5 & 6.7 & 6.2 & 7.1 \\
\hline $35-39$ & 5.7 & 6.0 & 5.5 & 6.1 & 5.9 & 6.2 \\
\hline $40-44$ & 4.5 & 4.4 & 4.6 & 5.0 & 5.1 & 4.8 \\
\hline $45-49$ & 4.3 & 4.3 & 4.2 & 4.1 & 4.0 & 4.2 \\
\hline $50-54$ & 3.4 & 3.6 & 3.2 & 3.2 & 3.5 & 2.9 \\
\hline $55-59$ & 2.7 & 2.7 & 2.7 & 2.5 & 2.3 & 2.7 \\
\hline $60-64$ & 2.8 & 3.0 & 2.6 & 2.6 & 2.6 & 2.5 \\
\hline $65-69$ & 1.9 & 2.0 & 1.7 & 1.6 & 1.6 & 1.7 \\
\hline $70-74$ & 1.3 & 1.6 & 1.0 & 1.3 & 1.3 & 1.2 \\
\hline 75 and above & 1.0 & 1.2 & 0.9 & 1.2 & 1.3 & 1.2 \\
\hline Age not stated & 0.0 & 0.0 & 0.0 & 0.2 & 0.2 & 0.2 \\
\hline Number & 94,320 & 46,412 & 47,904 & $74,316,709$ & $38,594,996$ & $35,721,713$ \\
\hline Median age (years) & 18.0 & 16.0 & 18.0 & 19.4 & 18.8 & 20.1 \\
\hline Sex ratio, all ages ${ }^{1}$ & 1,055 & NA & NA & 926 & NA & NA \\
\hline Sex ratio, age $0-6$ years ${ }^{1}$ & 938 & NA & $\mathrm{NA}$ & 944 & NA & NA \\
\hline
\end{tabular}

Note: All Ns are unweighted. NA: Not applicable. ${ }^{1}$ Sex ratio is defined as the number of females per 1,000 males. 
Overall, the sex ratio of the de jure population of the state was 1,043 females per 1,000 males, considerably higher than that observed in 2001 Census (919). Similar patterns were evident in both urban (926 versus 868) and rural areas (1,055 versus 926).The increase in the sex ratio is likely the result of increased employmentrelated out-migration of single young men. The child sex ratio of the state was 935 females per 1,000 males aged 0-6, close to that reported in the 2001 Census (942). While the child sex ratio in the rural areas was similar to that observed in the 2001 Census (938 and 944, respectively), the urban child sex ratio observed in the Youth Study was somewhat lower (901 and 924, respectively). Even in the urban areas, standard errors were relatively small and the $95 \%$ confidence interval ranged from 863 to 939 , suggesting no significant change in the urban child sex ratio between the census and the Youth Survey.

\subsection{Profile of the household population: Marital status}

Table 2.4 presents the marital status distribution of the surveyed population, classified by age, residence and sex. A comparison with the marital status distribution as obtained in the 2001 Census (data not shown in tabular form) suggests a similar distribution, except that proportions never married, particularly in the ages 10-19 for females and 10-24 for males, have increased somewhat in the period 2001-06 (Office of the Registrar General and Census Commissioner, 2001b). The currently married include both those who have married and cohabited with their spouse as well as those for whom cohabitation has not been initiated, that is, for whom gauna was not performed.

Findings suggest wide gender differences in marriage age distributions, notably between the ages of 15 and 29: of those aged 15-19 years, just $7 \%$ of young men compared to $39 \%$ of young women were currently married. This increased to $48 \%$ and $90 \%$, respectively, for those aged $20-24$ years and further to $83 \%$ and 97\% for those aged 25-29 years. Patterns were similar for both rural and urban areas, but larger percentages of both young men and women were married in each age group up to age 30 in rural versus urban areas.

Table 2.4 also provides estimates of the singulate mean age at marriage (SMAM), calculated from the age-specific proportion of never-married individuals obtained in the household survey. As suggested above, the singulate mean age at marriage was considerably higher among the male population compared to the female: 23 and 18 years, respectively, indicating that women tended to marry men who were an average of five years older than themselves. Differences were also observed by rural-urban residence, with the singulate mean age at marriage 3-4 years higher among urban youth compared to rural youth.

In order to assess age at marriage among those married more recently, the Youth Study household questionnaire asked specifically about marriages that had taken place in the three years prior to interview among the household's usual residents at that time. Table 2.5 suggests that the median age at marriage for those who married in the recent past was 21 years among young men and 17 years among young women. Rural-urban differences were evident; the median age at marriage for urban men was three years higher than for rural men (24 and 21 years, respectively), and for urban women, two years higher than for rural women (19 and 17 years, respectively). Findings also show that large proportions of both young women and men had married before the legal minimum age at marriage. Over half of young women $(52 \%)$ had married before they were aged 18-the legal minimum age at marriage for females. Over two-fifths of young men (44\%) had married before they were 21 , the legal minimum age at marriage for males. It is also notable that $12 \%$ of young men had married even earlier, that is, before age 18. Rural-urban differences were notable: rural females and males were twice as likely as their urban counterparts to marry before the legal minimum age at marriage (55\% and $28 \%$ of rural and urban females, respectively; $46 \%$ and $22 \%$ of rural and urban males, respectively). 
Table 2.4: Marital status of the surveyed population

Percent distribution of the surveyed population aged 6 years and above by marital status and sex, according to residence, Bihar, 2007

\begin{tabular}{|c|c|c|c|c|c|c|}
\hline \multirow[t]{3}{*}{ Age (years) (\%) } & \multicolumn{6}{|c|}{ Marital status } \\
\hline & \multicolumn{3}{|c|}{ Male } & \multicolumn{3}{|c|}{ Female } \\
\hline & $\begin{array}{c}\text { Never } \\
\text { married }\end{array}$ & $\begin{array}{l}\text { Currently } \\
\text { married }^{1}\end{array}$ & $\begin{array}{l}\text { Separated/ } \\
\text { divorced/ } \\
\text { widowed }\end{array}$ & $\begin{array}{c}\text { Never } \\
\text { married }\end{array}$ & $\begin{array}{l}\text { Currently } \\
\text { married }^{1}\end{array}$ & $\begin{array}{l}\text { Separated/ } \\
\text { divorced/ } \\
\text { widowed }\end{array}$ \\
\hline \multicolumn{7}{|c|}{ Combined } \\
\hline $6-9$ & 99.3 & 0.1 & 0.0 & 99.5 & 0.1 & 0.0 \\
\hline $10-14$ & 99.4 & 0.3 & 0.0 & 98.1 & 1.7 & 0.0 \\
\hline $15-19$ & 92.7 & 7.2 & 0.1 & 60.7 & 39.0 & 0.2 \\
\hline $20-24$ & 51.6 & 48.0 & 0.5 & 8.9 & 90.1 & 1.0 \\
\hline $25-29$ & 15.9 & 83.2 & 0.9 & 1.5 & 97.0 & 1.5 \\
\hline 30 and above & 1.3 & 91.5 & 7.2 & 0.2 & 82.0 & 17.7 \\
\hline Total & 48.3 & 48.4 & 3.1 & 36.9 & 55.6 & 7.4 \\
\hline SMAM $^{2}$ (years) & \multicolumn{3}{|c|}{22.8} & \multicolumn{3}{|c|}{18.1} \\
\hline \multicolumn{7}{|c|}{ Urban } \\
\hline $6-9$ & 99.2 & 0.3 & 0.0 & 99.4 & 0.0 & 0.0 \\
\hline $10-14$ & 99.6 & 0.2 & 0.0 & 99.3 & 0.6 & 0.0 \\
\hline $15-19$ & 98.1 & 1.9 & 0.0 & 81.1 & 18.6 & 0.1 \\
\hline $20-24$ & 77.4 & 22.6 & 0.0 & 31.6 & 67.1 & 1.3 \\
\hline $25-29$ & 42.8 & 56.6 & 0.6 & 6.3 & 91.6 & 2.0 \\
\hline 30 and above & 3.5 & 91.3 & 5.2 & 0.5 & 81.1 & 18.4 \\
\hline Total & 51.8 & 45.8 & 2.3 & 40.2 & 51.7 & 8.0 \\
\hline SMAM $^{2}$ (years) & \multicolumn{3}{|c|}{26.1} & \multicolumn{3}{|c|}{20.7} \\
\hline \multicolumn{7}{|c|}{ Rural } \\
\hline $6-9$ & 99.3 & 0.1 & 0.0 & 99.5 & 0.1 & 0.0 \\
\hline $10-14$ & 99.4 & 0.3 & 0.0 & 98.0 & 1.8 & 0.0 \\
\hline $15-19$ & 91.9 & 8.0 & 0.1 & 58.5 & 41.2 & 0.2 \\
\hline $20-24$ & 47.3 & 52.2 & 0.5 & 6.5 & 92.5 & 1.0 \\
\hline $25-29$ & 12.2 & 86.8 & 1.0 & 1.0 & 97.5 & 1.5 \\
\hline 30 and above & 1.1 & 91.5 & 7.4 & 0.2 & 82.0 & 17.7 \\
\hline Total & 47.9 & 48.7 & 3.2 & 36.6 & 55.9 & 7.3 \\
\hline SMAM $^{2}$ (years) & \multicolumn{3}{|c|}{22.3} & \multicolumn{3}{|c|}{17.8} \\
\hline
\end{tabular}

Note: Row totals may not equal 100\% due to missing cases or "don't know" responses. ${ }^{1}$ Includes both those who are currently married and cohabiting as well as those who have not yet initiated cohabitation. ${ }^{2}$ SMAM: Singulate mean age at marriage (for those whose first marriage occurred between the ages of 6 and 55 years). 
Table 2.5: Age at marriage of usual residents of households

Age at marriage of usual residents of surveyed households who were married in the three years preceding the interview, according to residence, Bihar, 2007

\begin{tabular}{|c|c|c|c|}
\hline Age at marriage & Combined & Urban & Rural \\
\hline \multicolumn{4}{|l|}{$\begin{array}{l}\text { Median age at marriage of usual residents married in } \\
\text { the } 3 \text { years preceding the interview (years) }\end{array}$} \\
\hline Male & 21.0 & 24.0 & 21.0 \\
\hline Female & 17.0 & 19.0 & 17.0 \\
\hline \multicolumn{4}{|l|}{ Of those married in last 3 years, males married (\%): } \\
\hline Before age 18 & 12.0 & 3.1 & 12.9 \\
\hline Before age 21 & 43.5 & 21.7 & 45.6 \\
\hline $\begin{array}{l}\text { Of those married in last } 3 \text { years, females married before } \\
\text { age } 18(\%)\end{array}$ & 52.2 & 28.0 & 54.6 \\
\hline
\end{tabular}

\subsection{Profile of the household population: Educational attainment}

Table 2.6 shows the percent distribution of the surveyed population aged 6 years and above by educational level and median years of schooling according to sex, age and residence. Findings highlight low levels of educational attainment of the state's population. Over half $(52 \%)$ of the population aged 6 years and above had no formal education. More females than males fell into this group: $65 \%$ and $40 \%$, respectively. Rural-urban differences were also wide: about one-third (30\%) of the urban population compared to over half (55\%) of the rural population had never been to school. Reaffirming the low levels of educational attainment in the state, findings also indicate that just $6 \%$ of the total population had received 12 or more years of education. Gender and rural-urban differences remained evident: $9 \%$ and 3\% of males and females, respectively, and $19 \%$ and $4 \%$ of the urban and rural populations, respectively, had reached this level of education. The median years of schooling was 3 years for males and, as discussed earlier, almost two-thirds of females had never been to school. Rural-urban differences show that while the urban population had completed on average six years of schooling, as discussed earlier, over half of the rural population had never been to school.

\subsection{Profile of the household population: Work participation}

Table 2.7 presents the percentage distribution of the surveyed population aged 6 years and above reported to have been working in the seven days prior to interview according to sex and residence. While $36 \%$ of the total population was reported as working, a considerably larger percentage of males (55\%) than females $(18 \%)$ were working. Rural-urban differences suggest that more rural than urban females were reported as working (19\% versus $8 \%)$. In comparison, percentages of working males were similar in urban and rural areas (54\% and 55\%, respectively). A positive association between age and work was observed: $6 \%$ of those aged 10-14 reported working, compared with $25 \%$ of those aged $15-19,39 \%$ in the $20-24$ year age group and continued increases thereafter (reaching 60\% among those aged 30 and above). Age-specific increases were much sharper among males than females. 
Table 2.6: Educational attainment

Percent distribution of the surveyed population aged 6 years and above by educational level and median years of schooling, according to age, sex and residence, Bihar, 2007

\begin{tabular}{|c|c|c|c|c|c|c|}
\hline \multirow[t]{2}{*}{ Age (years) } & \multicolumn{4}{|c|}{ Completed years of schooling (\%) } & \multirow{2}{*}{$\begin{array}{c}\text { No. of } \\
\text { persons }\end{array}$} & \multirow{2}{*}{$\begin{array}{l}\text { Median } \\
\text { years of } \\
\text { schooling }\end{array}$} \\
\hline & None $^{1}$ & $1-7$ & $8-11$ & $\begin{array}{c}12 \\
\text { and above }\end{array}$ & & \\
\hline \multicolumn{7}{|c|}{ Combined } \\
\hline \multicolumn{7}{|l|}{ Total } \\
\hline $6-9$ & 64.2 & 35.1 & 0.0 & 0.0 & 17,727 & $\mathrm{NC}$ \\
\hline $10-14$ & 28.2 & 66.9 & 4.6 & 0.0 & 20,320 & 3.0 \\
\hline $15-19$ & 34.0 & 27.7 & 33.6 & 4.4 & 15,959 & 6.0 \\
\hline $20-24$ & 45.6 & 18.0 & 23.5 & 12.8 & 12,447 & 4.0 \\
\hline $25-29$ & 51.4 & 15.8 & 21.6 & 11.0 & 10,207 & $\mathrm{NC}$ \\
\hline 30 and above & 64.4 & 13.1 & 14.5 & 7.7 & 54,018 & $\mathrm{NC}$ \\
\hline Total & 52.4 & 27.3 & 14.2 & 5.6 & 130,693 & NC \\
\hline \multicolumn{7}{|l|}{ Male } \\
\hline $6-9$ & 61.7 & 37.4 & 0.0 & 0.0 & 9,157 & $\mathrm{NC}$ \\
\hline $10-14$ & 21.1 & 73.1 & 5.4 & 0.0 & 10,801 & 3.0 \\
\hline $15-19$ & 22.0 & 29.8 & 42.3 & 5.7 & 7,739 & 7.0 \\
\hline $20-24$ & 26.0 & 21.5 & 31.1 & 21.3 & 5,364 & 8.0 \\
\hline $25-29$ & 32.9 & 19.6 & 29.3 & 18.0 & 4,810 & 7.0 \\
\hline 30 and above & 46.7 & 17.4 & 22.2 & 13.0 & 27,419 & 3.7 \\
\hline Total & 39.5 & 32.2 & 19.0 & 8.8 & 65,297 & 3.0 \\
\hline \multicolumn{7}{|l|}{ Female } \\
\hline $6-9$ & 66.8 & 32.7 & 0.0 & 0.0 & 8,570 & $\mathrm{NC}$ \\
\hline $10-14$ & 36.2 & 59.8 & 3.7 & 0.0 & 9,518 & 2.0 \\
\hline $15-19$ & 44.1 & 26.0 & 26.3 & 3.4 & 8,220 & 4.0 \\
\hline $20-24$ & 57.7 & 15.8 & 18.8 & 7.5 & 7,083 & $\mathrm{NC}$ \\
\hline $25-29$ & 65.2 & 13.0 & 15.8 & 5.8 & 5,396 & $\mathrm{NC}$ \\
\hline 30 and above & 81.7 & 8.8 & 6.8 & 2.5 & 26,597 & $\mathrm{NC}$ \\
\hline Total & 64.5 & 22.8 & 9.7 & 2.7 & 65,392 & NC \\
\hline \multicolumn{7}{|c|}{ Urban } \\
\hline \multicolumn{7}{|l|}{ Total } \\
\hline $6-9$ & 56.6 & 42.7 & 0.0 & 0.0 & 6,105 & $\mathrm{NC}$ \\
\hline $10-14$ & 16.1 & 72.4 & 11.3 & 0.0 & 7,851 & 4.0 \\
\hline $15-19$ & 16.6 & 19.7 & 49.1 & 14.4 & 7,033 & 9.0 \\
\hline $20-24$ & 20.3 & 13.8 & 29.0 & 36.7 & 5,625 & 10.0 \\
\hline $25-29$ & 22.6 & 13.5 & 28.7 & 34.8 & 4,421 & 10.0 \\
\hline 30 and above & 36.3 & 14.8 & 22.6 & 25.6 & 22,497 & 7.0 \\
\hline Total & 30.3 & 26.9 & 23.0 & 19.4 & 53,536 & 6.0 \\
\hline \multicolumn{7}{|l|}{ Male } \\
\hline $6-9$ & 56.9 & 42.5 & 0.0 & 0.0 & 3,264 & $\mathrm{NC}$ \\
\hline $10-14$ & 14.5 & 73.4 & 11.9 & 0.0 & 4,128 & 4.0 \\
\hline $15-19$ & 13.4 & 19.2 & 52.7 & 14.6 & 3,640 & 9.0 \\
\hline $20-24$ & 13.5 & 13.2 & 29.4 & 43.9 & 2,737 & 10.0 \\
\hline $25-29$ & 14.2 & 12.6 & 29.3 & 43.7 & 2,248 & 10.0 \\
\hline 30 and above & 22.2 & 14.0 & 26.4 & 36.5 & 11,757 & 10.0 \\
\hline Total & 22.5 & 26.7 & 25.1 & 25.2 & 27,775 & 8.0 \\
\hline
\end{tabular}

Cont'd on next page... 
Table 2.6: (Cont'd)

\begin{tabular}{|c|c|c|c|c|c|c|}
\hline \multirow[t]{2}{*}{ Age (years) } & \multicolumn{4}{|c|}{ Completed years of schooling (\%) } & \multirow{2}{*}{$\begin{array}{c}\text { No. of } \\
\text { persons }\end{array}$} & \multirow{2}{*}{$\begin{array}{c}\text { Median } \\
\text { years of } \\
\text { schooling }\end{array}$} \\
\hline & None $^{1}$ & $1-7$ & $8-11$ & $\begin{array}{c}12 \\
\text { and above }\end{array}$ & & \\
\hline \multicolumn{7}{|c|}{ Urban } \\
\hline \multicolumn{7}{|l|}{ Female } \\
\hline $6-9$ & 56.2 & 43.0 & 0.0 & 0.0 & 2,841 & $\mathrm{NC}$ \\
\hline $10-14$ & 17.9 & 71.4 & 10.6 & 0.0 & 3,723 & 4.0 \\
\hline $15-19$ & 20.0 & 20.3 & 45.3 & 14.3 & 3,393 & 9.0 \\
\hline $20-24$ & 26.7 & 14.4 & 28.6 & 30.0 & 2,888 & 9.0 \\
\hline $25-29$ & 31.2 & 14.5 & 28.2 & 25.9 & 2,172 & 8.0 \\
\hline 30 and above & 51.8 & 15.8 & 18.5 & 13.7 & 10,740 & $\mathrm{NC}$ \\
\hline Total & 38.6 & 27.2 & 20.8 & 13.1 & 25,760 & 4.0 \\
\hline \multicolumn{7}{|c|}{ Rural } \\
\hline Total & & & & & & \\
\hline $6-9$ & 64.8 & 34.5 & 0.0 & 0.0 & 11,622 & $\mathrm{NC}$ \\
\hline $10-14$ & 29.3 & 66.3 & 4.0 & 0.0 & 12,469 & 3.0 \\
\hline $15-19$ & 36.2 & 28.7 & 31.7 & 3.2 & 8,926 & 5.0 \\
\hline $20-24$ & 48.8 & 18.5 & 22.8 & 9.8 & 6,822 & 2.0 \\
\hline $25-29$ & 54.7 & 16.1 & 20.8 & 8.3 & 5,786 & $\mathrm{NC}$ \\
\hline 30 and above & 67.4 & 12.9 & 13.6 & 5.7 & 31,521 & $\mathrm{NC}$ \\
\hline Total & 54.7 & 27.4 & 13.3 & 4.2 & 77,157 & NC \\
\hline \multicolumn{7}{|l|}{ Male } \\
\hline $6-9$ & 62.1 & 37.0 & 0.0 & 0.0 & 5,893 & $\mathrm{NC}$ \\
\hline $10-14$ & 21.7 & 73.1 & 4.9 & 0.0 & 6,673 & 3.0 \\
\hline $15-19$ & 23.2 & 31.3 & 40.9 & 4.4 & 4,099 & 7.0 \\
\hline $20-24$ & 28.1 & 22.9 & 31.4 & 17.6 & 2,627 & 7.0 \\
\hline $25-29$ & 35.5 & 20.5 & 29.4 & 14.5 & 2,562 & 6.0 \\
\hline 30 and above & 49.5 & 17.8 & 21.7 & 10.3 & 15,662 & 2.0 \\
\hline Total & 41.4 & 32.8 & 18.3 & 6.9 & 37,522 & 2.0 \\
\hline \multicolumn{7}{|l|}{ Female } \\
\hline $6-9$ & 67.5 & 31.9 & 0.0 & 0.0 & 5,729 & $\mathrm{NC}$ \\
\hline $10-14$ & 38.0 & 58.7 & 3.0 & 0.0 & 5,795 & 2.0 \\
\hline $15-19$ & 46.7 & 26.6 & 24.2 & 2.2 & 4,827 & 3.0 \\
\hline $20-24$ & 60.9 & 15.9 & 17.8 & 5.2 & 4,195 & $\mathrm{NC}$ \\
\hline $25-29$ & 68.5 & 12.8 & 14.6 & 3.8 & 3,224 & $\mathrm{NC}$ \\
\hline 30 and above & 84.7 & 8.1 & 5.7 & 1.4 & 15,857 & NC \\
\hline Total & 67.0 & 22.4 & 8.7 & 1.7 & 39,632 & NC \\
\hline
\end{tabular}

Note: All Ns are unweighted. Row totals may not equal 100\% due to missing cases or "don't know" responses. NC: Median cannot be calculated as more than $50 \%$ had no formal education. ${ }^{1}$ Includes non-literate and literate with no formal schooling. 
Table 2.7: Work participation

Percent distribution of the surveyed population aged 6 years and above by work participation, according to age, sex and residence, Bihar, 2007

\begin{tabular}{|l|r|r|r|r|r|r|r|r|c|}
\hline \multirow{2}{*}{ Age (years) (\%) } & \multicolumn{3}{|c|}{ Combined } & \multicolumn{3}{c|}{ Urban } & \multicolumn{3}{c|}{ Rural } \\
\cline { 2 - 13 } & Total & Male & Female & Total & Male & Female & Total & Male & Female \\
\hline $6-9$ & 0.4 & 0.4 & 0.4 & 0.2 & 0.3 & 0.2 & 0.4 & 0.4 & 0.4 \\
$10-14$ & 5.8 & 7.1 & 4.4 & 3.6 & 6.1 & 1.0 & 6.0 & 7.2 & 4.8 \\
$15-19$ & 24.5 & 40.3 & 11.3 & 16.1 & 28.2 & 3.1 & 25.5 & 42.0 & 12.1 \\
$20-24$ & 39.4 & 76.7 & 16.3 & 31.4 & 58.7 & 5.3 & 40.4 & 79.6 & 17.4 \\
$25-29$ & 53.7 & 92.7 & 24.5 & 45.6 & 82.6 & 8.2 & 54.6 & 94.1 & 26.1 \\
30 and above & 59.6 & 88.9 & 30.8 & 52.0 & 86.8 & 14.0 & 60.4 & 89.1 & 32.5 \\
Total & $\mathbf{3 5 . 9}$ & $\mathbf{5 4 . 7}$ & $\mathbf{1 8 . 2}$ & $\mathbf{3 1 . 6}$ & $\mathbf{5 3 . 9}$ & $\mathbf{7 . 7}$ & $\mathbf{3 6 . 3}$ & $\mathbf{5 4 . 8}$ & $\mathbf{1 9 . 2}$ \\
\hline
\end{tabular}

Note: Work participation is defined as reported work activity in the seven days prior to interview.

\subsection{Socio-demographic characteristics of households and heads of households}

Table 2.8 presents selected characteristics pertaining to households and their heads, according to residence, for all households as well as for those containing youth eligible for interview (that is, all young women aged 15-24 years, unmarried young men aged 15-24 years and married young men aged 15-29 years).

Findings suggest that heads of households were overwhelmingly male and about half (49\%) were aged 45 years and above with similar distributions observed for heads of both rural and urban households. Age differences suggest that heads of households that contained youth eligible for interview in the Youth Study were somewhat older than heads of all households: for example, the age of the head of household was 45 years or more among $56 \%$ of households that contained youth eligible for interview compared to $49 \%$ of all households. These differences were more prominent in urban than rural areas.

Table 2.8: Socio-demographic characteristics of households and heads of households

Percent distribution of all surveyed households and households containing youth eligible for interview by selected socio-demographic characteristics of heads of households, household size and type of family, according to residence, Bihar, 2007

\begin{tabular}{|l|c|c|c|c|c|c|}
\hline \multirow{2}{*}{$\begin{array}{l}\text { Socio-demographic } \\
\text { characteristics (\%) }\end{array}$} & \multicolumn{2}{|c|}{ Combined } & \multicolumn{2}{c|}{ Urban } & \multicolumn{3}{c|}{ Rural } \\
\cline { 2 - 7 } & $\begin{array}{c}\text { All } \\
\text { households }\end{array}$ & $\begin{array}{c}\text { Households } \\
\text { with youth }\end{array}$ & $\begin{array}{c}\text { All } \\
\text { households }\end{array}$ & $\begin{array}{c}\text { Households } \\
\text { with youth }\end{array}$ & $\begin{array}{c}\text { All } \\
\text { households }\end{array}$ & $\begin{array}{l}\text { Households } \\
\text { with youth }\end{array}$ \\
\hline Sex of household head & & & & & & \\
Male & 83.4 & 88.7 & 89.5 & 90.0 & 82.9 & 88.6 \\
Female & 16.6 & 11.3 & 10.5 & 10.0 & 17.1 & \\
Current age of household & & & & & & \\
head (years) & & & & & & \\
Below 25 & 5.1 & 8.9 & 4.6 & 6.4 & 9.2 \\
25-34 & 19.6 & 19.0 & 15.2 & 13.1 & 20.0 & 19.6 \\
35-44 & 26.5 & 16.7 & 27.8 & 17.8 & 26.4 & 16.6 \\
$45-54$ & 22.3 & 28.3 & 23.8 & 32.0 & 22.1 & 27.8 \\
55 and above & 26.5 & 27.2 & 28.6 & 30.7 & 26.3 & 26.8
\end{tabular}

Cont'd on next page... 


\begin{tabular}{|c|c|c|c|c|c|c|}
\hline \multirow{2}{*}{$\begin{array}{l}\text { Socio-demographic } \\
\text { characteristics (\%) }\end{array}$} & \multicolumn{2}{|c|}{ Combined } & \multicolumn{2}{|c|}{ Urban } & \multicolumn{2}{|c|}{ Rural } \\
\hline & $\begin{array}{c}\text { All } \\
\text { households }\end{array}$ & $\begin{array}{l}\text { Households } \\
\text { with youth }\end{array}$ & $\begin{array}{c}\text { All } \\
\text { households }\end{array}$ & $\begin{array}{l}\text { Households } \\
\text { with youth }\end{array}$ & $\begin{array}{c}\text { All } \\
\text { households }\end{array}$ & $\begin{array}{l}\text { Households } \\
\text { with youth }\end{array}$ \\
\hline Religion of household head & & & & & & \\
\hline Hindu & $\begin{array}{l}84.2 \\
157\end{array}$ & 85.8 & 82.7 & 81.6 & 84.4 & 86.3 \\
\hline Christian & 0.1 & 0.0 & 0.2 & $\begin{array}{r}10.1 \\
0.1\end{array}$ & 0.0 & 0.0 \\
\hline Other ${ }^{1}$ & 0.0 & 0.0 & 0.2 & 0.2 & 0.0 & 0.0 \\
\hline $\begin{array}{l}\text { Caste/tribe of household } \\
\text { head }\end{array}$ & & & & & & \\
\hline SC & 20.7 & 21.4 & 11.3 & 12.5 & 21.6 & 22.4 \\
\hline ST & 1.1 & 0.9 & 0.5 & 0.4 & 1.1 & 1.0 \\
\hline OBC & 61.2 & 61.2 & 58.5 & 60.4 & 61.5 & 61.3 \\
\hline General $^{2}$ & 16.9 & 16.3 & 29.6 & 26.6 & 15.7 & 15.2 \\
\hline Caste/tribe unknown & 0.1 & 0.1 & 0.1 & 0.1 & 0.1 & 0.1 \\
\hline $\begin{array}{l}\text { Schooling of household } \\
\text { head (years) }\end{array}$ & & & & & & \\
\hline None $^{3}$ & 54.2 & 49.9 & 27.3 & 28.4 & 56.7 & 52.3 \\
\hline $1-7$ & 17.3 & 19.4 & 15.2 & 16.9 & 17.5 & 19.7 \\
\hline $8-10$ & 18.7 & 21.2 & 25.7 & 27.1 & 18.1 & 20.6 \\
\hline $11-12$ & 3.8 & 4.1 & 9.4 & 10.2 & 3.3 & 3.5 \\
\hline Above 12 & 5.3 & 4.9 & 21.7 & 16.7 & 3.8 & 3.6 \\
\hline $\begin{array}{l}\text { Current work status } \\
\text { of household head }\end{array}$ & & & & & & \\
\hline Working & 84.1 & 86.2 & 80.4 & 80.6 & 84.5 & 86.8 \\
\hline Not working & 15.8 & 13.7 & 19.5 & 19.3 & 15.4 & 13.1 \\
\hline $\begin{array}{l}\text { Number of members in the } \\
\text { household }\end{array}$ & & & & & & \\
\hline 1 & 3.2 & 0.5 & 4.0 & 1.3 & 3.1 & 0.4 \\
\hline 2 & 9.1 & 4.4 & 7.6 & 3.8 & 9.2 & 4.5 \\
\hline 3 & 10.8 & 10.2 & 9.3 & 7.0 & 10.9 & 10.5 \\
\hline 4 & 15.0 & 15.1 & 15.5 & 13.9 & 15.0 & 15.2 \\
\hline 5 & 18.0 & 16.9 & 17.3 & 16.6 & 18.0 & 16.9 \\
\hline 6 & 15.5 & 14.3 & 15.4 & 15.1 & 15.5 & 14.2 \\
\hline 7 or more & 28.5 & 38.7 & 30.7 & 42.3 & 28.3 & 38.3 \\
\hline Mean household size & 5.5 & 6.3 & 5.7 & 6.6 & 5.4 & 6.3 \\
\hline Type of family & & & & & & \\
\hline Nuclear & 59.1 & 42.8 & 55.9 & 44.8 & 59.4 & 42.6 \\
\hline Non-nuclear & 40.9 & 57.2 & 44.1 & 55.2 & 40.6 & 57.4 \\
\hline $\begin{array}{l}\text { Households with at least one } \\
\text { literate member aged } 18 \\
\text { and above }\end{array}$ & 59.6 & 70.5 & 84.9 & 88.6 & 57.2 & 68.5 \\
\hline Number of households & 28,205 & 11,810 & 10,887 & 4,794 & 17,318 & 7,016 \\
\hline
\end{tabular}

Note: All Ns are unweighted. Column totals may not equal 100\% due to missing cases or "don't know" responses. OBC: Other backward caste. SC: Scheduled caste. ST: Scheduled tribe. ${ }^{1}$ Includes Buddhist/Neo-Buddhist, Sikh, Jain, Jewish, Parsi/ Zoroastrian and no specified religion. ${ }^{2}$ Includes all those not belonging to SC, ST or OBC. ${ }^{3}$ Includes non-literate and literate with no formal schooling. ${ }^{4}$ Defined as reported work activity in the seven days prior to interview. 
Distributions by religion suggest that $84 \%$ of household heads were Hindu and $16 \%$ were Muslim. Distributions were similar among all households and those containing youth eligible for interview in the Youth Study, irrespective of rural and urban residence. As far as caste was concerned, the largest group belonged to other backward castes (61\%), followed by scheduled castes $(21 \%)$ and general castes (17\%). Rural-urban differences were evident. The rural population consisted of a much larger proportion of households belonging to scheduled castes than did urban population (22\% and $11 \%$, respectively). In contrast, the urban population consisted of a much larger proportion of households belonging to general castes than did the rural population (30\% and $16 \%$, respectively).

Educational attainment levels suggest that over half of all heads of households had no schooling and another one-sixth had only 1-7 years of schooling. Just as educational distributions differed for the general population, here too, heads of households in urban areas were better-educated than their rural counterparts; almost one-third of those in urban areas had 12 or more years of education compared to fewer than one-tenth of heads of households in rural areas. The vast majority of heads of households reported working in the last seven days $(84 \%)$, and rural-urban differences were modest.

Households contained an average of 5.5 members. This number was higher (6.3) among those containing youth eligible for interview in the Youth Study. Rural-urban differences were modest. As far as family type was concerned, about three-fifths of all households, irrespective of rural-urban residence, consisted of a nuclear family. However, among households containing youth eligible for interview in the Youth Study, fewer were nuclear: $43 \%$, with little rural-urban variation (43\% in rural areas and $45 \%$ in urban areas).

Finally, 60\% of all households contained at least one literate member aged 18 and above, a percentage that was somewhat higher $(71 \%)$ in households containing eligible youth. Rural-urban differences were quite wide: $85 \%$ and $57 \%$ of urban and rural households, respectively, contained at least one literate member aged 18 and above, as did $89 \%$ and $69 \%$, respectively, of those containing eligible youth.

\subsection{Profile of the household population: Housing characteristics}

Table 2.9 provides information on ownership of residence, housing quality, access to basic amenities and indicators of crowding. Information was obtained from responses to the household questionnaire and, in the case of housing type, interviewer observations. Information is presented by rural-urban residence separately for all surveyed households and households containing youth eligible for the Youth Study. The characteristics of both types of households are basically similar.

Almost all households (97\%), irrespective of whether or not they contained youth, owned the structure in which they resided. Considerably more rural than urban households, however, reported owning their residence (99\% and 74\%, respectively). Overall, interviewers observed that over half of all households (53\%) lived in kachcha houses (constructed from mud, thatch or other low-quality materials), 25\% lived in semi-pucca houses (constructed using a mix of low- and high-quality materials) and 22\% lived in pucca houses (constructed entirely from cement, masonry or other high-quality materials).

Most residential structures contained 1 (35\%) or 2-3 (49\%) rooms; urban households were more likely to report 4 or more rooms than rural households ( $27 \%$ and $15 \%$ respectively). The mean number of persons per room was 3.0 for all households and 3.1 for those containing eligible youth. Rural-urban differences were modest. 
Table 2.9: Housing characteristics

Percent distribution of all surveyed households and households containing youth eligible for interview by selected housing characteristics, according to residence, Bihar, 2007

\begin{tabular}{|c|c|c|c|c|c|c|}
\hline \multirow{2}{*}{$\begin{array}{l}\text { Housing } \\
\text { characteristics (\%) }\end{array}$} & \multicolumn{2}{|c|}{ Combined } & \multicolumn{2}{|c|}{ Urban } & \multicolumn{2}{|c|}{ Rural } \\
\hline & $\begin{array}{c}\text { All } \\
\text { households }\end{array}$ & $\begin{array}{l}\text { Households } \\
\text { with youth }\end{array}$ & $\begin{array}{l}\text { All } \\
\text { households }\end{array}$ & $\begin{array}{l}\text { Households } \\
\text { with youth }\end{array}$ & $\begin{array}{c}\text { All } \\
\text { households }\end{array}$ & $\begin{array}{l}\text { Households } \\
\text { with youth }\end{array}$ \\
\hline Ownership of residence & & & & & & \\
\hline Yes & 96.7 & 96.9 & 73.5 & 77.5 & 98.9 & 99.0 \\
\hline No & 3.3 & 3.1 & 26.5 & 22.4 & 1.1 & 1.0 \\
\hline Type of house & & & & & & \\
\hline Kachcha & 53.2 & 50.3 & 15.8 & 15.4 & 56.7 & 54.1 \\
\hline Semi-pucca & 24.9 & 25.7 & 22.4 & 23.6 & 25.1 & 26.0 \\
\hline Pucca & 21.8 & 23.9 & 61.7 & 61.0 & 18.1 & 19.9 \\
\hline $\begin{array}{l}\text { Number of rooms } \\
\text { in the house }{ }^{1}\end{array}$ & & & & & & \\
\hline 1 & 35.4 & 29.6 & 24.8 & 19.7 & 36.4 & 30.7 \\
\hline $2-3$ & 49.1 & 49.2 & 48.5 & 49.4 & 49.1 & 49.2 \\
\hline $4-5$ & 10.8 & 13.9 & 19.0 & 21.2 & 10.0 & 13.1 \\
\hline $\begin{array}{l}6 \text { or more } \\
\text { Average number of } \\
\text { persons per room }\end{array}$ & 4.8 & 7.2 & 7.7 & 9.7 & 4.5 & 6.9 \\
\hline Up to 2 & 53.3 & 51.5 & 64.9 & 63.2 & 52.2 & 50.3 \\
\hline $3-4$ & 28.5 & 31.6 & 21.8 & 24.1 & 29.2 & 32.4 \\
\hline $5-6$ & 13.0 & 12.0 & 9.2 & 8.5 & 13.3 & 12.3 \\
\hline More than 6 & 5.2 & 4.9 & 4.1 & 4.2 & 5.3 & 5.0 \\
\hline $\begin{array}{l}\text { Mean number of } \\
\text { persons per room }\end{array}$ & 3.0 & 3.1 & 2.6 & 2.7 & 3.0 & 3.1 \\
\hline Source of lighting & & & & & & \\
\hline Electricity & 13.9 & 15.8 & 71.2 & 70.8 & 8.5 & 9.8 \\
\hline Kerosene & 85.7 & 83.9 & 28.6 & 29.0 & 91.0 & 89.9 \\
\hline Other lighting sources ${ }^{2}$ & 0.4 & 0.3 & 0.1 & 0.2 & 0.4 & 0.3 \\
\hline Source of drinking water & & & & & & \\
\hline $\begin{array}{l}\text { Own piped water/ } \\
\text { hand-pump/covered well }\end{array}$ & 44.6 & 48.8 & 59.6 & 62.6 & 43.2 & 47.3 \\
\hline $\begin{array}{l}\text { Public piped water/ } \\
\text { hand-pump/covered well }\end{array}$ & 48.6 & 44.5 & 36.9 & 33.8 & 49.7 & 45.6 \\
\hline Own open well & 1.1 & 1.1 & 1.1 & 0.9 & 1.1 & 1.1 \\
\hline Public open well & 5.8 & 5.6 & 2.4 & 2.5 & 6.1 & 5.9 \\
\hline Surface water ${ }^{3}$ & 0.0 & 0.0 & 0.1 & 0.1 & 0.0 & 0.0 \\
\hline
\end{tabular}

Cont'd on next page... 
Table 2.9: (Cont'd)

\begin{tabular}{|c|c|c|c|c|c|c|}
\hline \multirow{2}{*}{$\begin{array}{l}\text { Housing } \\
\text { characteristics (\%) }\end{array}$} & \multicolumn{2}{|c|}{ Combined } & \multicolumn{2}{|c|}{ Urban } & \multicolumn{2}{|c|}{ Rural } \\
\hline & $\begin{array}{c}\text { All } \\
\text { households }\end{array}$ & $\begin{array}{l}\text { Households } \\
\text { with youth }\end{array}$ & $\begin{array}{c}\text { All } \\
\text { households }\end{array}$ & $\begin{array}{l}\text { Households } \\
\text { with youth }\end{array}$ & $\begin{array}{c}\text { All } \\
\text { households }\end{array}$ & $\begin{array}{l}\text { Households } \\
\text { with youth }\end{array}$ \\
\hline Toilet facility & & & & & & \\
\hline Own flush toilet & 9.4 & 11.2 & 43.6 & 45.1 & 6.2 & 7.5 \\
\hline Shared flush toilet & 3.5 & 3.2 & 17.6 & 15.4 & 2.1 & 1.9 \\
\hline Own pit toilet & 2.8 & 3.3 & 7.0 & 7.6 & 2.4 & 2.9 \\
\hline Shared pit toilet & 1.1 & 1.0 & 4.7 & 4.2 & 0.8 & 0.7 \\
\hline No toilet facility & 83.1 & 81.2 & 27.1 & 27.6 & 88.4 & 87.1 \\
\hline $\begin{array}{l}\text { Main type of fuel } \\
\text { used for cooking }\end{array}$ & & & & & & \\
\hline Liquid petroleum gas & 5.3 & 5.5 & 47.4 & 43.8 & 1.4 & 1.4 \\
\hline Bio-gas & 0.1 & 0.1 & 0.2 & 0.2 & 0.1 & 0.1 \\
\hline Kerosene & 0.4 & 0.3 & 1.6 & 1.0 & 0.3 & 0.2 \\
\hline $\begin{array}{l}\text { Wood/crop residue/ } \\
\text { dung cakes/coal/charcoal }\end{array}$ & 94.1 & 94.1 & 50.6 & 54.9 & 98.2 & 98.3 \\
\hline Other types of fuel ${ }^{4}$ & 0.0 & 0.0 & 0.1 & 0.1 & 0.0 & 0.0 \\
\hline Number of households & 28,205 & 11,810 & 10,887 & 4,794 & 17,318 & 7,016 \\
\hline
\end{tabular}

Note: All Ns are unweighted. Column totals may not equal 100\% due to missing cases or "don't know" responses. ${ }^{1}$ Excludes toilets/bathrooms but includes kitchen. ${ }^{2}$ Includes oil, gas, etc. ${ }^{3}$ Includes water of a spring, river, stream, pond, lake or dam. ${ }^{4}$ Includes electricity, straw, shrubs and grass.

Respondents were asked about their household's main source of lighting and drinking water. In addition, information was gathered on toilet facilities typically accessed, and cooking fuel generally used. As Table 2.9 shows, only $14 \%$ of households had electricity, including $71 \%$ of urban households and $9 \%$ of rural households, while percentages reporting electricity in urban areas are almost identical to those reported in NFHS-3 (74\%), percentages so reporting in rural areas is considerably higher in NFHS-3 (19\%)). The vast majority of households (93\%) reported that their main source of drinking water was either piped water, water obtained from a hand-pump or a covered well (while not entirely comparable, $96 \%$ of households reported that they have access to an improved source of drinking water, defined to include piped water, tube-well or borehole, protected well or spring, rainwater or bottled water in NFHS-3, IIPS and Macro International, 2008). These facilities were reported as self-owned for $45 \%$ and as public or shared facilities for the remaining $49 \%$. Rural-urban differences were negligible with respect to access to these safe sources of drinking water (97\% and $93 \%$ of urban and rural households, respectively); however, urban households were more likely to report self-owned facilities than rural households (60\% versus $43 \%)$.

Access to a toilet facility of any kind was reported by a few-just one-sixth of all households (17\%, compared to 25\% as assessed in NFHS-3; IIPS and Macro International, 2008). Large rural-urban differences were observed: $88 \%$ of rural households, compared to $27 \%$ of urban households had no access to toilet facilities.

Finally, the main source of cooking fuel was coal, charcoal, wood, crop residue or dung cakes, reported by $94 \%$ of all households (compared to $90 \%$ reported in NFHS-3; IIPS and Macro International, 2008), and $98 \%$ of all rural households compared to $51 \%$ of urban households. Liquid petroleum gas was used, in contrast, by just $5 \%$ of all households, that is, $1 \%$ in rural areas to $47 \%$ in urban areas. Patterns of access to 
these facilities in households containing youth eligible for interview in the Youth Study were similar to those observed for all households, described above.

\subsection{Profile of the household population: Ownership of agricultural land}

Table 2.10 presents information on ownership of agricultural land for households in both rural and urban areas (irrigated and non-irrigated). Most households, irrespective of whether or not they contained youth eligible for interview, either owned no land (50-53\%) or owned marginal holdings (35-37\%). These proportions were much higher for urban households ( $70 \%$ and $14 \%$, respectively) than for rural households $(51 \%$ and $37 \%$, respectively). About two-fifths of all households reported owning some irrigated land.

Table 2.10: Household ownership of agricultural land

Percent distribution of all surveyed households and households containing youth eligible for interview by ownership of agricultural land, according to residence, Bihar, 2007

\begin{tabular}{|c|c|c|c|c|c|c|}
\hline \multirow[t]{2}{*}{ Land holding (\%) } & \multicolumn{2}{|c|}{ Combined } & \multicolumn{2}{|c|}{ Urban } & \multicolumn{2}{|c|}{ Rural } \\
\hline & $\begin{array}{c}\text { All } \\
\text { households }\end{array}$ & $\begin{array}{l}\text { Households } \\
\text { with youth }\end{array}$ & $\begin{array}{c}\text { All } \\
\text { households }\end{array}$ & $\begin{array}{l}\text { Households } \\
\text { with youth }\end{array}$ & $\begin{array}{c}\text { All } \\
\text { households }\end{array}$ & $\begin{array}{l}\text { Households } \\
\text { with youth }\end{array}$ \\
\hline Land holding (in acres) & & & & & & \\
\hline Landless & 52.5 & 49.8 & 69.8 & 70.0 & 50.8 & 47.6 \\
\hline Marginal $(\leq 2.50)$ & 35.3 & 36.6 & 14.4 & 15.2 & 37.3 & 38.9 \\
\hline Small (2.51-5.00) & 4.1 & 5.2 & 4.0 & 4.1 & 4.1 & 5.3 \\
\hline Medium (5.01-10.00) & 1.7 & 2.2 & 2.5 & 2.2 & 1.7 & 2.2 \\
\hline Large $(>10.00)$ & 3.8 & 3.8 & 2.6 & 2.2 & 3.9 & 4.0 \\
\hline Own any irrigated land & 37.1 & 39.5 & 20.3 & 20.7 & 38.7 & 41.5 \\
\hline Number of households & 28,205 & 11,810 & 10,887 & 4,794 & 17,318 & 7,016 \\
\hline
\end{tabular}

Note: All Ns are unweighted. Column totals may not equal 100\% due to missing cases or "don't know" responses.

\subsection{Profile of the household population: Overall economic status}

Household economic status was measured using a wealth index, composed of household asset data on ownership of selected durable goods, including means of transportation, as well as data on access to a number of amenities. The wealth index was constructed by allocating the following scores to a household's reported assets or amenities:

Type of house: 2 for pucca; 1 for semi-pucca; 0 for kachcha

Agricultural land owned: 4 for more than 10 acres; 3 for 5.1-10.0 acres; 2 for 2.6-5.0 acres; 1 for less than 2.6 acres, or if the household owns some land but does not know how much; 0 for no land

Irrigated land owned: 1 for any irrigated land; 0 for no land

Access to toilet facility: 4 for own flush toilet; 2 for shared flush toilet or own pit toilet; 1 for shared pit toilet or other types of toilet; 0 for no toilet facility

Cooking fuel used: 2 for liquid petroleum gas, electricity or bio-gas; 1 for kerosene, wood, crop residue, dung cakes, coal or charcoal; 0 for other types of cooking fuel, for example, dry leaves 
Access to drinking water facility: 4 for own piped water, hand-pump or covered well; 3 for own open well; 2 for public or shared piped water, hand-pump or covered well; 1 for public or shared open well; 0 for other sources of drinking water, for example, surface water, tanker/truck or rainwater

Access to electricity: 3 for electricity; 0 for no electricity

Ownership of household assets: 4 for car or truck; 3 each for motor cycle or scooter, refrigerator, computer/ laptop, telephone (landline or mobile), colour television; 2 each for bicycle, electric fan, radio or transistor, black and white television, sewing machine, water pump, animal-drawn cart; 1 for watch or clock; 0 for each of the above items that the household does not possess.

Index scores, so constructed, ranged from 0 to 54. Households were then ranked according to the index score. This ranked sample was divided into quintiles-i.e., five groups, each containing an equal number of households - with the first quintile representing households of the lowest wealth status and the fifth quintile representing households with the highest wealth status. In the Youth Study, the wealth quintiles were developed at the state level on the basis of the weighted sample for the whole state.

Findings are presented in Table 2.11. As far as ownership of household assets was concerned, the items most likely to be owned by all households were a watch or clock $(55 \%)$ and a bicycle (45\%). One-sixth owned a radio $(17 \%)$. Other items owned by one-tenth or more of all households included an electric fan (14\%) and a telephone (10\%). Wide rural-urban differences were observed, with rural households far less likely than urban households to report ownership of most items. For example, while $71 \%$ of urban households owned an electric fan, just $8 \%$ of rural households did; and while $41 \%$ of urban households owned a telephone, just $7 \%$ of rural households did. Sizeable proportions of all households (35\%) and those containing eligible youth $(27 \%)$ did not own a single item; again, this proportion was much higher among rural households than urban (in rural areas, $38 \%$ and $29 \%$ of all households and those containing youth, respectively, did not own a single item; compared to $7 \%$ and $6 \%$, respectively, in urban areas).

The distribution of households by wealth quintiles shows that two-thirds of urban households were in the wealthiest (fifth) quintile; in contrast, only $16 \%$ of rural households were in this quintile. Likewise, one-fifth of rural households were in the poorest (first) quintile of the index compared to only $3 \%$ of urban households. In both urban and rural areas the distribution of households by wealth quintiles was, by and large, similar across all households and those that contained a youth eligible for interview in the Youth Study.

\subsection{Profile of surveyed youth: Background characteristics}

A total of 8,136 youth were interviewed. Table 2.12 presents the socio-demographic characteristics of surveyed youth. Age profiles suggest that larger proportions of young men and women were concentrated in the 15-19 year age group than in the 20-24 year age group (60-62\% compared to 38-40\%). Moreover, the unmarried were clearly younger than the married. While 57\% of married young women were aged 20-24, only 9\% of unmarried women fell into these ages. Gender differences were also wide. Among married young women, over two-fifths were between 15 and 19 years of age and slightly fewer than three-fifths were aged 20-24 years; in contrast, few married young men were between the ages of 15 and 19 (8\% of all respondents aged 15-29 and 18\% of those aged 15-24). Among the unmarried, gender differences were narrower, but young women were still more likely to be concentrated in the 15-19-year age group than young men (91\% and 78\%, respectively). Similar age profiles were observed by rural-urban residence for both young men and women.

The distribution of youth by religion was fairly similar to that observed in the household population: $86 \%$ of youth were Hindu and 14\% were Muslim. While no differences were evident by marital status among young men, a larger proportion of married women than unmarried women were Hindu ( $89 \%$ versus $80 \%)$. Rural-urban differences in the distribution of the population by religion were modest. 
Table 2.11: Household assets and wealth status

Percentage of all surveyed households and households containing youth eligible for interview owning selected household assets and percent distribution of households by wealth quintile, according to residence, Bihar, 2007

\begin{tabular}{|c|c|c|c|c|c|c|}
\hline \multirow{2}{*}{$\begin{array}{l}\text { Housing } \\
\text { characteristics (\%) }\end{array}$} & \multicolumn{2}{|c|}{ Combined } & \multicolumn{2}{|c|}{ Urban } & \multicolumn{2}{|c|}{ Rural } \\
\hline & $\begin{array}{c}\text { All } \\
\text { households }\end{array}$ & $\begin{array}{l}\text { Households } \\
\text { with youth }\end{array}$ & $\begin{array}{c}\text { All } \\
\text { households }\end{array}$ & $\begin{array}{l}\text { Households } \\
\text { with youth }\end{array}$ & $\begin{array}{c}\text { All } \\
\text { households }\end{array}$ & $\begin{array}{l}\text { Households } \\
\text { with youth }\end{array}$ \\
\hline Assets owned & & & & & & \\
\hline Watch/clock & 54.9 & 63.0 & 87.9 & 90.3 & 51.8 & 60.1 \\
\hline Electric fan & 13.6 & 15.8 & 71.2 & 71.7 & 8.2 & 9.7 \\
\hline Bicycle & 45.0 & 53.2 & 56.7 & 63.5 & 43.9 & 52.1 \\
\hline Radio and/or transistor & 17.0 & 20.4 & 29.1 & 30.2 & 15.9 & 19.3 \\
\hline Colour television & 3.8 & 4.6 & 28.9 & 28.6 & 1.4 & 1.9 \\
\hline $\mathrm{B} / \mathrm{W}$ television & 7.6 & 10.0 & 27.0 & 30.1 & 5.8 & 7.9 \\
\hline $\begin{array}{l}\text { Telephone (landline/ } \\
\text { mobile) }\end{array}$ & 10.2 & 13.0 & 40.7 & 41.6 & 7.3 & 9.9 \\
\hline Refrigerator & 1.6 & 1.8 & 14.4 & 12.8 & 0.4 & 0.6 \\
\hline Motorcycle/scooter & 4.7 & 6.2 & 17.7 & 16.3 & 3.5 & 5.1 \\
\hline Sewing machine & 7.6 & 9.4 & 25.1 & 27.8 & 5.9 & 7.4 \\
\hline Animal-drawn cart & 1.4 & 1.8 & 0.5 & 0.6 & 1.5 & 2.0 \\
\hline Water pump & 3.9 & 4.9 & 5.8 & 5.5 & 3.7 & 4.8 \\
\hline $\begin{array}{l}\text { Personal computer/ } \\
\text { laptop }\end{array}$ & 0.3 & 0.3 & 3.3 & 3.2 & 0.0 & 0.0 \\
\hline Car/truck & 0.4 & 0.5 & 2.4 & 2.0 & 0.2 & 0.4 \\
\hline Tractor & 1.0 & 1.5 & 0.6 & 0.7 & 1.0 & 1.6 \\
\hline Thresher & 1.3 & 1.8 & 0.5 & 0.7 & 1.4 & 1.9 \\
\hline None of the above & 35.0 & 27.1 & 7.4 & 5.5 & 37.6 & 29.4 \\
\hline Wealth quintile & & & & & & \\
\hline First & 20.0 & 16.5 & 2.8 & 2.0 & 21.6 & 18.0 \\
\hline Second & 20.0 & 17.6 & 6.9 & 6.0 & 21.2 & 18.9 \\
\hline Third & 20.0 & 19.0 & 7.7 & 7.5 & 21.2 & 20.3 \\
\hline Fourth & 20.0 & 22.5 & 14.6 & 15.0 & 20.5 & 23.3 \\
\hline Fifth & 20.0 & 24.4 & 68.0 & 69.6 & 15.5 & 19.5 \\
\hline Number of households & 28,205 & 11,810 & 10,887 & 4,794 & 17,318 & 7,016 \\
\hline
\end{tabular}

Note: All Ns are unweighted.

Caste-wise distributions were generally similar among young men and women, with $14-20 \%$ falling into general castes, $20-21 \%$ into scheduled castes and $58-64 \%$ into other backward castes. Differences by marital status show that more unmarried than married youth belonged to general castes ( $24 \%$ versus $11 \%$ among young men and $23 \%$ versus $10 \%$ among young women), and conversely more married than unmarried youth belonged to scheduled castes (27\% versus $17 \%$ among young men and $25 \%$ versus $15 \%$ among young women); differences are likely attributed to caste-wise differences in age at marriage. Rural-urban differences were also evident; urban youth were more likely than rural youth to belong to general castes $(28 \%$ versus $19 \%$ among young men and $25 \%$ versus $13 \%$ among young women) and conversely less likely to belong to scheduled castes (10\% versus $22 \%$ among young men and $13 \%$ versus $22 \%$ among young women). 
Table 2.12: Background characteristics of surveyed youth

Percent distribution of surveyed youth by selected background characteristics, according to residence, Bihar, 2007

\begin{tabular}{|c|c|c|c|c|c|c|c|c|c|c|c|c|}
\hline \multirow[t]{2}{*}{$\begin{array}{l}\text { Background } \\
\text { characteristics }\end{array}$} & \multicolumn{2}{|c|}{$\begin{array}{c}\text { Men } \\
(\mathrm{M})^{4} \\
15-24\end{array}$} & \multicolumn{2}{|c|}{$\begin{array}{l}\text { Women } \\
(\mathrm{W})^{4} \\
15-24\end{array}$} & \multicolumn{2}{|c|}{$\begin{array}{c}\text { Married } \\
\text { men }(\mathrm{MM})^{4} \\
15-29\end{array}$} & \multicolumn{2}{|c|}{$\begin{array}{c}\text { Married } \\
\text { women }(M W)^{4} \\
15-24\end{array}$} & \multicolumn{2}{|c|}{$\begin{array}{c}\text { Unmarried } \\
\text { men }(\mathrm{UM})^{4} \\
15-24\end{array}$} & \multicolumn{2}{|c|}{$\begin{array}{c}\text { Unmarried } \\
\text { women }(\mathrm{UW})^{4} \\
15-24 \\
\end{array}$} \\
\hline & Percent & Number & Percent & Number & Percent & Number & Percent & Number & Percent & Number & Percent & Number \\
\hline \multicolumn{13}{|c|}{ Combined } \\
\hline Age (years) & & & & & & & & & & & & \\
\hline $15-19$ & 62.1 & 1,144 & 59.6 & 3,615 & 7.7 & 63 & 43.0 & 842 & 77.8 & 1,081 & 91.4 & 2,773 \\
\hline $20-24$ & 37.9 & 798 & 40.4 & 1,914 & 34.6 & 387 & 57.0 & 1,499 & 22.2 & 411 & 8.6 & 415 \\
\hline $25-29$ & NA & NA & NA & NA & 57.7 & 665 & NA & NA & NA & NA & NA & NA \\
\hline \multicolumn{13}{|l|}{ Religion } \\
\hline Hindu & 86.0 & 1,649 & 85.6 & 4,553 & 87.9 & 963 & 88.6 & 2,004 & 85.9 & 1,261 & 80.3 & 2,549 \\
\hline Muslim & 13.9 & 289 & 14.3 & 969 & 12.1 & 152 & 11.4 & 336 & 13.9 & 227 & 19.6 & 633 \\
\hline Christian & 0.1 & 2 & 0.0 & 3 & 0.0 & 0 & 0.0 & 0 & 0.1 & 2 & 0.0 & 3 \\
\hline Other ${ }^{1}$ & 0.1 & 2 & 0.0 & 3 & 0.0 & 0 & 0.0 & 0 & 0.1 & 2 & 0.0 & 3 \\
\hline \multicolumn{13}{|l|}{ Caste } \\
\hline SC & 20.1 & 307 & 21.2 & 910 & 26.7 & 243 & 24.8 & 482 & 16.7 & 199 & 14.8 & 428 \\
\hline ST & 1.9 & 24 & 0.1 & 8 & 3.2 & 23 & 0.1 & 3 & 1.1 & 13 & 0.1 & 5 \\
\hline OBC & 58.0 & 1,160 & 64.3 & 3,489 & 58.9 & 685 & 65.6 & 1,540 & 58.2 & 885 & 62.2 & 1,949 \\
\hline General $^{2}$ & 19.9 & 446 & 14.4 & 1,120 & 10.9 & 163 & 9.5 & 316 & 23.7 & 390 & 22.9 & 804 \\
\hline No caste/do not know & 0.2 & 5 & 0.0 & 2 & 0.2 & 1 & 0.0 & 0 & 0.2 & 5 & 0.1 & 2 \\
\hline \multicolumn{13}{|l|}{ Educational level (years) } \\
\hline None $^{3}$ & 16.1 & 271 & 51.0 & 1,934 & 29.4 & 287 & 64.1 & 1,207 & 11.7 & 154 & 28.2 & 727 \\
\hline $1-7$ & 30.3 & 486 & 24.9 & 1,436 & 28.8 & 277 & 20.5 & 519 & 28.6 & 350 & 33.4 & 917 \\
\hline $8-11$ & 41.5 & 847 & 19.6 & 1,577 & 30.2 & 367 & 12.9 & 456 & 46.9 & 709 & 31.2 & 1,121 \\
\hline 12 and above & 12.2 & 338 & 4.5 & 582 & 11.7 & 184 & 2.6 & 159 & 12.8 & 279 & 7.2 & 423 \\
\hline \multicolumn{13}{|c|}{ Worked in last 12 months } \\
\hline Yes & 72.7 & 1,306 & 36.8 & 1,562 & 96.8 & 1,069 & 38.0 & 618 & 64.3 & 881 & 36.0 & 944 \\
\hline No & 27.1 & 634 & 63.2 & 3,966 & 2.9 & 44 & 62.0 & 1,722 & 35.5 & 610 & 64.0 & 2,244 \\
\hline \multicolumn{13}{|l|}{ Wealth quintile } \\
\hline First & 9.1 & 107 & 18.1 & 570 & 14.2 & 102 & 22.2 & 317 & 7.4 & 63 & 11.4 & 253 \\
\hline Second & 12.0 & 167 & 18.6 & 712 & 17.2 & 148 & 20.9 & 351 & 10.7 & 110 & 15.0 & 361 \\
\hline Third & 19.4 & 261 & 19.0 & 809 & 20.1 & 169 & 20.2 & 377 & 18.6 & 189 & 17.5 & 432 \\
\hline Fourth & 26.3 & 440 & 20.5 & 1,042 & 22.2 & 237 & 19.5 & 430 & 27.0 & 325 & 23.0 & 612 \\
\hline Fifth & 33.2 & 967 & 23.8 & 2,396 & 26.3 & 459 & 17.2 & 866 & 36.3 & 805 & 33.2 & 1,530 \\
\hline Total & 100.0 & 1,942 & 100.0 & 5,529 & 100.0 & 1,115 & 100.0 & 2,341 & 100.0 & 1,492 & 100.0 & 3,188 \\
\hline \multicolumn{13}{|c|}{ Urban } \\
\hline \multicolumn{13}{|l|}{ Age (years) } \\
\hline $15-19$ & 58.2 & 566 & 57.0 & 1,447 & 3.3 & 16 & 27.5 & 312 & 65.7 & 550 & 78.3 & 1,135 \\
\hline $20-24$ & 41.8 & 473 & 43.0 & 1,134 & 34.8 & 190 & 72.5 & 824 & 34.3 & 283 & 21.7 & 310 \\
\hline $25-29$ & NA & NA & NA & NA & 62.0 & 341 & NA & NA & NA & NA & NA & NA \\
\hline \multicolumn{13}{|l|}{ Religion } \\
\hline Hindu & 82.1 & 862 & 81.0 & 2,092 & 84.9 & 463 & 83.1 & 939 & 81.6 & 684 & 79.6 & 1,153 \\
\hline Muslim & 17.1 & 173 & 18.7 & 482 & 15.1 & 84 & 16.9 & 196 & 17.6 & 145 & 20.0 & 286 \\
\hline Christian & 0.4 & 2 & 0.2 & 3 & 0.0 & 0 & 0.0 & 0 & 0.4 & 2 & 0.2 & 3 \\
\hline Other $^{1}$ & 0.4 & 2 & 0.2 & 3 & 0.0 & 0 & 0.0 & 0 & 0.4 & 2 & 0.2 & 3 \\
\hline \multicolumn{13}{|l|}{ Caste } \\
\hline SC & 10.0 & 114 & 13.4 & 340 & 15.2 & 87 & 16.2 & 181 & 9.4 & 79 & 11.1 & 159 \\
\hline ST & 0.7 & 7 & 0.3 & 7 & 1.1 & 6 & 0.0 & 2 & 0.8 & 5 & 0.4 & 5 \\
\hline OBC & 61.3 & 634 & 61.0 & 1,586 & 64.1 & 349 & 65.5 & 742 & 60.4 & 500 & 58.1 & 844 \\
\hline General $^{2}$ & 27.6 & 280 & 25.3 & 647 & 19.6 & 105 & 18.3 & 211 & 29.0 & 245 & 30.4 & 436 \\
\hline No caste/do not know & 0.4 & 4 & 0.0 & 1 & 0.0 & 0 & 0.0 & 0 & 0.4 & 4 & 0.0 & 1 \\
\hline
\end{tabular}


Table 2.12: (Cont'd)

\begin{tabular}{|c|c|c|c|c|c|c|c|c|c|c|c|c|}
\hline \multirow[t]{2}{*}{$\begin{array}{l}\text { Background } \\
\text { characteristics }\end{array}$} & \multicolumn{2}{|c|}{$\begin{array}{c}\text { Men } \\
(M)^{4} \\
15-24\end{array}$} & \multicolumn{2}{|c|}{$\begin{array}{c}\text { Women } \\
(\mathrm{W})^{4} \\
15-24 \\
\end{array}$} & \multicolumn{2}{|c|}{$\begin{array}{c}\text { Married } \\
\text { men }(\mathrm{MM})^{4} \\
15-29 \\
\end{array}$} & \multicolumn{2}{|c|}{$\begin{array}{c}\text { Married } \\
\text { women }(\mathrm{MW})^{4} \\
15-24 \\
\end{array}$} & \multicolumn{2}{|c|}{$\begin{array}{c}\text { Unmarried } \\
\text { men }(\mathrm{UM})^{4} \\
15-24 \\
\end{array}$} & \multicolumn{2}{|c|}{$\begin{array}{c}\text { Unmarried } \\
\text { women }(\mathrm{UW})^{4} \\
15-24 \\
\end{array}$} \\
\hline & Percent & Number & Percent & Number & Percent & Number & Percent & Number & Percent & Number & Percent & Number \\
\hline \multicolumn{13}{|c|}{ Urban } \\
\hline $\begin{array}{l}\text { Educational level (years) } \\
\text { None }^{3}\end{array}$ & 10.4 & 122 & 223 & 591 & 20.7 & 116 & 35.9 & 414 & 8.6 & 76 & 121 & 177 \\
\hline $1-7$ & $\begin{array}{l}10.4 \\
17.9\end{array}$ & 196 & 21.5 & 563 & $\begin{array}{l}20.7 \\
19.6\end{array}$ & 109 & 23.9 & $\begin{array}{l}414 \\
272\end{array}$ & $\begin{array}{r}0.0 \\
17.1\end{array}$ & 146 & $\begin{array}{l}12.1 \\
19.8\end{array}$ & 291 \\
\hline $8-11$ & 46.6 & 473 & 36.4 & 933 & 37.0 & 199 & 28.2 & 315 & 48.2 & 398 & 42.7 & 618 \\
\hline 12 and above & 25.1 & 248 & 19.8 & 494 & 22.8 & 123 & 12.0 & 135 & 26.1 & 213 & 25.3 & 359 \\
\hline \multicolumn{13}{|l|}{ Worked in last 12 months } \\
\hline Yes & 58.8 & 638 & 15.1 & 388 & 94.6 & 518 & 12.6 & 140 & 53.7 & 448 & 17.0 & 248 \\
\hline No & 41.2 & 401 & 84.9 & 2,192 & 5.4 & 29 & 87.4 & 995 & 46.3 & 385 & 83.0 & 1,197 \\
\hline \multicolumn{13}{|l|}{ Wealth quintile } \\
\hline First & 1.1 & 14 & 2.2 & 59 & 3.3 & 16 & 3.5 & 37 & 0.4 & 5 & 1.4 & 22 \\
\hline Second & 4.3 & 49 & 5.7 & 148 & 7.6 & 47 & 7.7 & 88 & 3.7 & 30 & 4.0 & 60 \\
\hline Third & 6.1 & 65 & 8.2 & 216 & 8.7 & 48 & 11.2 & 127 & 5.7 & 48 & 6.1 & 89 \\
\hline Fourth & 16.8 & 187 & 14.1 & 369 & 19.6 & 110 & 16.8 & 193 & 15.6 & 133 & 12.1 & 176 \\
\hline Fifth & 71.8 & 724 & 69.9 & 1,789 & 60.9 & 326 & 60.8 & 691 & 74.6 & 617 & 76.3 & 1,098 \\
\hline Total & 100.0 & 1,039 & 100.0 & 2,581 & 100.0 & 547 & 100.0 & 1,136 & 100.0 & 833 & 100.0 & 1,445 \\
\hline \multicolumn{13}{|c|}{ Rural } \\
\hline \multicolumn{13}{|l|}{ Age (years) } \\
\hline $15-19$ & 62.7 & 578 & 60.0 & 2,168 & 8.2 & 47 & 44.0 & 530 & 80.2 & 531 & 93.8 & 1,638 \\
\hline $20-24$ & 37.3 & 325 & 40.0 & 780 & 34.6 & 197 & 56.0 & 675 & 19.8 & 128 & 6.2 & 105 \\
\hline $25-29$ & NA & NA & NA & NA & 57.2 & 324 & NA & NA & NA & NA & NA & NA \\
\hline \multicolumn{13}{|l|}{ Religion } \\
\hline Hindu & 86.6 & 787 & 86.2 & 2,461 & 88.1 & 500 & 88.9 & 1,065 & 86.8 & 577 & 80.5 & 1,396 \\
\hline Muslim & 13.4 & 116 & 13.8 & 487 & 11.9 & 68 & 11.1 & 140 & 13.2 & 82 & 19.5 & 347 \\
\hline Christian & 0.0 & 0 & 0.0 & 0 & 0.0 & 0 & 0.0 & 0 & 0.0 & 0 & 0.0 & 0 \\
\hline Other ${ }^{1}$ & 0.0 & 0 & 0.0 & 0 & 0.0 & 0 & 0.0 & 0 & 0.0 & 0 & 0.0 & 0 \\
\hline \multicolumn{13}{|l|}{ Caste } \\
\hline SC & 21.8 & 193 & 22.2 & 570 & 27.8 & 156 & 25.4 & 301 & 18.1 & 120 & 15.5 & 269 \\
\hline ST & 2.1 & 17 & 0.1 & 1 & 3.4 & 17 & 0.1 & 1 & 1.2 & 8 & 0.0 & 0 \\
\hline $\mathrm{OBC}$ & 57.4 & 526 & 64.7 & 1,903 & 58.5 & 336 & 65.6 & 798 & 57.8 & 385 & 62.9 & 1,105 \\
\hline General $^{2}$ & 18.6 & 166 & 13.0 & 473 & 10.2 & 58 & 8.9 & 105 & 22.7 & 145 & 21.5 & 368 \\
\hline No caste/do not know & 0.1 & 1 & 0.0 & 1 & 0.2 & 1 & 0.0 & 0 & 0.2 & 1 & 0.0 & 1 \\
\hline \multicolumn{13}{|l|}{ Educational level (years) } \\
\hline None $^{3}$ & 17.0 & 149 & 54.7 & 1,343 & 30.1 & 171 & 65.9 & 793 & 12.3 & 78 & 31.1 & 550 \\
\hline $1-7$ & 32.3 & 290 & 25.3 & 873 & 29.6 & 168 & 20.3 & 247 & 30.9 & 204 & 35.9 & 626 \\
\hline $8-11$ & 40.6 & 374 & 17.4 & 644 & 29.5 & 168 & 11.9 & 141 & 46.7 & 311 & 29.2 & 503 \\
\hline 12 and above & 10.1 & 90 & 2.6 & 88 & 10.7 & 61 & 2.0 & 24 & 10.2 & 66 & 3.8 & 64 \\
\hline \multicolumn{13}{|l|}{ Worked in last 12 months } \\
\hline Yes & 75.0 & 668 & 39.6 & 1,174 & 97.0 & 551 & 39.7 & 478 & 66.4 & 433 & 39.5 & 696 \\
\hline No & 24.7 & 233 & 60.4 & 1,774 & 2.6 & 15 & 60.3 & 727 & 33.4 & 225 & 60.5 & 1,047 \\
\hline \multicolumn{13}{|l|}{ Wealth quintile } \\
\hline First & 10.4 & 93 & 20.1 & 511 & 15.2 & 86 & 23.4 & 280 & 8.7 & 58 & 13.2 & 231 \\
\hline Second & 13.2 & 118 & 20.2 & 564 & 18.0 & 101 & 21.8 & 263 & 12.2 & 80 & 17.0 & 301 \\
\hline Third & 21.7 & 196 & 20.4 & 593 & 21.1 & 121 & 20.8 & 250 & 21.1 & 141 & 19.6 & 343 \\
\hline Fourth & 27.9 & 253 & 21.4 & 673 & 22.5 & 127 & 19.7 & 237 & 29.3 & 192 & 25.0 & 436 \\
\hline Fifth & 26.8 & 243 & 17.8 & 607 & 23.2 & 133 & 14.3 & 175 & 28.7 & 188 & 25.3 & 432 \\
\hline Total & 100.0 & 903 & 100.0 & 2,948 & 100.0 & 568 & 100.0 & 1,205 & 100.0 & 659 & 100.0 & 1,743 \\
\hline
\end{tabular}

Note: All Ns are unweighted. Column totals may not equal 100\% due to missing cases or "don't know" responses. NA: Not applicable. OBC: Other backward caste. SC: Scheduled caste. ST: Scheduled tribe. ${ }^{1}$ Includes Buddhist/Neo-Buddhist, Sikh, Jain, Jewish, Parsi/Zoroastrian and no specified religion. ${ }^{2}$ Includes all those not belonging to SC, ST or OBC. ${ }^{3}$ Includes non-literate and literate with no formal schooling. ${ }^{4}$ These abbreviations have been used in subsequent tables in this report. 
Educational distributions suggest that youth were better educated than the population at large. Even so, levels of educational attainment were low and gender differences were wide. In total, 16\% of young men and 51\% of young women had no formal education (compared to $40 \%$ and $65 \%$, respectively, of the general population described in Table 2.6) and just $12 \%$ and $5 \%$ had 12 or more years of education (compared to $9 \%$ and $3 \%$ of the general population). As can be seen from the above, gender differences were wide, with young women far more likely to be concentrated among the uneducated or poorly educated than young men. It is notable, however, that even among young men, educational attainment levels were modest. Differences by marital status indicate that as many as $29 \%$ of married young men and $64 \%$ of married young women had no formal education, compared to $12 \%$ and $28 \%$ of unmarried young men and women, respectively. Notwithstanding these differences in percentages who had no formal education, percentages of youth who had attained 12 or more years of education were negligible (12\% and 3\% among married young men and women; $13 \%$ and $7 \%$, respectively, among the unmarried). Urban youth were generally better educated than rural youth: for example, $10 \%$ of young men and $22 \%$ of young women in urban areas had no formal education compared to $17 \%$ and $55 \%$ of rural youth, respectively. Similarly, $25 \%$ and $20 \%$, respectively, of urban young men and women completed 12 or more years of education compared to $10 \%$ and $3 \%$ of rural youth, respectively.

Gender differences were evident with regard to work status; young men were almost twice as likely as young women to have ever worked in paid or unpaid activities in the 12 months preceding the interview $(73 \%$ versus 37\%). Differences by marital status were evident among young men, but not among young women; $97 \%$ of married young men had worked in the year preceding the interview compared to $64 \%$ of unmarried young men. Also evident was that rural youth were more likely to be engaged in economic activities than were urban youth and that gender differences were somewhat narrow in rural areas than in urban areas: $75 \%$ of young men and $40 \%$ of young women in rural areas were engaged in economic activities compared to $59 \%$ and $15 \%$, respectively, in urban areas.

Household economic status distributions, as measured by wealth quintiles, suggest that young men were somewhat less likely than young women to belong to households in the poorer quintiles. For example, $9 \%$ of young men compared to $18 \%$ of young women fell into households in the poorest (first) quintile. Conversely, young men were somewhat more likely than young women to belong to households in the wealthiest quintile. Likewise, the married were generally more likely to be concentrated in households in the poorer quintiles than were the unmarried. For example, $14 \%$ and $22 \%$ of married young men and women fell into households in the poorest (first) quintile, compared to $7 \%$ and $11 \%$, respectively, of the unmarried; conversely, $26 \%$ and $17 \%$ of married young men and women, respectively fell into households in the wealthiest (fifth) quintile, compared to $36 \%$ and $33 \%$ of unmarried young men and women, respectively. Rural-urban differences were wide, with rural youth more likely than their urban counterparts to belong to households in the poorer quintiles; conversely, more urban than rural youth belonged to households in the wealthiest quintile.

\subsection{Profile of surveyed youth: Parental characteristics}

The Youth Study inquired about the socio-demographic characteristics of respondents' parents, including their survival status, education and occupation. Findings, presented in Table 2.13, suggest that in about four in five respondents, both parents were surviving. While no gender differences were evident among the unmarried, married young men tended to be somewhat less likely to report that both parents were alive than married young women $(70 \%$ versus $79 \%$, respectively), clearly a function of the fact that married young men in our sample were older than married young women. Rural-urban differences were generally narrow. For those with just one parent surviving, this parent was more likely to be the mother (9-10\%) than the father (5-6\%). Finally, 1-2\% reported that neither parent was alive. 
Table 2.13: Parental characteristics of surveyed youth

Percent distribution of surveyed youth by parental characteristics, according to residence, Bihar, 2007

\begin{tabular}{|c|c|c|c|c|c|c|}
\hline Parental characteristics (\%) & $\begin{array}{c}\mathrm{M} \\
15-24\end{array}$ & $\begin{array}{c}\text { W } \\
15-24\end{array}$ & $\begin{array}{c}\text { MM } \\
15-29\end{array}$ & $\begin{array}{c}\text { MW } \\
15-24\end{array}$ & $\begin{array}{c}\text { UM } \\
15-24\end{array}$ & $\begin{array}{c}\text { UW } \\
15-24\end{array}$ \\
\hline \multicolumn{7}{|c|}{ Combined } \\
\hline \multicolumn{7}{|l|}{ Survival status } \\
\hline Both parents dead & 1.0 & 1.8 & 3.6 & 2.5 & 0.8 & 0.5 \\
\hline Only father alive & 5.1 & 5.8 & 10.4 & 7.0 & 3.8 & 3.5 \\
\hline Only mother alive & 9.7 & 9.4 & 16.1 & 11.2 & 8.6 & 6.0 \\
\hline Both parents alive & 84.2 & 82.9 & 69.9 & 79.3 & 86.8 & 89.9 \\
\hline \multicolumn{7}{|l|}{ Educational attainment level } \\
\hline Median years of schooling of father & 6.0 & 4.0 & $\mathrm{NC}$ & $\mathrm{NC}$ & 7.0 & 7.0 \\
\hline Median years of schooling of mother & $\mathrm{NC}$ & NC & $\mathrm{NC}$ & NC & $\mathrm{NC}$ & NC \\
\hline \multicolumn{7}{|l|}{ Current/last occupational status of father } \\
\hline Cultivator & 33.7 & 25.4 & 32.9 & 28.0 & 33.9 & 21.4 \\
\hline Agricultural labourer & 12.6 & 20.2 & 21.6 & 22.9 & 9.6 & 16.3 \\
\hline Administrative/executive/managerial/clerical & 7.8 & 6.7 & 5.3 & 4.5 & 9.6 & 10.0 \\
\hline Business & 7.2 & 6.6 & 5.7 & 4.9 & 7.2 & 9.1 \\
\hline Skilled manual/machinery & 17.1 & 19.2 & 13.3 & 17.9 & 18.3 & 21.2 \\
\hline Unskilled non-agricultural labourer & 20.1 & 20.7 & 18.7 & 20.7 & 20.3 & 20.5 \\
\hline Other & 1.1 & 0.4 & 1.5 & 0.3 & 0.8 & 0.6 \\
\hline Never worked & 0.2 & 0.6 & 0.6 & 0.6 & 0.3 & 0.8 \\
\hline \multicolumn{7}{|l|}{ Current/last occupational status of mother } \\
\hline Cultivator & 10.5 & 17.0 & 10.3 & 18.8 & 9.7 & 14.5 \\
\hline Agricultural labourer & 11.8 & 26.3 & 16.1 & 29.3 & 9.5 & 22.1 \\
\hline Administrative/executive/managerial/clerical & 0.6 & 0.9 & 0.4 & 0.4 & 0.7 & 1.6 \\
\hline Business & 0.5 & 0.9 & 0.6 & 0.8 & 0.5 & 1.1 \\
\hline Skilled manual/machinery & 0.8 & 2.0 & 0.4 & 1.7 & 0.9 & 2.4 \\
\hline Unskilled non-agricultural labourer & 3.2 & 3.7 & 5.5 & 4.1 & 2.7 & 2.7 \\
\hline Other & 0.3 & 0.3 & 0.4 & 0.4 & 0.1 & 0.3 \\
\hline Housewife/never worked & 72.3 & 48.8 & 66.4 & 44.4 & 76.0 & 55.3 \\
\hline Number of respondents & 1,942 & 5,529 & 1,115 & 2,341 & 1,492 & 3,188 \\
\hline \multicolumn{7}{|c|}{ Urban } \\
\hline \multicolumn{7}{|l|}{ Survival status } \\
\hline Both parents dead & 1.4 & 1.3 & 5.4 & 2.1 & 0.8 & 0.6 \\
\hline Only father alive & 4.7 & 5.2 & 6.5 & 7.0 & 4.5 & 3.8 \\
\hline Only mother alive & 12.5 & 9.7 & 17.4 & 12.0 & 11.9 & 7.9 \\
\hline Both parents alive & 81.4 & 83.8 & 70.7 & 78.9 & 82.8 & 87.7 \\
\hline \multicolumn{7}{|l|}{ Educational attainment level } \\
\hline Median years of schooling of father & 9.0 & 10.0 & 7.0 & 8.0 & 9.0 & 10.0 \\
\hline Median years of schooling of mother & $\mathrm{NC}$ & $\mathrm{NC}$ & NC & NC & NC & 5.0 \\
\hline \multicolumn{7}{|l|}{ Current/last occupational status of father } \\
\hline Cultivator & 13.2 & 11.2 & 12.0 & 17.6 & 13.5 & 6.5 \\
\hline Agricultural labourer & 2.1 & 4.2 & 6.5 & 7.0 & 1.6 & 2.4 \\
\hline Administrative/executive/managerial/clerical & 14.6 & 18.6 & 13.0 & 13.4 & 15.1 & 22.3 \\
\hline
\end{tabular}


Table 2.13: (Cont'd)

\begin{tabular}{|c|c|c|c|c|c|c|}
\hline Parental characteristics (\%) & $\begin{array}{c}\mathrm{M} \\
15-24\end{array}$ & $\begin{array}{c}\text { W } \\
15-24\end{array}$ & $\begin{array}{c}\text { MM } \\
15-29\end{array}$ & $\begin{array}{c}\text { MW } \\
15-24\end{array}$ & $\begin{array}{c}\text { UM } \\
15-24\end{array}$ & $\begin{array}{c}\text { UW } \\
15-24\end{array}$ \\
\hline \multicolumn{7}{|c|}{ Urban } \\
\hline Business & 15.0 & 15.9 & 13.0 & 13.4 & 15.9 & 17.8 \\
\hline Skilled manual/machinery & 28.2 & 25.9 & 25.0 & 24.6 & 28.2 & 26.7 \\
\hline Unskilled non-agricultural labourer & 24.3 & 22.6 & 28.3 & 22.5 & 23.3 & 22.7 \\
\hline Other & 2.1 & 0.8 & 2.2 & 0.7 & 2.0 & 0.8 \\
\hline Never worked & 0.4 & 0.8 & 0.0 & 0.7 & 0.4 & 0.8 \\
\hline \multicolumn{7}{|l|}{ Current/last occupational status of mother } \\
\hline Cultivator & 1.4 & 4.4 & 2.2 & 7.0 & 1.2 & 2.6 \\
\hline Agricultural labourer & 1.1 & 4.4 & 2.2 & 7.0 & 0.8 & 2.4 \\
\hline Administrative/executive/managerial/clerical & 1.4 & 3.3 & 1.1 & 2.1 & 1.6 & 4.3 \\
\hline Business & 1.1 & 2.0 & 1.1 & 2.1 & 1.2 & 2.2 \\
\hline Skilled manual/machinery & 1.4 & 4.1 & 1.1 & 3.5 & 1.6 & 4.5 \\
\hline Unskilled non-agricultural labourer & 4.7 & 6.4 & 4.3 & 6.3 & 4.1 & 6.7 \\
\hline Other & 0.4 & 0.2 & 1.1 & 0.0 & 0.0 & 0.2 \\
\hline Housewife/never worked & 88.5 & 75.2 & 87.0 & 72.0 & 89.3 & 77.1 \\
\hline Number of respondents & 1,039 & 2,581 & 547 & 1,136 & 833 & 1,445 \\
\hline \multicolumn{7}{|c|}{ Rural } \\
\hline Survival status & & & & & & \\
\hline Both parents dead & 0.9 & 1.9 & 3.4 & 2.5 & 0.8 & 0.4 \\
\hline Only father alive & 5.2 & 5.9 & 10.7 & 7.0 & 3.6 & 3.5 \\
\hline Only mother alive & 9.3 & 9.4 & 16.0 & 11.1 & 8.0 & 5.7 \\
\hline Both parents alive & 84.6 & 82.8 & 69.9 & 79.3 & 87.6 & 90.4 \\
\hline \multicolumn{7}{|l|}{ Educational attainment level } \\
\hline Median years of schooling of father & 5.0 & 1.0 & NC & $\mathrm{NC}$ & 7.0 & 6.0 \\
\hline Median years of schooling of mother & $\mathrm{NC}$ & NC & $\mathrm{NC}$ & $\mathrm{NC}$ & $\mathrm{NC}$ & $\mathrm{NC}$ \\
\hline \multicolumn{7}{|l|}{ Current/last occupational status of father } \\
\hline Cultivator & 37.2 & 27.2 & 34.8 & 28.7 & 37.9 & 24.1 \\
\hline Agricultural labourer & 14.3 & 22.3 & 23.1 & 23.9 & 11.2 & 18.8 \\
\hline Administrative/executive/managerial/clerical & 6.7 & 5.2 & 4.6 & 4.0 & 8.5 & 7.7 \\
\hline Business & 5.9 & 5.3 & 5.0 & 4.3 & 5.6 & 7.5 \\
\hline Skilled manual/machinery & 15.2 & 18.4 & 12.2 & 17.5 & 16.3 & 20.2 \\
\hline Unskilled non-agricultural labourer & 19.4 & 20.4 & 17.8 & 20.6 & 19.6 & 20.1 \\
\hline Other & 0.9 & 0.3 & 1.5 & 0.2 & 0.6 & 0.6 \\
\hline Never worked & 0.2 & 0.6 & 0.7 & 0.5 & 0.3 & 0.7 \\
\hline \multicolumn{7}{|l|}{ Current/last occupational status of mother } \\
\hline Cultivator & 12.1 & 18.6 & 11.1 & 19.5 & 11.2 & 16.7 \\
\hline Agricultural labourer & 13.5 & 29.1 & 17.3 & 30.8 & 11.2 & 25.7 \\
\hline Administrative/executive/managerial/clerical & 0.5 & 0.6 & 0.4 & 0.3 & 0.5 & 1.1 \\
\hline Business & 0.4 & 0.8 & 0.5 & 0.7 & 0.3 & 0.9 \\
\hline Skilled manual/machinery & 0.6 & 1.7 & 0.3 & 1.5 & 0.7 & 2.0 \\
\hline Unskilled non-agricultural labourer & 3.0 & 3.4 & 5.6 & 4.0 & 2.4 & 2.0 \\
\hline Other & 0.3 & 0.3 & 0.3 & 0.4 & 0.2 & 0.2 \\
\hline Housewife/never worked & 69.6 & 45.4 & 64.6 & 42.6 & 73.5 & 51.3 \\
\hline Number of respondents & 903 & 2,948 & 568 & 1,205 & 659 & 1,743 \\
\hline
\end{tabular}

Note: All Ns are unweighted. Column totals may not equal 100\% due to missing cases or "don't know" responses. NC: Not calculated, as more than $50 \%$ had no formal education. 
Parents' educational attainment was considerably lower than that of youth respondents. For example, the median number of years of education completed by fathers of young men and women was 6 and 4 years, respectively. Fathers of the unmarried were considerably better educated than fathers of the married; fathers of the unmarried typically had 7 years of schooling - in comparison, fathers of over half of married young men and women had never been to school. Differences in educational attainment of fathers by marital status of youth may be attributed to the fact that the better educated may be more likely than the poorly educated to delay the marriage of their children. Rural-urban differences were wide, with rural fathers having completed 1-5 years and urban fathers 9-10 years of schooling. As far as maternal education was concerned, over half of mothers of youth, irrespective of sex, marital status or rural-urban residence, had never been to school.

The Youth Study also inquired about the current or last main occupation of respondents' parents. Distributions of occupational status suggest that fathers of $25-34 \%$ of youth were working on their own farms, compared to mothers of $11-17 \%$ of youth. Fathers of far fewer youth were agricultural labourers: $13 \%$ and $20 \%$ of fathers of young men and women, respectively. A similar proportion—between $12 \%$ and $26 \%$-of mothers of young men and women were agricultural labourers. About one-fifth (17-19\%) of fathers and hardly any (1-2\%) mothers were engaged in skilled manual occupations, and $20-21 \%$ of fathers and $3-4 \%$ of mothers were unskilled non-agricultural labourers. Fewer than $10 \%$ of fathers and less than $1 \%$ of mothers were engaged in administrative, executive, managerial or clerical occupations; similar proportions were running their own business. Finally, mothers of $72 \%$ and $49 \%$ of young men and women, respectively, were housewives; hardly any fathers (less than 1\%) had never worked. Differences by marital status were evident; parents of married youth were more likely to have been engaged in agricultural activities than were parents of unmarried youth. Additionally, mothers of unmarried youth were more likely to have never worked than mothers of married youth. Rural-urban differences were also evident. While rural parents were largely cultivators and agricultural labourers, urban parents were more likely to be concentrated in skilled manual occupations, unskilled nonagricultural activities, administrative, managerial or clerical occupations, and business and in the case of mothers, in housework. That somewhat larger percentages of the fathers of married compared to unmarried youth in urban settings (particularly young women) were in agricultural occupations may be attributed to migration into urban areas by married youth. 
Young people in India are spending more of their adolescent years acquiring an education than ever before. Educational attainment levels have increased, the percentage that has never been to school has declined and gender differences in educational attainment levels have diminished (Office of the Registrar General and Census Commissioner, 2001f). This is not to say, however, that schooling is universal or that gender differences are no longer a concern. Attainment of primary school education is still far from universal, especially among girls. Differences by caste, religion, region and poverty levels persist; and the quality of education varies widely for different sub-groups of youth. This chapter examines the schooling experiences of youth in terms of educational attainment, reasons for school non-attendance or discontinuation, quality of schools and colleges attended and schooling experiences.

\subsection{Educational attainment}

The Youth Study obtained information on whether the respondent had ever been to school and, if so, the number of years of schooling successfully completed. Current schooling status was also assessed, and a Life Event Calendar inquired about the schooling status of all respondents from the age of 12 . Findings are presented in Table 3.1 .

Findings highlight that schooling was far from universal among young people, particularly among young women, in Bihar: $16 \%$ of young men and $51 \%$ of young women had never been to school. Wide differences by marital status were evident: $12 \%$ of unmarried young men compared to $29 \%$ of married young men had no formal education. The corresponding difference among young women was much wider: $28 \%$ of unmarried young women compared to $64 \%$ of married young women had never been to school. As expected, a larger percentage of rural than urban youth had never attended school. Rural-urban differences were much wider among young women than men: $55 \%$ of rural young women compared to $22 \%$ of urban young women, and $17 \%$ of rural young men compared to $10 \%$ of urban young men had never been to school. Indeed, two-thirds of married young women and almost one-third of unmarried young women in rural settings had never been to school.

Educational attainment levels of young people also reconfirm differences by sex, marital status and rural-urban residence. Young men, on average, had 8 years of schooling while, as discussed earlier, over half of young women had never been to school. Among young men, the unmarried had 2 more years of schooling than the married (8 versus 6); in contrast among young women, while the unmarried had completed 6 years of schooling, the majority of the married had never been to school. Rural-urban differences suggest that urban young men typically had 1 more year of schooling than their rural counterparts (9 versus 8 ). Among young women, while those in urban areas had typically attained 8 years of education, over half of those in rural areas had never attended school. Similar differences were evident in the proportion of youth who had completed high school (Class 10). Young men were more than twice as likely as young women to have completed high school (30\% versus 13\%). Married youth were less likely to have completed high school than the unmarried 
(26\% and 33\%, respectively, among young men; $9 \%$ and 19\%, respectively, among young women). We note that disparities by marital status may be wider than what is reflected here because the unmarried were younger and more likely to be pursuing their education at the time of interview.

Table 3.1: Educational attainment and current educational status

Percent distribution of youth by years of schooling successfully completed, median years of schooling and percentage currently in school, according to residence, Bihar, 2007

\begin{tabular}{|c|c|c|c|c|c|c|}
\hline Schooling status (\%) & $\begin{array}{c}\mathrm{M} \\
15-24\end{array}$ & $\begin{array}{c}\text { W } \\
15-24\end{array}$ & $\begin{array}{c}\text { MM } \\
15-29\end{array}$ & $\begin{array}{c}\text { MW } \\
15-24\end{array}$ & $\begin{array}{c}\text { UM } \\
15-24\end{array}$ & $\begin{array}{l}\text { UW } \\
15-24\end{array}$ \\
\hline \multicolumn{7}{|c|}{ Combined } \\
\hline \multicolumn{7}{|l|}{ Completed years of schooling } \\
\hline None $^{1}$ & 16.1 & 51.0 & 29.4 & 64.1 & 11.7 & 28.2 \\
\hline $1-4$ & 10.7 & 9.5 & 12.2 & 7.7 & 10.1 & 13.1 \\
\hline $5-7$ & 19.5 & 15.4 & 16.6 & 12.8 & 18.6 & 20.4 \\
\hline $8-9$ & 23.5 & 11.0 & 15.9 & 6.6 & 26.5 & 19.0 \\
\hline $10-11$ & 18.0 & 8.6 & 14.3 & 6.3 & 20.3 & 12.2 \\
\hline 12 and above & 12.2 & 4.5 & 11.7 & 2.6 & 12.8 & 7.2 \\
\hline Median years of schooling & 8.0 & NC & 6.0 & NC & 8.0 & 6.0 \\
\hline Currently in school/college & 41.7 & 18.9 & 4.9 & 4.7 & 54.0 & 44.5 \\
\hline Number of respondents & 1,942 & 5,529 & 1,115 & 2,341 & 1,492 & 3,188 \\
\hline \multicolumn{7}{|c|}{ Urban } \\
\hline \multicolumn{7}{|l|}{ Completed years of schooling } \\
\hline None $^{1}$ & 10.4 & 22.3 & 20.7 & 35.9 & 8.6 & 12.2 \\
\hline $1-4$ & 6.8 & 7.2 & 6.5 & 9.2 & 6.6 & 5.7 \\
\hline $5-7$ & 11.1 & 14.3 & 13.0 & 14.8 & 10.7 & 14.2 \\
\hline $8-9$ & 22.9 & 16.2 & 17.4 & 12.0 & 23.8 & 19.1 \\
\hline $10-11$ & 23.7 & 20.3 & 19.6 & 16.2 & 24.2 & 23.5 \\
\hline 12 and above & 25.1 & 19.8 & 22.8 & 12.0 & 26.2 & 25.4 \\
\hline Median years of schooling & 9.0 & 8.0 & 9.0 & 5.0 & 10.0 & 9.0 \\
\hline Currently in school/college & 51.3 & 38.3 & 5.4 & 7.7 & 57.8 & 60.5 \\
\hline Number of respondents & 1,039 & 2,581 & 547 & 1,136 & 833 & 1,445 \\
\hline \multicolumn{7}{|c|}{ Rural } \\
\hline Completed years of schooling & & & & & & \\
\hline None $^{1}$ & 17.0 & 54.7 & 30.1 & 65.9 & 12.3 & 31.1 \\
\hline $1-4$ & 11.4 & 9.8 & 12.7 & 7.6 & 10.7 & 14.4 \\
\hline $5-7$ & 20.9 & 15.5 & 16.9 & 12.7 & 20.2 & 21.5 \\
\hline $8-9$ & 23.5 & 10.3 & 15.8 & 6.2 & 27.1 & 19.0 \\
\hline $10-11$ & 17.1 & 7.1 & 13.8 & 5.7 & 19.6 & 10.1 \\
\hline 12 and above & 10.1 & 2.6 & 10.7 & 2.0 & 10.2 & 3.8 \\
\hline Median years of schooling & 8.0 & $\mathrm{NC}$ & 5.0 & NC & 8.0 & 5.0 \\
\hline Currently in school/college & 40.1 & 16.4 & 5.0 & 4.5 & 53.2 & 41.5 \\
\hline Number of respondents & 903 & 2,948 & 568 & 1,205 & 659 & 1,743 \\
\hline
\end{tabular}

Note: All Ns are unweighted. Column totals may not equal $100 \%$ due to missing cases or "don't know" responses ${ }^{1}$ Includes non-literate and literate with no formal schooling. NC: Median cannot be calculated as more than 50\% had no formal education. 
Rural youth were considerably less likely to have completed high school than urban youth $(27 \%$ and $49 \%$, respectively, among young men; $10 \%$ and $40 \%$ among young women, respectively). Rural-urban differences were widest among unmarried young women, with $49 \%$ of urban young women compared to $14 \%$ of rural young women having completed high school.

At the time of interview, $42 \%$ of young men compared to $19 \%$ of young women were in school or college. These gender differences were influenced by differences among unmarried youth. For example, 54\% of young men compared to $45 \%$ of young women were still in school/college at the time of the interview. In contrast, hardly any married youth (5\% each of young men and women, respectively) were still studying. Among the unmarried, patterns varied. Among unmarried young men, for example, rural-urban differences were narrow: $58 \%$ and $53 \%$ of those in urban and rural areas, respectively were studying at the time of interview. In contrast, rural-urban differences were pronounced among unmarried young women: $61 \%$ of urban young women compared to $42 \%$ of rural young women were studying at the time of interview.

\subsection{Differentials in educational attainment}

Differentials in educational levels of young men and women, measured with respect to completed years of schooling, are presented in Tables 3.2 and 3.3, respectively. Findings show a positive association between age and years of education completed among both young men and women, irrespective of marital status and rural-urban residence.

Table 3.2: Educational attainment of young men by selected background characteristics

Percent distribution of young men by educational level, according to selected background characteristics and residence, Bihar, 2007

\begin{tabular}{|c|c|c|c|c|c|c|c|c|c|c|c|c|}
\hline \multirow{3}{*}{$\begin{array}{l}\text { Background } \\
\text { characteristics } \\
\text { (\%) }\end{array}$} & \multicolumn{4}{|c|}{ M, 15-24 } & \multicolumn{4}{|c|}{ MM, 15-29 } & \multicolumn{4}{|c|}{ UM, 15-24 } \\
\hline & \multicolumn{12}{|c|}{ Completed years of schooling } \\
\hline & None $^{1}$ & $1-7$ & 8-9 & $10+$ & None $^{1}$ & $1-7$ & $8-9$ & $10+$ & None $^{1}$ & $1-7$ & $8-9$ & $10+$ \\
\hline \multicolumn{13}{|c|}{ Combined } \\
\hline \multicolumn{13}{|l|}{ Age (years) } \\
\hline $15-19$ & 13.3 & 31.0 & 29.1 & 26.6 & 29.1 & 36.0 & 15.1 & 19.8 & 12.1 & 30.6 & 30.3 & 27.0 \\
\hline $20-24$ & 20.5 & 29.1 & 14.2 & 36.1 & 28.0 & 34.7 & 15.0 & 22.3 & 10.6 & 21.5 & 13.3 & 54.5 \\
\hline $25-29$ & NA & NA & NA & NA & 30.2 & 24.3 & 16.6 & 28.9 & NA & $\mathrm{NA}$ & NA & NA \\
\hline \multicolumn{13}{|l|}{ Religion } \\
\hline Hindu & 14.6 & 29.2 & 24.1 & 32.1 & 27.0 & 28.2 & 16.7 & 28.1 & 10.8 & 27.2 & 27.4 & 34.6 \\
\hline Muslim & 25.4 & 36.8 & 19.5 & 18.4 & 46.3 & 33.8 & 9.6 & 10.3 & 17.8 & 37.5 & 21.2 & 23.6 \\
\hline \multicolumn{13}{|l|}{ Caste } \\
\hline SC & 23.8 & 35.2 & 19.7 & 21.3 & 37.8 & 27.4 & 15.4 & 19.4 & 16.9 & 34.3 & 22.6 & 26.2 \\
\hline $\mathrm{OBC}$ & 16.9 & 31.9 & 23.4 & 27.8 & 27.5 & 31.1 & 14.6 & 26.8 & 13.3 & 30.7 & 26.8 & 29.1 \\
\hline General $^{2}$ & 4.9 & 21.0 & 25.6 & 48.6 & 12.4 & 19.0 & 23.1 & 45.5 & 4.5 & 19.8 & 26.8 & 48.9 \\
\hline \multicolumn{13}{|l|}{ Wealth quintile } \\
\hline First & 38.8 & 38.2 & 12.9 & 10.1 & 61.0 & 23.9 & 5.0 & 10.1 & 28.2 & 46.4 & 15.5 & 10.0 \\
\hline Second & 29.4 & 42.6 & 17.0 & 11.1 & 48.4 & 34.4 & 11.5 & 5.7 & 21.9 & 45.0 & 18.8 & 14.4 \\
\hline Third & 20.7 & 37.3 & 26.8 & 15.2 & 30.4 & 34.8 & 18.3 & 16.5 & 15.5 & 35.7 & 32.9 & 15.9 \\
\hline Fourth & 14.5 & 33.4 & 25.9 & 26.3 & 22.5 & 34.5 & 19.3 & 23.7 & 12.4 & 30.0 & 28.3 & 29.3 \\
\hline Fifth & 3.7 & 17.0 & 24.8 & 54.4 & 4.8 & 18.8 & 19.8 & 56.7 & 3.0 & 15.3 & 26.6 & 55.1 \\
\hline Total & 16.1 & 30.2 & 23.5 & 30.2 & 29.4 & 28.8 & 15.9 & 25.9 & 11.7 & 28.6 & 26.5 & 33.1 \\
\hline
\end{tabular}

Cont'd on next page... 
Table 3.2: (Cont'd)

\begin{tabular}{|c|c|c|c|c|c|c|c|c|c|c|c|c|}
\hline \multirow{3}{*}{$\begin{array}{l}\text { Background } \\
\text { characteristics } \\
(\%)\end{array}$} & \multicolumn{4}{|c|}{ M, 15-24 } & \multicolumn{4}{|c|}{ MM, 15-29 } & \multicolumn{4}{|c|}{ UM, 15-24 } \\
\hline & \multicolumn{12}{|c|}{ Completed years of schooling } \\
\hline & None $^{1}$ & $1-7$ & 8-9 & $10+$ & None $^{1}$ & $1-7$ & 8-9 & $10+$ & None $^{1}$ & $1-7$ & 8-9 & $10+$ \\
\hline \multicolumn{13}{|c|}{ Urban } \\
\hline Age (years) & & & & & & & & & & & & \\
\hline $15-19$ & 9.2 & 19.0 & 28.2 & 43.6 & * & * & * & * & 8.8 & 18.8 & 28.8 & 43.8 \\
\hline $20-24$ & 12.7 & 16.9 & 15.3 & 55.1 & 21.9 & 21.9 & 18.8 & 37.5 & 8.3 & 14.3 & 14.3 & 63.1 \\
\hline $25-29$ & NA & NA & NA & NA & 20.3 & 16.9 & 16.9 & 45.8 & NA & NA & NA & $\mathrm{NA}$ \\
\hline \multicolumn{13}{|l|}{ Religion } \\
\hline Hindu & 8.2 & 16.5 & 23.8 & 51.5 & 17.7 & 19.0 & 16.5 & 46.8 & 6.5 & 15.0 & 25.0 & 53.5 \\
\hline Muslim & 21.3 & 25.5 & 21.3 & 31.9 & 42.9 & 21.4 & 14.3 & 21.4 & 18.6 & 25.6 & 20.9 & 34.9 \\
\hline \multicolumn{13}{|l|}{ Caste } \\
\hline SC & 20.7 & 24.1 & 17.2 & 37.9 & 35.7 & 21.4 & 21.4 & 21.4 & 14.3 & 23.8 & 14.3 & 47.6 \\
\hline OBC & 12.3 & 19.9 & 25.7 & 42.1 & 19.0 & 22.4 & 17.2 & 41.4 & 10.8 & 18.9 & 27.0 & 43.2 \\
\hline General $^{2}$ & 3.8 & 10.3 & 19.2 & 66.7 & 11.8 & 11.8 & 11.8 & 64.7 & 2.8 & 9.9 & 19.7 & 67.6 \\
\hline \multicolumn{13}{|l|}{ Wealth quintile } \\
\hline First & * & * & * & * & * & * & * & * & * & * & * & * \\
\hline Second & $(50.0)$ & $(33.3)$ & $(16.7)$ & $(0.0)$ & $(57.1)$ & $(28.6)$ & $(14.3)$ & $(0.0)$ & $(50.0)$ & $(37.5)$ & $(12.5)$ & $(0.0)$ \\
\hline Third & 25.0 & 37.5 & 31.3 & 6.3 & $(33.3)$ & $(33.3)$ & $(22.2)$ & (11.1) & $(28.6)$ & $(35.7)$ & $(28.6)$ & $(7.1)$ \\
\hline Fourth & 19.1 & 31.9 & 23.4 & 25.5 & 33.3 & 27.8 & 22.2 & 16.7 & 15.4 & 33.3 & 23.1 & 28.2 \\
\hline Fifth & 4.5 & 11.9 & 22.9 & 60.7 & 8.8 & 14.0 & 15.8 & 61.4 & 3.8 & 11.5 & 24.0 & 60.7 \\
\hline Total & 10.4 & 18.0 & 23.0 & 48.6 & 20.7 & 19.6 & 17.4 & 42.4 & 8.6 & 17.2 & 23.8 & 50.4 \\
\hline \multicolumn{13}{|c|}{ Rural } \\
\hline \multicolumn{13}{|l|}{ Age (years) } \\
\hline $15-19$ & 14.0 & 32.9 & 29.2 & 24.0 & $(28.9)$ & $(37.3)$ & $(14.5)$ & (19.3) & 12.5 & 32.5 & 30.5 & 24.4 \\
\hline $20-24$ & 22.1 & 31.3 & 14.1 & 32.4 & 28.6 & 35.7 & 14.7 & 21.0 & 11.3 & 24.3 & 13.0 & 51.4 \\
\hline $25-29$ & NA & NA & NA & NA & 31.2 & 24.9 & 16.6 & 27.3 & NA & NA & NA & NA \\
\hline \multicolumn{13}{|l|}{ Religion } \\
\hline Hindu & 15.6 & 31.3 & 24.1 & 29.0 & 27.9 & 29.0 & 16.6 & 26.5 & 11.5 & 29.4 & 28.0 & 31.1 \\
\hline Muslim & 26.1 & 38.9 & 19.5 & 15.5 & 46.3 & 35.0 & 8.9 & 9.8 & 17.7 & 40.2 & 21.3 & 20.7 \\
\hline \multicolumn{13}{|l|}{ Caste } \\
\hline SC & 24.3 & 36.0 & 19.9 & 19.9 & 37.9 & 27.7 & 15.1 & 19.3 & 17.2 & 35.2 & 23.3 & 24.2 \\
\hline $\mathrm{OBC}$ & 17.9 & 33.9 & 22.9 & 25.3 & 28.4 & 31.9 & 14.4 & 25.3 & 13.9 & 33.1 & 26.8 & 26.2 \\
\hline General $^{2}$ & 5.1 & 23.3 & 27.5 & 44.1 & 12.5 & 20.2 & 25.0 & 42.3 & 4.9 & 22.3 & 28.6 & 44.2 \\
\hline \multicolumn{13}{|l|}{ Wealth quintile } \\
\hline First & 38.5 & 38.5 & 12.6 & 10.3 & 60.6 & 23.9 & 5.2 & 10.3 & 27.5 & 46.8 & 15.6 & 10.1 \\
\hline Second & 28.3 & 43.0 & 17.0 & 11.7 & 48.1 & 34.6 & 11.4 & 5.9 & 20.3 & 45.8 & 19.0 & 15.0 \\
\hline Third & 20.6 & 37.4 & 26.6 & 15.4 & 30.6 & 34.7 & 18.1 & 16.7 & 14.9 & 35.9 & 33.2 & 16.0 \\
\hline Fourth & 14.0 & 33.6 & 26.0 & 26.4 & 21.7 & 35.2 & 19.1 & 23.9 & 11.8 & 29.9 & 29.0 & 29.3 \\
\hline Fifth & 3.3 & 19.3 & 25.7 & 51.7 & 4.2 & 19.3 & 21.0 & 55.5 & 2.5 & 17.3 & 27.9 & 52.2 \\
\hline Total & 17.0 & 32.3 & 23.5 & 27.2 & 30.1 & 29.6 & 15.8 & 24.5 & 12.3 & 30.9 & 27.1 & 29.8 \\
\hline
\end{tabular}

Note: Row totals may not equal 100\% due to missing cases or "don't know" responses. ( ) Based on 25-49 unweighted cases. ${ }^{*}$ Percentage not shown, based on fewer than 25 unweighted cases. NA: Not applicable. OBC: Other backward caste. SC: Scheduled caste. ST: Scheduled tribe. ${ }^{1}$ Includes non-literate and literate with no formal schooling. ${ }^{2}$ Includes all those not belonging to $S C, S T$ or $O B C$. 
Differences in educational attainment levels by religion, shown in Tables 3.2 and 3.3, indicate that Muslims tended to be more disadvantaged than Hindus, irrespective of sex, marital status and rural-urban residence. For example, $18 \%$ and $8 \%$ of Muslim young men and women, respectively, had completed at least 10 years of education, compared to $32 \%$ and $14 \%$ of Hindu young men and women, respectively. Caste differences suggest that those belonging to general castes were considerably more likely than others to have completed 10 or more years of schooling among both young men and women, irrespective of marital status and rural-urban residence; conversely, those belonging to scheduled castes were considerably less likely to have done so.

A positive association was consistently observed between the economic status of young people's households, measured in wealth quintiles, and young people's educational attainment levels. For example, among young men, just $10 \%$ of those from households in the poorest (first) quintile had completed 10 or more years of schooling, compared to $54 \%$ of those from households in the wealthiest (fifth) quintile. Among young women however, the pattern was quite different: differences in percentages of those who had completed 10 or more years of schooling were mild among those in the first to fourth quintiles (fewer than 10\%) and were apparent for the wealthiest quintile (fifth), among whom $39 \%$ had completed 10 or more years of education. Patterns were similar for both the unmarried and the married and those residing in rural and urban areas.

Table 3.3: Educational attainment of young women by selected background characteristics

Percent distribution of young women by educational level, according to selected background characteristics and residence, Bihar, 2007

\begin{tabular}{|c|c|c|c|c|c|c|c|c|c|c|c|c|}
\hline \multirow{3}{*}{$\begin{array}{l}\text { Background } \\
\text { characteristics } \\
(\%)\end{array}$} & \multicolumn{4}{|c|}{ W, 15-24 } & \multicolumn{4}{|c|}{ MW, 15-24 } & \multicolumn{4}{|c|}{ UW, 15-24 } \\
\hline & \multicolumn{12}{|c|}{ Completed years of schooling } \\
\hline & None $^{1}$ & $1-7$ & 8-9 & $10+$ & None $^{1}$ & $1-7$ & 8-9 & $10+$ & None $^{1}$ & $1-7$ & $8-9$ & $10+$ \\
\hline \multicolumn{13}{|c|}{ Combined } \\
\hline Age (years) & & & & & & & & & & & & \\
\hline $15-19$ & 48.0 & 28.1 & 13.4 & 10.4 & 69.6 & 20.2 & 5.6 & 4.7 & 30.0 & 35.4 & 20.2 & 14.4 \\
\hline $20-24$ & 55.4 & 20.1 & 7.3 & 17.2 & 59.9 & 20.8 & 7.3 & 12.0 & 8.4 & 12.5 & 7.0 & 72.2 \\
\hline \multicolumn{13}{|l|}{ Religion } \\
\hline Hindu & 50.6 & 24.0 & 11.5 & 13.9 & 63.8 & 20.0 & 6.9 & 9.3 & 25.3 & 32.7 & 20.6 & 21.5 \\
\hline Muslim & 53.5 & 30.3 & 8.1 & 8.2 & 66.0 & 25.0 & 3.7 & 5.2 & 40.2 & 36.7 & 12.7 & 10.4 \\
\hline \multicolumn{13}{|l|}{ Caste } \\
\hline SC & 68.5 & 21.0 & 5.1 & 5.4 & 75.9 & 16.5 & 3.4 & 4.1 & 46.7 & 35.1 & 9.9 & 8.2 \\
\hline $\mathrm{OBC}$ & 51.5 & 25.7 & 11.2 & 11.6 & 63.7 & 21.1 & 6.8 & 8.3 & 29.0 & 35.0 & 19.5 & 16.5 \\
\hline General $^{2}$ & 23.1 & 26.7 & 18.9 & 31.3 & 36.0 & 26.1 & 13.1 & 24.8 & 14.0 & 28.2 & 23.8 & 34.0 \\
\hline \multicolumn{13}{|l|}{ Wealth quintile } \\
\hline First & 75.8 & 18.4 & 3.4 & 2.4 & 81.3 & 13.7 & 2.3 & 2.7 & 54.8 & 35.8 & 7.4 & 1.9 \\
\hline Second & 69.5 & 23.9 & 4.0 & 2.6 & 77.1 & 18.6 & 2.2 & 2.0 & 49.6 & 37.7 & 8.6 & 4.2 \\
\hline Third & 56.2 & 29.3 & 9.0 & 5.4 & 66.2 & 23.3 & 6.3 & 4.2 & 34.3 & 42.7 & 15.1 & 7.9 \\
\hline Fourth & 46.8 & 29.9 & 14.1 & 9.3 & 58.9 & 24.9 & 7.7 & 8.5 & 27.3 & 38.0 & 24.5 & 10.2 \\
\hline Fifth & 17.5 & 22.6 & 21.0 & 38.9 & 29.4 & 23.4 & 16.4 & 30.8 & 6.7 & 22.7 & 26.1 & 44.5 \\
\hline Total & 51.0 & 24.9 & 11.0 & 13.1 & 64.1 & 20.5 & 6.6 & 8.8 & 28.2 & 33.4 & 19.0 & 19.4 \\
\hline
\end{tabular}

Cont'd on next page... 


\begin{tabular}{|c|c|c|c|c|c|c|c|c|c|c|c|c|}
\hline \multirow{3}{*}{$\begin{array}{l}\text { Background } \\
\text { characteristics } \\
(\%)\end{array}$} & \multicolumn{4}{|c|}{ W, 15-24 } & \multicolumn{4}{|c|}{ MW, 15-24 } & \multicolumn{4}{|c|}{ UW, 15-24 } \\
\hline & \multicolumn{12}{|c|}{ Completed years of schooling } \\
\hline & None $^{1}$ & $1-7$ & 8-9 & $10+$ & None $^{1}$ & $1-7$ & 8-9 & $10+$ & None $^{1}$ & $1-7$ & 8-9 & $10+$ \\
\hline \multicolumn{13}{|c|}{ Urban } \\
\hline Age (years) & & & & & & & & & & & & \\
\hline $15-19$ & 19.8 & 24.5 & 20.9 & 34.7 & 44.7 & 26.3 & 13.2 & 15.8 & 13.7 & 24.0 & 23.0 & 39.3 \\
\hline $20-24$ & 25.3 & 17.6 & 9.9 & 47.3 & 33.0 & 23.3 & 11.7 & 32.0 & 7.5 & 4.7 & 4.7 & 83.2 \\
\hline \multicolumn{13}{|l|}{ Religion } \\
\hline Hindu & 18.2 & 21.3 & 17.0 & 43.5 & 31.9 & 25.2 & 12.6 & 30.3 & 7.6 & 18.3 & 20.6 & 53.4 \\
\hline Muslim & 40.3 & 23.5 & 11.8 & 24.4 & 58.3 & 16.7 & 8.3 & 16.7 & 30.6 & 26.5 & 13.3 & 29.6 \\
\hline \multicolumn{13}{|l|}{ Caste } \\
\hline SC & 37.6 & 29.4 & 12.9 & 20.0 & 54.5 & 27.3 & 9.1 & 9.1 & 20.0 & 32.7 & 16.4 & 30.9 \\
\hline OBC & 22.9 & 23.9 & 17.2 & 36.0 & 34.4 & 25.8 & 11.8 & 28.0 & 13.2 & 22.6 & 21.3 & 42.9 \\
\hline General $^{2}$ & 12.3 & 11.7 & 15.4 & 60.5 & 23.1 & 15.4 & 15.4 & 46.2 & 7.4 & 9.4 & 15.4 & 67.8 \\
\hline \multicolumn{13}{|l|}{ Wealth quintile } \\
\hline First & 66.7 & 26.7 & 6.7 & 0.0 & $(100.0)$ & $(0.0)$ & $(0.0)$ & $(0.0)$ & * & * & * & * \\
\hline Second & 57.1 & 31.4 & 5.7 & 5.7 & 63.6 & 27.3 & 9.1 & 0.0 & 45.0 & 40.0 & 5.0 & 10.0 \\
\hline Third & 53.8 & 30.8 & 9.6 & 5.8 & 68.8 & 25.0 & 6.3 & 0.0 & 37.9 & 37.9 & 13.8 & 10.3 \\
\hline Fourth & 40.4 & 31.5 & 16.9 & 11.2 & 52.0 & 32.0 & 8.0 & 8.0 & 28.3 & 30.0 & 25.0 & 16.7 \\
\hline Fifth & 10.6 & 17.8 & 18.0 & 53.7 & 19.8 & 22.1 & 15.1 & 43.0 & 5.3 & 15.1 & 19.6 & 59.9 \\
\hline Total & 22.3 & 21.5 & 16.2 & 40.0 & 35.9 & 23.9 & 12.0 & 28.2 & 12.2 & 19.9 & 19.1 & 48.9 \\
\hline \multicolumn{13}{|c|}{ Rural } \\
\hline Age (years) & & & & & & & & & & & & \\
\hline $15-19$ & 51.5 & 28.6 & 12.5 & 7.4 & 70.6 & 19.9 & 5.3 & 4.2 & 32.5 & 37.2 & 19.7 & 10.6 \\
\hline $20-24$ & 59.5 & 20.4 & 7.0 & 13.0 & 62.1 & 20.6 & 7.0 & 10.3 & 9.6 & 17.5 & 7.8 & 65.1 \\
\hline \multicolumn{13}{|l|}{ Religion } \\
\hline Hindu & 54.6 & 24.3 & 10.8 & 10.3 & 65.7 & 19.6 & 6.6 & 8.0 & 28.4 & 35.3 & 20.6 & 15.7 \\
\hline Muslim & 55.6 & 31.5 & 7.4 & 5.5 & 67.4 & 25.6 & 2.9 & 4.1 & 42.0 & 38.6 & 12.5 & 6.8 \\
\hline \multicolumn{13}{|l|}{ Caste } \\
\hline SC & 71.0 & 20.3 & 4.4 & 4.2 & 76.7 & 16.1 & 3.2 & 3.9 & 50.4 & 35.5 & 8.9 & 5.3 \\
\hline OBC & 55.0 & 25.9 & 10.4 & 8.7 & 65.6 & 20.9 & 6.4 & 7.1 & 31.7 & 37.1 & 19.2 & 12.1 \\
\hline General $^{2}$ & 25.9 & 30.4 & 19.9 & 23.8 & 37.8 & 27.6 & 12.8 & 21.9 & 15.7 & 33.1 & 25.9 & 25.3 \\
\hline Wealth quintile & & & & & & & & & & & & \\
\hline First & 75.8 & 18.4 & 3.4 & 2.4 & 81.2 & 13.8 & 2.3 & 2.7 & 55.2 & 35.8 & 7.3 & 1.7 \\
\hline Second & 69.9 & 23.6 & 3.9 & 2.5 & 77.4 & 18.6 & 2.1 & 1.9 & 49.8 & 37.6 & 8.7 & 3.9 \\
\hline Third & 56.2 & 29.3 & 9.1 & 5.4 & 66.2 & 23.1 & 6.3 & 4.4 & 34.1 & 43.0 & 15.2 & 7.8 \\
\hline Fourth & 47.3 & 29.7 & 13.9 & 9.1 & 59.4 & 24.5 & 7.4 & 8.8 & 27.2 & 38.7 & 24.4 & 9.7 \\
\hline Fifth & 21.0 & 25.1 & 22.6 & 31.4 & 32.1 & 23.5 & 16.8 & 27.6 & 7.5 & 26.9 & 29.7 & 36.0 \\
\hline Total & 54.7 & 25.3 & 10.3 & 9.6 & 65.9 & 20.3 & 6.2 & 7.6 & 31.1 & 35.9 & 19.0 & 14.0 \\
\hline
\end{tabular}

Note: Row totals may not equal 100\% due to missing cases or "don't know" responses. ( ) Based on 25-49 unweighted cases. ${ }^{*}$ Percentage not shown, based on fewer than 25 unweighted cases. OBC: Other backward caste. SC: Scheduled caste. ST: Scheduled tribe. ${ }^{1}$ Includes non-literate and literate with no formal schooling. ${ }^{2}$ Includes all those not belonging to SC, ST or $O B C$. 


\subsection{School attendance}

Figure 3.1 presents schooling status at ages 12 and 15, representing, for many, periods before and after puberty was attained. Findings reconfirm the limited school attendance among young people in Bihar: $77 \%$ and $43 \%$ of young men and women, respectively, were in school at age 12 and far fewer-62\% and 31\%-at age 15. Married youth and rural youth were far less likely than their counterparts to be in school at ages 12 or 15 .

Figures 3.2a-c show graphically the cumulative percentages of youth (all youth who had completed at least one year of schooling) who had completed each year of education from Class 2 to Class 17, using life table techniques. Findings show substantial declines in school completion as early as Class 3; only $95 \%$ and $93 \%$ of young men and women respectively, who were ever enrolled in school had completed Class 3. Completion rates fell below $90 \%$ in Class 5 and Class 4 among young men and women, respectively. Both married and unmarried young women reported similar patterns of early discontinuation: completion rates for both fell below $90 \%$ by Class 4 . Marital status differences in school completion were evident among young men, among whom completion rates fell below $90 \%$ in Class 4 among the married and in Class 5 among the unmarried.

Declines in school attendance became progressively steeper, with differences between young men and women widening as the level of schooling increased. Among both young men and women, there were several notable declines in school completion levels. Among young women, declines of 5 points or more occurred between each class, with notable declines occurring between Classes 5 and $6(83 \%$ to $73 \%)$, Classes 6 and 7 (73\% to 67\%), Classes 7 and 8 (67\% to $59 \%)$, Classes 10 and 11 (46\% to $29 \%)$, Classes 12 and $13(28 \%$ to $19 \%)$ and finally, Classes 15 and 16 (19\% to $3 \%)$. Among young men, in contrast, notable declines occurred between Classes 5 and $6(88 \%$ to $82 \%)$, Classes 10 and 11 (60\% to $47 \%)$, Classes 12 and 13 (45\% to $30 \%)$, and Classes 15 and 16 (26\% to 5\%). These represent significant transitions: completion of a primary school, high school, a higher secondary school and a college degree, respectively. The additional decline between Classes 7 and 8 among young women may reflect withdrawal from school around the time of menarche.
Figure 3.1: Percentage of youth who were in school at ages 12 and 15 , according to residence, Bihar, 2007
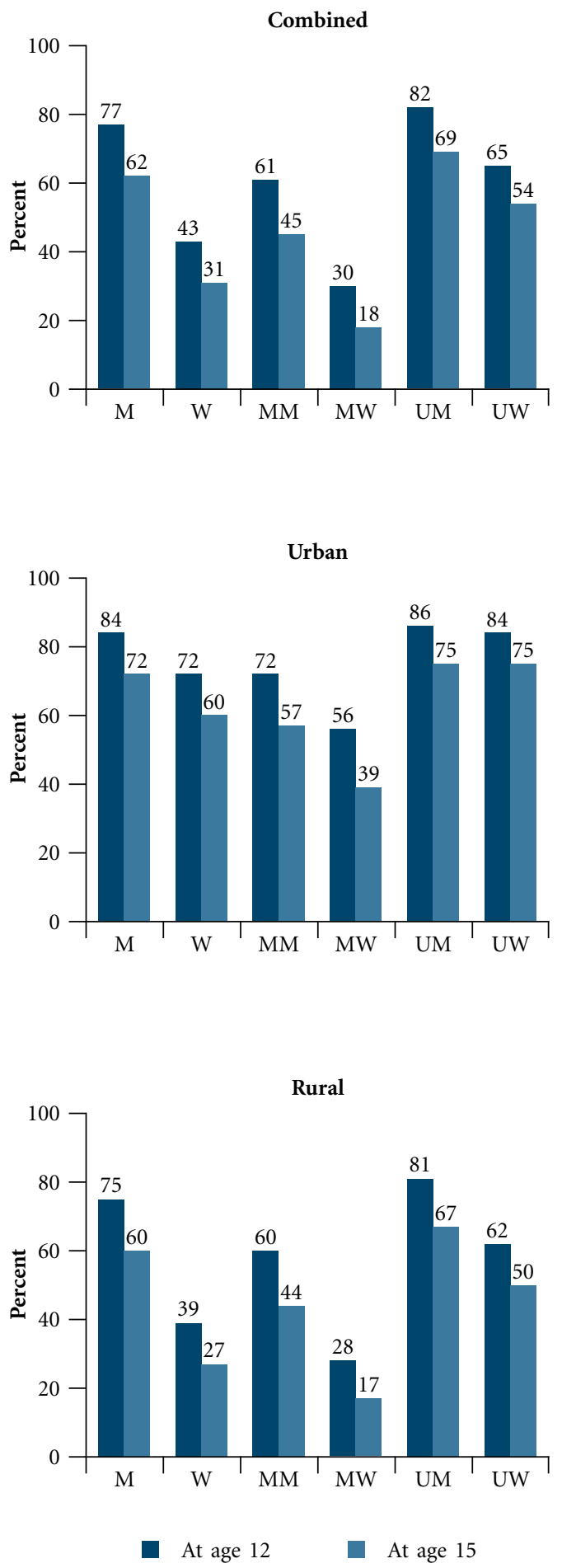
Findings also show significant differences between married and unmarried youth in school attendance levels. Among the married, declines of 5 points or more in completion rates occurred as early as between Classes 2 and 3 among both young men and women. School completion continued to decline steeply, by and large, thereafter, and gender differences were apparent. Among married young women, between Classes 4 and 10, annual declines of 7-17 points were observed. In contrast, among married young men, declines from one class to the next ranged from 4 to 14 points between Classes 4 and Class 10. Indeed, very few married youth continued their education after Class 11 (less than one-fifth of married young men and one in eight married young women). Among unmarried youth, declines were much more gradual. A decline of 5 points or more occurred between Classes 5 and 6 and a steep decline of 13-15 points occurred much later, that is, between Classes 10 and 11. Other notable declines occurred among unmarried youth between Classes 10 and 11 (13-15 points), Classes 12 and 13 (11-14 points) and Classes 15 and 16 (28-30 points).

Rural and urban patterns of school completion (Figures 3.2b and 3.2c) diverged considerably and findings suggest that rural youth, particularly rural young women, were markedly disadvantaged in terms of school retention even at the primary school level. For example, completion rates fell below $90 \%$ in Class 5 and 4 among young men and women, respectively, in rural areas. In contrast in urban areas, this occurred in Class 6 among both young men and women. Moreover, rural-urban differences became progressively wider from Class 6 onwards among young men (89\% of urban young men and $79 \%$ of rural young men had completed Class 6) and from Class 5 onwards among young women (91\% of urban young women and $81 \%$ of rural young women had completed Class 5). The corresponding percentages at Class 10 were $72 \%$ and $58 \%$, respectively, among young men, and $68 \%$ and $41 \%$, respectively, among young women.

Figure 3.2a: Cumulative percentage of youth who had completed each year of education (Classes 1 to 17 ), Bihar (combined), 2007

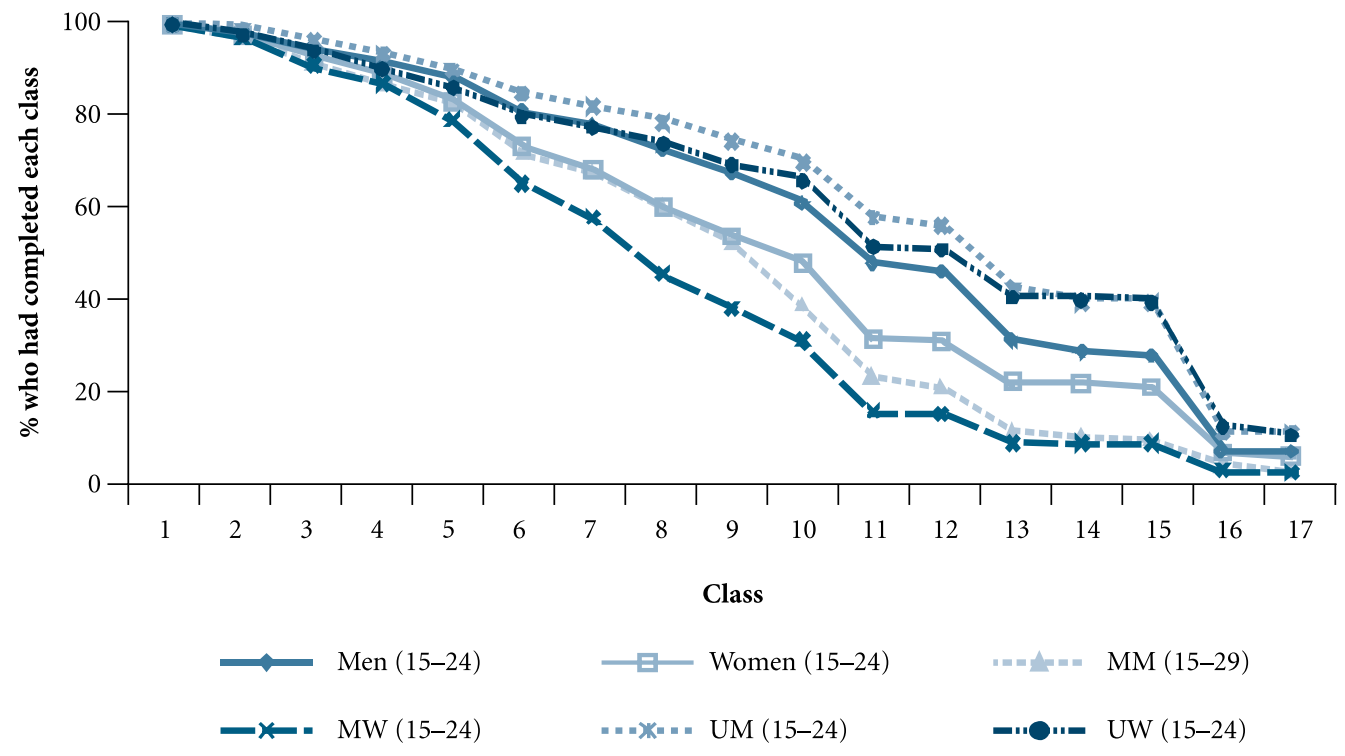


Figure 3.2b: Cumulative percentage of youth who had completed each year of education (Classes 1 to 17), Bihar (urban), 2007

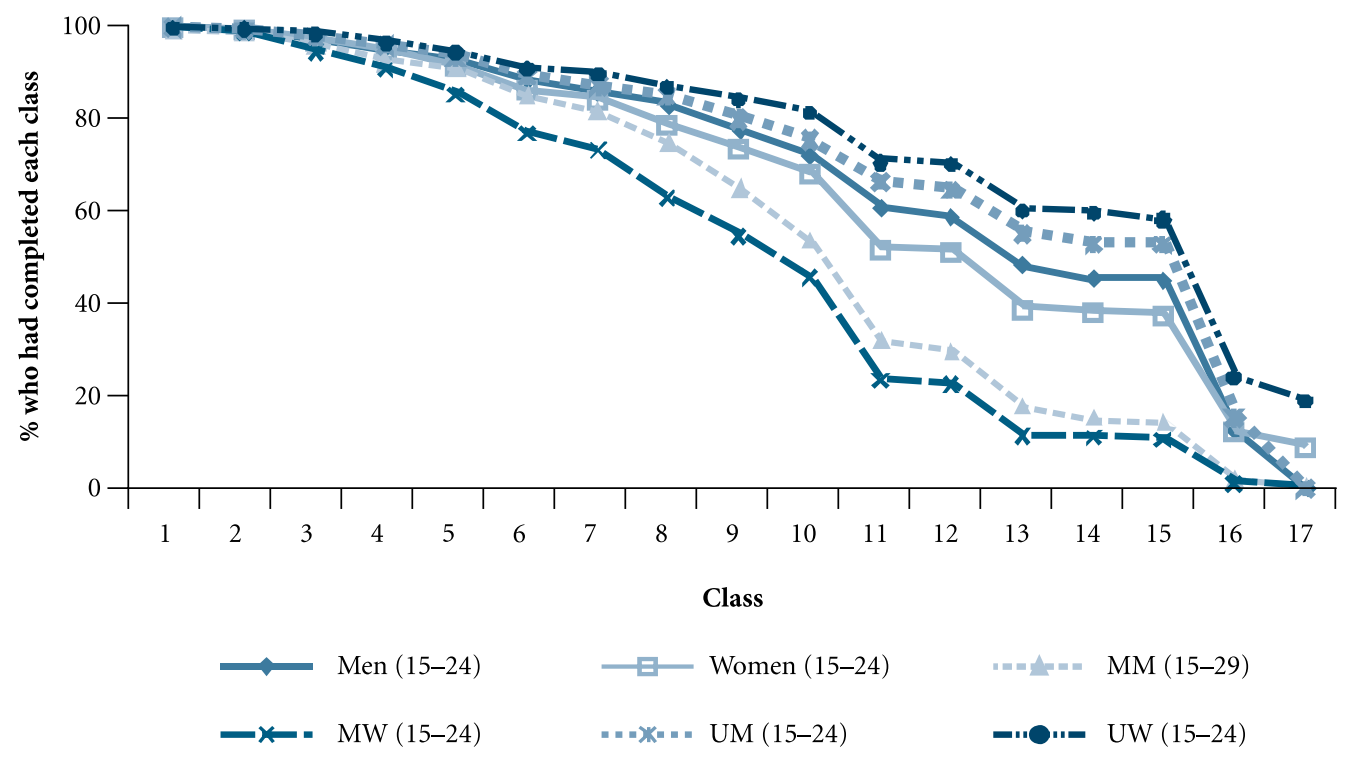

Figure 3.2c: Cumulative percentage of youth who had completed each year of education (Classes 1 to 17$)$, Bihar (rural), 2007

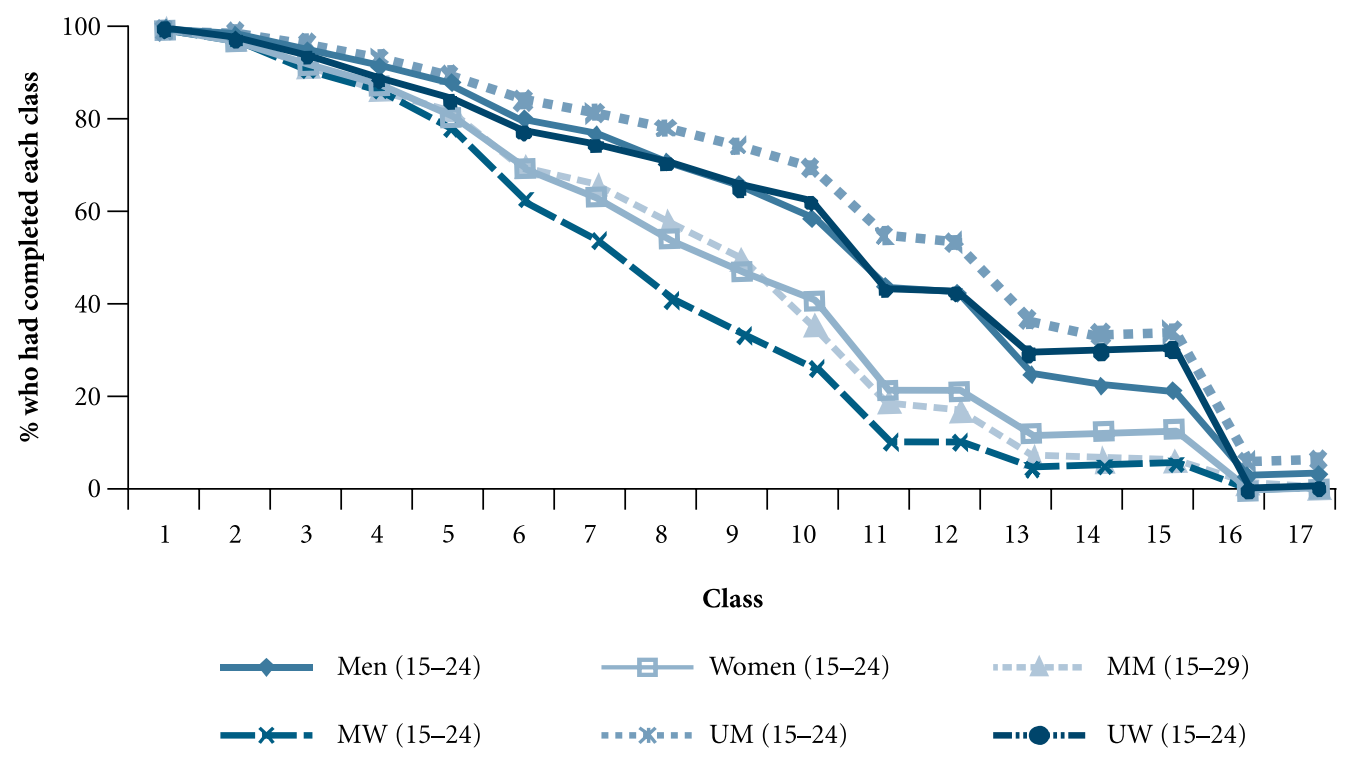




\subsection{Reasons for school non-attendance or discontinuation}

The Youth Study inquired about reasons for never going to school from all those who so reported, and reasons for discontinuing school from all those who had not completed Class 12. Responses are provided in Table 3.4a for those who had never gone to school and have been grouped into five categories: economic reasons (work on the family farm or business, wage earning work, family poverty, i.e., the family could not afford to keep the respondent in school); housework-related reasons (required for care of siblings or housework); attitude or perception-related reasons (unsafe to send children to school, education not considered necessary, respondent's lack of interest); school-related reasons (school located too far away, appropriate transport not available, poor school quality and infrastructure, poor quality of teaching); and health-related reasons (health problems of respondent, illness or death of a family member).

Findings suggest that key reasons for never going to school were economic, cited by $58 \%$ of young men and $65 \%$ of young women. Notably, about one-third of young men and half of young women reported that their families could not afford to send them to school. More young women than men had never been enrolled in school because they were required to work on the family farm or business and conversely more young men than women had never been enrolled in school because they were required to work for wages. Housework-related factors were reported by larger percentages of young women than men $(58 \%$ and $13 \%$, respectively). Attitude or perception-related reasons for never attending school were also reported by large percentages, particularly young men (39\% and $23 \%$ of young men and women, respectively). Of note is that lack of interest in studies was more likely to be reported by young men than women $(31 \%$ and $12 \%$, respectively). School-related reasons were cited by relatively few young men, and by a larger proportion of young women ( $7 \%$ of young men and 19\% of young women). Finally, $11-19 \%$ of youth cited health-related reasons (mostly the sickness or death of a family member) for never attending school.

Notable differences by marital status were evident among young men. Married young men were more likely than their unmarried counterparts to report economic reasons, particularly wage earning work (39\% versus $20 \%)$. While they were less likely to report attitude or perception-related reasons in general, they were more likely than their unmarried counterparts to indicate that they were never enrolled in school because education was not considered necessary (15\% versus $6 \%$ ), and less likely to report lack of interest (21\% versus $38 \%)$. Differences by marital status were narrow among young women. Rural-urban differences suggest that young men in urban settings were more likely than those in rural settings to report economic reasons $(64 \%$ and $57 \%$, respectively) and health-related reasons (25\% and $18 \%$, respectively). Rural-urban differences were relatively modest among young women, but suggest that more urban than rural young women cited healthrelated reasons (17\% versus $10 \%$ ) and more rural than urban young women mentioned work on the family farm or business as reasons for never having attended school (24\% versus $7 \%)$.

Table 3.4b reports findings for those who had discontinued their education before completing Class 12. In addition to the five sets of reasons included above, an additional category, early transition into adult roles, has been included, containing such reasons as marriage and employment. Reasons are presented separately for those who had dropped out of school before completing middle school (Class 7), high school (Class 10) and higher secondary education (Class 12), respectively. As evident also from Figures 3.3a and 3.3b, reasons varied considerably by sex and marital status of the respondent. 
Table 3.4a: Reasons for never attending school

Percentage of youth who never attended school by reasons for never attending school, according to residence, Bihar, 2007

\begin{tabular}{|c|c|c|c|c|c|c|}
\hline Reasons (\%) & $\begin{array}{c}\mathrm{M} \\
15-24\end{array}$ & $\begin{array}{c}\mathrm{W} \\
15-24\end{array}$ & $\begin{array}{c}\text { MM } \\
15-29\end{array}$ & $\begin{array}{c}\text { MW } \\
15-24\end{array}$ & $\begin{array}{c}\text { UM } \\
15-24\end{array}$ & $\begin{array}{c}\text { UW } \\
15-24\end{array}$ \\
\hline \multicolumn{7}{|c|}{ Combined } \\
\hline \multicolumn{7}{|l|}{ Economic reasons } \\
\hline Required for work on farm/family business & 13.7 & 22.8 & 12.9 & 23.3 & 18.5 & 21.5 \\
\hline Required for outside work for payment in cash/kind & 27.4 & 8.2 & 38.8 & 7.6 & 19.8 & 10.7 \\
\hline Family could not afford it (cost too much) & 31.3 & 50.3 & 33.6 & 49.5 & 30.4 & 53.3 \\
\hline At least one economic reason & 57.9 & 65.2 & 65.0 & 64.5 & 58.0 & 68.1 \\
\hline Housework-related reasons & 12.6 & 58.0 & 16.4 & 58.7 & 13.7 & 55.3 \\
\hline \multicolumn{7}{|l|}{ Parental or youth attitudes and perceptions } \\
\hline Not safe to send girls/boys to school & 0.0 & 4.5 & 0.0 & 4.2 & 0.0 & 6.1 \\
\hline Education not considered necessary & 9.8 & 7.7 & 14.7 & 7.6 & 5.6 & 8.1 \\
\hline Respondent not interested in studies & 30.9 & 12.2 & 21.3 & 12.6 & 37.7 & 10.1 \\
\hline At least one attitude/perception-related reason & 38.6 & 23.3 & 33.9 & 23.4 & 42.2 & 22.9 \\
\hline \multicolumn{7}{|l|}{ School-related reasons } \\
\hline School too far away/transport not available & 4.9 & 12.5 & 7.0 & 13.5 & 2.5 & 8.3 \\
\hline Poor quality of school facilities, teaching or education & 2.5 & 6.9 & 2.1 & 6.5 & 1.9 & 9.2 \\
\hline At least one school-related reason & 7.4 & 18.9 & 8.4 & 19.6 & 3.7 & 16.0 \\
\hline Health-related reasons & 19.0 & 10.6 & 22.0 & 10.8 & 19.3 & 9.3 \\
\hline Number who never attended school & 250 & 1,867 & 258 & 1,168 & 144 & 699 \\
\hline \multicolumn{7}{|c|}{ Urban } \\
\hline \multicolumn{7}{|l|}{ Economic reasons } \\
\hline Required for work on farm/family business & 14.3 & 6.6 & 16.7 & 6.1 & 10.0 & 5.1 \\
\hline Required for outside work for payment in cash/kind & 25.0 & 8.1 & 27.8 & 8.2 & 25.0 & 8.5 \\
\hline Family could not afford it (cost too much) & 37.0 & 56.9 & 33.3 & 53.1 & 38.1 & 66.1 \\
\hline At least one economic reason & 64.3 & 64.0 & 66.7 & 61.2 & 61.9 & 69.5 \\
\hline Housework-related reasons & 14.8 & 53.3 & 16.7 & 55.1 & 14.3 & 49.2 \\
\hline \multicolumn{7}{|l|}{ Parental or youth attitudes and perceptions } \\
\hline Not safe to send girls/boys to school & 0.0 & 5.1 & 0.0 & 6.0 & 0.0 & 5.0 \\
\hline Education not considered necessary & 10.7 & 8.0 & 16.7 & 8.2 & 10.0 & 8.5 \\
\hline Respondent not interested in studies & 32.1 & 15.3 & 27.8 & 16.3 & 33.3 & 11.9 \\
\hline $\begin{array}{l}\text { At least one attitude/perception-related reason } \\
\text { School-related reasons }\end{array}$ & 40.7 & 26.3 & 38.9 & 26.5 & 42.9 & 23.7 \\
\hline School too far away/transport not available & 0.0 & 8.0 & 0.0 & 10.2 & 0.0 & 3.4 \\
\hline Poor quality of school facilities, teaching or education & 3.6 & 5.8 & 0.0 & 6.0 & 0.0 & 6.7 \\
\hline At least one school-related reason & 3.6 & 13.1 & 0.0 & 14.3 & 0.0 & 10.2 \\
\hline Health-related reasons & 25.0 & 16.9 & 22.2 & 18.4 & 25.0 & 15.3 \\
\hline Number who never attended school & 116 & 570 & 109 & 396 & 72 & 174 \\
\hline
\end{tabular}




\begin{tabular}{|c|c|c|c|c|c|c|}
\hline Reasons (\%) & $\begin{array}{c}\mathrm{M} \\
15-24\end{array}$ & $\begin{array}{c}\text { W } \\
15-24\end{array}$ & $\begin{array}{c}\text { MM } \\
15-29\end{array}$ & $\begin{array}{c}\text { MW } \\
15-24\end{array}$ & $\begin{array}{c}\text { UM } \\
15-24\end{array}$ & $\begin{array}{c}\text { UW } \\
\text { 15-24 }\end{array}$ \\
\hline \multicolumn{7}{|c|}{ Rural } \\
\hline Economic reasons & & & & & & \\
\hline Required for work on farm/family business & 13.6 & 23.7 & 12.4 & 23.9 & 19.1 & 22.8 \\
\hline Required for outside work for payment in cash/kind & 27.2 & 8.2 & 39.6 & 7.6 & 19.1 & 10.9 \\
\hline Family could not afford it (cost too much) & 30.7 & 49.9 & 33.6 & 49.4 & 29.1 & 52.4 \\
\hline At least one economic reason & 57.2 & 65.3 & 64.9 & 64.7 & 57.4 & 68.0 \\
\hline Housework-related reasons & 12.1 & 58.3 & 16.4 & 58.8 & 14.2 & 55.8 \\
\hline \multicolumn{7}{|l|}{ Parental or youth attitudes and perceptions } \\
\hline Not safe to send girls/boys to school & 0.0 & 4.5 & 0.0 & 4.1 & 0.0 & 6.3 \\
\hline Education not considered necessary & 9.7 & 7.7 & 14.9 & 7.6 & 5.0 & 8.1 \\
\hline Respondent not interested in studies & 30.7 & 12.0 & 20.9 & 12.5 & 38.3 & 10.0 \\
\hline At least one attitude/perception-related reason & 38.5 & 23.2 & 33.6 & 23.3 & 42.6 & 22.9 \\
\hline \multicolumn{7}{|l|}{ School-related reasons } \\
\hline School too far away/transport not available & 5.4 & 12.8 & 7.5 & 13.7 & 2.8 & 8.6 \\
\hline Poor quality of school facilities, teaching or education & 2.3 & 7.0 & 1.9 & 6.5 & 1.4 & 9.4 \\
\hline At least one school-related reason & 7.8 & 19.2 & 9.0 & 19.8 & 4.3 & 16.5 \\
\hline Health-related reasons & 18.3 & 10.3 & 22.0 & 10.6 & 18.4 & 8.9 \\
\hline Number who never attended school & 134 & 1,297 & 149 & 772 & 72 & 525 \\
\hline
\end{tabular}

Note: All Ns are unweighted. Column totals may exceed 100\% due to multiple responses.

Among those who had completed just 1-6 years of schooling, economic considerations dominated reasons for school discontinuation for young men, irrespective of marital status or rural-urban residence. Two-thirds of all young men so reported. Fewer young men cited attitude or perception-related reasons (34\%) and housework-related reasons (20\%). Among young women, four key reasons were cited: housework-related (40\%), economic (37\%), attitude or perception-related (27\%) and school-related (26\%). Reasons reported by married and unmarried youth, and by rural and urban youth were roughly similar. However, some notable differences were evident. Among young men, for example, the married were more likely than the unmarried to cite at least one economic reason ( $78 \%$ versus $66 \%$ ), but less likely to cite school-related reasons ( $6 \%$ versus $14 \%)$. Among young women, in contrast, it was the unmarried who were more likely than the married to cite economic reasons (53\% versus $28 \%$ ); they were also more likely to cite housework-related reasons ( $45 \%$ versus $38 \%$ ). Notably, marriage was reported as the reason for school discontinuation by $23 \%$ of married young women. Rural-urban differences indicate that urban young men were less likely than their rural counterparts to report economic reasons (59\% versus $69 \%)$, housework-related reasons (12\% versus $21 \%)$ and school-related reasons (3\% versus 13\%). Conversely, they were more likely to report attitude or perception-related reasons (50\% versus $32 \%$ ) and health-related reasons (24\% versus $14 \%$ ). Among young women, in contrast, those from urban settings were more likely than their rural counterparts to report economic reasons ( $45 \%$ versus $36 \%$ ); they were also more likely to report health-related reasons ( $21 \%$ versus $11 \%)$, but less likely than young women from rural areas to attribute school discontinuation to school-related reasons $(15 \%$ versus $27 \%)$. 


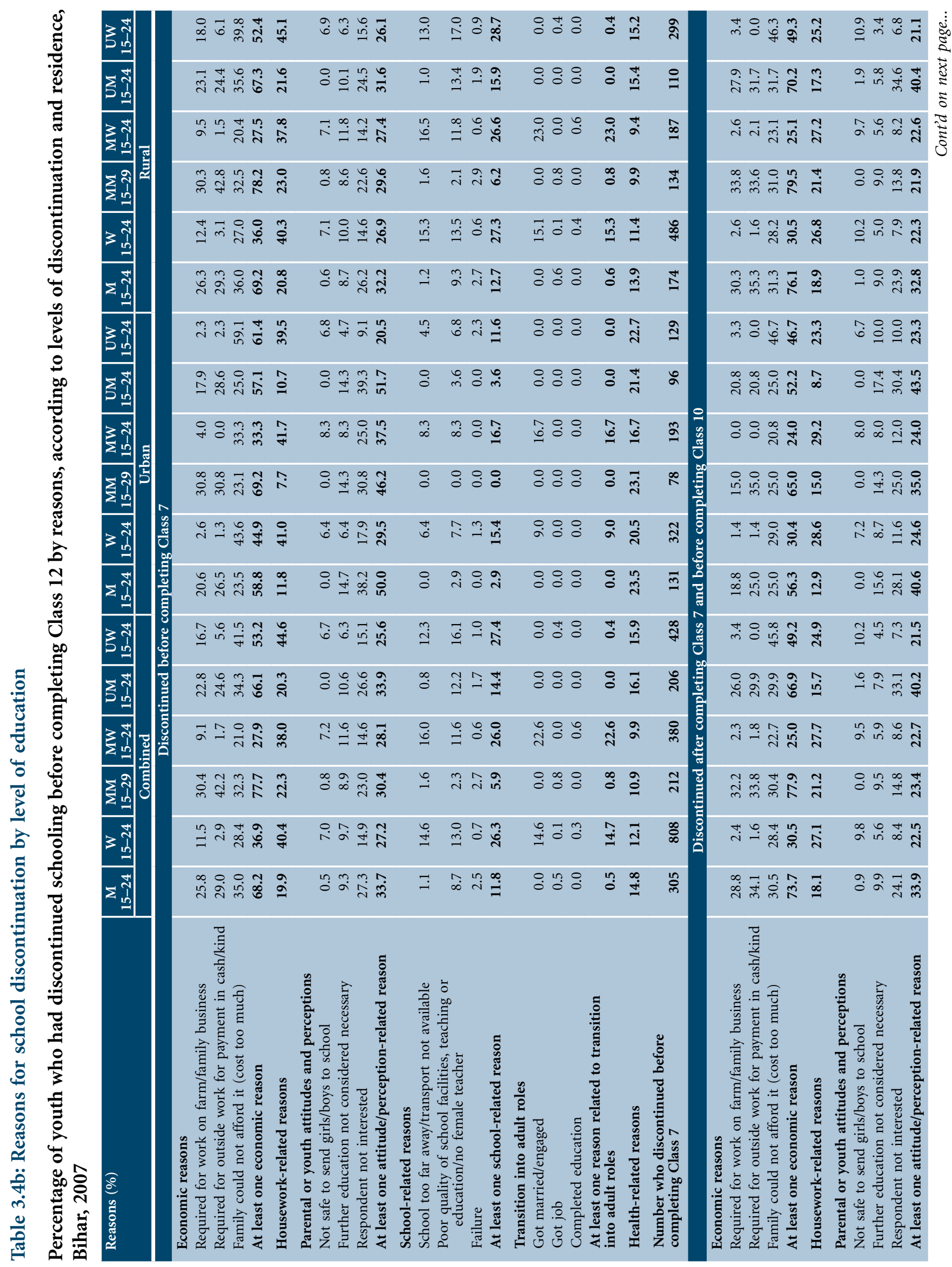




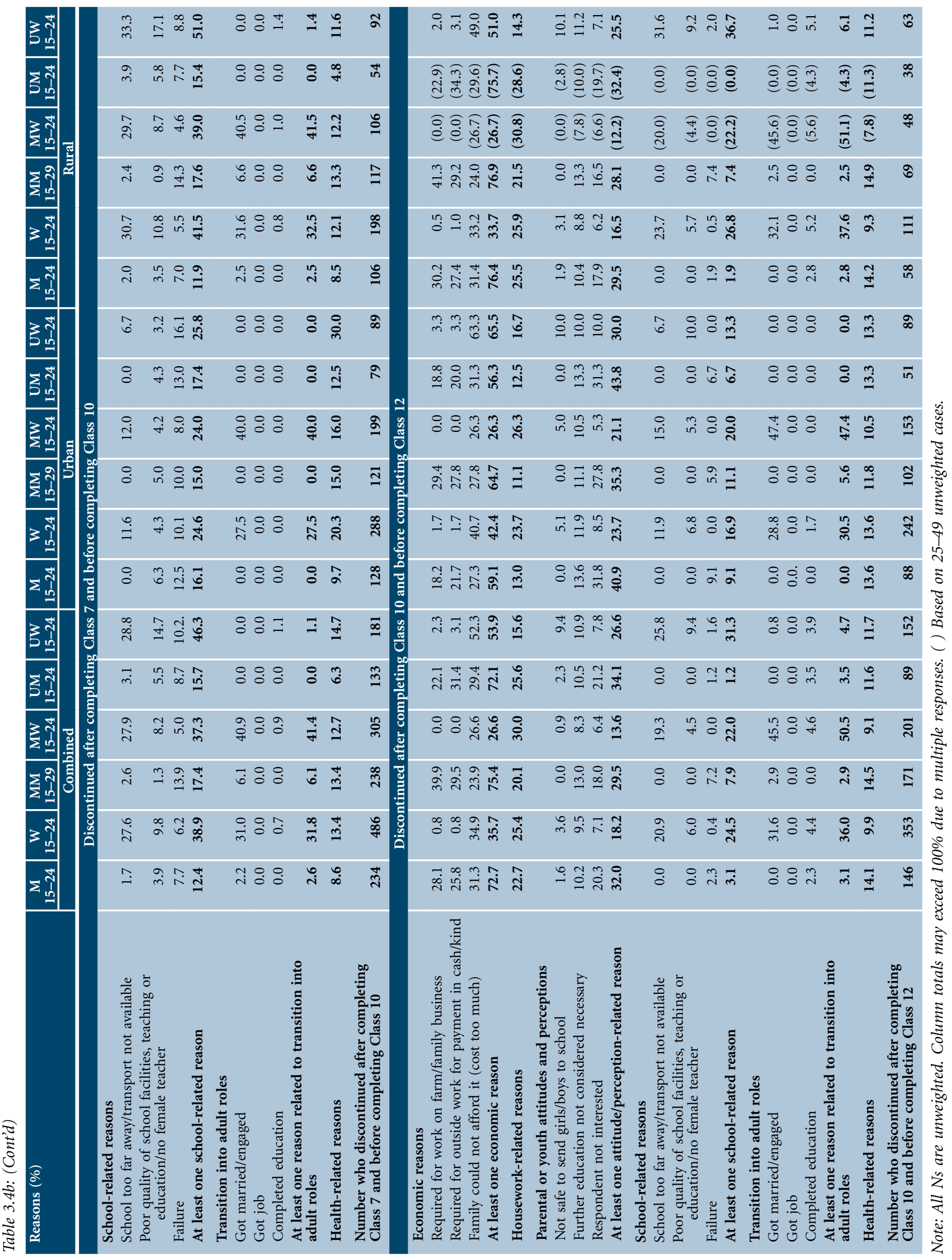


Figure 3.3a: Percentage of married youth who had discontinued schooling by class when discontinued and reasons for discontinuation, Bihar 2007

Married men

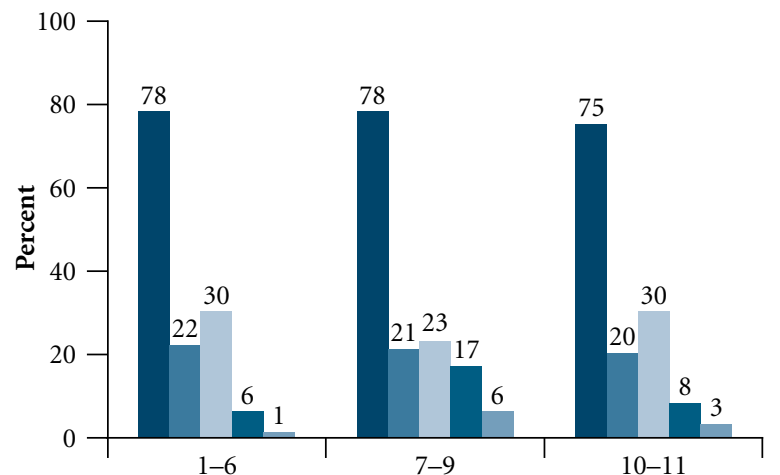

At least one economic reason

- At least one attitude/perception-related reason

At least one reason related to transition into adult roles
Married women

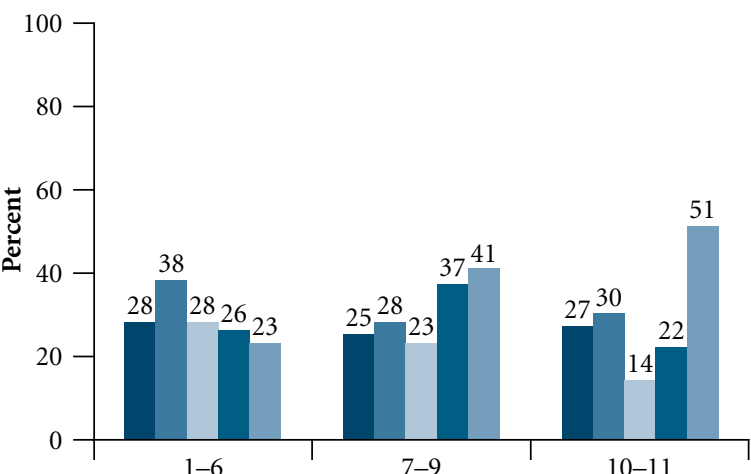

At least one housework-related reason

At least one school-related reason

Figure 3.3b: Percentage of unmarried youth who had discontinued schooling by class when discontinued and reasons for discontinuation, Bihar, 2007

Unmarried men

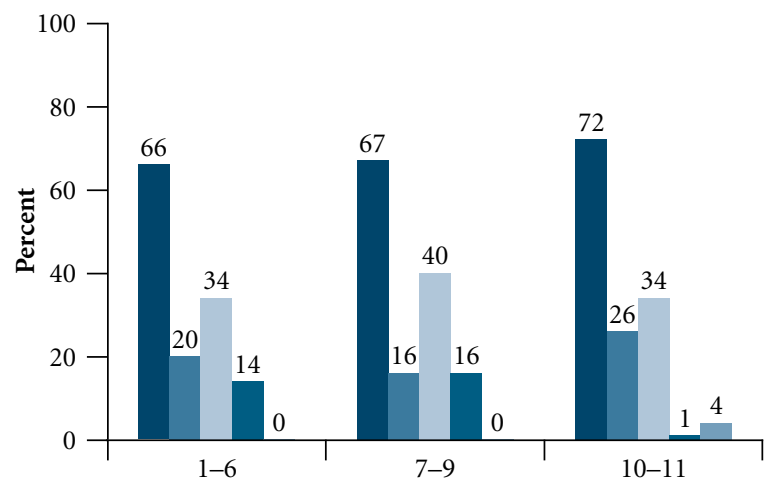

- At least one economic reason

- At least one attitude/perception-related reason

- At least one reason related to transition into adult roles
Unmarried women

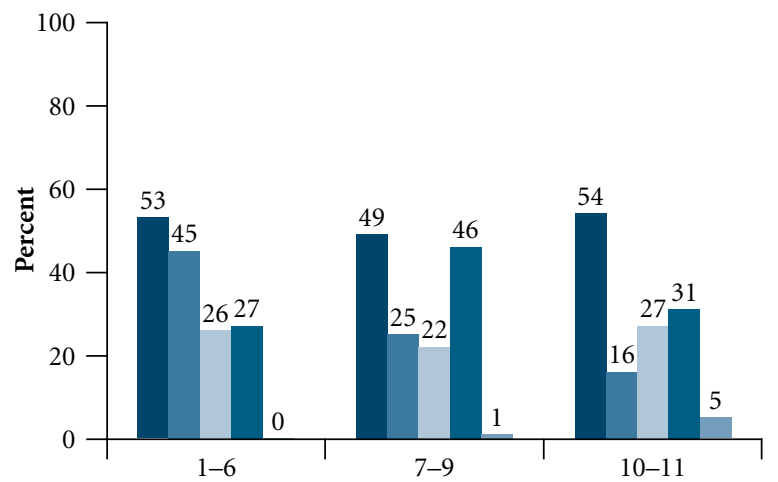

At least one housework-related reason

At least one school-related reason

Gender differences in reasons for school discontinuation were more pronounced among those who had completed Classes 7-9. The leading reason for young men continued to be economic, reported by threequarters. Attitude or perception-related reasons were cited by one-third and housework-related reasons by about one-fifth. Differences by marital status indicate that the married were more likely to report economic reasons and health-related reasons and less likely to report attitude or perception-related reasons compared to the unmarried. Rural-urban differences show that more urban than rural young men reported attitude or perception-related reasons, and more rural than urban young men reported economic reasons. Among young 
women in contrast, school-related factors (39\%), transition into adult roles (32\%) and economic reasons (31\%) were most often reported as reasons for discontinuation; housework-related and attitude or perceptionrelated factors were reported by $27 \%$ and $23 \%$, respectively. Differences by marital status indicate that more unmarried than married women reported economic and school-related reasons. However, transitions into adult roles were far more likely to be reported as a reason for school discontinuation by married women: $41 \%$ of married young women cited marriage or engagement as a reason for school discontinuation. Additionally in urban areas, more unmarried than married young women reported health-related reasons. Rural-urban differences were largely muted among young women; however, more rural than urban young women reported school-related reasons and more urban than rural young women reported health-related reasons.

Among those who had discontinued their education after completing Classes 10 or 11, gender differences continued to be wide. Among young men, leading reasons again were economic (73\%), attitude or perceptionrelated $(32 \%)$ and housework related reasons (23\%) Differences by marital status were negligible, but urban young men were more likely than rural young men to report attitude or perception-related and school-related reasons and less likely to cite economic and housework-related reasons. The leading reasons for school discontinuation among young women, in contrast, were economic and transition to adult roles-reasons reported by $36 \%$ each. Indeed, $32 \%$ of all young women and $46 \%$ of married young women reported discontinuing their education in order to marry. One-quarter each cited housework-related and school-related reasons. About one-fifth reported attitude or perception-related reasons. Differences by marital status suggest that the unmarried were more likely than the married to report economic, attitude or perception-related and school-related reasons for discontinuation. Conversely, they were less likely to report housework-related reasons and transition to adult roles. Rural-urban differences were evident. While urban young women were more likely than rural young women to cite economic and attitude or perception-related factors, rural young women were more likely to cite school-related reasons and transition to adult roles as reasons for school discontinuation.

\subsection{School/college type, quality and experiences}

All respondents were asked about the kind of school or college they had last attended or were attending at the time of interview and the facilities available in that school or college. They were also asked about their experiences: whether they attended classes regularly, their attitudes towards education and their performance in that school or college. Tables 3.5 and 3.6 present findings on type and quality of educational institutions most recently attended, and schooling experiences, respectively. Findings are presented separately for those who were still in school or college at the time of interview and for those who had discontinued their education before completing Class 12, in order to explore the extent to which school/college quality and experiences differed between these two groups. As school quality and experiences are unlikely to be different for the married and unmarried, Tables 3.5 and 3.6 present information by sex and rural-urban residence of respondents only. In addition, because experiences may vary according to level of education attained, findings are presented separately for primary or middle school, high school or higher secondary or college.

\subsubsection{School/college type and quality}

Table 3.5 shows a significant gender divide in terms of type of educational facility that youth attended, irrespective of rural-urban residence or current schooling status. The majority of young men reported attending co-educational facilities at all levels of education; $85-97 \%$ of young men who were currently attending a school or college reported as such. In contrast, young women were less likely to attend a co-educational facility at higher levels of schooling; for example among those who were currently attending a school or a college, the percentage attending a co-educational facility dropped from $90 \%$ at primary or middle school level to $78 \%$ at high school level to $63 \%$ at higher secondary and higher levels. 


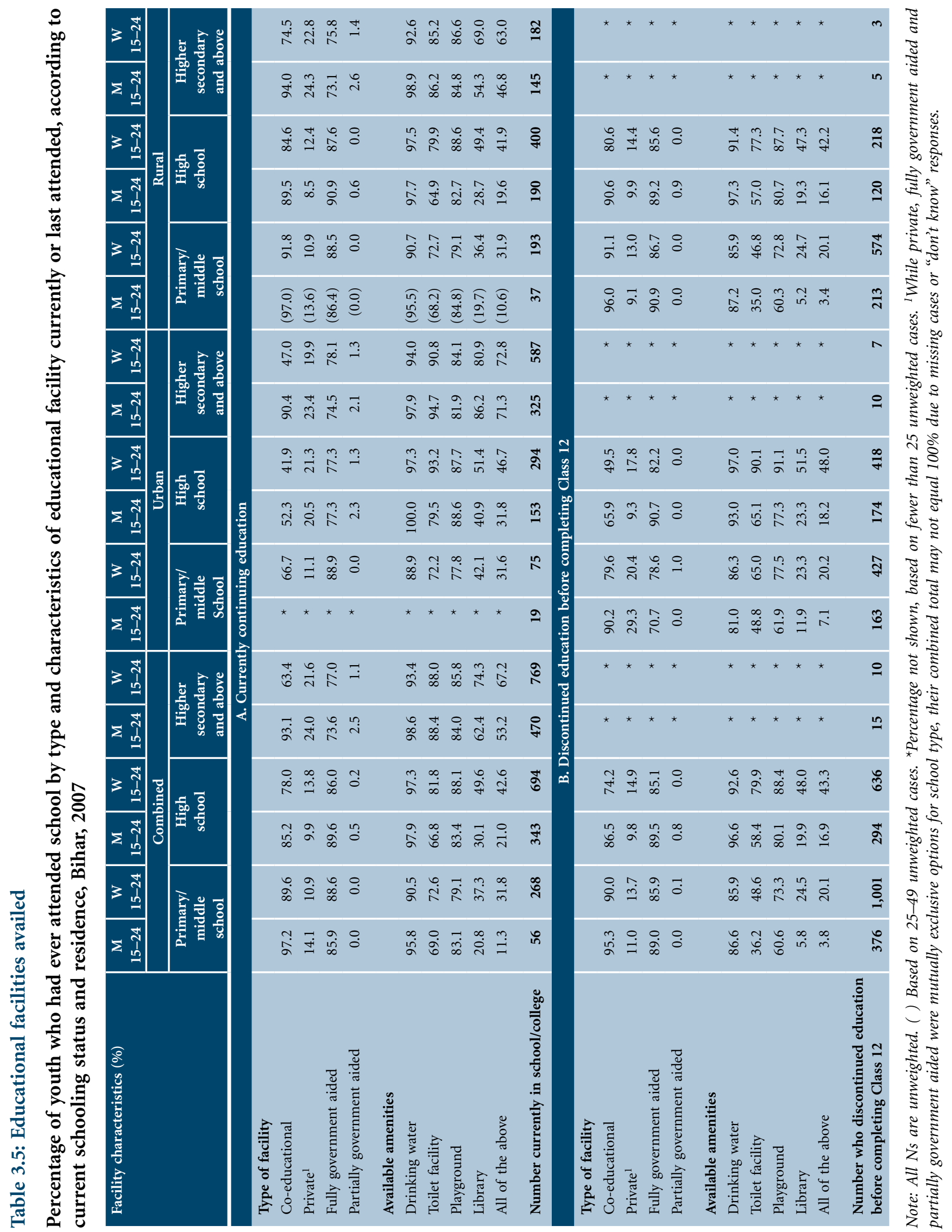


Rural-urban differences suggest that irrespective of sex and current schooling status of the respondent, rural youth were more likely than urban youth to attend a co-educational facility at all levels of education.

The majority of youth at all levels of education attended government schools or colleges. Even so, some patterns are notable. Youth were more likely to attend private educational facilities at higher secondary level than at any other levels; $24 \%$ of young men and $22 \%$ of young women pursuing their education at the time of interview attended a private school at higher secondary level compared to $11-14 \%$ of youth at primary or middle level and $10-14 \%$ at high school level. Second, while urban youth were more likely than rural youth to attend a private educational facility at high school levels, differences were negligible at higher secondary level.

School quality was assessed by questions on the availability of drinking water, toilets, playgrounds and library facilities. Findings from Table 3.5 show differences between those pursuing their education at the time of interview and those who had discontinued their education; but these differences varied by level of education attained and by type of amenity considered.

Among those pursuing their education at the time of interview, the overwhelming majority of youth (89-100\%), irrespective of sex, rural-urban residence or level of education attained, had access to drinking water. Playgrounds were available to somewhat fewer (78-89\%). Toilet and library facilities were available to fewer and differences by sex, rural-urban residence and level of education attained were evident. Toilet facilities were available to $69-73 \%$ of youth attending primary or middle school, $67-82 \%$ of those attending high school and $88 \%$ of those attending higher secondary or higher levels of education. Library facilities were even less likely to be reported, except among those who had reached higher secondary levels or more: for example, even among those at high school level, library facilities were available to just $30 \%$ of young men and half of young women. Rural-urban differences were also evident, urban youth were, for the most part, more likely than rural youth to report the availability of these two facilities.

Reported access to amenities among youth who had discontinued their education varied by the level at which schooling was discontinued. For example, those who had discontinued their education at primary or middle level were far less likely than those pursuing their education to report the availability of drinking water and playgrounds (86-87\% reported that drinking water was available and $61-73 \%$ reported the availability of playgrounds); these differences were less evident among, those who had discontinued their education at high school level (93-97\% and 80-88\%, respectively). Toilets and library facilities were far less likely to be reported by those who had discontinued their education than those who were pursuing their education at the time of interview, irrespective of the level of education. Only $36 \%$ of young men and $49 \%$ of young women who discontinued their education at primary or middle level reported that toilet facilities were available; $58 \%$ and $80 \%$, respectively who discontinued at high school level reported the availability of toilets. A similar situation was apparent with regard to the availability of library facilities, with $6 \%$ and $20 \%$, respectively, among young men who discontinued their education at primary or middle and high school levels and $25 \%$ and $48 \%$ among young women, respectively, reporting so. Rural-urban differences were largely negligible, except that urban youth were far more likely than rural youth to report having a toilet facility at their school.

Availability of all four amenities-drinking water, playgrounds, toilets and libraries-increased systematically with level of schooling attained for all youth, irrespective of whether or not they had discontinued their education. Among those still in school, all four amenities were available to $11-32 \%$ of youth at primary or middle school level, $21-43 \%$ of those at high school level and $53-67 \%$ of those at higher secondary level. Among those who had discontinued their education, availability of all four amenities increased from 4-20\% among those who discontinued at primary or middle school level to $17-43 \%$ of those who discontinued at 


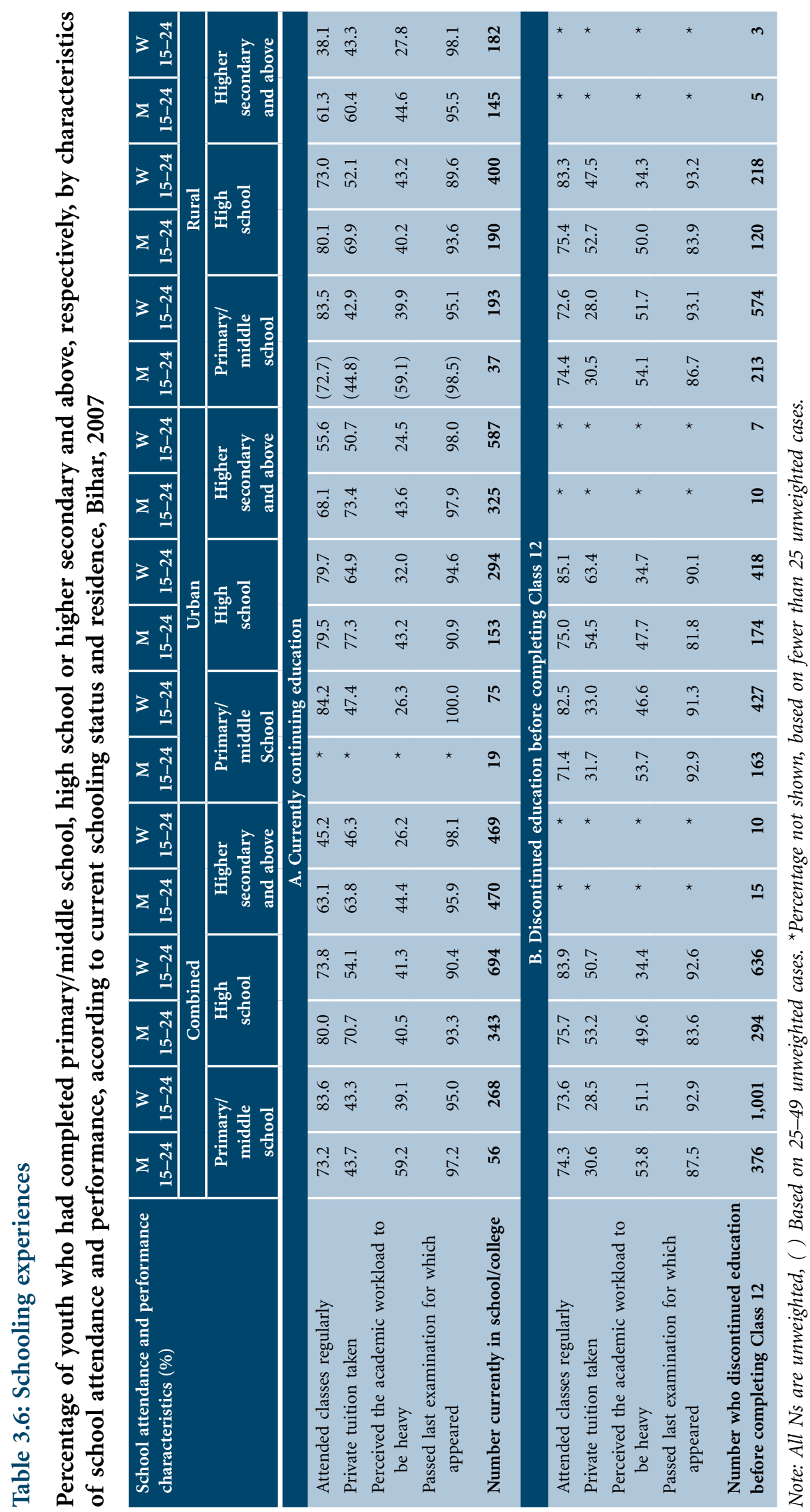


high school level. With the exception of young women at high school level, among whom no differences were observed, availability of all four amenities was typically more likely to be reported by those who were studying at the time of interview than those who had discontinued their education, raising the possibility that the availability of amenities may have played a role in school continuation. Gender differences were apparent, with larger percentages of young women than men reporting the availability of all four amenities, most likely because an adequate physical infrastructure, as reflected by the presence of these amenities, was considered a prerequisite for girls to be enrolled in school. Finally, urban youth who were still attending school at high school or higher secondary levels were considerably more likely to report the availability of all four amenities than their rural counterparts. Rural-urban differences among those who had discontinued schooling were negligible.

\subsubsection{School/college experiences}

Table 3.6 presents young people's schooling experiences, namely, whether or not they attended class regularly, took private tuition, considered the academic workload to be heavy and had passed the last examination for which they had appeared. Among youth who were still attending school, findings show a general decline in regular attendance with the level of schooling attended. Among young men, regular attendance was reported by $73-80 \%$ of those at the primary or middle levels and high school level and $63 \%$ at higher secondary level; among young women, regular attendance fell from $84 \%$ among those in primary or middle school to $74 \%$ and $45 \%$, respectively, among those in high school or pursuing a higher education. Rural-urban differences were evident only among young women at the high school level and among both young men and women at the higher secondary level, with those in urban areas more likely than their rural counterparts to report regular attendance.

Percentages of youth who had taken private tuition varied by the level of school attended; youth were more likely to have taken private coaching at high school level ( $71 \%$ and $54 \%$ of young men and women, respectively) than any other levels (43-44\% among those at primary or middle levels and $64 \%$ of young men and $46 \%$ of young women, at higher secondary or higher levels). As evident above, except at the primary or middle levels, young men were more likely than young women to have taken private coaching. Rural-urban differences were evident; larger proportions of urban than rural youth reported that they had attended coaching classes.

While approximately equal percentages of young men and women at high school level reported feeling that the academic workload was heavy, gender differences were wide among youth at lower and higher levels. At these levels, more young men than women reported feeling that the academic workload was heavy; for example, $59 \%$ of young men and 39\% of young women who were in primary or middle schools at the time of the survey reported as such, as did $44 \%$ and $26 \%$ of those at higher secondary levels. Rural-urban differences were negligible among young men; however, more urban than rural young women currently attending primary or middle schools and high schools perceived that academic workload was heavy.

Most youth over 90\% reported that they had passed the last school or college examinations for which they had appeared.

Among youth who had discontinued their education, young women who had done so at primary or middle level were less likely than those who discontinued later to attend school regularly. Corresponding differences were muted among young men. Percentages reporting private tuition increased with level of education at which schooling was discontinued $(29-31 \%$ and $51-53 \%$, respectively, among those who discontinued at primary or middle, or high school). Half of young men and between one-third and half of young women perceived the academic workload to be heavy. Finally, $84-93 \%$ of young men and women reported that they 
passed the last examination for which they had appeared, irrespective of the level at which schooling was discontinued. Differences by sex or rural-urban residence were modest.

Schooling experiences differed somewhat among those who had discontinued schooling and those who were studying at the time of interview. Differences in regular attendance did not differ much among young men, young women who were studying at primary or middle levels were somewhat more likely to report regular attendance than those who had discontinued their education at this stage. In contrast, those at high school level were less likely than those who had discontinued at this stage to report regular attendance. At the same time, youth who were continuing their education were considerably more likely to report private tuition (54-71\% compared to $51-53 \%$ of those who discontinued their education at high school level, for example). No clear pattern could be discerned in the case of perceptions about the academic workload, however, those pursuing their education were, for the most part, more likely to have passed the last examination for which they had appeared.

\subsection{Summary}

While youth in Bihar were better educated than the population at large in the state, schooling was far from universal among young people. As many as one in six young men and half of young women had never attended school. Findings show, moreover, that young women in rural areas and married young women in general were particularly disadvantaged; $55 \%$ of rural young women and $64 \%$ of married young women had never been to school.

Not only was school enrolment limited, but school completion rates were also low among young people. Findings show that of those who had completed Class 1, declines in class completion took place as early as Class 3; only $95 \%$ and $93 \%$ of young men and women, respectively, who had completed one year of schooling had gone on to complete Class 3. Declines in class completion became progressively steeper as the level of schooling increased, with differences between young men and women widening as the level of schooling increased. For example, notable declines occurred between Classes 5 and 6, suggesting that many youth discontinued their education even without completing elementary education. Indeed, just $30 \%$ of young men and $13 \%$ of young women had completed high school in the state.

What is notable is that, at the time of interview, over half of unmarried young men and fewer than half of unmarried young women (and very few married) were still in school or college.

Leading reasons for never attending school among young men and women were economic (child required for work on the family farm/business or for outside wage earning work, or the family could not afford schoolrelated expenses) and attitudes and perceptions (such as, for example, that education was unnecessary or that children were not interested). Housework-related reasons (required for care of siblings or housework) were additionally an important reason for young women never going to school.

Among those who had ever been to school, leading reasons for discontinuation among young women and men, irrespective of the level at which schooling was discontinued were economic issues and attitudes and perceptions. However, among young women, other factors were also important: school-related factors (academic failure, distance to school, poor school quality and infrastructure) and housework responsibilities continued to be significant motivating factors behind discontinuation at all levels. Of note particularly is that one in seven and two in five young women who had discontinued schooling at primary and high school levels, respectively, reported having done so in order to marry. 
Findings also suggest a gender divide in the type of educational facility that youth attended. While young men by and large attended co-educational facilities at all levels of education, young women were less likely to attend a co-educational facility at higher levels of schooling. At the same time, no apparent gender differences were observed in terms of the type of school—government or private-in which youth were enrolled.

Differences were observed in the educational facilities attended by youth who were still in school and those who had discontinued their education at various levels. For example, youth still studying were more likely for the most part to report the availability of water, toilets, playgrounds and libraries than were those who had discontinued. Schooling experiences also differed somewhat among those who had discontinued schooling and those who were studying at the time of interview. For example, young women pursuing their education were more likely than those who had discontinued their education at this level to report regular attendance. Youth who were continuing their education were considerably more likely to report private tuition, and more likely to have passed the last examination for which they had appeared. 


\section{Economic and non-economic} activity

The period between the ages of 15 and 29 marks, for many young people, entry into the labour market and economic independence, acquisition of professional and technical skills and new living arrangements. Economic uncertainty, however, dominates the lives of many youth. According to International Labour Organisation (ILO) estimates, although youth (aged 15-24) comprise around $25 \%$ of the world's working-age population, they constitute around $44 \%$ of the unemployed (ILO, 2006). The unemployment rate among youth has also been identified as one of the key indicators for monitoring the progress towards achieving the UN Millennium Development Goals (UNDP, 2000). For many young people, this period also marks the discontinuation of education and increasing acceptance of domestic responsibilities. This chapter explores the economic activity of young people, their work-related mobility, their participation in non-economic activities (domestic work) and their vocational skill-building experiences and preferences.

\subsection{Economic activity}

During the survey, a number of questions were asked to assess the economic activity and occupational status of youth. Youth were asked whether they had ever worked, either for or without remuneration. They were also asked whether they had worked in the 12 months preceding the interview, whether they were seeking employment, the type of work in which they engaged, and the number of months during which they had worked or sought work in the year preceding the interview.

Work profiles varied widely, as shown in Table 4.1. In total, three-quarters of young men and about one-half of young women reported that they had been engaged in paid or unpaid work at some point in their lives. Young men and women, particularly young men, were less likely to have engaged in unpaid than in paid work; while $63 \%$ of young men had engaged in paid work, only $38 \%$ had engaged in unpaid work. The corresponding percentages among young women were $33 \%$ and $25 \%$, respectively. Marital status differences indicate that the married were more likely than the unmarried to have engaged in paid or unpaid work. Differences were much wider among young men than young women: almost all married young men (98\%) compared to two-thirds of unmarried young men reported having ever worked. Among young women, $53 \%$ of the married compared to $38 \%$ of the unmarried had ever worked. Rural-urban differences suggest, moreover, that more rural than urban youth had ever worked- $77 \%$ versus $62 \%$ of young men and $50 \%$ versus $22 \%$ of young women.

For many, work was initiated in childhood, that is, by age 15. Over one-third of young men and women reported that they had initiated either paid or unpaid work by the time they were aged 15. More married than unmarried and more rural than urban youth had initiated economic activity from an early age, irrespective of sex. 
Table 4.1: Economic activity

Percentage of youth who ever worked and who worked in last 12 months, and percent distribution of youth by duration of work and main occupation in the last 12 months, according to residence, Bihar, 2007

\begin{tabular}{|c|c|c|c|c|c|c|}
\hline Economic activity (\%) & $\begin{array}{c}\mathrm{M} \\
15-24\end{array}$ & $\begin{array}{c}\text { W } \\
15-24\end{array}$ & $\begin{array}{c}\text { MM } \\
15-29\end{array}$ & $\begin{array}{c}\text { MW } \\
15-24\end{array}$ & $\begin{array}{c}\text { UM } \\
15-24\end{array}$ & $\begin{array}{c}\text { UW } \\
15-24\end{array}$ \\
\hline \multicolumn{7}{|c|}{ Combined } \\
\hline \multicolumn{7}{|l|}{ Ever worked } \\
\hline Paid work & 63.0 & 33.3 & 93.9 & 38.7 & 52.3 & 24.3 \\
\hline Unpaid work & 37.6 & 24.7 & 48.2 & 26.9 & 34.4 & 21.6 \\
\hline Either paid or unpaid work & 74.9 & 47.2 & 97.8 & 52.9 & 67.1 & 38.0 \\
\hline Started working before age 15 & 36.9 & 35.3 & 45.7 & 39.8 & 33.9 & 28.5 \\
\hline \multicolumn{7}{|l|}{ Ever worked in last 12 months } \\
\hline Paid work & 58.9 & 27.1 & 89.7 & 29.8 & 48.0 & 23.0 \\
\hline Unpaid work & 23.8 & 15.8 & 19.5 & 14.4 & 25.3 & 19.2 \\
\hline Either paid or unpaid work & 72.7 & 36.8 & 96.8 & 38.0 & 64.3 & 36.0 \\
\hline Number of respondents & 1,942 & 5,529 & 1,115 & 2,341 & 1,492 & 3,188 \\
\hline \multicolumn{7}{|l|}{ Duration of paid work in last 12 months } \\
\hline Most of the year (6 months or more) & 64.1 & 44.3 & 81.8 & 47.0 & 56.3 & 37.4 \\
\hline Part of the year ( $3-5$ months) & 25.3 & 33.4 & 15.0 & 34.2 & 30.2 & 31.9 \\
\hline Rarely (less than 3 months) & 9.9 & 20.5 & 2.7 & 16.9 & 13.3 & 29.1 \\
\hline \multicolumn{7}{|l|}{ Main occupation (paid work) } \\
\hline Cultivator & 10.1 & 0.9 & 13.0 & 0.9 & 10.3 & 1.4 \\
\hline Agricultural labourer & 17.6 & 83.0 & 17.9 & 84.8 & 15.8 & 80.3 \\
\hline Administrative/executive/managerial/clerical & 7.4 & 2.3 & 4.8 & 1.3 & 9.4 & 4.4 \\
\hline Business & 6.3 & 0.3 & 5.9 & 0.1 & 6.7 & 0.7 \\
\hline Skilled manual/machinery & 24.4 & 8.8 & 24.1 & 8.4 & 23.5 & 9.0 \\
\hline Unskilled non-agricultural labourer & 31.7 & 4.2 & 32.2 & 4.1 & 31.8 & 3.7 \\
\hline Other & 2.3 & 0.2 & 2.0 & 0.3 & 2.2 & 0.0 \\
\hline Number engaged in paid work in last 12 months & 1,106 & 1,085 & 999 & 477 & 705 & 608 \\
\hline \multicolumn{7}{|c|}{ Urban } \\
\hline \multicolumn{7}{|l|}{ Ever worked } \\
\hline Paid work & 54.8 & 15.4 & 93.5 & 17.6 & 49.0 & 13.6 \\
\hline Unpaid work & 21.8 & 11.0 & 32.6 & 12.7 & 20.1 & 9.7 \\
\hline Either paid or unpaid work & 62.4 & 22.3 & 96.7 & 25.9 & 57.6 & 20.0 \\
\hline Started working before age 15 & 24.7 & 10.4 & 32.6 & 14.1 & 23.3 & 7.7 \\
\hline \multicolumn{7}{|l|}{ Ever worked in last 12 months } \\
\hline Paid work & 52.3 & 10.4 & 89.1 & 9.2 & 46.7 & 11.3 \\
\hline Unpaid work & 11.8 & 6.4 & 12.0 & 4.2 & 11.9 & 7.9 \\
\hline Either paid or unpaid work & 58.8 & 15.1 & 94.6 & 12.6 & 53.7 & 17.0 \\
\hline Number of respondents & 1,039 & 2,581 & 547 & 1,136 & 833 & 1,445 \\
\hline
\end{tabular}


Table 4.1: (Cont'd)

\begin{tabular}{|c|c|c|c|c|c|c|}
\hline Economic activity (\%) & $\begin{array}{c}\mathrm{M} \\
15-24 \\
\end{array}$ & $\begin{array}{c}\mathrm{W} \\
15-24\end{array}$ & $\begin{array}{c}\text { MM } \\
15-29\end{array}$ & $\begin{array}{c}\text { MW } \\
15-24\end{array}$ & $\begin{array}{c}\text { UM } \\
15-24\end{array}$ & $\begin{array}{c}\text { UW } \\
15-24\end{array}$ \\
\hline \multicolumn{7}{|c|}{ Urban } \\
\hline \multicolumn{7}{|l|}{ Duration of paid work in last 12 months } \\
\hline Most of the year (6 months or more) & 77.4 & 52.3 & 90.2 & 46.2 & 75.4 & 56.4 \\
\hline Part of the year ( $3-5$ months) & 15.8 & 21.5 & 8.5 & 30.8 & 16.7 & 18.2 \\
\hline Rarely (less than 3 months) & 6.8 & 26.2 & 1.2 & 23.1 & 7.9 & 25.5 \\
\hline \multicolumn{7}{|l|}{ Main occupation (paid work) } \\
\hline Cultivator & 2.1 & 0.0 & 2.4 & 0.0 & 2.6 & 0.0 \\
\hline Agricultural labourer & 1.4 & 18.5 & 2.4 & 30.8 & 1.7 & 12.7 \\
\hline Administrative/executive/managerial/clerical & 14.4 & 24.6 & 9.8 & 7.7 & 16.5 & 32.7 \\
\hline Business & 11.6 & 4.6 & 13.4 & 7.7 & 11.3 & 3.6 \\
\hline Skilled manual/machinery & 30.8 & 27.7 & 34.1 & 23.1 & 30.4 & 29.1 \\
\hline Unskilled non-agricultural labourer & 36.3 & 24.6 & 35.4 & 30.8 & 33.9 & 21.8 \\
\hline Other & 3.4 & 0.0 & 2.4 & 0.0 & 3.5 & 0.0 \\
\hline Number engaged in paid work in last 12 months & 574 & 263 & 490 & 101 & 390 & 162 \\
\hline \multicolumn{7}{|c|}{ Rural } \\
\hline \multicolumn{7}{|l|}{ Ever worked } \\
\hline Paid work & 64.4 & 35.7 & 93.9 & 40.1 & 52.9 & 26.3 \\
\hline Unpaid work & 40.3 & 26.5 & 49.6 & 27.8 & 37.1 & 23.7 \\
\hline Either paid or unpaid work & 77.0 & 50.4 & 97.9 & 54.7 & 69.0 & 41.3 \\
\hline Started working before age 15 & 39.0 & 38.5 & 46.8 & 41.4 & 36.0 & 32.3 \\
\hline \multicolumn{7}{|l|}{ Ever worked in last 12 months } \\
\hline Paid work & 59.9 & 29.3 & 89.6 & 31.2 & 48.2 & 25.2 \\
\hline Unpaid work & 25.8 & 17.0 & 20.1 & 15.1 & 27.9 & 21.2 \\
\hline Either paid or unpaid work & 75.0 & 39.6 & 97.0 & 39.7 & 66.4 & 39.5 \\
\hline Number of respondents & 903 & 2,948 & 568 & 1,205 & 659 & 1,743 \\
\hline \multicolumn{7}{|l|}{ Duration of paid work in last 12 months } \\
\hline Most of the year (6 months or more) & 62.2 & 43.9 & 81.1 & 47.0 & 52.7 & 35.8 \\
\hline Part of the year ( $3-5$ months) & 26.7 & 34.0 & 15.6 & 34.3 & 32.7 & 33.0 \\
\hline Rarely (less than 3 months) & 10.3 & 20.3 & 2.7 & 16.8 & 14.3 & 29.4 \\
\hline \multicolumn{7}{|l|}{ Main occupation (paid work) } \\
\hline Cultivator & 11.3 & 1.0 & 14.1 & 0.9 & 11.8 & 1.3 \\
\hline Agricultural labourer & 19.9 & 85.9 & 19.3 & 85.9 & 18.4 & 86.1 \\
\hline Administrative/executive/managerial/clerical & 6.3 & 1.3 & 4.5 & 1.0 & 8.0 & 2.1 \\
\hline Business & 5.5 & 0.1 & 5.1 & 0.0 & 5.8 & 0.4 \\
\hline Skilled manual/machinery & 23.5 & 7.9 & 23.2 & 8.2 & 22.3 & 7.4 \\
\hline Unskilled non-agricultural labourer & 31.1 & 3.3 & 31.8 & 3.8 & 31.4 & 2.1 \\
\hline Other & 2.2 & 0.2 & 2.0 & 0.3 & 2.0 & 0.0 \\
\hline Number engaged in paid work in last 12 months & 532 & 822 & 509 & 376 & 315 & 446 \\
\hline
\end{tabular}

Note: All Ns are unweighted. Column totals may not equal 100\% due to missing cases or "don't know" responses. 
Table 4.1 also presents the percentages of youth reporting that they had worked any time in the 12 months prior to the interview. We note that the measure of work in the year prior to interview covers a wide range of experiences that go beyond what is typically considered an employment rate (for example, as per the usual principal status definition, employment is defined as those who worked for the major part of the year preceding the interview as a fraction of those in the labour force, that is, those who worked or sought work for the major part of the year). Included in our measure of work are youth who worked for any length of time during the year as a proportion of all youth, irrespective of whether they had worked or sought work in the year preceding the interview.

Percentages of youth who worked in the last 12 months largely mirrored lifetime economic activity for young men. Among young women, this was true for the unmarried. Fewer married young women, however, reported economic activity in the last 12 months compared to lifetime economic activity, a finding that may be attributable to conflict with childbearing and childrearing activities, on the one hand, and, on the other, the tendency of married young women to be secluded from outside work.

Findings also suggest that among young people who worked for remuneration in the year prior to the interview, the majority of young men $(64 \%)$ worked for at least six months of the year, while somewhat fewer young women (44\%) did so. Marital status differences indicate that more married than unmarried young people reported working for most of the year. While this pattern was observed among both men and women in rural areas and men in urban areas, among young women in urban areas the pattern differed: more unmarried than married young women reported having worked most of the year. Rural-urban differences suggest that larger proportions of urban than rural youth reported working for most of the year, $77 \%$ compared to $62 \%$ among young men and $52 \%$ compared to $44 \%$ among young women.

Occupational distributions of those engaged in remunerated work in the 12 months preceding the interview indicate that the distribution differed somewhat among young men and considerably among young women in rural and urban areas. The leading occupations among young men in rural areas, reported by $86 \%$ of young men, were agricultural work, unskilled non-agricultural labour and skilled labour. In urban areas, the leading occupations, reported by $67 \%$ of young men, were unskilled non-agricultural labour and skilled labour. Another $26 \%$ of young men in urban areas reported administrative, executive, managerial and clerical occupations (14\%) and business (12\%). Among young women in rural areas, the leading occupations were agricultural, particularly agricultural labour (86\%). In contrast, the leading occupations in urban areas were skilled labour, unskilled non-agricultural labour and administrative, executive, managerial and clerical occupations, together reported by $77 \%$ of young women. Notably, $19 \%$ of urban young women worked as agricultural labourers.

Differences by marital status were evident in urban areas only. Among young men, the unmarried were somewhat more likely to report administrative, executive, managerial and clerical occupations than the married (17\% versus $10 \%$ ). Among young women, the married were more likely than the unmarried to report agricultural and unskilled non-agricultural labour and less likely to report skilled labour and administrative, executive, managerial and clerical occupations. It is also notable that unmarried young women were less likely than other urban groups to report unskilled labour (22\% compared to $31-35 \%)$ and conversely, more likely to report administrative, executive, managerial or clerical occupations (33\% compared to $8-17 \%$ ).

Among youth reporting unpaid work in the 12 months preceding the interview, findings suggest that the majority of youth were engaged in agricultural activities, that is, on the family farm ( $72 \%$ and $86 \%$ of young men and women, respectively), with considerably fewer ( $28 \%$ and $14 \%$, respectively) working in family business related activities (not shown in tabular form). As expected, more rural than urban youth were engaged in agricultural activities. 


\subsection{Unemployment}

To measure unemployment rates among respondents, the Youth Study assessed (a) whether youth had worked in the 12 months preceding the interview and if so, the number of months worked; and (b) whether youth were seeking work and if so, the number of months during which they had been searching for work. Table 4.2 reports unemployment rates, defined as those seeking employment for the major part of the year preceding the interview as a fraction of those in the labour force. Labour force refers to those who were working or seeking work for the major part of the year. It does not, therefore, include those exclusively studying, those who may have worked for a short period in the year preceding the interview, or those who had sought work for a short period in the year preceding the interview.

Measured in this way, the percentage of unemployed youth was $22 \%$ among young men and $36 \%$ among young women, rates considerably higher than those observed by the National Sample Survey (NSS) (NSSO, 2006) among youth using the principal usual status definition. We note, however, that rates obtained in the Youth Study are not quite comparable to the NSS, not only because the questions were not identical, but also because of differences in the frequency with which information was obtained and corresponding differences in the recall period (quarterly in the NSS as compared to a 12-month recall period in the Youth Study) and differences in the household member eligible to provide information on youth unemployment (any household member in the NSS compared to the individual herself or himself in the Youth Study).

\section{Table 4.2: Unemployment}

Percentage of youth in the labour force who were unemployed, according to residence, Bihar, 2007

\begin{tabular}{|c|c|c|c|c|c|c|}
\hline Unemployment $(\%)^{1}$ & $\underset{15-24}{\mathrm{M}}$ & $\begin{array}{c}\text { W } \\
15-24\end{array}$ & $\begin{array}{c}\text { MM } \\
15-29\end{array}$ & $\begin{array}{c}\text { MW } \\
15-24\end{array}$ & $\begin{array}{c}\text { UM } \\
15-24\end{array}$ & $\begin{array}{c}\text { UW } \\
15-24\end{array}$ \\
\hline \multicolumn{7}{|c|}{ Combined } \\
\hline Unemployed & 22.4 & 35.7 & 9.8 & 34.5 & 28.8 & 37.6 \\
\hline Number in labour force & 1,126 & 1,029 & 1,002 & 487 & 740 & 542 \\
\hline \multicolumn{7}{|c|}{ Urban } \\
\hline Unemployed & 21.7 & 58.3 & 7.1 & 68.2 & 24.6 & 51.2 \\
\hline Number in labour force & 611 & 419 & 497 & 181 & 432 & 238 \\
\hline \multicolumn{7}{|c|}{ Rural } \\
\hline Unemployed & 22.7 & 33.7 & 10.0 & 33.2 & 29.6 & 35.3 \\
\hline Number in labour force & 515 & 610 & 505 & 306 & 308 & 304 \\
\hline
\end{tabular}

Note: All Ns are unweighted. ${ }^{1}$ Unemployment rate: Youth who were seeking work for the major part of the year preceding the interview as a proportion of those in the labour force (namely, those who were employed and/or seeking work for the major part of the year).

Findings suggest that while gender differences were evident in both urban and rural areas, these were much wider in urban areas: $58 \%$ of young women compared to $22 \%$ of young men reported unemployment in urban areas, and the corresponding percentages were $34 \%$ and $23 \%$, respectively, in rural areas. Differences by marital status indicate that unmarried young men were almost three times as likely as married young men to report unemployment. Among young women, differences by marital status were muted for the overall and rural population. In urban areas, however, the married were more likely than the unmarried to report unemployment. Unemployment rates in urban and rural areas were similar among young men (22-23\%), but 
differed considerably among young women. Larger proportions of urban than rural young women reported unemployment ( $58 \%$ versus $34 \%)$.

Table 4.3 describes socio-economic differentials in reported unemployment among young men and women. While unemployment appeared to be somewhat higher among younger (aged 15-19) than older (aged 20-24) men, the reverse was true among young women. Differences by religion suggest that while unemployment was higher among Hindu than Muslim young men, the reverse was true among young women. Caste-wise differences indicate that young men and women belonging to general castes reported the highest rate of unemployment. Unemployment rates increased consistently with education among young men; it increased from $11 \%$ among those with no formal education to $47 \%$ among those who had completed Class 12 . The patterns were less consistent among young women; even so, unemployment rate increased from $15 \%$ among those with no formal education to $77 \%$ among those who had completed $8-11$ years of schooling and $68 \%$ among those who had completed Class 12. The relative dearth of employment opportunities for the educated is reiterated in other studies as well (Chandrasekhar, Ghosh and Roychowdhury, 2006; NSSO, 2006). Differences by family economic status suggest that unemployment rates were the highest among young people in households belonging to the wealthiest quintile and the differences were much wider among young women than young men, possibly reflecting the dearth of employment opportunities for educated youth, who were considerably more likely to belong to households in the wealthiest quintile than to other households.

In general, similar patterns of unemployment were observed among both married and unmarried youth. However, the associations, described above, between caste and economic status with unemployment were not evident among married young men.

Patterns by rural-urban residence were, by and large, similar to the patterns observed for young men and women in general, except that some of the associations, described above, were not consistently observed among young people in urban areas (caste and unemployment among young men, and religion and unemployment among young women, for example).

\subsection{Work-related mobility}

Among young men who had ever worked, over two-fifths reported the experience of work-related mobility, as shown in Table 4.4. Just $2 \%$ of young women, in contrast, had lived away from home for work-related reasons. Gender differences may be attributed to the finding observed earlier that men were more likely than women to be engaged in such activities as non-agricultural labour and skilled manual labour, which entail mobility; they may also be attributed to the greater restrictions placed on the independent movement of young women than young men.

Differences by marital status indicate that married young men were considerably more likely to have experienced work-related mobility than unmarried men (63\% versus $34 \%$ ), perhaps a function of the fact that married men tended to be older and have more work experience than the unmarried. Rural-urban differences suggest that rural young men were more likely to report work-related mobility than their urban counterparts, perhaps because rural work opportunities were more seasonal than urban work opportunities, requiring rural young men to explore work opportunities outside their home settings. Almost four-fifths of young men (79\%) who reported work-related mobility had remained outside their home village or neighbourhood for three months or longer. Not only did few young women report having lived outside the home village or neighbourhood for work, but among those who had, considerably fewer than their male peers reported living away for three or more months $(28 \%)$. 
Table 4.3: Unemployment by selected background characteristics

Percentage of youth in the labour force who were unemployed by selected background characteristics, according to residence, Bihar, 2007

\begin{tabular}{|c|c|c|c|c|c|c|}
\hline Background characteristics (\%) & $\begin{array}{c}\mathrm{M} \\
15-24\end{array}$ & $\begin{array}{c}\text { W } \\
15-24\end{array}$ & $\begin{array}{c}\mathrm{MM} \\
15-29\end{array}$ & $\begin{array}{c}\text { MW } \\
15-24\end{array}$ & $\begin{array}{c}\text { UM } \\
15-24\end{array}$ & $\begin{array}{c}\text { UW } \\
\text { 15-24 }\end{array}$ \\
\hline \multicolumn{7}{|c|}{ Combined } \\
\hline \multicolumn{7}{|l|}{ Age (years) } \\
\hline $15-19$ & 25.7 & 33.2 & (14.5) & 31.7 & 27.6 & 34.5 \\
\hline $20-24$ & 19.9 & 38.1 & 12.4 & 36.0 & 30.8 & 56.8 \\
\hline $25-29$ & NA & NA & 7.8 & NA & NA & NA \\
\hline \multicolumn{7}{|l|}{ Religion } \\
\hline Hindu & 23.7 & 34.6 & 10.3 & 33.4 & 29.9 & 36.6 \\
\hline Muslim & 15.5 & 43.7 & 6.2 & 45.3 & 22.1 & 41.8 \\
\hline \multicolumn{7}{|l|}{ Caste } \\
\hline SC & 20.6 & 22.2 & 12.5 & 22.4 & 24.0 & 20.5 \\
\hline OBC & 20.5 & 37.4 & 8.3 & 37.3 & 26.3 & 36.5 \\
\hline General $^{1}$ & 33.7 & 63.9 & 9.6 & 64.9 & 40.4 & 61.7 \\
\hline \multicolumn{7}{|l|}{ Educational level (years) } \\
\hline None $^{2}$ & 10.5 & 15.4 & 7.0 & 15.9 & 10.2 & 12.1 \\
\hline $1-7$ & 15.0 & 48.8 & 6.3 & 55.8 & 18.1 & 35.8 \\
\hline $8-11$ & 27.6 & 77.4 & 12.2 & 79.3 & 33.9 & 75.0 \\
\hline 12 and above & 47.3 & 68.2 & 20.0 & $(70.4)$ & 56.1 & 65.6 \\
\hline \multicolumn{7}{|l|}{ Wealth quintile } \\
\hline First & 20.9 & 25.8 & 12.3 & 25.7 & $(20.4)$ & 24.2 \\
\hline Second & 15.3 & 21.1 & 7.4 & 19.1 & 20.3 & 27.6 \\
\hline Third & 16.5 & 29.6 & 9.5 & 30.1 & 17.9 & 26.5 \\
\hline Fourth & 21.7 & 41.6 & 7.0 & 44.0 & 29.6 & 36.6 \\
\hline Fifth & 30.5 & 71.3 & 12.5 & 76.3 & 38.3 & 64.8 \\
\hline Total & 22.4 & 35.7 & 9.8 & 34.5 & 28.8 & 37.6 \\
\hline \multicolumn{7}{|c|}{ Urban } \\
\hline \multicolumn{7}{|l|}{ Age (years) } \\
\hline $15-19$ & 25.4 & 50.0 & * & $(50.0)$ & 26.2 & 49.0 \\
\hline $20-24$ & 18.9 & 64.9 & 7.1 & 72.2 & 24.2 & 54.8 \\
\hline $25-29$ & NA & NA & 5.7 & NA & NA & NA \\
\hline \multicolumn{7}{|l|}{ Religion } \\
\hline Hindu & 23.2 & 62.0 & 8.5 & 66.7 & 27.3 & 57.7 \\
\hline Muslim & 15.6 & 50.0 & 0.0 & $(75.0)$ & 17.9 & 43.3 \\
\hline \multicolumn{7}{|l|}{ Caste } \\
\hline SC & 27.8 & 46.2 & 7.7 & $(50.0)$ & $(38.5)$ & * \\
\hline $\mathrm{OBC}$ & 17.5 & 56.3 & 5.7 & 69.2 & 19.5 & 49.1 \\
\hline Genearl $^{1}$ & 29.4 & 68.0 & 11.8 & $(80.0)$ & 33.3 & 61.9 \\
\hline
\end{tabular}


Table 4.3: (Cont'd)

\begin{tabular}{|c|c|c|c|c|c|c|}
\hline Background characteristics (\%) & $\begin{array}{c}\mathrm{M} \\
15-24\end{array}$ & $\begin{array}{c}\text { W } \\
15-24\end{array}$ & $\begin{array}{c}\text { MM } \\
15-29\end{array}$ & $\begin{array}{c}\text { MW } \\
15-24\end{array}$ & $\begin{array}{c}\text { UM } \\
15-24\end{array}$ & $\begin{array}{c}\text { UW } \\
15-24\end{array}$ \\
\hline \multicolumn{7}{|c|}{ Urban } \\
\hline \multicolumn{7}{|l|}{ Educational level (years) } \\
\hline None $^{2}$ & 7.7 & 32.1 & 5.6 & 42.9 & 5.9 & 25.0 \\
\hline $1-7$ & 5.7 & 55.6 & 0.0 & $(75.0)$ & 7.1 & $(41.7)$ \\
\hline $8-11$ & 22.2 & 74.1 & 6.5 & 85.7 & 25.6 & 65.0 \\
\hline 12 and above & 42.9 & 67.7 & 16.7 & $(75.0)$ & 45.9 & 63.3 \\
\hline \multicolumn{7}{|l|}{ Wealth quintile } \\
\hline First & * & * & * & * & * & * \\
\hline Second & $(11.1)$ & $(50.0)$ & $(0.0)$ & * & * & * \\
\hline Third & (9.1) & $(41.7)$ & $(0.0)$ & * & $(12.5)$ & (33.3) \\
\hline Fourth & 15.2 & 56.3 & 6.3 & $(66.7)$ & 20.0 & $(50.0)$ \\
\hline Fifth & 25.5 & 64.1 & 7.8 & 76.9 & 29.1 & 56.6 \\
\hline Total & 21.7 & 58.3 & 7.1 & 68.2 & 24.6 & 51.2 \\
\hline \multicolumn{7}{|c|}{ Rural } \\
\hline \multicolumn{7}{|l|}{ Age (years) } \\
\hline $15-19$ & 25.7 & 31.8 & $(13.3)$ & 31.3 & 27.9 & 32.5 \\
\hline $20-24$ & 20.1 & 35.4 & 12.6 & 34.3 & 32.8 & 57.1 \\
\hline $25-29$ & NA & NA & 8.0 & NA & NA & NA \\
\hline \multicolumn{7}{|l|}{ Religion } \\
\hline Hindu & 23.7 & 32.6 & 10.5 & 32.2 & 30.4 & 33.7 \\
\hline Muslim & 16.1 & 42.1 & 6.8 & $(42.9)$ & $(24.7)$ & 41.8 \\
\hline \multicolumn{7}{|l|}{ Caste } \\
\hline SC & 20.0 & 21.4 & 12.8 & 21.9 & $(22.0)$ & 18.4 \\
\hline OBC & 21.2 & 35.4 & 8.5 & 35.9 & 27.8 & 34.4 \\
\hline General $^{1}$ & 34.6 & 62.3 & $(10.2)$ & $(63.5)$ & 42.5 & $(61.6)$ \\
\hline \multicolumn{7}{|l|}{ Educational level (years) } \\
\hline None $^{2}$ & 10.8 & 14.7 & 7.1 & 15.4 & 10.1 & 11.2 \\
\hline $1-7$ & 15.7 & 48.3 & 6.3 & 55.6 & 19.8 & 35.3 \\
\hline $8-11$ & 28.6 & 78.0 & 12.8 & $(78.9)$ & 35.5 & 76.9 \\
\hline 12 and above & 48.8 & $(68.5)$ & 20.0 & * & $(60.5)$ & * \\
\hline \multicolumn{7}{|l|}{ Wealth quintile } \\
\hline First & 21.3 & 25.5 & 12.5 & 25.7 & $(20.8)$ & 24.5 \\
\hline Second & 15.6 & 20.5 & 7.7 & 18.8 & $(20.5)$ & 26.7 \\
\hline Third & 16.8 & 28.8 & 9.8 & 29.7 & 18.3 & 25.8 \\
\hline Fourth & 22.4 & 40.3 & 6.6 & $(43.2)$ & 31.0 & 35.5 \\
\hline Fifth & 32.5 & 74.3 & 13.6 & (76.1) & 43.4 & (70.7) \\
\hline Total & 22.7 & 33.7 & 10.0 & 33.2 & 29.6 & 35.3 \\
\hline
\end{tabular}

Note: ( ) Based on 25-49 unweighted cases. ${ }^{*}$ Percentage not shown, based on fewer than 25 unweighted cases. NA: Not applicable. OBC: Other backward caste. SC: Scheduled caste. ST: Scheduled tribe. ${ }^{1}$ Includes all those not belonging to SC, ST or $\mathrm{OBC} .{ }^{2}$ Includes non-literate and literate with no formal schooling. 
Table 4.4: Work-related mobility

Percentage of youth who had ever lived outside their home village/area for work, according to residence, Bihar, 2007

\begin{tabular}{|c|c|c|c|c|c|c|}
\hline Mobility characteristics (\%) & $\begin{array}{c}\mathrm{M} \\
15-24\end{array}$ & $\begin{array}{c}\text { W } \\
15-24\end{array}$ & $\begin{array}{c}\text { MM } \\
15-29\end{array}$ & $\begin{array}{c}\text { MW } \\
15-24\end{array}$ & $\begin{array}{c}\text { UM } \\
15-24\end{array}$ & $\begin{array}{c}\text { UW } \\
15-24\end{array}$ \\
\hline \multicolumn{7}{|c|}{ Combined } \\
\hline \multicolumn{7}{|l|}{ Work-related mobility } \\
\hline Ever stayed outside village/area for work & 43.8 & 2.3 & 62.9 & 2.5 & 34.2 & 2.0 \\
\hline Number ever worked & 1,360 & 1,965 & 1,088 & 948 & 929 & 1,017 \\
\hline Stayed outside village/area for 3 months or longer & 78.6 & $(27.6)$ & 78.5 & * & 82.7 & * \\
\hline Number ever stayed out of home village/area for work & 514 & 43 & 576 & 21 & 279 & 22 \\
\hline \multicolumn{7}{|c|}{ Urban } \\
\hline \multicolumn{7}{|l|}{ Work-related mobility } \\
\hline Ever stayed outside village/area for work & 28.7 & 2.8 & 40.4 & 2.7 & 24.8 & 3.1 \\
\hline Number ever worked & 674 & 582 & 532 & 292 & 479 & 290 \\
\hline Stayed outside village/area for 3 months or longer & 66.0 & * & 66.7 & * & 65.7 & * \\
\hline Number ever stayed out of home village/area for work & 204 & 15 & 215 & 6 & 120 & 9 \\
\hline \multicolumn{7}{|c|}{ Rural } \\
\hline Work-related mobility & & & & & & \\
\hline Ever stayed outside village/area for work & 45.8 & 2.3 & 64.9 & 2.5 & 35.8 & 1.8 \\
\hline Number ever worked & 686 & 1,383 & 556 & 656 & 450 & 727 \\
\hline Stayed outside village/area for 3 months or longer & 79.5 & $(24.1)$ & 79.1 & * & 84.7 & * \\
\hline Number ever stayed out of home village/area for work & 310 & 28 & 361 & 15 & 159 & 13 \\
\hline
\end{tabular}

Note: All Ns are unweighted. ( ) Based on 25-49 unweighted cases. ${ }^{*}$ Percentage not shown, based on fewer than 25 unweighted cases.

\subsection{Economic activity and schooling status}

While the period of transition to adulthood is marked by discontinuation of schooling and entry into the labour market for many young people, some combine schooling and work and others are neither in school nor working. Data collected through the Life Event Calendar component of the Youth Study provided an opportunity to explore the pattern of these events (that is, studying, working, both studying and working, and neither studying nor working) in young people's lives from the age of 12 , and these are presented in Figures $4.1 \mathrm{a}-\mathrm{c}$. Patterns varied widely by sex and marital status of the respondent. We note that Figures 4.1a and $4.1 \mathrm{~b}$ convey the situation both prior to and following marriage for married youth.

A comparison of the two panels of Figure 4.1a shows, first, that the proportion of youth reporting school attendance declined steadily across all groups as young people transitioned out of early adolescence to late adolescence and young adulthood. For example, while $77 \%$ of young men and $43 \%$ of young women were in school (a small minority of these were also working) at age 12, the percentage who remained in school at age 15 fell to $62 \%$ for young men and $31 \%$ for young women. Moreover, the rates of decline in school 
attendance were steepest between ages 16-19 for young men and 15-17 for young women. Second, between one in ten and one in five young men reported having combined studying and working during adolescence and the percentages of young men who so reported peaked at ages 15-17 and declined thereafter. Among young women, in contrast, very few (5\% or less) combined studying and working at any age. Third, exit from school was accompanied by a steady rise in work participation over the ages among young men, but not so among young women. Among young men, larger percentages were working than in school as early as by age 16. Among young women, work participation remained more or less steady at all ages. Finally, significant proportions of young women but not young men were neither in school nor working from age 12 onwards. Among young men, small proportions (one in 8 or fewer) were neither working nor in school at any age. Among young women, there was a steady increase by age. At age 12,32\% of young women were neither working nor in school; percentages increased to $40 \%$ at age 15 and $59 \%$ at age 20.

Figures $4.1 \mathrm{~b}$ and $4.1 \mathrm{c}$ suggest that patterns differed between married and unmarried youth. For one, the married were less likely than the unmarried to be in school at each age. For example, $61 \%$ and $30 \%$ of married young men and women, respectively, and $82 \%$ and $65 \%$ of the unmarried, respectively, were in school (a small minority of these were also working) at age 12, and the percentages of those who remained in school fell thereafter. At age 20, for example, only $11 \%$ of married young men and $4 \%$ of married young women were pursuing their education compared to $33 \%$ and $46 \%$ of unmarried young men and women, respectively. Second, the unmarried were more likely than the married to have combined schooling and work at most ages, and the differences between the married and the unmarried were particularly evident among young men at ages 15-23, that is high school and college going ages; differences were milder among young women. Third, while considerably larger percentages of married than unmarried young men were neither in school nor working in early adolescence, a reverse pattern was observed once young men transitioned out of adolescence to young adulthood. Among young women, more married than unmarried were neither in school nor working from age 12. The percentage of those neither in school nor working increased steadily till age 17 and thereafter plateaued among married young women; among unmarried young women, it continued to increase even at later ages. Finally, the ages at which more youth were working than in school were 15 and 17 among married and unmarried young men, respectively. Among young women, the pattern differed between the married and the unmarried: even at age 12, more were working than in school among the married and even at age 24, more were in school than working among the unmarried. We note, however that the sample contains few women aged 23-24 and that estimates in these ages may have been affected as a result.

\subsection{Participation in non-economic activity}

The Youth Study also inquired about the extent to which young men and women participated in domestic chores. All youth were asked whether and how frequently they were engaged in activities such as housework (cooking, cleaning, child/sibling care), shopping for groceries for the family and tasks such as collecting firewood or fetching water, and paying electricity or phone bills (as appropriate for urban and rural areas). Findings, reported in Table 4.5 and Figure 4.2, highlight the gendered nature of young people's participation in domestic chores. Young women were more likely than young men to be engaged in work inside the home, and less likely to be engaged in tasks that violated norms restricting their mobility outside the home. For example, the vast majority of young women (90\%) were often engaged in housework, compared with just $7 \%$ of young men. In contrast, $95 \%$ of young men reported sometimes or often shopping for groceries, compared with $33 \%$ of young women. Likewise, $85 \%$ of young men, compared to $40 \%$ of young women reported participating sometimes or often in tasks such as collecting firewood or fetching water, and paying electricity or phone bills. 
Figure 4.1a: Economic activity and schooling status among youth aged 15-24, by age, Bihar, 2007
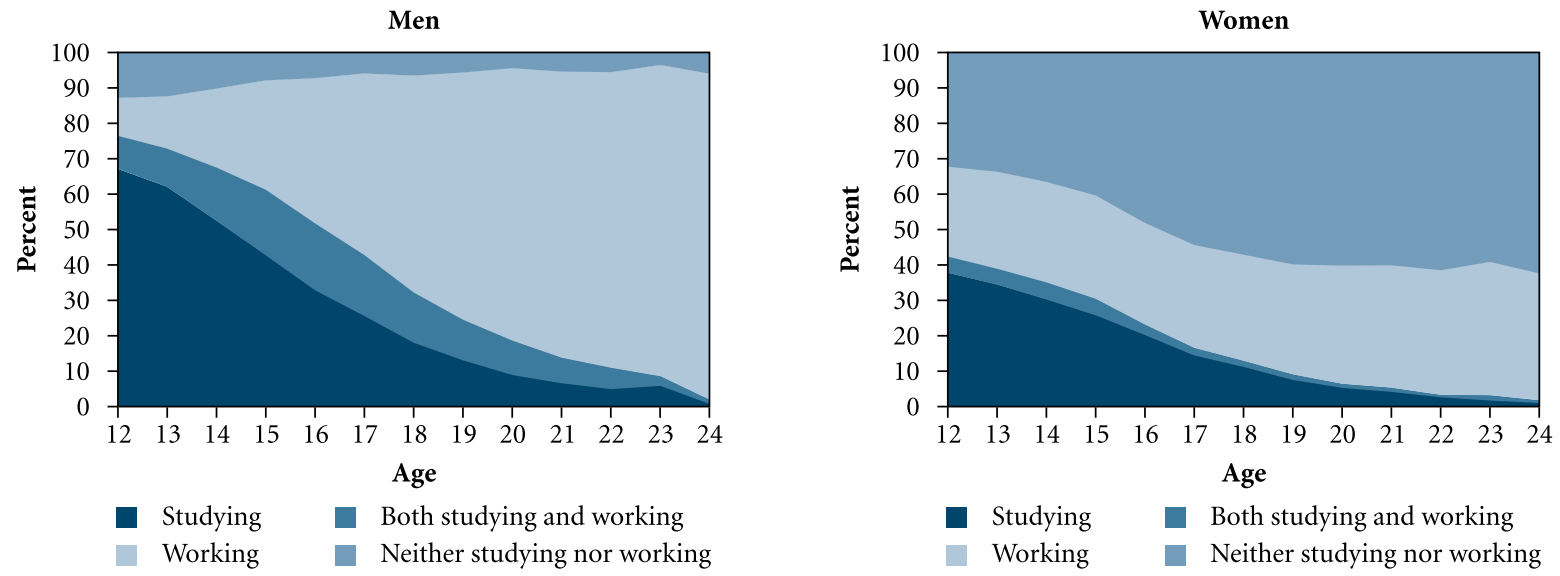

Note: For married youth, the figure conveys the situation prior to and following marriage.

Figure 4.1b: Economic activity and schooling status among married men aged 15-29 and married women aged 15-24, by age, Bihar, 2007
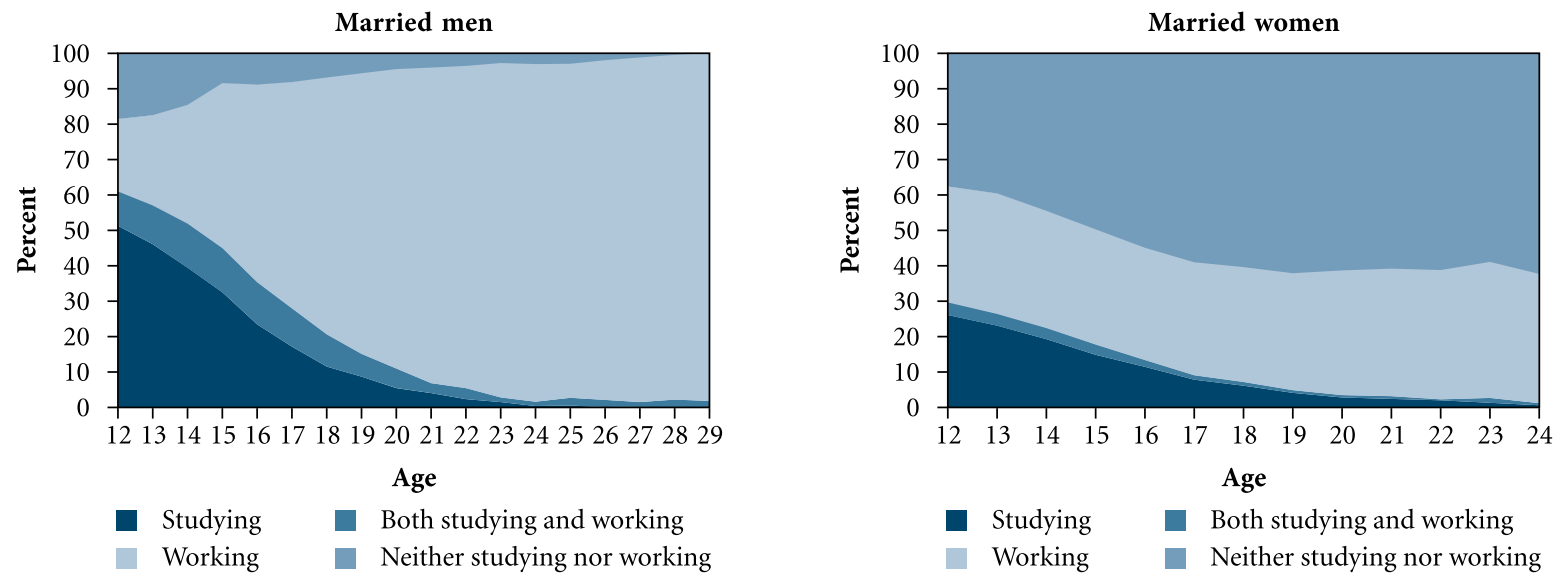

Note: For married youth, the figure conveys the situation prior to and following marriage.

Figure 4.1c: Economic activity and schooling status among unmarried men and women aged 15-24, by age, Bihar, 2007
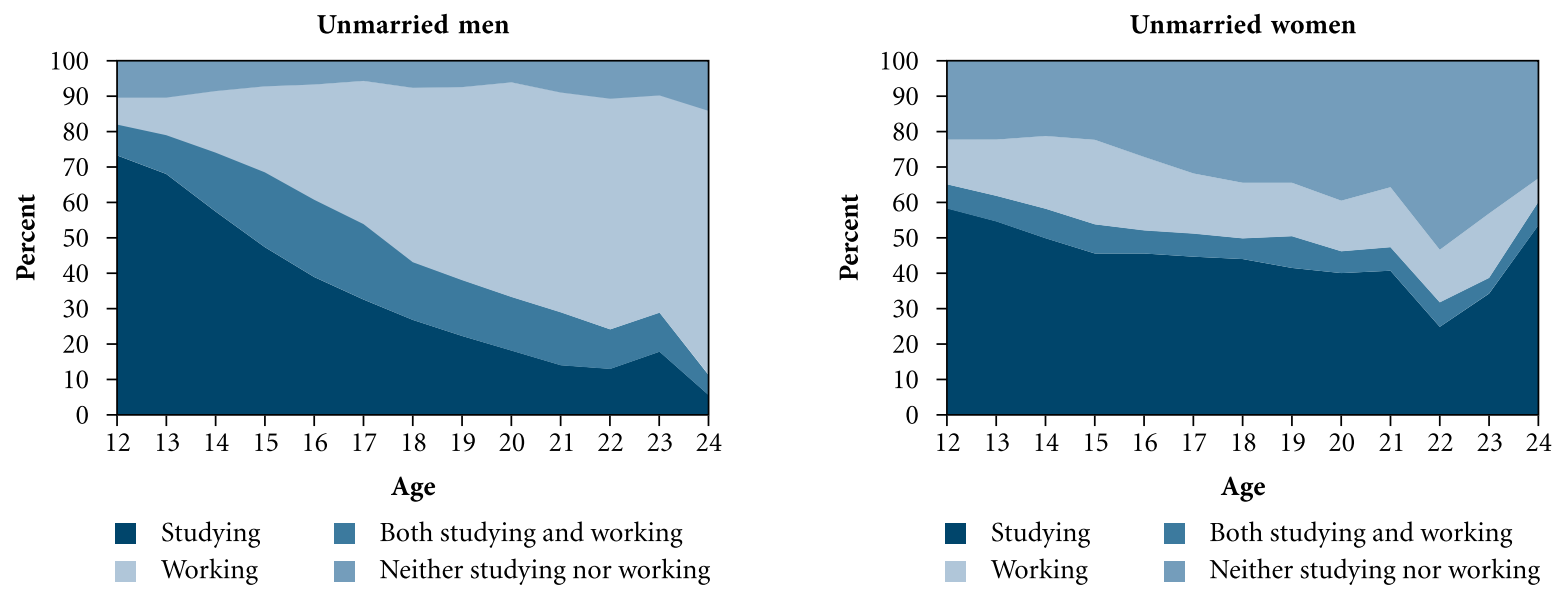
In terms of differences in participation in household chores by marital status, patterns varied by type of activity. For example, the unmarried were as likely as the married to engage in housework and shopping but somewhat less likely than the married to engage in tasks such as collecting firewood or fetching water (rural settings), and paying electricity or phone bills (urban settings). The patterns remained, by and large, similar in both urban and rural areas. However in urban areas, unmarried young women were considerably more likely than married young women to report shopping for groceries. Frequency of engaging in domestic activities varied. Larger proportions of married than unmarried young women reported engaging in housework and such tasks as collecting firewood or fetching water, and paying electricity or phone bills on a regular basis; likewise, married young men were more likely than the unmarried to report shopping and engaging in such tasks as collecting firewood or fetching water, and paying electricity or phone bills on a regular basis.

Rural-urban differences were moderate but suggest that urban youth were considerably less likely than their rural counterparts to report engaging in such tasks as collecting firewood or fetching water, and paying bills. Differences were particularly evident for young women upon whom much of the responsibility for collecting firewood and water rested in rural areas. In addition, urban young men were moderately more likely than rural young men to report engaging in housework.

\subsection{Participation in vocational training programmes}

A number of vocational training opportunities are available to youth through government, non-government and private organisations. Our survey inquired whether respondents had attended any such programmes, and the kinds of programmes they would like to attend, if offered. Findings, presented in Table 4.6 and Figure 4.3, indicate that $14 \%$ of young men and $10 \%$ of young women had ever attended a vocational training programme. While differences by marital status were modest, rural-urban differences were considerable. Urban youth were far more likely to have received training than their rural counterparts (26\% and $25 \%$ among young men and women, respectively, in urban areas, compared with $12 \%$ and $8 \%$, respectively, in rural areas).
Figure 4.2: Percentage of youth who participated in domestic chores, according to residence, Bihar, 2007
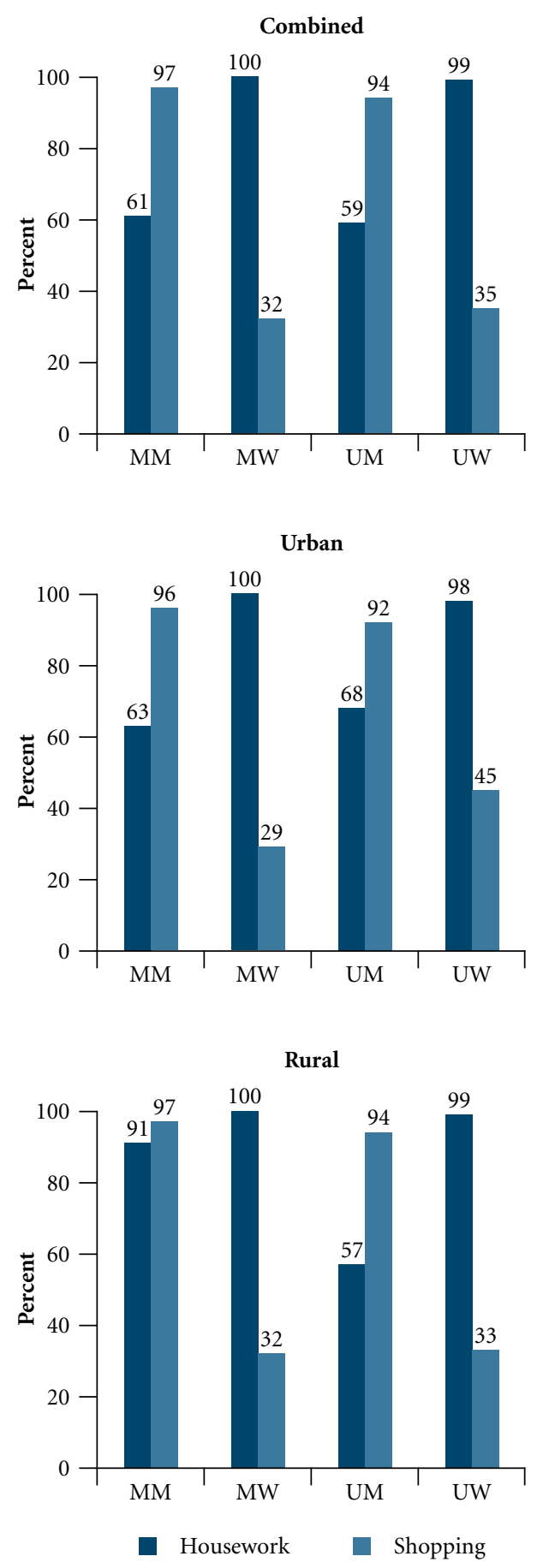
Table 4.5: Participation in household chores

Percent distribution of youth by extent of participation in various household chores, according to residence, Bihar, 2007

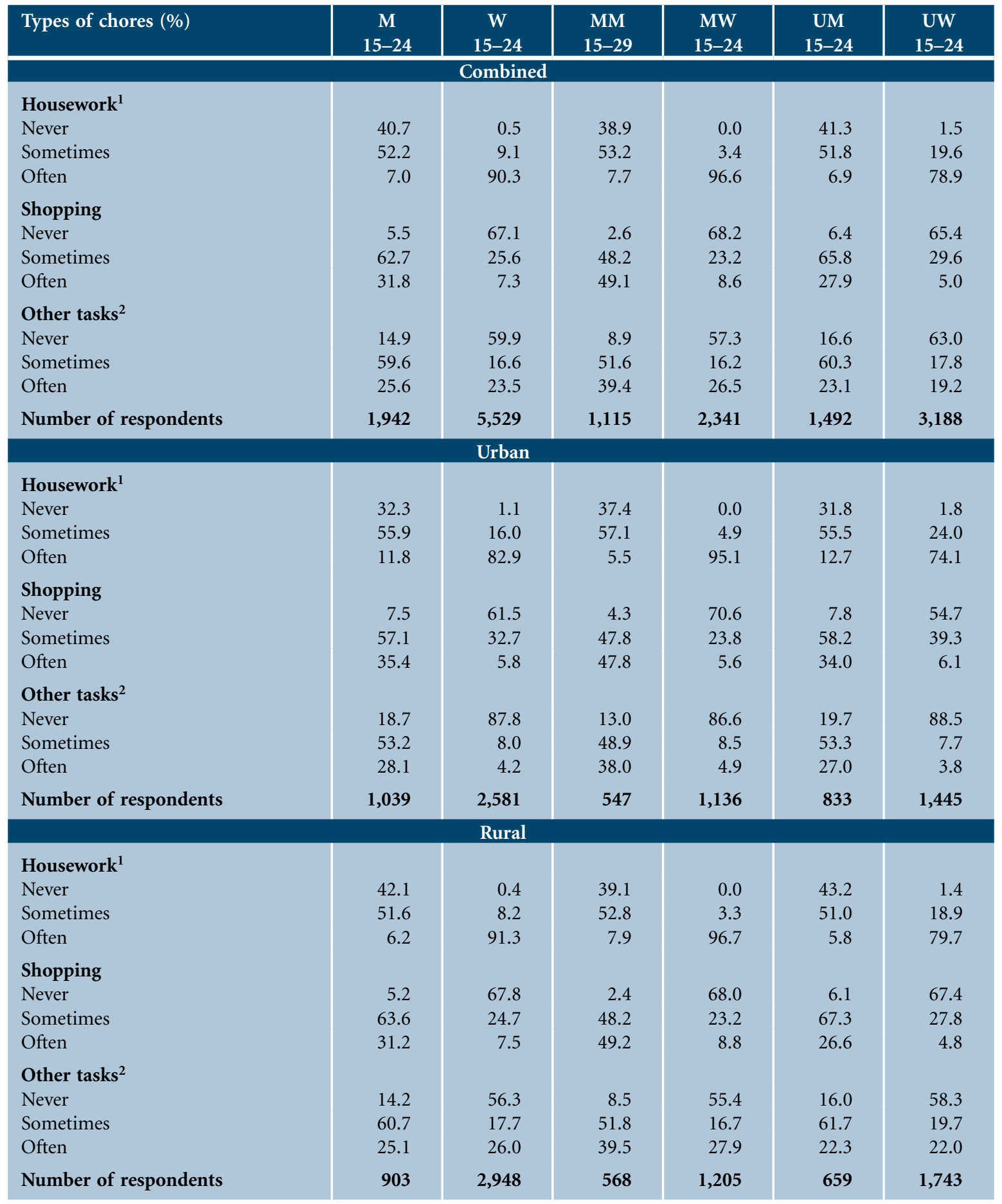

Note: All Ns are unweighted. Column totals may not equal 100\% due to missing cases or "don't know" responses. ${ }^{1}$ Includes cooking, cleaning, etc. ${ }^{2}$ Respondents were given examples of other tasks such as collecting firewood, fetching water, grazing livestock, paying bills, etc. 
The kind of training received varied by sex of the respondent and rural-urban residence. Among young men, leading training programmes reported were focused on computer skills (23\%) and auto mechanics or electrical work (20\%). Between one in ten and one in seven young men also reported having received training in tailoring, driving, plumbing or masonry, English language and secretarial skills and handicrafts. Key training received by young women was quite different: $82 \%$ reported training in tailoring and $31 \%$ in handicrafts. The unmarried were more likely to report training in new technologies than the married. For example, $27 \%$ of unmarried young men compared to $12 \%$ of married young men reported computer training; corresponding figures for young women were $11 \%$ and $2 \%$, respectively. Finally, training received by rural youth was more likely than that obtained by urban youth to fall into more traditional activities. For example, rural young men were far more likely than their urban counterparts to have received training in driving and plumbing or masonry, and, conversely, less likely to have been trained in computer skills or English language, typing or shorthand.

Figure 4.3: Percentage of youth who ever attended a vocational training programme and percentage who were interested in participating in such programmes, according to residence, Bihar, 2007

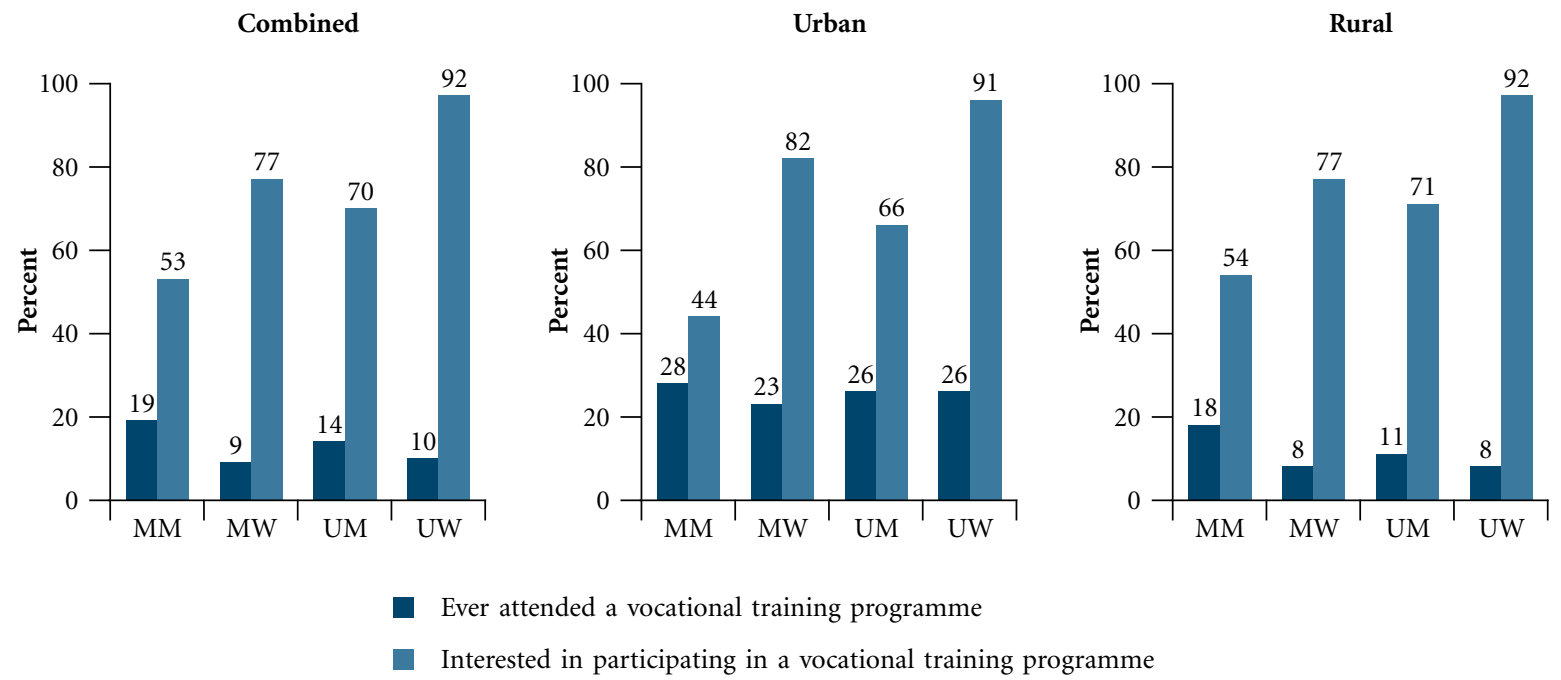

Large proportions of youth- $66 \%$ of young men and $82 \%$ of young women-reported interest in attending vocational training programmes, as shown in Table 4.7. Although more unmarried than married youth expressed interest in attending vocational training programmes, it is notable that over half of married men and over three-quarters of married women were interested in developing vocational skills. Rural-urban differences were modest, except that more married youth in rural areas than in urban areas expressed interest in acquiring vocational skills. Skills in which youth wished to be trained virtually mirrored the patterns revealed above. The majority of young women continued to wish to be trained in areas such as tailoring and handicrafts. Young men's preferences, in contrast, were focused on computer training, auto mechanics or electrical work and driving. 
Table 4.6: Participation in vocational training programmes

Percentage of youth who ever attended a vocational training programme and type of programme attended, according to residence, Bihar, 2007

\begin{tabular}{|c|c|c|c|c|c|c|}
\hline Programmes/courses attended (\%) & $\begin{array}{c}\mathrm{M} \\
15-24\end{array}$ & $\begin{array}{c}\mathrm{W} \\
15-24\end{array}$ & $\begin{array}{c}\text { MM } \\
15-29\end{array}$ & $\begin{array}{c}\text { MW } \\
15-24\end{array}$ & $\begin{array}{c}\text { UM } \\
15-24\end{array}$ & $\begin{array}{c}\text { UW } \\
15-24\end{array}$ \\
\hline \multicolumn{7}{|c|}{ Combined } \\
\hline Ever attended a vocational training programme & 14.0 & 10.0 & 18.9 & 9.3 & 13.7 & 10.4 \\
\hline Number of respondents & 1,942 & 5,529 & 1,115 & 2,341 & 1,492 & 3,188 \\
\hline \multicolumn{7}{|l|}{ Types of programmes/courses attended } \\
\hline Tailoring & 9.1 & 82.3 & 10.9 & 89.0 & 6.3 & 73.8 \\
\hline Auto mechanic/electrical work & 20.4 & 0.0 & 23.7 & 0.0 & 18.6 & 0.0 \\
\hline Driving & 10.9 & 0.0 & 17.1 & 0.0 & 8.8 & 0.0 \\
\hline Plumbing/masonry & 9.5 & 0.0 & 19.0 & 0.0 & 10.7 & 0.0 \\
\hline Poultry/goat farm & 0.7 & 0.0 & 0.9 & 0.0 & 0.0 & 0.0 \\
\hline Beauty parlour/salon & 1.8 & 4.3 & 2.8 & 3.2 & 0.5 & 5.7 \\
\hline Nurse's aid & 2.6 & 0.5 & 2.8 & 0.9 & 3.4 & 0.0 \\
\hline Computer training & 23.4 & 5.8 & 12.3 & 1.8 & 27.3 & 10.8 \\
\hline English language/typing/shorthand & 10.6 & 2.2 & 5.7 & 0.5 & 13.2 & 4.2 \\
\hline Handicrafts/painting/embroidery/cooking & 15.0 & 30.6 & 10.9 & 27.5 & 14.6 & 34.0 \\
\hline Other & 2.2 & 0.9 & 0.9 & 0.9 & 2.0 & 0.9 \\
\hline Number ever attended any vocational training & 372 & 865 & 258 & 363 & 291 & 502 \\
\hline \multicolumn{7}{|c|}{ Urban } \\
\hline Ever attended a vocational training programme & 25.8 & 24.8 & 28.3 & 23.2 & 26.2 & 25.9 \\
\hline Number of respondents & 1,039 & 2,581 & 547 & 1,136 & 833 & 1,445 \\
\hline \multicolumn{7}{|l|}{ Types of programmes/courses attended } \\
\hline Tailoring & 4.2 & 65.8 & 3.8 & 84.8 & 4.7 & 54.7 \\
\hline Auto mechanic/electrical work & 19.4 & 0.0 & 25.9 & 0.0 & 18.8 & 0.0 \\
\hline Driving & 4.2 & 0.0 & 15.4 & 0.0 & 3.1 & 0.0 \\
\hline Plumbing/masonry & 4.2 & 0.0 & 11.5 & 0.0 & 3.1 & 0.0 \\
\hline Poultry/goat farm & 0.0 & 0.0 & 0.0 & 0.0 & 0.0 & 0.0 \\
\hline Beauty parlour/salon & 1.4 & 7.6 & 0.0 & 6.1 & 1.6 & 9.4 \\
\hline Nurse's aid & 1.4 & 0.0 & 3.8 & 0.0 & 0.0 & 0.0 \\
\hline Computer training & 51.4 & 17.7 & 19.2 & 6.1 & 54.7 & 25.8 \\
\hline English language/typing/shorthand & 15.3 & 7.0 & 15.4 & 3.0 & 15.6 & 9.4 \\
\hline Handicrafts/painting/embroidery/cooking & 11.1 & 41.1 & 15.4 & 39.4 & 9.4 & 43.0 \\
\hline Other & 1.4 & 0.0 & 0.0 & 0.0 & 1.5 & 0.0 \\
\hline Number ever attended any vocational training & 265 & 634 & 155 & 262 & 217 & 372 \\
\hline \multicolumn{7}{|c|}{ Rural } \\
\hline Ever attended a vocational training programme & 12.0 & 8.1 & 18.1 & 8.4 & 11.2 & 7.6 \\
\hline Number of respondents & 903 & 2,948 & 568 & 1,205 & 659 & 1,743 \\
\hline \multicolumn{7}{|l|}{ Types of programmes/courses attended } \\
\hline Tailoring & 10.9 & 88.9 & 11.9 & 90.2 & 7.1 & 86.2 \\
\hline Auto mechanic/electrical work & 20.8 & 0.0 & 23.2 & 0.0 & 18.6 & 0.0 \\
\hline Driving & 12.9 & 0.0 & 17.8 & 0.0 & 10.7 & 0.0 \\
\hline Plumbing/masonry & 11.4 & 0.0 & 20.0 & 0.0 & 13.6 & 0.0 \\
\hline Poultry/goat farm & 1.0 & 0.0 & 1.1 & 0.0 & 0.0 & 0.0 \\
\hline Beauty parlour/salon & 2.0 & 3.0 & 2.7 & 3.2 & 0.0 & 2.9 \\
\hline Nurse's aid & 3.0 & 0.8 & 2.7 & 1.1 & 4.3 & 0.0 \\
\hline Computer training & 13.9 & 1.3 & 10.8 & 1.1 & 14.3 & 1.5 \\
\hline English language/typing/shorthand & 8.9 & 0.3 & 4.3 & 0.0 & 12.1 & 1.0 \\
\hline Handicrafts/painting/embroidery/cooking & 16.4 & 26.4 & 10.2 & 25.5 & 17.0 & 28.4 \\
\hline Other & 3.0 & 1.3 & 1.1 & 1.1 & 2.8 & 1.5 \\
\hline Number ever attended any vocational training & 107 & 231 & 103 & 101 & 74 & 130 \\
\hline
\end{tabular}

Note: All Ns are unweighted. Column totals may exceed 100\% due to multiple responses. 
Table 4.7: Willingness of youth to participate in vocational training programmes

Percentage of youth interested in participating in vocational training programmes and type of programme they were interested in participating in, according to residence, Bihar, 2007

\begin{tabular}{|c|c|c|c|c|c|c|}
\hline Programmes/courses (\%) & $\begin{array}{c}\mathrm{M} \\
15-24\end{array}$ & $\begin{array}{c}\text { W } \\
15-24\end{array}$ & $\begin{array}{c}\text { MM } \\
15-29\end{array}$ & $\begin{array}{c}\text { MW } \\
15-24\end{array}$ & $\begin{array}{c}\text { UM } \\
15-24\end{array}$ & $\begin{array}{c}\text { UW } \\
15-24\end{array}$ \\
\hline \multicolumn{7}{|c|}{ Combined } \\
\hline $\begin{array}{l}\text { Interested in participating in a vocational } \\
\text { training programme }\end{array}$ & 66.2 & 82.3 & 52.7 & 77.1 & 70.0 & 91.9 \\
\hline $\begin{array}{l}\text { Number of respondents } \\
\text { Types of programmes in which youth } \\
\text { wished to participate }\end{array}$ & 1,942 & 5,529 & 1,115 & 2,341 & 1,492 & 3,188 \\
\hline Tailoring & 9.2 & 89.7 & 8.8 & 92.7 & 8.6 & 86.2 \\
\hline Auto mechanic/electric work & 31.8 & 0.0 & 41.3 & 0.0 & 30.1 & 0.1 \\
\hline Driving & 14.2 & 0.1 & 22.6 & 0.0 & 12.5 & 0.1 \\
\hline Plumbing/masonry & 8.4 & 0.0 & 18.5 & 0.0 & 5.7 & 0.1 \\
\hline Poultry/goat farm & 1.1 & 0.1 & 0.9 & 0.1 & 1.1 & 0.0 \\
\hline Beauty parlour/salon & 0.3 & 4.0 & 0.3 & 2.4 & 0.2 & 6.0 \\
\hline Nurse's aid & 1.1 & 1.2 & 1.5 & 1.0 & 1.1 & 1.6 \\
\hline Computer training & 38.7 & 7.3 & 13.8 & 1.7 & 45.6 & 15.0 \\
\hline English language/typing/shorthand & 7.9 & 2.6 & 1.2 & 0.9 & 9.8 & 4.9 \\
\hline Handicrafts/painting/embroidery/cooking & 4.3 & 56.4 & 5.3 & 53.6 & 3.9 & 60.6 \\
\hline $\begin{array}{l}\text { Number interested in participating in a } \\
\text { vocational training programme }\end{array}$ & 1,258 & 4,780 & 540 & 1,855 & 1,017 & 2,925 \\
\hline \multicolumn{7}{|c|}{ Urban } \\
\hline $\begin{array}{l}\text { Interested in participating in a vocational } \\
\text { training programme }\end{array}$ & 64.2 & 87.3 & 43.5 & 81.7 & 66.1 & 91.3 \\
\hline $\begin{array}{l}\text { Number of respondents } \\
\text { Types of programmes in which youth } \\
\text { wished to participate }\end{array}$ & 1,039 & 2,581 & 547 & 1,136 & 833 & 1,445 \\
\hline Tailoring & 3.4 & 70.3 & 2.5 & 81.0 & 3.7 & 63.5 \\
\hline Auto mechanic/electric work & 29.6 & 0.2 & 40.0 & 0.0 & 28.0 & 0.2 \\
\hline Driving & 9.0 & 0.2 & 17.5 & 0.0 & 8.0 & 0.2 \\
\hline Plumbing/masonry & 2.8 & 0.0 & 7.5 & 0.0 & 1.9 & 0.0 \\
\hline Poultry/goat farm & 0.6 & 0.0 & 0.0 & 0.0 & 0.6 & 0.0 \\
\hline Beauty parlour/salon & 0.0 & 12.8 & 0.0 & 9.5 & 0.0 & 14.8 \\
\hline Nurse's aid & 0.6 & 1.4 & 0.0 & 0.9 & 0.6 & 1.6 \\
\hline Computer training & 56.4 & 24.3 & 32.5 & 7.8 & 58.6 & 35.0 \\
\hline English language/typing/shorthand & 11.7 & 8.8 & 5.0 & 1.7 & 13.0 & 13.1 \\
\hline Handicrafts/painting/embroidery/cooking & 5.6 & 60.9 & 7.5 & 62.1 & 5.6 & 60.0 \\
\hline $\begin{array}{l}\text { Number interested in participating in a } \\
\text { vocational training programme }\end{array}$ & 654 & 2,251 & 237 & 929 & 551 & 1,322 \\
\hline
\end{tabular}


Table 4.7: (Cont'd)

\begin{tabular}{|c|c|c|c|c|c|c|}
\hline Programmes/courses (\%) & $\begin{array}{c}\mathrm{M} \\
15-24\end{array}$ & $\begin{array}{c}\text { W } \\
15-24\end{array}$ & $\begin{array}{c}\text { MM } \\
15-29\end{array}$ & $\begin{array}{c}\text { MW } \\
15-24\end{array}$ & $\begin{array}{c}\text { UM } \\
15-24\end{array}$ & $\begin{array}{c}\text { UW } \\
15-24\end{array}$ \\
\hline \multicolumn{7}{|c|}{ Rural } \\
\hline $\begin{array}{l}\text { Interested in participating in a vocational } \\
\text { training programme }\end{array}$ & 66.6 & 81.7 & 53.6 & 76.9 & 70.8 & 92.0 \\
\hline $\begin{array}{l}\text { Number of respondents } \\
\text { Types of programmes in which youth } \\
\text { wished to participate }\end{array}$ & 903 & 2,948 & 568 & 1,205 & 659 & 1,743 \\
\hline Tailoring & 10.1 & 92.4 & 9.1 & 93.5 & 9.5 & 90.3 \\
\hline Auto mechanic/electric work & 32.2 & 0.0 & 41.4 & 0.0 & 30.6 & 0.1 \\
\hline Driving & 15.0 & 0.1 & 23.0 & 0.0 & 13.3 & 0.1 \\
\hline Plumbing/masonry & 9.3 & 0.0 & 19.3 & 0.0 & 6.5 & 0.1 \\
\hline Poultry/goat farm & 1.2 & 0.1 & 0.9 & 0.1 & 1.2 & 0.0 \\
\hline Beauty parlour/salon & 0.4 & 2.8 & 0.4 & 1.9 & 0.2 & 4.4 \\
\hline Nurse's aid & 1.1 & 1.2 & 1.6 & 1.0 & 1.2 & 1.6 \\
\hline Computer training & 35.9 & 5.0 & 12.2 & 1.4 & 43.1 & 11.4 \\
\hline English language/typing/shorthand & 7.3 & 1.8 & 0.9 & 0.8 & 9.2 & 3.4 \\
\hline Handicrafts/painting/embroidery/cooking & 4.2 & 55.8 & 4.9 & 53.0 & 3.6 & 60.8 \\
\hline $\begin{array}{l}\text { Number interested in participating in a } \\
\text { vocational training programme }\end{array}$ & 604 & 2,529 & 303 & 926 & 466 & 1,603 \\
\hline
\end{tabular}

Note: All Ns are unweighted. Column totals may exceed 100\% due to multiple responses.

\subsection{Summary}

Work profiles suggest that three-quarters of young men and about one-half of young women had at some time engaged in paid or unpaid work. Indeed, almost all married young men and two-thirds of unmarried young men had done so, compared with over half and about two-fifths of married and unmarried young women, respectively. Likewise, more youth in rural than urban areas had ever worked. While the majority of youth were engaged in paid work, considerable proportions of young men (38\%) and women (25\%) reported unpaid work on the family farm or business. Economic activity was often initiated at an early age: over one in three young men and women reported initiating work as children (before age 15). Data on work participation in the 12 months prior to interview indicate that the majority of young men (64\% of unmarried and $97 \%$ of married) and a substantial proportion of young women (36\% and $38 \%$, respectively) had engaged in paid or unpaid work at some point in the 12 months preceding the survey. The majority of young men (64\%) who worked in the year prior to the interview had done so for the major part (at least six months) of the year. In contrast among young women, over two-fifths (44\%) had done so.

Occupational profiles among those working for wages differed somewhat among young men and considerably among young women in rural and urban areas. In rural areas, among young men, the leading occupations were agricultural work, unskilled non-agricultural and skilled labour, together reported by $86 \%$ of young men. Among young women in rural areas, the leading occupations were agricultural, particularly agricultural labour in which as many as $86 \%$ were employed. In urban areas, leading occupations were similar for both young men and women and included unskilled non-agricultural labour, skilled labour and administrative, executive, managerial and clerical occupations together reported by $82 \%$ of young men and $77 \%$ of young 
women. It is also notable that more unmarried young women reported administrative, executive, managerial or clerical occupations, compared to other urban groups.

Findings also show substantial levels of unemployment among young men (22\%) and women (36\%). Unemployment tended to be considerably higher among unmarried than married young men, but similar among married and unmarried young women. Unemployment was particularly high among the educated and economically better off.

Youth were clearly interested in acquiring skills that would enable employment generation; two in three young men and four in five young women reported interest in vocational skill training. However, far fewer-just $14 \%$ of young men and $10 \%$ of young women — had attended even one vocational training programme. 


\section{Chapter 5}

\section{Media exposure and access to}

pornographic materials

Media may play an important role in shaping the attitudes and behaviours of youth. Youth gain access to new information through a variety of sources, including print and visual media and, increasingly, the internet. Many are also exposed to pornography through these channels. The Youth Study probed young people's exposure to various media sources, the extent of their exposure to pornographic materials by way of books/ magazines, films and the internet, and their perceptions about the influence of television and films on youth behaviours.

\subsection{Mass media exposure}

The survey asked a number of questions regarding youth exposure to mass media. These included whether and how frequently young people read newspapers, magazines or books, watched films, watched television programmes other than movies, and accessed the internet. Questions regarding exposure to print media and the internet were asked only of those who had attained at least five years of education, as this was considered a prerequisite for basic literacy and, thus, understanding such materials. Youth were asked to rate the frequency of their exposure to each medium according to the categories "never," "sometimes" and "often". If any young person did not respond in this format but rather, in terms of days per week, three or more exposures per week were classified as "often" and less frequent exposure as "sometimes".

Findings are presented in Table 5.1 and Figure 5.1. They suggest that youth were exposed to a variety of media, but that typically, more young men than women reported media exposure. The largest proportion of youth was exposed to print materials (newspapers, magazines or books) $-89 \%$ of young men and $81 \%$ of young women who had completed five or more years of education. Exposure to television was reported by $78 \%$ of all young men and $48 \%$ of all young women. A somewhat larger proportion of young men (89\%) and young women $(59 \%)$ watched films either on CD/DVD or at a theatre or video parlour. Few youth with five or more years of education accessed the internet: $6 \%$ of young men and $3 \%$ of young women.
Figure 5.1: Percentage of youth exposed to television, films, print media and the internet, Bihar, 2007

Combined

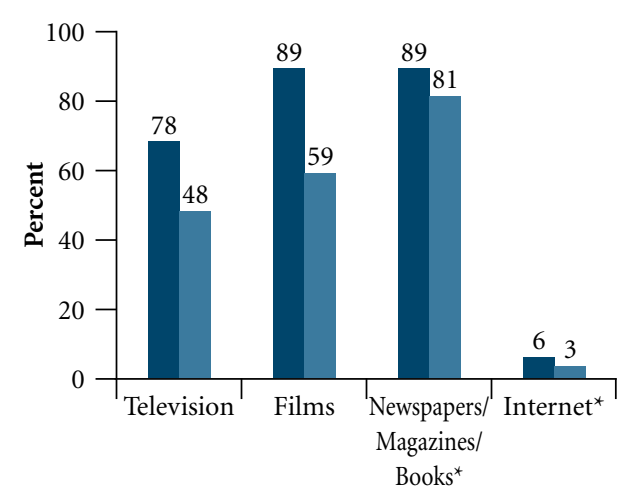

Urban

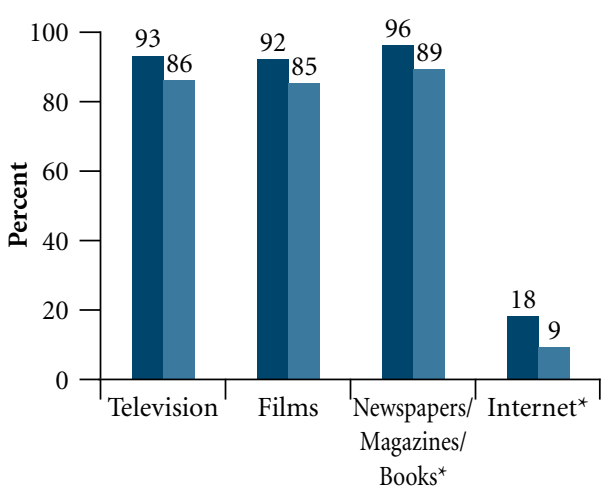

Rural

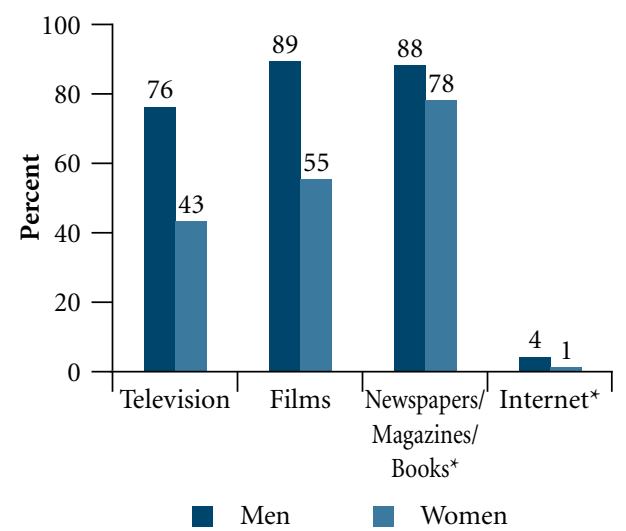

Note: ${ }^{*}$ Question asked only of respondents who had completed five or more years of education. 
Table 5.1: Mass media exposure

Percent distribution of youth exposed to various mass media by frequency of exposure, according to residence, Bihar, 2007

\begin{tabular}{|c|c|c|c|c|c|c|}
\hline Exposure indicators (\%) & $\begin{array}{c}M \\
15-24\end{array}$ & $\begin{array}{c}\text { W } \\
15-24\end{array}$ & $\begin{array}{c}\text { MM } \\
15-29\end{array}$ & $\begin{array}{l}\text { MW } \\
15-24 \\
\end{array}$ & $\begin{array}{c}\text { UM } \\
15-24\end{array}$ & $\begin{array}{l}\text { UW } \\
15-24\end{array}$ \\
\hline \multicolumn{7}{|c|}{ Combined } \\
\hline Frequency of watching television & & & & & & \\
\hline Never & 21.7 & 52.0 & 26.4 & 57.9 & 19.9 & 43.5 \\
\hline Sometimes & 68.8 & 38.8 & 68.0 & 36.5 & 68.5 & 43.1 \\
\hline Often & 9.5 & 9.1 & 5.7 & 5.6 & 11.6 & 13.4 \\
\hline \multicolumn{7}{|l|}{ Frequency of watching films } \\
\hline Never & 10.5 & 41.4 & 10.0 & 46.0 & 10.6 & 34.4 \\
\hline Sometimes & 87.3 & 57.0 & 87.8 & 53.0 & 86.9 & 63.2 \\
\hline Often & 2.0 & 1.6 & 1.8 & 1.0 & 2.5 & 2.4 \\
\hline Number of respondents & 1,942 & 5,529 & 1,115 & 2,341 & 1,492 & 3,188 \\
\hline \multicolumn{7}{|l|}{$\begin{array}{l}\text { Frequency of reading newspapers/ } \\
\text { magazines/books }\end{array}$} \\
\hline Never & 10.6 & 18.8 & 10.9 & 24.2 & 10.3 & 14.6 \\
\hline Sometimes & 55.0 & 58.1 & 66.7 & 65.1 & 51.8 & 52.3 \\
\hline Often & 34.0 & 22.6 & 22.1 & 10.1 & 37.4 & 32.6 \\
\hline \multicolumn{7}{|l|}{ Frequency of accessing the internet ${ }^{1}$} \\
\hline Never & 93.5 & 96.9 & 96.3 & 98.5 & 92.2 & 96.1 \\
\hline Sometimes & 5.2 & 2.2 & 2.6 & 0.9 & 6.3 & 2.8 \\
\hline Often & 0.8 & 0.3 & 0.8 & 0.0 & 1.0 & 0.4 \\
\hline Number with 5 or more years of education & 1,498 & 3,059 & 720 & 935 & 1,212 & 2,124 \\
\hline \multicolumn{7}{|c|}{ Urban } \\
\hline \multicolumn{7}{|l|}{ Frequency of watching television } \\
\hline Never & 7.1 & 14.0 & 7.7 & 21.1 & 6.9 & 8.9 \\
\hline Sometimes & 60.4 & 43.2 & 67.0 & 44.4 & 58.8 & 42.5 \\
\hline Often & 32.5 & 42.8 & 25.3 & 34.5 & 34.3 & 48.6 \\
\hline \multicolumn{7}{|l|}{ Frequency of watching films } \\
\hline Never & 7.9 & 15.2 & 6.5 & 18.3 & 8.2 & 13.0 \\
\hline Sometimes & 86.7 & 78.3 & 90.2 & 76.8 & 86.5 & 79.6 \\
\hline Often & 5.4 & 6.4 & 3.3 & 4.9 & 5.3 & 7.5 \\
\hline Number of respondents & 1,039 & 2,581 & 547 & 1,136 & 833 & 1,445 \\
\hline \multicolumn{7}{|l|}{$\begin{array}{l}\text { Frequency of reading newspapers/ } \\
\text { magazines } / \text { books }^{1}\end{array}$} \\
\hline Never & 4.3 & 10.5 & 6.0 & 19.5 & 3.9 & 6.4 \\
\hline Sometimes & 42.7 & 52.2 & 53.7 & 63.6 & 40.6 & 46.7 \\
\hline Often & 53.0 & 36.8 & 40.3 & 16.9 & 55.6 & 46.4 \\
\hline \multicolumn{7}{|l|}{ Frequency of accessing the internet ${ }^{1}$} \\
\hline Never & 82.3 & 90.4 & 91.0 & 96.2 & 80.7 & 87.7 \\
\hline Sometimes & 14.3 & 7.8 & 7.5 & 3.8 & 15.5 & 9.9 \\
\hline Often & 3.5 & 1.3 & 1.5 & 0.0 & 3.9 & 2.0 \\
\hline Number with 5 or more years of education & 845 & 1,801 & 394 & 617 & 701 & 1,184 \\
\hline
\end{tabular}

Cont'd on next page... 
Table 5.1: (Cont'd)

\begin{tabular}{|c|c|c|c|c|c|c|}
\hline Exposure indicators (\%) & $\begin{array}{c}\mathrm{M} \\
15-24\end{array}$ & $\begin{array}{c}\text { W } \\
15-24\end{array}$ & $\begin{array}{c}\text { MM } \\
15-29\end{array}$ & $\begin{array}{c}\text { MW } \\
15-24\end{array}$ & $\begin{array}{c}\text { UM } \\
15-24\end{array}$ & $\begin{array}{c}\text { UW } \\
15-24\end{array}$ \\
\hline \multicolumn{7}{|c|}{ Rural } \\
\hline Frequency of watching television & & & & & & \\
\hline Never & 24.2 & 57.0 & 28.0 & 60.3 & 22.4 & 49.8 \\
\hline Sometimes & 70.1 & 38.3 & 68.1 & 36.0 & 70.4 & 43.2 \\
\hline Often & 5.7 & 4.7 & 3.9 & 3.7 & 7.2 & 6.9 \\
\hline \multicolumn{7}{|l|}{ Frequency of watching films } \\
\hline Never & 10.9 & 44.8 & 10.3 & 47.8 & 11.1 & 38.3 \\
\hline Sometimes & 87.4 & 54.3 & 87.6 & 51.5 & 87.0 & 60.2 \\
\hline Often & 1.5 & 1.0 & 1.7 & 0.7 & 1.9 & 1.4 \\
\hline Number of respondents & 903 & 2,948 & 568 & 1,205 & 659 & 1,743 \\
\hline \multicolumn{7}{|l|}{$\begin{array}{l}\text { Frequency of reading newspapers/ } \\
\text { magazines/books }{ }^{1}\end{array}$} \\
\hline Never & 11.8 & 20.9 & 11.5 & 24.9 & 11.7 & 16.8 \\
\hline Sometimes & 57.3 & 59.6 & 68.2 & 65.2 & 54.1 & 53.8 \\
\hline Often & 30.4 & 18.8 & 20.0 & 9.3 & 33.6 & 28.7 \\
\hline \multicolumn{7}{|l|}{ Frequency of accessing the internet ${ }^{1}$} \\
\hline Never & 95.7 & 98.6 & 96.9 & 98.6 & 94.6 & 98.4 \\
\hline Sometimes & 3.5 & 0.7 & 2.1 & 0.7 & 4.4 & 0.9 \\
\hline Often & 0.3 & 0.0 & 0.7 & 0.0 & 0.4 & 0.0 \\
\hline Number with 5 or more years of education & 653 & 1,258 & 326 & 318 & 511 & 940 \\
\hline
\end{tabular}

Note: All Ns are unweighted. Column totals may not equal 100\% due to missing cases or "don't know" responses. ${ }^{1}$ Question asked only of respondents who had completed five or more years of education.

Differences by marital status varied between young men and young women. Among young men, more unmarried than married youth reported exposure to television; however, differences were muted with respect to exposure to other media. Among young women, the unmarried were consistently more likely to be exposed to each medium than the married and this was apparent, by and large, in both rural and urban areas. Frequency of media exposure also varied. For example, findings show that more unmarried than married youth reported frequent exposure to television and print media, irrespective of rural-urban residence. Differences by ruralurban residence were also evident, with urban youth-particularly young women-more likely than rural youth to be exposed to the media. Notably, some $9 \%$ of young women and $18 \%$ of young men in urban settings accessed the internet, compared to less than $1 \%$ and $4 \%$, respectively, of rural youth.

\subsection{Exposure to pornographic materials}

Youth were asked whether they were exposed to pornographic materials by way of films, books and magazines, and the internet (for those who accessed the internet). Table 5.2 reports that $26 \%$ of young men had watched "blue" or pornographic films, compared to $4 \%$ of young women. More married than unmarried youth were exposed to pornographic films (38\% versus $23 \%$ among young men and $6 \%$ versus $1 \%$ among young women). Differences by rural-urban residence were wide among young men, with more urban youth reporting exposure to pornographic films than rural youth, irrespective of marital status: $25 \%$ of rural young men compared to $35 \%$ of their urban counterparts had ever watched a pornographic film. Among young women, the differences were apparent among the married (5\% and $13 \%$, respectively, of rural and urban respondents), but muted among the unmarried (1-2\%). 
Table 5.2: Exposure to pornographic materials

Percentage of youth exposed to different pornographic materials, according to residence, Bihar, 2007

\begin{tabular}{|c|c|c|c|c|c|c|}
\hline Exposure indicators (\%) & $\begin{array}{c}\mathrm{M} \\
15-24\end{array}$ & $\begin{array}{c}\text { W } \\
15-24\end{array}$ & $\begin{array}{c}\text { MM } \\
15-29\end{array}$ & $\begin{array}{c}\text { MW } \\
15-24\end{array}$ & $\begin{array}{c}\text { UM } \\
15-24\end{array}$ & $\begin{array}{l}\text { UW } \\
15-24\end{array}$ \\
\hline \multicolumn{7}{|c|}{ Combined } \\
\hline Ever watched a "blue"/pornographic film & 26.0 & 4.1 & 37.5 & 5.6 & 23.0 & 0.9 \\
\hline Number of respondents & 1,942 & 5,529 & 1,115 & 2,341 & 1,492 & 3,188 \\
\hline Frequency of watching "blue"/pornographic films & & & & & & \\
\hline Rarely & 56.8 & 70.2 & 56.7 & 69.7 & 55.7 & $(79.3)$ \\
\hline Sometimes & 41.1 & 29.8 & 41.4 & 30.3 & 42.3 & $(20.7)$ \\
\hline Often & 1.6 & 0.0 & 0.5 & 0.0 & 1.7 & $(0.0)$ \\
\hline \multicolumn{7}{|l|}{$\begin{array}{l}\text { Person accompanying when watching "blue"/ } \\
\text { pornographic films }\end{array}$} \\
\hline Alone & 8.4 & 10.3 & 11.2 & 6.0 & 7.9 & $(55.2)$ \\
\hline $\operatorname{Peer}(\mathrm{s})$ & 89.0 & 12.5 & 84.7 & 11.3 & 89.2 & $(34.5)$ \\
\hline Spouse & NA & NA & 3.1 & 77.4 & NA & NA \\
\hline Other(s) & 1.8 & 4.0 & 1.0 & 3.8 & 2.9 & $(10.3)$ \\
\hline $\begin{array}{l}\text { Ever forced by anyone to watch "blue"/ } \\
\text { pornographic films }\end{array}$ & 21.5 & 34.7 & 20.5 & 37.1 & 21.6 & $(10.3)$ \\
\hline $\begin{array}{l}\text { Number who ever watched "blue"/ } \\
\text { pornographic films }\end{array}$ & 597 & 249 & 491 & 213 & 412 & 36 \\
\hline Ever read/looked at pornographic books/magazines & 20.9 & 5.7 & 25.7 & 6.3 & 19.3 & 4.3 \\
\hline Number of respondents & 1,942 & 5,529 & 1,115 & 2,341 & 1,492 & 3,188 \\
\hline \multicolumn{7}{|l|}{$\begin{array}{l}\text { Frequency of reading/looking at pornographic } \\
\text { books/ magazines }\end{array}$} \\
\hline Rarely & 63.8 & 48.7 & 61.9 & 57.7 & 68.2 & 27.1 \\
\hline Sometimes & 35.2 & 47.5 & 37.4 & 40.9 & 31.1 & 62.1 \\
\hline Often & 0.5 & 2.8 & 0.0 & 0.0 & 0.7 & 9.3 \\
\hline $\begin{array}{l}\text { Number who ever read/looked at pornographic } \\
\text { books/magazines }\end{array}$ & 457 & 345 & 328 & 177 & 334 & 168 \\
\hline Ever accessed pornographic materials on the internet & 32.6 & 10.9 & $(19.0)$ & * & 30.6 & 12.9 \\
\hline Number who ever accessed the internet & 166 & 167 & 43 & 22 & 156 & 145 \\
\hline \multicolumn{7}{|c|}{ Urban } \\
\hline Ever watched a "blue"/pornographic film & 35.1 & 6.4 & 51.6 & 13.4 & 33.1 & 1.6 \\
\hline Number of respondents & 1,039 & 2,581 & 547 & 1,136 & 833 & 1,445 \\
\hline Frequency of watching "blue"/pornographic films & & & & & & \\
\hline Rarely & 54.1 & 61.0 & 55.3 & 63.2 & 54.4 & * \\
\hline Sometimes & 42.9 & 39.0 & 44.7 & 36.8 & 43.0 & * \\
\hline Often & 2.0 & 0.0 & 0.0 & 0.0 & 1.3 & * \\
\hline \multicolumn{7}{|l|}{$\begin{array}{l}\text { Person accompanying when watching "blue"/ } \\
\text { pornographic films }\end{array}$} \\
\hline Alone & 9.2 & 14.6 & 19.1 & 5.6 & 8.6 & * \\
\hline $\operatorname{Peer}(\mathrm{s})$ & 88.8 & 7.3 & 76.6 & 0.0 & 90.1 & * \\
\hline Spouse & NA & NA & 4.3 & 94.4 & NA & NA \\
\hline Other(s) & 1.0 & 0.0 & 0.0 & 0.0 & 1.2 & * \\
\hline $\begin{array}{l}\text { Ever forced by anyone to watch "blue"/ } \\
\text { pornographic films }\end{array}$ & 19.4 & 29.3 & 14.6 & 31.6 & 18.8 & * \\
\hline $\begin{array}{l}\text { Number who ever watched "blue"/ } \\
\text { pornographic films }\end{array}$ & 376 & 174 & 284 & 152 & 273 & 22 \\
\hline
\end{tabular}


Table 5.2: (Cont'd)

\begin{tabular}{|c|c|c|c|c|c|c|}
\hline Exposure indicators (\%) & $\begin{array}{c}\text { M } \\
15-24\end{array}$ & $\begin{array}{c}\text { W } \\
15-24\end{array}$ & $\begin{array}{c}\text { MM } \\
15-29\end{array}$ & $\begin{array}{c}\text { MW } \\
15-24\end{array}$ & $\begin{array}{c}\text { UM } \\
15-24\end{array}$ & $\begin{array}{l}\text { UW } \\
15-24\end{array}$ \\
\hline \multicolumn{7}{|c|}{ Urban } \\
\hline Ever read/looked at pornographic books/magazines & 26.1 & 7.8 & 33.7 & 9.2 & 25.7 & 7.1 \\
\hline Number of respondents & 1,039 & 2,581 & 547 & 1,136 & 833 & 1,445 \\
\hline \multicolumn{7}{|l|}{$\begin{array}{l}\text { Frequency of reading/looking at pornographic } \\
\text { books/ magazines }\end{array}$} \\
\hline Rarely & 71.2 & 26.0 & 64.5 & 33.3 & 73.0 & 22.9 \\
\hline Sometimes & 28.8 & 70.0 & 35.5 & 66.7 & 27.0 & 71.4 \\
\hline Often & 0.0 & 4.0 & 0.0 & 0.0 & 0.0 & 5.7 \\
\hline $\begin{array}{l}\text { Number who ever read/looked at pornographic } \\
\text { books/magazines }\end{array}$ & 274 & 204 & 182 & 103 & 214 & 101 \\
\hline Ever accessed pornographic materials on the internet & 50.0 & 7.3 & $(50.0)$ & * & 50.0 & 4.2 \\
\hline Number who ever accessed the internet & 142 & 157 & 34 & 20 & 133 & 137 \\
\hline \multicolumn{7}{|c|}{ Rural } \\
\hline Ever watched a "blue"/pornographic film & 24.5 & 3.7 & 36.3 & 5.1 & 21.0 & 0.8 \\
\hline Number of respondents & 903 & 2,498 & 568 & 1,205 & 659 & 1,743 \\
\hline Frequency of watching "blue"/pornographic films & & & & & & \\
\hline Rarely & 57.4 & 72.3 & 56.9 & 71.7 & 56.1 & * \\
\hline Sometimes & 40.7 & 27.7 & 41.0 & 28.3 & 42.4 & * \\
\hline Often & 1.5 & 0.0 & 0.5 & 0.0 & 1.5 & * \\
\hline \multicolumn{7}{|l|}{$\begin{array}{l}\text { Person accompanying when watching "blue"/ } \\
\text { pornographic films }\end{array}$} \\
\hline Alone & 8.2 & 9.3 & 10.0 & 6.2 & 7.6 & * \\
\hline $\operatorname{Peer}(\mathrm{s})$ & 88.9 & 14.2 & 85.9 & 12.4 & 88.9 & * \\
\hline Spouse & NA & NA & 3.0 & 76.1 & NA & NA \\
\hline Other(s) & 2.2 & 4.4 & 1.1 & 3.5 & 3.4 & * \\
\hline $\begin{array}{l}\text { Ever forced by anyone to watch "blue"/ } \\
\text { pornographic films }\end{array}$ & 22.0 & 36.1 & 21.4 & 38.1 & 22.1 & * \\
\hline $\begin{array}{l}\text { Number who ever watched "blue"/ } \\
\text { pornographic films }\end{array}$ & 221 & 75 & 207 & 61 & 139 & 14 \\
\hline Ever read/looked at pornographic books/magazines & 20.0 & 5.4 & 25.0 & 6.1 & 18.1 & 3.9 \\
\hline Number of respondents & 903 & 2,948 & 568 & 1,205 & 659 & 1,743 \\
\hline \multicolumn{7}{|l|}{$\begin{array}{l}\text { Frequency of reading/looking at pornographic } \\
\text { books/ magazines }\end{array}$} \\
\hline Rarely & 62.2 & 52.8 & 61.8 & 59.9 & 66.8 & 28.6 \\
\hline Sometimes & 36.6 & 43.4 & 37.5 & 38.7 & 32.3 & 59.0 \\
\hline Often & 0.6 & 2.6 & 0.0 & 0.0 & 0.9 & 10.5 \\
\hline $\begin{array}{l}\text { Number who ever read/looked at pornographic } \\
\text { books/ magazines }\end{array}$ & 183 & 141 & 146 & 74 & 120 & 67 \\
\hline Ever accessed pornographic materials on the internet & * & * & * & * & * & * \\
\hline Number who ever accessed the internet & 24 & 10 & 9 & 2 & 23 & 8 \\
\hline
\end{tabular}

Note: All Ns are unweighted. Column totals may not equal 100\% due to missing cases or "don't know" responses. ( ) Based on 25-49 unweighted cases. ${ }^{*}$ Percentage not shown, based on fewer than 25 unweighted cases. NA: Not applicable. 
Among youth who had ever watched a pornographic film, 43\% of young men and 30\% of young women reported having viewed such films sometimes or frequently (see Table 5.2). Because almost no unmarried young women had ever watched a pornographic film, we restrict the remaining discussion to young men and married young women. Differences by marital status and residence were negligible among young men; among marred young women, those in urban settings were more likely than those in rural areas, to have viewed pornographic films sometimes or often. Most young men had watched pornographic films together with friends. Among young women, three-quarters of the married reported that they had watched such films together with their husbands. One in five young men (22\%) and over one in three married young women $(37 \%)$ reported that they had been pressured or forced, at least once, to do so.

Exposure to pornographic books and magazines was reported by $21 \%$ of young men and $6 \%$ of young women. Married young men were somewhat more likely than the unmarried to report exposure to pornographic books and magazines, but no such differences were evident among young women. Somewhat more urban youth, particularly young men, reported exposure to pornographic books and magazines, compared to rural youth. Over one-third of all young men and one-half of young women who reported reading or looking at pornographic books or magazines did so sometimes or often. Marital status differences suggest that while married young men were somewhat more likely than the unmarried to report as such $(37 \%$ and $32 \%$ of married and unmarried young men, respectively), the reverse was true for young women $(41 \%$ and $71 \%$ of married and unmarried young women, respectively). Finally, more rural than urban young men reported frequent exposure; among young women, particularly the married, a reverse pattern was evident.

Of those exposed to the internet, one-third of young men (33\%) and one-tenth of young women (11\%) had accessed pornographic materials on the internet.

\subsection{Youth perceptions about the influence of television and films on youth behaviours}

The survey also questioned youth about their perceptions of the influence of television and films on youth behaviours. Specifically, they were asked whether they believed that television and films influenced the way in which their friends dressed, whether violence on television and in films could make youth aggressive and whether they had ever felt like having sex after watching certain films. Table 5.3 suggests that about three-fifths of young men and half of young women believed that television and films influenced the way their friends dressed. About three-fifths of young men and women believed that violence on television and in films could make youth aggressive. One-quarter of young men and one-tenth of young women reported that watching certain films had made them desire sex. By and large, gender differences were narrow except that more young men than women felt that television and films influenced their friends' or their own behaviours.

Differences in reported perceptions by marital status suggest that by and large more unmarried than married youth believed that television and films influenced the way youth dress and more unmarried than married young women believed that television and films influenced youth aggressiveness. Conversely, more married than unmarried youth reported that watching certain films had made them desire sex. Rural-urban differences were mild among young men, with those in urban areas mildly more likely to report that television and films influenced youth dress and their own desire for sex. Differences were wider among young women with regard to media influence on their friends' behaviours. 
Table 5.3: Perceptions about the influence of television and films on youth behaviours

Percentage of youth reporting perceptions regarding the influence of television and films on youth behaviours, according to residence, Bihar, 2007

\begin{tabular}{|c|c|c|c|c|c|c|}
\hline $\begin{array}{l}\text { Perceptions about the influence of } \\
\text { television and films (\%) }\end{array}$ & $\underset{15-24}{M}$ & $\begin{array}{c}\text { W } \\
15-24\end{array}$ & $\begin{array}{c}\text { MM } \\
15-29\end{array}$ & $\begin{array}{c}\text { MW } \\
15-24\end{array}$ & $\begin{array}{c}\text { UM } \\
15-24\end{array}$ & $\begin{array}{l}\text { UW } \\
15-24\end{array}$ \\
\hline \multicolumn{7}{|c|}{ Combined } \\
\hline TV/films influence the way friends dress & 57.5 & 50.4 & 46.6 & 45.0 & 58.9 & 59.7 \\
\hline Violence on TV and in films can make youth aggressive & 57.9 & 57.4 & 58.8 & 54.1 & 57.5 & 62.8 \\
\hline Certain films make respondent want to have sex & 24.0 & 9.5 & 35.0 & 12.8 & 20.8 & 3.3 \\
\hline Number of respondents & 1,942 & 5,529 & 1,115 & 2,341 & 1,492 & 3,188 \\
\hline \multicolumn{7}{|c|}{ Urban } \\
\hline TV/films influence the way friends dress & 55.0 & 65.8 & 47.8 & 62.0 & 55.7 & 68.6 \\
\hline Violence on TV and in films can make youth aggressive & 63.1 & 72.6 & 67.0 & 68.5 & 62.9 & 75.6 \\
\hline Certain films make respondent want to have sex & 28.9 & 9.9 & 42.4 & 17.6 & 26.9 & 4.3 \\
\hline Number of respondents & 1,039 & 2,581 & 547 & 1,136 & 833 & 1,445 \\
\hline \multicolumn{7}{|c|}{ Rural } \\
\hline TV/films influence the way friends dress & 57.9 & 48.4 & 46.6 & 43.9 & 59.5 & 58.1 \\
\hline Violence on TV and in films can make youth aggressive & 57.1 & 55.5 & 58.2 & 53.1 & 56.4 & 60.4 \\
\hline Certain films make respondent want to have sex & 23.2 & 9.5 & 34.2 & 12.5 & 19.6 & 3.1 \\
\hline Number of respondents & 903 & 2,948 & 568 & 1,205 & 659 & 1,743 \\
\hline
\end{tabular}

Note: All Ns are unweighted.

\subsection{Summary}

Findings suggest that large proportions of all youth in Bihar were exposed to the media, typically newspapers, magazines or books ( $89 \%$ of young men and $81 \%$ of young women with five or more years of education), films ( $89 \%$ of all young men and $59 \%$ of all young women) and television ( $78 \%$ of all young men and $48 \%$ of all young women). Exposure to the internet was reported by far fewer (6\% of young men and 3\% of young women with five or more years of education). Gender differences were apparent, with young men typically more likely to be exposed to each medium than young women.

Findings also suggest that one in four young men and $4 \%$ of young women watched pornographic films, and of these, about two-fifths reported having viewed such films sometimes or frequently. One in five young men and $6 \%$ of young women accessed pornographic books and magazines, and of these, one-third of young men and half of young women reported that they accessed these materials sometimes or frequently. Of those exposed to the internet, one in three young men and one in ten young women had accessed pornographic materials on the internet. Finally, between half and three-fifths of young men and women acknowledged the influence that media have on youth behaviours. 


\section{Chapter 6}

\section{Growing up}

This chapter focuses on such experiences as puberty as well as youth interaction with parents and peers while growing up. Globally, studies have suggested a declining age at puberty for young men and women and stress that this, along with rising ages at marriage, provides a longer window in which young people can make same- and opposite-sex friends (National Research Council and Institute of Medicine, 2005). Several studies have highlighted the importance of close parental interaction for healthy development of young people (Laird et al., 2003; Marta, 1997; Sroufe, 1991). Others note that young people's interaction with parents is particularly limited when it comes to discussion of sensitive issues, for example, girl-boy relations or sexual and reproductive matters (Alexander et al., 2006a; 2006b; Lambert and Wood, 2005; Mehra, Savithri and Coutinho, 2002). In addition, a few studies have shown that the peer group is, for many youth, a central source of both information and support, and at the same time, a source of misinformation and pressure to adopt risky behaviours (Bhuiya et al., 2003; Sachdev, 1998; Ul Haque and Faizunnisa, 2003).

The Youth Study included several questions relating to each of these issues. This chapter begins by describing the ages at which young people experienced signs of puberty. It then explores aspects of their family life and interaction with parents on various matters of importance to youth. It also addresses peer networks and interaction, specifically, the size of the same- and opposite-sex peer network and peer activities in which respondents engaged. Finally, the chapter discusses young people's access to support networks for discussing personal matters.

\subsection{Puberty}

In order to examine ages at which puberty occurs among young men and women, the Youth Study included questions on age at menarche for young women and age at which voice change and growth of pubic hair were noticed for young men. Table 6.1a shows that mean age at menarche was 13.7 years for young women. Urban young women tended to experience menarche earlier than rural young women; for example, $18 \%$ of young women in urban areas compared to $5 \%$ of those in rural areas, attained menarche at or below age 12 .

Voice change and appearance of pubic hair for young men occurred somewhat later than did menarche for young women. Table $6.1 \mathrm{~b}$ shows that the average ages at which young men reported voice change and growth of pubic hair were 14.1 and 14.5 years, respectively. Rural-urban differences in the mean ages at which these changes occurred did not vary much.

\subsection{Family life and interaction with parents}

The Youth Study explored a variety of issues that capture the nature of family life, and youth interaction with parents in particular. Married respondents were specifically asked to recall the period before marriage. 
Table 6.1a: Age at puberty among young women

Percent distribution of young women aged 15-24 by age at puberty, according to residence, Bihar, 2007

\begin{tabular}{|c|c|c|c|}
\hline Puberty indicators (\%) & Combined & Urban & Rural \\
\hline Age at menarche (years) & & & \\
\hline Below 12 & 0.7 & 2.2 & 0.4 \\
\hline 12 & 5.5 & 15.5 & 4.2 \\
\hline 13 & 28.3 & 23.1 & 29.0 \\
\hline 14 & 17.6 & 24.8 & 16.7 \\
\hline 15 and above & 15.4 & 19.9 & 14.8 \\
\hline Not yet menstruated & 1.6 & 0.9 & 1.7 \\
\hline Mean age at menarche (years) ${ }^{1}$ & 13.7 & 13.6 & 13.7 \\
\hline Number of respondents & 5,529 & 2,581 & 2,948 \\
\hline
\end{tabular}

Note: All Ns are unweighted. Column totals may not equal 100\% due to missing cases or "don't know" responses. ${ }^{1}$ Excludes those who had not menstruated at the time of interview.

Table 6.1b: Age at puberty among young men

Percent distribution of young men aged 15-24 by age at puberty, according to residence, Bihar, 2007

\begin{tabular}{|c|c|c|c|}
\hline Puberty indicators (\%) & Combined & Urban & Rural \\
\hline \multicolumn{4}{|l|}{ Age at which voice change noticed (years) } \\
\hline Below 14 & 17.5 & 14.0 & 18.0 \\
\hline 14 & 14.1 & 15.4 & 13.8 \\
\hline 15 & 10.6 & 13.6 & 10.1 \\
\hline 16 & 6.0 & 8.6 & 5.5 \\
\hline 17 and above & 4.1 & 6.1 & 3.7 \\
\hline No voice change yet & 3.9 & 3.9 & 3.9 \\
\hline Did not notice/don't remember & 43.9 & 38.4 & 44.9 \\
\hline Mean age at voice change (years) ${ }^{1}$ & 14.1 & 14.5 & 14.1 \\
\hline \multicolumn{4}{|l|}{ Age at which pubic hair noticed (years) } \\
\hline Below 14 & 14.6 & 13.3 & 14.8 \\
\hline 14 & 25.6 & 28.7 & 25.1 \\
\hline 15 & 22.8 & 24.7 & 22.5 \\
\hline 16 & 10.9 & 12.5 & 10.6 \\
\hline 17 and above & 5.5 & 5.0 & 5.6 \\
\hline No pubic hair yet & 3.8 & 1.8 & 4.2 \\
\hline Mean age at which pubic hair noticed ${ }^{1}$ & 14.5 & 14.6 & 14.5 \\
\hline Number of respondents & 1,942 & 1,039 & 903 \\
\hline
\end{tabular}

Note: All Ns are unweighted. Column totals may not equal 100\% due to missing cases or "don't know" responses. ${ }^{1}$ Excludes those who had not noticed voice change/appearance of pubic hair at the time of interview or did not remember age at the time of voice changelappearance of pubic hair.

\subsubsection{Socialisation experiences}

Table 6.2 presents findings on the socialisation experiences of youth during their teenage years as compared with siblings, or cousins of the opposite sex if the respondent did not have an opposite-sex sibling. Wide gender differences were evident in relation to respondents' freedom to go out; for example, three-fifths (59\%) of young men reported that they had more freedom to go out than their sisters or female cousins did; a 
considerably larger proportion-almost four-fifths_of young women agreed that they had less freedom to go out than their brothers or male cousins. With respect to housework expectations, it appears that young men and women perceived their role in housework somewhat differently. Just one-third of young men reported that they were expected to do less housework than their sisters or female cousins, suggesting that large proportions of households did not discriminate between their sons and daughters in terms of expectations regarding housework. Young women's responses, however, suggest wide gender differences; about three-quarters $(71 \%)$ of young women reported that they were expected to do more housework than their brothers or male cousins.

Table 6.2: Socialisation experiences

Percent distribution of youth by degree of mobility and housework responsibilities relative to an opposite-sex sibling/cousin, according to residence, Bihar, 2007

\begin{tabular}{|c|c|c|c|c|c|c|}
\hline Socialisation experiences (\%) & $\begin{array}{c}M \\
15-24\end{array}$ & $\begin{array}{c}W \\
15-24\end{array}$ & $\begin{array}{c}\text { MM } \\
15-29\end{array}$ & $\begin{array}{l}\text { MW } \\
15-24\end{array}$ & $\begin{array}{c}\text { UM } \\
15-24\end{array}$ & $\begin{array}{c}\text { UW } \\
15-24\end{array}$ \\
\hline \multicolumn{7}{|c|}{$\begin{array}{ll} & \text { Combined }\end{array}$} \\
\hline \multicolumn{7}{|l|}{$\begin{array}{l}\text { Respondent had less freedom }(\mathrm{W}) / \text { more freedom }(\mathrm{M}) \text { to } \\
\text { roam/go out than opposite-sex sibling or cousin }\end{array}$} \\
\hline Yes & 59.1 & 77.8 & 60.4 & 79.4 & 58.3 & 75.4 \\
\hline No & 36.3 & 21.7 & 33.1 & 20.0 & 37.6 & 24.3 \\
\hline \multicolumn{7}{|l|}{$\begin{array}{l}\text { Respondent was expected to do more housework }(\mathrm{W}) / \\
\text { less housework }(\mathrm{M}) \text { than opposite-sex sibling or cousin }\end{array}$} \\
\hline Yes & 31.5 & 71.2 & 31.9 & 74.5 & 32.5 & 65.5 \\
\hline No & 60.6 & 25.8 & 60.0 & 21.7 & 59.6 & 32.7 \\
\hline Number of respondents & 1,942 & 5,529 & 1,115 & 2,341 & 1,492 & 3,188 \\
\hline \multicolumn{7}{|c|}{$\begin{array}{ll} & \text { Urban }\end{array}$} \\
\hline \multicolumn{7}{|l|}{$\begin{array}{l}\text { Respondent had less freedom }(\mathrm{W}) / \text { more freedom }(\mathrm{M}) \text { to } \\
\text { roam/go out than opposite-sex sibling or cousin }\end{array}$} \\
\hline Yes & 59.9 & 69.2 & 62.4 & 74.6 & 60.2 & 65.4 \\
\hline No & 38.4 & 30.6 & 35.5 & 25.4 & 38.1 & 34.4 \\
\hline \multicolumn{7}{|l|}{$\begin{array}{l}\text { Respondent was expected to do more housework }(\mathrm{W}) / \\
\text { less housework (M) than opposite-sex sibling or cousin }\end{array}$} \\
\hline Yes & 41.9 & 61.6 & 40.2 & 67.6 & 42.2 & 57.1 \\
\hline No & 54.5 & 37.7 & 55.4 & 31.0 & 54.1 & 42.7 \\
\hline Number of respondents & 1,039 & 2,581 & 547 & 1,136 & 833 & 1,445 \\
\hline \multicolumn{7}{|l|}{$\begin{array}{ll}\text { Rural } \\
\end{array}$} \\
\hline \multicolumn{7}{|l|}{$\begin{array}{l}\text { Respondent had less freedom }(\mathrm{W}) / \text { more freedom }(\mathrm{M}) \text { to } \\
\text { roam/go out than opposite-sex sibling or cousin }\end{array}$} \\
\hline Yes & 58.9 & 78.9 & 60.2 & 79.7 & 57.9 & 77.2 \\
\hline No & 35.9 & 20.5 & 32.9 & 19.6 & 37.5 & 22.4 \\
\hline \multicolumn{7}{|l|}{$\begin{array}{l}\text { Respondent was expected to do more housework (W)/ } \\
\text { less housework (M) than opposite-sex sibling or cousin }\end{array}$} \\
\hline Yes & 29.8 & 72.4 & 31.1 & 74.9 & 30.6 & 67.1 \\
\hline No & 61.6 & 24.2 & 60.4 & 21.1 & 60.7 & 30.8 \\
\hline Number of respondents & 903 & 2,948 & 568 & 1,205 & 659 & 1,743 \\
\hline
\end{tabular}

Note: All Ns are unweighted. Column totals may not equal 100\% due to "unsure" responses. For married respondents, questions referred to the period prior to marriage. 
Figure 6.1: Percentage of youth reporting gendered socialisation experiences relative to an opposite-sex sibling/cousin, according to residence, Bihar, 2007
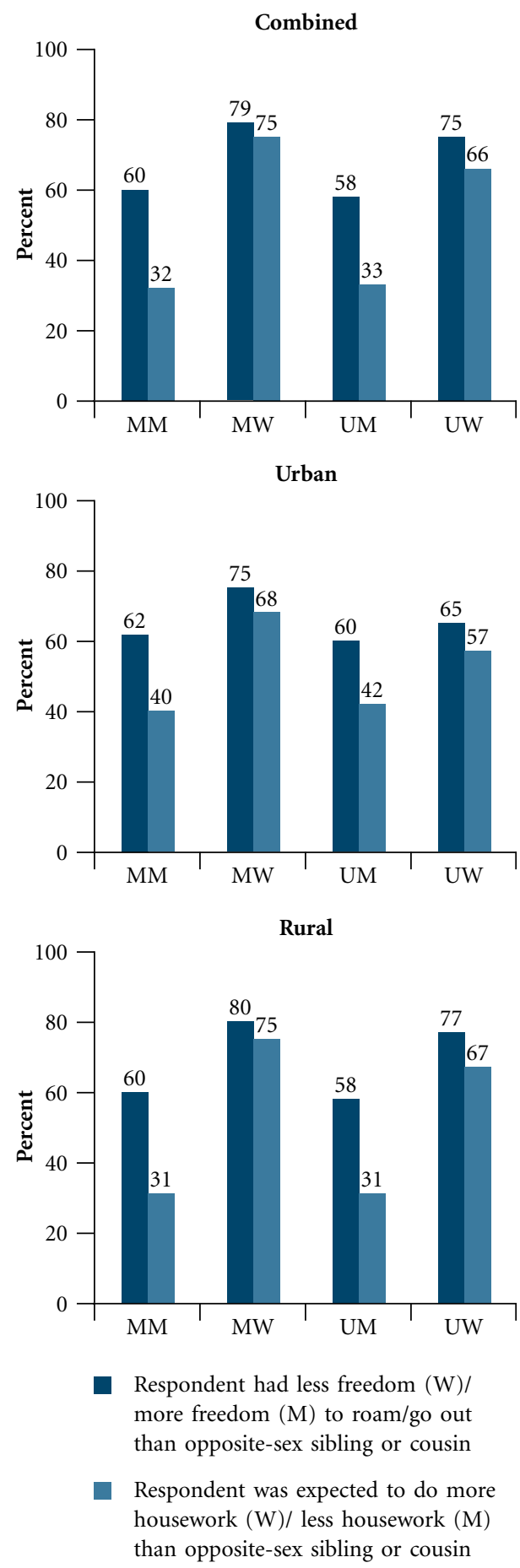

Note: For married respondents, questions referred to the period prior to marriage.
Differences by marital status were muted among young men. Among young women, even though larger proportions of both married and unmarried women reported gender unequal socialisation experiences, the unmarried, particularly in urban areas, were somewhat less likely to report so. Differences by rural-urban residence varied across young men and women. Among young men, while no differences were apparent in relation to freedom to go out, more urban than rural young men reported that they were expected to do less housework than their sisters or female cousins ( $42 \%$ and $30 \%$ respectively). Among young women, more rural than urban respondents reported gender unequal socialisation experiences on both matters (see Figure 6.1). Such findings may be explained by the likelihood that rural respondents and those who married early belonged to families adhering more closely to traditional gender norms than the families of other young women.

Parental attitudes towards youth friendships and social activities were probed by asking young men and women about whether their mothers and fathers, respectively, would disapprove if they engaged in a series of activities, ranging from bringing a same-sex friend to their home to having a love marriage. Married youth were asked to respond according to their experience prior to marriage. Findings, presented in Table 6.3, suggest considerable variation in youth perceptions by activity. What is clearly noticeable is that parents were most likely to be perceived to disapprove of love marriages for their children. This was reported by $93-94 \%$ of young men and $98-99 \%$ of young women. Also noticeable was that youth perceived parents to be far more likely to disapprove of activities conducted with members of the opposite sex than those conducted with same-sex individuals (see Figure 6.2). For example, just $9-10 \%$ of young men and $2 \%$ of young women reported that their mother or father would be angry if they brought same-sex friends to their home. In contrast, $74-80 \%$ of fathers and mothers of young men and $94-97 \%$ of fathers and mothers of young women were perceived to disapprove of activities in which youth may have engaged with an opposite sex friend, such as, for example, bringing an opposite-sex friend to their home, talking to a person of the opposite sex who did not belong to the family and going to a mela or film with someone of the opposite sex.

Also evident from Table 6.3 is that even though interactions with same-sex friends were less likely to be met with disapproval, $46-48 \%$ of young men and $28-37 \%$ of young women reported expecting parental disapproval if they went out with same-sex friends to a mela or film. It is notable, moreover, that more young men than women perceived parental disapproval if 
Table 6.3: Perceptions of parental reactions to selected activities

Percentage of youth who perceived that their parents would disapprove of them engaging in selected activities, according to residence, Bihar, 2007

\begin{tabular}{|c|c|c|c|c|c|c|}
\hline \multirow[t]{2}{*}{ Perceptions of parental reactions (\%) } & $\underset{15-24}{M}$ & $\begin{array}{c}\text { W } \\
15-24\end{array}$ & $\begin{array}{c}\text { MM } \\
15-29\end{array}$ & $\begin{array}{c}\text { MW } \\
15-24\end{array}$ & $\begin{array}{c}\text { UM } \\
15-24\end{array}$ & $\begin{array}{c}\text { UW } \\
15-24\end{array}$ \\
\hline & \multicolumn{6}{|c|}{ Father } \\
\hline \multicolumn{7}{|c|}{ Combined } \\
\hline \multicolumn{7}{|l|}{ Father would disapprove if respondent: } \\
\hline Brought opposite-sex friends home & 79.6 & 97.1 & 82.1 & 98.0 & 77.9 & 95.8 \\
\hline $\begin{array}{l}\text { Talked to a person of the opposite sex from } \\
\text { outside the home }\end{array}$ & 73.7 & 95.8 & 76.8 & 97.0 & 72.2 & 94.3 \\
\hline Went to a mela/film with same-sex friends & 46.3 & 37.4 & 45.7 & 37.8 & 48.3 & 36.9 \\
\hline Went to a mela/film with opposite-sex friends & 75.0 & 96.2 & 74.5 & 96.9 & 74.8 & 95.4 \\
\hline Joined a club or mandal & 25.6 & 49.5 & 25.9 & 50.7 & 25.9 & 47.5 \\
\hline Had a love marriage & 93.2 & 98.5 & 93.0 & 98.9 & 92.9 & 98.2 \\
\hline Found a job & NA & 14.6 & NA & 16.9 & NA & 10.8 \\
\hline Number of respondents ${ }^{1}$ & 1,704 & 4,973 & 878 & 2,012 & 1,332 & 2,961 \\
\hline \multicolumn{7}{|c|}{ Urban } \\
\hline \multicolumn{7}{|l|}{ Father would disapprove if respondent: } \\
\hline Brought same-sex friends home & 10.4 & 1.9 & 11.3 & 1.7 & 10.3 & 2.0 \\
\hline Brought opposite-sex friends home & 70.8 & 90.3 & 76.1 & 95.1 & 69.5 & 86.9 \\
\hline $\begin{array}{l}\text { Talked to a person of the opposite sex from } \\
\text { outside the home }\end{array}$ & 63.5 & 88.5 & 65.7 & 92.6 & 62.9 & 85.4 \\
\hline Went to a mela/film with same-sex friends & 42.5 & 36.9 & 42.3 & 36.9 & 43.2 & 36.9 \\
\hline Went to a mela/film with opposite-sex friends & 67.6 & 91.9 & 69.0 & 93.4 & 68.1 & 90.5 \\
\hline Joined a club or mandal & 30.8 & 45.7 & 26.8 & 45.9 & 31.0 & 45.6 \\
\hline Had a love marriage & 89.2 & 96.1 & 90.1 & 96.7 & 88.3 & 95.4 \\
\hline Found a job & NA & 12.7 & NA & 18.2 & NA & 8.6 \\
\hline Number of respondents ${ }^{1}$ & 890 & 2,298 & 421 & 973 & 728 & 1,325 \\
\hline \multicolumn{7}{|c|}{ Rural } \\
\hline Father would disapprove if respondent: & & & & & & \\
\hline Brought same-sex friends home & 10.2 & 2.4 & 11.9 & 2.9 & 10.1 & 1.3 \\
\hline Brought opposite-sex friends home & 81.0 & 97.9 & 82.6 & 98.2 & 79.4 & 97.4 \\
\hline $\begin{array}{l}\text { Talked to a person of the opposite sex from } \\
\text { outside the home }\end{array}$ & 75.2 & 96.8 & 77.9 & 97.3 & 74.0 & 95.8 \\
\hline Went to a mela/film with same-sex friends & 46.9 & 37.5 & 46.1 & 37.9 & 49.3 & 36.9 \\
\hline Went to a mela/film with opposite-sex friends & 76.1 & 96.8 & 75.0 & 97.0 & 76.0 & 96.2 \\
\hline Joined a club or mandal & 24.8 & 49.9 & 25.8 & 51.0 & 25.0 & 47.9 \\
\hline Had a love marriage & 93.8 & 98.8 & 93.2 & 98.9 & 93.7 & 98.7 \\
\hline Found a job & NA & 14.9 & NA & 16.8 & NA & 11.2 \\
\hline Number of respondents ${ }^{1}$ & 814 & 2,675 & 457 & 1,039 & 604 & 1,636 \\
\hline
\end{tabular}

Cont'd on next page... 
Table 6.3: (Cont'd)

\begin{tabular}{|c|c|c|c|c|c|c|}
\hline \multirow[t]{2}{*}{ Perceptions of parental reactions (\%) } & $\underset{15-24}{M}$ & $\begin{array}{c}\text { W } \\
15-24\end{array}$ & $\begin{array}{c}\text { MM } \\
15-29\end{array}$ & $\begin{array}{c}\text { MW } \\
15-24\end{array}$ & $\begin{array}{c}\text { UM } \\
15-24\end{array}$ & $\begin{array}{c}\text { UW } \\
15-24\end{array}$ \\
\hline & \multicolumn{6}{|c|}{ Mother } \\
\hline \multicolumn{7}{|c|}{ Combined } \\
\hline \multicolumn{7}{|l|}{ Mother would disapprove if respondent: } \\
\hline Brought opposite-sex friends home & 78.7 & 96.6 & 79.9 & 98.3 & 76.8 & 94.1 \\
\hline $\begin{array}{l}\text { Talked to a person of the opposite sex from } \\
\text { outside the home }\end{array}$ & 73.7 & 94.2 & 75.6 & 95.5 & 72.1 & 92.6 \\
\hline Went to a mela/film with same-sex friends & 47.6 & 28.0 & 46.0 & 26.8 & 49.5 & 29.9 \\
\hline Went to a mela/film with opposite-sex friends & 75.5 & 94.1 & 74.1 & 94.7 & 75.8 & 93.3 \\
\hline Joined a club or mandal & 23.8 & 43.1 & 23.5 & 44.0 & 24.5 & 41.7 \\
\hline Had a love marriage & 93.9 & 98.0 & 93.5 & 98.3 & 93.6 & 97.5 \\
\hline Found a job & NA & 10.5 & NA & 12.1 & NA & 7.7 \\
\hline Number of respondents ${ }^{1}$ & 1,819 & 5,172 & 968 & 2,121 & 1,417 & 3,051 \\
\hline \multicolumn{7}{|c|}{ Urban } \\
\hline $\begin{array}{l}\text { Mother would disapprove if respondent: } \\
\text { Brought same-sex friends home }\end{array}$ & 8.8 & 1.3 & 9.9 & 1.6 & 8.3 & 1.5 \\
\hline Brought opposite-sex friends home & 70.5 & 87.7 & 76.5 & 94.6 & 69.3 & 82.8 \\
\hline $\begin{array}{l}\text { Talked to a person of the opposite sex from } \\
\text { outside the home }\end{array}$ & 62.5 & 84.7 & 66.3 & 90.6 & 61.7 & 80.9 \\
\hline Went to a mela/film with same-sex friends & 41.6 & 31.4 & 40.7 & 29.5 & 42.2 & 33.1 \\
\hline Went to a mela/film with opposite-sex friends & 69.0 & 89.6 & 71.3 & 93.8 & 68.8 & 86.7 \\
\hline Joined a club or mandal & 29.1 & 41.2 & 25.9 & 43.4 & 29.4 & 39.6 \\
\hline Had a love marriage & 88.9 & 95.5 & 91.4 & 96.9 & 88.7 & 94.3 \\
\hline Found a job & NA & 9.8 & NA & 14.0 & NA & 6.8 \\
\hline Number of respondents ${ }^{1}$ & 971 & 2,409 & 482 & 1,031 & 787 & 1,378 \\
\hline \multicolumn{7}{|c|}{ Rural } \\
\hline Mother would disapprove if respondent: & & & & & & \\
\hline Brought same-sex friends home & 9.5 & 1.5 & 9.6 & 1.6 & 9.0 & 1.3 \\
\hline Brought opposite-sex friends home & 80.0 & 97.7 & 80.2 & 98.5 & 78.2 & 96.1 \\
\hline $\begin{array}{l}\text { Talked to a person of the opposite sex from } \\
\text { outside the home }\end{array}$ & 75.6 & 95.5 & 76.5 & 95.9 & 74.1 & 94.7 \\
\hline Went to a mela/film with same-sex friends & 48.5 & 27.6 & 46.4 & 26.7 & 50.9 & 29.3 \\
\hline Went to a mela/film with opposite-sex friends & 76.6 & 94.7 & 74.5 & 94.8 & 77.2 & 94.5 \\
\hline Joined a club or mandal & 23.0 & 43.4 & 23.3 & 44.1 & 23.6 & 42.1 \\
\hline Had a love marriage & 94.7 & 98.3 & 93.7 & 98.4 & 94.6 & 98.2 \\
\hline Found a job & NA & 10.6 & NA & 12.0 & NA & 7.8 \\
\hline Number of respondents ${ }^{1}$ & 848 & 2,763 & 486 & 1,090 & 630 & 1,673 \\
\hline
\end{tabular}

Note: All Ns are unweighted. NA: Not applicable. For married respondents, questions referred to the period prior to marriage.

${ }^{1}$ Includes only those respondents reporting that their father or mother, respectively, was alive at the time of interview. 
Figure 6.2: Percentage of youth reporting that their father and mother, respectively, would disapprove if they brought same- and opposite-sex friends home, according to residence, Bihar, 2007
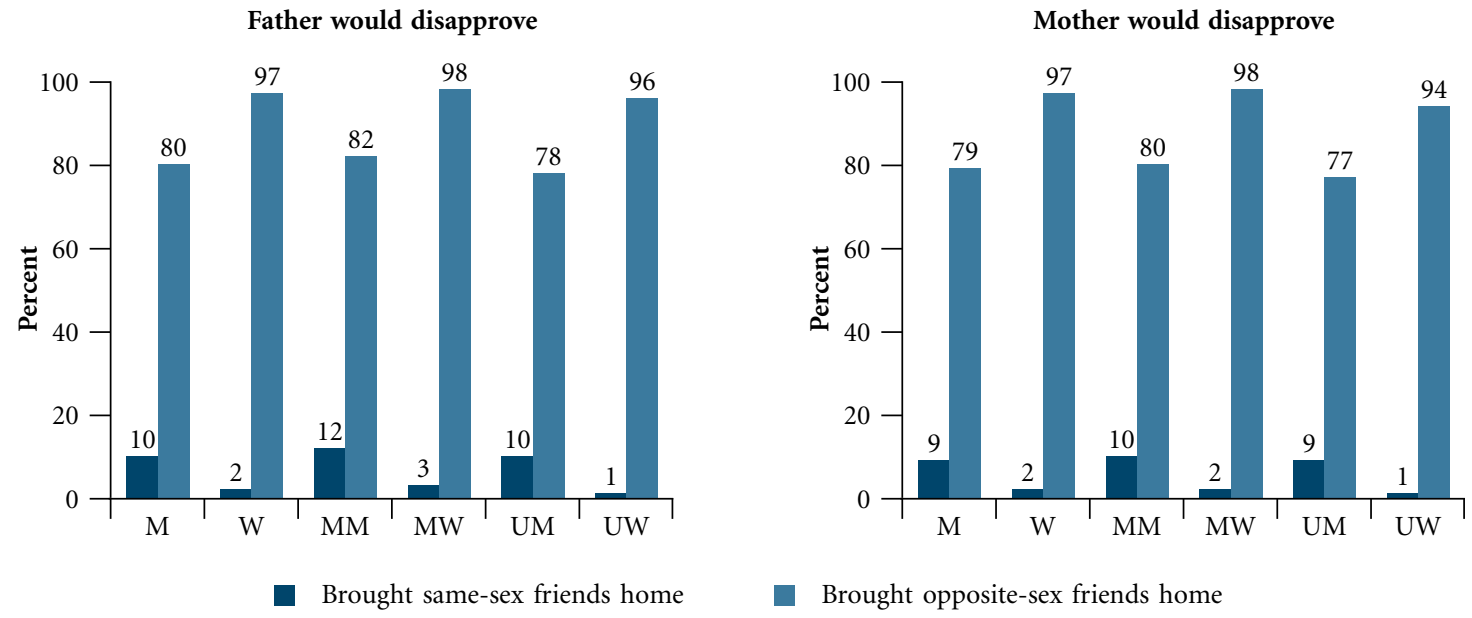

Note: For married respondents, questions referred to the period prior to marriage. Percentages were calculated only of those respondents reporting that their father or mother, respectively, was alive at the time of interview.

they brought same-sex friends home or went on outings with them. Fewer parents were perceived however to disapprove of their sons joining a club or mandal than their daughters:, $24-26 \%$ of young men and $43-50 \%$ of young women reported expecting parental disapproval if they joined a club or mandal. By and large, finally, differences in perceived reactions of fathers versus mothers were negligible, although somewhat larger proportions of young women perceived disapproval of selected activities from their fathers than their mothers. For instance, $37 \%$ and $28 \%$ of young women reported expecting disapproval from their fathers and mothers, respectively, if they went on outings with their same-sex friends, and 50\% and $43 \%$ reported disapproval from their fathers and mothers, respectively, if they joined a club.

Differences by marital status were by and large narrow, with few exceptions. Somewhat more married young women, irrespective of residence, reported parental disapproval if they found a job, compared to their unmarried counterparts. In addition in urban areas, married young people, particularly women, were somewhat more likely to perceive parental disapproval of interactions with opposite-sex individuals than unmarried youth.

In contrast, rural-urban differences were more consistently observed with urban youth less likely than rural youth to perceive parental disapproval of most activities. With regard to bringing an opposite-sex friend to their home, for example, $80-81 \%$ and $98 \%$ of rural young men and women, respectively, perceived that their parents would disapprove, compared to $71 \%$ and $88-90 \%$ of urban young men and women, respectively.

Youth were also asked about the extent to which family life was characterised by quarrels and domestic violence between parents, and whether they had witnessed their father beating their mother or vice versa. Findings are reported in Table 6.4. They suggest that about three-fifths of young men and over two-thirds of young women with both parents living acknowledged that they had ever witnessed quarrels between their parents. One percent reported that they had witnessed their mother beating their father. One in five young men and women reported ever witnessing their father beating their mother. Larger proportions of married youth reported witnessing their father beating their mother (28\% compared to $17 \%$ of married and unmarried young men, respectively; $22 \%$ and $16 \%$ of married and unmarried young women, respectively). 
Likewise, rural youth were more likely than urban youth to report having witnessed their father beating their mother: $21 \%$ of young men and $20 \%$ of young women in rural areas had witnessed their father beating their mother, compared to $13 \%$ and $15 \%$ of young men and women, respectively, in urban areas.

Youth were also asked whether one or both parents had ever beaten them since the age of 12. Findings, shown in Table 6.4, suggest that over half of young men $(55 \%)$ and $11 \%$ of young women with at least one parent alive at the time of the survey reported being beaten by a parent at any time since the age of 12 . Differences by marital status and rural-urban residence were negligible.

Table 6.4: Experience of domestic violence

Percentage of youth reporting violence between parents and being beaten by parents, according to residence, Bihar, 2007

\begin{tabular}{|c|c|c|c|c|c|c|}
\hline Experiences of domestic violence (\%) & $\begin{array}{c}M \\
15-24\end{array}$ & $\begin{array}{c}\mathrm{W} \\
15-24\end{array}$ & $\begin{array}{c}\text { MM } \\
15-29\end{array}$ & $\begin{array}{c}\text { MW } \\
15-24\end{array}$ & $\begin{array}{c}\text { UM } \\
15-24\end{array}$ & $\begin{array}{l}\text { UW } \\
15-24\end{array}$ \\
\hline \multicolumn{7}{|c|}{ Combined } \\
\hline Parents ever fought & 57.3 & 69.7 & 62.9 & 73.5 & 56.6 & 63.6 \\
\hline Mother ever beat father & 1.0 & 0.9 & 1.8 & 1.1 & 1.0 & 0.4 \\
\hline Father ever beat mother & 19.8 & 19.6 & 28.1 & 22.0 & 17.4 & 15.8 \\
\hline Number with both parents alive & 1,608 & 4,689 & 779 & 1,847 & 1,270 & 2,842 \\
\hline $\begin{array}{l}\text { Respondent beaten by father and/or mother } \\
\text { since age } 12\end{array}$ & 55.4 & 11.4 & 57.0 & 10.0 & 55.4 & 13.7 \\
\hline Number with at least one parent alive & 1,915 & 5,456 & 1,067 & 2,286 & 1,479 & 3,170 \\
\hline \multicolumn{7}{|c|}{ Urban } \\
\hline Parents ever fought & 52.9 & 65.7 & 65.6 & 67.9 & 50.7 & 64.4 \\
\hline Mother ever beat father & 1.3 & 0.6 & 1.6 & 0.9 & 1.5 & 0.5 \\
\hline Father ever beat mother & 13.3 & 15.4 & 19.0 & 18.9 & 12.4 & 13.0 \\
\hline Number with both parents alive & 841 & 2,160 & 384 & 892 & 690 & 1,268 \\
\hline $\begin{array}{l}\text { Respondent beaten by father and/or mother } \\
\text { since age } 12\end{array}$ & 55.3 & 14.2 & 60.2 & 10.1 & 55.0 & 17.1 \\
\hline Number with at least one parent alive & 1,020 & 2,547 & 519 & 1,112 & 825 & 1,435 \\
\hline \multicolumn{7}{|c|}{ Rural } \\
\hline Parents ever fought & 58.0 & 70.2 & 62.7 & 73.8 & 57.7 & 63.4 \\
\hline Mother ever beat father & 0.9 & 0.9 & 1.8 & 1.1 & 0.9 & 0.4 \\
\hline Father ever beat mother & 20.9 & 20.2 & 28.9 & 22.2 & 18.3 & 16.3 \\
\hline Number with both parents alive & 767 & 2,529 & 395 & 955 & 580 & 1,574 \\
\hline $\begin{array}{l}\text { Respondent beaten by father and/or mother } \\
\text { since age } 12\end{array}$ & 55.4 & 11.0 & 56.7 & 10.0 & 55.5 & 13.1 \\
\hline Number with at least one parent alive & 895 & 2,909 & 548 & 1,174 & 654 & 1,735 \\
\hline
\end{tabular}

Note: All Ns are unweighted. Domestic violence refers exclusively to physical violence. 


\subsubsection{Communication with parents}

Information regarding communication with parents on issues relevant to youth-such as school performance, friendships, romantic relationships, being teased or bullied, physical maturation, reproductive processes and contraception-was elicited from all respondents reporting that their mothers or fathers were alive at the time of the interview. Findings, presented in Table 6.5 and Figures 6.3a-b, reveal that communication on any topic was far from universal. In general, among all groups, sensitive topics—such as romantic relationships, reproduction and contraception and adolescent body changes among young men-were rarely discussed with either parent.

Table 6.5: Parental communication

Percentage of youth who discussed selected matters with parents, according to residence, Bihar, 2007

\begin{tabular}{|c|c|c|c|c|c|c|c|c|c|c|c|c|}
\hline \multirow[t]{2}{*}{$\begin{array}{l}\text { Issues } \\
\text { discussed (\%) }\end{array}$} & $\begin{array}{c}\text { M } \\
15-24 \\
\end{array}$ & $\begin{array}{c}\mathrm{W} \\
15-24 \\
\end{array}$ & $\begin{array}{c}\text { MM } \\
15-29 \\
\end{array}$ & $\begin{array}{c}\text { MW } \\
15-24 \\
\end{array}$ & $\begin{array}{c}\text { UM } \\
15-24 \\
\end{array}$ & $\begin{array}{l}\text { UW } \\
15-24\end{array}$ & $\begin{array}{c}M \\
15-24 \\
\end{array}$ & $\begin{array}{c}\text { W } \\
15-24 \\
\end{array}$ & $\begin{array}{c}\text { MM } \\
15-29 \\
\end{array}$ & $\begin{array}{c}\text { MW } \\
15-24 \\
\end{array}$ & $\begin{array}{c}\text { UM } \\
15-24 \\
\end{array}$ & $\begin{array}{l}\text { UW } \\
15-24\end{array}$ \\
\hline & \multicolumn{6}{|c|}{ Father } & \multicolumn{6}{|c|}{ Mother } \\
\hline \multicolumn{13}{|c|}{ Combined } \\
\hline School performance & 72.9 & 52.2 & 64.3 & 41.8 & 77.1 & 68.9 & 62.3 & 59.3 & 53.2 & 50.0 & 66.0 & 74.6 \\
\hline Friendships & 55.0 & 23.3 & 55.9 & 18.9 & 56.1 & 29.9 & 55.4 & 67.9 & 53.5 & 63.7 & 57.2 & 74.6 \\
\hline Romantic relationships & 0.6 & 0.4 & 0.2 & 0.2 & 0.8 & 0.5 & 0.8 & 7.2 & 0.2 & 5.6 & 1.0 & 9.5 \\
\hline Being teased/bullied & 3.5 & 5.5 & 2.7 & 5.2 & 3.5 & 5.9 & 2.9 & 39.3 & 2.0 & 35.5 & 3.4 & 45.1 \\
\hline Adolescent body changes & 4.1 & 1.1 & 2.0 & 1.1 & 4.7 & 1.1 & 3.8 & 74.2 & 2.7 & 71.8 & 4.2 & 78.0 \\
\hline Reproductive processes & 0.2 & 0.0 & 0.6 & 0.0 & 0.1 & 0.0 & 0.1 & 3.0 & 0.3 & 3.7 & 0.1 & 1.6 \\
\hline Contraception & 0.3 & 0.1 & 0.2 & 0.1 & 0.2 & 0.0 & 0.2 & 2.5 & 0.0 & 2.5 & 0.2 & 2.4 \\
\hline Number of respondents ${ }^{1}$ & 1,704 & 4,973 & 878 & 2,012 & 1,332 & 2,961 & 1,819 & 5,172 & 968 & 2,121 & 1,417 & 3,051 \\
\hline \multicolumn{13}{|c|}{ Urban } \\
\hline School performance & 79.3 & 75.3 & 66.2 & 62.3 & 80.8 & 83.8 & 68.7 & 80.5 & 51.9 & 69.0 & 71.4 & 88.3 \\
\hline Friendships & 59.2 & 40.1 & 52.1 & 30.3 & 60.1 & 46.9 & 60.5 & 80.0 & 53.1 & 73.6 & 61.9 & 84.5 \\
\hline Romantic relationships & 2.1 & 1.2 & 0.0 & 0.0 & 2.3 & 1.8 & 2.3 & 14.5 & 0.0 & 10.1 & 2.6 & 17.6 \\
\hline Being teased/bullied & 4.1 & 7.8 & 2.9 & 5.7 & 4.2 & 8.9 & 4.6 & 51.9 & 2.5 & 42.6 & 5.2 & 58.2 \\
\hline Adolescent body changes & 7.5 & 1.6 & 4.2 & 0.8 & 8.0 & 2.2 & 7.3 & 83.0 & 3.7 & 78.3 & 7.8 & 86.4 \\
\hline Reproductive processes & 0.4 & 0.0 & 0.0 & 0.0 & 0.5 & 0.0 & 0.4 & 3.7 & 0.0 & 4.6 & 0.4 & 3.4 \\
\hline Contraception & 0.4 & 0.0 & 0.0 & 0.0 & 0.5 & 0.0 & 0.4 & 4.7 & 0.0 & 4.7 & 0.4 & 4.7 \\
\hline Number of respondents ${ }^{1}$ & 890 & 2,298 & 421 & 973 & 728 & 1,325 & 971 & 2,409 & 482 & 1,031 & 787 & 1,378 \\
\hline \multicolumn{13}{|c|}{ Rural } \\
\hline School performance & 71.9 & 49.2 & 64.2 & 40.4 & 76.3 & 66.3 & 61.2 & 56.6 & 53.3 & 48.8 & 65.0 & 72.1 \\
\hline Friendships & 54.3 & 21.1 & 56.3 & 18.1 & 55.3 & 26.9 & 54.6 & 66.3 & 53.6 & 63.0 & 56.3 & 72.8 \\
\hline Romantic relationships & 0.3 & 0.3 & 0.2 & 0.3 & 0.4 & 0.3 & 0.4 & 6.2 & 0.2 & 5.3 & 0.7 & 8.1 \\
\hline Being teased/bullied & 3.4 & 5.2 & 2.6 & 5.1 & 3.3 & 5.4 & 2.6 & 37.6 & 1.9 & 35.1 & 3.0 & 42.8 \\
\hline Adolescent body changes & 3.6 & 1.1 & 1.9 & 1.2 & 4.0 & 0.9 & 3.3 & 73.0 & 2.6 & 71.3 & 3.5 & 76.5 \\
\hline Reproductive processes & 0.2 & 0.0 & 0.5 & 0.0 & 0.0 & 0.0 & 0.0 & 2.9 & 0.2 & 3.7 & 0.0 & 1.2 \\
\hline Contraception & 0.3 & 0.1 & 0.2 & 0.1 & 0.2 & 0.0 & 0.1 & 2.2 & 0.0 & 2.4 & 0.2 & 1.9 \\
\hline Number of respondents ${ }^{1}$ & 814 & 2,675 & 457 & 1,039 & 604 & 1,636 & 848 & 2,763 & 486 & 1,090 & 630 & 1,673 \\
\hline
\end{tabular}

Note: All Ns are unweighted. For married respondents questions referred to the period prior to marriage. ${ }^{1}$ Includes only those respondents reporting that their father or mother, respectively, was alive at the time of interview. 
Figure 6.3a: Percentage of youth who discussed various matters with their father, according to residence, Bihar, 2007
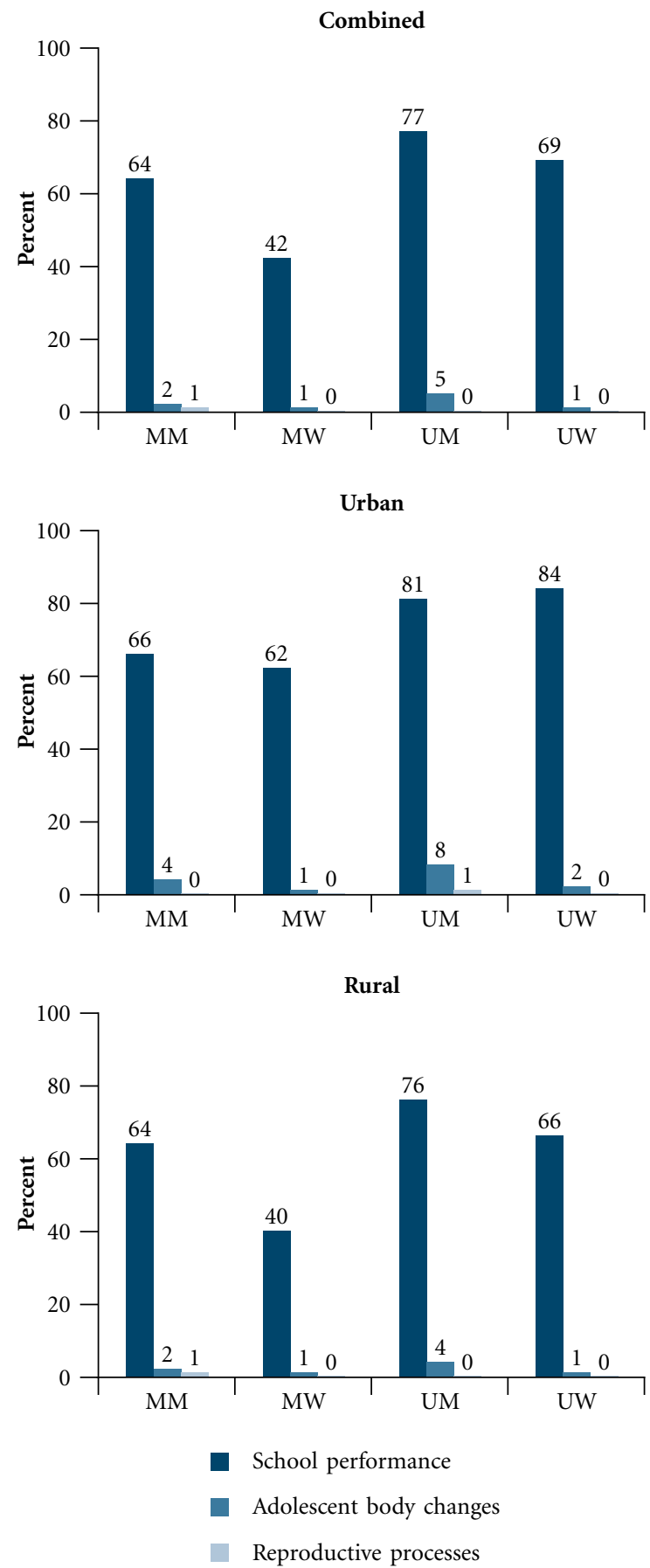

Note: For married respondents, questions referred to the period prior to marriage. Percentages were calculated only of those respondents reporting that their father was alive at the time of the interview.
Figure 6.3b: Percentage of youth who discussed various matters with their mother, according to residence, Bihar, 2007
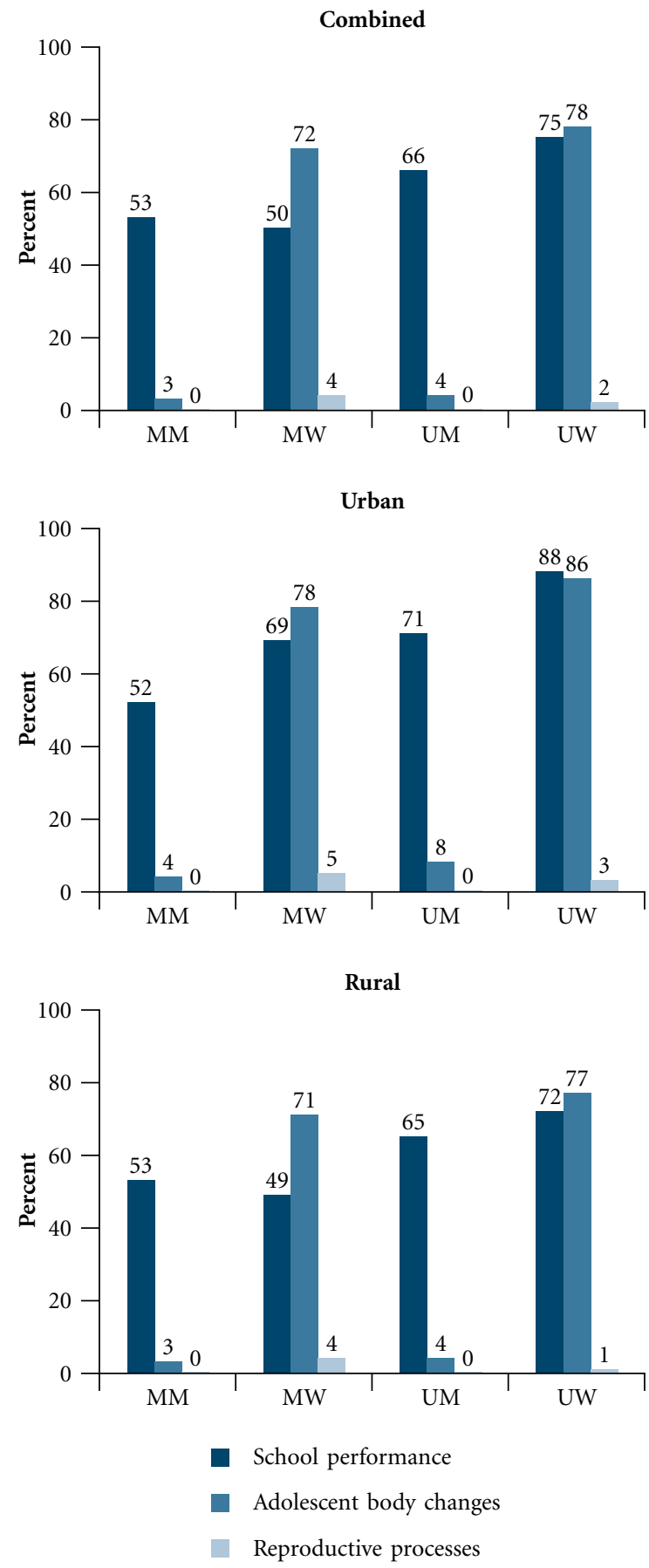

Note: For married respondents, questions referred to the period prior to marriage. Percentages were calculated only of those respondents reporting that their mother was alive at the time of the interview. 
Among both young men and young women, topics most likely to be discussed with their father were schooling and friendships: about three-quarters of young men and half of young women reported discussing schooling ( $73 \%$ and $52 \%$, respectively) and over half of young men and about one-quarter of young women reported discussing friendships (55\% and 23\%, respectively) with their father. Six percent or fewer young men and women reported discussing such topics as being teased/ bullied, adolescent body changes, romantic relationships, reproduction and contraception with their father.

The extent to which young men and young women discussed different matters with their mother differed widely. Young women were more likely than young men to have discussed six of the seven topics with their mother. The topics that young men were most likely to have discussed with their mother were identical to those discussed with their father: schooling (62\%) and friendships (55\%). All other topics were rarely discussed $(0-4 \%)$. In contrast, large proportions of young women discussed not only schooling and friendships with their mother (59\% and $68 \%$ respectively) but also being teased or bullied and adolescent body changes (39\% and $74 \%$, respectively). However, only $3-7 \%$ of young women reported discussing romantic relationships, reproduction and contraception with their mother.

Findings suggest that communication with a parent was more likely to be reported by young women than young men. While young men were about as likely to discuss each issue with their mothers as their fathers, with the exception of school performance, young women were far more likely to discuss almost all matters with their mothers than with their fathers.

Differences by marital status varied across young men and women. Among young men, these differences were largely muted, except that more unmarried than married youth discussed school performance with their parents. Among young women, the unmarried were more likely than the married to discuss both school performance and friendships with both parents. In addition, they were more likely to discuss such topics as being teased/bullied and adolescent body changes with their mothers. Differences by rural-urban residence suggest greater openness with parents among urban compared to rural youth. However, this openness was largely restricted to non-sensitive topics-schooling and friendships. Additionally, urban young women were more likely than their rural counterparts to share such topics as being teased/bullied, romantic relationships and adolescent body changes with their mothers.

\subsection{Peer networks and interaction}

In order to assess the size of peer networks and the nature of peer interaction, the Youth Study asked young people about the number of same-sex friends they had, whether they had opposite-sex friends and the kinds of activities in which they engaged with their same- and opposite-sex friends. Married respondents were asked to recall the situation prior to marriage.

Table 6.6 reports findings on the size of peer networks. The median number of same-sex friends reported by young men and women were identical ( 3 friends). Nonetheless, young men were more likely than young women to report five or more same-sex friends (29\% versus $19 \%$ ). No differences by marital status were evident in the median number of same-sex friends reported by young men and women. In terms of rural-urban differences, the median number of same-sex friends reported by unmarried young men in urban settings (4) exceeded, by one, the median number reported by those in rural settings (3). The reverse was true for unmarried young women ( 2 and 3 in urban and rural areas, respectively). Among the married, no rural-urban differences were evident (all reported a median number of three friends). 
Table 6.6: Size of peer networks

Percent distribution of youth by number of same- and opposite-sex friends, according to residence, Bihar, 2007

\begin{tabular}{|c|c|c|c|c|c|c|}
\hline Number of friends (\%) & $\begin{array}{c}\mathrm{M} \\
15-24\end{array}$ & $\begin{array}{c}\text { W } \\
15-24\end{array}$ & $\begin{array}{c}\text { MM } \\
15-29\end{array}$ & $\begin{array}{c}\text { MW } \\
15-24\end{array}$ & $\begin{array}{c}\text { UM } \\
15-24\end{array}$ & $\begin{array}{c}\text { UW } \\
\text { 15-24 }\end{array}$ \\
\hline \multicolumn{7}{|c|}{ Combined } \\
\hline \multicolumn{7}{|l|}{ Number of same-sex friends } \\
\hline None & 3.6 & 2.1 & 3.8 & 1.9 & 3.8 & 2.4 \\
\hline 1 & 16.8 & 16.4 & 19.5 & 14.9 & 16.2 & 19.2 \\
\hline 2 & 25.7 & 28.7 & 23.9 & 29.6 & 26.3 & 27.3 \\
\hline 3 & 12.0 & 18.5 & 10.6 & 18.5 & 12.7 & 18.7 \\
\hline 4 & 12.5 & 15.4 & 12.9 & 15.8 & 12.3 & 14.7 \\
\hline 5 or more & 29.4 & 18.8 & 29.4 & 19.4 & 28.7 & 17.7 \\
\hline Median number of same-sex friends & 3.0 & 3.0 & 3.0 & 3.0 & 3.0 & 3.0 \\
\hline At least one opposite-sex friend (\%) & 16.2 & 4.8 & 14.7 & 3.2 & 16.2 & 7.3 \\
\hline Number of respondents & 1,942 & 5,529 & 1,115 & 2,341 & 1,492 & 3,188 \\
\hline \multicolumn{7}{|c|}{ Urban } \\
\hline \multicolumn{7}{|l|}{ Number of same-sex friends } \\
\hline None & 1.4 & 2.5 & 2.2 & 2.8 & 1.6 & 2.4 \\
\hline 1 & 11.1 & 18.7 & 12.9 & 16.9 & 10.2 & 20.2 \\
\hline 2 & 21.1 & 27.8 & 24.7 & 28.2 & 20.7 & 27.5 \\
\hline 3 & 12.9 & 17.0 & 11.8 & 19.0 & 13.0 & 15.6 \\
\hline 4 & 14.3 & 14.3 & 14.0 & 14.8 & 14.6 & 13.9 \\
\hline 5 or more & 39.3 & 19.7 & 34.4 & 18.3 & 39.8 & 20.4 \\
\hline Median number of same-sex friends & 4.0 & 3.0 & 3.0 & 3.0 & 4.0 & 2.0 \\
\hline At least one opposite-sex friend (\%) & 27.6 & 12.4 & 23.9 & 8.5 & 28.2 & 15.4 \\
\hline Number of respondents & 1,039 & 2,581 & 547 & 1,136 & 833 & 1,445 \\
\hline \multicolumn{7}{|c|}{ Rural } \\
\hline \multicolumn{7}{|l|}{ Number of same-sex friends } \\
\hline None & 3.9 & 2.0 & 3.9 & 1.9 & 4.2 & 2.3 \\
\hline 1 & 17.8 & 16.1 & 20.1 & 14.7 & 17.3 & 19.0 \\
\hline 2 & 26.5 & 28.9 & 23.8 & 29.6 & 27.4 & 27.2 \\
\hline 3 & 11.9 & 18.7 & 10.5 & 18.5 & 12.7 & 19.3 \\
\hline 4 & 12.2 & 15.5 & 12.9 & 15.8 & 11.9 & 14.9 \\
\hline 5 or more & 27.8 & 18.7 & 28.9 & 19.4 & 26.5 & 17.2 \\
\hline Median number of same-sex friends & 3.0 & 3.0 & 3.0 & 3.0 & 3.0 & 3.0 \\
\hline At least one opposite-sex friend (\%) & 14.3 & 3.8 & 13.9 & 2.9 & 13.8 & 5.8 \\
\hline Number of respondents & 903 & 2,948 & 568 & 1,205 & 659 & 1,743 \\
\hline
\end{tabular}

Note: All Ns are unweighted. For married respondents, questions referred to the period prior to marriage. 
Figure 6.4: Percentage of youth reporting at least one opposite-sex friend, according to residence, Bihar, 2007
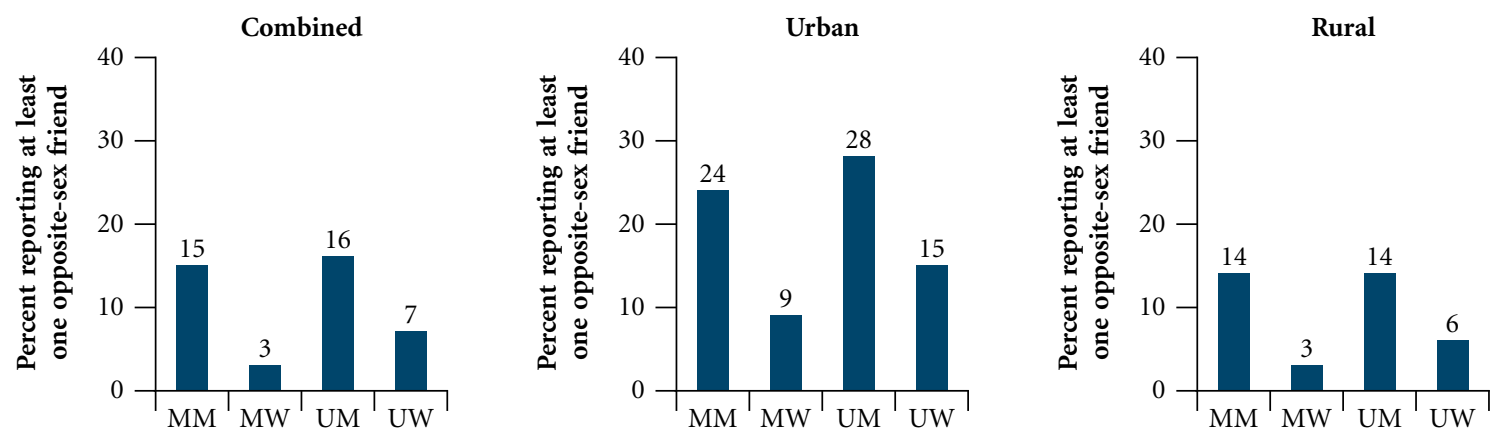

Note: For married respondents, questions referred to the period prior to marriage.

Opposite-sex peer networks were reported by sizeable proportions of young people and gender differences were apparent. Young men were somewhat more likely than young women to report having at least one opposite-sex friend (16\% of young men compared to $5 \%$ of young women). Differences by marital status were modest (see Figure 6.4). Nonetheless, in urban areas, more unmarried young women reported an opposite-sex friend, compared to married young women. Finally, rural young men and women were considerably less likely than their urban counterparts to report an opposite-sex friend.

Table 6.7 reports the nature of interaction with same- and opposite-sex friends. Again, the married were asked to recall the situation prior to marriage. Respondents were asked if they went on picnics or to films with their peers, studied together, spent time chatting, engaged in sporting activities or drank and gambled with their friends.

The activity in which almost all young people engaged was chatting with their same-sex friends. Also, $89 \%$ of young men and $76 \%$ of young women reported engaging in sports or outdoor games with their same-sex friends. Significant gender differences were evident in other forms of interaction: young men were considerably more likely than young women to report going on picnics or to films ( $72 \%$ and $19 \%$ respectively) or studying together with their same-sex peers (66\% and $42 \%$ respectively). It was notable that one in eight young men (12\%) reported drinking and gambling with their same-sex friends; hardly any young women so reported.

Among young men, the unmarried were more likely than the married to report studying ( $72 \%$ versus $49 \%$ ) and engaging in sports (91\% versus $79 \%)$ with their same-sex friends. Conversely, the married were more likely than the unmarried to report drinking or gambling with their same-sex friends ( $25 \%$ versus $8 \%$ ). As in the case of young men, unmarried young women were more likely than the married to report studying $(60 \%$ versus $31 \%)$ with their friends, presumably a function of the higher levels of school attainment of the unmarried. However, the married were more likely to report engaging in sports or outdoor games ( $83 \%$ versus $65 \%)$. Rural-urban patterns were, by and large, similar. However, urban young men and women were more likely than their rural counterparts to go on picnics or to films ( $82 \%$ versus $70 \%$ among young men; $24 \%$ versus $18 \%$ among young women). Additionally, urban young women were more likely to study with their same-sex friends (68\% versus $38 \%$ among young women). Rural young women, however, were somewhat more likely than urban young women to be engaged in sports activities with them ( $77 \%$ versus $71 \%)$. 
Table 6.7: Interaction with same- and opposite-sex friends

Percentage of youth reporting interaction with same- and opposite-sex friends by types of activities, according to residence, Bihar, 2007

\begin{tabular}{|c|c|c|c|c|c|c|}
\hline Activities (\%) & $\begin{array}{c}\mathrm{M} \\
15-24\end{array}$ & $\begin{array}{c}\text { W } \\
15-24\end{array}$ & $\begin{array}{c}\text { MM } \\
15-29\end{array}$ & $\begin{array}{c}\text { MW } \\
15-24\end{array}$ & $\begin{array}{c}\text { UM } \\
15-24\end{array}$ & $\begin{array}{c}\text { UW } \\
15-24\end{array}$ \\
\hline \multicolumn{7}{|c|}{ A. Activities with same-sex friends } \\
\hline \multicolumn{7}{|c|}{ Combined } \\
\hline Going on picnics/to see films & 72.1 & 18.6 & 74.8 & 16.5 & 71.4 & 22.3 \\
\hline Studying together & 66.2 & 41.5 & 49.3 & 30.8 & 72.4 & 60.0 \\
\hline Spending time chatting/gossiping & 99.6 & 99.9 & 99.8 & 100.0 & 99.5 & 99.9 \\
\hline Playing sports & 88.8 & 76.4 & 79.3 & 82.6 & 91.3 & 64.8 \\
\hline Drinking or gambling & 12.2 & 0.1 & 24.7 & 0.1 & 7.9 & 0.2 \\
\hline Number with at least one same-sex friend & 1,892 & 5,402 & 1,080 & 2,290 & 1,452 & 3,112 \\
\hline \multicolumn{7}{|c|}{ Urban } \\
\hline Going on picnics/to see films & 81.8 & 23.7 & 89.0 & 22.5 & 80.9 & 24.5 \\
\hline Studying together & 69.0 & 67.5 & 55.6 & 56.8 & 70.5 & 75.1 \\
\hline Spending time chatting/gossiping & 99.6 & 99.7 & 100.0 & 100.0 & 99.6 & 99.8 \\
\hline Playing sports & 86.9 & 71.0 & 80.2 & 83.3 & 88.0 & 62.2 \\
\hline Drinking or gambling & 12.4 & 0.2 & 30.0 & 0.0 & 10.4 & 0.2 \\
\hline Number with at least one same-sex friend & 1,024 & 2,517 & 535 & 1,108 & 820 & 1,409 \\
\hline \multicolumn{7}{|c|}{ Rural } \\
\hline Going on picnics/to see films & 70.4 & 18.0 & 73.5 & 16.1 & 69.5 & 21.9 \\
\hline Studying together & 65.7 & 38.1 & 48.7 & 29.2 & 72.7 & 57.2 \\
\hline Spending time chatting/gossiping & 99.6 & 100.0 & 99.9 & 100.0 & 99.5 & 99.9 \\
\hline Playing sports & 89.1 & 77.1 & 79.2 & 82.6 & 92.1 & 65.3 \\
\hline Drinking or gambling & 12.2 & 0.1 & 24.2 & 0.1 & 7.5 & 0.2 \\
\hline Number with at least one same-sex friend & 868 & 2,885 & 545 & 1,182 & 632 & 1,703 \\
\hline \multicolumn{7}{|c|}{ B. Activities with opposite-sex friends } \\
\hline \multicolumn{7}{|c|}{ Combined } \\
\hline Going on picnics/to see films & 36.0 & 16.4 & 39.6 & 18.4 & 36.9 & 15.5 \\
\hline Studying together & 53.1 & 47.8 & 41.5 & 32.9 & 57.7 & 57.8 \\
\hline Spending time chatting/gossiping & 98.4 & 97.0 & 97.6 & 100.0 & 97.9 & 95.3 \\
\hline Playing sports & 26.5 & 24.0 & 36.0 & 28.9 & 22.4 & 19.3 \\
\hline Drinking or gambling & 0.9 & 0.4 & 1.8 & 0.0 & 1.2 & 0.9 \\
\hline Number with at least one opposite-sex friend & 414 & 452 & 212 & 133 & 324 & 319 \\
\hline \multicolumn{7}{|c|}{ Urban } \\
\hline Going on picnics/to see films & 39.0 & 16.5 & 40.9 & 16.7 & 37.7 & 16.0 \\
\hline Studying together & 53.2 & 63.8 & 45.5 & 58.3 & 55.1 & 67.1 \\
\hline Spending time chatting/gossiping & 98.7 & 97.5 & 100.0 & 100.0 & 98.6 & 98.7 \\
\hline Playing sports & 26.0 & 25.3 & 27.3 & 33.3 & 25.0 & 22.4 \\
\hline Drinking or gambling & 1.3 & 0.0 & 0.0 & 0.0 & 1.4 & 0.0 \\
\hline Number with at least one opposite-sex friend & 284 & 315 & 133 & 97 & 232 & 218 \\
\hline \multicolumn{7}{|c|}{ Rural } \\
\hline Going on picnics/to see films & 34.9 & 16.4 & 39.0 & $(17.2)$ & 36.6 & 14.7 \\
\hline Studying together & 52.9 & 41.0 & 40.8 & $(29.2)$ & 58.7 & 53.5 \\
\hline Spending time chatting/gossiping & 98.3 & 96.8 & 97.2 & $(100.0)$ & 97.7 & 93.6 \\
\hline Playing sports & 26.6 & 23.4 & 37.3 & $(28.1)$ & 20.9 & 17.8 \\
\hline Drinking or gambling & 0.8 & 0.5 & 2.1 & $(0.0)$ & 1.2 & 1.3 \\
\hline Number with at least one opposite-sex friend & 130 & 137 & 79 & 36 & 92 & 101 \\
\hline
\end{tabular}

Note: All Ns are unweighted. For married respondents, questions referred to the period prior to marriage. ( ) Based on 25-49 unweighted cases. 
The range of activities in which youth engaged with their opposite-sex peers was much narrower. As shown in Panel B of Table 6.7, the only activity in which about as many youth engaged with their opposite-sex friends as their same-sex friends was chatting and gossiping, mentioned by $97-98 \%$ of youth reporting at least one opposite-sex friend. As in the case of interactions with same-sex friends, young men tended to be more likely than young women to report that they went to picnic or films (36\% versus $16 \%$ ) or studied (53\% versus $48 \%$ ) with their opposite-sex friends. Differences by marital status were apparent in only a couple of activities (for example, studying and playing sports). More unmarried than married youth reported studying with their opposite-sex friends. Finally, rural-urban differences were largely muted, except that urban young women were more likely than rural young women to study with their opposite-sex friends (64\% versus $41 \%$ ).

\subsection{Support networks}

The Youth Study also asked respondents about the individual with whom they would most likely discuss a range of personal matters, namely, taking a job, menstrual problems (females) and nocturnal emission or swapnadosh (males) and boy-girl relationships. All those aged 20 and above were asked to think back to the time they were aged 15-18 while responding to these questions.

Findings, reported in Table 6.8a, indicate that the person with whom youth would most likely discuss personal matters varied considerably by sex of the respondent and type of topic. Young women tended to consider their mothers as their leading confidante on matters relating to taking a job (34\%) and menstrual problems (49\%). Matters pertaining to boy-girl relationships, however, were most likely to be confided in peers (39\%). Although hardly any young women reported their fathers as the main confidante for boy-girl relationships or menstrual problems, one-fifth of young women reported their fathers as a leading confidante on issues relating to taking a job. Young men, in contrast, were less likely to consider a parent as a key confidante, except on the non-sensitive issue of taking a job (on which $22 \%$ cited their mothers and $43 \%$ cited their fathers). Parents were rarely cited as key confidantes (less than $2 \%$ ) by young men on such issues as nocturnal emission or swapnadosh, and boy-girl relationships, for which most young men reported peers as their leading confidantes (54\% and $79 \%$, respectively). Differences by marital status were negligible among young men, except that more married than unmarried young men reported a friend as a confidante on taking a job. Among young women, these differences were more notable. For example, the unmarried were more likely than the married to report a parent - the mother in particular — as a confidante on all three matters and a friend as a confidante on boy-girl relationship issues. Of note was that while between two-fifths and half of married young women reported their spouse as a leading confidante on all three topics, $1 \%$ or fewer of married young men reported so, presumably because more young women than men were married at ages 15-18. Also notable is the finding that $9-20 \%$ of married young women reported someone else as a confidante, usually a sister- or brother-in law and even a mother-in-law. Patterns in rural and urban settings remained similar.

Notably, substantial proportions of young men reported that they would not confide in anyone on these topics, ranging from $9 \%$ for taking a job to $20 \%$ for boy-girl relationship to $37 \%$ for anxiety about nocturnal emission. The corresponding percentages among young women ranged from $2 \%$ for taking a job and menstrual problems to $11 \%$ for boy-girl relationships.

The Youth Study also asked young women about the individual in whom they were most likely to confide if they were teased by a boy. Findings, reported in Table $6.8 \mathrm{~b}$, show that over one-third reported that they would confide in their mothers, compared to just $2 \%$ who reported that they would confide in their fathers, $11 \%$ who would confide in a friend and $10 \%$ who would confide in someone else. Married young women reported that they would have been most likely to have confided in their husbands (50\%) on this matter, compared to $23 \%$ and $8 \%$ respectively, who would have confided in a parent or a peer and $11 \%$ who would 


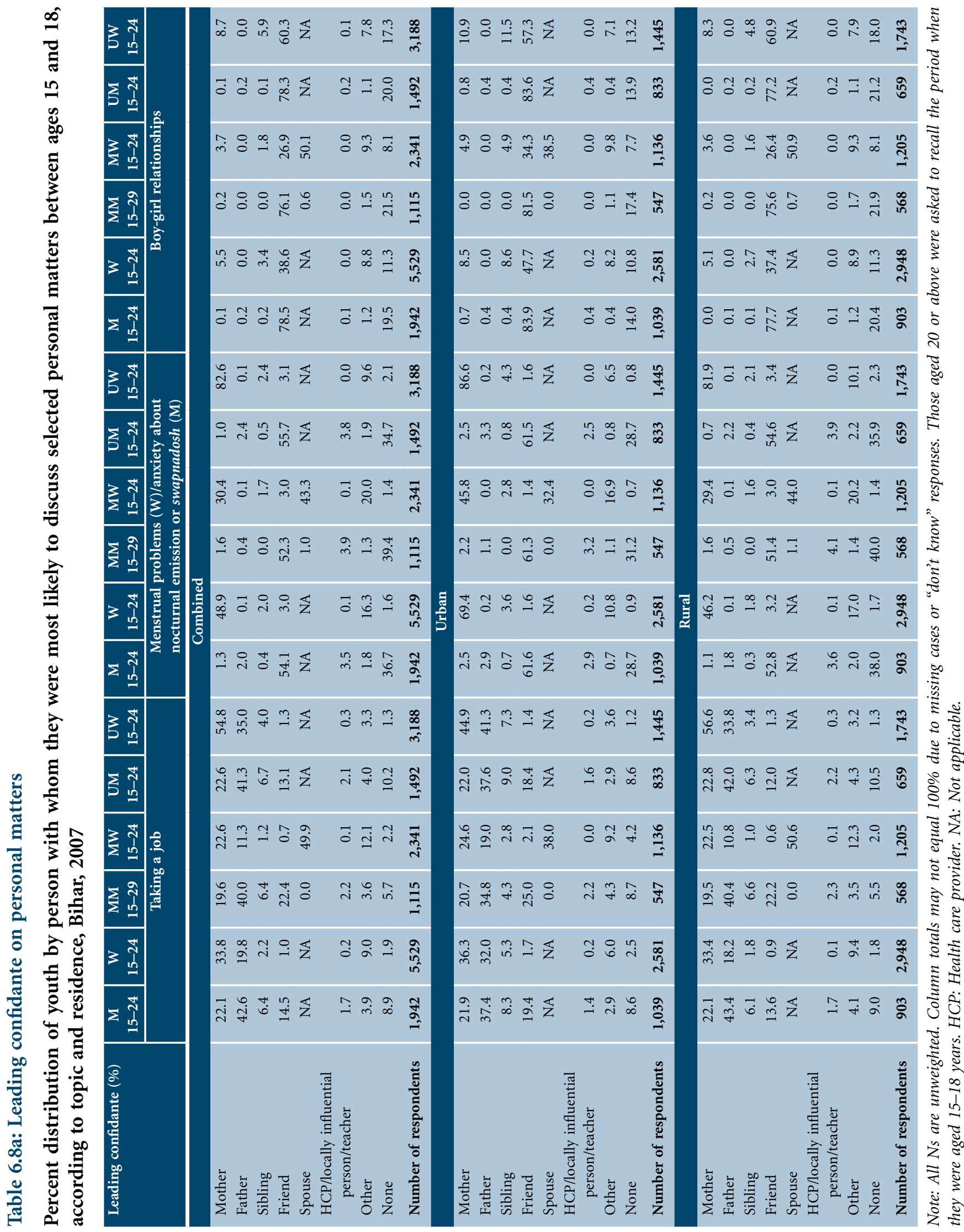


have confided in, as above, the brother- or sister-in-law and even the mother-in-law. In contrast, four-fifths of unmarried young women would confide in a parent $(64 \%)$ or friend $(18 \%)$ or someone else (usually a sister-in-law, 9\%). Findings also show that young women in urban settings were more likely than those in rural settings to report a parent or sibling as a confidante on matters relating to the experience of teasing. However, larger proportions of married young women in rural areas than in urban areas reported their husbands as a confidante on this issue.

Table 6.8b: Leading confidante on matters relating to the experience of teasing among young women

Percent distribution of young women by person with whom they were most likely to discuss being teased by a boy between ages 15 and 18, according to residence, Bihar, 2007

\begin{tabular}{|c|c|c|c|c|c|c|c|c|c|}
\hline \multirow[t]{2}{*}{ Leading confidante (\%) } & $\begin{array}{c}\text { W } \\
15-24\end{array}$ & $\begin{array}{l}\text { MW } \\
15-24\end{array}$ & $\begin{array}{l}\text { UW } \\
15-24\end{array}$ & $\begin{array}{c}\text { W } \\
15-24\end{array}$ & $\begin{array}{l}\text { MW } \\
15-24\end{array}$ & $\begin{array}{l}\text { UW } \\
15-24\end{array}$ & $\begin{array}{c}\text { W } \\
15-24\end{array}$ & $\begin{array}{l}\text { MW } \\
15-24\end{array}$ & $\begin{array}{l}\text { UW } \\
15-24\end{array}$ \\
\hline & \multicolumn{3}{|c|}{ Combined } & \multicolumn{3}{|c|}{ Urban } & \multicolumn{3}{|c|}{ Rural } \\
\hline Mother & 35.9 & 22.1 & 61.1 & 48.4 & 32.4 & 60.0 & 34.2 & 21.5 & 61.2 \\
\hline Father & 1.7 & 1.0 & 2.7 & 3.5 & 2.1 & 4.6 & 1.4 & 1.0 & 2.4 \\
\hline Sibling & 3.1 & 1.8 & 5.3 & 9.0 & 5.6 & 11.3 & 2.4 & 1.5 & 4.2 \\
\hline Friend & 11.4 & 8.0 & 17.8 & 12.1 & 8.5 & 14.7 & 11.3 & 8.0 & 18.4 \\
\hline Spouse & NA & 50.3 & NA & NA & 39.4 & NA & NA & 50.9 & NA \\
\hline $\begin{array}{l}\mathrm{HCP} / \text { locally influential } \\
\text { person/teacher }\end{array}$ & 0.1 & 0.1 & 0.1 & 0.0 & 0.0 & 0.0 & 0.1 & 0.1 & 0.1 \\
\hline Other person & 10.0 & 10.9 & 8.6 & 8.0 & 8.5 & 7.5 & 10.3 & 11.0 & 8.8 \\
\hline None & 5.3 & 5.8 & 4.4 & 2.5 & 3.5 & 1.8 & 5.6 & 6.0 & 4.9 \\
\hline Number of respondents & 5,529 & 2,341 & 3,188 & 2,581 & 1,136 & 1,445 & 2,948 & 1,205 & 1,743 \\
\hline
\end{tabular}

Note: All Ns are unweighted. Column totals may not equal 100\% due to missing cases or "don't know" responses. Those aged 20 or above were asked to recall the period when they were aged 15-18 years. HCP: Health care provider. NA: Not applicable.

\subsection{Summary}

Youth Study findings suggest the gendered nature of socialisation of youth. For example, responses from both young men and women indicate that unequal gender norms regarding freedom of movement prevailed in most study households, with three-fifths of young men acknowledging that they had more freedom to go out than their sisters or female cousins did, and almost four-fifths of young women agreeing that they had less freedom to go out than their brothers or male cousins. Moreover, although young men's responses to questions related to housework expectations suggest a gender equal socialisation experience, it is notable that about three-quarters of young women reported that they were expected to do more housework than their brothers or male cousins. Similarly, while parents appeared to control both young men's and women's social interactions, particularly those involving members of the opposite sex, young women were more likely than young men to experience such restrictions. For example, $79-80 \%$ of young men and $97 \%$ of young women reported expecting parental disapproval if they brought an opposite-sex friend to their home.Nonetheless, it is notable that while young women were brought up with more restrictions, parents appeared to place considerable restrictions on young men's interactions as well, including with same-sex peers.

Findings regarding communication with parents on issues relevant to youth-such as school performance, friendships, being teased or bullied, physical maturation, romantic relationships and reproductive processes- 
reiterate those from other studies, showing that such communication is far from universal. Moreover, among all youth, sensitive topics—such as romantic relationships, reproduction and contraception, and adolescent body changes issues among young men-were rarely discussed with either parent.

That parent-child communication was restricted was also evident from responses to questions probing the most likely confidante on a range of topics from taking a job to boy-girl relationships. While parents were mentioned as leading confidantes on topics such as taking a job, they were rarely cited as leading confidantes on more sensitive matters. While young women identified their mothers as the most likely confidante on such matters as menstrual problems and experience of teasing, young men rarely identified a parent as a leading confidante on matters relating to nocturnal emission or swapnadosh. And neither young men nor women identified a parent as a leading confidante on boy-girl matters.

Young people's family lives were marked by violence, both experienced and witnessed. One in five youth had observed their fathers beating their mothers. Many respondents reported experiencing a beating by a parent during adolescence; over half of young men and $11 \%$ of young women reported such experiences.

In contrast, growing up was associated with close peer networks. Almost all youth reported having same-sex friends. Opposite-sex peer networks were less common but nonetheless reported by $16 \%$ of young men and $5 \%$ of young women. Interaction with friends tended to be restricted to activities such as chatting and engaging in sports, although young men did tend to report studying and going on picnics or to films. Indeed, findings suggest that youth derived an important measure of support from their peer networks on sensitive matters: friends were by far the leading confidante on boy-girl relationships for both young men and women and on nocturnal emission for young men. 


\section{Agency and gender role attitudes}

Evidence on agency and gender role attitudes among youth, although sparse, suggests that in traditional settings such as India, young women and even some young men have limited agency in terms of decision-making on matters affecting their own lives, freedom of movement and access to resources. Gender role attitudes, similarly, tend to be traditional, assigning greater value to young men than young women (Alexander et al., 2006a; 2006b; Ram et al., 2006; Santhya et al., 2008; Sebastian, Grant and Mensch, 2005). This chapter discusses Youth Study findings on agency and gender role attitudes.

\subsection{Decision-making}

In order to assess young people's involvement in decision-making, the Youth Study asked all respondents about their involvement in decisions related to three specific matters: choice of friends, spending one's own money and buying clothes for oneself. If youth reported that they were involved in decision-making on any issue, they were asked whether they made the decision entirely on their own or jointly with other family members.

Findings, presented in Table 7.1 and Figure 7.1, reveal that irrespective of sex, marital status and place of residence, youth were overwhelmingly likely to choose their friends on their own; $97 \%$ of young men and 96\% of young women so reported. Fewer youth were involved in making decisions on spending their own money than on choice of friends, and this was particularly evident among young women. For example, while 75\% of young men reported that they made decisions on their own about spending money, only $57 \%$ of young women so reported. Findings further suggest that a considerable proportion of both young men (11\%) and women $(20 \%)$ reported that it was other family members who made decisions on spending money without their involvement. As shown in Figure 7.1, marital status differences suggest that among young men, the married were more likely than the unmarried ( $85 \%$ versus $72 \%$ ) to make this decision independently. Among young women, these differences were muted for the overall population, but differed across urban and rural young women. While the unmarried were more likely than the married to have made independent decisions on spending money in urban areas ( $76 \%$ versus $67 \%$ ), a reverse pattern was evident in rural areas (50\% and $57 \%$ of unmarried and married women, respectively). Rural-urban differences were negligible among young men, but notable among young women; urban young women were more likely than rural women to report independent decisions related to spending their own money and conversely, rural young women were more likely than urban young women to be entirely excluded from such decisions.

Even fewer youth were involved in making decisions about the purchase of clothes for themselves, and differences were particularly pronounced among young women. For example, while $53 \%$ of young men decided on their own about purchasing clothes, only $30 \%$ of young women did so. One-third of young women (31\%) compared to one-fifth of young men $(21 \%)$ reported that they did not have any say in decisions to buy clothes for themselves and that it was other family members who made this decision for them. Differences by marital status were also observed. Married young men were more likely than unmarried young men to 
Table 7.1: Decision-making

Percent distribution of youth by participation in decision-making on selected matters, according to residence, Bihar, 2007

\begin{tabular}{|c|c|c|c|c|c|c|}
\hline Participation in decision-making (\%) & $\begin{array}{c}\mathrm{M} \\
15-24\end{array}$ & $\begin{array}{c}\mathrm{W} \\
15-24\end{array}$ & $\begin{array}{c}\text { MM } \\
15-29\end{array}$ & $\begin{array}{c}\text { MW } \\
15-24\end{array}$ & $\begin{array}{c}\text { UM } \\
15-24\end{array}$ & $\begin{array}{c}\text { UW } \\
\text { 15-24 }\end{array}$ \\
\hline \multicolumn{7}{|c|}{ A. Choice of friends } \\
\hline \multicolumn{7}{|c|}{ Combined } \\
\hline Respondent only & 97.3 & 95.5 & 98.7 & 95.6 & 97.0 & 95.3 \\
\hline Jointly with others & 1.5 & 2.0 & 0.4 & 1.8 & 1.8 & 2.4 \\
\hline Others only & 1.2 & 2.5 & 1.0 & 2.6 & 1.2 & 2.4 \\
\hline Number of respondents & 1,942 & 5,529 & 1,115 & 2,341 & 1,492 & 3,188 \\
\hline \multicolumn{7}{|c|}{ Urban } \\
\hline Respondent only & 97.5 & 97.6 & 97.8 & 97.9 & 97.5 & 97.6 \\
\hline Jointly with others & 1.1 & 1.4 & 1.1 & 1.4 & 1.2 & 1.4 \\
\hline Others only & 1.4 & 0.9 & 1.1 & 0.7 & 1.2 & 1.0 \\
\hline Number of respondents & 1,039 & 2,581 & 547 & 1,136 & 833 & 1,445 \\
\hline \multicolumn{7}{|c|}{ Rural } \\
\hline Respondent only & 97.2 & 95.3 & 98.6 & 95.5 & 96.9 & 94.8 \\
\hline Jointly with others & 1.5 & 2.0 & 0.3 & 1.8 & 1.9 & 2.6 \\
\hline Others only & 1.2 & 2.7 & 1.1 & 2.8 & 1.2 & 2.6 \\
\hline Number of respondents & 903 & 2,948 & 568 & 1,205 & 659 & 1,743 \\
\hline \multicolumn{7}{|c|}{ B. Spending money } \\
\hline \multicolumn{7}{|c|}{ Combined } \\
\hline Respondent only & 75.1 & 56.5 & 85.2 & 57.3 & 72.1 & 53.6 \\
\hline Jointly with others & 14.2 & 23.9 & 9.5 & 24.1 & 15.8 & 24.2 \\
\hline Others only & 10.6 & 19.6 & 5.3 & 18.6 & 12.2 & 22.2 \\
\hline Number of respondents & 1,942 & 5,529 & 1,115 & 2,341 & 1,492 & 3,188 \\
\hline \multicolumn{7}{|c|}{ Urban } \\
\hline Respondent only & 78.6 & 71.9 & 83.7 & 66.9 & 78.3 & 75.5 \\
\hline Jointly with others & 11.1 & 15.9 & 12.0 & 20.4 & 11.1 & 12.8 \\
\hline Others only & 10.4 & 12.2 & 4.3 & 12.7 & 10.7 & 11.7 \\
\hline Number of respondents & 1,039 & 2,581 & 547 & 1,136 & 833 & 1,445 \\
\hline \multicolumn{7}{|c|}{ Rural } \\
\hline Respondent only & 74.5 & 54.4 & 85.3 & 56.7 & 70.9 & 49.6 \\
\hline Jointly with others & 14.8 & 25.0 & 9.3 & 24.4 & 16.7 & 26.2 \\
\hline Others only & 10.7 & 20.6 & 5.4 & 18.9 & 12.4 & 24.1 \\
\hline Number of respondents & 903 & 2,948 & 568 & 1,205 & 659 & 1,743 \\
\hline
\end{tabular}


Table 7.1: (Cont'd)

\begin{tabular}{|c|c|c|c|c|c|c|}
\hline Participation in decision-making (\%) & $\begin{array}{c}M \\
15-24\end{array}$ & $\begin{array}{c}\text { W } \\
15-24\end{array}$ & $\begin{array}{c}\text { MM } \\
15-29\end{array}$ & $\begin{array}{c}\text { MW } \\
15-24\end{array}$ & $\begin{array}{c}\text { UM } \\
15-24\end{array}$ & $\begin{array}{c}\text { UW } \\
15-24\end{array}$ \\
\hline \multicolumn{7}{|c|}{ C. Buying clothes for oneself } \\
\hline \multicolumn{7}{|c|}{ Combined } \\
\hline Respondent only & 53.1 & 29.7 & 81.1 & 29.0 & 44.5 & 29.9 \\
\hline Jointly with others & 25.6 & 39.1 & 11.5 & 40.0 & 30.4 & 37.8 \\
\hline Others only & 21.3 & 31.1 & 7.4 & 30.9 & 25.1 & 32.4 \\
\hline Number of respondents & 1,942 & 5,529 & 1,115 & 2,341 & 1,492 & 3,188 \\
\hline \multicolumn{7}{|c|}{ Urban } \\
\hline Respondent only & 63.8 & 46.2 & 83.7 & 40.1 & 61.1 & 50.4 \\
\hline Jointly with others & 17.6 & 34.5 & 9.8 & 38.0 & 18.9 & 32.0 \\
\hline Others only & 18.6 & 19.3 & 6.5 & 21.8 & 20.1 & 17.6 \\
\hline Number of respondents & 1,039 & 2,581 & 547 & 1,136 & 833 & 1,445 \\
\hline \multicolumn{7}{|c|}{ Rural } \\
\hline Respondent only & 51.3 & 27.6 & 80.8 & 28.3 & 41.3 & 26.1 \\
\hline Jointly with others & 26.9 & 39.7 & 11.6 & 40.2 & 32.6 & 38.8 \\
\hline Others only & 21.7 & 32.7 & 7.5 & 31.5 & 26.1 & 35.1 \\
\hline Number of respondents & 903 & 2,948 & 568 & 1,205 & 659 & 1,743 \\
\hline
\end{tabular}

Note: All Ns are unweighted. Column totals may not equal 100\% due to missing cases or "don't know" responses.

Figure 7.1: Percent distribution of youth by participation in decision-making on selected matters, Bihar, 2007
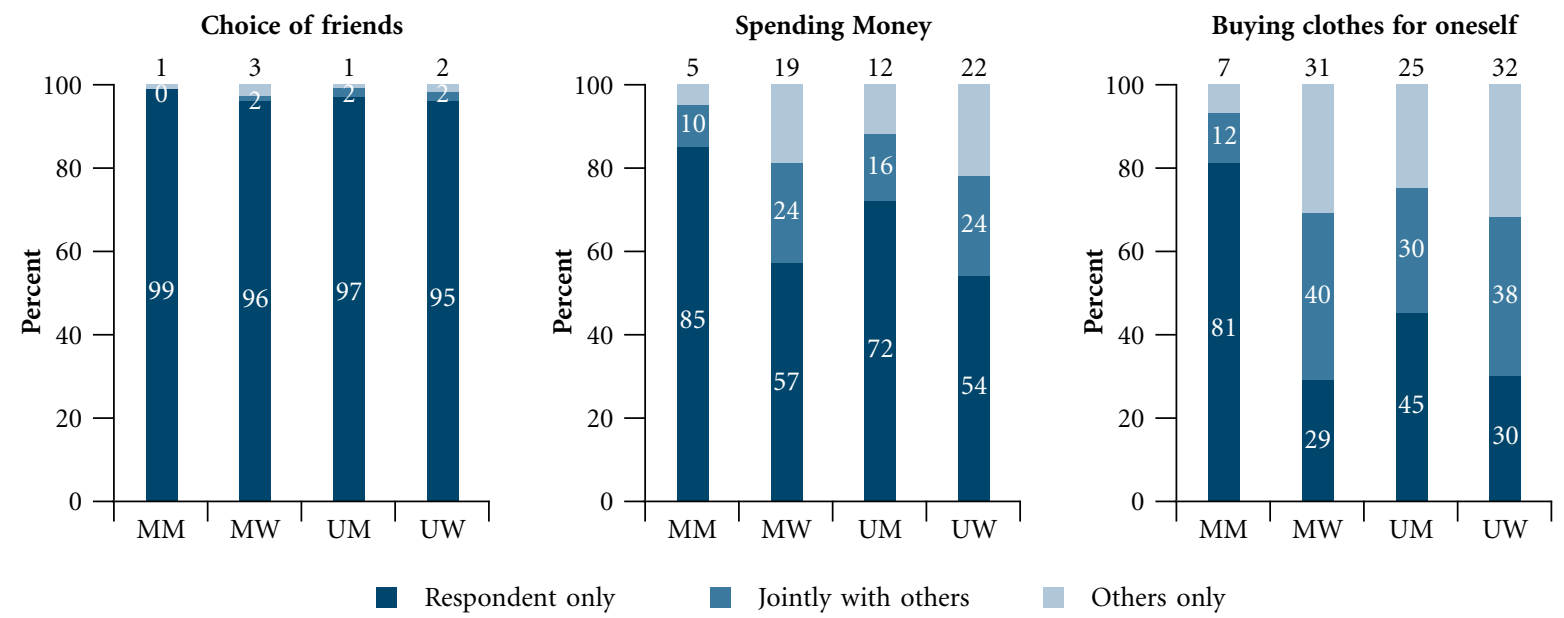

Note: Percentages may not equal 100.0 because of rounding.

make independent decisions related to buying clothes on their own (81\% versus $45 \%)$. Among young women, while these differences were muted for overall and rural population, it was the unmarried who were more likely than the married to have made independent decisions in urban areas (50\% versus $40 \%$ ). Finally, ruralurban differences reiterate that larger proportions of urban youth made independent decisions relating to the purchase of clothes. Additionally, rural young women, particularly unmarried young women, were more likely to be entirely excluded from such decisions, compared to their urban counterparts. 
In order to assess the extent to which youth had independent decision-making power on all three matters. Table 7.2 presents the percentage of youth who reported that they independently made decisions on choice of friends, spending money and purchase of clothes. In total, $46 \%$ of young men compared to $25 \%$ of young women reported independent decision-making on all three issues. While married young men were more likely than their unmarried counterparts to make decisions independently, these differences were muted among young women. As observed above, urban youth were more likely than rural youth to report independent decision-making on all three matters.

Table 7.2 also presents combined responses on independent decision-making by selected background characteristics. Findings reveal that independent decision-making on all three matters was indeed higher among older than younger respondents, irrespective of sex and marital status. By and large, a similar pattern prevailed in both urban and rural areas. Differences by religion were, in general, modest; however, Hindu young women in urban areas were more likely than Muslim women to report decision-making authority, irrespective of marital status. Caste-wise differences were modest among young men, but suggest that those

Table 7.2: Decision-making autonomy by selected background characteristics

Percentage of youth who independently made decisions on choice of friends, spending money and buying clothes for themselves by selected background characteristics, according to residence, Bihar, 2007

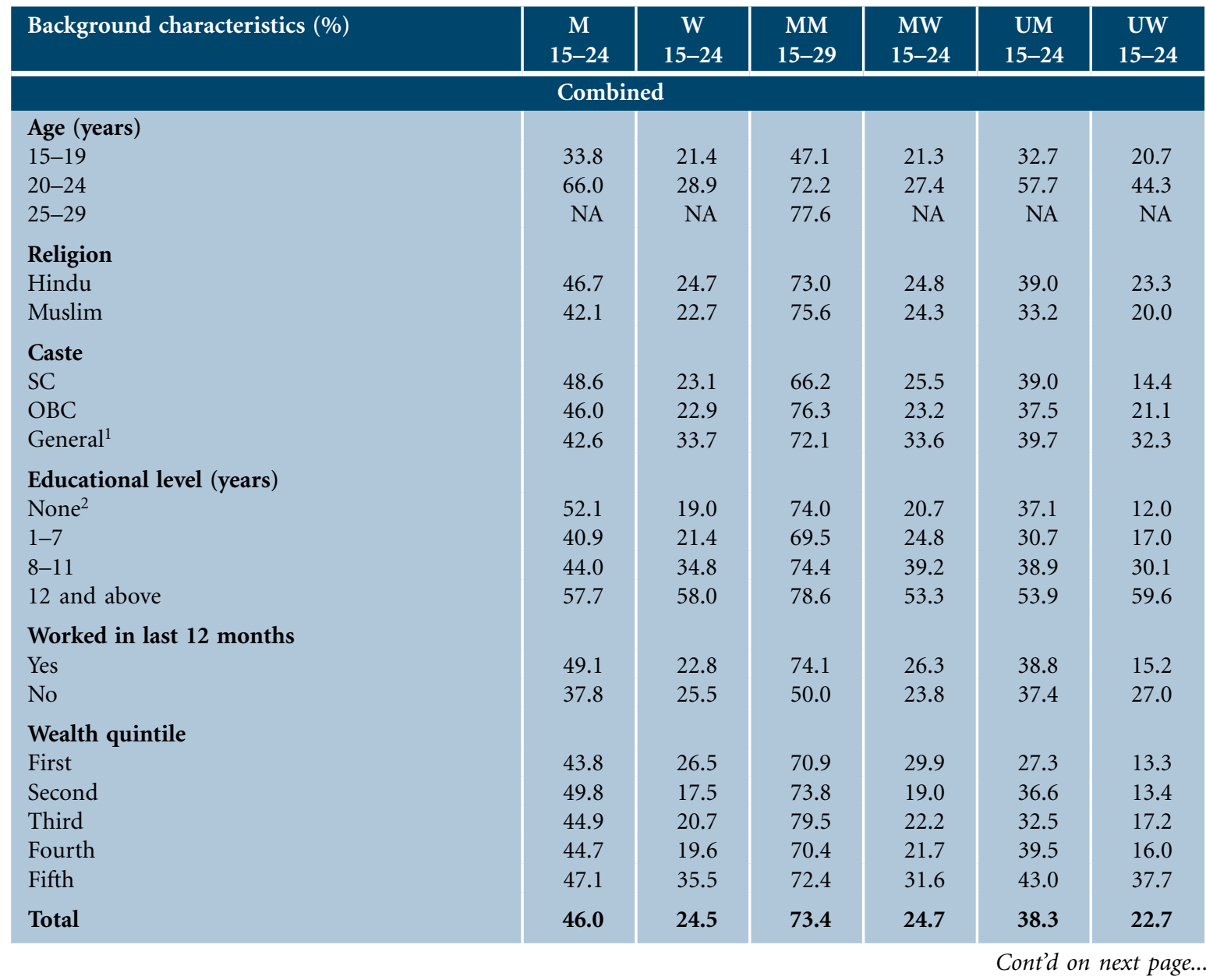


Table 7.2: (Cont'd)

\begin{tabular}{|c|c|c|c|c|c|c|}
\hline Background characteristics (\%) & $\begin{array}{c}\mathrm{M} \\
15-24\end{array}$ & $\begin{array}{c}\text { W } \\
15-24\end{array}$ & $\begin{array}{c}\text { MM } \\
15-29\end{array}$ & $\begin{array}{c}\text { MW } \\
15-24\end{array}$ & $\begin{array}{c}\text { UM } \\
15-24\end{array}$ & $\begin{array}{c}\text { UW } \\
15-24\end{array}$ \\
\hline \multicolumn{7}{|c|}{ Urban } \\
\hline \multicolumn{7}{|l|}{ Age (years) } \\
\hline $15-19$ & 45.4 & 39.7 & * & 30.8 & 45.3 & 41.9 \\
\hline $20-24$ & 70.1 & 42.7 & 75.0 & 35.9 & 69.0 & 58.9 \\
\hline 25-29 & NA & NA & 74.1 & NA & NA & NA \\
\hline \multicolumn{7}{|l|}{ Religion } \\
\hline Hindu & 56.1 & 42.6 & 73.4 & 36.1 & 53.5 & 47.5 \\
\hline Muslim & 52.1 & 33.6 & 71.4 & 29.2 & 51.2 & 36.7 \\
\hline \multicolumn{7}{|l|}{ Caste } \\
\hline SC & 62.1 & 36.5 & 78.6 & 30.4 & 59.1 & 40.0 \\
\hline OBC & 55.9 & 37.9 & 72.9 & 31.5 & 53.4 & 43.0 \\
\hline General $^{1}$ & 53.2 & 50.9 & 72.2 & 48.1 & 51.4 & 52.0 \\
\hline \multicolumn{7}{|l|}{ Educational level (years) } \\
\hline None $^{2}$ & 51.7 & 21.1 & 73.7 & 23.1 & 47.6 & 18.3 \\
\hline $1-7$ & 42.0 & 29.7 & 66.7 & 29.4 & 36.6 & 30.6 \\
\hline $8-11$ & 53.1 & 47.4 & 73.5 & 43.6 & 50.4 & 49.0 \\
\hline 12 and above & 72.5 & 63.5 & 81.0 & 58.8 & 71.9 & 64.8 \\
\hline \multicolumn{7}{|l|}{ Worked in last 12 months } \\
\hline Yes & 59.1 & 37.5 & 73.6 & 35.3 & 56.1 & 39.3 \\
\hline No & 50.4 & 41.7 & 60.0 & 34.7 & 50.4 & 46.8 \\
\hline \multicolumn{7}{|l|}{ Wealth quintile } \\
\hline First & * & 28.6 & * & $(25.0)$ & * & * \\
\hline Second & $(58.3)$ & 22.9 & $(75.0)$ & 27.3 & $(44.4)$ & 20.0 \\
\hline Third & 43.8 & 25.5 & $(75.0)$ & 25.0 & $(35.7)$ & 27.6 \\
\hline Fourth & 53.2 & 31.1 & 72.2 & 29.2 & 50.0 & 33.9 \\
\hline Fifth & 57.2 & 46.6 & 73.7 & 40.2 & 55.7 & 50.4 \\
\hline Total & 55.7 & 41.0 & 73.9 & 34.5 & 53.3 & 45.5 \\
\hline \multicolumn{7}{|c|}{ Rural } \\
\hline \multicolumn{7}{|l|}{ Age (years) } \\
\hline $15-19$ & 32.1 & 19.2 & $(47.0)$ & 20.9 & 30.7 & 17.5 \\
\hline $20-24$ & 65.2 & 27.0 & 72.0 & 26.6 & 54.3 & 34.9 \\
\hline 25-29 & NA & NA & 77.8 & NA & NA & NA \\
\hline \multicolumn{7}{|l|}{ Religion } \\
\hline Hindu & 45.2 & 22.6 & 72.9 & 24.1 & 36.3 & 19.0 \\
\hline Muslim & 39.8 & 20.8 & 76.0 & 23.9 & 28.7 & 16.9 \\
\hline \multicolumn{7}{|l|}{ Caste } \\
\hline SC & 47.5 & 22.1 & 65.5 & 25.3 & 37.2 & 11.0 \\
\hline OBC & 44.2 & 21.0 & 76.6 & 22.7 & 34.2 & 17.5 \\
\hline General $^{1}$ & 39.9 & 29.2 & 72.1 & 31.6 & 36.7 & 27.2 \\
\hline \multicolumn{7}{|l|}{ Educational level (years) } \\
\hline None $^{2}$ & 52.1 & 18.9 & 74.0 & 20.6 & 35.9 & 11.6 \\
\hline $1-7$ & 40.8 & 20.5 & 69.7 & 24.4 & 29.9 & 15.6 \\
\hline $8-11$ & 42.3 & 31.2 & 74.2 & 38.3 & 36.6 & 25.1 \\
\hline 12 and above & 51.8 & 52.0 & 78.9 & * & 44.9 & 53.4 \\
\hline \multicolumn{7}{|l|}{ Worked in last 12 months } \\
\hline Yes & 47.8 & 22.0 & 74.1 & 26.1 & 36.1 & 13.3 \\
\hline No & 34.3 & 22.5 & * & 22.8 & 34.1 & 22.0 \\
\hline \multicolumn{7}{|l|}{ Wealth quintile } \\
\hline First & 43.4 & 26.4 & 70.5 & 30.0 & 27.5 & 13.0 \\
\hline Second & 49.6 & 17.3 & 73.9 & 18.8 & 36.2 & 13.1 \\
\hline Third & 44.8 & 20.5 & 79.6 & 22.1 & 32.3 & 16.7 \\
\hline Fourth & 43.8 & 18.7 & 70.0 & 21.2 & 38.4 & 14.4 \\
\hline Fifth & 42.5 & 29.8 & 72.2 & 29.2 & 36.5 & 30.6 \\
\hline Total & 44.5 & 22.3 & 73.3 & 24.1 & 35.3 & 18.6 \\
\hline
\end{tabular}

Note: ${ }^{*}$ Percentage not shown, based on fewer than 25 unweighted cases. ( ) Based on 25-49 unweighted cases. NA: Not applicable. OBC: Other backward caste. SC: Scheduled caste. ST: Scheduled tribe. ${ }^{1}$ Includes all those not belonging to SC, ST or OBC. ${ }^{2}$ Includes non-literate and literate with no formal schooling. 
belonging to scheduled castes were most likely and those belonging to general castes least likely to report independent decision-making, irrespective of residence. However, the patterns were less consistent when analysed separately for the married and the unmarried. Among young women, those belonging to general castes were more likely than others to report independent decision-making, irrespective of marital status and residence.

Independent decision-making increased consistently with level of education among young women, irrespective of marital status and residence. For example, $58 \%$ of those with 12 or more years of schooling decided independently on all three issues, compared with just $19 \%$ of young women without any formal education. Among young men, in contrast, differences by education were typically narrow and patterns less consistent. While young men with 12 or more years of education were most likely to report independent decision-making, those with 1-7 years and 8-11 years of schooling were far less likely than those with no formal education to report independent decision-making.

Economic activity status was associated with independent decision-making among young men; those who had worked in the last 12 months tended to be more likely than others to make decisions independently, irrespective of residence. While a similar pattern was evident among married young men, the differences were muted among unmarried young men. Among young women, the association was unclear, except that unmarried working women were less likely than other women to report independent decision-making, irrespective of residence.

Differences in independent decision-making by economic status of households were, in general, narrow among young men, irrespective of residence. Even so among the unmarried, those from households in the poorest quintile were least likely and those from households in the wealthiest quintile most likely to report independent decision making. Among young women, those belonging to households in the wealthiest quintile were more likely than others to report independent decision making. At the same time, it is notable that young women from households in the poorest quintile were more likely than those from households belonging to second to fourth quintiles to report independent decision making. It is also notable that even young men from households in the poorest (first) quintile were typically more likely to report independent decisionmaking than young women in the wealthiest quintile. Findings, moreover, suggest that the patterns differed for the married and the unmarried. Among the married, young women belonging to the poorest and the wealthiest quintiles were more likely than others to report independent decision making. In contrast among the unmarried, differences in percentages who reported independent decision making were mild among those in the first to fourth quintiles (13-17\%) and were apparent for the wealthiest quintile, among whom $38 \%$ reported independent decision making.

\subsection{Freedom of movement}

Freedom of movement was assessed only for all young women and unmarried young men because married young men generally have unrestricted mobility. Mobility was measured by a number of questions relating to whether the respondent perceived that he or she was permitted to visit places within and outside the village (rural) or neighbourhood (urban) unescorted, only if accompanied by someone else, or was not permitted to visit the place at all. Places within the village or neighbourhood included a shop/market, the home of a friend/relative and a community programme. Places outside the village or neighbourhood included the home of a relative or friend, movie theatre, video parlour or other place of entertainment and a community programme. Finally, all respondents were asked if they could go to a health facility unescorted if required. Table 7.3 and Figure 7.2 report findings relating to mobility. 
Findings confirm that freedom of movement even within the village or neighbourhood was not universal, although the mobility of young women, both married and unmarried, was far more limited than that of young men. For example, findings suggest that $53 \%$ of young women $-49 \%$ and $61 \%$ of the married and the unmarried, respectively-could go unescorted to a shop or market within the village or neighbourhood compared with $98 \%$ of unmarried young men. Notably, $19 \%$ of married young women and $8 \%$ of unmarried young women were not at all allowed to go to a shop or market within the village or neighbourhood. Mobility to attend programmes within the village or neighbourhood was more restricted than the above, particularly among young women. Only $12 \%$ of young women were allowed to attend community programmes within the village or neighbourhood unescorted compared with $77 \%$ of unmarried young men. Moreover, $15 \%$ of young women were not allowed to attend such programmes at all.

Freedom to visit places outside the village or neighbourhood unescorted was even more restricted than mobility within the village or neighbourhood. Of the three sites, freedom to visit a place of entertainment or to attend a programme was more curtailed than freedom to visit a friend or relative residing outside the village or neighbourhood. Young women's mobility was particularly limited: fewer than $2 \%$ were permitted to visit a place of entertainment or to attend a programme conducted outside the village or neighbourhood unescorted. While $65 \%$ and $75 \%$ were allowed to visit a place of entertainment or attend a programme, respectively, if accompanied, as many as one in four and one in three young women were not allowed to attend a programme or to visit a place of entertainment outside the home village or neighbourhood, respectively, under any circumstances. While young men's mobility was not curtailed to the same extent as young women, it was far from universal: findings show that $48-56 \%$ of unmarried young men were allowed to visit a place of entertainment or to attend a programme conducted outside their village or neighbourhood unescorted and the large majority $(83 \%)$ were allowed to visit friends or relatives outside the village or neighbourhood unescorted.

With regard to freedom to visit a health facility unescorted, findings, presented in Table 7.3, reveal that just one-tenth of young women, compared with $70 \%$ of unmarried young men, reported that they could do so.

Differences by marital status in young women's mobility were evident in relation to selected locations; the unmarried were more likely than the married to report freedom to visit shops and friends within the village or neighbourhood. Conversely, the married were somewhat more likely to report freedom to visit a health facility. With regard to freedom to visit other locations, differences were muted.

Rural-urban differentials in freedom of movement were muted among young men, but notable among young women. A larger proportion of those in urban compared to rural areas were allowed to go unescorted to such locations within their villages or neighbourhoods as a shop or market ( $62 \%$ and $52 \%$, respectively) or a friend or relative (65\% and $56 \%$, respectively) and even to visit friends or relatives outside the respondent's village or urban neighbourhood ( $17 \%$ and $8 \%$, respectively). With regard to all other locations, these differences were modest; however, young women in urban areas were somewhat more likely to report freedom of movement than their rural counterparts. Conversely, rural young women were twice as likely as urban young women to report that they were not at all allowed to visit many of these locations, including a shop or market, a place of entertainment outside their village or neighbourhood and community programmes inside or outside their village or neighbourhood. Findings, moreover, show that rural-urban differentials in young women's freedom of movement were much wider among the unmarried than the married. 
Table 7.3: Freedom of movement

Percent distribution of youth by extent of freedom to visit selected locations within or outside the village/neighbourhood, according to residence, Bihar, 2007

\begin{tabular}{|c|c|c|c|c|c|c|c|c|c|c|c|c|}
\hline \multirow[t]{2}{*}{ Mobility indicators (\%) } & $\begin{array}{c}\text { W } \\
15-24\end{array}$ & $\begin{array}{l}\text { MW } \\
15-24\end{array}$ & $\begin{array}{l}\text { UW } \\
15-24\end{array}$ & $\begin{array}{c}\text { UM } \\
15-24\end{array}$ & $\begin{array}{c}\text { W } \\
15-24\end{array}$ & $\begin{array}{c}\text { MW } \\
15-24\end{array}$ & $\begin{array}{l}\text { UW } \\
15-24\end{array}$ & $\begin{array}{c}\text { UM } \\
15-24\end{array}$ & $\begin{array}{c}\text { W } \\
15-24\end{array}$ & $\begin{array}{l}\text { MW } \\
15-24\end{array}$ & $\begin{array}{l}\text { UW } \\
15-24\end{array}$ & $\begin{array}{c}\text { UM } \\
15-24\end{array}$ \\
\hline & \multicolumn{4}{|c|}{ Combined } & \multicolumn{4}{|c|}{ Urban } & \multicolumn{4}{|c|}{ Rural } \\
\hline \multicolumn{13}{|l|}{ Permitted to: } \\
\hline $\begin{array}{l}\text { Visit shop/market within } \\
\text { village/neighbourhood }\end{array}$ & & & & & & & & & & & & \\
\hline Alone & 53.0 & 48.5 & 61.0 & 97.7 & 61.9 & 50.3 & 70.0 & 99.6 & 51.9 & 48.4 & 59.3 & 97.3 \\
\hline Only with someone else & 32.4 & 33.0 & 31.4 & 2.3 & 29.6 & 35.7 & 25.3 & 0.4 & 32.7 & 32.8 & 32.6 & 2.7 \\
\hline Not allowed & 14.6 & 18.5 & 7.6 & 0.0 & 8.5 & 14.0 & 4.7 & 0.0 & 15.4 & 18.7 & 8.1 & 0.0 \\
\hline \multicolumn{13}{|l|}{$\begin{array}{l}\text { Visit friend/relative within } \\
\text { village/neighbourhood }\end{array}$} \\
\hline Alone & 56.8 & 52.5 & 64.4 & 94.6 & 65.0 & 52.8 & 74.1 & 95.9 & 55.7 & 52.5 & 62.6 & 94.2 \\
\hline Only with someone else & 38.9 & 41.7 & 34.1 & 5.1 & 31.7 & 40.8 & 24.9 & 3.3 & 39.9 & 41.7 & 35.8 & 5.4 \\
\hline Not allowed & 4.2 & 5.7 & 1.5 & 0.3 & 3.3 & 6.3 & 1.0 & 0.8 & 4.4 & 5.6 & 1.6 & 0.3 \\
\hline \multicolumn{13}{|l|}{$\begin{array}{l}\text { Attend programme within } \\
\text { village/neighbourhood }\end{array}$} \\
\hline Alone & 12.1 & 11.8 & 12.6 & 77.0 & 14.0 & 12.0 & 15.4 & 77.6 & 11.9 & 11.8 & 12.1 & 76.9 \\
\hline Only with someone else & 73.0 & 71.5 & 75.5 & 19.4 & 78.5 & 76.8 & 79.6 & 18.4 & 72.3 & 71.1 & 74.7 & 19.6 \\
\hline Not allowed & 14.9 & 16.7 & 11.9 & 3.6 & 7.5 & 11.3 & 5.1 & 4.1 & 15.8 & 17.1 & 13.1 & 3.5 \\
\hline \multicolumn{13}{|l|}{$\begin{array}{l}\text { Visit friend/relative outside } \\
\text { village/neighbourhood }\end{array}$} \\
\hline Alone & 9.0 & 9.5 & 7.3 & 82.5 & 17.1 & 11.9 & 20.9 & 84.4 & 7.9 & 9.4 & 4.8 & 82.1 \\
\hline Only with someone else & 86.8 & 85.9 & 88.9 & 12.9 & 81.3 & 85.3 & 78.3 & 12.3 & 87.5 & 85.9 & 90.8 & 13.0 \\
\hline Not allowed & 4.2 & 4.6 & 3.8 & 4.6 & 1.6 & 2.8 & 0.8 & 3.3 & 4.6 & 4.7 & 4.4 & 4.9 \\
\hline \multicolumn{13}{|l|}{$\begin{array}{l}\text { Visit nearby village/ } \\
\text { neighbourhood for } \\
\text { entertainment }\end{array}$} \\
\hline Alone & 1.5 & 0.9 & 2.3 & 48.4 & 6.8 & 3.5 & 8.9 & 50.2 & 0.9 & 0.7 & 1.1 & 48.1 \\
\hline Only with someone else & 65.0 & 64.2 & 65.6 & 25.5 & 78.6 & 81.0 & 77.2 & 32.2 & 63.3 & 63.2 & 63.5 & 24.1 \\
\hline Not allowed & 33.4 & 34.9 & 32.1 & 26.1 & 14.6 & 15.5 & 13.9 & 17.6 & 35.9 & 36.1 & 35.4 & 27.7 \\
\hline \multicolumn{13}{|l|}{$\begin{array}{l}\text { Attend programme outside } \\
\text { village/neighbourhood }\end{array}$} \\
\hline Alone & 0.6 & 0.4 & 0.7 & 55.6 & 2.2 & 2.1 & 2.4 & 58.6 & 0.4 & 0.4 & 0.4 & 55.0 \\
\hline Only with someone else & 74.6 & 72.7 & 77.5 & 25.3 & 85.7 & 83.2 & 87.3 & 22.5 & 73.2 & 72.0 & 75.7 & 25.8 \\
\hline Not allowed & 24.8 & 26.9 & 21.7 & 19.1 & 12.1 & 14.7 & 10.3 & 18.9 & 26.4 & 27.6 & 23.9 & 19.2 \\
\hline \multicolumn{13}{|l|}{ Visit health facility } \\
\hline Alone & 9.5 & 11.2 & 5.9 & 70.3 & 13.7 & 14.7 & 13.0 & 74.2 & 8.9 & 11.0 & 4.6 & 69.6 \\
\hline Only with someone else & 88.6 & 87.6 & 90.9 & 29.1 & 85.4 & 84.6 & 86.0 & 25.8 & 89.1 & 87.8 & 91.8 & 29.7 \\
\hline Not allowed & 1.9 & 1.2 & 3.2 & 0.6 & 0.9 & 0.7 & 1.0 & 0.0 & 2.0 & 1.3 & 3.6 & 0.7 \\
\hline Number of respondents & 5,529 & 2,341 & 3,188 & 1,492 & 2,581 & 1,136 & 1,445 & 833 & 2,948 & 1,205 & 1,743 & 659 \\
\hline
\end{tabular}

Note: All Ns are unweighted. Column totals may not equal 100\% due to missing cases or "don't know" responses. Questions regarding freedom of movement were not asked of married males, as their mobility is generally unrestricted.

Summary measures have been created from the range of questions relating to freedom to visit places within and outside the village or neighbourhood, namely, the percentage who were free to visit at least one place within the village or neighbourhood, on one hand, and outside the village or neighbourhood, on the other. As shown in Table 7.4, 99\% and $86 \%$ of unmarried young men had freedom to visit at least one place within and outside the village or neighbourhood, respectively and $70 \%$ to visit a health facility. In comparison, only $64 \%$ of young women reported freedom to visit at least one place within the village or neighbourhood and 
Figure 7.2: Percentage of youth allowed to visit selected places within and outside the village/ neighbourhood unescorted, Bihar, 2007

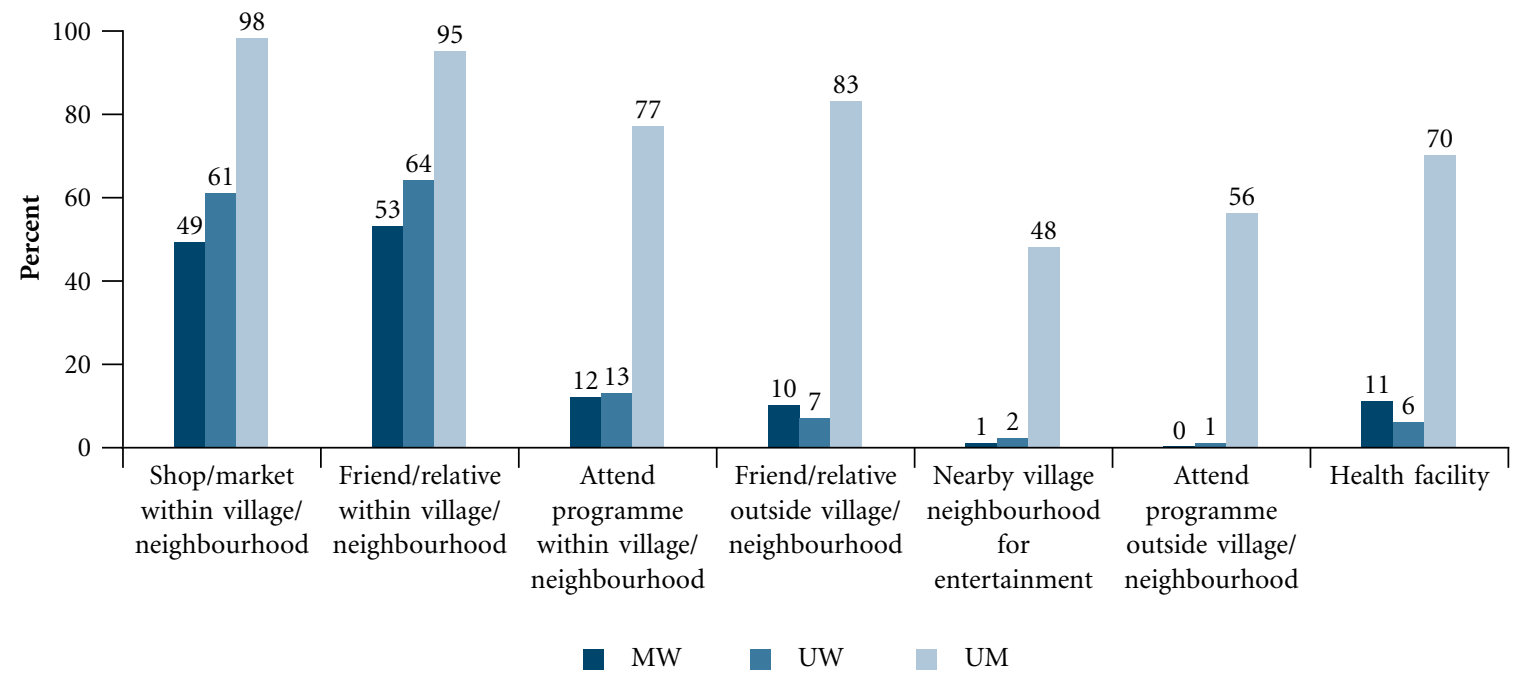

Note: Questions regarding freedom of movement were not asked of married males, as their mobility is generally unrestricted.

$10 \%$ each to visit at least one place outside the village or neighbourhood and a health facility. Differences by marital status were wide with respect to freedom to visit at least one place within the village or neighbourhood, with the unmarried more likely than the married to report freedom to do so; however, the differences were modest with regard to freedom to visit at least one place outside the village or neighbourhood. Likewise, urban young women were more likely than rural young women to report freedom to visit at least one location within or outside the village or neighbourhood. Table 7.4 presents percentages of youth reporting each of these summary measures of freedom of movement as well as freedom to visit a health facility by selected socio-economic and demographic characteristics.

Findings reveal that among unmarried young men, socio-demographic differentials were narrow in the case of mobility within the village. Freedom to visit places outside the village and a health centre unescorted, however, increased with age and work status. Additionally, somewhat more Muslim young men compared to Hindu men and more young men belonging to general castes compared to those belonging to scheduled castes reported freedom to visit locations outside the village or neighbourhood. Similarly, better educated young men were more likely than others to report freedom to visit a health facility. Other associations were modest and less consistent.

Among young women, socio-demographic differentials were evident. Even so, they were typically quite narrow and inconsistent with some exceptions. With regard to mobility within the village or neighbourhood, for instance, more working than non-working women reported freedom to visit locations within the village or neighbourhood; and a generally inverse association was observed between household economic status and freedom to visit locations within the village or neighbourhood, with households in the poorest quintile more likely than others to report mobility. With regard to freedom to visit locations outside the village or urban neighbourhood and to visit a health facility, socio-demographic differentials were apparent in more instances. For example, older women were somewhat more likely to report freedom than younger women. Findings also suggest that even though mobility did not increase steadily with years of schooling, those who had completed 12 years of schooling or more were consistently more likely than others to report freedom of movement. 
Table 7.4: Freedom of movement by selected background characteristics

Percentage of youth who could visit various places unescorted by selected background characteristics, according to residence, Bihar, 2007

\begin{tabular}{|c|c|c|c|c|c|c|c|c|c|c|c|c|}
\hline \multirow[t]{2}{*}{$\begin{array}{l}\text { Background } \\
\text { characteristics (\%) }\end{array}$} & $\begin{array}{c}W \\
15-24 \\
\end{array}$ & $\begin{array}{c}\text { MW } \\
15-24 \\
\end{array}$ & $\begin{array}{c}\text { UW } \\
15-24 \\
\end{array}$ & $\begin{array}{c}\text { UM } \\
15-24 \\
\end{array}$ & $\begin{array}{c}W \\
15-24 \\
\end{array}$ & $\begin{array}{c}\text { MW } \\
15-24 \\
\end{array}$ & $\begin{array}{c}\text { UW } \\
15-24 \\
\end{array}$ & $\begin{array}{c}\text { UM } \\
15-24 \\
\end{array}$ & $\begin{array}{c}\mathrm{W} \\
15-24 \\
\end{array}$ & $\begin{array}{c}\text { MW } \\
15-24 \\
\end{array}$ & $\begin{array}{c}\text { UW } \\
15-24 \\
\end{array}$ & $\begin{array}{c}\text { UM } \\
15-24 \\
\end{array}$ \\
\hline & \multicolumn{4}{|c|}{ Within village/neighbourhood } & \multicolumn{4}{|c|}{ Outside village/neighbourhood } & \multicolumn{4}{|c|}{ Health facility } \\
\hline \multicolumn{13}{|c|}{ Combined } \\
\hline \multicolumn{13}{|l|}{ Age (years) } \\
\hline $15-19$ & 66.4 & 58.2 & 73.3 & 98.8 & 6.9 & 5.7 & 7.4 & 83.5 & 5.4 & 5.9 & 4.7 & 68.5 \\
\hline $20-24$ & 60.3 & 59.1 & 74.0 & 99.4 & 13.4 & 12.9 & 17.6 & 94.3 & 15.5 & 15.2 & 18.8 & 77.0 \\
\hline \multicolumn{13}{|l|}{ Religion } \\
\hline Hindu & 63.8 & 58.8 & 74.0 & 99.1 & 10.1 & 10.2 & 9.0 & 85.0 & 10.0 & 11.5 & 6.4 & 70.8 \\
\hline Muslim & 64.2 & 58.2 & 70.8 & 98.1 & 6.3 & 6.7 & 5.3 & 90.8 & 6.5 & 8.6 & 4.0 & 67.6 \\
\hline \multicolumn{13}{|l|}{ Caste } \\
\hline SC & 67.3 & 66.1 & 71.0 & 98.4 & 10.8 & 12.7 & 4.7 & 79.9 & 9.3 & 10.8 & 4.0 & 73.9 \\
\hline $\mathrm{OBC}$ & 62.8 & 56.8 & 74.4 & 99.3 & 8.7 & 8.9 & 7.8 & 85.8 & 9.0 & 10.8 & 4.9 & 69.5 \\
\hline Genearl $^{1}$ & 63.6 & 51.8 & 72.2 & 98.3 & 11.2 & 9.0 & 11.6 & 89.5 & 12.1 & 14.3 & 9.9 & 69.8 \\
\hline \multicolumn{13}{|c|}{ Educational level (years) } \\
\hline None $^{2}$ & 64.9 & 63.6 & 70.5 & 100.0 & 10.0 & 11.5 & 3.2 & 90.3 & 9.7 & 11.5 & 2.1 & 66.9 \\
\hline $1-7$ & 62.9 & 53.3 & 73.9 & 98.1 & 5.1 & 5.6 & 4.1 & 82.2 & 6.8 & 10.4 & 2.5 & 62.5 \\
\hline $8-11$ & 62.0 & 45.0 & 74.4 & 98.9 & 11.2 & 8.0 & 12.7 & 85.4 & 9.9 & 11.0 & 8.6 & 71.7 \\
\hline 12 and above & 66.4 & 46.7 & 77.2 & 100.0 & 22.0 & 8.3 & 28.2 & 91.1 & 19.6 & 11.7 & 24.6 & 85.9 \\
\hline \multicolumn{13}{|c|}{ Worked in last 12 months } \\
\hline Yes & 76.0 & 75.5 & 76.8 & 99.1 & 12.5 & 15.8 & 5.7 & 88.9 & 11.8 & 15.7 & 3.9 & 72.8 \\
\hline No & 56.8 & 48.3 & 71.4 & 98.7 & 7.8 & 6.1 & 9.8 & 80.4 & 8.1 & 8.4 & 7.0 & 65.7 \\
\hline \multicolumn{13}{|l|}{ Wealth quintile } \\
\hline First & 72.8 & 71.1 & 79.3 & 98.2 & 11.5 & 14.1 & 2.5 & 85.6 & 12.1 & 14.8 & 1.7 & 70.3 \\
\hline Second & 67.6 & 65.9 & 72.0 & 100.0 & 8.2 & 9.8 & 4.0 & 81.4 & 6.9 & 9.0 & 1.7 & 61.3 \\
\hline Third & 62.5 & 59.4 & 69.4 & 98.6 & 9.8 & 12.0 & 5.0 & 87.0 & 6.9 & 8.7 & 2.7 & 64.0 \\
\hline Fourth & 57.4 & 45.7 & 75.7 & 98.5 & 6.3 & 6.1 & 6.3 & 85.9 & 10.0 & 13.1 & 5.1 & 70.8 \\
\hline Fifth & 60.9 & 47.8 & 72.3 & 99.3 & 11.6 & 6.0 & 15.3 & 86.5 & 11.0 & 9.9 & 11.5 & 75.9 \\
\hline Total & 63.9 & 58.7 & 73.3 & 98.9 & 9.5 & 9.8 & 8.3 & 85.9 & 9.5 & 11.2 & 5.9 & 70.3 \\
\hline \multicolumn{13}{|c|}{ Urban } \\
\hline \multicolumn{13}{|l|}{ Age (years) } \\
\hline $15-19$ & 73.6 & 51.3 & 79.1 & 99.4 & 20.1 & 10.3 & 22.7 & 82.0 & 9.9 & 10.3 & 10.1 & 68.1 \\
\hline $20-24$ & 65.3 & 59.2 & 80.4 & 100.0 & 20.1 & 14.6 & 32.7 & 95.2 & 18.2 & 16.3 & 23.4 & 85.7 \\
\hline \multicolumn{13}{|l|}{ Religion } \\
\hline Hindu & 71.1 & 58.0 & 80.9 & 99.5 & 22.1 & 13.6 & 28.2 & 87.0 & 14.9 & 16.1 & 14.2 & 73.4 \\
\hline Muslim & 64.7 & 50.0 & 73.5 & 100.0 & 10.9 & 12.5 & 11.1 & 86.0 & 7.6 & 8.3 & 8.1 & 77.3 \\
\hline \multicolumn{13}{|l|}{ Caste } \\
\hline SC & 70.2 & 62.5 & 76.8 & 100.0 & 11.9 & 8.7 & 14.5 & 90.9 & 10.6 & 16.7 & 5.5 & 82.6 \\
\hline OBC & 68.8 & 55.4 & 79.8 & 99.3 & 18.3 & 11.8 & 23.3 & 85.8 & 13.1 & 13.0 & 13.2 & 70.7 \\
\hline Genearl $^{1}$ & 72.8 & 57.7 & 79.3 & 100.0 & 28.0 & 22.2 & 30.7 & 87.5 & 16.1 & 19.2 & 15.3 & 78.9 \\
\hline \multicolumn{13}{|c|}{ Educational level (years) } \\
\hline None $^{2}$ & 62.7 & 57.7 & 72.1 & 100.0 & 9.2 & 9.8 & 5.0 & 95.2 & 8.5 & 11.5 & 3.3 & 81.0 \\
\hline $1-7$ & 65.9 & 52.9 & 77.8 & 97.6 & 12.4 & 11.8 & 14.1 & 78.0 & 9.4 & 11.8 & 6.1 & 66.7 \\
\hline $8-11$ & 71.6 & 59.0 & 78.1 & 100.0 & 22.8 & 15.4 & 25.7 & 84.6 & 14.7 & 17.9 & 12.8 & 70.3 \\
\hline 12 and above & 79.4 & 58.8 & 86.4 & 100.0 & 36.0 & 23.5 & 40.8 & 95.3 & 22.4 & 17.6 & 23.2 & 84.1 \\
\hline Worked in last $12 \mathrm{n}$ & & & & & & & & & & & & \\
\hline Yes & 78.1 & 70.6 & 82.1 & 100.0 & 20.8 & 17.6 & 21.4 & 89.3 & 15.6 & 17.6 & 14.1 & 77.1 \\
\hline No & 68.5 & 55.2 & 78.8 & 99.1 & 20.0 & 12.1 & 25.6 & 84.1 & 13.3 & 13.7 & 12.9 & 70.8 \\
\hline Wealth quintile & & & & & & & & & & & & \\
\hline First & 64.3 & $(60.0)$ & * & * & 13.3 & $(20.0)$ & * & * & 7.1 & (20.0) & * & * \\
\hline Second & 69.4 & 63.6 & 85.0 & (100.0) & 8.6 & 9.1 & 10.0 & $(88.9)$ & 8.3 & 9.1 & 5.0 & $(77.8)$ \\
\hline Third & 67.3 & 60.0 & 76.7 & $(100.0)$ & 11.5 & 12.5 & 6.9 & (71.4) & 9.6 & 12.5 & 3.4 & (64.3) \\
\hline Fourth & 70.0 & 62.5 & 76.7 & 100.0 & 13.3 & 12.5 & 15.0 & 86.8 & 12.2 & 16.7 & 8.5 & 76.9 \\
\hline Fifth & 70.6 & 54.0 & 80.2 & 99.5 & 23.6 & 13.8 & 28.9 & 88.0 & 15.0 & 14.9 & 14.9 & 74.2 \\
\hline Total & 70.0 & 57.0 & 79.4 & 99.6 & 20.1 & 13.4 & 24.9 & 86.9 & 13.7 & 14.7 & 13.0 & 74.2 \\
\hline
\end{tabular}


Table 7.4: (Cont'd)

\begin{tabular}{|c|c|c|c|c|c|c|c|c|c|c|c|c|}
\hline \multirow[t]{2}{*}{$\begin{array}{l}\text { Background } \\
\text { characteristics (\%) }\end{array}$} & $\begin{array}{c}\text { W } \\
15-24\end{array}$ & $\begin{array}{c}\text { MW } \\
\text { 15-24 }\end{array}$ & $\begin{array}{c}\text { UW } \\
15-24\end{array}$ & $\begin{array}{c}\text { UM } \\
15-24\end{array}$ & $\begin{array}{c}\text { W } \\
15-24\end{array}$ & $\begin{array}{c}\text { MW } \\
15-24\end{array}$ & $\begin{array}{c}\text { UW } \\
\text { 15-24 }\end{array}$ & $\begin{array}{c}\text { UM } \\
15-24\end{array}$ & $\begin{array}{c}\text { W } \\
15-24\end{array}$ & $\begin{array}{c}\text { MW } \\
15-24\end{array}$ & $\begin{array}{c}\text { UW } \\
15-24\end{array}$ & $\begin{array}{c}\text { UM } \\
15-24\end{array}$ \\
\hline & Withir & village/ & neighbor & rhood & Outsid & village & neighbo & $\overline{\text { irhood }}$ & & Health & facility & \\
\hline \multicolumn{13}{|c|}{ Rural } \\
\hline \multicolumn{13}{|l|}{ Age (years) } \\
\hline $15-19$ & 65.5 & 58.5 & 72.4 & 98.8 & 5.3 & 5.6 & 5.1 & 83.6 & 4.8 & 5.7 & 3.8 & 68.5 \\
\hline $20-24$ & 59.6 & 59.1 & 69.9 & 99.2 & 12.5 & 12.8 & 7.8 & 93.9 & 15.2 & 15.1 & 15.7 & 74.0 \\
\hline \multicolumn{13}{|l|}{ Religion } \\
\hline Hindu & 63.0 & 58.8 & 72.7 & 99.0 & 8.6 & 9.9 & 5.5 & 84.7 & 9.4 & 11.3 & 4.9 & 70.3 \\
\hline Muslim & 64.0 & 58.8 & 70.3 & 97.6 & 5.5 & 6.6 & 4.2 & 92.1 & 6.2 & 8.6 & 3.2 & 64.8 \\
\hline \multicolumn{13}{|l|}{ Caste } \\
\hline SC & 67.1 & 66.2 & 70.0 & 98.7 & 10.7 & 12.7 & 3.4 & 78.9 & 9.2 & 10.8 & 3.8 & 73.5 \\
\hline OBC & 62.1 & 57.0 & 73.5 & 99.3 & 7.5 & 8.7 & 5.2 & 85.9 & 8.5 & 10.7 & 3.5 & 69.3 \\
\hline Genearl $^{1}$ & 61.2 & 51.0 & 70.3 & 97.9 & 6.9 & 7.1 & 6.7 & 90.1 & 10.9 & 13.8 & 8.4 & 67.5 \\
\hline \multicolumn{13}{|c|}{ Educational level(years) } \\
\hline None $^{2}$ & 65.0 & 63.8 & 70.4 & 100.0 & 10.0 & 11.6 & 3.0 & 90.2 & 9.7 & 11.5 & 2.0 & 64.7 \\
\hline $1-7$ & 62.6 & 53.4 & 73.5 & 98.2 & 4.3 & 5.2 & 3.2 & 82.9 & 6.6 & 10.3 & 2.1 & 62.3 \\
\hline $8-11$ & 59.3 & 42.9 & 73.4 & 98.6 & 8.0 & 6.9 & 9.2 & 85.6 & 8.6 & 10.0 & 7.6 & 71.8 \\
\hline 12 and above & 53.6 & * & 66.0 & 100.0 & 8.0 & * & 12.6 & 89.8 & 16.8 & * & 26.2 & 87.4 \\
\hline \multicolumn{13}{|c|}{ Worked in last 12 months } \\
\hline Yes & 75.9 & 75.7 & 76.4 & 98.9 & 12.1 & 15.7 & 4.4 & 88.8 & 11.7 & 15.6 & 3.2 & 72.1 \\
\hline No & 54.7 & 47.7 & 69.5 & 98.8 & 5.6 & 5.5 & 5.7 & 79.4 & 7.1 & 7.9 & 5.5 & 64.3 \\
\hline \multicolumn{13}{|l|}{ Wealth quintile } \\
\hline First & 73.0 & 71.2 & 79.7 & 98.2 & 11.6 & 14.0 & 2.5 & 85.3 & 12.1 & 15.0 & 1.7 & 70.6 \\
\hline Second & 67.5 & 66.0 & 71.6 & 100.0 & 8.2 & 9.8 & 3.5 & 80.9 & 6.9 & 9.0 & 1.5 & 59.9 \\
\hline Third & 62.3 & 59.4 & 69.0 & 98.5 & 9.8 & 12.0 & 4.9 & 87.8 & 6.7 & 8.5 & 2.7 & 64.0 \\
\hline Fourth & 56.4 & 44.8 & 75.6 & 98.4 & 5.6 & 5.8 & 5.6 & 86.0 & 9.9 & 12.9 & 4.8 & 70.1 \\
\hline Fifth & 56.0 & 46.0 & 68.0 & 98.9 & 5.5 & 3.8 & 7.6 & 85.5 & 9.0 & 8.5 & 9.7 & 76.8 \\
\hline Total & 63.1 & 58.8 & 72.2 & 98.9 & 8.2 & 9.5 & 5.2 & 85.6 & 8.9 & 11.0 & 4.6 & 69.6 \\
\hline
\end{tabular}

Note: ( ) Based on 25-49 unweighted cases. ${ }^{*}$ Percentage not shown, based on fewer than 25 unweighted cases. Questions regarding freedom of movement were not asked of married males, as their mobility is generally unrestricted. OBC: Other backward caste. SC: Scheduled caste. ST: Scheduled tribe. ${ }^{1}$ Includes all those not belonging to SC, ST or OBC. ${ }^{2}$ Includes non-literate and literate with no formal schooling.

Patterns of socio-demographic differentials in measures of mobility differed among married and unmarried young women. For example, while unmarried young women's mobility within the village or neighbourhood did not differ much by any of the selected background characteristics, mobility of married young women differed considerably by such background characteristics as caste, education, work participation and household economic status. Interestingly, findings consistently suggest that progressively better educated women and those from economically better off families had less freedom of movement than other women. Patterns also differed with regard to associations between background characteristics and freedom to visit locations outside the village and a health facility. Indeed, while age conferred freedom on both the married and the unmarried, associations observed among the married did not hold true among the unmarried and vice-versa. For example, young women's freedom to visit locations outside the village or neighbourhood and a health facility was positively associated with work participation among the married, but not among the unmarried. Similarly, while among the unmarried, young women belonging to households in the wealthiest quintile were more likely than others to report freedom to visit locations outside the village or neighbourhood and a health facility, no such pattern was observed among the married. Finally, freedom to move outside the village or neighbourhood and to visit a health facility increased systematically with education among the unmarried but not among the married. The fact that differences by education were wide among the unmarried but not observed among the married suggests that marriage may have limited the positive association between education and mobility. 
Socio-demographic differentials in mobility, described above for the overall population were observed, by and large, among rural and urban respondents, as seen in Table 7.4. Even so, some exceptions were notable. For example, while mobility within the village or neighbourhood increased with education among urban young women, a reverse pattern was observed among rural young women. Similarly, while the association between mobility within the village or neighbourhood and economic status of the household was inconsistent among young women in urban settings, it declined with increasing economic status among young women in rural areas.

\subsection{Access to money}

In order to understand access to financial resources among youth, information was obtained on whether they had any savings, from any source, ranging from wages to gifts and pocket money. They were also asked whether they owned an account in a bank or a post office and if so, whether they operated the account themselves. Results are presented in Table 7.5.

Wide gender differences were observed. For example, young women were twice as likely as young men to have reported savings ( $44 \%$ of young women compared to $20 \%$ of young men). Differences by marital status were narrow among young women, but wide among young men; 30\% and 16\% of married and unmarried young men, respectively, reported some savings. Somewhat more urban than rural youth reported savings, and the difference was pronounced among young women (24\% versus $19 \%$ among young men, and $56 \%$ versus $43 \%$ among young women).

Findings on ownership of a bank/post office account reveal a different picture. Only a minority of youth reported owning a bank/post office account; even so, more young men than women so reported-11\% of young men and $5 \%$ of young women. Gender differences were wider among the married. For example, married young men were four times as likely as married young women to own an account $(17 \%$ and $4 \%$, respectively), highlighting married young women's limited access to resources. Differences by marital status suggest that married young men were somewhat more likely than unmarried young men to own an account ( $17 \%$ and $10 \%$, respectively). Among young women, marital status differences were negligible. Rural-urban differences were apparent as well, with urban residents more likely than rural respondents to report owning a bank account (21\% versus $10 \%$ among young men and $14 \%$ versus $4 \%$ among young women).

With regard to operation of the account too, gender differences were stark. Over three-quarters of young men (77\%) who owned an account operated it themselves. In contrast, only about two-fifths of young women who owned an account did so $(38 \%)$. Marital status differences suggest that the married were more likely than the unmarried to operate their accounts on their own. Rural-urban differences were negligible for young men, but wider for young women, among whom rural women were more likely than their urban counterparts to operate their own accounts ( $41 \%$ and $32 \%$, respectively); we note however, that among unmarried young women, it was the urban who were more likely than the rural to operate their own accounts.

\subsection{Gender role attitudes}

In order to understand gender role attitudes, youth were asked seven questions reflecting attitudes, including the relative importance attached to educating boys versus girls, the role of husbands as main decision-makers with regard to spending money, girls' participation in decisions about their own marriages, a woman's need to take permission from her husband for any activity, the comparative performance of girls versus boys in studies, gender roles in domestic work and whether girls who dress provocatively deserve to be teased. Findings, presented in Table 7.6, suggest a mixed scenario. 
Table 7.5: Access to money

Percentage of youth who reported having any savings, owning an account in a bank or post office and operating the account themselves, according to residence, Bihar, 2007

\begin{tabular}{|c|c|c|c|c|c|c|}
\hline Savings indicators (\%) & $\begin{array}{c}\mathrm{M} \\
15-24\end{array}$ & $\begin{array}{c}\text { W } \\
15-24\end{array}$ & $\begin{array}{c}\text { MM } \\
15-29\end{array}$ & $\begin{array}{c}\text { MW } \\
15-24\end{array}$ & $\begin{array}{c}\text { UM } \\
15-24\end{array}$ & $\begin{array}{c}\text { UW } \\
15-24\end{array}$ \\
\hline \multicolumn{7}{|c|}{ Combined } \\
\hline Has savings of any amount & 19.5 & 44.3 & 30.3 & 45.2 & 16.4 & 41.8 \\
\hline Ownership of a bank/post office account: & & & & & & \\
\hline In own (respondent's) name & 8.7 & 2.6 & 14.3 & 2.1 & 7.8 & 3.0 \\
\hline Jointly with someone else & 2.7 & 2.3 & 3.1 & 2.2 & 2.6 & 2.4 \\
\hline No account & 88.7 & 95.1 & 82.6 & 95.7 & 89.7 & 94.7 \\
\hline Number of respondents & 1,942 & 5,529 & 1,115 & 2,341 & 1,492 & 3,188 \\
\hline Operates bank/post office account themselves & 76.8 & 38.2 & 89.7 & 46.0 & 70.8 & 27.7 \\
\hline Number with an account & 309 & 469 & 270 & 182 & 221 & 287 \\
\hline \multicolumn{7}{|c|}{ Urban } \\
\hline Has savings of any amount & 23.7 & 56.4 & 32.6 & 55.9 & 22.5 & 56.7 \\
\hline Ownership of a bank/post office account: & & & & & & \\
\hline In own (respondent's) name & 15.4 & 8.2 & 30.4 & 5.6 & 14.3 & 10.1 \\
\hline Jointly with someone else & 5.4 & 6.1 & 3.3 & 7.0 & 5.7 & 5.7 \\
\hline No account & 79.2 & 85.9 & 67.4 & 88.0 & 80.0 & 84.4 \\
\hline Number of respondents & 1,039 & 2,581 & 547 & 1,136 & 833 & 1,445 \\
\hline Operates bank/post office account themselves & 73.7 & 32.2 & 90.3 & 35.3 & 70.8 & 31.2 \\
\hline Number with an account & 222 & 363 & 179 & 137 & 165 & 226 \\
\hline \multicolumn{7}{|c|}{ Rural } \\
\hline Has savings of any amount & 18.8 & 42.8 & 30.1 & 44.5 & 15.2 & 39.1 \\
\hline Ownership of a bank/post office account: & & & & & & \\
\hline In own (respondent's) name & 7.5 & 1.9 & 12.9 & 2.0 & 6.6 & 1.7 \\
\hline Jointly with someone else & 2.2 & 1.8 & 3.1 & 1.9 & 2.0 & 1.7 \\
\hline No account & 90.4 & 96.3 & 84.0 & 96.2 & 91.6 & 96.6 \\
\hline Number of respondents & 903 & 2,948 & 568 & 1,205 & 659 & 1,743 \\
\hline Operates bank/post office account themselves & 77.9 & 41.2 & 89.6 & $(48.8)$ & 70.5 & 24.2 \\
\hline Number with an account & 87 & 106 & 91 & 45 & 56 & 61 \\
\hline
\end{tabular}

Note: All Ns are unweighted. Column totals may not equal 100\% due to missing cases or "don't know" responses. ( ) Based on 25-49 unweighted cases. 
Questions that were most likely to elicit egalitarian attitudes from both young men and women included whether educating boys was more important than educating girls, whether girls are usually as good as boys in studies and whether husbands should be the main decision-makers with regard to spending money; $61-78 \%$ of young men and $82-88 \%$ of young women expressed egalitarian views on these matters. In addition, almost two-thirds of young women (65\%), but many fewer young men (36\%) expressed egalitarian attitudes about whether girls should be allowed to decide about their own marriage. Questions that were least likely to elicit egalitarian responses from youth included whether women should obtain their husbands' permission for most things ( $15 \%$ of young men and $31 \%$ of young women disagreed with the statement) and whether girls who dress provocatively deserve to be teased (35\% of young men and $37 \%$ of young women disagreed with the statement). Variation in reporting of egalitarian attitudes by topic is highlighted in Figure 7.3.

Table 7.6: Gender role attitudes

Percent distribution of youth by attitudes towards gender roles, according to residence, Bihar, 2007

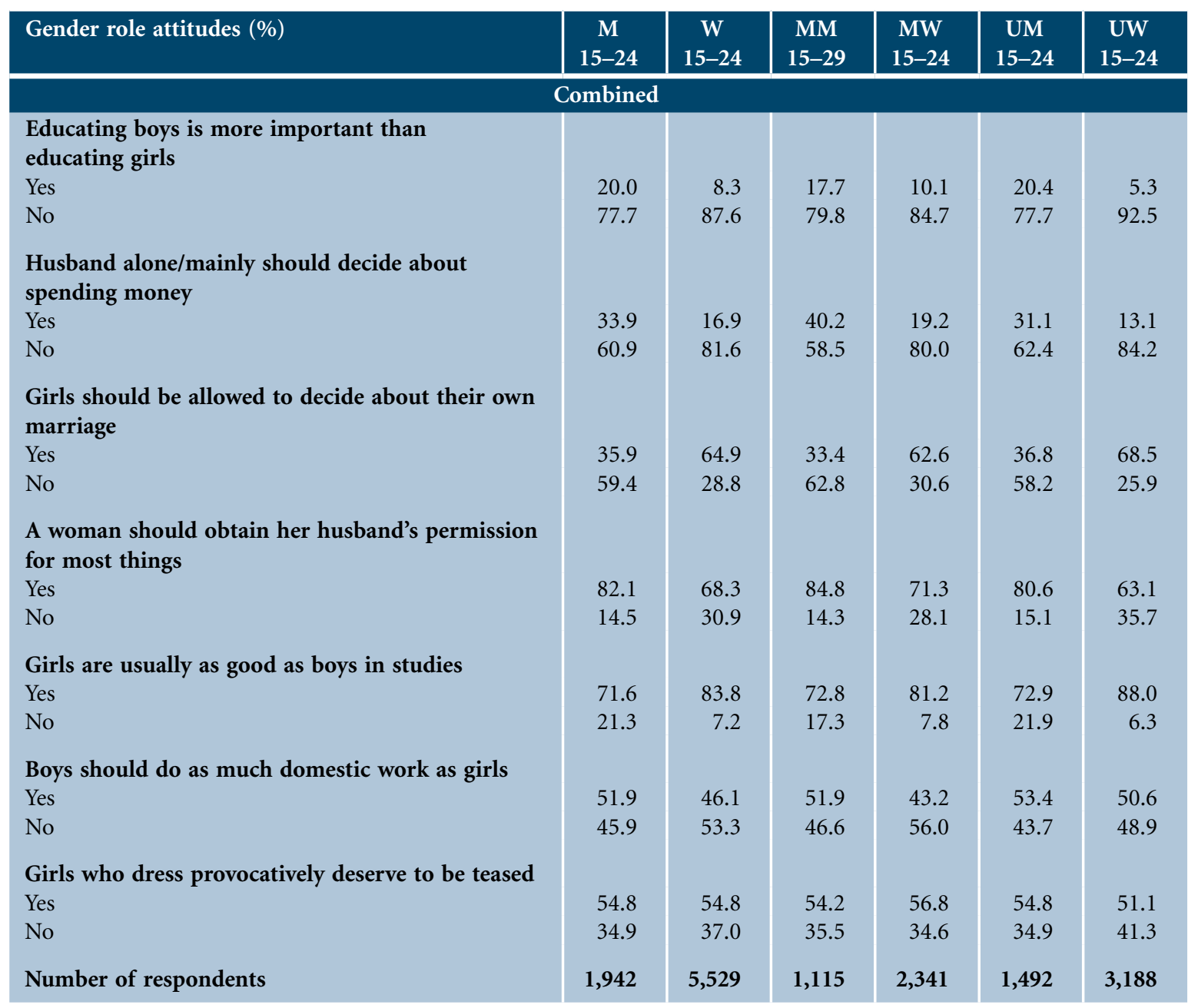




\begin{tabular}{|c|c|c|c|c|c|c|}
\hline Gender role attitudes (\%) & $\underset{15-24}{M}$ & $\begin{array}{c}W \\
15-24\end{array}$ & $\begin{array}{c}\text { MM } \\
15-29\end{array}$ & $\begin{array}{c}\text { MW } \\
15-24\end{array}$ & $\begin{array}{c}\text { UM } \\
15-24\end{array}$ & $\begin{array}{l}\text { UW } \\
15-24\end{array}$ \\
\hline \multicolumn{7}{|c|}{ Urban } \\
\hline \multicolumn{7}{|c|}{$\begin{array}{l}\text { Educating boys is more important than } \\
\text { educating girls }\end{array}$} \\
\hline Yes & 16.8 & 3.0 & 15.2 & 4.2 & 16.8 & 2.0 \\
\hline No & 81.1 & 95.0 & 83.7 & 93.0 & 81.1 & 96.6 \\
\hline \multicolumn{7}{|c|}{$\begin{array}{l}\text { Husband alone/mainly should decide about } \\
\text { spending money }\end{array}$} \\
\hline Yes & 30.8 & 8.5 & 37.0 & 11.3 & 29.9 & 6.7 \\
\hline No & 65.6 & 90.7 & 62.0 & 88.7 & 66.4 & 92.1 \\
\hline \multicolumn{7}{|c|}{$\begin{array}{l}\text { Girls should be allowed to decide about their } \\
\text { own marriage }\end{array}$} \\
\hline Yes & 46.6 & 77.1 & 44.6 & 72.7 & 47.5 & 80.0 \\
\hline No & 49.1 & 19.8 & 53.3 & 22.4 & 48.0 & 18.0 \\
\hline \multicolumn{7}{|c|}{$\begin{array}{l}\text { A woman should obtain her husband's permission } \\
\text { for most things }\end{array}$} \\
\hline Yes & 80.2 & 60.7 & 84.9 & 66.2 & 79.1 & 56.9 \\
\hline No & 17.3 & 38.5 & 14.0 & 33.8 & 18.0 & 41.9 \\
\hline \multicolumn{7}{|c|}{ Girls are usually as good as boys in studies } \\
\hline Yes & 77.3 & 92.6 & 78.3 & 88.7 & 77.1 & 95.3 \\
\hline No & 17.3 & 3.9 & 16.3 & 5.6 & 17.1 & 2.6 \\
\hline \multicolumn{7}{|c|}{ Boys should do as much domestic work as girls } \\
\hline Yes & 62.4 & 59.8 & 63.0 & 51.4 & 62.7 & 65.7 \\
\hline No & 36.9 & 39.9 & 35.9 & 47.9 & 36.5 & 34.1 \\
\hline \multicolumn{7}{|c|}{ Girls who dress provocatively deserve to be teased } \\
\hline Yes & 59.5 & 53.6 & 63.0 & 55.2 & 58.2 & 52.4 \\
\hline No & 33.3 & 43.1 & 31.5 & 41.3 & 34.4 & 44.5 \\
\hline Number of respondents & 1,039 & 2,581 & 547 & 1,136 & 833 & 1,445 \\
\hline \multicolumn{7}{|c|}{ Rural } \\
\hline \multicolumn{7}{|c|}{$\begin{array}{l}\text { Educating boys is more important than } \\
\text { educating girls }\end{array}$} \\
\hline Yes & 20.5 & 9.0 & 17.8 & 10.5 & 21.2 & 5.9 \\
\hline No & 77.1 & 86.6 & 79.6 & 84.2 & 77.0 & 91.8 \\
\hline \multicolumn{7}{|c|}{$\begin{array}{l}\text { Husband alone/mainly should decide about } \\
\text { spending money }\end{array}$} \\
\hline Yes & 34.4 & 18.0 & 40.5 & 19.7 & 31.3 & 14.3 \\
\hline No & 60.1 & 80.5 & 58.1 & 79.4 & 61.6 & 82.8 \\
\hline \multicolumn{7}{|c|}{$\begin{array}{l}\text { Girls should be allowed to decide about their } \\
\text { own marriage }\end{array}$} \\
\hline Yes & 34.1 & 63.3 & 32.4 & 61.9 & 34.7 & 66.4 \\
\hline No & 61.2 & 29.9 & 63.6 & 31.1 & 60.2 & 27.4 \\
\hline \multicolumn{7}{|c|}{$\begin{array}{l}\text { A woman should obtain her husband's permission } \\
\text { for most things }\end{array}$} \\
\hline Yes & 82.4 & 69.2 & 84.7 & 71.6 & 80.9 & 64.3 \\
\hline No & 14.1 & 29.9 & 14.4 & 27.7 & 14.5 & 34.6 \\
\hline \multicolumn{7}{|c|}{ Girls are usually as good as boys in studies } \\
\hline Yes & 70.7 & 82.6 & 72.3 & 80.7 & 72.0 & 86.7 \\
\hline No & 22.0 & 7.6 & 17.4 & 8.0 & 22.8 & 7.0 \\
\hline \multicolumn{7}{|c|}{ Boys should do as much domestic work as girls } \\
\hline Yes & 50.1 & 44.3 & 50.9 & 42.7 & 51.7 & 47.8 \\
\hline No & 47.4 & 55.0 & 47.5 & 56.6 & 45.1 & 51.7 \\
\hline \multicolumn{7}{|c|}{ Girls who dress provocatively deserve to be teased } \\
\hline Yes & 54.1 & 55.0 & 53.4 & 56.9 & 54.1 & 50.8 \\
\hline No & 35.2 & 36.2 & 35.9 & 34.2 & 35.0 & 40.7 \\
\hline Number of respondents & 903 & 2,948 & 568 & 1,205 & 659 & 1,743 \\
\hline
\end{tabular}

Note: All Ns are unweighted. Column totals may not equal 100\% due to missing cases, "don't know" or "unsure" responses. 
Young men were consistently more likely than young women to report unequal gender role attitudes in relation to most topics. For example, even though large proportions of youth believed that husbands alone should not be the main decision-makers with regard to spending money, more young men than women (34\% and $17 \%$, respectively) expressed the traditional attitude that husbands alone should decide about spending money. Similarly, $59 \%$ of young men compared to $29 \%$ of young women reported that girls should not be allowed to make marriage-related decisions. The issues on which young men were more likely than or as

Figure 7.3: Percentage of youth who expressed egalitarian gender role attitudes on selected issues, Bihar, 2007

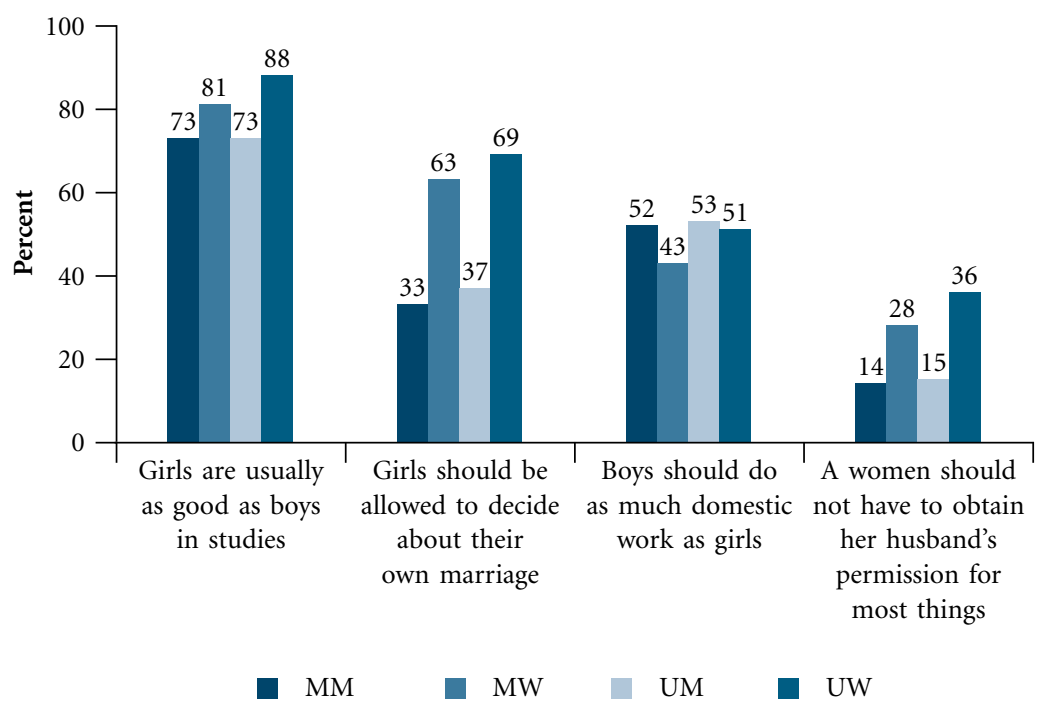

likely as young women to express egalitarian views were whether boys should do as much domestic work as girls $(52 \%$ and $46 \%$ of young men and women agreed with the statement, respectively) and whether girls who dress provocatively deserve to be teased (35\% of young men and $37 \%$ of young women disagreed with the statement).

No differences by marital status were evident among young men; however among young women, the unmarried reported more egalitarian attitudes than the married on almost all statements. Rural-urban differences were consistently observed, with urban youth more likely to reveal gender egalitarian attitudes than their rural counterparts. The only exception was that young men in urban settings were more likely than their rural counterparts to agree that girls who dress provocatively deserve to be teased (60\% and $54 \%$, respectively).

\subsection{Attitudes towards wife beating}

Youth were asked a number of questions to gauge the extent to which beating one's wife was perceived to be an acceptable behaviour. Young people were asked whether they agreed that wife beating was a way of expressing love, and whether wife beating was justified in four situations, including refusal to have sex with the husband. Findings are presented in Table 7.7. Although large proportions of youth (76\% of young men and $84 \%$ of young women) disagreed that wife beating was a sign of love, it is notable that $10-12 \%$ of young people did conform to this view. Differences by marital status and residence were negligible. 
Findings show, moreover, that over two-fifths of young men (44\%) and about three-fifths of young women $(58 \%)$ did justify wife beating. While marital status differences were muted among young men, a somewhat larger percentage of married than unmarried young women perceived that wife beating was justified in at least one of the four situations about which information was sought (61\% versus 53\%). Rural-urban differences suggest that considerably more rural than urban youth, particularly young women, justified wife beating.

The situations under which young people were most likely to perceive that wife beating was justified were relatively similar among young men and women. A similar proportion of young men $(30 \%$ each) perceived wife beating was justified in three of the four situations posed, namely, if the husband suspected that the wife had been unfaithful, if the wife went out without telling her husband and if the wife disagreed with her husband's opinion. A slightly larger proportion of young women (34-40\%) justified wife beating in these situations. Both young men and women were least likely to justify wife beating if a woman refused to have sexual relations with her husband ( $12 \%$ and $7 \%$ among young men and women, respectively). Marital status differences were typically muted among young men, but unmarried women were less likely than the married to justify wife-beating in at least two of the four situations. Rural-urban differences were wide and consistent: a larger percentage of rural than urban youth agreed that wife beating was justified in each of the four situations posed.

Table 7.7: Attitudes towards wife beating

Percent distribution of youth by attitudes towards wife beating in selected situations, according to residence, Bihar, 2007

\begin{tabular}{|c|c|c|c|c|c|c|}
\hline Attitudes towards wife beating (\%) & $\underset{15-24}{\mathrm{M}}$ & $\begin{array}{c}\text { W } \\
15-24\end{array}$ & $\begin{array}{c}\text { MM } \\
15-29\end{array}$ & $\begin{array}{c}\text { MW } \\
15-24\end{array}$ & $\begin{array}{c}\text { UM } \\
15-24\end{array}$ & $\begin{array}{c}\text { UW } \\
15-24\end{array}$ \\
\hline \multicolumn{7}{|c|}{ Combined } \\
\hline Beating wife means husband loves her & 102 & 117 & 139 & 125 & 95 & 105 \\
\hline Disagree & 75.9 & 84.3 & 78.2 & 84.9 & 74.8 & 82.7 \\
\hline Don't know/can't say & 13.9 & 4.0 & 7.4 & 2.6 & 15.8 & 6.8 \\
\hline \multicolumn{7}{|l|}{ Beating wife is justified if: } \\
\hline Husband suspects wife has been unfaithful & & & & & & \\
\hline Yes & 30.1 & 34.2 & 34.2 & 38.0 & 29.6 & 27.7 \\
\hline No & 65.5 & 64.8 & 64.0 & 61.7 & 64.6 & 69.9 \\
\hline Don't know/can't say & 4.4 & 1.0 & 1.6 & 0.3 & 5.8 & 2.3 \\
\hline \multicolumn{7}{|l|}{ Wife goes out without telling husband } \\
\hline Yes & 29.5 & 39.3 & 31.6 & 41.1 & 29.8 & 36.4 \\
\hline No & 64.0 & 59.8 & 63.2 & 58.4 & 63.6 & 61.7 \\
\hline Don’t know/can't say & 6.4 & 0.9 & 5.0 & 0.4 & 6.6 & 1.8 \\
\hline \multicolumn{7}{|l|}{ Wife disagrees with husband's opinion } \\
\hline Yes & 30.0 & 38.8 & 30.7 & 39.9 & 30.4 & 37.4 \\
\hline No & 63.2 & 59.3 & 64.2 & 59.1 & 62.1 & 59.2 \\
\hline Don’t know/can’t say & 6.8 & 1.8 & 4.8 & 1.1 & 7.5 & 3.4 \\
\hline \multicolumn{7}{|l|}{ Wife refuses to have sexual relations with husband } \\
\hline 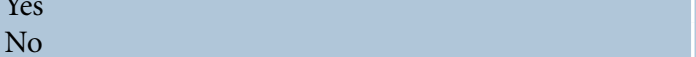 & $\begin{array}{l}12.2 \\
77.6\end{array}$ & $\begin{array}{r}6.5 \\
89.0\end{array}$ & $\begin{array}{l}11.8 \\
86.3\end{array}$ & $\begin{array}{r}7.0 \\
91.5\end{array}$ & $\begin{array}{l}12.2 \\
74.6\end{array}$ & $\begin{array}{r}5.8 \\
84.1\end{array}$ \\
\hline Don't know/can't say & 10.2 & 4.4 & 1.7 & 1.5 & 13.2 & 10.0 \\
\hline $\begin{array}{l}\text { Believed that wife beating is justified in at least one } \\
\text { of the above situations }\end{array}$ & 44.3 & 57.7 & 46.5 & 60.5 & 44.2 & 53.3 \\
\hline Number of respondents & 1,942 & 5,529 & 1,115 & 2,341 & 1,492 & 3,188 \\
\hline
\end{tabular}


Table 7.7: (Cont'd)

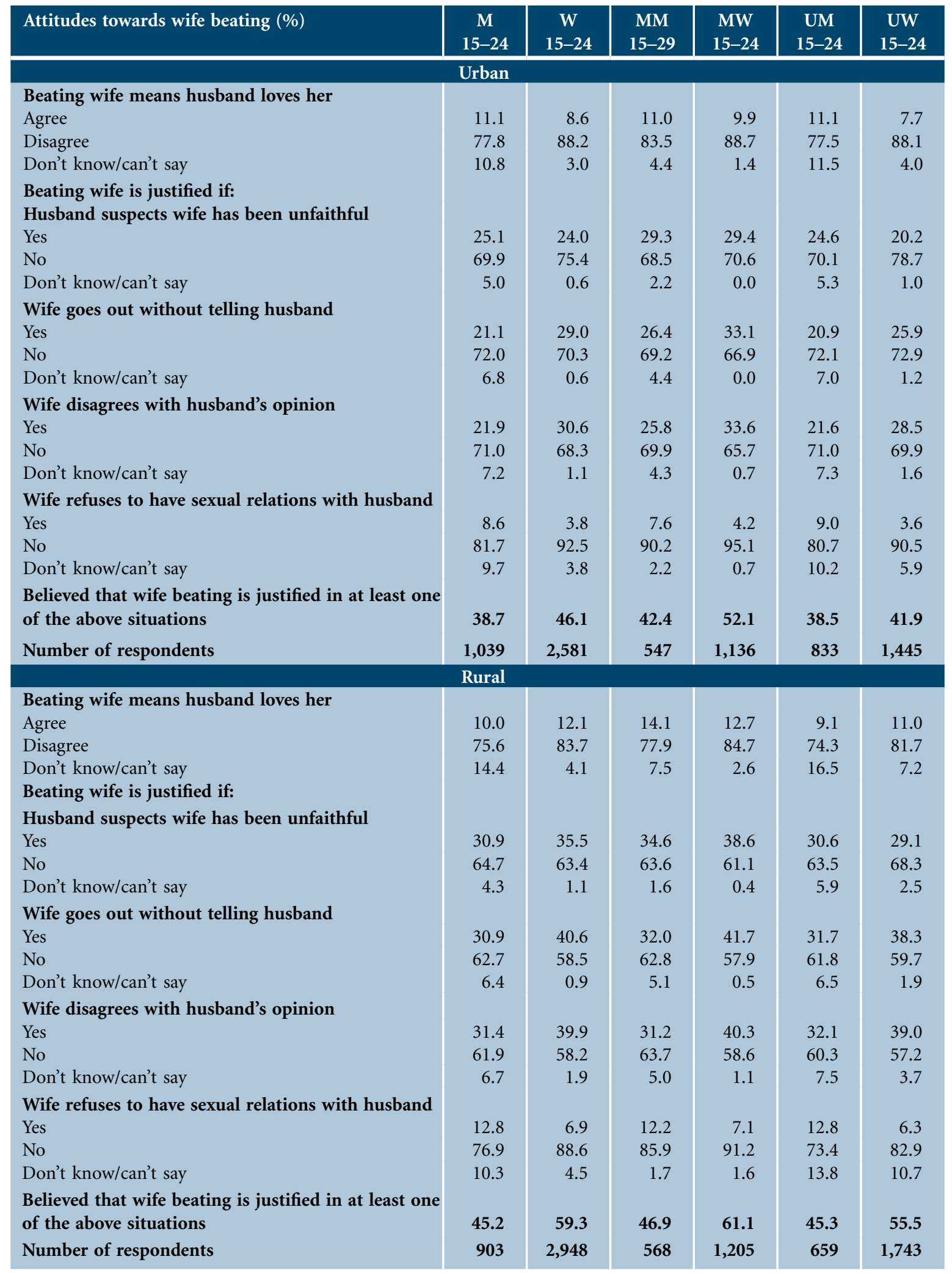

Note: All Ns are unweighted. Column totals may not equal 100\% due to missing cases. 
Figure 7.4: Percentage of youth who believed wife beating is justified in selected situations, Bihar, 2007

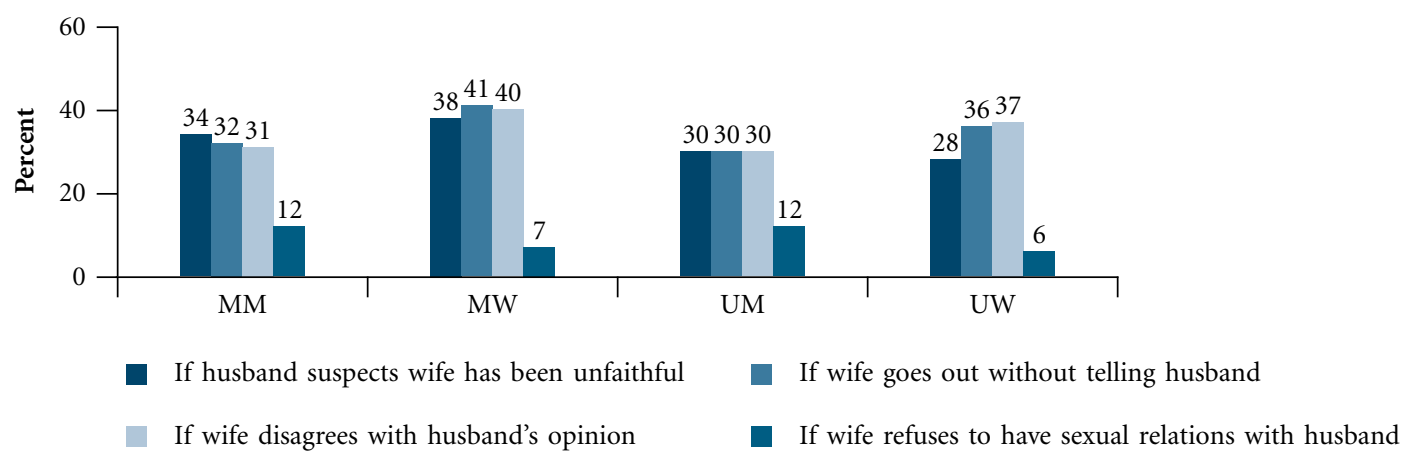

\subsection{Summary}

Findings clearly highlight young women's extremely limited agency. For example, just one in four young women reported independent decision-making on all three issues explored in the survey, namely, decisions on choice of friends, spending money and purchase of clothes. Likewise, freedom of movement even within the village or neighbourhood was not universal among young women; only about two in three young women had the freedom to visit locations within their own village or neighbourhood unescorted. Moreover, just one in ten young women reported freedom to visit at least one place outside the village or neighbourhood and a health facility unescorted. Access to and control over financial resources tended to be limited among young women; just over two in five reported some savings and 5\% owned a bank or post office savings account. Of those who owned an account, about two-fifths operated it themselves.

Also notable from the findings is the striking gender divide in all these dimensions of young people's agency. Young women were far more disadvantaged in terms of decision-making autonomy and mobility than young men. Likewise, although young women were more likely than young men to have money saved ( $44 \%$ and $20 \%$, respectively), they were less likely than young men to own a bank or post office savings account (5\% and $11 \%)$. Moreover, they were much less likely than their male counterparts to operate these accounts themselves (38\% and $77 \%$ of those who had an account).

While young men were clearly not as disadvantaged as young women, findings indicate that many young men were also unable to exercise agency in their everyday lives. For example, only $46 \%$ of young men reported independent decision-making on all three issues explored in the survey. Similarly, young men's mobility was far from universal; for example, just $48-56 \%$ of unmarried young men were allowed to visit a place of entertainment or to attend a programme conducted outside their village or neighbourhood unescorted, and two in three were allowed to visit a health facility unescorted.

Although over two-fifths of young men and about three-fifths of young women justified wife beating in at least one situation, relatively large proportions of youth espoused egalitarian gender role attitudes on other issues explored. Even so, it is notable that young men were consistently more likely than young women to report unequal gender role attitudes on these issues. 


\section{Chapter 8}

\section{Awareness of sexual and}

reproductive health matters

A considerable body of research, including the NFHS (IIPS and Macro International, 2007a), has highlighted relatively low levels of awareness regarding selected sexual and reproductive health issues in both the general and youth populations. The Youth Study sought to explore awareness of a wide range of issues relating to sex, pregnancy, contraception and STI, including HIV/AIDS, as well as knowledge of laws governing age at marriage and abortion. Where possible, further questions were posed to assess the extent of in-depth awareness of these matters. Along with the results of these items, this chapter explores findings on communication about and sources of information for sexual and reproductive health matters, as well as youth perceptions and experiences of family life or sex education.

\subsection{Awareness of sexual and reproductive health matters}

In this section, we present evidence of the extent to which young people are aware of or hold misconceptions about various issues related to sex and pregnancy, contraception, STI and HIV.

\subsubsection{Sex and pregnancy}

In order to assess young people's knowledge about sex and pregnancy, the Youth Study asked youth whether they agreed or disagreed with four statements: (a) a woman can get pregnant after kissing or hugging; (b) a woman is most likely to get pregnant if she has sex half-way between her periods; (c) a woman has to bleed at first intercourse; and (d) a woman can get pregnant at first sex. Given the prevalence of sex-selective abortions in the country (Bhat and Zavier, 2007; Dagar, 2007), we also asked whether youth were aware of any tests that could determine the sex of the foetus.

Findings, presented in Table 8.1, clearly suggest that awareness of sex- and pregnancy-related matters was limited. The one exception was knowledge that women cannot become pregnant after kissing or hugging; $97 \%$ of young men and women were aware of this. Even so, it is notable that $8 \%$ of unmarried young women were either unsure or believed it to be possible.

Awareness of other matters was reported by far smaller proportions of youth and considerable differences were evident by sex, marital status and rural-urban residence of respondents. Just one-third of young men and one-fifth of young women were aware that women are most likely to become pregnant if they engage in sexual relations mid-cycle. More married than unmarried youth $(58 \%$ and $24 \%$ of married and unmarried young men, respectively, and $28 \%$ and $8 \%$ of married and unmarried young women, respectively) reported correct knowledge of this issue (see Figure 8.1).Differences by rural-urban residence were muted. When analysed separately for the married and the unmarried among young women, however, those in urban areas were more likely than those in rural areas to report knowledge of this issue. 
Table 8.1: Awareness of sex- and pregnancy-related matters

Percent distribution of youth by awareness of sex- and pregnancy-related matters, according to residence, Bihar, 2007

\begin{tabular}{|c|c|c|c|c|c|c|}
\hline Awareness indicators (\%) & $\begin{array}{c}\text { M } \\
15-24\end{array}$ & $\begin{array}{c}W \\
15-24\end{array}$ & $\begin{array}{c}\text { MM } \\
15-29\end{array}$ & $\begin{array}{c}\text { MW } \\
15-24\end{array}$ & $\begin{array}{l}\text { UM } \\
15-24\end{array}$ & $\begin{array}{l}\text { UW } \\
15-24\end{array}$ \\
\hline \multicolumn{7}{|c|}{ Combined } \\
\hline $\begin{array}{l}\text { A woman can get pregnant after kissing/hugging } \\
\text { True }\end{array}$ & 0.2 & 0.5 & 0.4 & 0.5 & 0.3 & 0.5 \\
\hline False & 97.0 & 96.6 & 99.2 & 99.0 & 96.0 & 91.9 \\
\hline Don't know/not sure & 2.9 & 2.9 & 0.2 & 0.4 & 3.8 & 7.6 \\
\hline \multicolumn{7}{|l|}{$\begin{array}{l}\text { A woman is most likely to get pregnant if she has } \\
\text { sex half-way between her periods }\end{array}$} \\
\hline True & 32.2 & 21.1 & 57.8 & 27.7 & 24.2 & 8.4 \\
\hline False & 12.1 & 7.8 & 17.8 & 10.1 & 10.6 & 3.6 \\
\hline Don't know/not sure & 55.7 & 71.1 & 24.4 & 62.2 & 65.2 & 88.0 \\
\hline \multicolumn{7}{|l|}{ A woman has to bleed at first intercourse } \\
\hline True & 41.6 & 41.9 & 57.2 & 57.0 & 34.6 & 13.9 \\
\hline False & 21.2 & 28.4 & 33.9 & 35.2 & 18.6 & 15.7 \\
\hline Don't know/not sure & 37.1 & 29.7 & 8.9 & 7.8 & 46.8 & 70.4 \\
\hline \multicolumn{7}{|l|}{ A woman can get pregnant at first sex } \\
\hline True & 27.4 & 33.2 & 40.0 & 37.0 & 24.2 & 25.6 \\
\hline False & 38.3 & 39.3 & 48.9 & 48.1 & 33.0 & 23.0 \\
\hline Don't know/not sure & 34.2 & 27.5 & 11.1 & 14.9 & 42.8 & 51.5 \\
\hline \multicolumn{7}{|l|}{$\begin{array}{l}\text { It is possible to do a medical test to know the } \\
\text { sex of a foetus }\end{array}$} \\
\hline True & 68.5 & 79.6 & 73.3 & 82.0 & 67.6 & 74.3 \\
\hline False & 13.4 & 7.0 & 12.8 & 6.7 & 13.2 & 7.6 \\
\hline Don't know/not sure & 18.0 & 13.4 & 13.9 & 11.3 & 19.1 & 18.0 \\
\hline Had correct knowledge of all of the above & 3.7 & 2.1 & 6.6 & 2.7 & 2.8 & 0.8 \\
\hline Number of respondents & 1,942 & 5,529 & 1,115 & 2,341 & 1,492 & 3,188 \\
\hline \multicolumn{7}{|c|}{ Urban } \\
\hline $\begin{array}{l}\text { A woman can get pregnant after kissing/hugging } \\
\text { True }\end{array}$ & 0.0 & 0.3 & 0.0 & 0.7 & 0.0 & 0.2 \\
\hline False & 98.2 & 97.2 & 100.0 & 99.3 & 98.4 & 95.5 \\
\hline Don't know/not sure & 1.8 & 2.5 & 0.0 & 0.0 & 1.6 & 4.3 \\
\hline \multicolumn{7}{|l|}{$\begin{array}{l}\text { A woman is most likely to get pregnant if she has } \\
\text { sex half-way between her periods }\end{array}$} \\
\hline True & 31.2 & 23.4 & 62.0 & 37.1 & 27.3 & 13.5 \\
\hline False & 12.9 & 6.9 & 17.4 & 11.2 & 12.7 & 4.0 \\
\hline Don't know/not sure & 55.9 & 69.7 & 20.7 & 51.7 & 60.0 & 82.4 \\
\hline \multicolumn{7}{|l|}{ A woman has to bleed at first intercourse } \\
\hline True & 34.4 & 29.8 & 47.8 & 53.1 & 32.4 & 12.8 \\
\hline False & 29.4 & 27.5 & 45.7 & 40.6 & 27.5 & 18.2 \\
\hline Don't know/not sure & 36.2 & 42.7 & 6.5 & 6.3 & 40.2 & 69.0 \\
\hline \multicolumn{7}{|l|}{ A woman can get pregnant at first sex } \\
\hline True & 31.5 & 39.8 & 46.7 & 45.8 & 30.7 & 35.4 \\
\hline False & 29.4 & 32.4 & 41.3 & 44.4 & 26.6 & 23.8 \\
\hline Don’t know/not sure & 39.1 & 27.8 & 12.0 & 9.9 & 42.6 & 40.8 \\
\hline \multicolumn{7}{|l|}{$\begin{array}{l}\text { It is possible to do a medical test to know the } \\
\text { sex of a foetus }\end{array}$} \\
\hline True & 80.4 & 87.3 & 83.7 & 88.1 & 80.4 & 86.4 \\
\hline False & 6.4 & 5.5 & 7.6 & 4.9 & 6.1 & 6.1 \\
\hline Don't know/not sure & 12.9 & 7.2 & 8.7 & 7.0 & 13.1 & 7.5 \\
\hline Had correct knowledge of all of the above & 6.1 & 4.9 & 13.0 & 7.7 & 5.3 & 2.4 \\
\hline Number of respondents & 1,039 & 2,581 & 547 & 1,136 & 833 & 1,445 \\
\hline
\end{tabular}


Table 8.1: (Cont'd)

\begin{tabular}{|c|c|c|c|c|c|c|}
\hline Awareness indicators (\%) & $\begin{array}{c}M \\
15-24\end{array}$ & $\begin{array}{c}\text { W } \\
15-24\end{array}$ & $\begin{array}{c}\text { MM } \\
15-29\end{array}$ & $\begin{array}{c}\text { MW } \\
15-24\end{array}$ & $\begin{array}{l}\text { UM } \\
15-24\end{array}$ & $\begin{array}{l}\text { UW } \\
15-24\end{array}$ \\
\hline \multicolumn{7}{|c|}{ Rural } \\
\hline A woman can get pregnant after kissing/hugging & & & & & & \\
\hline True & 0.2 & 0.5 & 0.5 & 0.5 & 0.3 & 0.6 \\
\hline False & 96.8 & 96.5 & 99.1 & 99.0 & 95.6 & 91.3 \\
\hline Don't know/not sure & 3.0 & 2.9 & 0.2 & 0.4 & 4.1 & 8.2 \\
\hline \multicolumn{7}{|l|}{$\begin{array}{l}\text { A woman is most likely to get pregnant if she has } \\
\text { sex half-way between her periods }\end{array}$} \\
\hline True & 32.4 & 20.8 & 57.5 & 27.1 & 23.6 & 7.4 \\
\hline False & 11.9 & 7.9 & 17.8 & 10.0 & 10.3 & 3.5 \\
\hline Don't know/not sure & 55.7 & 71.3 & 24.7 & 62.9 & 66.1 & 89.1 \\
\hline \multicolumn{7}{|l|}{ A woman has to bleed at first intercourse } \\
\hline True & 42.8 & 43.4 & 58.1 & 57.3 & 35.0 & 14.1 \\
\hline False & 19.9 & 28.6 & 32.8 & 34.8 & 16.9 & 15.3 \\
\hline Don’t know/not sure & 37.3 & 28.0 & 9.1 & 7.9 & 48.1 & 70.7 \\
\hline \multicolumn{7}{|l|}{ A woman can get pregnant at first sex } \\
\hline True & 26.8 & 32.4 & 39.4 & 36.4 & 23.0 & 23.8 \\
\hline False & 39.8 & 40.2 & 49.6 & 48.4 & 34.2 & 22.8 \\
\hline Don't know/not sure & 33.4 & 27.4 & 11.0 & 15.2 & 42.8 & 53.4 \\
\hline \multicolumn{7}{|l|}{$\begin{array}{l}\text { It is possible to do a medical test to know the } \\
\text { sex of a foetus }\end{array}$} \\
\hline True & 66.5 & 78.6 & 72.3 & 81.6 & 65.1 & 72.2 \\
\hline False & 14.6 & 7.2 & 13.4 & 6.8 & 14.6 & 7.8 \\
\hline Don't know/not sure & 18.9 & 14.3 & 14.4 & 11.6 & 20.3 & 20.0 \\
\hline Had correct knowledge of all of the above & 3.3 & 1.8 & 6.1 & 2.4 & 2.3 & 0.5 \\
\hline Number of respondents & 903 & 2,948 & 568 & 1,205 & 659 & 1,743 \\
\hline
\end{tabular}

Note: All Ns are unweighted. Column totals may not equal 100\% due to missing cases.

Awareness that a woman does not have to bleed at first intercourse was reported by even fewer $(21-28 \%)$. Notably, two in five youth believed that a woman has to bleed at first intercourse. Marital status differences were notable, with married youth better informed than unmarried youth (34-35\% compared to 16\%-19\%). Rural-urban differences were wide among young men, with urban young men more likely to be correctly informed about this issue than rural young men (29\% versus $20 \%$ ); differences were muted among young women.

Awareness that a woman can get pregnant at first sex was also limited, correctly reported by just $27-33 \%$ of youth. Again, more married than unmarried youth (37-40\% compared to $24-26 \%$ ), and somewhat more urban than rural youth (32-40\% compared to $27-32 \%)$ reported correctly that a woman could become pregnant at first sex.

Two-thirds of young men (69\%) and four-fifths of young women $(80 \%)$ were aware of the availability of tests to determine the sex of the foetus. Married youth were somewhat more likely than unmarried youth to report awareness of sex determination testing. Differences by rural-urban residence were pronounced, with larger percentages of urban than rural youth reporting awareness of sex determination testing (80\% and $87 \%$ of young men and women in urban areas, compared to $67 \%$ and $79 \%$, respectively, in rural areas).

In order to examine overall knowledge regarding sex and pregnancy, a summary measure was computed that assessed the percentage of youth who were aware of all five matters, and is presented in Table 8.1. 
Figure 8.1: Percentage of youth reporting awareness of selected sex- and pregnancy-related matters, according to residence, Bihar, 2007

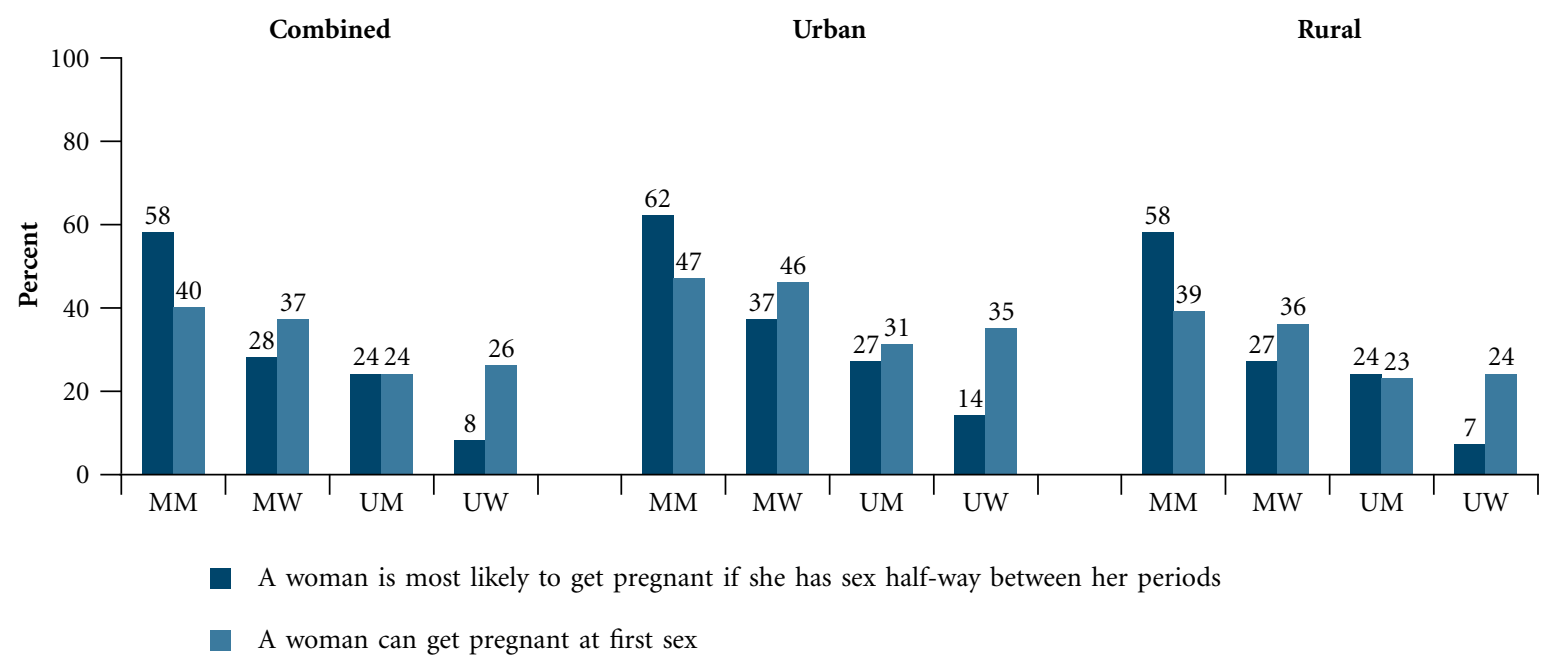

Findings show that just 2-4\% of youth had correct knowledge of all five issues. Differences by marital status and residence were modest. The only somewhat notable difference was that married youth in urban settings were more knowledgeable than married youth in rural settings (8-13\% compared to $2-6 \%$ ). Indeed, the highest levels of awareness of the five matters combined were reported by married young men in urban settings, among whom 13\% reported awareness of all unmarried young women in rural settings, among whom just $0.5 \%$ were correctly aware of all issues; in contrast, the poorest levels of awareness were reported by five matters.

\subsubsection{Socio-demographic differentials in awareness of sex- and pregnancy-related matters}

Differentials in awareness, measured with respect to percentage aware of all five issues relating to sex and pregnancy discussed above, are presented in Table 8.2. Level of awareness did not differ much by background characteristics, which is not surprising given the extremely low level of awareness observed for the overall population. However, some notable differences were apparent, particularly among married youth. For example, a larger percentage of married young men belonging to general castes than others reported awareness of all five issues (18\% compared to 5-6\%). Findings also show a consistent positive association between education and household economic status with awareness of sex- and pregnancy-related matters. For example, awareness of all five matters increased from 1-2\% among uneducated married youth to $12-18 \%$ among those with 12 or more years of schooling. Similarly, awareness increased from 3\% among married young men belonging to the poorest (first) quintile to $11 \%$ among those belonging to the wealthiest (fifth) quintile; corresponding increases were mild among married young women. Similar patterns of socio-demographic differentials were, by and large, evident in both rural and urban areas. In addition, findings suggest that in urban areas, somewhat larger proportions of married young men aged 25-29 years compared to those aged 20-24, and those belonging to the Hindu religion compared to those belonging to the Muslim religion reported awareness of sex- and pregnancy-related matters. 
Table 8.2: Awareness of sex- and pregnancy-related matters by selected background characteristics

Percentage of youth who had correct knowledge of all five sex- and pregnancy-related matters by selected background characteristics, according to residence, Bihar, 2007

\begin{tabular}{|c|c|c|c|c|c|c|}
\hline Background characteristics (\%) & $\begin{array}{c}\mathrm{M} \\
15-24\end{array}$ & $\begin{array}{c}\text { W } \\
15-24\end{array}$ & $\begin{array}{c}\text { MM } \\
15-29\end{array}$ & $\begin{array}{c}\text { MW } \\
15-24\end{array}$ & $\begin{array}{c}\text { UM } \\
15-24\end{array}$ & $\begin{array}{c}\text { UW } \\
15-24\end{array}$ \\
\hline \multicolumn{7}{|c|}{ Combined } \\
\hline \multicolumn{7}{|l|}{ Age (years) } \\
\hline $15-19$ & 2.0 & 1.2 & 2.3 & 1.9 & 2.0 & 0.6 \\
\hline $20-24$ & 6.3 & 3.4 & 6.8 & 3.3 & 5.7 & 2.9 \\
\hline $25-29$ & NA & NA & 7.2 & NA & NA & NA \\
\hline \multicolumn{7}{|l|}{ Religion } \\
\hline Hindu & 3.9 & 2.2 & 7.0 & 2.8 & 2.8 & 0.8 \\
\hline Muslim & 2.2 & 1.5 & 3.7 & 1.9 & 2.9 & 0.8 \\
\hline \multicolumn{7}{|l|}{ Caste } \\
\hline SC & 2.5 & 2.0 & 4.7 & 2.6 & 0.8 & 0.4 \\
\hline OBC & 3.1 & 1.9 & 5.8 & 2.5 & 2.4 & 0.5 \\
\hline General $^{1}$ & 6.7 & 3.3 & 18.2 & 4.5 & 5.1 & 2.1 \\
\hline \multicolumn{7}{|l|}{ Educational level (years) } \\
\hline None $^{2}$ & 1.6 & 1.7 & 0.9 & 2.1 & 2.3 & 0.3 \\
\hline $1-7$ & 2.4 & 2.0 & 6.8 & 3.3 & 0.7 & 0.3 \\
\hline $8-11$ & 4.1 & 2.2 & 7.7 & 3.3 & 3.3 & 1.0 \\
\hline 12 and above & 8.4 & 7.2 & 17.6 & 11.7 & 5.8 & 3.9 \\
\hline \multicolumn{7}{|l|}{ Worked in last 12 months } \\
\hline Yes & 3.6 & 1.5 & 6.5 & 1.7 & 2.5 & 1.0 \\
\hline No & 3.8 & 2.5 & $(15.6)$ & 3.3 & 3.4 & 0.7 \\
\hline \multicolumn{7}{|l|}{ Wealth quintile } \\
\hline First & 3.4 & 1.2 & 2.5 & 1.5 & 2.7 & 0.0 \\
\hline Second & 4.3 & 1.3 & 4.7 & 1.6 & 2.5 & 0.0 \\
\hline Third & 2.4 & 2.2 & 5.4 & 2.7 & 1.1 & 0.9 \\
\hline Fourth & 2.7 & 1.5 & 6.9 & 2.2 & 1.5 & 0.4 \\
\hline Fifth & 5.1 & 3.9 & 10.9 & 6.0 & 4.6 & 1.7 \\
\hline Total & 3.7 & 2.1 & 6.6 & 2.7 & 2.8 & 0.8 \\
\hline \multicolumn{7}{|c|}{ Urban } \\
\hline \multicolumn{7}{|l|}{ Age (years) } \\
\hline $15-19$ & 4.3 & 2.2 & * & 5.1 & 4.3 & 1.6 \\
\hline $20-24$ & 8.5 & 8.4 & 9.4 & 9.6 & 7.2 & 5.7 \\
\hline $25-29$ & NA & NA & 15.5 & NA & NA & NA \\
\hline \multicolumn{7}{|l|}{ Religion } \\
\hline Hindu & 6.1 & 5.0 & 13.9 & 8.5 & 5.5 & 2.3 \\
\hline Muslim & 4.3 & 4.2 & 7.7 & 8.3 & 4.7 & 2.0 \\
\hline \multicolumn{7}{|l|}{ Caste } \\
\hline SC & 3.6 & 3.5 & 7.1 & 4.3 & 4.3 & 0.0 \\
\hline OBC & 5.3 & 4.9 & 13.6 & 7.6 & 4.7 & 2.1 \\
\hline General $^{1}$ & 7.8 & 5.6 & 16.7 & 11.1 & 7.0 & 4.0 \\
\hline
\end{tabular}


Table 8.2: (Cont'd)

\begin{tabular}{|c|c|c|c|c|c|c|}
\hline Background characteristics (\%) & $\begin{array}{c}M \\
15-24\end{array}$ & $\begin{array}{c}\text { W } \\
15-24\end{array}$ & $\begin{array}{c}\text { MM } \\
15-29\end{array}$ & $\begin{array}{c}\text { MW } \\
15-24\end{array}$ & $\begin{array}{c}\text { UM } \\
15-24\end{array}$ & $\begin{array}{c}\text { UW } \\
15-24\end{array}$ \\
\hline \multicolumn{7}{|c|}{ Urban } \\
\hline \multicolumn{7}{|l|}{ Educational level (years) } \\
\hline None $^{2}$ & 3.4 & 2.1 & 0.0 & 3.8 & 4.5 & 0.0 \\
\hline $1-7$ & 2.0 & 2.9 & 5.6 & 5.9 & 2.4 & 0.0 \\
\hline $8-11$ & 4.6 & 5.6 & 17.6 & 12.5 & 3.4 & 2.8 \\
\hline 12 and above & 12.9 & 8.8 & 23.8 & 17.6 & 10.9 & 4.8 \\
\hline \multicolumn{7}{|l|}{ Worked in last 12 months } \\
\hline Yes & 5.5 & 5.2 & 12.6 & 5.6 & 4.6 & 4.8 \\
\hline No & 7.0 & 4.8 & $(20.0)$ & 8.8 & 7.0 & 2.0 \\
\hline \multicolumn{7}{|l|}{ Wealth quintile } \\
\hline First & * & 0.0 & * & $(0.0)$ & * & * \\
\hline Second & $(8.3)$ & 5.6 & $(0.0)$ & 9.1 & $(11.1)$ & 0.0 \\
\hline Third & 5.9 & 3.8 & $(0.0)$ & 6.3 & $(7.1)$ & 0.0 \\
\hline Fourth & 2.1 & 2.2 & 11.1 & 4.2 & 2.6 & 0.0 \\
\hline Fifth & 7.0 & 5.6 & 17.5 & 10.3 & 6.0 & 3.2 \\
\hline Total & 6.1 & 4.9 & 13.0 & 7.7 & 5.3 & 2.4 \\
\hline \multicolumn{7}{|c|}{ Rural } \\
\hline \multicolumn{7}{|l|}{ Age (years) } \\
\hline $15-19$ & 1.6 & 1.1 & (2.4) & 1.8 & 1.6 & 0.5 \\
\hline $20-24$ & 6.0 & 2.7 & 6.5 & 2.8 & 5.3 & 1.2 \\
\hline $25-29$ & NA & NA & 6.3 & NA & NA & NA \\
\hline \multicolumn{7}{|l|}{ Religion } \\
\hline Hindu & 3.6 & 1.9 & 6.5 & 2.5 & 2.3 & 0.5 \\
\hline Muslim & 1.3 & 1.0 & 2.5 & 1.6 & 1.8 & 0.6 \\
\hline \multicolumn{7}{|l|}{ Caste } \\
\hline SC & 2.5 & 1.9 & 4.2 & 2.3 & 0.9 & 0.2 \\
\hline $\mathrm{OBC}$ & 2.7 & 1.5 & 5.0 & 2.1 & 1.9 & 0.2 \\
\hline General $^{1}$ & 6.1 & 2.5 & 18.3 & 3.6 & 4.6 & 1.6 \\
\hline \multicolumn{7}{|l|}{ Educational level (years) } \\
\hline None $^{2}$ & 1.0 & 1.7 & 1.0 & 2.0 & 2.6 & 0.4 \\
\hline $1-7$ & 2.2 & 1.9 & 6.9 & 3.1 & 0.5 & 0.3 \\
\hline $8-11$ & 3.9 & 1.2 & 7.0 & 1.9 & 3.3 & 0.5 \\
\hline 12 and above & 7.1 & 5.6 & 15.6 & * & 3.1 & 2.9 \\
\hline \multicolumn{7}{|l|}{ Worked in last 12 months } \\
\hline Yes & 3.4 & 1.3 & 5.9 & 1.6 & 2.2 & 0.7 \\
\hline No & 2.9 & 2.0 & * & 2.8 & 2.6 & 0.4 \\
\hline \multicolumn{7}{|l|}{ Wealth quintile } \\
\hline First & 3.4 & 1.2 & 2.6 & 1.6 & 2.8 & 0.0 \\
\hline Second & 4.0 & 1.2 & 4.9 & 1.7 & 2.0 & 0.0 \\
\hline Third & 2.2 & 2.1 & 5.6 & 2.6 & 0.8 & 0.8 \\
\hline Fourth & 2.8 & 1.4 & 6.5 & 2.1 & 1.6 & 0.4 \\
\hline Fifth & 4.2 & 3.0 & 9.7 & 4.8 & 3.9 & 0.9 \\
\hline Total & 3.3 & 1.8 & 6.1 & 2.4 & 2.3 & 0.5 \\
\hline
\end{tabular}

Note: ( ) Based on 25-49 unweighted cases. ${ }^{*}$ Percentage not shown, based on fewer than 25 unweighted cases. NA: Not applicable. OBC: Other backward caste. SC: Scheduled caste. ST: Scheduled tribe. ${ }^{1}$ Includes all those not belonging to SC, ST or OBC. ${ }^{2}$ Includes non-literate and literate with no formal schooling. 


\subsubsection{Awareness of contraceptive methods}

The Youth Study explored young people's awareness of contraceptive methods in several ways. First, they were asked to list all contraceptive methods about which they had heard; second, interviewers gave respondents a brief description of a variety of non-terminal contraceptive methods not mentioned spontaneously and inquired whether the respondent had heard of each; and third, further questioning probed for specific knowledge regarding the use of oral pills, emergency contraception, condoms, the intra-uterine device (IUD) and withdrawal. Table 8.3 presents percentages of youth reporting awareness-spontaneously or on prompting — of condoms, oral contraceptives, emergency contraception, the IUD and withdrawal; and those spontaneously reporting awareness of such methods as sterilisation, implants, vaginal methods, injectables and herbal and other traditional methods. Also presented are percentages of respondents reporting correct specific knowledge of the five methods indicated above.

The vast majority of youth (93\% of young men and $99 \%$ of young women) reported awareness (spontaneous or prompted) of at least one method of contraception and a similar range of youth were aware of at least one modern contraceptive method. Even so, it is notable that $8 \%$ of unmarried young men were not aware of at least one contraceptive method. The most widely known spacing methods were oral contraceptives (77\% and $84 \%$ of young men and women, respectively) and condoms (90\% and 62\%, respectively). Fewer youth reported awareness of the IUD (32\% of young men and $45 \%$ of young women), emergency contraception ( $10 \%$ and $4 \%$, respectively) or vaginal methods, implants or injectables ( $4 \%$ and $16 \%$, respectively). Terminal method awareness was not probed, hence, while female sterilisation was spontaneously reported by $57 \%$ of young men and $97 \%$ of young women, just $24 \%$ and $46 \%$, respectively, spontaneously reported awareness of male sterilisation. Differences in awareness of both any method and any modern method by marital status and rural-urban residence were modest.

Compared to awareness of modern methods, awareness of traditional methods was reported by far fewer youth $-8 \%$ of young men and $31 \%$ of young women. Gender differences were muted among unmarried youth, but pronounced among married youth: $45 \%$ of married young women compared to $15 \%$ of married young men reported awareness of traditional methods. We note that the surprisingly high levels of awareness of withdrawal reported by married women were corroborated by findings from the National Family Health Survey (NFHS-3) and the District Level Household Survey-Reproductive and Child Health Survey (DLHS-RCH). In contrast, it appears that awareness levels reported by married young men in the Youth Study are considerably lower than those reported by young men of similar ages in the National Family Health Survey (NFHS-3). Differences by marital status indicate that married youth, particularly young women were more likely than the unmarried to report awareness of at least one traditional method (15\% versus $5 \%$ among young men and $45 \%$ versus 5\% among young women). Rural-urban differences were narrow, except that married young women in urban areas were more likely than their rural counterparts to report awareness of traditional methods.

Findings also show significant gender differences in terms of awareness of individual contraceptive methods; larger percentages of young women than men were aware of most methods (oral contraceptives, IUDs, female sterilisation, male sterilisation, implants/vaginal methods/injectables and withdrawal). Men, in contrast, were more likely than women to be aware of condoms and emergency contraceptive pills. More married than unmarried youth reported awareness of most methods. Rural-urban differences show that urban youth were more likely than rural youth to be aware of most modern methods. 
In order to assess the extent to which youth had correct specific knowledge of contraceptive methods, and had not just heard of various methods, the Youth Study inquired whether youth were aware of the frequency with which oral contraceptives must be consumed (daily or weekly); the number of sex acts for which one condom could be used (one); the number of hours following sex that emergency contraceptive pills could be consumed (72 hours); where the IUD is placed (uterus); and when a man practising withdrawal should pull out of a woman (prior to ejaculation). Panel B of Table 8.3 presents percentages of youth reporting correct specific knowledge of these five methods. Correct specific knowledge of at least one of these five methods was reported by $65 \%$ of all young men and $62 \%$ of all young women. Gender differences were narrow for both the overall and married samples; however, they were pronounced among the unmarried sample, among whom more young men than women reported correct specific knowledge of at least one method (62\% versus $44 \%)$. More married than unmarried, and more urban than rural youth reported correct specific knowledge of at least one method.

Differentials in correct specific knowledge by sex, marital status and rural-urban residence are evident from findings presented in Table 8.3. In general, young women were more likely than young men to report correct specific knowledge of female-oriented methods such as oral contraceptives (48\% compared to $26 \%$ ) and the IUD $(23 \%$ compared to $9 \%)$. Young men, in contrast, were considerably more likely than young women to report correct specific knowledge about condoms (62\% and 30\%, respectively). Notably, more young women than men reported correct specific knowledge about withdrawal (23\% compared to $2 \%)$. Few young men and women had correct specific knowledge of emergency contraception (1-2\%). Differences by marital status suggest that the married were typically more likely than the unmarried to report correct specific knowledge of every method (see Figure 8.2). Likewise, urban respondents were more likely than their rural counterparts to report correct specific knowledge of every modern method.

Figure 8.2: Percentage of youth who reported correct specific knowledge of oral pills and condoms, according to residence, Bihar, 2007

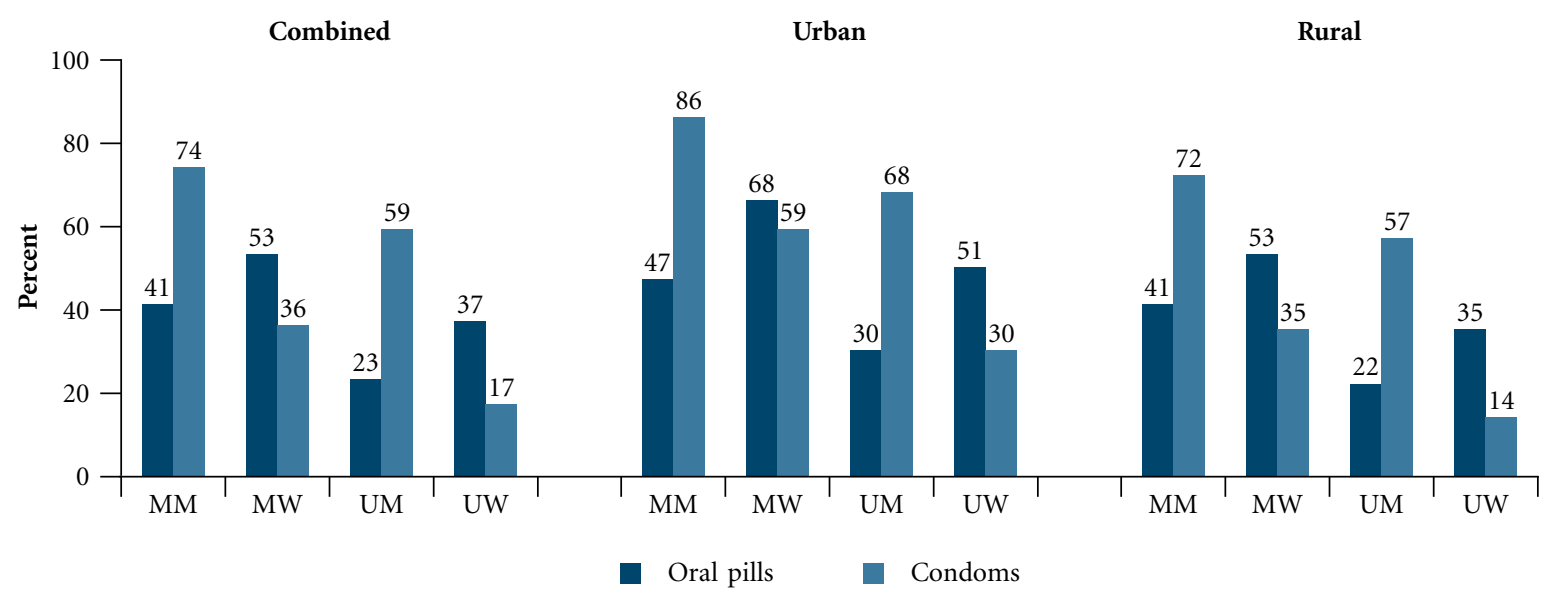


Table 8.3: Awareness of contraceptive methods

Percentage of youth who reported awareness and correct specific knowledge of various contraceptive methods, according to residence, Bihar, 2007

\begin{tabular}{|c|c|c|c|c|c|c|}
\hline Awareness indicators (\%) & $\begin{array}{c}\mathrm{M} \\
15-24\end{array}$ & $\begin{array}{c}\text { W } \\
15-24\end{array}$ & $\begin{array}{c}\text { MM } \\
15-29\end{array}$ & $\begin{array}{c}\text { MW } \\
15-24\end{array}$ & $\begin{array}{c}\text { UM } \\
15-24\end{array}$ & $\begin{array}{c}\text { UW } \\
\text { 15-24 }\end{array}$ \\
\hline \multicolumn{7}{|c|}{ A. Awareness } \\
\hline \multicolumn{7}{|c|}{ Combined } \\
\hline Any method & 93.2 & 98.8 & 97.6 & 99.6 & 91.8 & 97.1 \\
\hline Any modern method & 93.2 & 98.8 & 97.5 & 99.6 & 91.8 & 97.1 \\
\hline Oral pills & 77.2 & 84.0 & 86.5 & 86.8 & 75.4 & 78.1 \\
\hline Emergency contraceptive pills & 9.6 & 3.5 & 14.9 & 3.8 & 9.3 & 2.8 \\
\hline Condom & 89.6 & 62.0 & 92.5 & 67.2 & 88.5 & 50.5 \\
\hline IUD & 31.9 & 45.0 & 38.4 & 48.9 & 32.3 & 36.3 \\
\hline Female sterilisation & 57.3 & 97.2 & 67.3 & 98.0 & 54.8 & 95.5 \\
\hline Male sterilisation & 23.7 & 46.1 & 29.6 & 50.1 & 23.2 & 38.1 \\
\hline Implant/vaginal methods/injectables & 3.8 & 15.7 & 7.1 & 18.2 & 3.8 & 10.8 \\
\hline Any traditional method & 7.5 & 31.4 & 14.9 & 45.3 & 5.3 & 5.2 \\
\hline Withdrawal & 3.4 & 28.4 & 6.4 & 42.2 & 2.9 & 2.4 \\
\hline Safe period & 4.0 & 8.0 & 7.9 & 10.6 & 2.5 & 2.8 \\
\hline Traditional/herbal methods & 0.6 & 0.8 & 2.3 & 1.0 & 0.3 & 0.5 \\
\hline Number of respondents & 1,942 & 5,529 & 1,115 & 2,341 & 1,492 & 3,188 \\
\hline \multicolumn{7}{|c|}{ Urban } \\
\hline Any method & 97.8 & 99.4 & 98.9 & 100.0 & 97.6 & 99.2 \\
\hline Any modern method & 97.8 & 99.4 & 98.9 & 100.0 & 97.6 & 99.2 \\
\hline Oral pills & 88.2 & 92.9 & 94.6 & 94.4 & 87.3 & 91.9 \\
\hline Emergency contraceptive pills & 12.2 & 6.3 & 19.6 & 7.0 & 11.5 & 5.5 \\
\hline Condom & 96.8 & 82.5 & 97.8 & 85.9 & 96.7 & 80.2 \\
\hline IUD & 46.1 & 63.3 & 55.4 & 74.1 & 45.7 & 55.5 \\
\hline Female sterilisation & 58.1 & 98.4 & 74.2 & 98.6 & 56.1 & 98.0 \\
\hline Male sterilisation & 31.9 & 53.2 & 40.2 & 61.5 & 31.0 & 47.1 \\
\hline Implant/vaginal methods/injectables & 5.0 & 21.0 & 9.8 & 29.6 & 4.9 & 14.8 \\
\hline Any traditional method & 6.1 & 28.9 & 17.4 & 57.0 & 5.3 & 8.7 \\
\hline Withdrawal & 4.3 & 23.4 & 10.9 & 51.4 & 4.1 & 3.4 \\
\hline Safe period & 1.8 & 12.4 & 9.7 & 21.7 & 1.2 & 5.9 \\
\hline Traditional/herbal methods & 0.0 & 0.6 & 0.0 & 0.7 & 0.0 & 0.6 \\
\hline Number of respondents & 1,039 & 2,581 & 547 & 1,136 & 833 & 1,445 \\
\hline \multicolumn{7}{|c|}{ Rural } \\
\hline Any method & 92.5 & 98.7 & 97.5 & 99.6 & 90.6 & 96.8 \\
\hline Any modern method & 92.5 & 98.7 & 97.3 & 99.6 & 90.6 & 96.8 \\
\hline Oral pills & 75.3 & 82.8 & 85.7 & 86.3 & 73.0 & 75.5 \\
\hline Emergency contraceptive pills & 9.1 & 3.2 & 14.5 & 3.6 & 8.8 & 2.2 \\
\hline Condom & 88.4 & 59.3 & 92.0 & 66.0 & 86.9 & 45.1 \\
\hline IUD & 29.5 & 42.6 & 36.9 & 47.2 & 29.7 & 32.8 \\
\hline Female sterilisation & 57.2 & 97.0 & 66.7 & 97.9 & 54.5 & 95.0 \\
\hline Male sterilisation & 22.3 & 45.2 & 28.6 & 49.3 & 21.7 & 36.5 \\
\hline Implant/vaginal methods/injectables & 3.6 & 15.0 & 6.8 & 17.4 & 3.5 & 10.0 \\
\hline Any traditional method & 7.8 & 31.7 & 14.7 & 44.5 & 5.4 & 4.5 \\
\hline Withdrawal & 3.2 & 29.1 & 6.1 & 41.7 & 2.7 & 2.3 \\
\hline Safe period & 4.4 & 7.5 & 7.7 & 10.0 & 2.6 & 2.3 \\
\hline Traditional/herbal methods & 0.7 & 0.8 & 2.5 & 1.0 & 0.3 & 0.4 \\
\hline Number of respondents & 903 & 2,948 & 568 & 1,205 & 659 & 1,743 \\
\hline
\end{tabular}


Table 8.3: (Cont'd)

\begin{tabular}{|c|c|c|c|c|c|c|}
\hline Awareness indicators (\%) & $\begin{array}{c}\text { M } \\
15-24\end{array}$ & $\begin{array}{c}\text { W } \\
15-24\end{array}$ & $\begin{array}{c}\text { MM } \\
15-29\end{array}$ & $\begin{array}{l}\text { MW } \\
15-24\end{array}$ & $\begin{array}{c}\text { UM } \\
15-24\end{array}$ & $\begin{array}{l}\text { UW } \\
15-24\end{array}$ \\
\hline \multicolumn{7}{|c|}{ B. Correct specific knowledge ${ }^{1}$} \\
\hline \multicolumn{7}{|c|}{ Combined } \\
\hline Any method & 65.1 & 62.2 & 77.1 & 71.6 & 61.6 & 43.8 \\
\hline At least one modern method & 64.9 & 57.3 & 76.8 & 64.0 & 61.4 & 43.7 \\
\hline Oral pills & 26.2 & 48.1 & 41.2 & 53.4 & 23.1 & 37.3 \\
\hline Emergency contraceptive pills & 2.2 & 1.0 & 3.0 & 1.2 & 2.2 & 0.6 \\
\hline Condoms & 62.1 & 29.9 & 73.5 & 36.4 & 58.7 & 16.7 \\
\hline IUD & 9.0 & 22.6 & 17.0 & 27.3 & 8.4 & 12.9 \\
\hline $\begin{array}{l}\text { Any traditional method } \\
\text { Withdrawal }\end{array}$ & 2.2 & 22.6 & 5.3 & 33.8 & 17 & 13 \\
\hline Number of respondents & 1,942 & 5,529 & 1,115 & 2,341 & 1,492 & 3,188 \\
\hline \multicolumn{7}{|c|}{ Urban } \\
\hline Any method & 71.7 & 71.4 & 87.1 & 85.2 & 69.4 & 61.3 \\
\hline At least one modern method & 71.7 & 69.5 & 87.0 & 81.0 & 69.4 & 61.1 \\
\hline Oral pills & 31.8 & 57.8 & 46.7 & 67.6 & 29.5 & 50.7 \\
\hline Emergency contraceptive pills & 3.6 & 2.8 & 4.3 & 3.5 & 3.3 & 2.2 \\
\hline Condoms & 70.3 & 42.1 & 86.0 & 58.7 & 68.0 & 30.0 \\
\hline IUD & 15.1 & 34.3 & 30.4 & 47.2 & 13.9 & 24.9 \\
\hline $\begin{array}{l}\text { Any traditional method } \\
\text { Withdrawal }\end{array}$ & 3.2 & 19.8 & 8.7 & 44.4 & 2.9 & 2.0 \\
\hline Number of respondents & 1,039 & 2,581 & 547 & 1,136 & 833 & 1,445 \\
\hline \multicolumn{7}{|c|}{ Rural } \\
\hline Any method & 64.0 & 61.0 & 76.1 & 70.7 & 60.1 & 40.5 \\
\hline At least one modern method & 63.8 & 55.7 & 75.9 & 62.9 & 59.8 & 40.5 \\
\hline Oral pills & 25.3 & 46.9 & 40.7 & 52.5 & 21.8 & 34.9 \\
\hline Emergency contraceptive pills & 2.0 & 0.8 & 2.9 & 1.0 & 2.0 & 0.3 \\
\hline Condoms & 60.7 & 28.3 & 72.3 & 34.9 & 56.9 & 14.3 \\
\hline IUD & 8.0 & 21.1 & 15.7 & 26.0 & 7.4 & 10.7 \\
\hline $\begin{array}{l}\text { Any traditional method } \\
\text { Withdrawal }\end{array}$ & 2.1 & 22.9 & 5.0 & 33.2 & 1.4 & 1.3 \\
\hline Number of respondents & 903 & 2,948 & 568 & 1,205 & 659 & 1,743 \\
\hline
\end{tabular}

Note: All Ns are unweighted. ${ }^{1}$ Correct specific knowledge was assessed for oral pills, emergency contraceptive pills, condoms, IUD and withdrawal. The following questions were asked (correct answers in brackets)—Oral pills: How often should a woman take pills? [Daily/Weekly]; Emergency contraceptive pills: How soon after sexual intercourse should these pills be taken? [72 hours]; Condoms: For how many acts of sexual intercourse can one condom be used? [One]; IUD: Where is the IUD placed? [Uterus]; Withdrawal: When should a man pull out of a woman during sexual intercourse? [Prior to ejaculation].

\subsubsection{Condom-related perceptions}

Among those who reported awareness of condoms, the Youth Study probed youth who reported awareness of three specific aspects of this method, namely, whether condoms are a suitable method for preventing pregnancy, whether condoms can slip off the man and disappear inside the woman's body and whether condoms reduce sexual pleasure. Findings, presented in Table 8.4, show that $72-78 \%$ agreed that condoms were a suitable method for preventing pregnancy, but that awareness of other issues was reported by many fewer. Just 33\% and $25 \%$ of young men and women were aware that condoms cannot disappear into the woman's body and just $18-20 \%$ of youth felt that condoms do not reduce sexual pleasure. Notably, three-fifths of young men and two-thirds of young women reported that they were unsure about these two aspects. 
Table 8.4: Perceptions of selected issues related to condom use

Percent distribution of youth by their perceptions of condom use, according to residence, Bihar, 2007

\begin{tabular}{|c|c|c|c|c|c|c|}
\hline Perceptions (\%) & $\stackrel{M}{15-24}$ & $\begin{array}{c}\text { W } \\
15-24\end{array}$ & $\begin{array}{c}\text { MM } \\
15-29\end{array}$ & $\begin{array}{c}\text { MW } \\
15-24\end{array}$ & $\begin{array}{c}\text { UM } \\
15-24\end{array}$ & $\begin{array}{l}\text { UW } \\
15-24\end{array}$ \\
\hline \multicolumn{7}{|c|}{ Combined } \\
\hline \multicolumn{7}{|c|}{$\begin{array}{l}\text { Condoms are a suitable method for } \\
\text { preventing pregnancy }\end{array}$} \\
\hline Agree & 71.5 & 78.3 & 76.9 & 82.8 & 69.5 & 67.4 \\
\hline Disagree & 7.1 & 4.0 & 9.2 & 4.3 & 6.8 & 3.2 \\
\hline Don't know/can't say & 21.4 & 17.5 & 13.7 & 12.7 & 23.6 & 29.1 \\
\hline \multicolumn{7}{|c|}{$\begin{array}{l}\text { Condoms can slip off a man and disappear } \\
\text { inside a woman's body }\end{array}$} \\
\hline Agree & 4.7 & 8.5 & 9.4 & 10.3 & 4.1 & 4.0 \\
\hline Disagree & 32.9 & 25.1 & 44.4 & 29.8 & 29.4 & 13.0 \\
\hline Don't know/can't say & 62.3 & 66.2 & 46.0 & 59.8 & 66.3 & 82.6 \\
\hline \multicolumn{7}{|c|}{ Condoms reduce sexual pleasure } \\
\hline Agree & 20.3 & 12.9 & 31.4 & 16.3 & 16.2 & 4.7 \\
\hline Disagree & 18.1 & 19.9 & 28.5 & 22.7 & 15.9 & 12.7 \\
\hline Don't know/can't say & 61.5 & 66.9 & 39.9 & 60.9 & 67.9 & 82.2 \\
\hline Number aware of condoms & 1,804 & 3,701 & 1,057 & 1,772 & 1,379 & 1,929 \\
\hline \multicolumn{7}{|c|}{ Urban } \\
\hline \multicolumn{7}{|c|}{$\begin{array}{l}\text { Condoms are a suitable method for } \\
\text { preventing pregnancy }\end{array}$} \\
\hline Agree & 74.2 & 76.0 & 82.2 & 86.9 & 73.0 & 67.7 \\
\hline Disagree & 7.7 & 3.2 & 8.9 & 4.1 & 7.2 & 2.5 \\
\hline Don't know/can't say & 18.1 & 20.6 & 8.9 & 9.0 & 19.8 & 29.5 \\
\hline \multicolumn{7}{|c|}{$\begin{array}{l}\text { Condoms can slip off a man and disappear } \\
\text { inside a woman's body }\end{array}$} \\
\hline Agree & 7.0 & 7.6 & 11.1 & 12.3 & 6.4 & 4.3 \\
\hline Disagree & 35.8 & 27.0 & 55.6 & 41.8 & 32.6 & 15.4 \\
\hline Don't know/can't say & 57.2 & 65.2 & 33.3 & 45.9 & 61.0 & 80.1 \\
\hline \multicolumn{7}{|c|}{ Condoms reduce sexual pleasure } \\
\hline Agree & 21.0 & 10.1 & 35.2 & 18.0 & 19.0 & 4.0 \\
\hline Disagree & 23.2 & 23.0 & 35.2 & 33.6 & 21.1 & 14.9 \\
\hline Don't know/can't say & 55.7 & 66.7 & 29.7 & 48.4 & 59.9 & 80.8 \\
\hline Number aware of condoms & 1,007 & 2,127 & 536 & 976 & 806 & 1,151 \\
\hline \multicolumn{7}{|c|}{ Rural } \\
\hline \multicolumn{7}{|c|}{$\begin{array}{l}\text { Condoms are a suitable method for } \\
\text { preventing pregnancy }\end{array}$} \\
\hline Agree & 70.9 & 78.7 & 76.4 & 82.5 & 68.7 & 67.3 \\
\hline Disagree & 7.1 & 4.1 & 9.3 & 4.3 & 6.7 & 3.5 \\
\hline Don’t know/can’t say & 22.0 & 16.9 & 14.1 & 13.0 & 24.5 & 29.0 \\
\hline \multicolumn{7}{|c|}{$\begin{array}{l}\text { Condoms can slip off a man and disappear } \\
\text { inside a woman's body }\end{array}$} \\
\hline Agree & 4.3 & 8.6 & 9.4 & 10.1 & 3.5 & 3.9 \\
\hline Disagree & 32.3 & 24.7 & 43.3 & 28.7 & 28.8 & 12.3 \\
\hline Don't know/can't say & 63.2 & 66.4 & 47.2 & 60.9 & 67.5 & 83.5 \\
\hline \multicolumn{7}{|c|}{ Condoms reduce sexual pleasure } \\
\hline Agree & 20.3 & 13.4 & 31.0 & 16.1 & 15.6 & 4.9 \\
\hline Disagree & 17.2 & 19.4 & 27.9 & 21.7 & 14.8 & 11.9 \\
\hline Don’t know/can't say & 62.5 & 67.0 & 40.8 & 62.0 & 69.6 & 82.7 \\
\hline Number aware of condoms & 797 & 1,574 & 521 & 796 & 573 & 778 \\
\hline
\end{tabular}

Note: All Ns are unweighted. Column totals may not equal 100\% due to missing cases. 
Marital status differences suggest that the married were better informed about condoms than were the unmarried. For example, $44 \%$ of married young men compared to $29 \%$ of unmarried young men were aware that condoms cannot disappear into the woman's body; the corresponding percentages among young women were $30 \%$ and $13 \%$, respectively. Rural-urban differences were negligible. However when examined separately for married and unmarried youth in rural and urban settings, findings suggest that married youth in urban areas were better informed about condoms than their rural counterparts.

\subsubsection{Awareness of contraception prior to marriage}

Married youth were specifically asked whether they had been aware of contraception or had known where to obtain contraceptives prior to their marriage. Findings, presented in Table 8.5, suggest that of those who were aware of at least one method of contraception at the time of interview, just three-fifths of young men (59\%) compared to one-third of young women $(33 \%)$ had been aware of any contraceptive method before marriage. More urban than rural youth had been aware of any contraceptive method before marriage (69\% and $58 \%$ of urban and rural young men, respectively; and $49 \%$ and $32 \%$ of urban and rural young women, respectively). Somewhat fewer married youth knew, before marriage, about where to obtain contraceptives-53\% of young men and $24 \%$ of young women. Rural-urban differentials, noted above, persisted.

Table 8.5: Awareness of contraception prior to marriage

Percentage of married youth aware of any contraceptive method prior to marriage and percentage aware of a source of contraceptive supplies at that time, according to residence, Bihar, 2007

\begin{tabular}{|c|c|c|c|c|c|c|}
\hline \multirow[t]{2}{*}{ Knowledge (\%) } & $\begin{array}{c}\text { MM } \\
15-29\end{array}$ & $\begin{array}{c}\text { MW } \\
15-24\end{array}$ & $\begin{array}{c}\text { MM } \\
15-29\end{array}$ & $\begin{array}{c}\text { MW } \\
15-24\end{array}$ & $\begin{array}{c}\text { MM } \\
15-29\end{array}$ & $\begin{array}{c}\text { MW } \\
15-24\end{array}$ \\
\hline & \multicolumn{2}{|c|}{ Combined } & \multicolumn{2}{|c|}{ Urban } & \multicolumn{2}{|c|}{ Rural } \\
\hline $\begin{array}{l}\text { Aware of any contraceptive method } \\
\text { before marriage }\end{array}$ & 58.5 & 33.2 & 69.2 & 48.6 & 57.5 & 32.3 \\
\hline Aware of a contraceptive source before marriage & 52.7 & 24.0 & 67.4 & 37.3 & 51.4 & 23.2 \\
\hline $\begin{array}{l}\text { Number currently aware of at least one } \\
\text { contraceptive method }\end{array}$ & 1,096 & 2,334 & 543 & 1,134 & 553 & 1,200 \\
\hline
\end{tabular}

Note: All Ns are unweighted.

\subsubsection{Awareness of medical abortion}

Given that medical abortion has been legal since 2002, youth were asked if they were aware of "any pills" that a woman could take to terminate a pregnancy. As evident from Figure 8.3, one-fifth of youth reported that they were aware of such a method (since we did not probe further, some of these positive responses may not have been specifically referring to the mifepristone-misoprostol combination but rather to the variety of herbal and ayurvedic pills and other home remedies available). As many as $70-73 \%$ of young men and women reported that they were unsure whether such a means of inducing abortion existed. More married than unmarried youth were aware of medical abortion $(25 \%$ and $17 \%$ among young men and $23 \%$ and $14 \%$ among young women). Rural-urban differences were modest for the overall sample; however when analysed separately for married and unmarried youth in urban and rural areas, the urban youth were somewhat more likely than their rural counterparts to report awareness. 
Figure 8.3: Percent distribution of youth by awareness of medical abortion, according to residence, Bihar, 2007

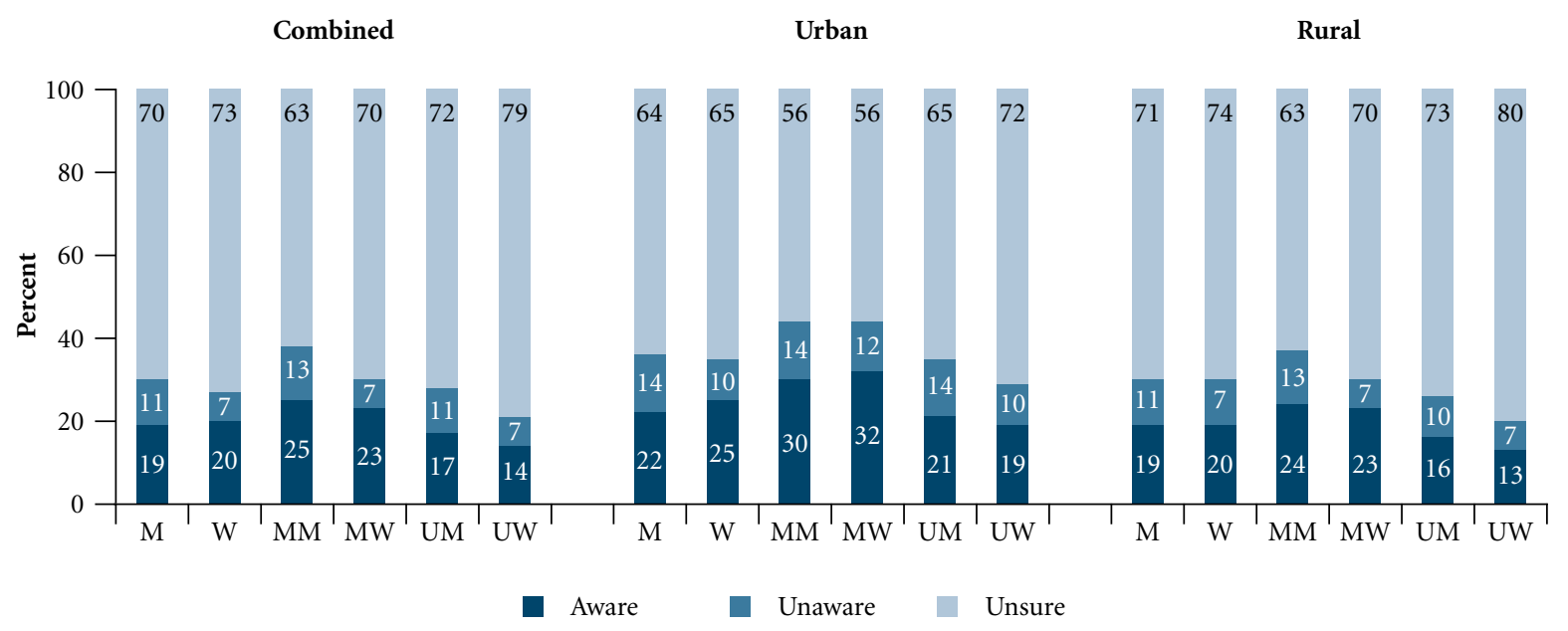

Note: Percentages may not equal 100.0 because of rounding.

\subsubsection{Awareness of sexually transmitted infections (STIs) and HIV/AIDS}

The Youth Study inquired whether youth had ever heard of infections that were transmitted through sexual contact. Findings, presented in Table 8.6, suggest that awareness of STIs other than HIV/AIDS was extremely limited. Indeed, just $11-12 \%$ of youth reported awareness of STIs. Differences by sex and residence were negligible; however, married youth were more likely than unmarried youth to be aware of STIs. Such differences were more pronounced among young women.

Among those who were aware of STIs other than HIV, awareness of at least one symptom of infection was far from universal, particularly among young men: just $37 \%$ of young men and $87 \%$ of young women could identify at least one such symptom; we note that the symptom most likely to be indicated by young women was vaginal discharge. This wide gender difference may be attributed to the finding that large proportions of young women (69\%) reported awareness of vaginal discharge as a symptom of infection, compared to just $19 \%$ of young men (not presented in tabular form). More married than unmarried youth, particularly among young women could identify at least one symptom (45\% versus 33\% among young men and 90\% versus $62 \%$ among young women) and the patterns were quite similar among urban and rural respondents. Rural-urban differences differed across young men and women. Among young men, somewhat more urban than rural young men, irrespective of marital status, were aware of at least one symptom (46\% versus 35\%). Among young women, the patterns differed between the married and the unmarried. Among the married, more rural than urban women were aware of at least one symptom (91\% versus $76 \%)$. In contrast among the unmarried, a reverse pattern was evident (59\% versus $71 \%$ ).

Questions exploring young people's awareness of HIV/AIDS were adapted from those used in the NFHS (IIPS and Macro International, 2007b). Findings, presented in Table 8.6, show that $87 \%$ of young men, compared to $47 \%$ of young women, had heard of HIV/AIDS (almost identical to levels- $83 \%$ and $44 \%$, respectivelyreported in NFHS-3; IIPS and Macro International, 2008). Differences by marital status were muted among young men, but apparent among young women, among whom more unmarried than married respondents reported awareness of HIV/AIDS, irrespective of residence (53\% versus 43\%). Urban respondents were more likely than their rural counterparts to report HIV/AIDS awareness, and the differences were much wider among young women ( $96 \%$ versus $86 \%$ among young men, and $79 \%$ versus $43 \%$ among young women). 
Table 8.6: Awareness of STIs and HIV/AIDS

Percent distribution of youth who had heard of and had specific knowledge about STIs and HIV/AIDS, according to residence, Bihar, 2007

\begin{tabular}{|c|c|c|c|c|c|c|c|c|c|}
\hline \multirow[t]{2}{*}{ Awareness among young men (\%) } & $\begin{array}{c}\text { M } \\
15-24\end{array}$ & $\begin{array}{c}\text { MM } \\
15-29\end{array}$ & $\begin{array}{c}\text { UM } \\
15-24\end{array}$ & $\begin{array}{c}\text { M } \\
15-24\end{array}$ & $\begin{array}{c}\text { MM } \\
15-29\end{array}$ & $\begin{array}{c}\text { UM } \\
15-24\end{array}$ & $\begin{array}{c}M \\
15-24\end{array}$ & $\begin{array}{c}\text { MM } \\
15-29\end{array}$ & $\begin{array}{c}\text { UM } \\
15-24\end{array}$ \\
\hline & \multicolumn{3}{|c|}{ Combined } & \multicolumn{3}{|c|}{ Urban } & \multicolumn{3}{|c|}{ Rural } \\
\hline Heard about STIs ${ }^{1}$ & 11.4 & 18.9 & 10.6 & 14.0 & 23.9 & 12.7 & 11.0 & 18.5 & 10.1 \\
\hline Number of respondents & 1,942 & 1,115 & 1,492 & 1,039 & 547 & 833 & 903 & 568 & 659 \\
\hline Could identify at least one symptom of STIs & 36.6 & 44.5 & 32.9 & 46.2 & 54.5 & 41.9 & 34.6 & 43.1 & 30.2 \\
\hline Number who had heard about STIs & 249 & 237 & 173 & 151 & 134 & 107 & 98 & 103 & 66 \\
\hline Heard about HIV/AIDS & 87.3 & 86.5 & 87.7 & 95.7 & 95.7 & 95.9 & 85.9 & 85.7 & 86.0 \\
\hline $\begin{array}{l}\text { Number of respondents } \\
\text { Of respondents who had heard about } \\
\text { HIV/AIDS, those reporting that: }\end{array}$ & 1,942 & 1,115 & 1,492 & 1,039 & 547 & 833 & 903 & 568 & 659 \\
\hline $\begin{array}{l}\text { One can reduce one's chances of getting HIV } \\
\text { by having a single sexual partner }\end{array}$ & 81.2 & 86.6 & 79.7 & 83.5 & 87.5 & 83.0 & 80.9 & 86.5 & 79.0 \\
\hline $\begin{array}{l}\text { One can reduce one's chances of getting HIV } \\
\text { by consistent use of condoms }\end{array}$ & 76.4 & 80.7 & 74.8 & 81.3 & 85.1 & 81.2 & 75.5 & 80.4 & 73.4 \\
\hline One cannot get HIV through mosquito bites & 51.9 & 49.0 & 53.1 & 64.4 & 58.0 & 65.1 & 49.6 & 48.1 & 50.5 \\
\hline $\begin{array}{l}\text { One cannot get HIV by sharing food with an } \\
\text { HIV-positive person }\end{array}$ & 68.9 & 64.6 & 69.6 & 82.4 & 79.3 & 82.1 & 66.4 & 63.1 & 66.9 \\
\hline $\begin{array}{l}\text { One cannot get HIV by hugging an } \\
\text { HIV-positive person }\end{array}$ & 75.9 & 74.8 & 75.8 & 85.4 & 80.7 & 85.5 & 74.2 & 74.0 & 73.7 \\
\hline $\begin{array}{l}\text { One cannot tell if a person is HIV-positive by } \\
\text { just looking at him/her }\end{array}$ & 82.6 & 80.4 & 83.6 & 87.3 & 86.2 & 87.2 & 81.7 & 79.8 & 82.8 \\
\hline Number who had heard about HIV/AIDS & 1,768 & 1,004 & 1,366 & 993 & 519 & 799 & 775 & 485 & 567 \\
\hline \multirow[t]{2}{*}{ Awareness among young women (\%) } & $\begin{array}{c}\text { W } \\
15-24\end{array}$ & $\begin{array}{c}\text { MW } \\
15-24\end{array}$ & $\begin{array}{c}\text { UW } \\
15-24\end{array}$ & $\begin{array}{c}\text { W } \\
15-24\end{array}$ & $\begin{array}{c}\text { MW } \\
\text { 15-24 }\end{array}$ & $\begin{array}{c}\text { UW } \\
\text { 15-24 }\end{array}$ & $\begin{array}{c}\text { W } \\
15-24\end{array}$ & $\begin{array}{c}\text { MW } \\
\text { 15-24 }\end{array}$ & $\begin{array}{c}\text { UW } \\
15-24\end{array}$ \\
\hline & \multicolumn{3}{|c|}{ Combined } & \multicolumn{3}{|c|}{ Urban } & \multicolumn{3}{|c|}{ Rural } \\
\hline Heard about STIs ${ }^{1}$ & 11.6 & 15.8 & 3.7 & 10.0 & 14.1 & 6.9 & 11.8 & 15.9 & 3.1 \\
\hline Number of respondents & 5,529 & 2,341 & 3,188 & 2,581 & 1,136 & 1,445 & 2,948 & 1,205 & 1,743 \\
\hline Could identify at least one symptom of STIs & 86.7 & 90.0 & 62.4 & 74.6 & 76.2 & 70.6 & 88.0 & 90.5 & 59.0 \\
\hline Number who had heard about STIs & 508 & 356 & 152 & 260 & 162 & 98 & 248 & 194 & 54 \\
\hline Heard about HIV/AIDS & 46.9 & 42.6 & 52.8 & 79.3 & 71.1 & 85.3 & 42.7 & 40.8 & 46.8 \\
\hline $\begin{array}{l}\text { Number of respondents } \\
\text { Of respondents who had heard about } \\
\text { HIV/AIDS, those reporting that: }\end{array}$ & 5,529 & 2,341 & 3,188 & 2,584 & 1,136 & 1,445 & 2,498 & 1,205 & 1,743 \\
\hline $\begin{array}{l}\text { One can reduce one's chances of getting HIV } \\
\text { by having single sexual partner }\end{array}$ & 80.0 & 82.5 & 75.6 & 85.3 & 90.0 & 82.5 & 78.7 & 81.7 & 73.3 \\
\hline $\begin{array}{l}\text { One can reduce one's chances of getting HIV } \\
\text { by consistent use of condoms }\end{array}$ & 58.1 & 58.7 & 56.0 & 69.2 & 73.3 & 66.8 & 55.4 & 57.0 & 52.4 \\
\hline One cannot get HIV through mosquito bites & 56.3 & 53.4 & 59.6 & 68.5 & 65.0 & 70.9 & 53.4 & 52.1 & 55.8 \\
\hline $\begin{array}{l}\text { One cannot get HIV by sharing food with an } \\
\text { HIV-positive person }\end{array}$ & 66.9 & 61.6 & 73.5 & 81.0 & 78.0 & 82.9 & 63.5 & 59.9 & 70.3 \\
\hline $\begin{array}{l}\text { One cannot get HIV by hugging an } \\
\text { HIV-positive person }\end{array}$ & 79.7 & 76.2 & 84.4 & 87.7 & 86.1 & 88.9 & 77.8 & 75.0 & 82.9 \\
\hline $\begin{array}{l}\text { One cannot tell if a person is HIV-positive by } \\
\text { just looking at him/her }\end{array}$ & 89.9 & 88.6 & 91.6 & 94.3 & 93.1 & 95.0 & 88.9 & 88.1 & 90.4 \\
\hline Number who had heard about HIV/AIDS & 3,334 & 1,299 & 2,035 & 2,032 & 805 & 1,227 & 1,302 & 494 & 808 \\
\hline
\end{tabular}

Note: All Ns are unweighted. ${ }^{1}$ Other than HIV. 
Among those who reported awareness of HIV/AIDS, knowledge of specific aspects of the disease was by no means complete. For example, just $80-81 \%$ of youth who had heard of HIV/AIDS were aware that one could reduce the chances of getting HIV by being faithful to a single partner. Awareness that one can reduce the chances of contracting HIV by using a condom every time one has sex was reported by $76 \%$ of young men and $58 \%$ of young women. Differences by marital status were modest, but consistently suggested that more married than unmarried youth reported awareness of staying faithful to a single partner and condom use as ways of reducing HIV transmission. These differences persisted among young men and women in both rural and urban settings. Rural-urban differences were also evident; more urban than rural youth reported awareness of both ways of reducing HIV transmission.

Misconceptions about modes of transmission were prevalent among substantial proportion of youth who reported awareness of HIV. For example, among those aware of HIV/AIDS, only 52\% of young men and $56 \%$ of young women knew that one cannot get HIV through mosquito bites and $69 \%$ of young men and $67 \%$ of young women knew that one could not acquire HIV by sharing food with an HIV infected person. Differences by marital status were modest among young men, but among young women, more married than unmarried held these misconceptions. For example, only $62 \%$ of married young women compared to $74 \%$ of unmarried young women knew that one could not acquire HIV by sharing food with an HIV infected person. Rural-urban differences were modest, but more rural than urban youth were consistently observed to hold these misconceptions.

\subsubsection{Comprehensive awareness of HIV/AIDS}

We measured comprehensive awareness of HIV/AIDS on the basis of information obtained regarding respondents' knowledge of HIV prevention and transmission. Comprehensive awareness is defined as knowledge of two ways of preventing HIV (specifically, condom use and single partner relations), rejection of common misconceptions about HIV transmission (namely, that HIV can be transmitted through mosquito bites, sharing food or hugging) and awareness that one cannot tell by looking at a person whether he or she has HIV. Findings are presented in Table 8.7 and suggest limited comprehensive awareness of HIV/AIDS and wide variation by sex and rural-urban residence. For example, while $28 \%$ of young men reported comprehensive awareness, only $15 \%$ of young women did so. Marital status differences were muted among young men; however, among young women, a somewhat larger percentage of the unmarried than the married reported comprehensive awareness (18\% versus 13\%). Likewise, urban youth were far more likely to report comprehensive awareness than rural youth. While $42 \%$ and $37 \%$ of young men and women in urban settings, respectively, reported comprehensive awareness, just $25 \%$ and $13 \%$ of young men and women, respectively, in rural settings did so.

Comprehensive awareness of HIV/AIDS was greater among older, better educated, and economically better off youth than others. For example, as shown in Figure $8.4 \mathrm{a}, 12 \%$ of married young men with no formal education displayed comprehensive HIV/AIDS awareness, compared to $55 \%$ of those with 12 or more years of education; comprehensive HIV/AIDS awareness was reported likewise by $3 \%$ of married young women with no education compared to $68 \%$ of those with 12 or more years of education. Likewise, Figure $8.4 \mathrm{~b}$ shows that comprehensive awareness increased from $9 \%$ among unmarried young men in the poorest (first) wealth quintile to $41 \%$ among those in the wealthiest (fifth) quintile, and from $4 \%$ to $38 \%$, respectively, among unmarried young women. While differentials by religion were inconsistent, caste-wise differences indicate that comprehensive awareness of HIV/AIDS was greater among youth belonging to general castes compared to those belonging to other castes and these differences were wider among young women than men. Differences by current economic activity indicate that those not engaged in any economic activity were better informed about HIV/AIDS than those who were engaged in work (33\% and $26 \%$, respectively, among young men, and 
Table 8.7: Comprehensive knowledge of HIV/AIDS by selected background characteristics

Percentage of youth who had comprehensive knowledge of HIV/AIDS by selected background characteristics, according to residence, Bihar, 2007

\begin{tabular}{|c|c|c|c|c|c|c|}
\hline Background characteristics (\%) & $\begin{array}{c}\mathrm{M} \\
15-24\end{array}$ & $\begin{array}{c}\mathrm{W} \\
15-24\end{array}$ & $\begin{array}{c}\text { MM } \\
15-29\end{array}$ & $\begin{array}{c}\text { MW } \\
15-24\end{array}$ & $\begin{array}{c}\text { UM } \\
15-24\end{array}$ & $\begin{array}{c}\text { UW } \\
15-24\end{array}$ \\
\hline \multicolumn{7}{|c|}{ Combined } \\
\hline \multicolumn{7}{|l|}{ Age (years) } \\
\hline $15-19$ & 24.5 & 13.4 & 15.1 & 9.7 & 25.2 & 15.8 \\
\hline $20-24$ & 33.2 & 18.3 & 30.9 & 15.8 & 36.0 & 41.4 \\
\hline $25-29$ & NA & NA & 26.1 & NA & NA & NA \\
\hline \multicolumn{7}{|l|}{ Religion } \\
\hline Hindu & 27.0 & 15.6 & 27.0 & 12.9 & 27.2 & 19.4 \\
\hline Muslim & 32.6 & 14.1 & 26.7 & 15.0 & 29.8 & 12.2 \\
\hline \multicolumn{7}{|l|}{ Caste } \\
\hline SC & 22.0 & 8.5 & 23.2 & 8.6 & 21.8 & 7.4 \\
\hline OBC & 26.8 & 13.9 & 26.9 & 12.1 & 25.9 & 15.9 \\
\hline General $^{1}$ & 35.6 & 32.0 & 40.2 & 32.7 & 34.7 & 30.3 \\
\hline \multicolumn{7}{|l|}{ Educational level (years) } \\
\hline None $^{2}$ & 9.2 & 3.3 & 12.2 & 3.4 & 5.1 & 2.6 \\
\hline $1-7$ & 13.3 & 14.5 & 20.5 & 19.2 & 9.8 & 8.5 \\
\hline $8-11$ & 36.1 & 36.3 & 36.9 & 41.5 & 35.2 & 31.5 \\
\hline 12 and above & 59.8 & 65.6 & 55.0 & 68.3 & 60.2 & 64.0 \\
\hline \multicolumn{7}{|l|}{ Worked in last 12 months } \\
\hline Yes & 26.0 & 7.8 & 26.9 & 7.1 & 24.6 & 8.5 \\
\hline No & 32.5 & 19.8 & $(35.5)$ & 17.0 & 32.8 & 23.3 \\
\hline \multicolumn{7}{|l|}{ Wealth quintile } \\
\hline First & 9.0 & 5.5 & 8.9 & 6.0 & 9.1 & 3.6 \\
\hline Second & 24.6 & 5.8 & 24.0 & 5.9 & 18.8 & 5.4 \\
\hline Third & 17.3 & 11.6 & 18.8 & 13.3 & 16.2 & 8.1 \\
\hline Fourth & 27.5 & 12.6 & 28.6 & 12.7 & 25.7 & 12.3 \\
\hline Fifth & 40.3 & 35.8 & 43.7 & 31.8 & 41.2 & 37.9 \\
\hline Total & 27.8 & 15.4 & 27.0 & 13.2 & 27.6 & 18.0 \\
\hline \multicolumn{7}{|c|}{ Urban } \\
\hline \multicolumn{7}{|l|}{ Age (years) } \\
\hline $15-19$ & 38.0 & 32.8 & * & 18.4 & 37.9 & 36.1 \\
\hline $20-24$ & 47.0 & 42.3 & 40.6 & 36.9 & 50.0 & 55.1 \\
\hline $25-29$ & NA & NA & 40.4 & NA & NA & NA \\
\hline \multicolumn{7}{|l|}{ Religion } \\
\hline Hindu & 42.2 & 39.3 & 43.6 & 34.7 & 42.0 & 43.0 \\
\hline Muslim & 39.6 & 25.2 & 23.1 & 20.8 & 41.9 & 27.3 \\
\hline \multicolumn{7}{|l|}{ Caste } \\
\hline SC & 34.5 & 27.1 & 35.7 & 21.7 & 34.8 & 30.4 \\
\hline OBC & 37.6 & 34.3 & 40.0 & 31.2 & 37.2 & 36.6 \\
\hline General $^{1}$ & 54.5 & 48.1 & 50.0 & 42.3 & 54.2 & 50.7 \\
\hline \multicolumn{7}{|l|}{ Educational level (years) } \\
\hline None $^{2}$ & 13.8 & 9.9 & 10.5 & 9.8 & 14.3 & 10.0 \\
\hline $1-7$ & 22.0 & 22.5 & 27.8 & 26.5 & 21.4 & 18.4 \\
\hline $8-11$ & 45.0 & 46.1 & 50.0 & 50.0 & 44.4 & 44.5 \\
\hline 12 and above & 61.4 & 65.9 & 61.9 & 70.6 & 60.9 & 64.0 \\
\hline
\end{tabular}


Table 8.7: (Cont'd)

\begin{tabular}{|c|c|c|c|c|c|c|}
\hline Background characteristics (\%) & $\begin{array}{c}M \\
15-24\end{array}$ & $\begin{array}{c}\text { W } \\
15-24\end{array}$ & $\begin{array}{c}\text { MM } \\
15-29\end{array}$ & $\begin{array}{c}\text { MW } \\
15-24\end{array}$ & $\begin{array}{c}\text { UM } \\
15-24\end{array}$ & $\begin{array}{c}\text { UW } \\
15-24\end{array}$ \\
\hline \multicolumn{7}{|c|}{ Urban } \\
\hline \multicolumn{7}{|l|}{ Worked in last 12 months } \\
\hline Yes & 36.6 & 32.3 & 39.1 & 27.8 & 35.9 & 35.7 \\
\hline No & 49.6 & 37.6 & $(60.0)$ & 33.1 & 49.6 & 41.0 \\
\hline \multicolumn{7}{|l|}{ Wealth quintile } \\
\hline First & * & 7.1 & * & $(0.0)$ & * & * \\
\hline Second & $(16.7)$ & 8.3 & $(14.3)$ & 9.1 & $(12.5)$ & 10.0 \\
\hline Third & 23.5 & 9.6 & $(12.5)$ & 6.7 & $(21.4)$ & 10.3 \\
\hline Fourth & 27.7 & 18.9 & 27.8 & 20.8 & 26.3 & 16.7 \\
\hline Fifth & 48.5 & 46.7 & 52.6 & 44.8 & 48.1 & 48.3 \\
\hline Total & 41.9 & 36.9 & 40.2 & 32.4 & 42.0 & 40.1 \\
\hline \multicolumn{7}{|c|}{ Rural } \\
\hline \multicolumn{7}{|l|}{ Age (years) } \\
\hline $15-19$ & 22.4 & 11.0 & $(14.3)$ & 9.3 & 23.2 & 12.7 \\
\hline $20-24$ & 30.5 & 14.9 & 30.2 & 14.0 & 31.2 & 32.5 \\
\hline $25-29$ & NA & NA & 24.7 & NA & NA & NA \\
\hline \multicolumn{7}{|l|}{ Religion } \\
\hline Hindu & 24.6 & 12.7 & 25.5 & 11.6 & 24.4 & 15.0 \\
\hline Muslim & 31.1 & 12.1 & 27.0 & 14.3 & 26.8 & 9.3 \\
\hline \multicolumn{7}{|l|}{ Caste } \\
\hline SC & 21.3 & 7.2 & 22.8 & 7.9 & 20.4 & 4.3 \\
\hline OBC & 24.9 & 11.4 & 25.6 & 10.9 & 23.6 & 12.4 \\
\hline General $^{1}$ & 31.0 & 27.9 & 38.5 & 31.1 & 29.7 & 25.0 \\
\hline \multicolumn{7}{|l|}{ Educational level (years) } \\
\hline None $^{2}$ & 8.7 & 2.9 & 12.0 & 3.1 & 3.9 & 2.0 \\
\hline $1-7$ & 12.5 & 13.6 & 19.8 & 18.6 & 8.6 & 7.5 \\
\hline $8-11$ & 34.4 & 33.7 & 35.4 & 40.2 & 33.3 & 28.0 \\
\hline 12 and above & 59.4 & 65.3 & 53.6 & * & 59.5 & 63.7 \\
\hline \multicolumn{7}{|l|}{ Worked in last 12 months } \\
\hline Yes & 24.6 & 6.5 & 25.7 & 6.6 & 22.8 & 6.4 \\
\hline No & 27.6 & 16.5 & * & 15.4 & 28.3 & 18.9 \\
\hline \multicolumn{7}{|l|}{ Wealth quintile } \\
\hline First & 9.1 & 5.4 & 9.0 & 6.0 & 9.2 & 3.4 \\
\hline Second & 24.7 & 5.7 & 23.9 & 5.8 & 19.1 & 5.0 \\
\hline Third & 17.3 & 11.8 & 19.0 & 13.5 & 15.6 & 7.8 \\
\hline Fourth & 27.4 & 12.0 & 28.7 & 12.0 & 25.8 & 11.9 \\
\hline Fifth & 36.7 & 30.2 & 41.6 & 28.6 & 37.7 & 32.2 \\
\hline Total & 25.4 & 12.6 & 25.8 & 12.0 & 24.8 & 13.9 \\
\hline
\end{tabular}

Note: ( ) Based on 25-49 unweighted cases. ${ }^{*}$ Percentage not shown, based on fewer than 25 unweighted cases. Comprehensive knowledge of HIV/AIDS includes: (1) identifying two major ways of preventing HIV (using condoms and limiting sex to one partner); (2) rejecting three common misconceptions about HIV transmission (that HIV can be transmitted through mosquito bites, sharing food with a person who has HIV and hugging someone who has HIV); and (3) knowing that a healthy looking person can be HIV-positive. NA: Not applicable. OBC: Other backward caste. SC: Scheduled caste. ST: Scheduled tribe. ${ }^{1}$ Includes all those not belonging to SC, ST or OBC. ${ }^{2}$ Includes non-literate and literate with no formal schooling. 
Figure 8.4a: Comprehensive knowledge of HIV/AIDS by educational level, Bihar, 2007

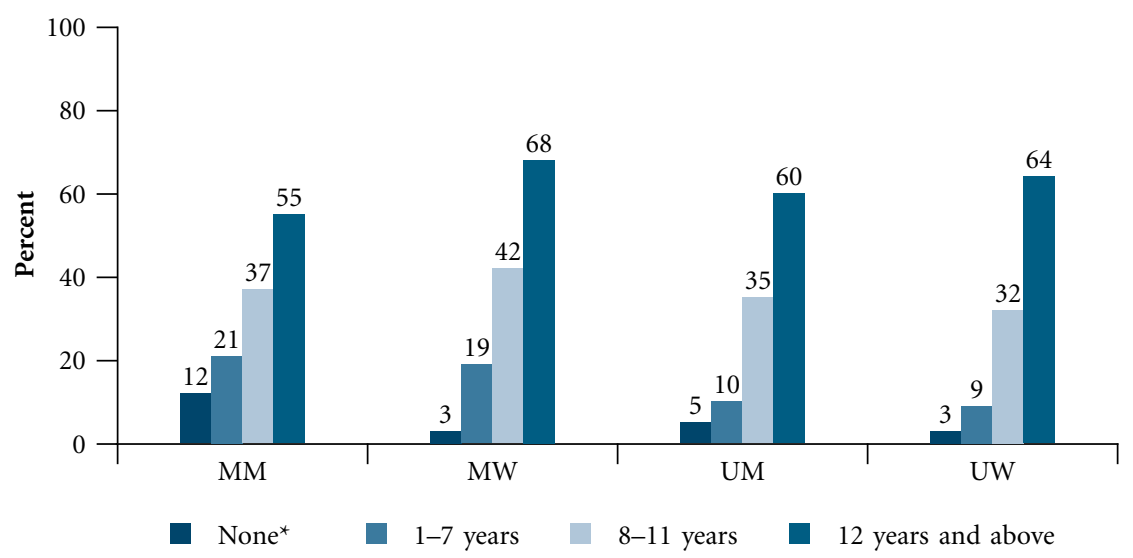

Note: ${ }^{\star}$ Includes non-literate and literate with no formal schooling.

Figure 8.4b: Comprehensive knowledge of HIV/AIDS by wealth quintile, Bihar, 2007

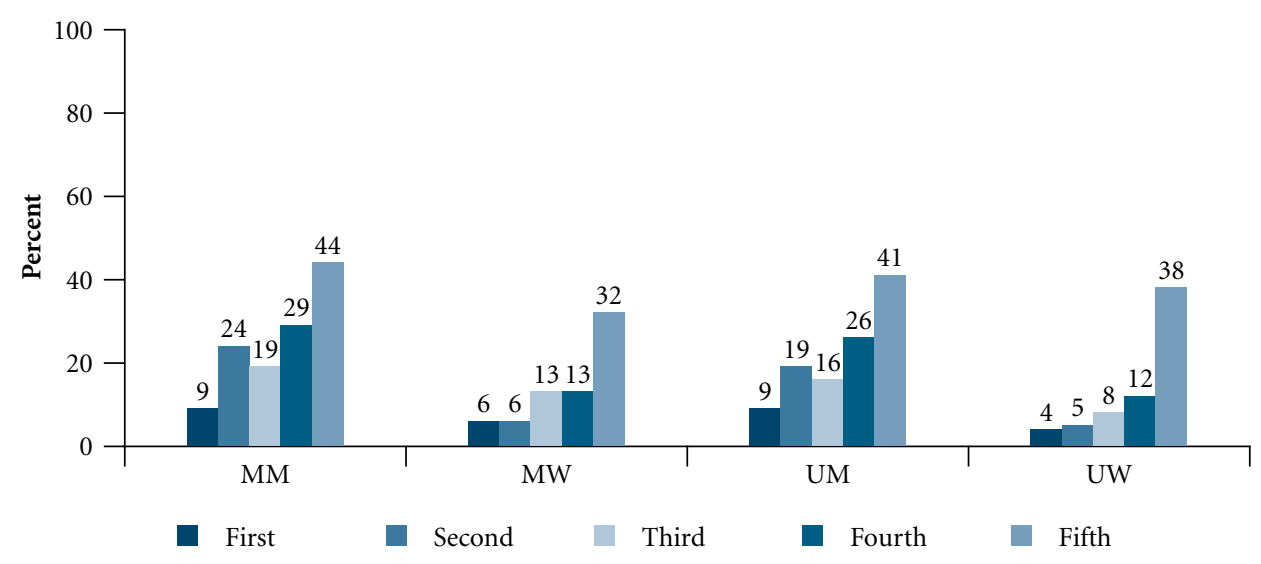

$20 \%$ and $8 \%$, respectively, among young women), perhaps because many youth who were not working were in school or college and thereby more likely to be exposed to HIV-related information.

Socio-demographic differences among married and unmarried youth, and rural and urban youth more or less mirrored the pattern observed for the combined sample. However, differences by religion suggest that in urban settings Muslim youth, particularly young women, tended to lag behind Hindu youth in terms of comprehensive awareness of HIV/AIDS; in rural areas, more Muslim than Hindu men reported comprehensive awareness.

A comparison of awareness of HIV and other STIs, presented in Figure 8.5, stresses that awareness of STIs other than HIV was far more limited than awareness of HIV/AIDS and even comprehensive awareness of HIV/AIDS. 
Figure 8.5: Percentage of youth by awareness of HIV/AIDS, comprehensive knowledge about HIV/AIDS and awareness of STIs, Bihar, 2007

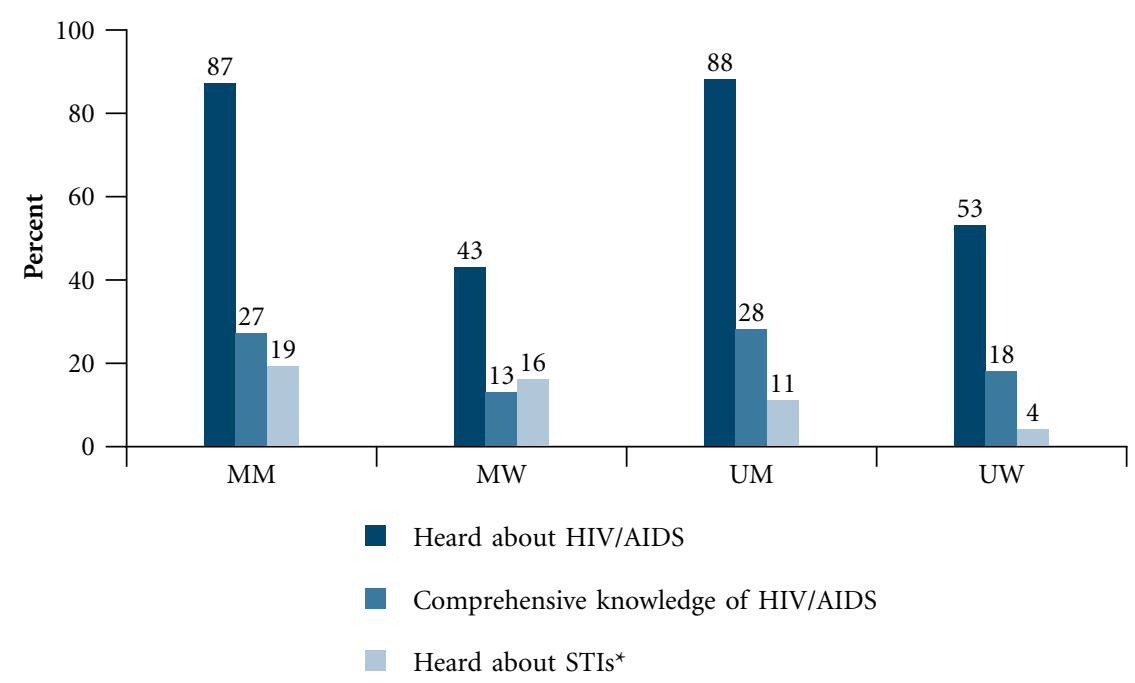

Note: ${ }^{\star}$ Other than HIV.

\subsection{Knowledge of legal issues related to marriage and abortion}

Lack of awareness of such issues as the legal minimum age for marriage and the fact that abortion services are legally available may pose barriers to health promoting behaviours. In this section, we present young people's awareness about the law on each of these issues.

\subsubsection{Knowledge of the legal minimum age at marriage}

The Youth Study collected information on whether youth were aware of the existence of laws relating to the legal minimum age at marriage for males and females in India and probed specific knowledge of these laws. Findings are presented in Table 8.8 and suggest that the majority of youth were aware of the existence of laws governing minimum age at marriage for both girls and boys. Wide gender differences were, however, apparent; young men were more likely than young women to be aware of such laws, with $90 \%$ of young men compared to $71-74 \%$ of young women reporting that there is a legal age at marriage for girls and boys. Differences by marital status were negligible among young men, but wide among young women. Indeed, many more unmarried than married young women were aware of such laws (77-79\% compared to $67-70 \%)$. Likewise, larger percentages of urban than rural youth were aware of such laws and rural-urban differences were pronounced among young women.

The exact legal minimum age at marriage for girls and especially for boys was far less likely to be known. Young women were less likely than young men to be aware of the correct legal minimum age at marriage. For example, $72 \%$ of young men compared to $58 \%$ of young women correctly reported that 18 years was the legal minimum age at marriage for females; however, only $49 \%$ of young men and $36 \%$ of young women correctly reported that 21 was the legal minimum age at marriage for males. Unmarried youth were more likely than married youth to be aware of the correct legal minimum age at marriage for females $(74 \%$ and 
$68 \%$, respectively, among young men; $68 \%$ and $53 \%$ respectively among young women) and males (51\% and $40 \%$, respectively, among young men; $49 \%$ and $29 \%$, respectively, among young women). Rural-urban differences were modest among young men; even so, more urban than rural youth reported awareness of correct legal minimum age at marriage for females ( $78 \%$ versus $71 \%)$. Among young women, these differences were wide and apparent with respect to awareness of legal minimum age at marriage for both females and males; $54 \%$ and $79 \%$ of urban young women were aware of the legal minimum age at marriage for males and females, respectively, compared to $34 \%$ and $56 \%$ of rural young women, respectively.

\subsubsection{Awareness of the conditions under which abortion is legal}

The Youth Study posed a number of questions to gauge youth awareness of conditions under which abortion is legal, for example, if the woman is married, if the woman is unmarried, if the pregnancy exceeds 20 weeks and if the foetus is female but the couple wants a son. Findings are presented in Table 8.9.

Table 8.8: Knowledge of the legal minimum age at marriage

Percentage of youth who had correct knowledge of the legal minimum age at marriage in India, according to residence, Bihar, 2007

\begin{tabular}{|c|c|c|c|c|c|c|}
\hline Knowledge (\%) & $\begin{array}{c}M \\
15-24\end{array}$ & $\begin{array}{c}\text { W } \\
15-24\end{array}$ & $\begin{array}{c}\text { MM } \\
15-29\end{array}$ & $\begin{array}{c}\text { MW } \\
15-24\end{array}$ & $\begin{array}{c}\text { UM } \\
15-24\end{array}$ & $\begin{array}{l}\text { UW } \\
15-24\end{array}$ \\
\hline \multicolumn{7}{|c|}{ Combined } \\
\hline $\begin{array}{l}\text { Aware that there is a legal age at marriage for: } \\
\text { Males }\end{array}$ & 90.2 & 70.8 & 89.2 & 66.9 & 91.2 & 76.9 \\
\hline Females & 90.2 & 73.8 & 89.2 & 70.4 & 91.0 & 79.4 \\
\hline Aware of correct legal age at marriage for: & 486 & 361 & 396 & 290 & 511 & 485 \\
\hline Females & 71.7 & 58.1 & 68.3 & 52.5 & 73.5 & 67.5 \\
\hline Number of respondents & 1,942 & 5,529 & 1,115 & 2,341 & 1,492 & 3,188 \\
\hline \multicolumn{7}{|c|}{ Urban } \\
\hline $\begin{array}{l}\text { Aware that there is a legal age at marriage for: } \\
\text { Males }\end{array}$ & 94.6 & 87.8 & 92.5 & 83.8 & 95.1 & 90.7 \\
\hline Females & 95.0 & 89.5 & 93.5 & 85.9 & 95.1 & 91.9 \\
\hline Aware of correct legal age at marriage for: & & & & & & 593 \\
\hline Females & 77.8 & 78.6 & 77.2 & 73.2 & 71.0 & 82.8 \\
\hline Number of respondents & 1,039 & 2,581 & 547 & 1,136 & 833 & 1,445 \\
\hline \multicolumn{7}{|c|}{ Rural } \\
\hline $\begin{array}{l}\text { Aware that there is a legal age at marriage for: } \\
\text { Males }\end{array}$ & 89.4 & 68.6 & 88.9 & 65.9 & 90.5 & 74.4 \\
\hline Females & 89.4 & 71.8 & 88.8 & 69.3 & 90.2 & 77.1 \\
\hline Aware of correct legal age at marriage for: & & & & & & \\
\hline $\begin{array}{l}\text { Males } \\
\text { Females }\end{array}$ & $\begin{array}{l}48.2 \\
70.7\end{array}$ & $\begin{array}{l}33.9 \\
55.5\end{array}$ & $\begin{array}{l}38.9 \\
67.4\end{array}$ & $\begin{array}{l}27.9 \\
51.1\end{array}$ & $\begin{array}{l}51.0 \\
72.7\end{array}$ & $\begin{array}{l}46.5 \\
64.7\end{array}$ \\
\hline Number of respondents & 903 & 2,948 & 568 & 1,205 & 659 & 1,743 \\
\hline
\end{tabular}

Note: All Ns are unweighted. 
Of the four conditions probed, the largest percentages of youth- $71 \%$ of young men and $79 \%$ of young women-were aware that sex-selective abortion is illegal, presumably the result of widespread information campaigns against sex-selective abortion. A second issue about which large percentages of respondents were aware was that it is illegal to terminate a pregnancy that has gone beyond 20 weeks, reported by $67 \%$ of young men and $83 \%$ of young women. Many fewer were aware that an unmarried woman is legally entitled to undergo an abortion (24-40\%) and even fewer were aware that a married woman is legally entitled to undergo abortion (16-25\%) (see Figure 8.6). Young men were more likely than young women to be aware that married women (25\% versus $16 \%)$ and unmarried women ( $40 \%$ versus $24 \%$ ) were legally entitled to undergo abortion. Conversely, young men were less likely than young women to be aware that sex-selective abortion is illegal and that it is illegal to terminate a pregnancy that has gone beyond 20 weeks. Differences by marital status were negligible among young women, but wide among young men, with more married than unmarried young men reporting awareness of three of the four legal conditions under which abortion is permitted. Rural-urban differences were muted among young men, but wide among young women, with more urban than rural young women reporting awareness of three of the four legal conditions under which abortion is permitted.

Figure 8.6: Percentage of youth who were aware of selected conditions under which abortion is legal, Bihar, 2007

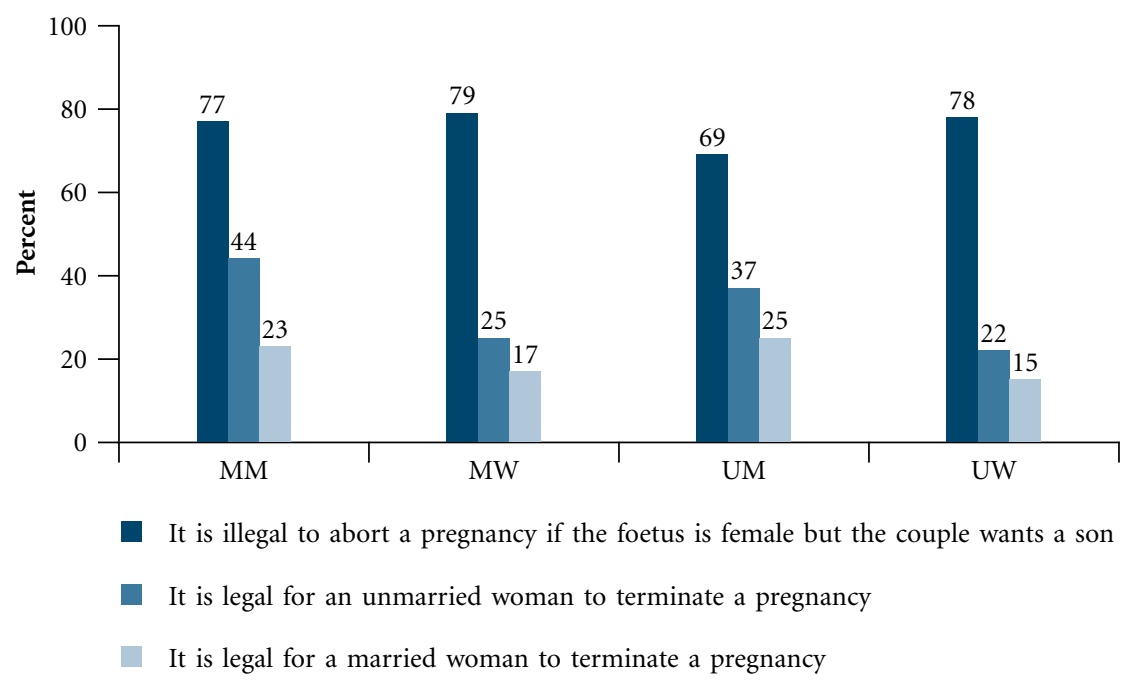

As is evident from Table 8.9 , few youth (6\% of young men and 3\% of young women) could correctly report the legality of all four conditions probed. Differences were mild by marital status and rural-urban residence.

\subsection{Sources of information on sex and reproduction}

The Youth Study questionnaire asked respondents about their sources of information on sexual matters and contraception. For the married, questions about sources of information on sexual matters referred to the situation prior to marriage; in contrast, questions relating to sources of information about contraception related to the current situation, that is, around the time of interview. 
Table 8.9: Awareness of the conditions under which abortion is legal

Percent distribution of youth by knowledge of the conditions under which abortion is legal, according to residence, Bihar, 2007

\begin{tabular}{|c|c|c|c|c|c|c|}
\hline Knowledge (\%) & $\begin{array}{c}M \\
15-24\end{array}$ & $\begin{array}{c}\text { W } \\
15-24\end{array}$ & $\begin{array}{c}\text { MM } \\
15-29\end{array}$ & $\begin{array}{c}\text { MW } \\
15-24\end{array}$ & $\begin{array}{c}\text { UM } \\
15-24\end{array}$ & $\begin{array}{l}\text { UW } \\
15-24\end{array}$ \\
\hline \multicolumn{7}{|c|}{ Combined } \\
\hline $\begin{array}{l}\text { Agree that it is legal for a married woman to } \\
\text { terminate a pregnancy }\end{array}$ & 24.7 & 16.2 & 22.9 & 16.7 & 24.8 & 15.2 \\
\hline $\begin{array}{l}\text { Agree that it is legal for an unmarried woman to } \\
\text { terminate a pregnancy }\end{array}$ & 40.4 & 23.8 & 43.6 & 24.8 & 37.3 & 21.5 \\
\hline $\begin{array}{l}\text { Agree that it is illegal to undergo abortion after } 20 \\
\text { weeks of gestation }\end{array}$ & 66.6 & 83.0 & 73.3 & 83.9 & 64.5 & 80.8 \\
\hline $\begin{array}{l}\text { Disagree that it is legal to abort a pregnancy if the } \\
\text { foetus is female but the couple wants a son }\end{array}$ & 71.2 & 78.5 & 76.5 & 78.7 & 69.4 & 77.6 \\
\hline Had correct knowledge of all of the above & 6.4 & 2.8 & 6.5 & 2.6 & 5.9 & 3.0 \\
\hline Number of respondents & 1,942 & 5,529 & 1,115 & 2,341 & 1,492 & 3,188 \\
\hline \multicolumn{7}{|c|}{ Urban } \\
\hline $\begin{array}{l}\text { Agree that it is legal for a married woman to } \\
\text { terminate a pregnancy }\end{array}$ & 27.5 & 16.0 & 25.0 & 15.5 & 27.9 & 16.6 \\
\hline $\begin{array}{l}\text { Agree that it is legal for an unmarried woman to } \\
\text { terminate a pregnancy }\end{array}$ & 40.9 & 29.7 & 43.5 & 29.6 & 40.2 & 30.0 \\
\hline $\begin{array}{l}\text { Agree that it is illegal to undergo abortion after } 20 \\
\text { weeks of gestation }\end{array}$ & 71.0 & 90.3 & 79.3 & 89.5 & 70.1 & 90.5 \\
\hline $\begin{array}{l}\text { Disagree that it is legal to abort a pregnancy if the } \\
\text { foetus is female but the couple wants a son }\end{array}$ & 74.2 & 86.0 & 76.1 & 84.6 & 73.5 & 86.8 \\
\hline Had correct knowledge of all of the above & 7.2 & 3.8 & 6.5 & 3.5 & 7.3 & 3.8 \\
\hline Number of respondents & 1,039 & 2,581 & 547 & 1,136 & 833 & 1,445 \\
\hline \multicolumn{7}{|c|}{ Rural } \\
\hline $\begin{array}{l}\text { Agree that it is legal for a married woman to } \\
\text { terminate a pregnancy }\end{array}$ & 24.3 & 16.2 & 22.6 & 16.8 & 24.3 & 15.0 \\
\hline $\begin{array}{l}\text { Agree that it is legal for an unmarried woman to } \\
\text { terminate a pregnancy }\end{array}$ & 40.4 & 23.1 & 43.6 & 24.5 & 36.8 & 20.0 \\
\hline $\begin{array}{l}\text { Agree that it is illegal to undergo abortion after } 20 \\
\text { weeks of gestation }\end{array}$ & 65.9 & 82.1 & 72.9 & 83.5 & 63.5 & 79.0 \\
\hline $\begin{array}{l}\text { Disagree that it is legal to abort a pregnancy if the } \\
\text { foetus is female but the couple wants a son }\end{array}$ & 70.7 & 77.5 & 76.4 & 78.3 & 68.5 & 75.9 \\
\hline Had correct knowledge of all of the above & 6.2 & 2.6 & 6.5 & 2.5 & 5.6 & 2.8 \\
\hline Number of respondents & 903 & 2,498 & 568 & 1,205 & 659 & 1,743 \\
\hline
\end{tabular}

Note: All Ns are unweighted.

\subsubsection{Sources of information on sexual matters}

Findings, presented in Table 8.10, suggest that young women had few sources of information on sex and reproduction. Indeed, over two-fifths of young women (44\%) reported that they had never received any information on sexual matters (prior to marriage among the married). While young men were far more likely to have been informed, $16 \%$ reported that they had never received information on sex or reproduction (prior to marriage among the married). Among young men the married were as likely as the unmarried to report that they had never received any information on sexual matters; however among young women, the 
married were more likely to so report (51\% versus 33\%). Larger percentages of rural young men and women were uninformed about sexual matters compared to their urban counterparts (17\% and $10 \%$ among young men, and $46 \%$ versus $28 \%$ among young women, respectively).

Leading sources of information on sex and reproduction were somewhat similar among young men and women and included friends and neighbours (reported by $62 \%$ and $31 \%$ of young men and women, respectively) and the media (reported by $49 \%$ and 12\%, respectively). Young women, in addition, reported family members other than spouse $(19 \%)$ as a major source of information. Other sources were mentioned by fewer than five percent of both young men and women. Notably, very few youth (0-4\%) cited teachers and schools, health care providers or youth or women's group or NGO worker as a source of information on sex and reproduction. Differences by marital status were modest; even so, the unmarried were more likely than the married to cite the mass media as a source of information (51\% versus $41 \%$ among young men, and $21 \%$ versus $6 \%$ among young women). Among young women, in addition, the unmarried were more likely than the married to mention family members other than spouse (24\% versus $16 \%)$ and friends and neighbours as a source of information (37\% versus $28 \%)$.

Patterns were, by and large, similar in urban and rural areas, yet some differences were notable. Urban youth were more likely than rural youth to cite mass media as the main source of information $(65 \%$ versus $46 \%$ among young men and 38\% versus $8 \%$ among young women). Additionally, urban young women were more likely than their rural counterparts to report family members other than spouse (25\% versus $18 \%)$ as sources of information.

\subsubsection{Current sources of information on contraception}

Table 8.11 describes current sources of information on contraception as reported by youth who were aware of at least one contraceptive method. Findings reiterate, as above, that friends and the media played important roles in conveying contraception-related information to young people.

However, sources of information varied by sex, marital status and place of residence of respondents. For example, key sources of information for young men were mass media (70\%) and male friends (66\%). One in eight reported health care providers as sources of information on contraception. Among married young men, wives were reported as a source of information by just $10 \%$. Differences by marital status suggest that unmarried young men were less likely than married young men to obtain contraceptive information from family members other than spouse (6\% versus $11 \%)$, peers $(63 \%$ versus $72 \%)$ and health care providers ( $11 \%$ versus $24 \%$ ), but more likely to obtain information from mass media (73\% versus $59 \%$ ) and schools or teachers (6\% versus $1 \%$ ). Rural-urban differences suggest that young men in rural settings were less likely than those in urban settings to obtain information from the media (68\% and $81 \%$, respectively).

Among young women, patterns were different. Clearly, young women had a greater variety of sources of information on contraceptives than men. While friends and neighbours continued to be the main source of information on contraception among young women as well (53\%), such sources as family members other than spouse $(44 \%)$ and media (34\%) were also common sources of information. Differences by marital status were evident among young women. Almost three-fifths (59\%) of married young women reported that they obtained information on contraception from their husbands. Considerable percentages of married young women also received information on contraception from female friends/neighbours (56\%), family members other than spouse (44\%) and the media (25\%). Compared to married young women, unmarried young women's leading sources of information on contraceptives were female friends/neighbours (50\%), followed by the mass media (47\%) and family members (44\%). Rural-urban differences suggest that young women in 
Table 8.10: Sources of information on sexual matters before marriage

Percentage of youth by sources of information on sexual matters before marriage, according to residence, Bihar, 2007

\begin{tabular}{|c|c|c|c|c|c|c|}
\hline Sources of information (\%) & $\begin{array}{c}\mathrm{M} \\
15-24\end{array}$ & $\begin{array}{c}\mathrm{W} \\
15-24\end{array}$ & $\begin{array}{c}\text { MM } \\
15-29\end{array}$ & $\begin{array}{c}\text { MW } \\
15-24\end{array}$ & $\begin{array}{c}\text { UM } \\
15-24\end{array}$ & $\begin{array}{c}\text { UW } \\
15-24\end{array}$ \\
\hline \multicolumn{7}{|c|}{ Combined } \\
\hline Never received information & 15.7 & 44.1 & 15.5 & 50.7 & 15.3 & 32.7 \\
\hline A family member other than spouse & 2.7 & 18.7 & 5.2 & 16.0 & 2.1 & 23.6 \\
\hline Spouse/partner & 1.2 & 1.2 & 2.8 & 1.5 & 0.3 & 0.4 \\
\hline Friend/neighbour & 62.0 & 30.6 & 65.0 & 27.5 & 60.8 & 36.5 \\
\hline Teacher/school & 4.0 & 1.4 & 1.3 & 0.3 & 5.3 & 3.1 \\
\hline Health care provider & 4.1 & 0.2 & 7.2 & 0.2 & 3.8 & 0.4 \\
\hline Mass media ${ }^{1}$ & 48.5 & 11.6 & 41.1 & 5.6 & 50.8 & 21.0 \\
\hline Youth/mahila mandal/NGO worker & 0.2 & 0.1 & 0.3 & 0.1 & 0.2 & 0.3 \\
\hline Poster/billboard & 2.1 & 0.0 & 1.3 & 0.0 & 2.5 & 0.1 \\
\hline Don't remember & 2.0 & 7.4 & 2.8 & 9.1 & 1.9 & 4.6 \\
\hline Number of respondents & 1,942 & 5,529 & 1,115 & 2,341 & 1,492 & 3,188 \\
\hline \multicolumn{7}{|c|}{ Urban } \\
\hline Never received information & 9.7 & 27.8 & 8.6 & 38.7 & 10.2 & 20.0 \\
\hline A family member other than spouse & 1.4 & 25.0 & 3.3 & 22.5 & 1.2 & 26.7 \\
\hline Spouse/partner & 1.1 & 1.4 & 2.2 & 2.8 & 0.8 & 0.4 \\
\hline Friend/neighbour & 63.8 & 32.0 & 71.7 & 32.4 & 62.3 & 31.6 \\
\hline Teacher/school & 5.0 & 5.8 & 2.2 & 1.4 & 5.3 & 8.7 \\
\hline Health care provider & 4.6 & 0.3 & 6.5 & 0.0 & 4.1 & 0.4 \\
\hline Mass media $^{1}$ & 64.5 & 37.7 & 57.6 & 21.1 & 66.4 & 49.6 \\
\hline Youth/mahila mandal/NGO worker & 0.4 & 0.0 & 1.1 & 0.0 & 0.4 & 0.0 \\
\hline Poster/billboard & 3.6 & 0.2 & 3.3 & 0.0 & 3.7 & 0.2 \\
\hline Don't remember & 1.1 & 3.1 & 1.1 & 4.9 & 1.2 & 1.8 \\
\hline Number of respondents & 1,039 & 2,581 & 547 & 1,136 & 833 & 1,445 \\
\hline \multicolumn{7}{|c|}{ Rural } \\
\hline Never received information & 16.7 & 46.3 & 16.1 & 51.5 & 16.3 & 35.1 \\
\hline A family member other than spouse & 3.0 & 17.9 & 5.4 & 15.6 & 2.3 & 23.0 \\
\hline Spouse/partner & 1.2 & 1.1 & 2.8 & 1.5 & 0.2 & 0.3 \\
\hline Friend/neighbour & 61.7 & 30.5 & 64.4 & 27.2 & 60.5 & 37.4 \\
\hline Teacher/school & 3.8 & 0.8 & 1.3 & 0.3 & 5.3 & 2.1 \\
\hline Health care provider & 4.0 & 0.2 & 7.2 & 0.1 & 3.8 & 0.4 \\
\hline Mass media ${ }^{1}$ & 45.8 & 8.1 & 39.6 & 4.5 & 47.8 & 15.8 \\
\hline Youth/mahila mandal/NGO worker & 0.1 & 0.2 & 0.2 & 0.1 & 0.2 & 0.4 \\
\hline Poster/billboard & 1.8 & 0.0 & 1.2 & 0.0 & 2.2 & 0.1 \\
\hline Don't remember & 2.2 & 8.0 & 2.9 & 9.3 & 2.0 & 5.2 \\
\hline Number of respondents & 903 & 2,498 & 568 & 1,205 & 659 & 1,743 \\
\hline
\end{tabular}

Note: All Ns are unweighted. Column totals may exceed 100\% due to multiple responses. For married respondents, questions referred to the period prior to marriage. ${ }^{1}$ Include newspapers, books/magazines, radio/television and the internet. 
Table 8.11: Current sources of information on contraception

Percentage of youth reporting awareness of contraceptives by current sources of information, according to residence, Bihar, 2007

\begin{tabular}{|c|c|c|c|c|c|c|}
\hline Current sources of information (\%) & $\underset{15-24}{M}$ & $\begin{array}{c}\text { W } \\
15-24\end{array}$ & $\begin{array}{c}\text { MM } \\
15-29\end{array}$ & $\begin{array}{c}\text { MW } \\
\text { 15-24 }\end{array}$ & $\begin{array}{c}\text { UM } \\
15-24\end{array}$ & $\begin{array}{c}\text { UW } \\
15-24\end{array}$ \\
\hline \multicolumn{7}{|c|}{ Combined } \\
\hline Family member other than spouse & 7.8 & 43.8 & 10.8 & 43.8 & 5.9 & 44.0 \\
\hline Spouse/partner & 3.3 & 38.7 & 10.1 & 58.9 & 0.9 & 0.1 \\
\hline Female friend/neighbour & 1.7 & 53.2 & 1.6 & 55.6 & 2.0 & 49.6 \\
\hline Male friend/neighbour & 66.0 & 0.8 & 71.5 & 0.8 & 63.1 & 1.0 \\
\hline Teacher/school/college & 4.2 & 0.7 & 0.7 & 0.1 & 5.7 & 1.7 \\
\hline Health care provider & 12.9 & 2.9 & 24.2 & 3.9 & 10.7 & 1.0 \\
\hline Mass media ${ }^{1}$ & 69.5 & 33.5 & 58.5 & 25.4 & 72.9 & 46.7 \\
\hline Poster/billboard & 7.0 & 1.4 & 5.3 & 1.4 & 8.2 & 1.4 \\
\hline Youth/mahila mandal/NGO worker & 0.3 & 0.4 & 0.5 & 0.4 & 0.2 & 0.4 \\
\hline Other sources & 0.5 & 0.1 & 1.5 & 0.1 & 0.3 & 0.0 \\
\hline Number aware of contraceptives & 1,850 & 5,452 & 1,096 & 2,334 & 1,411 & 3,118 \\
\hline \multicolumn{7}{|c|}{ Urban } \\
\hline Family member other than spouse & 4.4 & 43.3 & 5.4 & 49.3 & 4.2 & 39.0 \\
\hline Spouse/partner & 2.2 & 29.1 & 10.9 & 69.0 & 0.4 & 0.4 \\
\hline Female friend/neighbour & 0.7 & 39.3 & 2.2 & 45.8 & 0.8 & 34.7 \\
\hline Male friend/neighbour & 65.6 & 0.3 & 76.1 & 0.0 & 63.2 & 0.2 \\
\hline Teacher/school/college & 5.5 & 2.8 & 2.2 & 0.0 & 5.9 & 4.5 \\
\hline Health care provider & 12.5 & 3.3 & 22.0 & 5.6 & 11.3 & 2.0 \\
\hline Mass media ${ }^{1}$ & 81.0 & 68.2 & 68.5 & 53.5 & 83.7 & 78.9 \\
\hline Poster/billboard & 9.9 & 3.3 & 12.1 & 2.1 & 9.2 & 4.3 \\
\hline Youth/mahila mandal/NGO worker & 0.7 & 0.2 & 1.1 & 0.0 & 0.4 & 0.2 \\
\hline Other sources & 0.7 & 0.0 & 1.1 & 0.0 & 0.8 & 0.0 \\
\hline Number aware of contraceptives & 1,016 & 2,566 & 543 & 1,134 & 813 & 1,432 \\
\hline \multicolumn{7}{|c|}{ Rural } \\
\hline Family member other than spouse & 8.3 & 43.9 & 11.3 & 43.4 & 6.3 & 44.9 \\
\hline Spouse/partner & 3.5 & 40.0 & 10.1 & 58.2 & 1.0 & 0.0 \\
\hline Female friend/neighbour & 1.9 & 55.0 & 1.5 & 56.2 & 2.3 & 52.4 \\
\hline Male friend/neighbour & 66.0 & 0.9 & 71.1 & 0.8 & 63.0 & 1.1 \\
\hline Teacher/school/college & 4.0 & 0.4 & 0.5 & 0.1 & 5.7 & 1.2 \\
\hline Health care provider & 12.9 & 2.9 & 24.4 & 3.8 & 10.5 & 0.9 \\
\hline Mass media ${ }^{1}$ & 67.5 & 29.0 & 57.6 & 23.6 & 70.6 & 40.7 \\
\hline Poster/billboard & 6.5 & 1.2 & 4.7 & 1.3 & 8.0 & 0.8 \\
\hline Youth/mahila mandal/NGO worker & 0.3 & 0.4 & 0.4 & 0.4 & 0.2 & 0.4 \\
\hline Other sources & 0.5 & 0.0 & 1.5 & 0.1 & 0.2 & 0.0 \\
\hline Number aware of contraceptives & 834 & 2,886 & 553 & 1,200 & 598 & 1,686 \\
\hline
\end{tabular}

Note: All Ns are unweighted. Column totals may not equal 100\% due to multiple responses. ${ }^{1}$ Include newspapers, books/ magazines, radio/television and the internet. 
rural settings were somewhat more likely than those in urban settings to rely on female friends (55\% versus $39 \%$ ), and much less likely to rely on the mass media ( $29 \%$ versus $68 \%$ ), for information on contraception. Among the married, however, urban young women were more likely than their rural counterparts to cite a husband as a source of information on contraception (69\% versus $58 \%$ ).

Of note with regard to both young men and women were findings suggesting that health care providers reached just one-quarter of married young men, one-tenth of unmarried young men and even fewer married and unmarried young women (1-4\%). This is likely a consequence of the lack of attention that the RCH Programme has paid, thus far, to young people, including married young women. Teachers, likewise, reached a minority of the unmarried (2-6\%).

A comparison of sources of information on sex and reproduction (prior to marriage for the married) and contraception suggests that leading sources of information on both matters were peers and the media, which are not necessarily the most reliable sources of information. In contrast, family members, teachers and health care providers-often assumed to be more reliable sources of information-were not necessarily reported as such. For example, health care providers were important sources of information on contraception just for married young men; they were far less likely to have provided information to the unmarried and even to married young women. No more than $6 \%$ of unmarried and hardly any married youth cited teachers as sources of information on sexual matters or contraception. No more than $8 \%$ of young men cited a family member as a source of information on sex or contraception.

\subsection{Perceptions and experience of family life or sex education}

In the Youth Study, we asked respondents about their views on the importance of imparting family life or sex education to youth, the best age at which youth should receive information about sexual matters and the best person to provide that information. We also asked youth whether they had received formal family life or sex education and if so, the source of this education and their opinion about its quality.

Table 8.12 presents findings on young people's perceptions of family life or sex education. The majority of youth felt that it is important to impart family life or sex education to youth $-81 \%$ of young men and $77 \%$ of young women. Differences by marital status were modest; however, somewhat more unmarried than married young women reported this perception ( $81 \%$ versus $74 \%)$. Urban youth, irrespective of sex and marital status, were more likely than their rural counterparts to report this perception ( $88-89 \%$ versus $75-80 \%)$.

Of those who perceived family life or sex education to be important, almost two-fifths of young men and over one-third of young women reported that such education should be provided to young people at ages between 15 and 17 years. Young men were more likely than young women to believe that information on sexual matters should be provided at age 18 years or later (33\% versus 19\%). However, considerably larger percentages of young women than men were not sure about the appropriate age for provision of family life or sex education. By and large, differences by marital status and rural-urban residence were narrow; it would appear however that unmarried young men were more likely than the married to consider ages below 18 as appropriate.

In terms of youth perceptions about the best person to impart education on sex or family life matters, young men and women revealed quite different preferences. Findings suggest that while young women would like to obtain information on sex or family life matters from their parents or siblings, this was not the case for young men, who would prefer to consult teachers, health care providers or other knowledgeable persons, on one hand, and peers, on the other. As shown in Table 8.12, among young men who perceived family life 
Table 8.12: Perceptions about family life or sex education

Percentage of youth by perceptions about family life or sex education, according to residence, Bihar, 2007

\begin{tabular}{|c|c|c|c|c|c|c|}
\hline Perceptions (\%) & $\begin{array}{c}\text { M } \\
15-24\end{array}$ & $\begin{array}{c}\text { W } \\
15-24\end{array}$ & $\begin{array}{c}\text { MM } \\
15-29\end{array}$ & $\begin{array}{c}\text { MW } \\
15-24\end{array}$ & $\begin{array}{c}\text { UM } \\
15-24\end{array}$ & $\begin{array}{l}\text { UW } \\
15-24\end{array}$ \\
\hline \multicolumn{7}{|c|}{ Combined } \\
\hline Perceived family life/sex education to be important & 81.3 & 76.8 & 81.9 & 74.4 & 80.8 & 80.5 \\
\hline Number of respondents & 1,942 & 5,529 & 1,115 & 2,341 & 1,492 & 3,188 \\
\hline \multicolumn{7}{|l|}{$\begin{array}{l}\text { Perceived that family life/sex education } \\
\text { should be provided at age (years): }\end{array}$} \\
\hline Below 12 & 5.5 & 3.6 & 5.9 & 4.2 & 6.1 & 2.7 \\
\hline $12-14$ & 19.0 & 25.4 & 14.9 & 26.2 & 20.8 & 24.1 \\
\hline $15-17$ & 38.4 & 36.1 & 33.8 & 35.3 & 38.8 & 37.1 \\
\hline 18 or above & 32.8 & 19.2 & 40.9 & 17.3 & 30.7 & 21.9 \\
\hline Don't know & 4.3 & 15.7 & 4.5 & 16.9 & 3.6 & 14.2 \\
\hline \multicolumn{7}{|l|}{$\begin{array}{l}\text { Perceived that the best person to provide family } \\
\text { life/sex education was: }\end{array}$} \\
\hline Parent & 11.2 & 34.1 & 11.9 & 32.5 & 12.2 & 36.3 \\
\hline Sibling/sister-in-law & 0.6 & 18.8 & 0.4 & 18.9 & 0.8 & 18.7 \\
\hline Spouse/partner & 0.3 & 4.8 & 0.7 & 7.4 & 0.4 & 0.5 \\
\hline Teacher & 29.1 & 7.3 & 23.5 & 5.3 & 33.0 & 10.7 \\
\hline Friend & 28.0 & 16.5 & 26.4 & 15.8 & 25.2 & 17.7 \\
\hline Health care provider/expert & 22.3 & 9.0 & 28.4 & 9.7 & 19.8 & 7.9 \\
\hline Youth club/mandal/NGO worker & 0.6 & 0.0 & 1.0 & 0.0 & 0.4 & 0.2 \\
\hline Others & 0.3 & 0.2 & 1.6 & 0.1 & 0.1 & 0.4 \\
\hline Don't know & 7.5 & 9.2 & 5.8 & 10.3 & 7.7 & 7.5 \\
\hline $\begin{array}{l}\text { Number who perceived family life/sex } \\
\text { education to be important }\end{array}$ & 1,631 & 4,553 & 932 & 1,859 & 1,254 & 2,696 \\
\hline \multicolumn{7}{|c|}{ Urban } \\
\hline Perceived family life/sex education to be important & 87.9 & 89.3 & 85.9 & 85.2 & 88.2 & 92.1 \\
\hline Number of respondents & 1,039 & 2,581 & 547 & 1,136 & 833 & 1,445 \\
\hline \multicolumn{7}{|l|}{$\begin{array}{l}\text { Perceived that family life/sex education } \\
\text { should be provided at age (years): }\end{array}$} \\
\hline Below 12 & 4.1 & 1.4 & 3.8 & 1.7 & 3.7 & 1.5 \\
\hline $12-14$ & 19.9 & 23.6 & 15.0 & 25.6 & 21.3 & 22.1 \\
\hline $15-17$ & 42.3 & 39.6 & 40.0 & 38.8 & 42.1 & 40.4 \\
\hline 18 or above & 30.1 & 27.8 & 37.5 & 24.0 & 29.2 & 30.3 \\
\hline Don't know & 3.7 & 7.6 & 3.8 & 9.9 & 3.7 & 5.7 \\
\hline \multicolumn{7}{|l|}{$\begin{array}{l}\text { Perceived that the best person to provide family } \\
\text { life/sex education was: }\end{array}$} \\
\hline Parent & 15.8 & 42.7 & 15.2 & 37.7 & 16.2 & 46.2 \\
\hline Sibling/sister-in-law & 0.8 & 17.2 & 0.0 & 21.3 & 0.9 & 14.5 \\
\hline Spouse/partner & 0.8 & 1.6 & 0.0 & 3.3 & 0.5 & 0.7 \\
\hline Teacher & 32.0 & 9.5 & 27.8 & 7.4 & 33.8 & 10.8 \\
\hline Friend & 26.7 & 16.3 & 29.1 & 17.2 & 25.5 & 15.6 \\
\hline Health care provider/expert & 17.4 & 8.6 & 22.8 & 8.2 & 16.7 & 8.8 \\
\hline Youth club/mandal/NGO worker & 0.4 & 0.0 & 0.0 & 0.0 & 0.5 & 0.2 \\
\hline Others & 0.4 & 0.2 & 1.3 & 0.0 & 0.5 & 0.2 \\
\hline Don't know & 5.7 & 3.9 & 3.8 & 4.9 & 5.6 & 3.1 \\
\hline $\begin{array}{l}\text { Number who perceived family life/sex } \\
\text { education to be important }\end{array}$ & 910 & 2,300 & 471 & 969 & 733 & 1,331 \\
\hline
\end{tabular}


Table 8.12: (Cont'd)

\begin{tabular}{|c|c|c|c|c|c|c|}
\hline Perceptions (\%) & $\begin{array}{c}\mathrm{M} \\
15-24\end{array}$ & $\begin{array}{c}\text { W } \\
15-24\end{array}$ & $\begin{array}{c}\text { MM } \\
15-29\end{array}$ & $\begin{array}{c}\text { MW } \\
15-24\end{array}$ & $\begin{array}{c}\text { UM } \\
15-24\end{array}$ & $\begin{array}{c}\text { UW } \\
15-24\end{array}$ \\
\hline \multicolumn{7}{|c|}{ Rural } \\
\hline Perceived family life/sex education to be important & 80.2 & 75.2 & 81.5 & 73.7 & 79.3 & 78.4 \\
\hline Number of respondents & 903 & 2,498 & 568 & 1,205 & 659 & 1,743 \\
\hline \multicolumn{7}{|l|}{$\begin{array}{l}\text { Perceived that family life/sex education } \\
\text { should be provided at age (years): }\end{array}$} \\
\hline Below 12 & 5.8 & 3.9 & 6.1 & 4.4 & 6.5 & 2.9 \\
\hline $12-14$ & 18.9 & 25.6 & 14.9 & 26.1 & 20.8 & 24.6 \\
\hline $15-17$ & 37.7 & 35.5 & 33.3 & 35.1 & 38.1 & 36.4 \\
\hline 18 or above & 33.3 & 17.9 & 41.1 & 16.8 & 31.0 & 20.0 \\
\hline Don't know & 4.4 & 17.0 & 4.6 & 17.4 & 3.6 & 16.0 \\
\hline \multicolumn{7}{|l|}{$\begin{array}{l}\text { Perceived that the best person to provide family } \\
\text { life/sex education was: }\end{array}$} \\
\hline Parent & 10.4 & 32.8 & 11.6 & 32.1 & 11.3 & 34.2 \\
\hline Sibling/sister-in-law & 0.6 & 19.0 & 0.4 & 18.8 & 0.9 & 19.6 \\
\hline Spouse/partner & 0.2 & 5.3 & 0.7 & 7.7 & 0.3 & 0.5 \\
\hline Teacher & 28.5 & 7.0 & 23.1 & 5.1 & 32.8 & 10.7 \\
\hline Friend & 28.2 & 16.5 & 26.1 & 15.7 & 25.2 & 18.2 \\
\hline Health care provider/expert & 23.1 & 9.1 & 29.0 & 9.8 & 20.4 & 7.7 \\
\hline Youth club/mandal/NGO worker & 0.6 & 0.1 & 1.1 & 0.0 & 0.4 & 0.1 \\
\hline Others & 0.3 & 0.2 & 1.7 & 0.1 & 0.0 & 0.4 \\
\hline Don't know & 7.9 & 10.0 & 6.0 & 10.7 & 8.3 & 8.5 \\
\hline $\begin{array}{l}\text { Number who perceived family life/sex } \\
\text { education to be important }\end{array}$ & 721 & 2,255 & 461 & 890 & 521 & 1,365 \\
\hline
\end{tabular}

Note: All Ns are unweighted. Column totals may not equal 100\% due to missing cases.

or sex education to be important, leading preferred sources of such education were teachers (29\%), friends $(28 \%)$ and health care providers and other experts (22\%). Just $12 \%$ cited a parent or sibling. Among young women who perceived family life or sex education to be important, in contrast, the most commonly cited preferred sources were parents (34\%), siblings (19\%) and friends (17\%). Teachers and health care providers or experts were cited by $7 \%$ and $9 \%$, respectively.

Differences by marital status were, by and large, modest among young women. Among young men, larger proportions of the married than the unmarried considered health care providers best equipped to provide family life or sex education (29\% versus $20 \%$ ); conversely, more of the unmarried than the married considered teachers best equipped to provide family life or sex education (33\% versus $24 \%$ ). Rural-urban differences were negligible, except that more urban than rural youth cited parents best equipped to provide family life or sex education (16\% versus $10 \%$ among young men; $43 \%$ versus $33 \%$ among young women).

Very few youth reported that they had received family life or sex education in school or through special programmes sponsored by the government or NGOs. As seen in Table 8.13, just 7\% of young men and 3\% of young women had received any formal family life or sex education. Differences by marital status were modest; however the unmarried were slightly more likely to have obtained family life or sex education than the married (see also Figure 8.7). Rural-urban differences were mild: slightly more urban than rural young men had ever received family life or sex education ( $11 \%$ and $7 \%$, respectively) and considerably more young women in urban than in rural settings had received this education ( $9 \%$ and $2 \%$, respectively). 
Table 8.13: Experiences of family life or sex education

Percentage of youth by experiences of family life or sex education, according to residence, Bihar, 2007

\begin{tabular}{|c|c|c|c|c|c|c|}
\hline Experiences (\%) & $\begin{array}{c}M \\
15-24\end{array}$ & $\begin{array}{c}\text { W } \\
15-24\end{array}$ & $\begin{array}{c}\text { MM } \\
15-29\end{array}$ & $\begin{array}{c}\text { MW } \\
15-24\end{array}$ & $\begin{array}{c}\text { UM } \\
15-24\end{array}$ & $\begin{array}{l}\text { UW } \\
15-24\end{array}$ \\
\hline \multicolumn{7}{|c|}{ Combined } \\
\hline Received formal family life/sex education & 7.2 & 2.5 & 3.9 & 0.9 & 8.3 & 5.1 \\
\hline Number of respondents & 1,942 & 5,529 & 1,115 & 2,341 & 1,492 & 3,188 \\
\hline \multicolumn{7}{|l|}{ Source of family life/sex education } \\
\hline NGO programme/camp & 4.2 & 5.5 & 9.3 & $(15.4)$ & 3.2 & 1.8 \\
\hline Government programme/camp & 14.0 & 6.9 & 30.2 & $(7.7)$ & 8.7 & 7.9 \\
\hline School/college & 77.6 & 81.4 & 60.5 & $(61.5)$ & 84.8 & 87.8 \\
\hline Others & 2.8 & 1.4 & 4.7 & $(0.0)$ & 2.4 & 1.8 \\
\hline $\begin{array}{l}\text { Opinion about family life/sex education received } \\
\text { It answered many queries }\end{array}$ & 74.8 & 75.3 & 85.7 & $(72.0)$ & 72.2 & 76.8 \\
\hline Teacher/trainer explained well & 83.2 & 78.1 & 88.4 & $(72.0)$ & 81.0 & 81.0 \\
\hline Respondent felt embarrassed & 44.4 & 56.2 & 44.2 & $(61.5)$ & 42.9 & 54.9 \\
\hline Number who received family life/sex education & 171 & 288 & 52 & 46 & 148 & 242 \\
\hline \multicolumn{7}{|c|}{ Urban } \\
\hline Received formal family life/sex education & 10.8 & 8.5 & 5.4 & 2.8 & 11.4 & 12.3 \\
\hline Number of respondents & 1,039 & 2,581 & 547 & 1,136 & 833 & 1,445 \\
\hline \multicolumn{7}{|l|}{ Source of family life/sex education } \\
\hline NGO programme/camp & 3.3 & 0.0 & $(16.7)$ & $(0.0)$ & 3.6 & 0.0 \\
\hline Government programme/camp & 6.7 & 1.9 & $(16.7)$ & $(0.0)$ & 7.1 & 1.6 \\
\hline School/college & 86.7 & 98.1 & $(80.0)$ & $(100.0)$ & 88.9 & 98.4 \\
\hline Others & 3.3 & 1.9 & $(0.0)$ & $(0.0)$ & 3.6 & 0.0 \\
\hline $\begin{array}{l}\text { Opinion about family life/sex education received } \\
\text { It answered many queries }\end{array}$ & 86.7 & 79.6 & $(80.0)$ & $(75.0)$ & 88.9 & 80.3 \\
\hline Teacher/trainer explained well & 86.2 & 84.9 & $(80.0)$ & $(75.0)$ & 85.7 & 86.7 \\
\hline Respondent felt embarrassed & 33.3 & 50.0 & $(40.0)$ & $(60.0)$ & 35.7 & 47.5 \\
\hline Number who received family life/sex education & 108 & 212 & 31 & 35 & 95 & 177 \\
\hline \multicolumn{7}{|c|}{ Rural } \\
\hline Received formal family life/sex education & 6.6 & 1.7 & 3.7 & 0.8 & 7.7 & 3.7 \\
\hline Number of respondents & 903 & 2,498 & 568 & 1,205 & 659 & 1,743 \\
\hline \multicolumn{7}{|l|}{ Source of family life/sex education } \\
\hline NGO programme/camp & 4.4 & 8.7 & * & * & 3.1 & 2.9 \\
\hline Government programme/camp & 15.9 & 9.9 & * & * & 9.2 & 11.5 \\
\hline School/college & 75.2 & 71.7 & * & * & 83.7 & 80.8 \\
\hline Others & 2.7 & 2.2 & * & * & 2.0 & 2.9 \\
\hline $\begin{array}{l}\text { Opinion about family life/sex education received } \\
\text { It answered many queries }\end{array}$ & 71.7 & 73.6 & * & * & 67.3 & 74.8 \\
\hline Teacher/trainer explained well & 83.2 & 75.0 & * & * & 79.6 & 78.4 \\
\hline Respondent felt embarrassed & 46.9 & 60.4 & * & * & 44.9 & 59.2 \\
\hline Number who received family life/sex education & 63 & 76 & 21 & 11 & 53 & 65 \\
\hline
\end{tabular}

Note: All Ns are unweighted. Column totals may not equal 100\% due to missing cases or "don't know" responses. ( ) Based on 25-49 unweighted cases. ${ }^{*}$ Percentage not shown, based on fewer than 25 unweighted cases. 
Figure 8.7: Percentage of youth who received family life or sex education, according to residence, Bihar, 2007
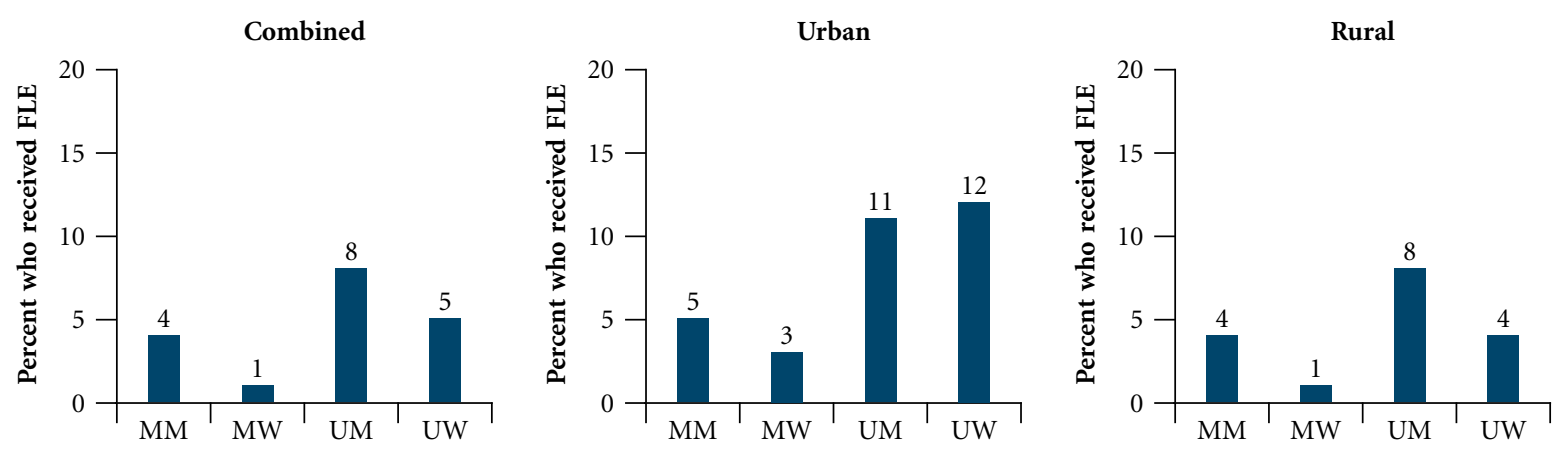

Note: FLE: Family life or sex education.

The large majority of youth who had received family life or sex education had done so in school or college ( $78 \%$ of young men and $81 \%$ of young women); married youth were less likely than unmarried youth to have obtained such education in school/college (61-62\% versus $85-88 \%)$, and conversely more likely to have received such education through NGO or government programmes (23-40\% versus $10-12 \%)$. Rural youth were somewhat less likely than urban youth to have received this education in school/college $(72-75 \%$ versus $87-98 \%$ ) and somewhat more likely to have received it in NGO or government programmes or camps (19-20\% versus $2-10 \%)$.

Of those who reported receiving formal family life or sex education, three-quarters felt that it had answered many of their questions and 78-83\% reported that teachers or trainers had explained matters well. Differences by marital status show that more married than unmarried young men felt that the family life or sex education they had received had answered many of their questions (86\% versus $72 \%)$ and that trainers had explained matters well (88\% versus $81 \%)$. Among young women, a reverse pattern was evident, with more unmarried than married young women reported as such (77-81\% versus $72 \%)$. Rural-urban differences indicate that in general, more urban than rural youth gave positive assessment of the education that they had received. Despite the fact that youth gave a generally positive assessment of the education they had received, $44 \%$ of young men and $56 \%$ of young women reported feeling uncomfortable or embarrassed in the course of family life or sex education, raising questions about the extent to which they were indeed able to participate freely and clarify doubts. More married than unmarried young women reported feeling uncomfortable or embarrassed (62\% versus 55\%), but the differences were muted among young men. Rural respondents were more likely to report embarrassment than urban respondents (47\% versus 33\% among young men and $60 \%$ versus $50 \%$ among young women).

Figure 8.8 compares the extent to which those who had received family life or sex education differed in terms of correct knowledge of selected sexual and reproductive health matters with those who had not. Findings suggest that youth who had received family life or sex education were more likely than those who had not to report in-depth awareness of contraception and comprehensive awareness of HIV/AIDS (see Sections 8.1.1, 8.1.3 and 8.1.8 for details of items considered in each summary measure). Differences were moderate in regard to correct knowledge of sex- and pregnancy-related matters. Differences were, by and large, evident among both rural and urban respondents, but wider in urban than rural areas (not shown in figure). 
Figure 8.8: Percentage of youth reporting knowledge of selected sexual and reproductive health matters according to whether they had or had not received family life or sex education, Bihar, 2007

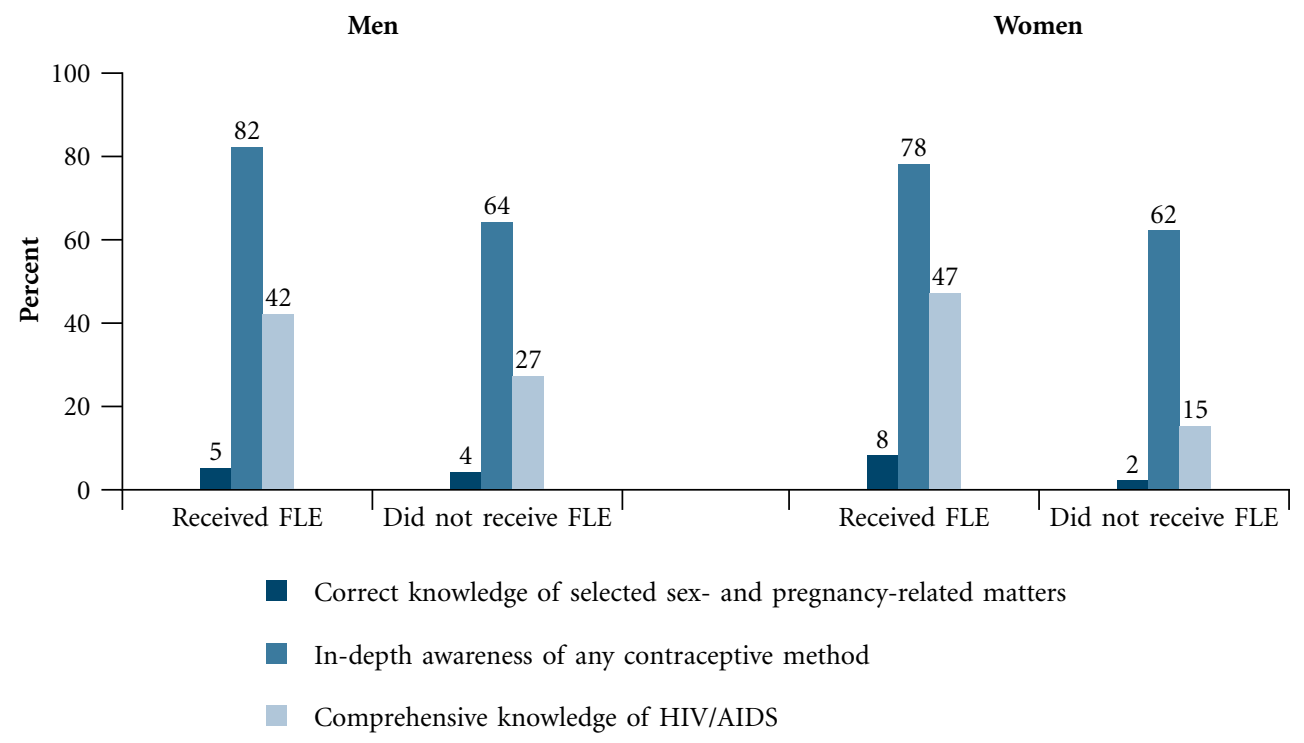

Note: FLE: Family life or sex education.

\subsection{Summary}

Findings presented in this chapter underscore young people's extremely limited awareness of most sexual and reproductive matters, ranging from how pregnancy occurs to contraception, HIV and safe sex practices. For example, just $27-33 \%$ of youth were even aware that a woman can get pregnant at first sex. Just $11-12 \%$ of youth reported awareness of STIs other than HIV. Even on topics about which young people were generally aware, awareness was far from universal. For example, only $72 \%$ of young men and $58 \%$ of young women knew that 18 years was the legal minimum age at marriage for females.

Moreover, even on topics about which young people were generally aware, findings show that in-depth understanding was limited. For example, while $93-99 \%$ reported awareness of at least one contraceptive method, in-depth awareness of condoms and oral contraceptives, the methods most familiar to youth, was reported by just $62 \%$ and $26 \%$ of young men and $30 \%$ and $48 \%$ of young women, respectively. Likewise, while $87 \%$ of young men and $47 \%$ of young women had heard of HIV, only $28 \%$ of young men and $15 \%$ of young women were fully aware of HIV and its transmission routes.

Not surprisingly, youth reported few reliable sources of information about sexual matters or contraception. Indeed, $16 \%$ of young men and $44 \%$ of young women reported that they had never received any information on sexual matters (prior to marriage among the married). Friends and the media were leading sources of information on both issues for both young men and women. Neither of these is necessarily a reliable source of information. For young women, in addition, family members were a leading source of information; they were rarely cited as a source of information by young men. Fewer than $6 \%$ of unmarried and hardly any married youth cited teachers as sources of information on sexual matters or contraception. Health care providers were important sources of information on contraception just for married young men; they were far 
less likely to have provided information to the unmarried and even to married young women. In short, health care providers, teachers and family members-often assumed to be a more reliable source of information than peers or the media-were infrequently and inconsistently cited as sources of information on sensitive topics such as sexual matters and contraception by young people.

Few youth had attended family life or sex education programmes either in or outside the school setting-just $7 \%$ of young men and 3\% young women. Despite this, youth were overwhelmingly in favour of the provision of family life or sex education; while young men preferred to receive this education from a professional (health care provider, teacher and so on), young women preferred parents or siblings. Findings suggest, moreover, that youth who had undergone family life or sex education were indeed more knowledgeable about sexual and reproductive matters than those not exposed to this education. 


\section{Pre-marital romantic and} sexual relationships

While evidence is sparse, several studies have noted that despite socio-cultural taboos, youth in India do find opportunities to mix and form romantic relationships, and to engage in pre-marital sex with a range of partners and in a variety of situations (Abraham, 2001; 2002; Abraham and Kumar, 1999; Alexander et al., 2006a; 2006b; Awasthi, Nichter and Pande, 2000). This chapter begins by describing the development of questions intended to capture these youth relationships. The chapter then explores young people's attitudes towards pre-marital physical intimacy and sex, and the extent and nature of their pre-marital romantic experiences, followed by a description of their pre-marital sexual experiences, including those within romantic partnerships and other situations. Finally, the chapter compares reports of pre-marital romantic and sexual experiences derived using three different methodological approaches, that is, face-to-face interviews, anonymous reporting of respondents' own experiences using a sealed envelope and anonymous third-party reporting of the experiences of respondents' friends.

\subsection{Development of the questionnaire module on pre-marital romantic and sexual relationships}

In view of the fact that social norms prohibiting pre-marital opposite-sex mixing may result in serious under-reporting of romantic and sexual relationships by youth, the Youth Study initiated the development of this module with a series of focus group discussions among married and unmarried young men and women. In the course of these focus group discussions, youth confirmed that romantic relationships were indeed formed, and mapped a range of places in which youth met their romantic partners secretly. They also listed the vocabulary used by youth to describe their romantic relationships, including the commonly used term "to give a proposal" to describe the act of conveying romantic intentions to opposite-sex individuals.

Building on these insights, a romantic relationship was defined as one comprising a boyfriend-girlfriend relationship (worded culturally appropriately) in which an emotional, physical or sexual relationship was experienced; one in which a "proposal" was accepted, or one in which the couple spent time together alone and secretly. Correspondingly, all respondents were asked questions on whether or not they had ever had a boy- or girl-friend; whether they had "proposed" to anyone of the opposite sex or someone of the opposite sex had "proposed" to them and the "proposal" was accepted, and whether they had spent time alone and secretly with an opposite-sex person. Youth who reported any of the above experiences were considered to have experienced a romantic relationship. We note that our definition of romantic relationships precluded the possibility of reporting same-sex romantic relationships.

All respondents who had reported a romantic partner were then probed regarding the nature of the relationship and the extent of physical contact experienced in the relationship. Questions probing respondents' experience with physical intimacy were posed on a continuum, starting with hand-holding and extending to sexual relations. Thus, the instrument sought to ask potentially sensitive or embarrassing questions in a gradual way, 
thereby also enabling the interviewer to build rapport with the respondent. Detailed questions concerning the nature of the relationship were asked with reference to the first romantic partner as well as the most recent, if more than one was reported.

Pre-survey focus group discussions also probed the nature of situations in which sex was experienced. Participants discussed an array of partners with whom youth engaged in sexual relations, including romantic and casual, heterosexual and homosexual, sex workers and older married women. Situations of forced and exchange sex were also discussed. Our survey, correspondingly, inquired about each of these different types of relationships after we had obtained detailed information on the nature of relationships with romantic partners.

Additionally, recognising the reluctance of youth to disclose sexual experience in a survey situation, at the conclusion of the interview, all respondents were asked a single question ("Have you ever had sex with anyone [for the unmarried]/Did you ever have sex with anyone before marriage [for the married]?") and asked to mark a blank card with a " $\checkmark$ " or a "X," place the card in an envelope, seal it and return it to the interviewer. Respondents were informed that the envelope would not be opened in the field, and that only the principal investigators would be able to link the information provided in the envelope with what was provided in the main body of the questionnaire.

We also recognised that despite significant rapport building and a well-developed sequence of questions eliciting sexual behaviours, young people may not have wished to disclose sexual activity in either of the above formats. Other researchers have observed that respondents may be more forthcoming about reporting sensitive behaviours among their peer networks than about themselves and that responses relating to the peer network correspond closely to their own experiences (Rossier, 2003). Hence the Youth Study incorporated anonymous third-party reporting questions, in which respondents reported the romantic and sexual experiences of up to five same-sex peers.

In addition, efforts were made to ensure that youth were comfortable revealing sensitive behaviours. Interviewers were young and trained to build rapport, discuss sensitive experiences in empathetic and matter-of-fact ways and generally make respondents feel comfortable about the topics to be discussed during the interview. As far as possible, interviews were held at times and places that assured the respondent maximum confidentiality. In cases in which family members attempted to participate in or overhear the interview, another interviewer was called upon to conduct an informal discussion or interview with other family members so as to ensure privacy for the interview. Nevertheless, we acknowledge that ensuring privacy may have been a problem, especially in low-income urban settings characterised by cramped housing conditions or that some youth may not have felt entirely at ease despite the extensive efforts made to ensure confidentiality. While findings are indeed in line with those observed in other small-scale and less representative studies (see Jejeebhoy and Sebastian, 2004 for a review), we acknowledge that romantic and sexual experiences may have been under-reported in the survey, notably by young women, and suggest that percentages presented here may be interpreted as conservative estimates.

\subsection{Attitudes toward pre-marital physical intimacy and sexual relations}

The Youth Study included a number of questions to assess young people's attitudes regarding the acceptability of pre-marital physical intimacy and sexual activity. Findings, presented in Table 9.1, suggest that young people's attitudes towards pre-marital physical intimacy and sex were generally negative. Indeed, few young men and women considered even pre-marital kissing to be acceptable. Young men were, by and large, more likely than young women to report accepting attitudes towards pre-marital kissing and sex. For example, 15\% 
Table 9.1: Attitudes toward pre-marital physical intimacy and sexual relations

Percent distribution of youth by attitudes towards pre-marital physical intimacy and sexual relations, according to residence, Bihar, 2007

\begin{tabular}{|c|c|c|c|c|c|c|}
\hline Attitudes (\%) & $\begin{array}{c}\mathrm{M} \\
15-24\end{array}$ & $\begin{array}{c}\text { W } \\
15-24\end{array}$ & $\begin{array}{c}\mathrm{MM} \\
15-29\end{array}$ & $\begin{array}{c}\text { MW } \\
15-24\end{array}$ & $\begin{array}{c}\text { UM } \\
15-24\end{array}$ & $\begin{array}{c}\text { UW } \\
\text { 15-24 }\end{array}$ \\
\hline \multicolumn{7}{|c|}{ Combined } \\
\hline \multicolumn{7}{|c|}{ Kissing before marriage is all right } \\
\hline Agree & 15.2 & 4.7 & 14.0 & 3.9 & 15.0 & 6.0 \\
\hline Disagree & 75.4 & 93.9 & 79.8 & 95.0 & 74.6 & 91.9 \\
\hline \multicolumn{7}{|c|}{$\begin{array}{l}\text { A boy's future would be ruined if he has sex } \\
\text { before marriage }\end{array}$} \\
\hline Agree & 68.1 & 52.4 & 74.2 & 52.1 & 68.1 & 53.1 \\
\hline Disagree & 24.7 & 44.4 & 22.2 & 45.2 & 23.9 & 42.5 \\
\hline \multicolumn{7}{|c|}{$\begin{array}{l}\text { A girl's future would be ruined if she has sex } \\
\text { before marriage }\end{array}$} \\
\hline Agree & 83.8 & 96.1 & 88.4 & 96.9 & 83.4 & 94.6 \\
\hline Disagree & 10.6 & 3.2 & 8.9 & 2.8 & 10.4 & 3.8 \\
\hline Number of respondents & 1,942 & 5,529 & 1,115 & 2,341 & 1,492 & 3,188 \\
\hline \multicolumn{7}{|c|}{ Urban } \\
\hline \multicolumn{7}{|c|}{ Kissing before marriage is all right } \\
\hline Agree & 21.9 & 8.2 & 15.1 & 5.6 & 23.0 & 10.1 \\
\hline Disagree & 70.3 & 90.7 & 79.6 & 93.7 & 68.9 & 88.5 \\
\hline \multicolumn{7}{|c|}{$\begin{array}{l}\text { A boy's future would be ruined if he has sex } \\
\text { before marriage }\end{array}$} \\
\hline Agree & 65.9 & 47.3 & 70.7 & 44.4 & 65.6 & 49.2 \\
\hline Disagree & 28.3 & 50.4 & 26.1 & 53.5 & 28.3 & 48.2 \\
\hline \multicolumn{7}{|c|}{$\begin{array}{l}\text { A girl's future would be ruined if she has sex } \\
\text { before marriage }\end{array}$} \\
\hline Agree & 81.4 & 95.8 & 84.9 & 97.2 & 80.8 & 94.9 \\
\hline Disagree & 14.3 & 3.6 & 11.8 & 2.8 & 14.7 & 4.2 \\
\hline Number of respondents & 1,039 & 2,581 & 547 & 1,136 & 833 & 1,445 \\
\hline \multicolumn{7}{|c|}{ Rural } \\
\hline \multicolumn{7}{|c|}{ Kissing before marriage is all right } \\
\hline Agree & 14.1 & 4.3 & 13.9 & 3.8 & 13.4 & 5.2 \\
\hline Disagree & 76.2 & 94.3 & 79.8 & 95.1 & 75.9 & 92.5 \\
\hline \multicolumn{7}{|c|}{$\begin{array}{l}\text { A boy's future would be ruined if he has sex } \\
\text { before marriage }\end{array}$} \\
\hline Agree & 68.5 & 53.0 & 74.6 & 52.6 & 68.6 & 53.9 \\
\hline Disagree & 24.2 & 43.7 & 21.8 & 44.7 & 23.1 & 41.5 \\
\hline \multicolumn{7}{|c|}{$\begin{array}{l}\text { A girl's future would be ruined if she has sex } \\
\text { before marriage }\end{array}$} \\
\hline Agree & 84.2 & 96.1 & 88.7 & 96.9 & 83.9 & 94.5 \\
\hline Disagree & 10.0 & 3.1 & 8.7 & 2.8 & 9.5 & 3.7 \\
\hline Number of respondents & 903 & 2,948 & 568 & 1,205 & 659 & 1,743 \\
\hline
\end{tabular}

Note: All Ns are unweighted. Column totals may not equal 100\% due to missing cases or "can't say" responses. 
of young men compared to $5 \%$ of young women felt that it is all right for a boy and girl to kiss each other before marriage. Findings also indicate gendered attitudes towards pre-marital physical intimacy and sex. While one-quarter of young men and over two-fifths of young women condoned pre-marital sexual activity among young men, far fewer- $11 \%$ of young men and $3 \%$ of young women—considered such behaviour acceptable among young women.

Differences by marital status of the respondent were negligible. Rural-urban differences were, however, notable on several issues. Youth in urban areas, particularly the unmarried, reported more liberal attitudes to pre-marital physical intimacy than those in rural areas. For example, $22 \%$ of urban young men compared to $14 \%$ of rural young men and $8 \%$ of urban young women compared to $4 \%$ of rural young women expressed the attitude that it is all right for a boy and girl to kiss each other before marriage. Moreover, somewhat larger percentages of urban than rural youth condoned pre-marital sexual activity among young men (28\% versus $24 \%$ among young men and $50 \%$ versus $44 \%$ among young women). However, rural-urban differences were negligible with regard to the acceptability of pre-marital sexual activity among young women.

\subsection{Pre-marital romantic relationships}

In this section we present the prevalence of pre-marital opposite-sex romantic relationships among youth and a profile of those who engaged in such relationships. The section also describes parent and peer awareness of pre-marital romantic relationships, youth intentions regarding marriage with their romantic partners and the extent of physical contact experienced in these relationships.

\subsubsection{Prevalence of pre-marital romantic relationships}

Despite the fact that youth tended to report relatively traditional attitudes, opportunities to form romantic relationships did exist for them, irrespective of sex or rural-urban residence of respondents. As shown in Table 9.2, many youth had either made a romantic "proposal" to an opposite-sex individual or had received such a "proposal". In total, $20 \%$ of young men and $12 \%$ of young women reported that they had ever made or received such a "proposal". Very few young women reported "proposing" to a man $(0.3 \%)$; among young men, however, almost as many reported making a "proposal" as receiving one (10\% and $12 \%$, respectively, made and received a proposal) (not shown in tabular form), perhaps revealing a tendency either on the part of young men to exaggerate the extent of their interaction with women or on the part of young women to conceal behaviour that may be considered socially unacceptable.

Patterns of experience in initiating pre-marital romantic relationships by marital status indicate that while no differences were apparent among young men, somewhat fewer married than unmarried young women reported making or receiving a "proposal" (9\% versus 15\%) (see also Figure 9.1), a difference attributable perhaps to the limited number of years they spent prior to marriage as an adolescent. Rural-urban differences suggest that larger percentages of urban youth received or made a "proposal" compared to their rural counterparts ( $25 \%$ versus $19 \%$ among young men and $21 \%$ versus $10 \%$ among young women).

"Proposals" were conveyed through an intermediary-a friend, relative or sibling-in many cases; 3-6\% of all youth reported that the "proposal" was conveyed through a mediator. This corresponds to over one-quarter of young men and women who had ever made or received a "proposal". Differences by marital status and rural-urban residence were negligible. Even so, married young women were somewhat more likely than the unmarried to report that the "proposals" they had made or received had been conveyed through an intermediary; $32 \%$ and $26 \%$ of married and unmarried young women who had ever made or received a "proposal", respectively, reported having used an intermediary. 
Figure 9.1: Percentage of youth who had made or received a "proposal" for romantic partnership formation and percentage who had an opposite-sex romantic partner, according to residence, Bihar, 2007

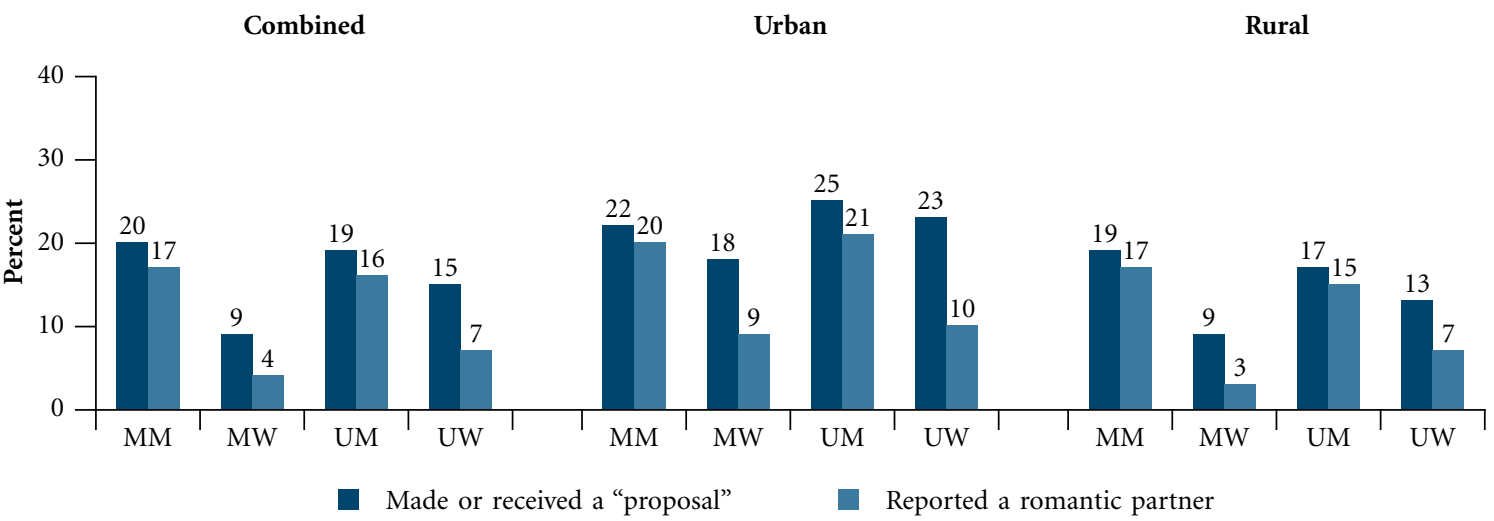

Compared to those who had made or received "proposals," fewer youth, particularly young women, reported the acceptance of such a "proposal"; $16 \%$ of young men and 5\% of young women reported that they had accepted a "proposal" or that their own "proposal" had been accepted. A roughly equal percentage reported that they had met an opposite-sex individual secretly. In total, in response to the direct or indirect questions, $17 \%$ of young men and 5\% of young women acknowledged the experience of a romantic partnership. Differences by marital status and rural-urban residence were mild. Even so, urban youth, particularly married young women and unmarried young men, were somewhat more likely than their rural counterparts to report that they had ever had a romantic partner. Few youth-just $4 \%$ of young men and hardly any young women-reported that they had more than one romantic partner.

Table 9.3 presents the percentage of youth reporting pre-marital romantic experience by background characteristics. Age profiles indicate a positive association with the formation of romantic relationship among young men; while a strong positive association was observed among unmarried young men, the association was inconsistent among married young men. In contrast among young women, age differences were narrow, irrespective of marital status.

Differentials by religion were narrow among both young men and women, irrespective of marital status. Even though caste-wise differences were narrow, those belonging to general castes were more likely than others to report a pre-marital romantic relationship. Similar patterns were observed among both the married and the unmarried.

Findings also show a positive association between schooling and the formation of romantic relationship, perhaps a result of greater opportunities for mobility and social mixing offered by schooling. For example, the percentage of young men who reported a romantic partner increased from $8 \%$ among those without formal schooling to $30 \%$ among those who had completed 12 or more years of schooling. The corresponding percentages among young women were $3 \%$ and $12 \%$, respectively. Patterns were similar for the married and the unmarried. Differences by work participation were narrow among both young men and women, irrespective of marital status.

Associations between household economic status and the formation of romantic partnership were inconsistent; even so, those belonging to households in the wealthiest quintile were more likely than others to report a romantic partnership. 
Table 9.2: Pre-marital romantic relationships

Percentage of youth reporting a pre-marital romantic relationship by relationship characteristics, according to residence, Bihar, 2007

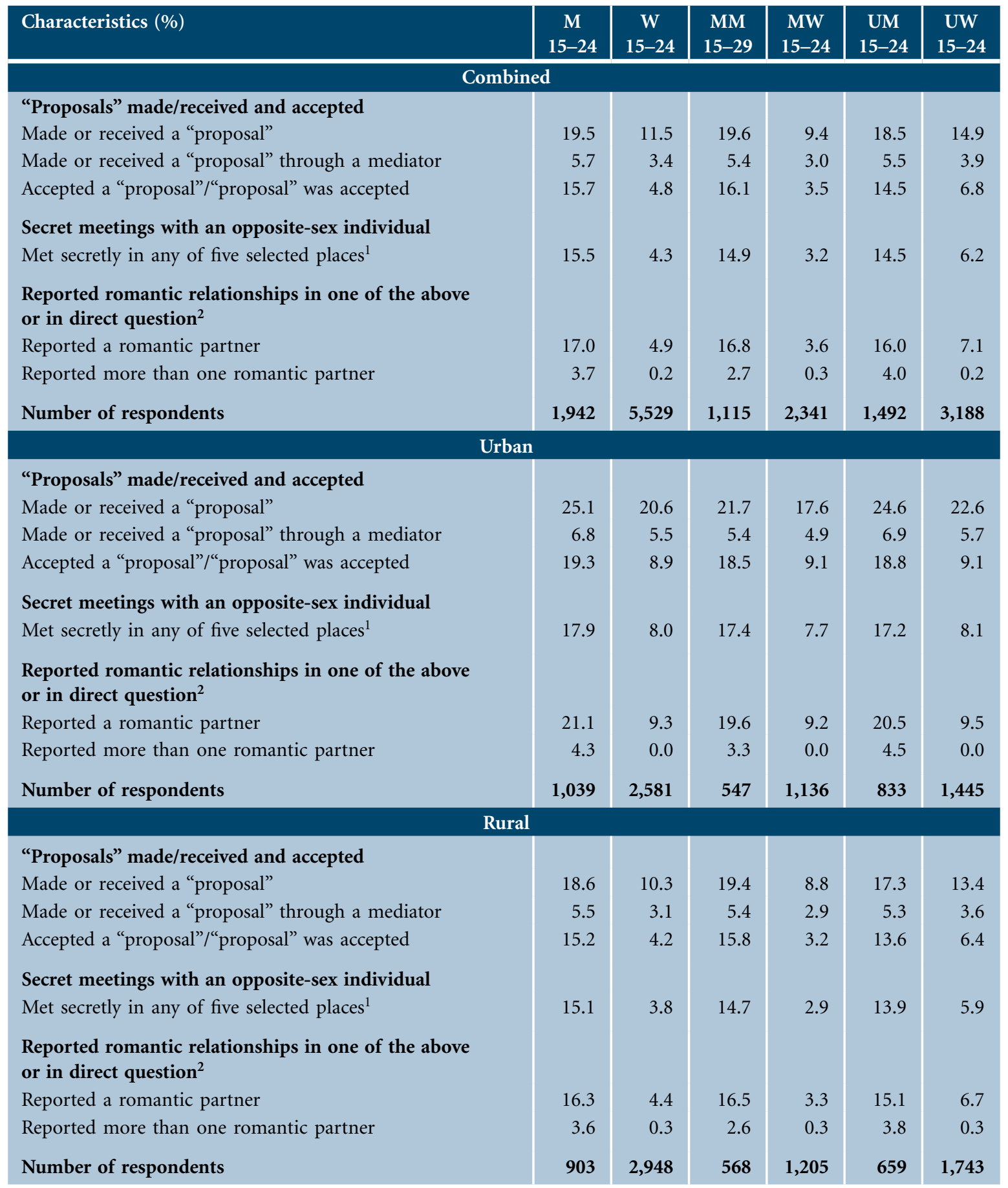

Note: All Ns are unweighted. ${ }^{1}$ Behind or around a temple/mosque/church; around a school/college; at own or someone else's home in the absence of parents; in fields/grazing areas (rural) and restaurants (urban); or in a garden/park/maidan/market or haat. ${ }^{2}$ Respondents were asked a direct question on whether or not they had ever had a boyfriend/girlfriend. 
Table 9.3: Prevalence of pre-marital romantic relationships by selected background characteristics

Percentage of youth reporting a pre-marital romantic relationship by selected background characteristics, according to residence, Bihar, 2007

\begin{tabular}{|c|c|c|c|c|c|c|}
\hline Background characteristics (\%) & $\begin{array}{c}\mathrm{M} \\
15-24\end{array}$ & $\begin{array}{c}\text { W } \\
15-24\end{array}$ & $\begin{array}{c}\text { MM } \\
15-29\end{array}$ & $\begin{array}{c}\text { MW } \\
15-24\end{array}$ & $\begin{array}{c}\text { UM } \\
15-24\end{array}$ & $\begin{array}{c}\text { UW } \\
\text { 15-24 }\end{array}$ \\
\hline \multicolumn{7}{|c|}{ Combined } \\
\hline \multicolumn{7}{|l|}{ Age (years) } \\
\hline $15-19$ & 13.3 & 5.6 & 16.3 & 4.1 & 13.0 & 6.9 \\
\hline $20-24$ & 23.1 & 3.9 & 20.5 & 3.3 & 26.6 & 9.5 \\
\hline $25-29$ & NA & NA & 14.5 & NA & NA & NA \\
\hline \multicolumn{7}{|l|}{ Religion } \\
\hline Hindu & 17.5 & 4.8 & 17.0 & 3.3 & 16.2 & 7.5 \\
\hline Muslim & 13.9 & 5.8 & 14.7 & 6.0 & 14.4 & 5.4 \\
\hline \multicolumn{7}{|l|}{ Caste } \\
\hline SC & 17.5 & 4.5 & 16.4 & 3.4 & 14.9 & 7.6 \\
\hline $\mathrm{OBC}$ & 15.3 & 4.0 & 15.1 & 2.7 & 14.5 & 6.4 \\
\hline General $^{1}$ & 20.8 & 9.6 & 23.8 & 10.8 & 19.2 & 8.5 \\
\hline \multicolumn{7}{|l|}{ Educational level (years) } \\
\hline None $^{2}$ & 7.6 & 2.9 & 9.2 & 2.5 & 8.6 & 4.7 \\
\hline $1-7$ & 14.6 & 4.9 & 20.2 & 3.1 & 11.0 & 6.8 \\
\hline $8-11$ & 18.4 & 8.4 & 17.0 & 8.0 & 18.0 & 8.7 \\
\hline 12 or more & 30.4 & 12.4 & 26.7 & 15.0 & 26.7 & 11.0 \\
\hline \multicolumn{7}{|l|}{ Worked in last 12 months } \\
\hline Yes & 18.1 & 4.7 & 16.6 & 3.6 & 17.2 & 6.7 \\
\hline No & 14.3 & 5.1 & $(25.0)$ & 3.7 & 13.8 & 7.4 \\
\hline \multicolumn{7}{|l|}{ Wealth quintile } \\
\hline First & 11.8 & 3.4 & 8.3 & 2.5 & 15.3 & 6.9 \\
\hline Second & 15.3 & 4.2 & 13.5 & 3.5 & 13.7 & 6.3 \\
\hline Third & 13.6 & 3.7 & 18.3 & 3.2 & 9.0 & 4.7 \\
\hline Fourth & 17.2 & 4.4 & 16.1 & 3.1 & 17.6 & 6.4 \\
\hline Fifth & 20.7 & 8.1 & 22.5 & 6.5 & 19.1 & 9.4 \\
\hline Total & 17.0 & 4.9 & 16.8 & 3.6 & 16.0 & 7.1 \\
\hline \multicolumn{7}{|c|}{ Urban } \\
\hline \multicolumn{7}{|l|}{ Age (years) } \\
\hline $15-19$ & 17.2 & 9.1 & * & 10.3 & 17.4 & 8.8 \\
\hline $20-24$ & 26.5 & 9.5 & 25.0 & 8.7 & 27.4 & 12.1 \\
\hline $25-29$ & NA & NA & 17.2 & NA & NA & NA \\
\hline \multicolumn{7}{|l|}{ Religion } \\
\hline Hindu & 20.9 & 9.3 & 20.3 & 9.2 & 20.5 & 9.6 \\
\hline Muslim & 21.3 & 9.2 & 15.4 & 8.3 & 20.9 & 9.1 \\
\hline \multicolumn{7}{|l|}{ Caste } \\
\hline SC & 17.9 & 11.8 & 21.4 & 13.0 & 17.4 & 12.5 \\
\hline $\mathrm{OBC}$ & 19.9 & 8.2 & 15.3 & 8.6 & 19.7 & 8.4 \\
\hline General $^{1}$ & 23.4 & 10.5 & 27.8 & 11.1 & 22.5 & 10.7 \\
\hline
\end{tabular}


Table 9.3: (Cont'd)

\begin{tabular}{|c|c|c|c|c|c|c|}
\hline Background characteristics (\%) & $\begin{array}{c}\mathrm{M} \\
15-24\end{array}$ & $\begin{array}{c}\text { W } \\
15-24\end{array}$ & $\begin{array}{c}\text { MM } \\
15-29\end{array}$ & $\begin{array}{c}\text { MW } \\
\text { 15-24 }\end{array}$ & $\begin{array}{c}\text { UM } \\
15-24\end{array}$ & $\begin{array}{l}\text { UW } \\
15-24\end{array}$ \\
\hline \multicolumn{7}{|c|}{ Urban } \\
\hline Educational level (years) & & & & & & \\
\hline None $^{2}$ & 13.8 & 8.5 & 10.5 & 7.8 & 14.3 & 8.3 \\
\hline $1-7$ & 20.0 & 7.2 & 16.7 & 5.9 & 19.0 & 7.1 \\
\hline $8-11$ & 19.8 & 10.3 & 20.6 & 12.5 & 18.8 & 9.5 \\
\hline 12 or more & 27.1 & 11.1 & 28.6 & 11.1 & 26.6 & 12.0 \\
\hline \multicolumn{7}{|l|}{ Worked in last 12 months } \\
\hline Yes & 23.3 & 11.5 & 18.4 & 5.9 & 23.5 & 11.9 \\
\hline No & 17.4 & 8.9 & $(40.0)$ & 8.9 & 17.7 & 8.8 \\
\hline \multicolumn{7}{|l|}{ Wealth quintile } \\
\hline First & * & 14.3 & * & $(20.0)$ & * & * \\
\hline Second & $(33.3)$ & 8.6 & $(14.3)$ & 9.1 & $(33.3)$ & 10.0 \\
\hline Third & 12.5 & 5.9 & $(12.5)$ & 6.3 & $(14.3)$ & 6.7 \\
\hline Fourth & 14.6 & 8.9 & 11.8 & 8.3 & 15.4 & 8.3 \\
\hline Fifth & 22.4 & 9.6 & 22.8 & 9.2 & 21.9 & 9.8 \\
\hline Total & 21.1 & 9.3 & 19.6 & 9.2 & 20.5 & 9.5 \\
\hline \multicolumn{7}{|c|}{ Rural } \\
\hline \multicolumn{7}{|l|}{ Age (years) } \\
\hline $15-19$ & 12.7 & 5.2 & $(16.9)$ & 3.8 & 12.3 & 6.6 \\
\hline $20-24$ & 22.4 & 3.1 & 20.1 & 2.8 & 26.3 & 7.8 \\
\hline 25-29 & NA & NA & 14.3 & NA & NA & NA \\
\hline \multicolumn{7}{|l|}{ Religion } \\
\hline Hindu & 16.9 & 4.2 & 16.8 & 3.0 & 15.4 & 7.1 \\
\hline Muslim & 12.4 & 5.2 & 14.8 & 5.3 & 12.7 & 4.8 \\
\hline \multicolumn{7}{|l|}{ Caste } \\
\hline SC & 17.2 & 4.0 & 16.1 & 3.0 & 14.6 & 7.0 \\
\hline $\mathrm{OBC}$ & 14.6 & 3.5 & 15.0 & 2.4 & 13.5 & 6.1 \\
\hline General $^{1}$ & 20.1 & 9.3 & 23.1 & 11.2 & 18.4 & 7.9 \\
\hline \multicolumn{7}{|l|}{ Educational level (years) } \\
\hline None $^{2}$ & 7.0 & 2.7 & 9.1 & 2.3 & 7.8 & 4.4 \\
\hline $1-7$ & 14.3 & 4.7 & 20.5 & 2.9 & 9.9 & 6.8 \\
\hline $8-11$ & 18.1 & 7.9 & 16.6 & 7.3 & 17.9 & 8.5 \\
\hline 12 or more & 31.8 & 13.6 & 25.7 & 16.3 & 26.8 & 9.7 \\
\hline \multicolumn{7}{|l|}{ Worked in last 12 months } \\
\hline Yes & 17.4 & 4.4 & 16.4 & 3.4 & 16.3 & 6.3 \\
\hline No & 13.2 & 4.4 & * & 3.2 & 12.7 & 7.0 \\
\hline \multicolumn{7}{|l|}{ Wealth quintile } \\
\hline First & 11.4 & 3.3 & 8.4 & 2.3 & 15.5 & 6.8 \\
\hline Second & 14.3 & 4.0 & 13.0 & 3.3 & 12.5 & 6.1 \\
\hline Third & 13.7 & 3.6 & 18.5 & 3.1 & 8.7 & 4.7 \\
\hline Fourth & 17.7 & 4.1 & 16.5 & 2.8 & 18.1 & 6.2 \\
\hline Fifth & 19.9 & 7.3 & 22.7 & 5.7 & 17.8 & 9.1 \\
\hline Total & 16.3 & 4.4 & 16.5 & 3.3 & 15.1 & 6.7 \\
\hline
\end{tabular}

Note: ( ) Based on 25-49 unweighted cases. ${ }^{*}$ Percentage not shown, based on fewer than 25 unweighted cases. NA: Not applicable. OBC: Other backward caste. SC: Scheduled caste. ST: Scheduled tribe. ${ }^{1}$ Includes all those not belonging to SC, ST or OBC. ${ }^{2}$ Includes non-literate and literate with no formal schooling. 
Similar patterns of socio-demographic differentials were observed, by and large, among rural and urban respondents.

\subsubsection{Characteristics of pre-marital romantic relationships}

Selected characteristics of reported pre-marital romantic relationships are presented in Table 9.4; in cases in which more than one romantic partner was reported, only information relating to the respondent's first romantic relationship was included. Age at initiation of pre-marital romantic relationships was measured by the age at which they first spent time alone with their partner.

Findings indicate that relationships were initiated at a young age for considerably large proportions of those who had experienced pre-marital romantic relationships. Almost half of young men (47\%) and almost three-fifths of young women $(58 \%)$ reported that they had spent time alone with their first romantic partner at age 15 or below. Unmarried young men were somewhat more likely than married young men to have initiated a romantic relationship at age 15 or below ( $47 \%$ versus $41 \%$ ); in contrast, among young women, it was the married who were more likely to have initiated their relationship at age 15 or below (64\% versus $53 \%$ ). Youth in rural areas were more likely than those in urban areas to have initiated a pre-marital romantic relationship at age 15 or below (49\% compared to $39 \%$ among young men and $61 \%$ compared to $44 \%$ among young women). Median ages of respondents when they first spent time alone with their pre-marital romantic partner were approximately one year older among young men than among young women (16 years and 15 years, respectively), and one year older among unmarried compared to married young women (15 and 14 , respectively), but identical among married and unmarried young men (16 years). Rural-urban differences suggest that median age at initiation of romantic relationships was identical among young men in both rural and urban areas (16 years) and one-and-a-half years later among urban young women compared to their rural counterparts ( 15.5 years versus 14 years). Information on the relative ages of reported partners suggests that male partners were, for the most part, older than female partners. For example, $71 \%$ of young men reported a female partner who was younger than they were, while $78 \%$ of young women reported a male partner who was older than they were. It is also notable that $10 \%$ of young men reported a female partner who was older than they were. Overwhelmingly, the partner was unmarried.

The first reported romantic partner was typically a neighbour or friend from the village or urban community (37\% among young men and $34 \%$ among young women). One-third of young men compared to almost one-fifth of young women reported an acquaintance from outside the village/urban community, and almost one-fifth of young men and one in eight young women reported a fellow student or colleague as the initial partner. Conversely, almost one-third of young women compared to $9 \%$ of young men reported that their first pre-marital partner was a relative. These gender differences may be attributed to the more limited mobility and access to social mixing opportunities that young women experienced in relation to young men, described in Chapter 7.

These patterns were fairly consistent among both married and unmarried youth. Nonetheless, married young men were less likely than unmarried young men to report a fellow student or colleague (11\% versus $21 \%$ ) and more likely to report a neighbour or friend from the village or urban community ( $45 \%$ versus $37 \%$ ) as the first romantic partner. Among young women, the married were more likely than the unmarried to report a relative (37\% versus $25 \%$ ) and less likely to report an acquaintance from outside the village/urban community ( $12 \%$ versus $25 \%)$. 
Rural-urban differences were apparent. Rural young men were more likely to report a neighbour or friend from the village or urban community or an acquaintance from outside of the home community than were urban young men. Conversely, urban young men were more likely than their rural counterparts to report a fellow student or colleague (36\% versus 14\%). Moreover, married young men in urban settings were somewhat more likely than their rural counterparts to report a relative (17\% versus $11 \%)$. Rural-urban differences were modest for the overall sample of young women. However, among the married, those in rural areas were more likely to report a relative (39\% versus 23\%) and less likely to report a neighbour or friend from the village/ urban community (33\% versus $39 \%$ ) than were those in urban areas. Among the unmarried, rural young women were more likely than urban young women to report an acquaintance from outside the village/urban community ( $27 \%$ versus $15 \%)$.

Respondents had typically been acquainted with their first romantic partner for one year or more before becoming romantically linked; this was consistently observed in all groups, irrespective of sex, marital status and rural-urban residence. Many-18\% of young men and 11\% of young women-reported that they had been acquainted with the partner since childhood, a finding not surprising given that a sizeable proportion of partners were either from the same neighbourhood or, among young women, relatives. Differences by sex, marital status and place of residence were modest. Even so, somewhat more married than unmarried young men reported shorter duration acquaintance (less than 12 months of acquaintance, 27\% compared to 21\%). Likewise, more rural young women, particularly the unmarried, reported shorter duration acquaintance than were urban young women.

The majority of youth reported that their partners came from religious backgrounds similar to their own, irrespective of sex, marital status or place of residence; $92-95 \%$ reported as such. Even so, somewhat more unmarried youth in urban areas reported a partner from a different religion, compared to those in rural areas $(8-13 \%$ versus $3-4 \%)$. With respect to partner's caste, findings suggest that even though the majority of youth reported a partner from similar caste backgrounds, as many as $37 \%$ of young men and $27 \%$ of young women did engage in a romantic relationship with someone of a different caste. While married young men were more likely than the unmarried to so report ( $46 \%$ versus $33 \%$ ), differences were modest among young women $(25-27 \%)$. Urban youth were more likely than rural youth to report as such, irrespective of marital status (54\% versus 33\% among young men and 39\% versus $23 \%$ among young women). With respect to household economic status, almost half of young men (47\%) and one-third of young women reported that the partner was from a family with a similar socio-economic status to their own. Of note, however, is that $35 \%$ of young men and $57 \%$ of young women reported a romantic partner from a better-off family. Unmarried youth were more likely than their married counterparts to report a partner from a different economic background (53\% versus $48 \%$ among young men and $68 \%$ versus $62 \%$ among young women). Likewise, more rural than urban youth reported a partner from a different economic background (52\% versus $48 \%$ among young men and $67 \%$ versus $56 \%$ among young women).

Table 9.5 presents youth responses to questions regarding places in which youth met their first romantic partner secretly, without adults present. The vast majority of youth who reported romantic relationships met secretly in each other's homes when other family members were absent ( $46 \%$ of young men and $48 \%$ of young women). Sizeable proportions of youth reported having met their romantic partner secretly in other places that offered them privacy or anonymity, including parks and gardens (13-29\%), fields or grazing areas (15-24\%), cinema/theatre halls (6-12\%) and behind temples/mosques/churches (10-13\%). One-tenth of young men and one in eight young women reported not meeting their romantic partner anywhere in secret. Differences by marital status and place of residence were modest, except that more urban than rural young men reported not meeting their romantic partner anywhere in secret (17\% versus $8 \%)$. 
Table 9.4: Characteristics of pre-marital romantic relationships and partners

Percentage of youth reporting a pre-marital romantic relationship by age at initiation of relationship, partner's socio-economic and demographic characteristics, and nature and duration of prior acquaintance, according to residence, Bihar, 2007

\begin{tabular}{|c|c|c|c|c|c|c|}
\hline Characteristics $(\%)^{1}$ & $\underset{15-24}{M}$ & $\begin{array}{c}\text { W } \\
15-24\end{array}$ & $\begin{array}{c}\text { MM } \\
15-29\end{array}$ & $\begin{array}{c}\text { MW } \\
15-24\end{array}$ & $\begin{array}{c}\text { UM } \\
15-24\end{array}$ & $\begin{array}{c}\text { UW } \\
15-24\end{array}$ \\
\hline \multicolumn{7}{|c|}{ Combined } \\
\hline \multicolumn{7}{|c|}{$\begin{array}{l}\text { Age when respondent first spent time alone with } \\
\text { partner (years) }\end{array}$} \\
\hline 15 or below & 47.0 & 57.9 & 41.2 & 63.5 & 47.1 & 53.3 \\
\hline $\begin{array}{l}\text { Median age when respondent first spent time } \\
\text { with partner }\end{array}$ & 16.0 & 15.0 & 16.0 & 14.0 & 16.0 & 15.0 \\
\hline \multicolumn{7}{|l|}{ Age of partner } \\
\hline Younger than respondent & 70.6 & 0.7 & 72.2 & 0.0 & 67.6 & 0.4 \\
\hline Same age as respondent & 16.8 & 7.0 & 18.7 & 4.7 & 18.9 & 9.3 \\
\hline Older than respondent & 9.9 & 77.9 & 5.3 & 84.7 & 10.9 & 71.7 \\
\hline Don't remember & 2.7 & 14.3 & 3.7 & 10.6 & 2.5 & 18.6 \\
\hline \multicolumn{7}{|l|}{ Partner's marital status } \\
\hline Unmarried & 97.0 & 94.9 & 96.3 & 94.0 & 96.6 & 95.6 \\
\hline Married & 3.0 & 5.1 & 2.7 & 6.0 & 3.4 & 4.4 \\
\hline \multicolumn{7}{|l|}{ Nature of prior acquaintance with first partner } \\
\hline Relative & 9.3 & 30.8 & 11.2 & 36.9 & 7.6 & 25.1 \\
\hline Fellow student/colleague & 17.7 & 13.2 & 11.2 & 11.9 & 20.6 & 14.1 \\
\hline Neighbour/friend & 37.2 & 33.7 & 44.9 & 34.5 & 37.0 & 33.0 \\
\hline Family friend & 0.0 & 0.4 & 0.0 & 0.0 & 0.0 & 0.4 \\
\hline Person from outside village/neighbourhood & 33.3 & 18.3 & 29.4 & 11.9 & 32.4 & 25.1 \\
\hline Other $^{2}$ & 2.4 & 3.7 & 3.2 & 4.8 & 2.5 & 2.2 \\
\hline \multicolumn{7}{|l|}{ Duration of acquaintance } \\
\hline Less than 1 month & 9.3 & 8.1 & 10.7 & 9.5 & 7.6 & 6.6 \\
\hline 1-11 months & 15.3 & 16.8 & 16.6 & 14.3 & 13.0 & 19.4 \\
\hline 12 months or more & 57.8 & 63.7 & 46.5 & 63.1 & 58.4 & 64.8 \\
\hline Since childhood & 17.7 & 11.4 & 26.2 & 13.1 & 21.0 & 9.3 \\
\hline \multicolumn{7}{|l|}{ Partner's religion } \\
\hline Same as respondent & 95.2 & 92.3 & 91.0 & 91.8 & 94.6 & 93.8 \\
\hline Different from respondent & 4.5 & 7.3 & 8.0 & 8.2 & 5.0 & 5.7 \\
\hline \multicolumn{7}{|l|}{ Partner's caste } \\
\hline Same as respondent & 62.3 & 72.2 & 52.4 & 72.9 & 66.8 & 72.1 \\
\hline Different from respondent & 36.5 & 26.7 & 45.5 & 24.7 & 32.8 & 27.4 \\
\hline \multicolumn{7}{|l|}{ Partner's socio-economic status } \\
\hline Same as respondent & 46.6 & 34.4 & 48.7 & 37.6 & 45.8 & 30.8 \\
\hline Better than respondent & 35.2 & 57.1 & 30.5 & 55.3 & 37.8 & 59.9 \\
\hline Worse than respondent & 15.8 & 8.1 & 17.1 & 7.1 & 14.7 & 8.4 \\
\hline Number reporting a romantic relationship & 368 & 395 & 203 & 143 & 270 & 252 \\
\hline
\end{tabular}


Table 9.4: (Cont'd)

\begin{tabular}{|c|c|c|c|c|c|c|}
\hline Characteristics $(\%)^{1}$ & $\begin{array}{c}\text { M } \\
15-24\end{array}$ & $\begin{array}{c}\text { W } \\
15-24\end{array}$ & $\begin{array}{c}\text { MM } \\
15-29\end{array}$ & $\begin{array}{c}\text { MW } \\
15-24\end{array}$ & $\begin{array}{l}\text { UM } \\
15-24\end{array}$ & $\begin{array}{l}\text { UW } \\
15-24\end{array}$ \\
\hline \multicolumn{7}{|c|}{ Urban } \\
\hline \multicolumn{7}{|c|}{$\begin{array}{l}\text { Age when respondent first spent time alone with } \\
\text { partner (years) }\end{array}$} \\
\hline 15 or below & 39.0 & 44.1 & 27.8 & 53.8 & 40.0 & 39.1 \\
\hline $\begin{array}{l}\text { Median age when respondent first spent time } \\
\text { with partner }\end{array}$ & 16.0 & 15.5 & 17.0 & 15.0 & 16.0 & 16.0 \\
\hline \multicolumn{7}{|l|}{ Age of partner } \\
\hline Younger than respondent & 67.8 & 3.4 & 72.2 & 0.0 & 66.0 & 2.1 \\
\hline Same age as respondent & 25.4 & 6.8 & 22.2 & 7.7 & 26.0 & 8.5 \\
\hline Older than respondent & 5.1 & 76.3 & 5.6 & 69.2 & 6.0 & 80.9 \\
\hline Don't remember & 1.7 & 13.6 & 0.0 & 23.1 & 2.0 & 8.5 \\
\hline \multicolumn{7}{|l|}{ Partner's marital status } \\
\hline Unmarried & 100.0 & 98.3 & 94.4 & 100.0 & 100.0 & 100.0 \\
\hline Married & 0.0 & 1.7 & 5.6 & 0.0 & 0.0 & 0.0 \\
\hline \multicolumn{7}{|l|}{ Nature of prior acquaintance with first partner } \\
\hline Relative & 6.8 & 26.7 & 16.7 & 23.1 & 5.9 & 27.1 \\
\hline Fellow student/colleague & 35.6 & 15.0 & 16.7 & 15.4 & 39.2 & 16.7 \\
\hline Neighbour/friend & 32.2 & 35.0 & 38.9 & 38.5 & 31.4 & 31.3 \\
\hline Family friend & 0.0 & 1.7 & 0.0 & 0.0 & 0.0 & 2.1 \\
\hline Person from outside village/neighbourhood & 23.7 & 15.0 & 27.8 & 15.4 & 21.6 & 14.6 \\
\hline Other $^{2}$ & 1.7 & 6.7 & 0.0 & 7.7 & 2.0 & 8.3 \\
\hline \multicolumn{7}{|l|}{ Duration of acquaintance } \\
\hline Less than 1 month & 6.8 & 8.3 & 11.1 & 7.7 & 6.0 & 6.4 \\
\hline $1-11$ months & 16.9 & 13.3 & 22.2 & 15.4 & 18.0 & 12.8 \\
\hline 12 months or more & 62.7 & 66.7 & 55.6 & 61.5 & 62.0 & 70.2 \\
\hline Since childhood & 13.6 & 11.7 & 11.1 & 15.4 & 14.0 & 10.6 \\
\hline \multicolumn{7}{|l|}{ Partner's religion } \\
\hline Same as respondent & 89.8 & 89.8 & 88.9 & 92.3 & 90.2 & 87.0 \\
\hline Different from respondent & 8.5 & 10.2 & 11.1 & 7.7 & 7.8 & 13.0 \\
\hline \multicolumn{7}{|l|}{ Partner's caste } \\
\hline Same as respondent & 44.1 & 61.0 & 47.1 & 69.2 & 45.1 & 53.2 \\
\hline Different from respondent & 54.2 & 39.0 & 52.9 & 30.8 & 52.9 & 46.8 \\
\hline \multicolumn{7}{|l|}{ Partner's socio-economic status } \\
\hline Same as respondent & 49.2 & 42.4 & 55.6 & 46.2 & 47.1 & 41.3 \\
\hline Better than respondent & 33.9 & 47.5 & 33.3 & 46.2 & 33.3 & 50.0 \\
\hline Worse than respondent & 13.6 & 8.5 & 11.1 & 7.7 & 15.7 & 8.7 \\
\hline Number reporting a romantic relationship & 220 & 238 & 109 & 102 & 170 & 136 \\
\hline
\end{tabular}

Cont'd on next page... 
Table 9.4: (Cont'd)

\begin{tabular}{|c|c|c|c|c|c|c|}
\hline Characteristics $(\%)^{1}$ & $\begin{array}{c}M \\
15-24\end{array}$ & $\begin{array}{c}\text { W } \\
15-24\end{array}$ & $\begin{array}{c}\text { MM } \\
15-29\end{array}$ & $\begin{array}{c}\text { MW } \\
15-24\end{array}$ & $\begin{array}{c}\text { UM } \\
15-24\end{array}$ & $\begin{array}{l}\text { UW } \\
15-24\end{array}$ \\
\hline \multicolumn{7}{|c|}{ Rural } \\
\hline \multicolumn{7}{|c|}{$\begin{array}{l}\text { Age when respondent first spent time alone with } \\
\text { partner (years) }\end{array}$} \\
\hline 15 or below & 48.6 & 61.4 & 42.0 & $(65.3)$ & 48.9 & 57.2 \\
\hline $\begin{array}{l}\text { Median age when respondent first spent tim } \\
\text { with partner }\end{array}$ & 16.0 & 14.0 & 16.0 & $(13.0)$ & 16.0 & 15.0 \\
\hline \multicolumn{7}{|l|}{ Age of partner } \\
\hline Younger than respondent & 70.9 & 0.0 & 72.2 & $(0.0)$ & 67.9 & 0.0 \\
\hline Same age as respondent & 15.3 & 7.0 & 18.9 & $(4.2)$ & 17.1 & 9.6 \\
\hline Older than respondent & 10.9 & 78.4 & 5.3 & $(87.5)$ & 12.3 & 69.1 \\
\hline Don't remember & 2.9 & 14.6 & 3.6 & $(8.3)$ & 2.7 & 21.3 \\
\hline \multicolumn{7}{|l|}{ Partner's marital status } \\
\hline Unmarried & 96.4 & 93.9 & 96.4 & $(93.1)$ & 95.7 & 95.0 \\
\hline Married & 3.6 & 6.1 & 2.4 & $(6.9)$ & 4.3 & 5.0 \\
\hline \multicolumn{7}{|l|}{ Nature of prior acquaintance with first partner } \\
\hline Relative & 9.9 & 31.9 & 10.7 & $(38.9)$ & 8.0 & 24.9 \\
\hline Fellow student/colleague & 13.9 & 12.7 & 11.2 & $(12.5)$ & 15.5 & 13.8 \\
\hline Neighbour/friend & 38.0 & 33.3 & 45.6 & $(33.3)$ & 38.5 & 33.1 \\
\hline Family friend & 0.0 & 0.0 & 0.0 & $(0.0)$ & 0.0 & 0.0 \\
\hline Person from outside village/neighbourhood & 35.4 & 19.2 & 29.0 & $(11.1)$ & 35.3 & 27.1 \\
\hline Other $^{2}$ & 2.9 & 2.8 & 3.6 & $(4.2)$ & 2.7 & 1.1 \\
\hline \multicolumn{7}{|l|}{ Duration of acquaintance } \\
\hline Less than 1 month & 9.8 & 7.9 & 11.2 & $(9.6)$ & 8.0 & 6.1 \\
\hline 1-11 months & 14.9 & 18.2 & 16.0 & $(15.1)$ & 11.7 & 21.1 \\
\hline 12 months or more & 56.7 & 62.6 & 45.6 & $(61.6)$ & 57.4 & 63.3 \\
\hline Since childhood & 18.5 & 11.2 & 27.2 & $(13.7)$ & 22.9 & 9.4 \\
\hline \multicolumn{7}{|l|}{ Partner's religion } \\
\hline Same as respondent & 96.4 & 93.0 & 91.2 & $(90.4)$ & 95.7 & 96.1 \\
\hline Different from respondent & 3.6 & 6.5 & 7.6 & $(9.6)$ & 4.3 & 3.3 \\
\hline \multicolumn{7}{|l|}{ Partner's caste } \\
\hline Same as respondent & 66.1 & 75.2 & 53.0 & $(72.6)$ & 72.3 & 77.2 \\
\hline Different from respondent & 32.8 & 23.4 & 44.6 & $(24.7)$ & 27.7 & 22.2 \\
\hline \multicolumn{7}{|l|}{ Partner's socio-economic status } \\
\hline Same as respondent & 46.0 & 32.1 & 47.3 & $(36.1)$ & 45.2 & 28.2 \\
\hline Better than respondent & 35.5 & 59.5 & 30.2 & $(56.9)$ & 38.8 & 62.4 \\
\hline Worse than respondent & 16.3 & 7.9 & 18.3 & $(6.9)$ & 14.9 & 8.8 \\
\hline Number reporting a romantic relationship & 148 & 157 & 94 & 41 & 100 & 116 \\
\hline
\end{tabular}

Note: All Ns are unweighted. Column totals may not equal 100\% due to missing cases or "don't know" responses. ( ) Based on 25-49 unweighted cases. ${ }^{1}$ First romantic partner, if more than one romantic partner reported. ${ }^{2}$ Includes employee, employer, teacher, other acquaintance and stranger. 
Table 9.5: Meeting places with pre-marital romantic partners

Percentage of youth reporting a pre-marital romantic relationship by places where they met their partner secretly, according to residence, Bihar, 2007

\begin{tabular}{|c|c|c|c|c|c|c|}
\hline Meeting places $(\%)^{1}$ & $\begin{array}{c}\mathrm{M} \\
15-24\end{array}$ & $\begin{array}{c}\text { W } \\
15-24\end{array}$ & $\begin{array}{c}\text { MM } \\
15-29\end{array}$ & $\begin{array}{c}\text { MW } \\
15-24\end{array}$ & $\begin{array}{c}\text { UM } \\
15-24\end{array}$ & $\begin{array}{c}\text { UW } \\
15-24\end{array}$ \\
\hline \multicolumn{7}{|c|}{ Combined } \\
\hline Each other's home & 46.4 & 48.4 & 40.6 & 50.6 & 47.9 & 46.3 \\
\hline Temple/mosque/church & 9.6 & 12.8 & 4.3 & 9.4 & 10.9 & 15.0 \\
\hline Cinema/theatre & 12.3 & 5.9 & 14.4 & 8.2 & 10.5 & 4.0 \\
\hline Park/garden & 29.0 & 12.8 & 34.8 & 11.8 & 29.0 & 13.7 \\
\hline Restaurant/eating place & 7.2 & 9.9 & 4.8 & 10.6 & 7.1 & 7.5 \\
\hline Jungle/riverside & 4.8 & 4.8 & 4.3 & 5.9 & 4.2 & 4.4 \\
\hline Field/grazing area & 24.3 & 14.7 & 36.4 & 9.4 & 19.7 & 20.7 \\
\hline Other places & 0.6 & 0.7 & 1.1 & 2.4 & 0.8 & 0.0 \\
\hline Never met unaccompanied & 9.6 & 12.5 & 7.0 & 11.9 & 11.3 & 12.8 \\
\hline Number reporting a romantic relationship & 368 & 395 & 203 & 143 & 270 & 252 \\
\hline \multicolumn{7}{|c|}{ Urban } \\
\hline Each other's home & 39.0 & 50.8 & 33.3 & 53.8 & 40.0 & 51.1 \\
\hline Temple/mosque/church & 15.3 & 16.9 & 16.7 & 16.7 & 15.7 & 17.0 \\
\hline Cinema/theatre & 11.9 & 8.5 & 16.7 & 15.4 & 11.8 & 6.4 \\
\hline Park/garden & 39.0 & 13.8 & 44.4 & 15.4 & 36.0 & 14.9 \\
\hline Restaurant/eating place & 18.6 & 20.3 & 22.2 & 15.4 & 18.0 & 23.4 \\
\hline Jungle/riverside & 1.7 & 1.7 & 5.6 & 0.0 & 2.0 & 0.0 \\
\hline Field/grazing area & 5.1 & 5.1 & 11.1 & 8.3 & 4.0 & 0.0 \\
\hline Other places & 0.0 & 0.0 & 0.0 & 0.0 & 0.0 & 0.0 \\
\hline Never met unaccompanied & 16.9 & 13.6 & 11.1 & 15.4 & 19.6 & 14.9 \\
\hline Number reporting a romantic relationship & 220 & 238 & 109 & 102 & 170 & 136 \\
\hline \multicolumn{7}{|c|}{ Rural } \\
\hline Each other's home & 48.0 & 47.7 & 41.4 & $(50.0)$ & 50.3 & 45.0 \\
\hline Temple/mosque/church & 8.4 & 11.2 & 3.0 & $(8.3)$ & 9.6 & 14.4 \\
\hline Cinema/theatre & 12.4 & 5.1 & 14.2 & $(6.9)$ & 10.1 & 3.3 \\
\hline Park/garden & 26.9 & 12.1 & 33.7 & $(11.1)$ & 26.7 & 13.3 \\
\hline Restaurant/eating place & 4.7 & 6.6 & 3.0 & $(9.7)$ & 3.7 & 3.3 \\
\hline Jungle/riverside & 5.5 & 6.1 & 4.1 & $(6.9)$ & 4.8 & 5.0 \\
\hline Field/grazing area & 28.4 & 17.4 & 39.1 & $(9.7)$ & 23.9 & 25.6 \\
\hline Other places & 0.7 & 0.9 & 1.2 & $(2.7)$ & 1.1 & 0.0 \\
\hline Never met unaccompanied & 8.0 & 12.1 & 6.5 & $(12.5)$ & 9.6 & 12.2 \\
\hline Number reporting a romantic relationship & 148 & 157 & 94 & 41 & 100 & 116 \\
\hline
\end{tabular}

Note: All Ns are unweighted. Column totals may exceed 100\% due to multiple responses. ( ) Based on 25-49 unweighted cases. ${ }^{1}$ First romantic partner, if more than one romantic partner reported. 


\subsubsection{Parental and peer awareness of romantic partnerships}

Table 9.6 reports findings on peer and parental awareness of young people's romantic partnerships. Youth overwhelmingly (77-78\%) reported that their peers were aware of their romantic relationships. Differences by marital status and rural-urban residence were muted, except that more urban than rural young men reported peer awareness of romantic relationship (85\% compared to $77 \%$ ).

Relatively few youth reported that their parents were aware of these partnerships. Young women were more likely than young men to report that parents were aware of their relationship (37\% and 23\%, respectively). The married were more likely to report parental awareness than the unmarried and the differences were more pronounced among young women $(28 \%$ and $23 \%$ of young men and $46 \%$ and $27 \%$ of young women, respectively). Moreover, urban youth were more likely to report parental awareness than their rural counterparts (36\% versus $20 \%$ among young men and $46 \%$ versus $34 \%$ among young women). Gender differences may be explained by the fact that young women, who tend to be more strictly supervised, would have had fewer opportunities to hide a relationship from their parents than young men. Differences by marital status may be attributed to the fact that revelation of the relationship could itself have triggered marriage, either to the same person or someone else, as seen in the panel on parental reactions. Rural-urban differences may be attributable to the fact that activities associated with rural life-fetching water and fuel, agricultural tasks and so on-required youth and their parents to be away from home for extended periods on a routine basis and may have provided rural youth more opportunities to pursue relationships without parental awareness.

The reported reactions of those parents who became aware of their children's pre-marital romantic relationships are presented in Table 9.6. Given the small numbers, we provide findings for the rural and urban populations together. Parents' reported reactions varied between young men and young women. Larger percentages of young women than men reported negative reactions. Two-fifths of young women (41\%) and one-third of young men (35\%) reported that their parents had shouted at them and $19 \%$ and $13 \%$, respectively, reported that their parents had beaten them. Almost one-fifth of young women (18\%) reported that they were not permitted to go out. In comparison, not a single young man reported as such. Another one-fifth of young women $(20 \%)$ and almost one-quarter $(23 \%)$ of young men were forbidden from meeting the partner. Among young women, $3 \%$ were withdrawn from school as a result.

For considerable percentages of young women, parents reacted by arranging their marriages to the romantic partner $(15 \%)$ or to someone else $(16 \%)$, perhaps in order to protect the family's honour. In contrast, few young men reported that their parents reacted in this way $(3-5 \%)$.

Notably, many parents either accepted the situation or did not react at all to the knowledge of their child's romantic relationship (39\% and 35\% of young men and women, respectively). One in three parents of young men $(30 \%)$ compared to few parents of young women $(13 \%)$ reacted by advising their sons or daughters, for example, not to let school performance suffer as a result of the relationship.

\subsubsection{Marriage intentions and duration of pre-marital romantic relationships}

The questionnaire probed all respondents who reported a relationship about their intentions to marry their romantic partner. Findings are reported in Table 9.7 and suggest young women were twice as likely as young men to report that they intended to marry either the first or most recent partner ( $81 \%$ versus $40 \%$ ), a finding observed in other studies as well (Alexander et al., 2006a; 2006b). Differences by marital status suggest that married youth were somewhat more likely to have reported this intention than the unmarried (47\% versus $41 \%$ among young men and $84 \%$ versus $78 \%$ among young women). Urban young men were more likely to report marital intentions than rural young men (51\% versus $38 \%)$, but no such differences were evident among young women. 
Table 9.6: Peer and parental awareness of first pre-marital romantic relationship

Percentage of youth reporting a pre-marital romantic relationship by peer and parental awareness of the first romantic relationship and parents' reaction, according to residence, Bihar, 2007

\begin{tabular}{|c|c|c|c|c|c|c|}
\hline Awareness and reactions $(\%)^{1}$ & $\begin{array}{c}\mathrm{M} \\
15-24\end{array}$ & $\begin{array}{c}\text { W } \\
15-24\end{array}$ & $\begin{array}{c}\text { MM } \\
15-29\end{array}$ & $\begin{array}{c}\text { MW } \\
\text { 15-24 }\end{array}$ & $\begin{array}{c}\text { UM } \\
15-24\end{array}$ & $\begin{array}{c}\text { UW } \\
15-24\end{array}$ \\
\hline \multicolumn{7}{|c|}{ Combined } \\
\hline Friends aware of relationship & 78.1 & 77.3 & 76.5 & 75.3 & 77.8 & 78.9 \\
\hline Parents aware of relationship & 23.1 & 36.6 & 27.8 & 45.9 & 23.1 & 26.9 \\
\hline Number reporting a romantic relationship & 368 & 395 & 203 & 143 & 270 & 252 \\
\hline \multicolumn{7}{|c|}{ Urban } \\
\hline Friends aware of relationship & 84.7 & 78.0 & 83.3 & 76.9 & 84.0 & 78.3 \\
\hline Parents aware of relationship & 35.6 & 45.8 & 44.4 & 50.0 & 34.0 & 42.6 \\
\hline Number reporting a romantic relationship & 220 & 238 & 109 & 102 & 170 & 136 \\
\hline \multicolumn{7}{|c|}{ Rural } \\
\hline Friends aware of relationship & 76.7 & 77.1 & 75.7 & $(75.3)$ & 76.1 & 79.0 \\
\hline Parents aware of relationship & 20.4 & 34.1 & 26.6 & $(45.2)$ & 19.8 & 22.8 \\
\hline Number reporting a romantic relationship & 148 & 157 & 94 & 41 & 100 & 116 \\
\hline \multicolumn{7}{|c|}{ Combined } \\
\hline Parents' reaction & & & & & & \\
\hline Shouted at respondent & 35.1 & 41.0 & 42.3 & 41.0 & 37.0 & 41.7 \\
\hline Beat respondent & 13.0 & 19.2 & 13.5 & 20.5 & 16.4 & 20.0 \\
\hline Did not allow respondent to go out & 0.0 & 18.2 & 0.0 & 15.4 & 0.0 & 23.3 \\
\hline Stopped respondent from meeting partner & 23.1 & 20.0 & 19.2 & 15.4 & 18.2 & 26.7 \\
\hline Forced respondent to discontinue education & 0.0 & 3.0 & 3.8 & 5.1 & 0.0 & 0.0 \\
\hline Reported to/shouted at partner's family & 0.0 & 5.0 & 0.0 & 5.1 & 0.0 & 5.0 \\
\hline Arranged marriage with partner & 5.1 & 15.2 & 3.8 & 17.9 & 3.6 & 9.8 \\
\hline Arranged marriage with someone else & 2.6 & 16.2 & 3.8 & 28.2 & 0.0 & 0.0 \\
\hline No reaction/accepted the situation & 38.5 & 35.0 & 28.8 & 30.8 & 32.7 & 40.0 \\
\hline $\begin{array}{l}\text { Advised respondent, including not to let school/college } \\
\text { performance suffer }\end{array}$ & 29.5 & 13.0 & 34.6 & 12.8 & 34.5 & 11.5 \\
\hline Number whose parents were aware of relationship & 112 & 153 & 71 & 70 & 79 & 83 \\
\hline
\end{tabular}

Note: All Ns are unweighted. Column totals may exceed 100\% due to multiple responses. Reporting of parents' reactions is presented for rural and urban combined due to small numbers. ( ) Based on 25-49 unweighted cases. ${ }^{1}$ First romantic partner, if more than one romantic partner reported.

Reality, in terms of outcomes of romantic relationships, was different from intention. For example, while 84\% of married young women had intended to marry their pre-marital partners, just $32 \%$ reported having done so; among married young men $47 \%$ reported an intention to marry their pre-marital partner, yet just $4 \%$ reported doing so. The rural-urban differences observed for intentions persisted in outcomes as well: among married young women, $46 \%$ of the urban compared to $30 \%$ of the rural, had married their pre-marital romantic partner. Corresponding percentages among married young men were $17 \%$ and $3 \%$, respectively.

The majority of unmarried youth were still in a relationship at the time of interview (56\% of young men and $82 \%$ of young women); rural-urban differences were modest. Of note was that among the married, $18 \%$ of young men and $8 \%$ of young women reported continuing a relationship with their pre-marital partner even after marriage, with more urban married young women reporting as such than their rural counterparts. 
Table 9.7: Marriage intentions and duration of pre-marital romantic relationships

Percentage of youth reporting a pre-marital romantic relationship by intention to marry partner, current relationship status and duration of relationship, according to residence, Bihar, 2007

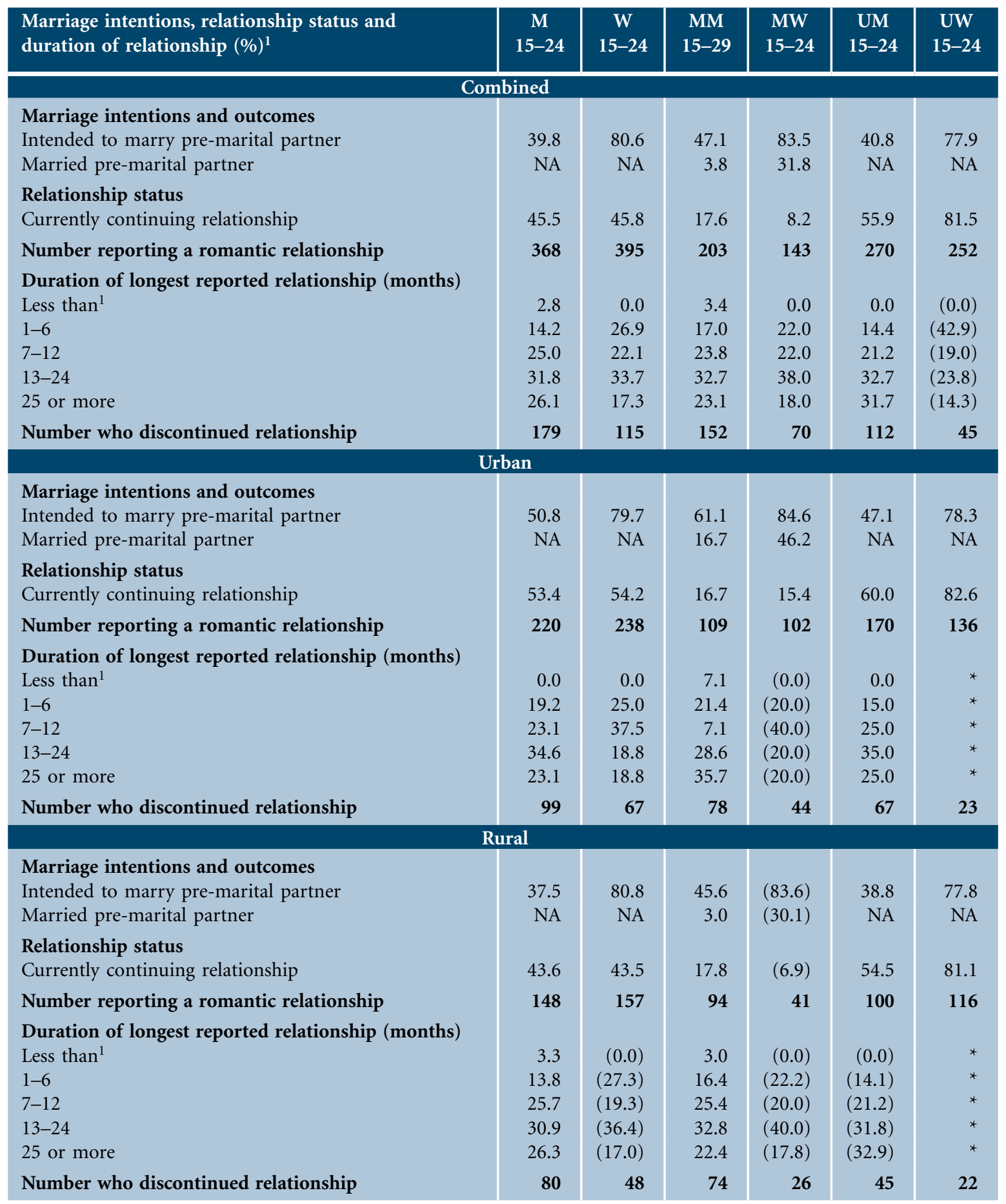

Note: All Ns are unweighted. Column totals may not equal 100\% due to missing cases or "don't know" responses. ( ) Based on 25-49 unweighted cases. ${ }^{*}$ Percentage not shown, based on fewer than 25 unweighted cases. NA: Not applicable. ${ }^{1}$ Data on marriage intentions, relationship status and duration of relationship were collected only with regard to the first and/or most recent partner. These data were not available for 96 young men and 3 young women who reported more than two romantic partners. 
Among those who had discontinued their romantic relationship (the longest relationship, if more than one romantic partner was reported), relationships extended over fairly long time periods (more than one year for $58 \%$ and $51 \%$ of young men and women, respectively). Gender differences were more pronounced for urban than for overall or rural samples, with $58 \%$ of young men compared to $38 \%$ of young women in urban areas reporting longer duration relationships. While unmarried young men were more likely than the married to report that relationships extended over a year (64\% versus 56\%), a reverse pattern was evident among young women ( $38 \%$ versus $56 \%$ ). While rural-urban differences were muted among young men, these were wide among young women, with more rural than urban young women reporting longer duration relationships (53\% versus $38 \%)$.

\subsubsection{Pre-marital physical intimacy and sex with a romantic partner}

Respondents who reported a pre-marital romantic relationship were asked whether they had engaged in a number of intimate behaviours with their romantic partner. These behaviours ranged from those reflecting minimal physical intimacy (hand-holding, hugging) to those reflecting increased physical intimacy (kissing on the lips) and finally, engaging in sexual relations. Findings, presented in Table 9.8, refer to youth experiences of physical intimacy with their first and/or most recent romantic partner, if more than one.

While the large majority of youth had held hands with any romantic partner, consistently fewer reported progressively more intimate behaviours. Gender differences in reporting of such experiences narrowed somewhat between reports of hand-holding and progressively more intimate forms of behaviour. For example, $87 \%$ of young men compared to $70 \%$ of young women reported holding hands with any romantic partner. In comparison, $63 \%$ of young men compared to $48 \%$ of young women, reported kissing their romantic partners, and $40 \%$ of young men compared to $30 \%$ of young women reported engaging in sex with one or more romantic partners.

Differences by marital status suggest that more married than unmarried young men reported each of these intimate behaviours (see also Figure 9.2). Similar patterns prevailed among young women as well, except in respect of holding hands, which roughly similar proportions of both married and unmarried young women reported. Rural-urban differences suggest that, aside from handholding, reported by equal proportions of young men and women, rural youth were more likely than urban youth to report more intimate behaviours including hugging, kissing and engaging in sex. The differences were widest in regard to engaging in sex; $45 \%$ and $35 \%$ of rural young men and women, respectively, compared to $19 \%$ and $15 \%$ of urban young men and women, respectively, reported pre-marital sex with a romantic partner, a finding that may be attributed to the greater opportunities for privacy in rural areas.

In short, findings confirm that pre-marital romantic relationships among youth almost always included some form of physical intimacy. Moreover, two-fifths of young men and almost one-third of young women who reported pre-marital romantic relationships had experienced sex with a romantic partner.

\subsubsection{Characteristics of sexual experiences within pre-marital romantic relationships}

The Youth Study asked all respondents reporting pre-marital sex with a romantic partner about fears of pregnancy or infection at the time of first sex, condom and contraceptive decision-making and use at first and subsequent sexual encounters with a romantic sexual partner, and the consensual nature of first sex. Findings are presented in Table 9.9. Given the small numbers of respondents reporting this experience, particularly among young women, we provide combined rural-urban findings for married and unmarried young men and women. 
Figure 9.2: Percentage of youth reporting experiences of physical intimacy and sex with a pre-marital romantic partner, Bihar, 2007

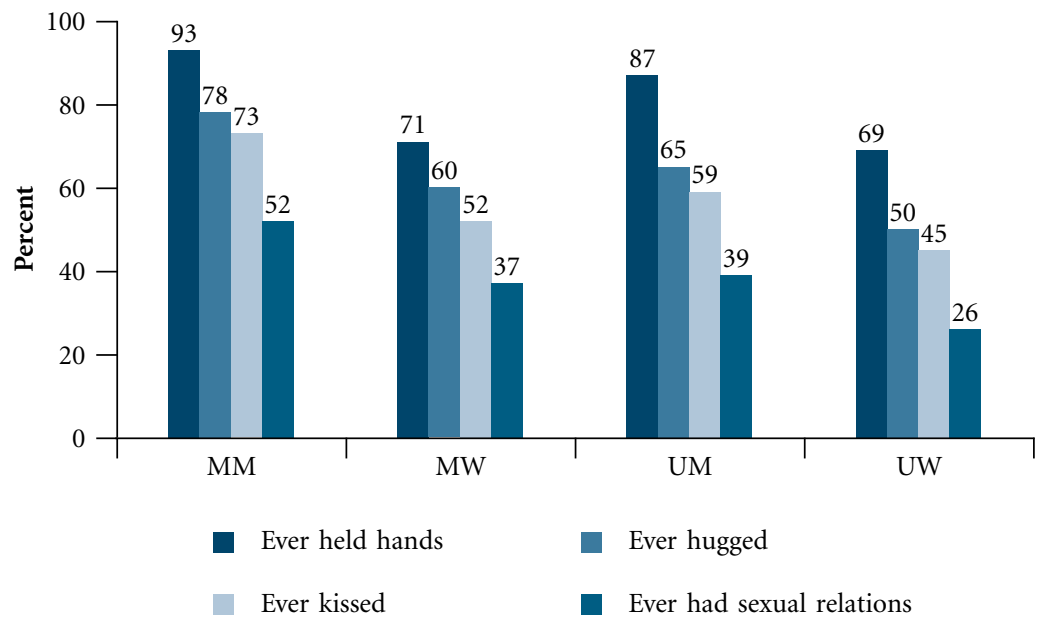

Table 9.8: Physical intimacy and sexual experiences in pre-marital romantic relationships

Percentage of youth reporting a pre-marital romantic relationship by experiences of physical intimacy and sex with their partner, according to residence, Bihar, 2007

\begin{tabular}{|c|c|c|c|c|c|c|}
\hline Physical intimacy $(\%)^{1}$ & $\begin{array}{c}\mathrm{M} \\
15-24\end{array}$ & $\begin{array}{c}\text { W } \\
15-24\end{array}$ & $\begin{array}{c}\text { MM } \\
15-29\end{array}$ & $\begin{array}{c}\text { MW } \\
15-24\end{array}$ & $\begin{array}{c}\text { UM } \\
15-24\end{array}$ & $\begin{array}{c}\text { UW } \\
15-24\end{array}$ \\
\hline \multicolumn{7}{|c|}{ Combined } \\
\hline Ever held hands & 87.4 & 69.6 & 93.0 & 70.6 & 86.6 & 68.7 \\
\hline Ever hugged & 66.8 & 54.4 & 77.5 & 59.5 & 65.1 & 50.2 \\
\hline Ever kissed & 62.6 & 47.6 & 73.3 & 51.8 & 59.2 & 44.5 \\
\hline Ever had sexual relations & 40.1 & 30.0 & 52.4 & 36.5 & 38.7 & 25.6 \\
\hline Number reporting a romantic relationship & 368 & 395 & 203 & 143 & 270 & 252 \\
\hline \multicolumn{7}{|c|}{ Urban } \\
\hline Ever held hands & 86.4 & 69.5 & 94.4 & 75.0 & 86.0 & 68.1 \\
\hline Ever hugged & 55.9 & 48.3 & 66.7 & 53.8 & 52.9 & 44.7 \\
\hline Ever kissed & 49.2 & 40.7 & 61.1 & 46.2 & 46.0 & 38.3 \\
\hline Ever had sexual relations & 18.6 & 15.3 & 27.8 & 23.1 & 18.0 & 8.5 \\
\hline Number reporting a romantic relationship & 220 & 238 & 109 & 102 & 170 & 136 \\
\hline \multicolumn{7}{|c|}{ Rural } \\
\hline Ever held hands & 87.6 & 69.2 & 93.5 & $(69.4)$ & 87.2 & 68.9 \\
\hline Ever hugged & 68.8 & 56.1 & 78.6 & $(61.1)$ & 68.4 & 51.4 \\
\hline Ever kissed & 65.5 & 49.3 & 74.6 & (52.8) & 62.8 & 46.1 \\
\hline Ever had sexual relations & 44.7 & 34.6 & 55.0 & $(38.9)$ & 44.1 & 30.0 \\
\hline Number reporting a romantic relationship & 148 & 157 & 94 & 41 & 100 & 116 \\
\hline
\end{tabular}

Note: All Ns are unweighted. ( ) Based on 25-49 unweighted cases. ${ }^{1}$ Data on ever held hands, ever hugged and ever kissed pertain to the first or most recent partner, if more than one partner was reported. Data on pre-marital sexual relations pertain not only to the first or most recent partner, but also to other romantic partners, if more than two romantic partners were reported. 
Among those who reported sexual experience within pre-marital romantic relationships, many more young women than men reported fear of pregnancy or infection. For example, fear of pregnancy was reported by $43 \%$ of young men and $59 \%$ of young women; fear of infection was reported by $18 \%$ of young men and $35 \%$ of young women. Married young men were as likely as the unmarried to report fear of pregnancy (43-45\%), but were more likely to report fear of infection (21\% versus $12 \%)$.

Reported contraceptive use at first pre-marital sex with a romantic partner and consistent contraceptive use in subsequent sexual encounters were limited. In total, just $23 \%$ of young men and $6 \%$ of young women reported using contraception at first sex and $19 \%$ and 6\%, respectively, reported that it was consistently practised in all sexual encounters with their romantic opposite-sex partner(s). More unmarried than married young men reported using contraception at first sex (25\% compared to 17\%). Consistent contraceptive use was also more likely to be reported by the unmarried than the married.

Condom use was limited. Just $17 \%$ of young men and 5\% of young women reported having used a condom during their first sexual encounter with a romantic partner. Even so, it is clear that the majority of those who practised contraception at first sex used a condom ( $74 \%$ of young men and $80 \%$ of young women).

Most youth reporting condom use at first sex reported that they had continued to use condoms in all sexual encounters with their romantic partner(s) $-16 \%$ of young men and $4 \%$ of young women. All young men and three-quarters of young women who used a condom at first sex reported doing so for pregnancy prevention. In comparison, $86 \%$ of young men and all young women who reported condom use at first sex reported doing so to prevent infection.

Youth reports of decision-making regarding contraceptive use at first pre-marital sex with an opposite-sex romantic partner reveal that young women were relatively disadvantaged. For example, responses of both young men and women suggest that the decision to practise contraception at first sex typically did not involve the female partner, either as the sole or joint decision-maker. While $23 \%$ of young men and $6 \%$ of young women had practised contraception at first sex with an opposite-sex romantic partner, just $8 \%$ and $2 \%$ of young men and women, respectively, reported that the female partner had participated in the decision.

While the majority of young men and women reported that their first experience of pre-marital sex with an opposite-sex romantic partner was consensual, several youth reported that it occurred without consent. Gender differences were wide in this respect. For example, $78 \%$ of young men reported that sex was consensual, compared to $51 \%$ of young women. Over one-fifth of young women (22\%) reported that their opposite-sex romantic partner had forced them to engage in sex the first time; in contrast, $5 \%$ of young men acknowledged that they had forced their partner to do so. One-quarter of young women $(26 \%)$ and $8 \%$ of young men reported that the male partner had persuaded the female partner to engage in sexual relations. Very few youth reported that the female partner had persuaded $(7 \%$ and $1 \%$ of young men and women, respectively) or forced ( $3 \%$ and none, respectively) the male partner to engage in sex.

Differences by marital status suggest that somewhat more unmarried than married young men reported that their first experience of pre-marital sex with an opposite-sex romantic partner was consensual $(83 \%$ versus $77 \%)$. Conversely, more married than unmarried young men reported that the female partner had persuaded the male partner to engage in sexual relations (13\% versus $4 \%)$. 
Table 9.9: Characteristics of sexual experiences within pre-marital romantic relationships

Percentage of youth reporting pre-marital sexual experiences with an opposite-sex romantic partner by selected characteristics of their first and subsequent sexual encounters with the partner, Bihar, 2007

\begin{tabular}{|c|c|c|c|c|c|c|}
\hline Characteristics (\%) ${ }^{1}$ & $\underset{15-24}{M}$ & $\begin{array}{c}W \\
15-24\end{array}$ & $\begin{array}{c}\text { MM } \\
15-29\end{array}$ & $\begin{array}{c}\text { MW } \\
15-24\end{array}$ & $\begin{array}{c}\text { UM } \\
15-24\end{array}$ & $\begin{array}{l}\text { UW } \\
15-24\end{array}$ \\
\hline Anxiety associated with first sex & & & & & & \\
\hline Afraid of getting pregnant at first sex & 42.9 & 59.0 & 43.3 & $(60.0)$ & 44.6 & $(58.6)$ \\
\hline Afraid of getting infection at first sex & 18.3 & 35.4 & 21.1 & $(38.7)$ & 12.0 & $(32.2)$ \\
\hline Contraceptive use & & & & & & \\
\hline Practised contraception at first sex & 23.3 & 6.1 & 16.5 & $(0.0)$ & 25.0 & $(13.8)$ \\
\hline Practised contraception in all sexual encounters ${ }^{2}$ & 18.8 & 6.1 & 12.4 & $(0.0)$ & 18.5 & $(13.8)$ \\
\hline Condom use & & & & & & \\
\hline Used a condom at first sex to: & 17.3 & 4.9 & 11.2 & $(0.0)$ & 20.7 & $(10.3)$ \\
\hline Avoid pregnancy & 17.3 & 3.7 & 11.2 & $(0.0)$ & 20.7 & $(8.5)$ \\
\hline Avoid infection & 14.9 & 4.9 & 7.1 & $(0.0)$ & 16.5 & $(10.3)$ \\
\hline Used condoms in all sexual encounters ${ }^{2}$ & 15.7 & 3.7 & 9.2 & $(0.0)$ & 18.5 & $(6.9)$ \\
\hline Decision to use contraception at first sex taken by: & & & & & & \\
\hline Respondent & 15.0 & 1.2 & 10.3 & $(0.0)$ & 17.4 & $(1.8)$ \\
\hline Partner & 4.5 & 3.7 & 6.2 & $(0.0)$ & 2.2 & $(8.8)$ \\
\hline Jointly & 3.8 & 1.2 & 0.0 & $(0.0)$ & 5.4 & (1.8) \\
\hline Consensuality of first sex & & & & & & \\
\hline Mutual consent & 78.4 & 51.2 & 76.5 & $(54.8)$ & 82.6 & $(41.7)$ \\
\hline Male partner forced & 4.5 & 22.0 & 2.0 & $(29.0)$ & 4.3 & $(15.0)$ \\
\hline Female partner forced & 3.0 & 0.0 & 2.0 & $(0.0)$ & 4.3 & $(0.0)$ \\
\hline Male partner persuaded & 7.5 & 25.6 & 6.1 & $(16.1)$ & 4.3 & $(40.0)$ \\
\hline Female partner persuaded & 6.7 & 1.2 & 13.3 & $(0.0)$ & 4.3 & (3.3) \\
\hline $\begin{array}{l}\text { Number reporting pre-marital sex with an opposite-sex } \\
\text { romantic partner }\end{array}$ & 108 & 87 & 80 & 40 & 73 & 47 \\
\hline
\end{tabular}

Note: All Ns are unweighted. Column totals may not equal 100\% due to missing cases or "don't know" responses. ( ) Based on 25-49 unweighted cases. ${ }^{1}$ In-depth probing of sexual experiences was restricted to respondents' first or most recent romantic partner. Therefore, if a respondent reported his/her first sexual experience as occurring with a romantic partner other than the first or the most recent, then age, consensuality and other characteristics at first sex were unknown. Information was not available in 1 such case. ${ }^{2}$ Data were missing for 15 young men who reported sexual experiences with a romantic partner other than the first or most recent partner.

\subsection{Pre-marital sexual experiences within romantic and other relationships}

Aside from the heterosexual romantic partnerships discussed in previous sections, the Youth Study also probed youth experiences of pre-marital sex with other partners, including casual partners and spouse before marriage, and in situations characterised by force and exchange of gifts or favours. In addition, in the case of male respondents, questions were asked about pre-marital sex with same-sex partners, sex workers and married women. In this and subsequent sections of this chapter, we present findings on the prevalence of pre-marital sexual experiences (irrespective of whether such experiences took place within romantic or other partnerships) among all youth in the sample. 


\subsubsection{Extent of pre-marital sexual experiences}

Table 9.10 reports percentages of respondents reporting pre-marital sex in any of the situations described above. Findings confirm that pre-marital sex profiles of young men and women varied widely. For $7 \%$ of young men and $1 \%$ of young women, pre-marital sex occurred in a romantic relationship with a person of the opposite sex. In contrast, $0.5-0.6 \%$ of youth reported that they were forced to engage in sex, and $0.4 \%$ of young men reported that they had forced sex on a female partner. Sex in exchange for money or favours was reported by $0.1-0.2 \%$ or fewer youth. Casual sex was reported by $2.2 \%$ of young men and $0.1 \%$ of young women.

Young men were asked, in addition, about same-sex relations as well as relations with sex workers and married women (excluding their own wives, if married). Findings suggest that $0.4 \%$ of young men had experienced same-sex relations. Relations with sex workers and married women were reported by $0.9 \%$ and $3.4 \%$ of young men. Just $0.3-0.4 \%$ of married young men and women reported sex with their spouse before marriage (some of these included those who had sex with a romantic partner whom they later married). In this way, a total of $12 \%$ of young men and $2 \%$ of young women reported pre-marital sexual relations in the course of face-to-face interviews.

Several youth, particularly young men who had not admitted sexual experience in the face-to-face interview did so in the anonymous sealed envelope. Including these, in total, 14\% of young men and 3\% of young women had reported any pre-marital sexual experience. Although the Youth Study findings fall on the low side of ranges observed in a variety of small case studies (15-30\% for males and fewer than 10\% for females; Jejeebhoy and Sebastian, 2004), the possibility that youth opted not to disclose sexual experience in various situations cannot be discounted, particularly in the case of reporting by young women, and in the reporting of forced, same-sex or sex worker relations.

The percentages reporting pre-marital sexual experience were similar among married and unmarried young women $(2 \%$ and $3 \%)$; however, married young men were more likely than unmarried young men to report pre-marital sexual experience (18\% versus 11\%) (see also Figure 9.3). Marital status differences in types of partners with whom sexual relations were experienced were generally negligible with one exception; married young men were considerably more likely than their unmarried counterparts to have experienced sexual relations with a married woman (6\% and 1\%, respectively). Rural-urban differences were modest (15\% and $10 \%$ of rural and urban young men, respectively and $3 \%$ and $2 \%$, respectively, of young women).

Table 9.11 presents percentages reporting pre-marital sexual experience by selected socio-demographic characteristics of youth. In view of the small numbers of respondents reporting such experience, findings are presented for all married and unmarried young men and women; findings for rural and urban respondents are not separately provided.

Findings suggest that percentages reporting pre-marital sexual experience did not differ much by most background characteristics. Even so, young men, particularly unmarried young men belonging to scheduled castes were more likely than others to report pre-marital sexual experience (21\% versus $11-12 \%)$. Young men who had worked in the last year were twice as likely as non-working young men to have experienced pre-marital sex (16\% versus $8 \%)$. This pattern was evident among unmarried young men, but not among married young men. In addition, an inverse association was observed between age and pre-marital sexual experiences among married young men. All other differentials were narrow. 
Table 9.10: Overall pre-marital sexual experiences

Percentage of youth reporting pre-marital sexual experiences with any partner and via different reporting methods, according to residence, Bihar, 2007

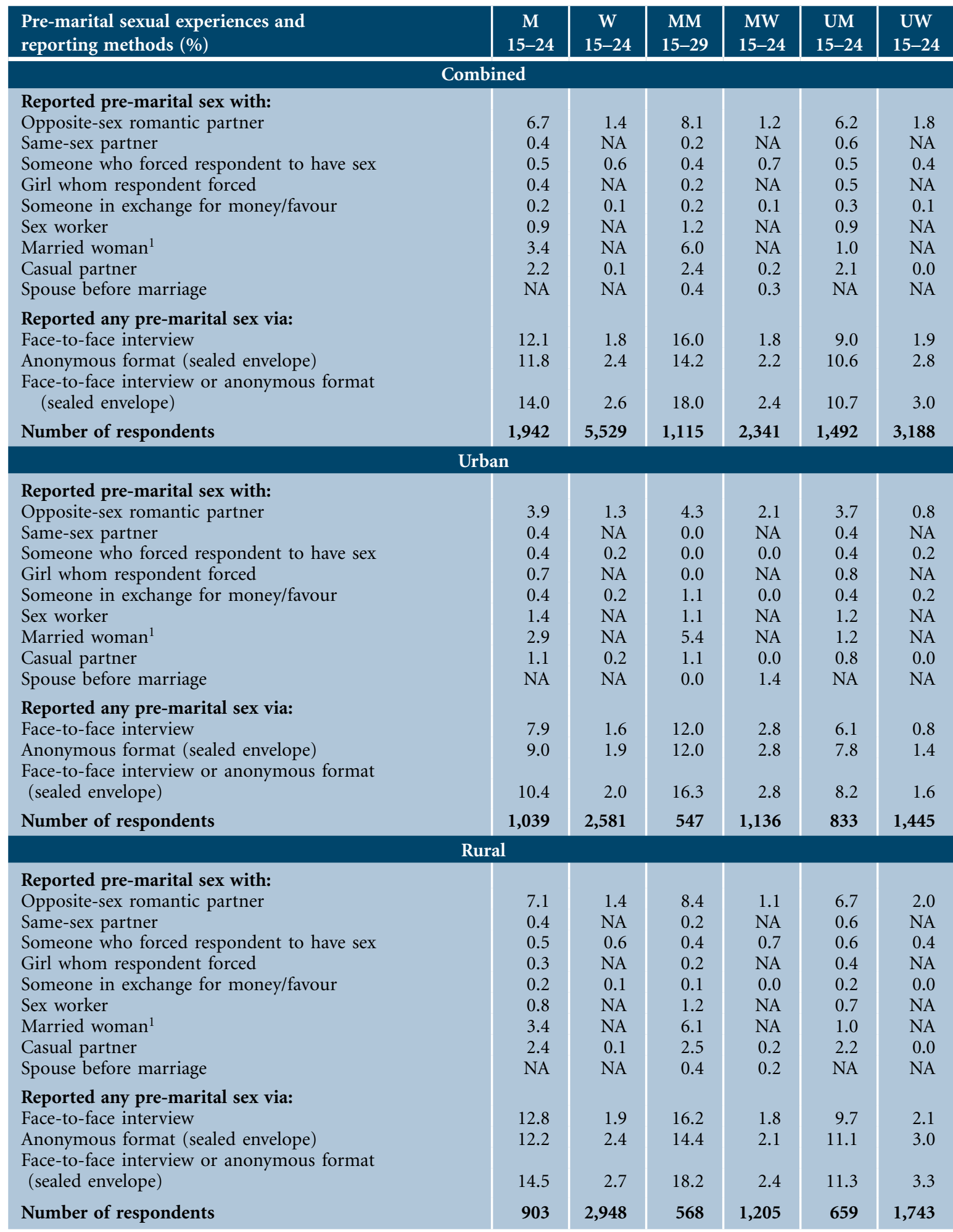

Note: All Ns are unweighted. NA: Not applicable. ${ }^{1}$ Sex with a married woman excludes sex with wife before marriage. 
Figure 9.3: Percentage of youth reporting any pre-marital sexual experiences (in face-to-face interview or sealed envelope), according to residence, Bihar, 2007
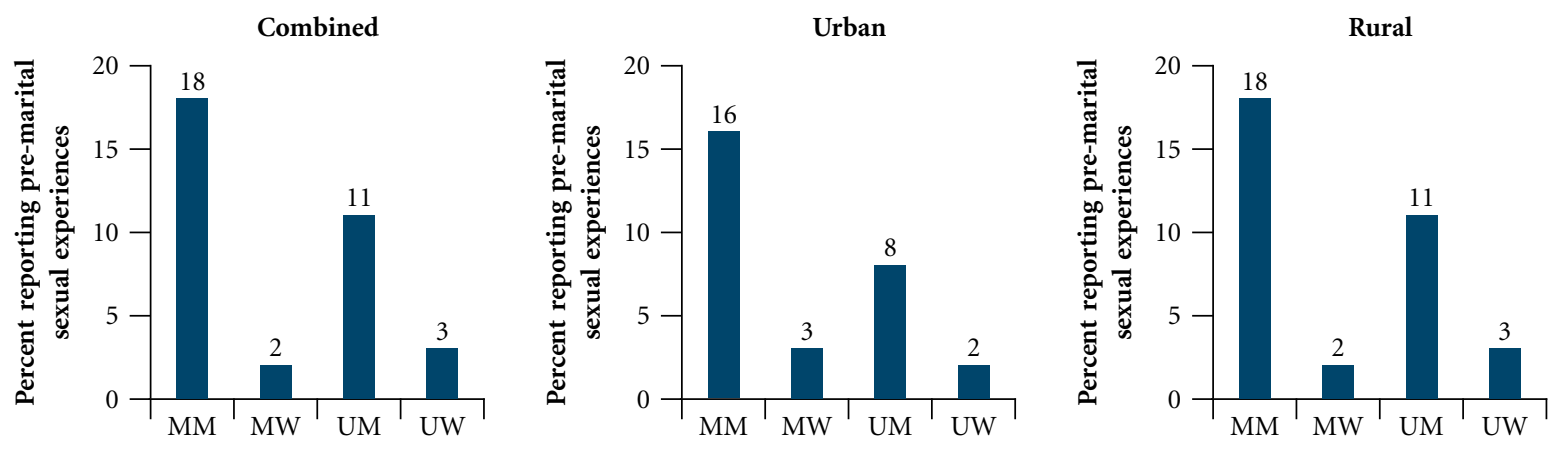

Table 9.11: Overall pre-marital sexual experiences by selected background characteristics

Percentage of youth reporting any pre-marital sexual experiences by selected background characteristics, Bihar, 2007

\begin{tabular}{|c|c|c|c|c|c|c|}
\hline Background characteristics (\%) & $\begin{array}{c}\text { M } \\
15-24\end{array}$ & $\begin{array}{c}\text { W } \\
15-24\end{array}$ & $\begin{array}{c}\text { MM } \\
15-29\end{array}$ & $\begin{array}{c}\text { MW } \\
15-24\end{array}$ & $\begin{array}{c}\text { UM } \\
15-24\end{array}$ & $\begin{array}{c}\text { UW } \\
15-24\end{array}$ \\
\hline \multicolumn{7}{|l|}{ Age (years) } \\
\hline $15-19$ & 12.5 & 2.9 & 44.2 & 2.7 & 9.8 & 3.2 \\
\hline $20-24$ & 16.4 & 2.2 & 18.1 & 2.2 & 13.9 & 1.5 \\
\hline $25-29$ & NA & NA & 14.6 & NA & NA & NA \\
\hline \multicolumn{7}{|l|}{ Religion } \\
\hline Hindu & 14.1 & 2.5 & 18.4 & 2.2 & 10.7 & 3.2 \\
\hline Muslim & 13.2 & 3.2 & 15.6 & 4.1 & 11.1 & 2.2 \\
\hline \multicolumn{7}{|l|}{ Caste } \\
\hline SC & 20.8 & 3.2 & 19.7 & 3.6 & 14.5 & 2.1 \\
\hline $\mathrm{OBC}$ & 12.2 & 2.3 & 16.0 & 1.8 & 10.0 & 3.3 \\
\hline General $^{1}$ & 10.5 & 3.0 & 18.9 & 3.2 & 9.3 & 2.7 \\
\hline \multicolumn{7}{|l|}{ Educational level (years) } \\
\hline None $^{2}$ & 16.8 & 2.0 & 18.0 & 1.9 & 8.0 & 2.1 \\
\hline $1-7$ & 14.3 & 3.2 & 20.2 & 2.7 & 10.6 & 3.8 \\
\hline $8-11$ & 12.5 & 3.5 & 15.5 & 4.3 & 11.4 & 2.9 \\
\hline 12 and above & 14.2 & 2.8 & 18.5 & 3.3 & 10.5 & 3.1 \\
\hline \multicolumn{7}{|l|}{ Worked in last 12 months } \\
\hline Yes & 16.0 & 3.2 & 18.1 & 3.0 & 12.6 & 3.7 \\
\hline No & 8.4 & 2.3 & $(21.9)$ & 2.1 & 7.4 & 2.6 \\
\hline \multicolumn{7}{|l|}{ Wealth quintile } \\
\hline First & 13.0 & 1.7 & 16.5 & 1.3 & 10.8 & 3.3 \\
\hline Second & 19.6 & 3.9 & 15.6 & 4.3 & 13.8 & 2.7 \\
\hline Third & 14.2 & 2.3 & 21.0 & 2.1 & 10.1 & 2.9 \\
\hline Fourth & 12.6 & 3.1 & 17.3 & 2.8 & 10.9 & 3.6 \\
\hline Fifth & 13.0 & 2.1 & 19.0 & 1.7 & 10.0 & 2.6 \\
\hline Total & 14.0 & 2.6 & 18.0 & 2.4 & 10.7 & 3.0 \\
\hline
\end{tabular}

Note: ( ) Based on 25-49 unweighted cases. NA: Not applicable. OBC: Other backward caste. SC: Scheduled caste. ST: Scheduled tribe. ${ }^{1}$ Includes all those not belonging to SC, ST or OBC. ${ }^{2}$ Includes non-literate and literate with no formal schooling. 


\subsubsection{Age at initiation of pre-marital sex}

Table 9.12 presents cumulative percentages of youth who experienced first pre-marital sex at selected ages (among all youth in the sample) calculated using life table techniques, with censoring taking place at the time of marriage for married youth and at the time of the survey for unmarried youth. For youth who reported pre-marital sex only through the anonymous sealed envelope method, age at first premarital sex was imputed conservatively, using age at marriage (for the married) and current age (for the unmarried) as age at initiation of pre-marital sex.

Several findings are notable. First, young men tended to initiate pre-marital sexual relations earlier than young women. Specifically, $7 \%$ of young men compared to $3 \%$ of young women had initiated first sex before age 18. Second, youth in rural areas were more likely to initiate pre-marital sexual relations earlier than their urban counterparts; for example, 6-15\% of rural youth compared to 3-9\% of urban youth had their sexual debut in adolescence. Third, findings indicate notable increases in initiation of pre-marital sexual activity as young people transitioned from early adolescence (before age 15) to late adolescence (before ages 18 and 20 ) to young adulthood (before ages 21 and 25). While just 1.5\% of young men and $0.7 \%$ of young women initiated pre-marital sexual relations in early adolescence (before age 15), 14\% of young men and 5\% of young women experienced first pre-marital sex before they transitioned out of adolescence (before age 20), and $27 \%$ of young men and $12 \%$ of young women had initiated pre-marital sexual relations before they transitioned into adulthood (before age 25).

The age-specific increase in cumulative percentages of those who had initiated pre-marital sexual relations was steeper among rural than among urban youth. Among rural young men, for example, while just $1.7 \%$ had experienced first sex in early adolescence (before age 15), as many as 15\% had experienced pre-marital sex before they transitioned out of adolescence (before age 20) and 29\% by the time they transitioned out of the youth ages to adulthood (before age 25). The corresponding percentages among urban youth were $0.4 \%$, $9 \%$ and 20\%, respectively. Although levels of pre-marital sex were lower among young women, the same pattern held true. Among rural women, $0.8 \%$ had initiated sex before age 15 and this percentage increased to $6 \%$ and further to $18 \%$ before ages 20 and 25 , respectively. Increases among young women in urban areas, in contrast, were relatively mild (from $0.3 \%$ before age 15 to $2.6 \%$ before age 20 and $3.3 \%$ before age 25 ).

Table 9.12: Age at initiation of pre-marital sex

\section{Cumulative percentage of youth by age at first pre-marital sexual experience, according to residence, Bihar, 2007}

\begin{tabular}{|c|c|c|c|c|c|c|}
\hline \multirow[t]{2}{*}{ Age at first pre-marital sex $(\%)^{1}$} & $\begin{array}{c}M \\
15-24\end{array}$ & $\begin{array}{c}\text { W } \\
15-24\end{array}$ & $\underset{15-24}{M}$ & $\begin{array}{c}\text { W } \\
15-24\end{array}$ & $\begin{array}{c}M \\
15-24\end{array}$ & $\begin{array}{c}\text { W } \\
15-24\end{array}$ \\
\hline & \multicolumn{2}{|c|}{ Combined } & \multicolumn{2}{|c|}{ Urban } & \multicolumn{2}{|c|}{ Rural } \\
\hline $\begin{array}{l}\text { First pre-marital sex occurred before age (years): } \\
15\end{array}$ & 1.5 & 0.7 & 0.4 & 0.3 & 1.7 & 0.8 \\
\hline 18 & 7.2 & 3.1 & 4.7 & 1.5 & 7.6 & 3.4 \\
\hline 20 & 14.1 & 5.3 & 8.8 & 2.6 & 15.1 & 6.0 \\
\hline 21 & 16.6 & 5.9 & 10.2 & 2.9 & 17.8 & 6.7 \\
\hline 25 & 27.3 & 11.9 & 19.6 & 3.3 & 28.9 & 18.1 \\
\hline Number of respondents & 1,942 & 5,529 & 1,039 & 2,581 & 903 & 2,948 \\
\hline
\end{tabular}

Note: All Ns are unweighted. ${ }^{1}$ Calculated using life table techniques. Age at first pre-marital sex among those who reported pre-marital sex only through the anonymous sealed envelope method was imputed conservatively, using age at marriage (for the married) and current age (for the unmarried). 


\subsubsection{Pre-marital sexual risk behaviours}

Table 9.13 presents findings relating to sexual risk behaviours of those reporting pre-marital sexual experiences, including multiple partner relations and inconsistent condom use. Findings confirm that where youth engaged in pre-marital sex, it was generally under unsafe conditions.

Sizeable proportions of sexually experienced youth had indeed engaged in sex with multiple partners before marriage; for example, one-fifth of young men reported two or more partners. While fewer young women reported that they had engaged in pre-marital sex, a relatively large proportion of these young women (28\%) reported multiple pre-marital partners. Among young men, the unmarried were more likely than the married to report multiple partner relationships (29\% versus $21 \%$ ); in contrast, the unmarried and the married were about as likely to report multiple partner relationships among young women. Unmarried youth were further probed about the number of partners with whom they had engaged in sex over the 12 months preceding the interview. One in six and one in eight sexually experienced unmarried young men and women, respectively, reported multiple sexual partners in the last year.

The Youth Study questionnaire probed consistent condom use only with regard to sex with the first and/ or most recent romantic partner, in exchange sex encounters, with sex workers and with married women. Information on condom use was not obtained for pre-marital sexual experiences with romantic partners other than the first or most recent, same-sex romantic partners, casual sex partners, spouse before marriage or among those who reported the experience of forced sex. Although few youth reported these latter relationships, we acknowledge that our consistent condom use indicator may not be comprehensive.

Table 9.13: Pre-marital sexual risk behaviours

\section{Percentage of sexually experienced youth who had pre-marital sex by number of partners and condom use, Bihar, 2007}

\begin{tabular}{|c|c|c|c|c|c|c|}
\hline Sexual behaviours (\%) & $\begin{array}{c}\mathrm{M} \\
15-24\end{array}$ & $\begin{array}{c}W \\
15-24\end{array}$ & $\begin{array}{c}\text { MM } \\
15-29\end{array}$ & $\begin{array}{c}\text { MW } \\
15-24\end{array}$ & $\begin{array}{c}\text { UM } \\
15-24\end{array}$ & $\begin{array}{l}\text { UW } \\
15-24\end{array}$ \\
\hline Total number of pre-marital sexual partners & & & & & & \\
\hline 1 & 78.2 & 72.3 & 79.2 & 72.1 & 70.6 & $(70.5)$ \\
\hline 2 or more & 21.8 & 27.7 & 20.8 & 27.9 & 29.4 & $(29.5)$ \\
\hline Consistent condom use with pre-marital sexual partners ${ }^{1}$ & 6.3 & 2.0 & 4.5 & 0.0 & 9.6 & $(5.0)$ \\
\hline $\begin{array}{l}\text { Number reporting pre-marital sex in face-to-face } \\
\text { interview }\end{array}$ & 206 & 99 & 159 & 50 & 113 & 49 \\
\hline Number of sexual partners in last 12 months & & & & & & \\
\hline None & NA & NA & NA & NA & 46.3 & $(30.0)$ \\
\hline 1 & NA & NA & NA & NA & 37.5 & $(58.3)$ \\
\hline 2 or more & NA & NA & NA & NA & 16.2 & $(11.7)$ \\
\hline Condom used at last pre-marital sex & NA & NA & NA & NA & 20.0 & $(13.3)$ \\
\hline $\begin{array}{l}\text { Number of unmarried respondents reporting pre-marital } \\
\text { sex in face-to-face interview }\end{array}$ & NA & NA & NA & NA & 113 & 49 \\
\hline
\end{tabular}

Note: All Ns are unweighted. NA: Not applicable. ${ }^{1}$ Questions on consistent condom use were asked only with regard to sexual relationships with first and/or most recent romantic partner, exchange sex partner, sex worker or married woman and excluded experiences with romantic partners other than first or most recent romantic partner, same-sex romantic partner, casual partner, spouse before marriage and experiences of forced sex. 
Findings suggest that among youth who reported pre-marital sex in the face-to-face interview, consistent condom use was extremely limited; only $6 \%$ of young men and $2 \%$ of young women reported that they had always used a condom. The unmarried were more likely than the married to report consistent condom use ( $10 \%$ versus $5 \%$ among young men and $5 \%$ versus $0 \%$ among young women). Condom use during the last pre-marital sexual encounter, assessed for unmarried respondents, suggests that only $20 \%$ of unmarried young men and $13 \%$ of unmarried young women reported condom use at last sex.

\subsubsection{Non-consensual sexual experiences}

The Youth Study questionnaire also probed the extent to which young people had experienced such nonconsensual sexual experiences as verbal harassment, non-consensual sexual touch or forced sex. In addition, young men were asked if they had ever verbally harassed a girl or perpetrated non-consensual sexual touch or forced sex. Findings on non-consensual sexual experiences are presented in Table 9.14. For the married, these refer to the period before marriage. We acknowledge that forced sex is an extremely sensitive issue and hence, very likely to have been under-reported.

Verbal sexual harassment was experienced by substantial minorities of young women $(23 \%)$ and few young men $(3 \%)$. Marital status differences were modest among young men, but wide among young women; $28 \%$ of unmarried young women compared to $19 \%$ of married young women reported having experienced verbal sexual harassment. Rural-urban differences suggest, moreover, that young women in urban settings were almost twice as likely to have reported the experience of verbal harassment as their rural counterparts (38\% in urban areas compared with $21 \%$ in rural areas). Rural-urban differences were muted among young men.

Non-consensual sexual touch was measured by questions that probed whether the respondent had ever been the victim of unwanted hugging or kissing in a sexual way, whether someone had touched their private parts without consent or had forced them to touch the perpetrator's private parts, and finally, whether someone had attempted to have sex with the respondent against her/his will using physical force or threats. As shown in Table 9.14, few respondents-3\%-admitted the experience of unwanted touch measured in these ways. Surprisingly, gender differences were not observed. Differences by marital status and rural-urban residence were negligible.

Questions on forced sex were posed in two ways: with regard to the consensual nature of first sex with a romantic opposite- or same-sex partner, on the one hand, and forced sex by any non-romantic partner, on the other. Even measured in this way, forced sex was rarely reported, that is, by $0.5 \%$ of young men and $0.6 \%$ of young women.

Young men's reports of perpetration of these acts are presented in Table 9.14. One-quarter of young men admitted that they had ever harassed a girl verbally. Moreover, one in eight young men admitted touching or brushing past a girl without her consent. Perpetration of verbal harassment and unwanted touch was about equally reported by both married and unmarried young men, and both urban and rural young men. Finally, $0.4 \%$ of young men reported that they had forced sex on a girl. 
Table 9.14: Pre-marital non-consensual sexual experiences

Percentage of youth reporting various pre-marital non-consensual sexual experiences, according to residence, Bihar, 2007

\begin{tabular}{|c|c|c|c|c|c|c|}
\hline Non-consensual sexual experiences (\%) & $\begin{array}{c}M \\
15-24\end{array}$ & $\begin{array}{c}\text { W } \\
15-24\end{array}$ & $\begin{array}{c}\text { MM } \\
15-29\end{array}$ & $\begin{array}{c}\text { MW } \\
15-24\end{array}$ & $\begin{array}{c}\text { UM } \\
15-24\end{array}$ & $\begin{array}{c}\text { UW } \\
15-24\end{array}$ \\
\hline \multicolumn{7}{|c|}{ Combined } \\
\hline \multicolumn{7}{|l|}{ Ever experienced } \\
\hline Verbal harassment & 3.3 & 22.6 & 2.1 & 19.0 & 3.7 & 28.4 \\
\hline Any non-consensual sexual touch ${ }^{1}$ & 2.7 & 3.4 & 2.2 & 2.9 & 2.9 & 4.1 \\
\hline Any forced sex & 0.5 & 0.6 & 0.4 & 0.7 & 0.5 & 0.4 \\
\hline \multicolumn{7}{|l|}{ Ever perpetrated the following: } \\
\hline Verbally harassed anyone ${ }^{2}$ & 24.0 & NA & 23.8 & NA & 23.5 & NA \\
\hline Touched or brushed past a girl ${ }^{2}$ & 12.4 & NA & 12.6 & NA & 11.6 & NA \\
\hline Forced sex on a girl & 0.4 & NA & 0.2 & NA & 0.5 & NA \\
\hline Number of respondents & 1,942 & 5,529 & 1,115 & 2,341 & 1,492 & 3,188 \\
\hline \multicolumn{7}{|c|}{ Urban } \\
\hline \multicolumn{7}{|l|}{ Ever experienced } \\
\hline Verbal harassment & 1.8 & 38.0 & 1.1 & 30.8 & 1.6 & 43.2 \\
\hline Any non-consensual sexual touch ${ }^{1}$ & 2.5 & 5.3 & 2.2 & 3.5 & 2.5 & 6.7 \\
\hline Any forced sex & 0.4 & 0.2 & 0.0 & 0.0 & 0.4 & 0.2 \\
\hline \multicolumn{7}{|l|}{ Ever perpetrated the following: } \\
\hline Verbally harassed anyone ${ }^{2}$ & 24.4 & NA & 28.3 & NA & 23.4 & NA \\
\hline Touched or brushed past a girl ${ }^{2}$ & 12.2 & NA & 14.1 & NA & 11.5 & NA \\
\hline Forced sex on a girl & 0.7 & NA & 0.0 & NA & 0.8 & NA \\
\hline Number of respondents & 1,039 & 2,581 & 547 & 1,136 & 833 & 1,445 \\
\hline \multicolumn{7}{|c|}{ Rural } \\
\hline \multicolumn{7}{|l|}{ Ever experienced } \\
\hline Verbal harassment & 3.6 & 20.6 & 2.2 & 18.2 & 4.1 & 25.7 \\
\hline Any non-consensual sexual touch ${ }^{1}$ & 2.8 & 3.1 & 2.2 & 2.9 & 3.0 & 3.7 \\
\hline Any forced sex & 0.5 & 0.6 & 0.4 & 0.7 & 0.6 & 0.4 \\
\hline \multicolumn{7}{|l|}{ Ever perpetrated the following: } \\
\hline Verbally harassed anyone ${ }^{2}$ & 24.0 & NA & 23.4 & NA & 23.5 & NA \\
\hline Touched or brushed past a girl ${ }^{2}$ & 12.4 & NA & 12.5 & NA & 11.6 & NA \\
\hline Forced sex on a girl & 0.3 & NA & 0.2 & NA & 0.4 & NA \\
\hline Number of respondents & 903 & 2,948 & 568 & 1,205 & 659 & 1,743 \\
\hline
\end{tabular}

Note: All Ns are unweighted. NA: Not applicable. ${ }^{1}$ Includes hugging in a sexual way, kissing in sexual way, touching of private parts and attempted forced sex. ${ }^{2}$ It is possible that married young men may have reported the occurrence of these events post-marriage since age at occurrence was not probed. 


\subsection{Triangulation of data on pre-marital sexual experiences among young people}

Acknowledging that young people may have been reluctant to disclose behaviours perceived as socially unacceptable such as pre-marital sex, the Youth Study included three approaches to elicit data on sexual behaviours. These were face-to-face interviews, anonymous reporting of respondents' own experiences via the sealed envelope and anonymous third-party reporting of peer experiences. Anonymous third-party reporting of peer experiences is a useful methodology by which to gauge sensitive behaviours that individuals are reluctant to express about themselves; findings are intended to shed light on behaviours of social networks and not necessarily the individual himself or herself (Rossier 2003).

We note that in anonymous third-party reporting, respondents may have reported as peers individuals whose ages fell outside our sample ages (15-24 and, in the case of married males, 15-29); therefore, in estimating pre-marital romantic and sexual experiences of young people using this reporting method, these were excluded. In addition, we recognise that in anonymous third-party reporting, friends reported by one respondent may also be reported by others. In estimating pre-marital romantic and sexual experiences of young people using this reporting method, our analysis sought to minimise chances that the experience of an individual belonging to more than one peer network would be included multiple times. Specifically, we inversely weighted the total sample of friends by the number of friends reported by each respondent. As a result, each respondent's network was given equal weight irrespective of its size.

Findings presented in Table 9.15 compare the levels of pre-marital romantic and sexual experiences obtained through these different approaches. Specifically, three indicators are presented: (a) percent reporting a premarital romantic relationship, (b) percent reporting the experience of pre-marital sex with a romantic opposite-sex partner, and (c) percent reporting any pre-marital sexual experience. For indicators a-b, we compare two sets of estimates derived from the face-to-face interview: respondents' reports of their own experiences as well as third-party reporting of the experiences of their peers. For indicator c, we compare three sets of estimates: any pre-marital sex as reported in the face-to-face format; any pre-marital sex among peers as assessed through anonymous third-party reporting; and any pre-marital sex as reported in the face-to-face interview supplemented by reports of pre-marital sexual experience recorded in the anonymous format, using the sealed envelope.

Comparisons indicate differences in reporting level by sex of the respondent and type of behaviour under consideration. In terms of pre-marital romantic relationships, anonymous third-party reporting yielded higher rates than did face-to-face reporting for young women, but not for young men (10\% and 5\%, respectively, among young women; $18 \%$ and $17 \%$, respectively, among young men). When analysed separately for the married and the unmarried, findings suggest that while anonymous third-party reporting provided higher estimates for married and unmarried young women and unmarried young men, the reverse was true for married young men, the group with perhaps the least to lose by admitting to a pre-marital romantic partnership.

As far as reporting of experience of pre-marital sex with a romantic partner was concerned, differences were narrower. Even so, young women were somewhat more likely to report sexual relations with a romantic partner among peers in the anonymous third-party reporting method than in the face-to-face interview. While the estimates derived from both methods were similar among the unmarried, these differed among the married. While the anonymous third-party reporting method provided higher estimates of sexual experiences with a romantic partner among peers than did the face-to-face interview for married young women, the reverse was true for married young men. 
Table 9.15: Levels of pre-marital romantic and sexual experiences by different reporting methods

Percentage of youth reporting pre-marital romantic relationships and percentage reporting sexual experiences within pre-marital romantic and other relationships by reporting method, according to residence, Bihar, 2007

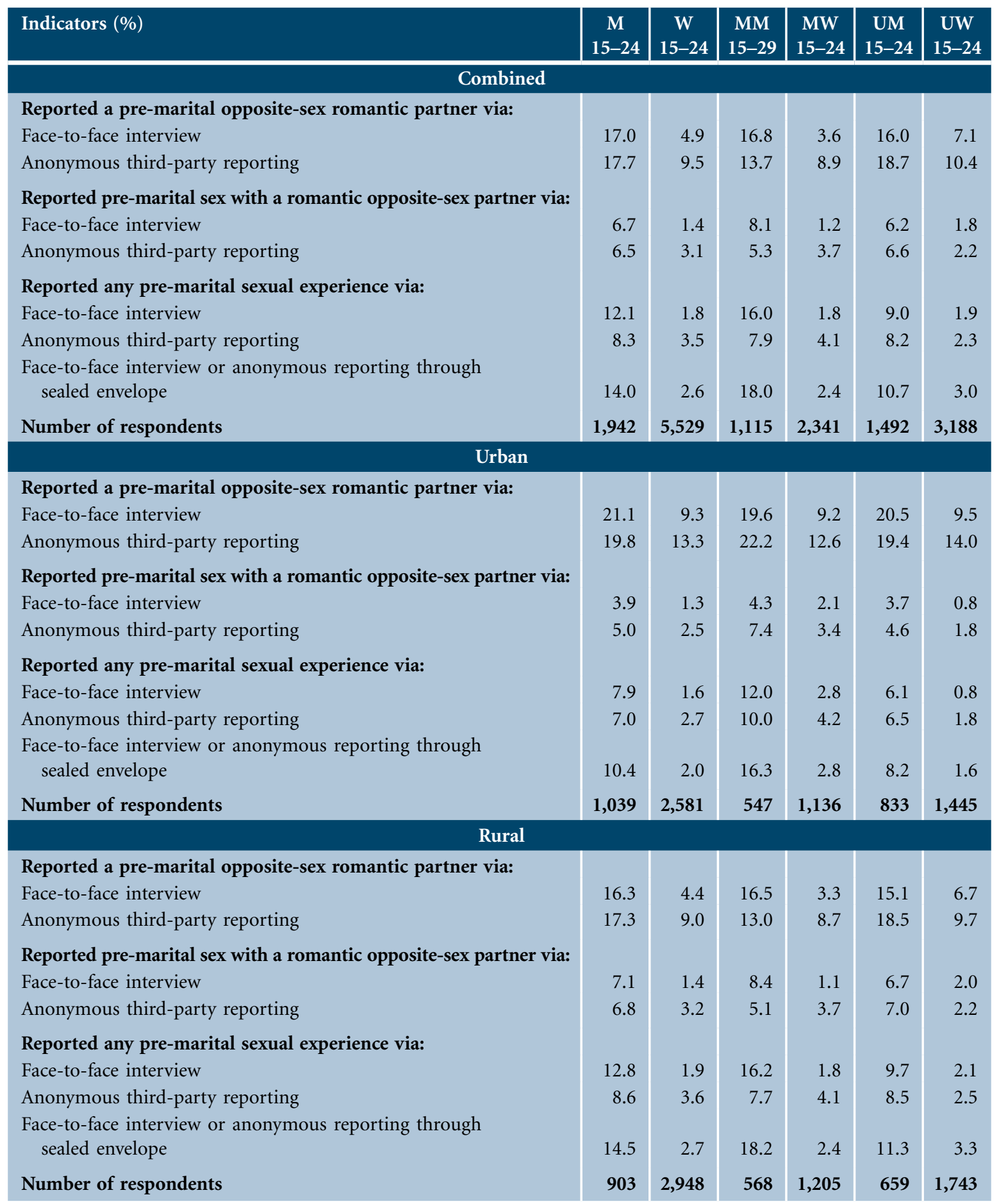

Note: All Ns are unweighted. Detailed information on friends' romantic and sexual experiences was collected for up to five of the respondent's closest same-sex friends. 
As far as reporting of any pre-marital sexual experience was concerned, patterns differed across young men and women. While anonymous third-party reporting of peer behaviours yielded rates lower than those selfreported in response to questions posed face-to-face for young men, the reverse was true for young women. As earlier, the estimates derived from both methods were similar among the unmarried; however, while the anonymous third-party reporting method provided higher estimates of pre-marital sexual experiences than did the face-to-face interview for married young women, the reverse was true for married young men. A comparison of any pre-marital sexual experience reported in face-to-face interviews and via the anonymous sealed envelope format suggests that some youth who had not admitted sexual experience in the face-to-face interview did so in the anonymous format: $2 \%$ of young men and less than $1 \%$ of young women. Indeed, $13 \%$ of young men and $27 \%$ of young women who reported sexual experience did so only in this more anonymous format (not shown in tabular form). Finally, it appears that self-reports of sexual experience, supplemented by self-reported experience in an anonymous format, provide consistently higher estimates of pre-marital sex among young men than does anonymous third-party reporting of pre-marital sexual experience among peers: for example, while $8 \%$ of young men reported pre-marital sex through anonymous third-party reporting, 14\% acknowledged the experience through direct reporting supplemented by anonymous reporting through the sealed envelope. Among young women, differences were negligible, but anonymous third-party reporting provided slightly higher estimates than self-reports (3.5\% and $2.6 \%$, respectively). The patterns remained more or less similar in both rural and urban areas.

\subsection{Summary}

Findings confirm that despite strict norms prohibiting pre-marital opposite-sex mixing, opportunities do exist for the formation of pre-marital romantic relations. Indeed, significant minorities of young men and women have received or made a "proposal" for a romantic relationship (20\% of young men and $12 \%$ of young women), and noteworthy, if smaller, percentages reported that they had been involved in a romantic partnership (17\% and $5 \%$ of young men and women, respectively). Patterns of pre-marital romantic partnerships suggest that where partnerships occurred, they were initiated at an early age and were usually hidden from parents but not from peers. Notable disparities in expectations of a longer-term commitment emerged that show that young women were considerably more likely than young men to have expected a romantic relationship to lead to marriage. The experiences of the married suggest, moreover, a disconnect between intentions and reality: among those who reported the intention to marry their pre-marital partner, $38 \%$ of young women and $8 \%$ of young men did so.

There is a clear progression in reported physical intimacy and sexual experience with romantic partners: while $87 \%$ of young men had held hands with a romantic partner, two-fifths had engaged in sexual relations; and among young women, while $70 \%$ had held hands with a romantic partner, 30\% had engaged in sexual relations with this partner. Partner communication and negotiation regarding safe sex were rare, and the vast majority had engaged in unprotected sex. Over one in five young women who had engaged in sexual relations with a romantic partner reported that their opposite-sex romantic partner had forced them to engage in sex the first time.

In total, $14 \%$ of young men and $3 \%$ of young women reported the experience of pre-marital sex within romantic and/or other partnerships. In general, first pre-marital sex took place earlier among young men than young women, and among rural than urban youth. Moreover, initiation into pre-marital sexual activity increased as young people transitioned from early to late adolescence and further as they transitioned into young adulthood. 
While sex with a romantic partner characterised pre-marital experiences for many of the sexually experienced young men and women, findings suggest that small proportions of young men also engaged in sex in other contexts. Other partners reported by them included, mainly, sex workers, married women and casual partners. Many of the pre-marital sexual experiences reported by youth were risky, for example, one-fifth of young men and one-quarter of young women reporting pre-marital sex had engaged in sex with more than one partner. Moreover, consistent condom use was extremely limited-only $6 \%$ of young men and $2 \%$ of young women reported condom use in all pre-marital encounters reported.

We acknowledge that youth, especially young women, may not report sexual experience in a survey situation. Hence, the Youth Study supplemented a series of direct questions with an opportunity to report sexual experience in an anonymous format. In total, direct questioning supplemented by self-reporting in an anonymous format provided considerably higher estimates of sexual experience among young men than did face-to-face questioning alone or anonymous third-party reporting of peer behaviours among young men. However among young women, anonymous third-party reporting provided slightly higher estimates than self-reports. 


\section{Transitions to marriage and} early married life

As is well known, the transition to marriage occurs early in India, both for young men and young women. The recent NFHS (IIPS and Macro International, 2007a) shows, for example, that in India, 47\% of young women aged 20-24 had married before the age of 18, the minimum legal age at marriage for females; $32 \%$ of young men aged 25-29 had, likewise, married before they reached the age of 21 , the legal minimum age at marriage for males. While marriage occurs early, marriage-related planning occurs even earlier, often as soon as a girl reaches menarche and, in many cases, even before she does so and without her participation. Moreover, while the consummation of marriage generally occurs following menarche, early married life tends to be isolated and frightening for many adolescent girls and young women. This chapter captures some of these experiences, including young people's preferences about the timing and type of marriage, marriage preparation and planning, and young people's participation in these processes, as well as their experiences in early married life and the fertility and contraceptive behaviours of young couples.

\subsection{Young people's preferences regarding timing and type of marriage}

The Youth Study sought to assess young people's preferences about the age at which to marry and, among the unmarried, their preferences for love or arranged marriages. While it is possible that youth who were married in adolescence might have reported the age at which they married as the preferred age, findings, presented in Table 10.1, indicate a gender divide in young people's preferences regarding timing of marriage. Most young women indicated a preference to marry in adolescence; almost one-quarter preferred to marry before 18 years and as many as three-fifths preferred to marry before age 20, that is, while still adolescent. In contrast, just $6 \%$ of young men preferred to marry before age 20 and over two-fifths of young men (44\%) preferred to marry at age 25 or later, a preference articulated by just $6 \%$ of young women.

Differentials by marital status and rural-urban residence of respondents were notable. Married youth were more likely than the unmarried to report that they preferred to marry before age 20 (71\% versus $45 \%$ among young women and $12 \%$ and $2 \%$ among young men). Conversely, more unmarried than married youth preferred to marry at age 25 or later (10\% versus $4 \%$ among young women, and $51 \%$ versus $32 \%$ among young men, respectively). A larger proportion of rural than urban young women expressed a preference to marry before age 20 ; for example, $65 \%$ of women in rural areas compared to $34 \%$ in urban areas. A smaller proportion of rural youth expressed a preference to marry late; for example, $40 \%$ of rural young men compared to $64 \%$ of urban young men preferred to marry at age 25 or later, as did $5 \%$ and $15 \%$, respectively, of young women.

Findings also show that almost all unmarried youth preferred to have an arranged rather than love marriage. For example, just $3-4 \%$ of young men and women reported that they would prefer a love marriage. Ruralurban differences were muted, nevertheless they suggest that urban youth were more likely than their rural counterparts to prefer a love marriage. 
Table 10.1: Preferences regarding timing and type of marriage

Percentage of youth reporting preferences regarding timing of marriage and percentage preferring a love marriage, according to residence, Bihar, 2007

\begin{tabular}{|c|c|c|c|c|c|c|}
\hline Indicators (\%) & $\begin{array}{c}\mathrm{M} \\
15-24\end{array}$ & $\begin{array}{c}\text { W } \\
15-24\end{array}$ & $\begin{array}{c}\text { MM } \\
15-29\end{array}$ & $\begin{array}{c}\text { MW } \\
15-24\end{array}$ & $\begin{array}{c}\text { UM } \\
15-24\end{array}$ & $\begin{array}{c}\text { UW } \\
15-24\end{array}$ \\
\hline \multicolumn{7}{|c|}{ Combined } \\
\hline \multicolumn{7}{|l|}{ Preferred to marry at age: } \\
\hline 17 or below & 1.4 & 23.8 & 3.0 & 32.0 & 0.5 & 9.4 \\
\hline 18 & 2.9 & 29.2 & 6.5 & 30.9 & 0.5 & 26.8 \\
\hline 19 & 1.8 & 8.2 & 2.6 & 7.7 & 1.0 & 9.1 \\
\hline 20 & 10.7 & 17.7 & 16.8 & 15.9 & 8.6 & 21.3 \\
\hline 21 & 9.8 & 5.5 & 9.6 & 4.1 & 9.3 & 8.0 \\
\hline 22 & 15.0 & 5.3 & 14.8 & 3.5 & 14.8 & 8.1 \\
\hline 23 & 6.1 & 1.5 & 6.2 & 0.6 & 5.8 & 3.1 \\
\hline 24 & 6.6 & 1.6 & 6.3 & 0.9 & 6.9 & 2.6 \\
\hline 25 or above & 43.8 & 6.0 & 31.9 & 3.5 & 50.5 & 10.0 \\
\hline Preferred not to marry & 1.9 & 1.2 & 2.4 & 1.0 & 2.1 & 1.6 \\
\hline Preferred a love marriage ${ }^{1}$ & NA & NA & NA & NA & 3.2 & 3.8 \\
\hline Number of respondents & 1,942 & 5,529 & 1,115 & 2,341 & 1,492 & 3,188 \\
\hline \multicolumn{7}{|c|}{ Urban } \\
\hline \multicolumn{7}{|l|}{ Preferred to marry at age: } \\
\hline 17 or below & 0.4 & 7.1 & 1.1 & 14.1 & 0.0 & 2.2 \\
\hline 18 & 1.4 & 19.7 & 5.4 & 29.6 & 0.4 & 12.6 \\
\hline 19 & 0.7 & 7.2 & 1.1 & 9.2 & 0.4 & 5.9 \\
\hline 20 & 5.7 & 18.6 & 13.0 & 21.1 & 4.1 & 16.8 \\
\hline 21 & 5.0 & 9.6 & 7.6 & 7.7 & 4.1 & 10.7 \\
\hline 22 & 10.3 & 11.0 & 12.0 & 7.7 & 10.2 & 13.4 \\
\hline 23 & 4.6 & 4.6 & 5.4 & 2.1 & 4.1 & 6.1 \\
\hline 24 & 6.0 & 5.3 & 7.6 & 2.1 & 6.1 & 7.7 \\
\hline 25 or above & 64.1 & 15.4 & 44.6 & 4.9 & 69.0 & 22.9 \\
\hline Preferred not to marry & 1.8 & 1.6 & 2.2 & 1.4 & 1.6 & 1.8 \\
\hline Preferred a love marriage ${ }^{1}$ & NA & NA & NA & NA & 6.1 & 6.7 \\
\hline Number of respondents & 1,039 & 2,581 & 547 & 1,136 & 833 & 1,445 \\
\hline \multicolumn{7}{|c|}{ Rural } \\
\hline \multicolumn{7}{|l|}{ Preferred to marry at age: } \\
\hline 17 or below & 1.6 & 26.0 & 3.1 & 33.1 & 0.6 & 10.8 \\
\hline 18 & 3.1 & 30.4 & 6.6 & 30.9 & 0.6 & 29.4 \\
\hline 19 & 2.0 & 8.3 & 2.7 & 7.6 & 1.1 & 9.7 \\
\hline 20 & 11.6 & 17.6 & 17.1 & 15.5 & 9.5 & 22.1 \\
\hline 21 & 10.6 & 5.0 & 9.8 & 3.9 & 10.3 & 7.5 \\
\hline 22 & 15.8 & 4.5 & 15.1 & 3.3 & 15.8 & 7.1 \\
\hline 23 & 6.3 & 1.1 & 6.3 & 0.5 & 6.1 & 2.5 \\
\hline 24 & 6.7 & 1.0 & 6.2 & 0.8 & 7.1 & 1.7 \\
\hline 25 or above & 40.4 & 4.8 & 30.7 & 3.4 & 46.8 & 7.7 \\
\hline Preferred not to marry & 2.0 & 1.2 & 2.4 & 1.0 & 2.1 & 1.6 \\
\hline Preferred a love marriage ${ }^{1}$ & NA & NA & NA & NA & 2.6 & 3.3 \\
\hline Number of respondents & 903 & 2,948 & 568 & 1,205 & 659 & 1,743 \\
\hline
\end{tabular}

Note: All Ns are unweighted. NA: Not applicable. ${ }^{1}$ Excludes those who reported a preference not to marry. 


\subsection{Marriage planning and extent of youth involvement}

Several questions were put to both married and unmarried youth to understand the process of marriage planning as well as their involvement in it. While questions on marriage planning were similar for the married and the unmarried, some questions were unique to one or the other group. For example, the Youth Study asked unmarried respondents whether their parents or family members had begun discussing plans for their marriage; and asked all married youth, and those unmarried youth for whom discussion had been initiated, about their age at that time and whether their parents sought their opinion about the age at which they wished to marry.

Findings presented in Table 10.2 indicate that marriage related discussions were initiated at young ages not only for young women but also for considerable proportions of young men. At the same time, findings reiterate vast gender differences in the age of young people when discussions were initiated regarding the planning of their marriages. Among those whose parents had ever initiated marriage-related discussion (almost all married youth and $37 \%$ and $76 \%$ of unmarried young men and women, respectively), findings indicate that four-fifths of all young women (82\%) and one-third of young men (35\%) reported that marriage-related discussion was initiated before age 18. Indeed, for $13 \%$ of young men and $61 \%$ of young women, such discussions were initiated as early as 15 years or below and for as many as $28 \%$ of young women, even as early as age 13. Marital status differences were muted for young men, but discussion was initiated earlier for currently married young women compared to the unmarried. For example, for $65 \%$ of married young women, compared to $37 \%$ of the unmarried, discussions were initiated at age 15 or earlier. Discussion on marriage was initiated earlier for rural respondents than urban; here too, wide differences were apparent between young women and young men.

Among those whose parents had initiated marriage-related discussion, young people were rarely consulted on the timing of marriage; even so, young men were more likely than young women to be consulted on such issues (28\% versus $5 \%$ ). Differences by marital status and rural-urban residence were observed (see Figure 10.1). For example, irrespective of the sex of respondents, a much smaller proportion of married than unmarried youth were consulted about when they wished to marry $(21 \%$ and $36 \%$, respectively, of married and unmarried young men; and $4 \%$ and $11 \%$ of young women, respectively), a finding that may be attributable to the more traditional characteristics of families of young people who were married earlier. Rural-urban differences suggest that a somewhat larger proportion of urban youth compared to rural youth reported that their opinions were sought ( $34 \%$ versus $27 \%$ among young men and $12 \%$ versus $4 \%$ among young women).

Figure 10.1: Percentage of youth reporting that their parents had ever sought their opinion on timing of marriage, according to residence, Bihar, 2007
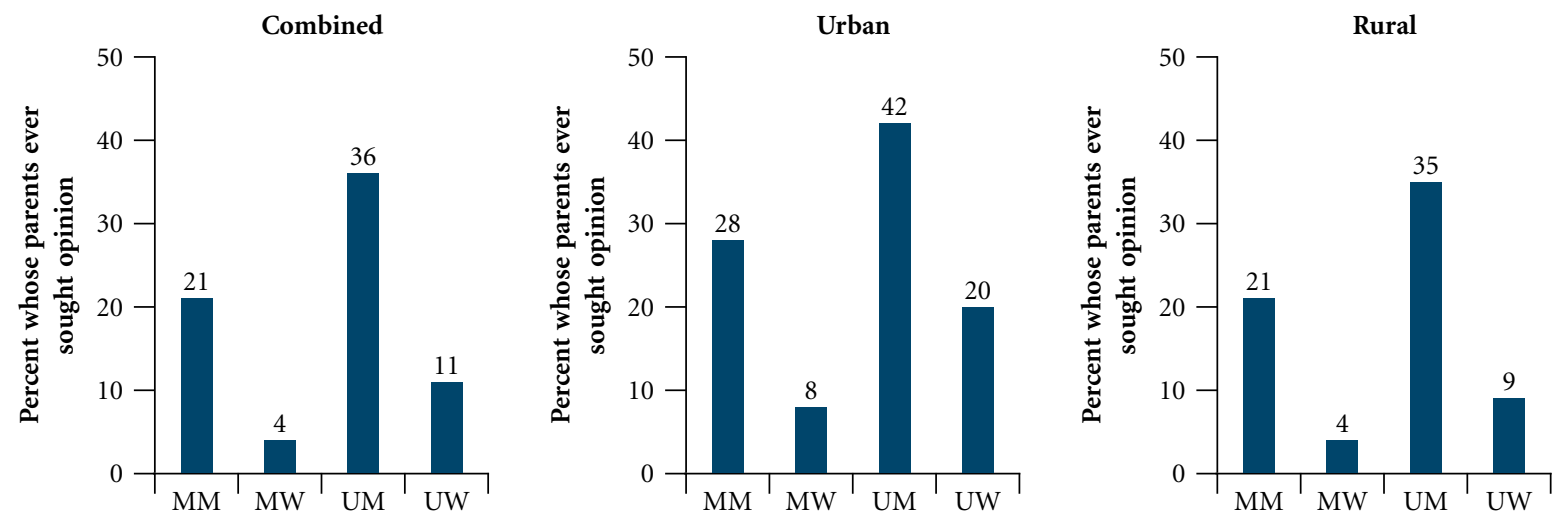

Note: Includes respondents whose parents had initiated marriage-related discussion. 
Table 10.2: Initiation of discussion on marriage and extent of youth involvement

Percentage of youth by age at initiation of marriage-related discussion, percentage whose opinion had been sought on timing of marriage and percentage who would find it difficult to tell parents if they did not like the match chosen, according to residence, Bihar, 2007

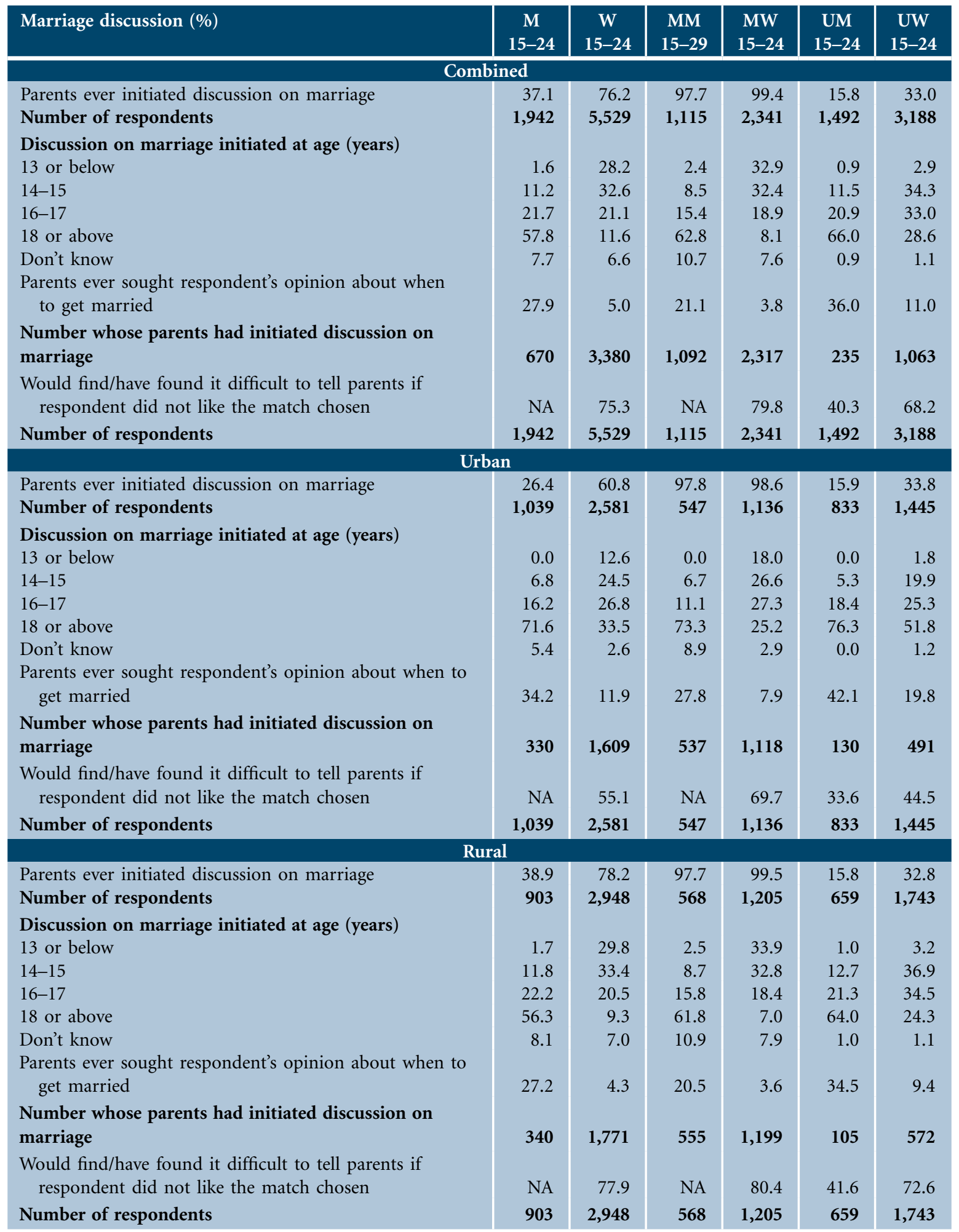

Note: All Ns are unweighted. Column totals may not equal 100\% due to missing cases. NA: Not applicable. 
The Youth Study also sought to assess the extent to which youth (other than married young men) perceived they could express or, among the married, could have expressed to their parents their preference not to marry the prospective spouse selected for them. Large proportions of youth perceived that it would be, or was difficult to oppose their parents if they did not approve of the match chosen for them. Although young women were more likely than unmarried young men to report difficulty, considerable percentages of unmarried young men also reported so (75\% of young women and $40 \%$ of unmarried young men). Marital status differences suggest that unmarried young women were less likely than the married to report difficulty in opposing their parents (68\% and $80 \%$, respectively). This difference may reflect the tendency for the married to report actual experiences and for many unmarried whose families had not yet initiated discussion to report perceptions. At the same time, it may reflect the fact that women who were married at younger ages may have come from more traditional backgrounds or, conversely, suggest a trend towards greater self-determination among the unmarried. Finally, and perhaps for reasons pertaining to the traditional nature of family life in rural areas, rural youth were more likely than their urban counterparts to report difficulty in confronting their parents on marriage-related issues ( $42 \%$ compared to $34 \%$ of unmarried young men; $78 \%$ compared to $55 \%$ of young women). In short, these findings confirm that large proportions of youth did not expect to play a role in decision-making with regard to their own marriage.

\subsection{Age at marriage and cohabitation}

Youth Study findings underscore the early age at marriage among young women in Bihar (Table 10.3). Of those aged $20-24$, as many as $46 \%$ of young women were married before age $15,77 \%$ before age 18 and $87 \%$ before age 20 . In rural areas, as many as $82 \%$ and $91 \%$ of women aged $20-24$ years were married before age 18 and 20, respectively; the corresponding percentages in urban areas were 45\% and 59\%. Findings from the NFHS-3, however, indicate that as many as $69 \%$ of 20-24 year-old women in Bihar were married before age 18 (IIPS and Macro International, 2008) Findings, moreover, indicate that even though there were indications of a decline in very early marriage (before age 15) among young women, sizeable numbers continued to marry before age 15: $46 \%$ of those aged 20-24 were married before age 15 compared to $25 \%$ of those aged 15-19.

Even though early marriage was less prevalent among young men, one in eight (13\%) young men aged 20-24 years were married before age 18 and almost one in three (31\%) before age 20 compared to $21 \%$ and $33 \%$, respectively, as reported in NFHS-3 (IIPS and Macro International, 2008). In rural areas, $15 \%$ and $34 \%$ of men aged 20-24 years were married before age 18 and 20, respectively.

The overwhelming majority of youth (99\% young men and women) had been married just once (not shown in tabular form). The mean age at marriage among those who were married was 19.6 years among young men and 14.7 years among young women. As expected, rural youth married earlier than urban youth; the mean age at marriage among rural youth was one-and-a-half years earlier than that of urban youth (19.5 years versus 20.9 years for young men and 14.6 years versus 16.2 years for young women). Mean age at cohabitation was one year later than mean age at marriage, suggesting that the practice of gauna was relatively common in Bihar.

\subsection{Marriage preparedness}

Several questions were put to both married and unmarried youth who were engaged to be married to understand their preparedness for marriage. Questions ranged from whether the proposed spouse was chosen by the young person or by his/her parents; whether the young person's approval of the prospective spouse was sought, if chosen by parents; and how much contact the young person and the prospective spouse had prior 
Table 10.3: Age at marriage and cohabitation

Percentage of youth aged 15-24 who were married before selected ages, percentage never married and mean age at marriage and cohabitation among those married, according to current age and residence, Bihar, 2007

\begin{tabular}{|c|c|c|c|c|c|c|c|}
\hline \multirow[t]{2}{*}{$\begin{array}{l}\text { Current age } \\
\text { (years) }(\%)\end{array}$} & \multicolumn{3}{|c|}{$\begin{array}{l}\text { Percentage first married before age } \\
\text { (years): }\end{array}$} & \multirow{2}{*}{$\begin{array}{c}\text { Percentage } \\
\text { never } \\
\text { married }\end{array}$} & \multicolumn{2}{|c|}{ Among those married: } & \multirow[t]{2}{*}{$\begin{array}{l}\text { Number of } \\
\text { respondents }\end{array}$} \\
\hline & 15 & 18 & 20 & & $\begin{array}{c}\text { Mean age } \\
\text { at marriage } \\
\text { (years) }\end{array}$ & $\begin{array}{c}\text { Mean age at } \\
\text { cohabitation } \\
\text { (years) }\end{array}$ & \\
\hline \multicolumn{8}{|c|}{ Combined } \\
\hline \multicolumn{8}{|l|}{ Men } \\
\hline $15-19$ & 1.7 & NA & NA & 92.2 & 16.0 & $(17.1)$ & 1,144 \\
\hline $20-24$ & 1.1 & 13.3 & 30.7 & 43.1 & 19.1 & 19.9 & 798 \\
\hline $15-24$ & 1.5 & NA & NA & 73.6 & $19.6^{1}$ & $20.6^{1}$ & 1,942 \\
\hline \multicolumn{8}{|l|}{ Women } \\
\hline $15-19$ & 24.8 & NA & NA & 53.4 & 14.4 & 15.3 & 3,615 \\
\hline $20-24$ & 46.0 & 77.0 & 87.3 & 7.9 & 14.9 & 15.9 & 1,914 \\
\hline $15-24$ & 33.4 & NA & NA & 35.0 & 14.7 & 15.7 & 5,529 \\
\hline \multicolumn{8}{|c|}{ Urban } \\
\hline \multicolumn{8}{|l|}{ Men } \\
\hline $15-19$ & 0.6 & NA & NA & 98.2 & * & * & 566 \\
\hline $20-24$ & 0.9 & 6.0 & 13.8 & 71.6 & 19.5 & 20.1 & 473 \\
\hline $15-24$ & 0.7 & NA & NA & 87.1 & $20.9^{1}$ & $21.6^{1}$ & 1,039 \\
\hline \multicolumn{8}{|l|}{ Women } \\
\hline $15-19$ & 7.2 & NA & NA & 79.9 & 15.2 & 15.8 & 1,447 \\
\hline $20-24$ & 17.2 & 45.3 & 59.1 & 29.2 & 16.6 & 17.2 & 1,134 \\
\hline $15-24$ & 11.5 & NA & NA & 58.1 & 16.2 & 16.9 & 2,581 \\
\hline \multicolumn{8}{|c|}{ Rural } \\
\hline \multicolumn{8}{|l|}{ Men } \\
\hline $15-19$ & 1.9 & NA & NA & 91.3 & $(16.0)$ & $(17.1)$ & 578 \\
\hline $20-24$ & 1.1 & 14.6 & 33.9 & 37.8 & 19.1 & 19.9 & 325 \\
\hline $15-24$ & 1.6 & NA & NA & 71.3 & $19.5^{1}$ & $20.5^{1}$ & 903 \\
\hline \multicolumn{8}{|l|}{ Women } \\
\hline $15-19$ & 27.0 & NA & NA & 50.1 & 14.4 & 15.3 & 2,168 \\
\hline $20-24$ & 50.1 & 81.5 & 91.2 & 5.0 & 14.7 & 15.8 & 780 \\
\hline $15-24$ & 36.2 & NA & NA & 32.0 & 14.6 & 15.6 & 2,948 \\
\hline
\end{tabular}

Note: All Ns are unweighted. ( ) Based on 25-49 unweighted cases. ${ }^{*}$ Mean not shown, based on fewer than 25 unweighted cases. NA: Not applicable due to censoring. ${ }^{1}$ Includes married men aged 25-29 years.

to marriage. As only $1 \%$ and $4 \%$ of unmarried young men and women, respectively, reported that they were engaged to be married (not shown in tabular form), we restrict our discussion to the currently married.

Table 10.4 describes marriage-related preparedness among the married. Almost all youth (99\%), irrespective of sex and place of residence, had married a partner chosen by their parents. While $88 \%$ of young men reported that their parents sought their approval while determining their marriage partners, only $56 \%$ of young women so reported. As many as one in ten young men and over two in five young women reported that their parents had not sought their approval. Just $1 \%$ of youth-3-4\% in urban areas and $1 \%$ in rural 
areas-reported having chosen their marriage partner on their own. Also indicative of lack of preparedness was the short gap between the point at which marriage arrangements were fixed and the date of the actual marriage: on average two months; about three in four respondents reporting the occurrence of their marriage within three months of the completion of marriage negotiations, irrespective of sex of the respondent (not shown in tabular form).

\section{Table 10.4: Marriage preparedness}

Percent distribution of married youth by type of marriage and selected indicators of their preparedness for marriage, according to residence, Bihar, 2007

\begin{tabular}{|c|c|c|c|c|c|c|}
\hline \multirow[t]{2}{*}{ Marriage indicators (\%) } & $\begin{array}{c}\mathrm{MM} \\
15-29\end{array}$ & $\begin{array}{c}\text { MW } \\
15-24\end{array}$ & $\begin{array}{c}\mathrm{MM} \\
15-29\end{array}$ & $\begin{array}{c}\text { MW } \\
\text { 15-24 }\end{array}$ & $\begin{array}{c}\text { MM } \\
15-29\end{array}$ & $\begin{array}{c}\text { MW } \\
15-24\end{array}$ \\
\hline & \multicolumn{2}{|c|}{ Combined } & \multicolumn{2}{|c|}{ Urban } & \multicolumn{2}{|c|}{ Rural } \\
\hline Type of marriage & & & & & & \\
\hline $\begin{array}{l}\text { Marriage fixed by respondent himself/herself } \\
\text { (love marriage) }\end{array}$ & 1.0 & 1.1 & 3.3 & 3.5 & 0.8 & 0.9 \\
\hline $\begin{array}{l}\text { Marriage arranged by parents, with respondent's } \\
\text { approval of choice of spouse }\end{array}$ & 88.4 & 55.8 & 89.1 & 57.7 & 88.5 & 55.7 \\
\hline Marriage fixed by parents without respondent's approval & 10.6 & 43.1 & 7.6 & 38.7 & 10.8 & 43.4 \\
\hline $\begin{array}{l}\text { Ever had a chance to meet/talk with fiancé/fiancée alone } \\
\text { Acquaintance with spouse before marrige }\end{array}$ & 7.1 & 3.5 & 12.0 & 7.8 & 6.7 & 3.2 \\
\hline Met on wedding day & 90.6 & 94.0 & 85.9 & 89.4 & 91.0 & 94.3 \\
\hline Knew somewhat before wedding day & 6.6 & 3.9 & 8.7 & 6.4 & 6.5 & 3.7 \\
\hline Knew well before wedding day & 2.8 & 2.1 & 5.4 & 4.3 & 2.5 & 1.9 \\
\hline Feelings about getting married & & & & & & \\
\hline Excited/looked forward to it & 48.7 & 9.0 & 52.2 & 12.7 & 48.3 & 8.7 \\
\hline Nothing special & 38.0 & 17.3 & 39.1 & 20.4 & 37.9 & 17.1 \\
\hline Very scared & 5.7 & 55.4 & 3.3 & 45.1 & 6.0 & 56.0 \\
\hline Anxious & 5.4 & 16.8 & 4.3 & 19.0 & 5.5 & 16.6 \\
\hline Unhappy & 2.1 & 1.6 & 1.1 & 2.8 & 2.2 & 1.5 \\
\hline Number of respondents & 1,115 & 2,341 & 547 & 1,136 & 568 & 1,205 \\
\hline Did not know what to expect of married life & 63.3 & 80.8 & 54.5 & 79.0 & 64.2 & 81.0 \\
\hline $\begin{array}{l}\text { Agree that youth do not get accurate information } \\
\text { about married life before marriage }\end{array}$ & 65.3 & 84.8 & 59.6 & 84.1 & 65.8 & 84.9 \\
\hline Number who had begun cohabiting & 1,072 & 2,236 & 525 & 1,102 & 547 & 1,134 \\
\hline
\end{tabular}

Note: All Ns are unweighted. Column totals may not equal 100\% due to missing cases or "don't know" responses.

Reported pre-marital acquaintance was extremely limited, and highlights that even those who reported that they had approved their parents' choice of spouse had rarely had an opportunity to meet the prospective spouse prior to marriage. Indeed, just $4-7 \%$ of youth reported that they had ever had a chance to meet and interact with their spouse-to-be alone prior to marriage. While gender differences were negligible, urban youth were somewhat more likely than their rural counterparts to report so (8-12\% versus $3-8 \%)$. Over $90 \%$ of married youth reported that they met their spouses for the first time on the wedding day. Just $4-7 \%$ youth reported that they knew their spouses only somewhat and 2-3\% reported that they knew their spouses well 
prior to marriage (see Figure 10.2). Rural-urban differences indicate that urban youth were somewhat more likely than their rural counterparts to report pre-marital acquaintance (11-14\% versus 6-9\%). In short, findings underscore the extent to which youth were excluded from marriage-related decision-making and were married to relative strangers.

Figure 10.2: Percent distribution of married youth by degree of acquaintance with future spouse before marriage, according to residence, Bihar, 2007

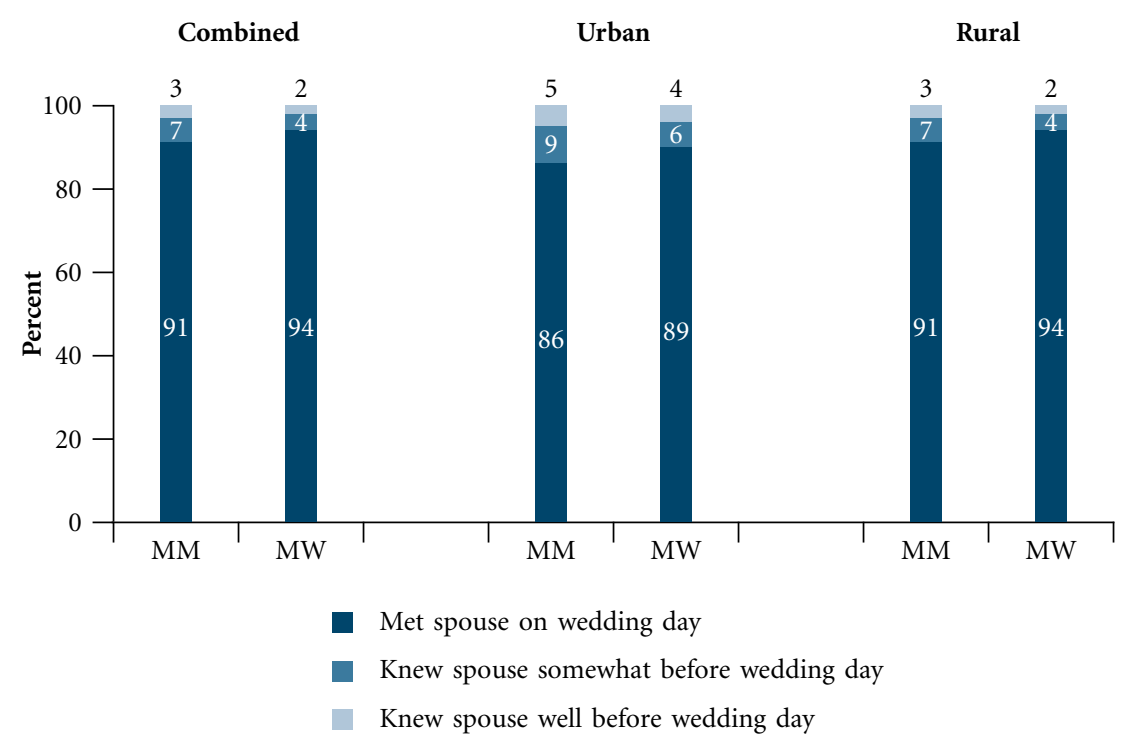

Note: Percentages may not equal 100.0 because of rounding.

Compounding this lack of pre-marital acquaintance, large proportions of youth-63\% of young men and $81 \%$ of young women - who had begun cohabiting with their spouses reported that they were unaware at the time of their marriage of what to expect of married life. Rural-urban differences were negligible among young women, but young men in urban areas $(55 \%)$ were considerably more likely than those in rural areas (64\%) to have reported as such. Two-thirds (65\%) of young men and over four-fifths (85\%) of young women reported that young people in general were poorly informed about married life prior to marriage, highlighting the need for family life or sex education and pre-marital counselling for young people. Somewhat more rural than urban young men so reported (66\% versus $60 \%$ ); the differences among young women were muted.

Commensurate with low levels of marriage preparedness, not all youth reported that they had looked forward to or had been excited about their marriages. Gender differences were pronounced: while $49 \%$ of young men said that they had been excited about their marriages, only $9 \%$ of young women so reported. Rural-urban differences were negligible. More than half of young women (55\%) reported that they had been very scared about getting married, compared to few $(6 \%)$ young men; more rural than urban young women so reported (56\% versus $45 \%)$.

\subsection{Payment of dowry}

Despite the existence of laws against dowry, Figure 10.3 shows that $68 \%$ of youth reported giving or taking dowry. Rural-urban differences in dowry reporting were modest among young men, but wide among young women; more urban than rural young women reported that their families had given dowry $(85 \%$ versus $67 \%$ ). These findings highlight that the practice of dowry remained just as strong among families of urban youth as among their rural counterparts. 
Figure 10.3: Percentage of married youth who reported receiving or giving dowry, according to residence, Bihar, 2007

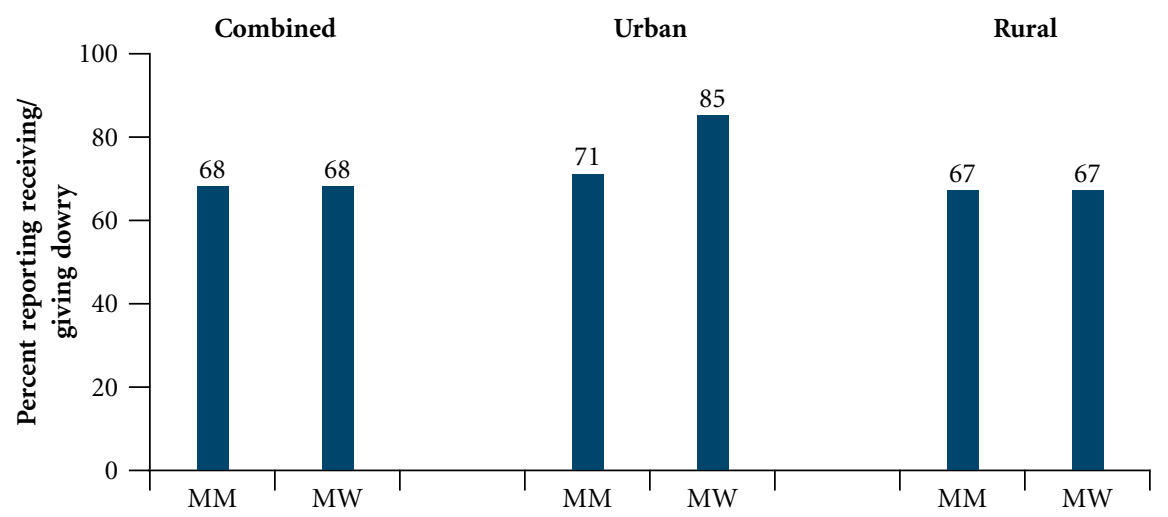

\subsection{Early marital experiences: Spousal communication and interaction}

Table 10.5 describes the extent of communication and interaction among young couples and confirms that communication even on everyday matters was far from universal (see also Figure 10.4). Between $78 \%$ and $88 \%$ of youth reported regularly discussing how to spend money and in-law issues. On both these matters, differences in reported communication by sex and place of residence of the respondent were negligible.

Likewise, $78-84 \%$ reported that they had ever communicated with their spouse on when and/or whether to have children or how many children to have, with little variation by sex or place of residence of the respondents. In contrast, discussion was more limited on the topic of contraception and notably, fewer young men $(38 \%)$ than women $(72 \%)$ reported that they had ever discussed contraception with their spouse. Rural-urban differences were somewhat wide; $46 \%$ of urban young men compared to $37 \%$ of rural young men reported such communication. The corresponding percentages among young women were $80 \%$ and $71 \%$, respectively.

Spousal interaction was also measured by questions regarding whether, in the six months preceding the interview, respondents had gone with their spouse to a movie, been on an outing or gone to their own (for young women) or wife's (for young men) natal home. Interaction of these types was clearly limited. Of the three places indicated, the majority had visited their own/ wife's natal home together with the spouse, but even this was not universal, reported by just $56 \%$ of young men and $45 \%$ of young women. Rural-urban differences were apparent, with urban youth somewhat more likely to report visiting their own/ wife's natal homes with the spouse than rural youth $(63 \%$ versus $56 \%$ among young men and $57 \%$ versus $45 \%$ among young women). Visiting places of entertainment was reported by relatively few youth, particularly young women. For example, while $37 \%$ of young men reported that they had been together with their wife on an outing (festival, picnic, etc.), only $15 \%$ of young women reported that they had done so with their husband; while $45 \%$ and $29 \%$ of urban young men and women, respectively, reported that they had been on an outing with their spouse, only $36 \%$ and $14 \%$ of rural young men and women, respectively, so reported.

Youth were also asked to assess their relative contentment with married life. Almost half of young men (47\%) and over one-quarter of young women (28\%) reported that they were very happy. Another half of young men $(49 \%)$ and two-thirds of young women (66\%) reported they were reasonably happy. 
Table 10.5: Early marital experiences

Percentage of married youth by selected characteristics of the marital relationship, according to residence, Bihar, 2007

\begin{tabular}{|c|c|c|c|c|c|c|}
\hline \multirow[t]{2}{*}{ Characteristics (\%) } & $\begin{array}{c}\text { MM } \\
15-29\end{array}$ & $\begin{array}{c}\text { MW } \\
15-24\end{array}$ & $\begin{array}{c}\text { MM } \\
15-29\end{array}$ & $\begin{array}{c}\text { MW } \\
15-24\end{array}$ & $\begin{array}{c}\text { MM } \\
15-29\end{array}$ & $\begin{array}{c}\text { MW } \\
15-24\end{array}$ \\
\hline & \multicolumn{2}{|c|}{ Combined } & \multicolumn{2}{|c|}{ Urban } & \multicolumn{2}{|c|}{ Rural } \\
\hline \multicolumn{7}{|l|}{ Usually communicates with spouse on: } \\
\hline How to spend money & 86.3 & 88.2 & 87.5 & 92.0 & 86.3 & 88.0 \\
\hline In-law issues & 79.6 & 78.1 & 80.9 & 78.1 & 79.4 & 78.1 \\
\hline \multicolumn{7}{|l|}{ Ever communicated with spouse on: } \\
\hline When/whether to have a baby & 77.9 & 84.0 & 79.5 & 88.4 & 77.8 & 83.7 \\
\hline Number of children to have & 79.7 & 83.2 & 80.7 & 88.4 & 79.6 & 82.9 \\
\hline Contraceptive use & 37.5 & 71.6 & 46.1 & 79.7 & 36.7 & 71.0 \\
\hline \multicolumn{7}{|l|}{ Went with spouse to the following in last 6 months: } \\
\hline Theatre/video parlour & 10.2 & 6.7 & 22.7 & 19.0 & 9.0 & 5.9 \\
\hline Festival/yatra/tamasha/play/tour/picnic/restaurant & 36.8 & 15.3 & 44.9 & 29.2 & 36.1 & 14.3 \\
\hline Woman's/wife's natal home & 56.3 & 45.3 & 62.5 & 56.5 & 55.7 & 44.5 \\
\hline \multicolumn{7}{|l|}{ Assessment of married life } \\
\hline Very happy & 46.6 & 27.7 & 46.6 & 34.8 & 46.7 & 27.2 \\
\hline Reasonably happy & 49.1 & 65.7 & 50.0 & 58.7 & 48.9 & 66.1 \\
\hline Unhappy & 2.9 & 3.5 & 2.3 & 3.6 & 3.0 & 3.5 \\
\hline Very unhappy & 1.4 & 3.1 & 1.1 & 2.9 & 1.4 & 3.1 \\
\hline Number who had begun cohabiting & 1,072 & 2,236 & 525 & 1,102 & 547 & 1,134 \\
\hline
\end{tabular}

Note: All Ns are unweighted. Column totals may not equal 100\% due to missing cases or "don't know" responses.

Figure 10.4: Percentage of married youth who reported spousal communication on selected topics, according to residence, Bihar, 2007

Combined

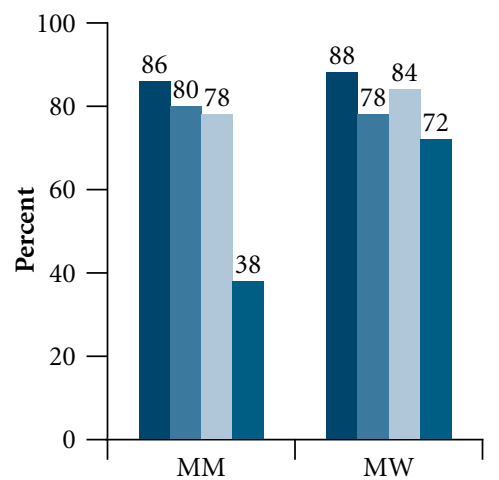

How to spend money

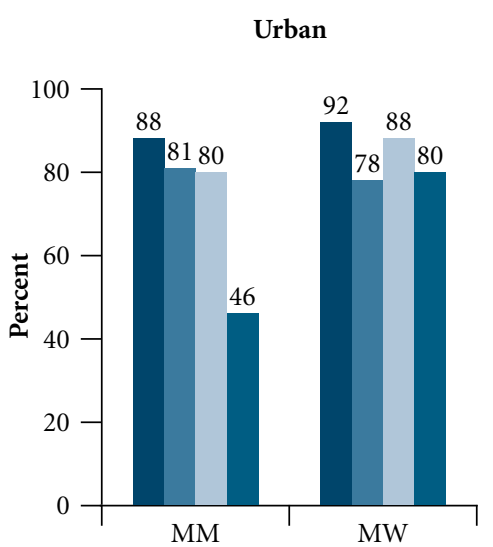

In-law issues

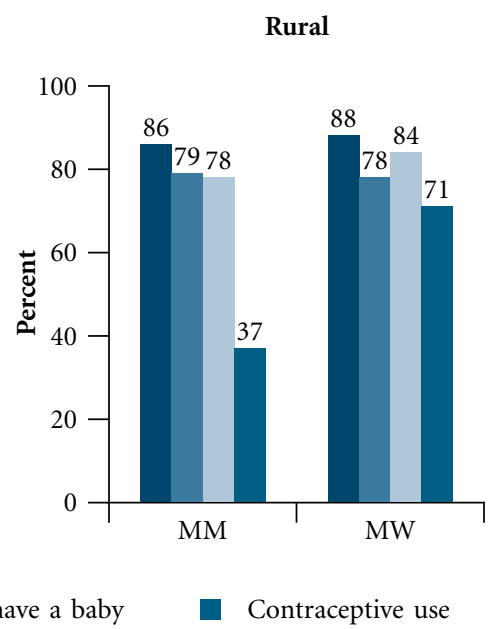




\subsection{Nature of marital sexual experiences}

In several previous studies, significant minorities of young women reported the experience of forced sex within marriage, including at initiation (see for example, Santhya and Jejeebhoy, 2006; Santhya et al., 2007). The Youth Study explored the extent to which early marital sexual experiences were enjoyable or forced. Findings, presented in Table 10.6, suggest that while virtually all young men reported enjoying their first marital sexual experience, far fewer young women so reported: $97 \%$ compared to $32 \%$. While rural-urban differences were muted among young men, somewhat more urban than rural young women reported enjoying the first sexual experience. A comparison of responses to questions regarding whether the spouse enjoyed the first sexual experience suggests that young men may have been unaware of their wives' negative experience and that young women may have been unaware of their husbands' positive experience: $49 \%$ of young men perceived that their wives had enjoyed the first sexual experience (compared to $32 \%$ of young women who reported that they had enjoyed the first sexual experience) and $51 \%$ young women perceived that their husbands had enjoyed the experience (far less than that reported by men themselves (97\%).

For many young women, the first marital sexual experience was painful or non-consensual; many fewer young men, however, perceived that the first experience was painful or non-consensual for their wives. For example, while over four-fifths of young women reported that the experience was painful, just half of young men reported that the experience had been painful for their wives.

Forced sex at initiation was reported by almost half of young women (49\%) with somewhat larger proportions of rural than urban women reporting so ( $49 \%$ and $43 \%$, respectively). In contrast, only $13 \%$ of young men reported that they had forced their wives to engage in sex the first time, with little rural-urban variation. Over the course of their marital lives, somewhat more young people acknowledged the experience (young women) or perpetration (young men) of forced sex within marriage: 54\% of young women reported that they had ever experienced forced sex within marriage and $25 \%$ of young men reported that they had ever perpetrated it. Rural-urban differences were modest. Recent experience of such an incident, that is, within the 12 months preceding the interview, was reported by $11 \%$ of young men and $27 \%$ of young women who had cohabited for at least one year, with only modest rural-urban differences.

Table 10.6: Sexual experiences within marriage

Percentage of married youth by nature of first and lifetime sexual experiences with spouse, according to residence, Bihar, 2007

\begin{tabular}{|c|c|c|c|c|c|c|}
\hline \multirow[t]{2}{*}{ Experiences (\%) } & $\begin{array}{c}\text { MM } \\
15-29\end{array}$ & $\begin{array}{c}\text { MW } \\
15-24\end{array}$ & $\begin{array}{c}\text { MM } \\
15-29\end{array}$ & $\begin{array}{c}\text { MW } \\
15-24\end{array}$ & $\begin{array}{c}\text { MM } \\
15-29\end{array}$ & $\begin{array}{c}\text { MW } \\
15-24\end{array}$ \\
\hline & \multicolumn{2}{|c|}{ Combined } & \multicolumn{2}{|c|}{ Urban } & \multicolumn{2}{|c|}{ Rural } \\
\hline Nature of first sexual experience with spouse & & & & & & \\
\hline Respondent enjoyed it & 97.2 & 32.2 & 97.8 & 38.4 & 97.1 & 31.8 \\
\hline Spouse enjoyed it & 49.3 & 50.6 & 56.2 & 49.3 & 48.6 & 50.7 \\
\hline Wife cried & 40.5 & 67.9 & 42.0 & 57.7 & 40.4 & 68.6 \\
\hline Painful for wife & 49.8 & 87.1 & 51.1 & 84.1 & 49.7 & 87.3 \\
\hline Wife unwilling and husband forced her & 13.1 & 48.7 & 10.2 & 43.2 & 13.3 & 49.0 \\
\hline Husband ever forced wife to have sex & 24.8 & 53.7 & 21.3 & 50.0 & 25.1 & 54.0 \\
\hline Number who had begun cohabiting & 1,072 & 2,236 & 525 & 1,102 & 547 & 1,134 \\
\hline Husband forced wife to have sex in last 12 months & 11.0 & 27.3 & 7.7 & 30.6 & 11.3 & 27.0 \\
\hline Number who had cohabited for at least 12 months & 955 & 2,017 & 462 & 990 & 493 & 1,027 \\
\hline
\end{tabular}

Note: All Ns are unweighted. 


\subsection{Experience of domestic violence within marriage}

The Youth Study explored the extent of domestic violence or verbal abuse perpetrated by young men on their wives and in less detail, by women on their husbands. Young men's and women's reports of both overall levels of violence and types of violence experienced or perpetrated were remarkably similar.

Both young men and women agreed that women rarely humiliate or perpetrate violence on their husbands. Table 10.7 shows that $5 \%$ or fewer young women had either verbally humiliated their husbands in the presence of others or perpetrated violence on them in any way. No differences were observed by respondents' sex or place of residence.

In contrast, considerably larger proportions of young men were reported to have humiliated their wives verbally in the presence of others or perpetrated physical violence in some form on their wives. Verbal humiliation was reported by a minority ( $4 \%$ and $8 \%$ of young men and women, respectively). Many more-over one-quarter of youth (30\%)-reported the experience (women) or perpetration (men) of some form of physical violence, with somewhat more rural youth reporting the experience (women) or perpetration (men) than urban youth (30\% of rural youth versus $24-25 \%$ of urban youth) (see also Figure 10.5).

Of all forms of physical violence, slapping was most commonly reported (29\%), followed by twisting of the wife's arm or pulling her hair (12-15\%). Between $7 \%$ and $9 \%$ of young women also reported the experience of being pushed, shaken or having something thrown at them, punched, kicked, dragged or beaten; somewhat fewer, between 3\% and 7\% of young men reported perpetrating these forms of violence on their wives. It is notable that $2 \%$ of young women reported that they had been burnt or choked by their husbands. Also notable is the finding that $14 \%$ of young men reported perpetrating more than one form of violence on their wives and $16 \%$ of young women reported experiencing more than one form of violence at the hands of their husbands.

Perpetration or experience of any form of physical violence within marriage in the 12 months preceding the interview was reported by $19 \%$ of young men and $24 \%$ of young women. As earlier, slapping was most commonly reported; $17 \%$ of young men reported slapping their wives and $23 \%$ of young women reported being slapped by their husbands in the 12 months preceding the interview.

Figure 10.5 Percentage of married young women reporting experience of physical violence perpetrated by their husband and percentage of married young men reporting perpetration of physical violence against their wife, according to residence, Bihar, 2007

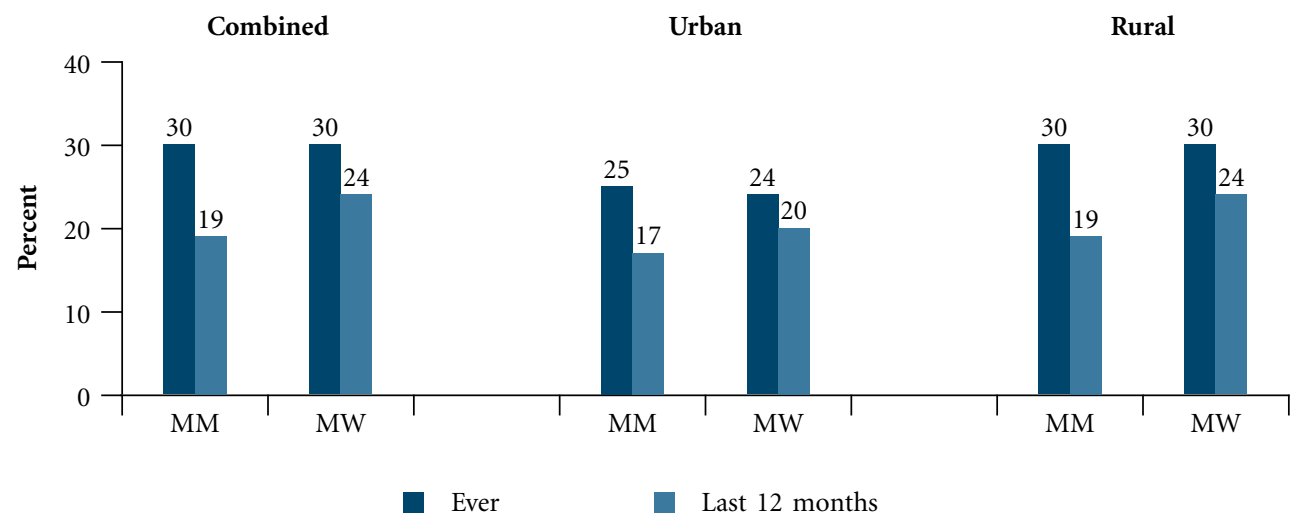


Table 10.7: Domestic violence within marriage

Percentage of married youth reporting experience of verbal abuse or physical violence within marriage by type of violence, according to residence, Bihar, 2007

\begin{tabular}{|c|c|c|c|c|c|c|}
\hline \multirow[t]{2}{*}{ Types of violence $(\%)$} & $\begin{array}{c}\text { MM } \\
15-29\end{array}$ & $\begin{array}{c}\text { MW } \\
15-24\end{array}$ & $\begin{array}{c}\text { MM } \\
15-29\end{array}$ & $\begin{array}{l}\text { MW } \\
15-24\end{array}$ & $\begin{array}{c}\text { MM } \\
15-29\end{array}$ & $\begin{array}{c}\text { MW } \\
15-24\end{array}$ \\
\hline & \multicolumn{2}{|c|}{ Combined } & \multicolumn{2}{|c|}{ Urban } & \multicolumn{2}{|c|}{ Rural } \\
\hline \multicolumn{7}{|c|}{ A. Verbal abuse or physical violence perpetrated by wife } \\
\hline Wife verbally abused husband in the presence of others & 3.9 & 4.5 & 3.4 & 5.1 & 4.1 & 4.5 \\
\hline Wife ever perpetrated any physical violence on husband & 0.4 & 0.5 & 0.0 & 0.7 & 0.4 & 0.5 \\
\hline $\begin{array}{l}\text { Wife perpetrated any physical violence on husband in } \\
\text { last } 12 \text { months }\end{array}$ & 0.2 & 0.1 & 0.0 & 0.0 & 0.2 & 0.1 \\
\hline Number who had begun cohabiting & 1,072 & 2,236 & 525 & 1,102 & 547 & 1,134 \\
\hline \multicolumn{7}{|c|}{ B. Verbal abuse or physical violence perpetrated by husband } \\
\hline Husband verbally abused wife in the presence of others & 3.5 & 7.8 & 3.4 & 8.0 & 3.7 & 7.7 \\
\hline Physical violence ever perpetrated by husband & & & & & & \\
\hline Slapped wife & 28.8 & 29.2 & 24.7 & 23.9 & 29.2 & 29.6 \\
\hline Twisted wife's arm or pulled her hair & 11.5 & 14.9 & 11.2 & 13.0 & 11.5 & 15.0 \\
\hline Pushed/shook or threw something at wife & 7.2 & 9.4 & 5.6 & 9.4 & 7.4 & 9.4 \\
\hline Punched wife & 5.0 & 7.0 & 2.3 & 7.2 & 5.3 & 7.0 \\
\hline Kicked, dragged or beat wife & 2.6 & 7.0 & 1.1 & 6.5 & 2.7 & 7.1 \\
\hline Choked or burnt wife on purpose & 0.4 & 2.0 & 0.0 & 2.2 & 0.4 & 2.0 \\
\hline Threatened or attacked wife with knife/gun & 0.2 & 0.5 & 0.0 & 0.0 & 0.2 & 0.5 \\
\hline $\begin{array}{l}\text { Perpetrated/experienced at least one of the above } \\
\text { forms of violence }\end{array}$ & 29.7 & 29.7 & 25.0 & 23.9 & 30.1 & 30.1 \\
\hline $\begin{array}{l}\text { Perpetrated/experienced more than one of the above } \\
\text { forms of violence }\end{array}$ & 13.9 & 16.4 & 11.4 & 14.5 & 14.1 & 16.6 \\
\hline \multirow{2}{*}{\multicolumn{7}{|c|}{$\begin{array}{l}\text { Experience of violence perpetrated by husband in } \\
\text { last } 12 \text { months } \\
\text { Slapped wife }\end{array}$}} \\
\hline & & & & & & \\
\hline Never & 82.5 & 77.3 & 83.1 & 81.2 & 82.4 & 77.0 \\
\hline Sometimes & 16.9 & 20.6 & 15.7 & 17.4 & 17.0 & 20.8 \\
\hline Often & 0.0 & 2.0 & 0.0 & 1.4 & 0.0 & 2.0 \\
\hline \multicolumn{7}{|l|}{ Twisted wife's arm or pulled her hair } \\
\hline Never & 92.8 & 87.7 & 93.2 & 89.9 & 92.9 & 87.5 \\
\hline Sometimes & 6.8 & 10.6 & 6.8 & 8.7 & 6.7 & 10.7 \\
\hline Often & 0.0 & 1.6 & 0.0 & 1.4 & 0.0 & 1.6 \\
\hline \multicolumn{7}{|l|}{ Pushed/shook or threw something at wife } \\
\hline Never & 96.5 & 92.1 & 96.6 & 92.8 & 96.4 & 92.0 \\
\hline Sometimes & 3.5 & 6.8 & 3.4 & 6.5 & 3.6 & 6.8 \\
\hline Often & 0.0 & 0.8 & 0.0 & 0.7 & 0.0 & 0.8 \\
\hline \multicolumn{7}{|l|}{ Punched wife } \\
\hline Never & 97.3 & 94.4 & 98.9 & 94.2 & 97.2 & 94.4 \\
\hline Sometimes & 2.7 & 5.0 & 1.1 & 5.1 & 2.8 & 5.0 \\
\hline Often & 0.0 & 0.6 & 0.0 & 0.7 & 0.0 & 0.6 \\
\hline
\end{tabular}


Table 10.7: (Cont'd)

\begin{tabular}{|c|c|c|c|c|c|c|}
\hline \multirow[t]{2}{*}{ Types of violence (\%) } & $\begin{array}{c}\text { MM } \\
15-29\end{array}$ & $\begin{array}{c}\text { MW } \\
15-24\end{array}$ & $\begin{array}{c}\text { MM } \\
15-29\end{array}$ & $\begin{array}{c}\text { MW } \\
15-24\end{array}$ & $\begin{array}{c}\text { MM } \\
15-29\end{array}$ & $\begin{array}{c}\text { MW } \\
15-24\end{array}$ \\
\hline & \multicolumn{2}{|c|}{ Combined } & \multicolumn{2}{|c|}{ Urban } & \multicolumn{2}{|c|}{ Rural } \\
\hline \multicolumn{7}{|c|}{ B. Verbal abuse or physical violence perpetrated by husband } \\
\hline \multicolumn{7}{|l|}{ Kicked, dragged or beat wife } \\
\hline Never & 99.0 & 94.3 & 100.0 & 94.9 & 98.9 & 94.3 \\
\hline Sometimes & 1.0 & 5.1 & 0.0 & 4.3 & 1.1 & 5.1 \\
\hline Often & 0.0 & 0.6 & 0.0 & 0.7 & 0.0 & 0.6 \\
\hline \multicolumn{7}{|l|}{ Choked or burnt wife on purpose } \\
\hline Never & 100.0 & 98.7 & 100.0 & 98.6 & 100.0 & 98.7 \\
\hline Sometimes & 0.0 & 1.0 & 0.0 & 1.4 & 0.0 & 1.0 \\
\hline Often & 0.0 & 0.3 & 0.0 & 0.0 & 0.0 & 0.3 \\
\hline \multicolumn{7}{|l|}{ Threatened or attacked wife with knife/gun } \\
\hline Never & 100.0 & 99.7 & 100.0 & 100.0 & 100.0 & 99.7 \\
\hline Sometimes & 0.0 & 0.2 & 0.0 & 0.0 & 0.0 & 0.2 \\
\hline Often & 0.0 & 0.1 & 0.0 & 0.0 & 0.0 & 0.1 \\
\hline $\begin{array}{l}\text { Perpetrated/experienced at least one of the above for } \\
\text { of violence in last } 12 \text { months }\end{array}$ & 18.7 & 23.6 & 17.0 & 19.6 & 18.8 & 23.9 \\
\hline Number who had begun cohabiting & 1,072 & 2,236 & 525 & 1,102 & 547 & 1,134 \\
\hline Experienced violence in first 12 months of marriage & 8.4 & 9.4 & 7.8 & 9.8 & 8.5 & 9.3 \\
\hline Number who had cohabited for at least 12 months & 955 & 2,017 & 462 & 990 & 493 & 1,027 \\
\hline
\end{tabular}

Note: All Ns are unweighted. Column totals may not equal 100\% due to missing cases or "don't know" responses.

Findings on the duration between marriage and the first occurrence of physical violence (among those who had cohabited for at least one year) indicate that a small minority of youth (8-9\%) reported perpetration or experience of physical violence within a year of marriage. Rural-urban differences were muted.

\subsection{Extent and nature of extra-marital sexual relations}

The Youth Study did not probe as extensively about extra-marital sexual experiences as it did about premarital sex, discussed in Chapter 9. A single direct question was asked to all married youth about whether they had experienced sexual relations with someone other than their spouse following marriage. In addition, youth reporting same-sex, exchange, forced or sex worker sex were probed about the timing of the first such encounter; for very few, it occurred following marriage. Given the lack of extensive probing, we caution readers that percentages of youth reporting extra-marital sexual experience, indicated in Table 10.8, may be particularly under-reported.

Hardly any $(0.4 \%)$ young women reported an extra-marital sexual encounter. In contrast, $4 \%$ of young men-including $3 \%$ of urban young men and $4 \%$ of rural young men-reported an extra-marital sexual encounter. Among young men, $1 \%$ reported extra-marital sex in the one year (or months since marriage for those married for less than one year) preceding the interview. 
Table 10.8: Extent of extra-marital sexual experiences

Percentage of married youth by extent of extra-marital sexual experiences, according to residence,
Bihar, 2007
\begin{tabular}{|l|r|r|r|r|r|r|r|}
\hline Experiences (\%) & MM & MW & MM & MW & MM & MW \\
& $\mathbf{1 5 - 2 9}$ & $\mathbf{1 5 - 2 4}$ & $\mathbf{1 5 - 2 9}$ & $\mathbf{1 5 - 2 4}$ & $\mathbf{1 5 - 2 9}$ & $\mathbf{1 5 - 2 4}$ \\
\cline { 2 - 8 } & Combined & \multicolumn{2}{|c|}{ Urban } & \multicolumn{3}{|c|}{ Rural } \\
\hline $\begin{array}{l}\text { Had sex with someone other than spouse after marriage } \\
\text { Reported at least one extra-marital sexual partner in last }\end{array}$ & 3.7 & 0.4 & 3.4 & 0.0 & 3.9 & 0.4 \\
$\quad 12$ months & 0.9 & 0.2 & 1.1 & 0.0 & 0.9 & 0.2 \\
Number who had begun cohabiting & $\mathbf{1 , 0 7 2}$ & $\mathbf{2 , 2 3 6}$ & $\mathbf{5 2 5}$ & $\mathbf{1 , 1 0 2}$ & $\mathbf{5 4 7}$ & $\mathbf{1 , 1 3 4}$
\end{tabular}

Note: All Ns are unweighted.

\subsection{Contraceptive practice within marriage: Lifetime, current and prior to first pregnancy}

The practise of contraception at any time during marital life was limited, reported by $23 \%$ of young men and $20 \%$ of young women (see Table 10.9 and Figure 10.6). Urban youth were more likely than rural youth to report ever use of contraceptives (33\% versus $22 \%$ among young men and $31 \%$ versus $19 \%$ among young women). Somewhat fewer youth reported ever use of modern contraceptive methods ( $21 \%$ of young men and $15 \%$ of young women); $28-32 \%$ of urban youth and $14-20 \%$ of rural youth reported as such. Modern methods most likely to have been reported by both men and women were condoms (reported by $15 \%$ of young men and $8 \%$ of young women) and oral pills (reported by $7 \%$ of young men and $4 \%$ of young women). Despite the young age of female respondents, some $4 \%$ of young women and $3 \%$ of young men reported they/their wives had been sterilised. Traditional contraceptive method use was somewhat more likely to be reported by young women than men ( $6 \%$ versus $3 \%$ ). Rural-urban differences in methods ever used were negligible, except that more urban than rural youth reported ever use of condoms (17-26\% versus $8-14 \%)$.

Relatively fewer youth (12\%) reported practising contraception at the time of the interview, with urban youth were more likely than their rural counterparts to report current contraceptive use (21\% versus $11 \%)$. Condoms and female sterilisation were the leading methods reported (6\% and $3 \%$ of young men, respectively and $3 \%$ and $4 \%$ of young women, respectively). Young women, in addition, also reported practice of traditional methods (3\%). Differences by residence were narrow, except that more urban youth reported use of condoms than did rural youth $(7-13 \%$ versus $3-5 \%)$.

The duration between marriage and first use of contraception was also explored. Very few youth reported that they had practiced contraception early within marriage, just $8 \%$ of young men and $3 \%$ of young women reported that they had initiated contraceptive use in the first six months of marriage. Rural-urban differences were modest, with somewhat more urban than rural young men reporting contraceptive use in the first six months of marriage (14\% versus $7 \%$ ). Moreover, just $6 \%$ of youth reported initiation of contraceptive use between six months and three years following marriage. Despite these small proportions, we note that of those who report ever having practiced contraception, one in three young men and one in eight young women reported initiating contraceptive use within the first six months following marriage.

Consistent with this profile, just $8 \%$ of young men and $4 \%$ of young women reported the practise of contraception to delay the first pregnancy. Again, the method most likely to have been used was condoms ( $5 \%$ of young men and $2 \%$ of young women). Somewhat larger percentages of urban than rural young men reported the practice of contraception to delay the first pregnancy ( $15 \%$ versus $8 \%)$; differences were negligible among young women ( $7 \%$ and $4 \%$, respectively). 
Table 10.9: Contraceptive use within marriage

Percentage of married youth by ever and current contraceptive use, percent distribution by duration between marriage and initiation of contraceptive use and percentage who used different contraceptive methods to delay first pregnancy, according to residence, Bihar, 2007

\begin{tabular}{|c|c|c|c|c|c|c|}
\hline \multirow[t]{2}{*}{ Contraceptive use (\%) } & $\begin{array}{c}\text { MM } \\
15-29\end{array}$ & $\begin{array}{c}\text { MW } \\
15-24\end{array}$ & $\begin{array}{c}\text { MM } \\
15-29\end{array}$ & $\begin{array}{c}\text { MW } \\
15-24\end{array}$ & $\begin{array}{c}\text { MM } \\
15-29\end{array}$ & $\begin{array}{c}\text { MW } \\
15-24\end{array}$ \\
\hline & \multicolumn{2}{|c|}{ Combined } & \multicolumn{2}{|c|}{ Urban } & \multicolumn{2}{|c|}{ Rural } \\
\hline \multicolumn{7}{|c|}{ Ever use of contraception } \\
\hline Any method & 23.1 & 20.0 & 33.0 & 31.2 & 22.3 & 19.3 \\
\hline Any modern method & 20.7 & 15.2 & 31.5 & 27.5 & 19.7 & 14.4 \\
\hline Female sterilisation & 3.3 & 4.1 & 4.5 & 5.8 & 3.2 & 4.0 \\
\hline Male sterilisation & 0.0 & 0.0 & 0.0 & 0.0 & 0.0 & 0.0 \\
\hline Oral pills & 6.7 & 4.3 & 5.7 & 7.2 & 6.8 & 4.1 \\
\hline IUD & 0.6 & 0.3 & 1.1 & 2.2 & 0.5 & 0.2 \\
\hline Condom & 15.2 & 8.4 & 26.1 & 16.7 & 14.2 & 7.8 \\
\hline Other $^{1}$ & 0.0 & 0.1 & 0.0 & 0.7 & 0.0 & 0.1 \\
\hline Any traditional method ${ }^{2}$ & 3.4 & 6.4 & 3.4 & 6.5 & 3.4 & 6.4 \\
\hline Number who had begun cohabiting & 1,072 & 2,236 & 525 & 1,102 & 547 & 1,134 \\
\hline \multicolumn{7}{|c|}{ Current use of contraception } \\
\hline Any method & 12.2 & 11.8 & 20.5 & 21.0 & 11.4 & 11.1 \\
\hline Any modern method & 10.9 & 8.7 & 19.3 & 17.4 & 10.2 & 8.1 \\
\hline Female sterilisation & 3.3 & 4.1 & 4.5 & 5.8 & 3.2 & 4.0 \\
\hline Male sterilisation & 0.0 & 0.0 & 0.0 & 0.0 & 0.0 & 0.0 \\
\hline Oral pills & 1.7 & 1.1 & 2.3 & 2.9 & 1.5 & 1.0 \\
\hline IUD & 0.5 & 0.1 & 0.0 & 0.7 & 0.5 & 0.1 \\
\hline Condom & 5.7 & 3.3 & 12.5 & 7.2 & 5.1 & 3.0 \\
\hline Other $^{1}$ & 0.0 & 0.0 & 0.0 & 0.0 & 0.0 & 0.0 \\
\hline Any traditional method ${ }^{2}$ & 1.3 & 3.3 & 2.2 & 3.6 & 1.2 & 3.2 \\
\hline Number who had begun cohabiting & 1,072 & 2,236 & 525 & 1,102 & 547 & 1,134 \\
\hline \multicolumn{7}{|c|}{ Duration between marriage and first use of contraception } \\
\hline \multicolumn{7}{|l|}{ Duration } \\
\hline Less than 6 months & 7.6 & 2.6 & 13.8 & 5.1 & 7.0 & 2.4 \\
\hline $6-11$ months & 1.0 & 0.7 & 1.1 & 0.7 & 1.0 & 0.7 \\
\hline 12 months to 3 years & 5.2 & 4.8 & 8.0 & 8.0 & 4.9 & 4.5 \\
\hline More than 3 years & 3.8 & 5.2 & 4.6 & 5.1 & 3.8 & 5.2 \\
\hline Don't know/don't remember & 5.5 & 6.7 & 4.6 & 11.7 & 5.6 & 6.4 \\
\hline Never used contraception & 76.9 & 80.0 & 67.8 & 69.3 & 77.7 & 80.8 \\
\hline Number who had begun cohabiting & 1,072 & 2,236 & 525 & 1,102 & 547 & 1,134 \\
\hline \multicolumn{7}{|c|}{ Ever use of contraception to delay first pregnancy } \\
\hline Any method & 8.2 & 4.3 & 14.8 & 6.5 & 7.6 & 4.2 \\
\hline Any modern method & 6.8 & 2.5 & 13.6 & 5.8 & 6.2 & 2.3 \\
\hline Oral pills & 2.2 & 0.5 & 3.4 & 0.7 & 2.1 & 0.5 \\
\hline IUD & 0.0 & 0.0 & 0.0 & 0.0 & 0.0 & 0.0 \\
\hline Condom & 5.4 & 2.3 & 12.5 & 5.1 & 4.8 & 2.1 \\
\hline Other $^{1}$ & 0.0 & 0.0 & 0.0 & 0.0 & 0.0 & 0.0 \\
\hline Any traditional method ${ }^{2}$ & 2.0 & 1.9 & 1.1 & 1.4 & 2.0 & 1.9 \\
\hline Number who had begun cohabiting & 1,072 & 2,236 & 525 & 1,102 & 547 & 1,134 \\
\hline
\end{tabular}

Note: All Ns are unweighted. Column totals may not equal $100 \%$ due to missing cases. ${ }^{1}$ Includes female condoms, injectables, implants, diaphragm and foam/jelly. ${ }^{2}$ Includes periodic abstinence/rhythm and withdrawal. 
Figure 10.6: Percentage of married youth reporting lifetime and current use of contraceptive methods within marriage, Bihar, 2007

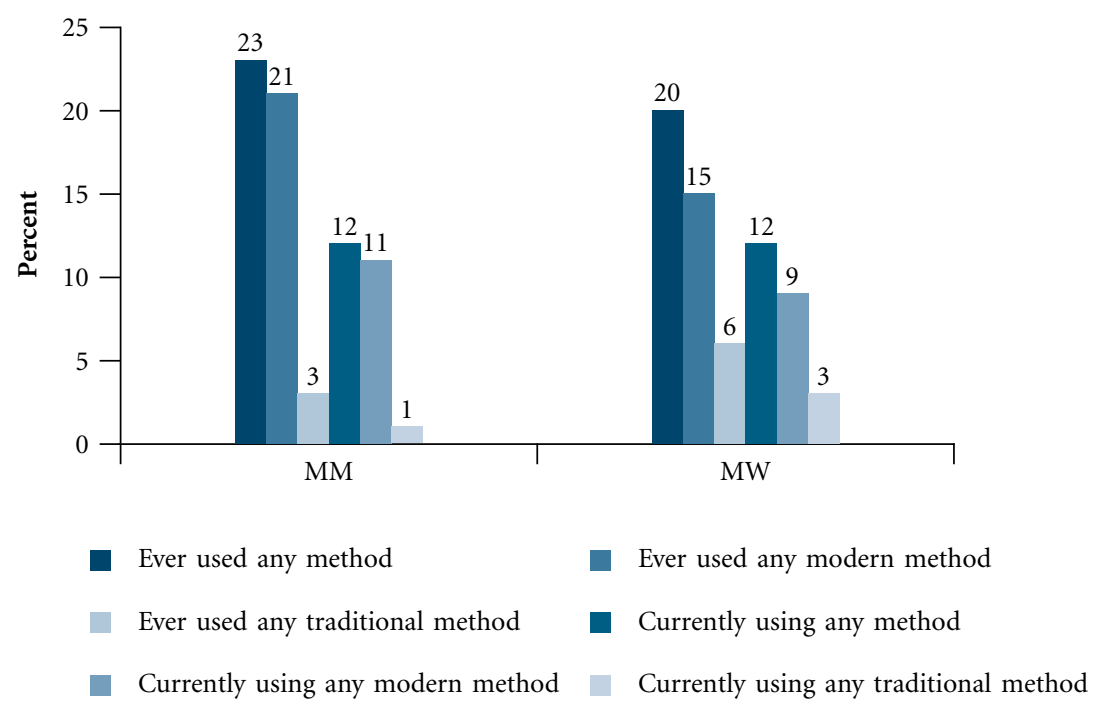

\subsection{Reproductive history}

This section addresses young people's reproductive histories, namely, the first pregnancy and its outcome, children ever born and surviving, experience of pregnancy loss and the wantedness of recent pregnancies. As reported in Table 10.10, $80 \%$ of young women and $79 \%$ of young men reported that they or their wives had experienced at least one pregnancy. Rural-urban differences were narrow.

\subsubsection{First pregnancy experiences}

Of those who reported that they or their wives had ever become pregnant, significant minorities reported current first pregnancy ( $7 \%$ of young women and $8 \%$ of young men, respectively). Among those who had experienced at least one pregnancy, the first pregnancy occurred within a year of marriage for half of young men and almost two-fifths of young women. We note that $34 \%$ of young women and $16 \%$ of young men reported that they could not remember the duration between cohabitation and the first pregnancy, making gender comparisons difficult. Rural-urban differences suggest that the first pregnancy occurred within a year of marriage for a larger proportion of urban than rural youth (55-65\% versus $37-50 \%$ ). The median duration between marriage and first pregnancy was 10 months for both young women and the wives of young men. Rural youth reported somewhat longer median duration than their urban counterparts (10-11 months reported by rural youth compared to $6-8$ months reported by urban youth).

Pregnancy outcomes were reported by all respondents who had completed their first pregnancy. Although the vast majority had experienced a live birth in every group, considerable proportions had experienced miscarriages and stillbirths: for 4-5\% the pregnancy ended in a stillbirth and for $7-9 \%$ in a miscarriage. In contrast, just $0.1 \%$ of young women had aborted their first pregnancy (not a single young man reported an induced abortion). Differences by sex and rural-urban residence were negligible. 
Table 10.10: First pregnancy experience

Percentage of married youth by duration from cohabitation to first pregnancy, outcome of first pregnancy, place of first delivery and type of attendance at first delivery, according to residence, Bihar, 2007

\begin{tabular}{|c|c|c|c|c|c|c|}
\hline \multirow[t]{2}{*}{ First pregnancy experience (\%) } & $\begin{array}{c}\text { MM } \\
15-29\end{array}$ & $\begin{array}{c}\text { MW } \\
15-24\end{array}$ & $\begin{array}{c}\text { MM } \\
15-29\end{array}$ & $\begin{array}{c}\text { MW } \\
\text { 15-24 }\end{array}$ & $\begin{array}{c}\text { MM } \\
15-29\end{array}$ & $\begin{array}{c}\text { MW } \\
15-24\end{array}$ \\
\hline & \multicolumn{2}{|c|}{ Combined } & \multicolumn{2}{|c|}{ Urban } & \multicolumn{2}{|c|}{ Rural } \\
\hline Ever been pregnant & 78.5 & 80.0 & 79.5 & 82.6 & 78.5 & 79.8 \\
\hline Number who had begun cohabiting & 1,072 & 2,236 & 525 & 1,102 & 547 & 1,134 \\
\hline Currently pregnant for the first time & 7.8 & 7.2 & 7.1 & 7.9 & 7.9 & 7.2 \\
\hline \multicolumn{7}{|l|}{ Duration from cohabitation to first pregnancy (months) } \\
\hline Up to 3 & 12.1 & 9.8 & 17.4 & 17.7 & 11.5 & 9.2 \\
\hline $3-6$ & 18.9 & 12.9 & 23.2 & 21.2 & 18.5 & 12.3 \\
\hline $7-12$ & 20.3 & 15.7 & 24.6 & 15.9 & 19.9 & 15.6 \\
\hline $13-24$ & 19.1 & 17.8 & 14.5 & 15.0 & 19.5 & 18.0 \\
\hline More than 24 & 13.9 & 10.1 & 8.7 & 7.1 & 14.5 & 10.3 \\
\hline Do not know/can't remember & 15.7 & 33.7 & 11.6 & 23.0 & 16.0 & 34.4 \\
\hline Median duration & 10.0 & 10.0 & 8.0 & 6.0 & 10.0 & 11.0 \\
\hline Number who had ever been pregnant & 849 & 1,820 & 420 & 914 & 429 & 906 \\
\hline \multicolumn{7}{|l|}{ Outcome of first pregnancy } \\
\hline Live birth & 86.8 & 89.2 & 90.6 & 86.8 & 86.5 & 89.2 \\
\hline Still birth & 4.6 & 3.9 & 1.6 & 3.8 & 4.9 & 3.9 \\
\hline Induced abortion & 0.0 & 0.1 & 0.0 & 0.9 & 0.0 & 0.1 \\
\hline Miscarriage & 8.5 & 6.7 & 7.8 & 8.5 & 8.6 & 6.6 \\
\hline Number who completed first pregnancy & 784 & 1,680 & 390 & 839 & 394 & 841 \\
\hline \multicolumn{7}{|l|}{ Place of first delivery } \\
\hline Respondent's parental home & 46.5 & 36.3 & 35.0 & 23.2 & 47.5 & 37.2 \\
\hline Spouse's parental home & 30.3 & 38.7 & 25.0 & 26.3 & 30.7 & 39.5 \\
\hline Health institution & 23.0 & 24.9 & 40.0 & 50.5 & 21.5 & 23.2 \\
\hline In transit & 0.0 & 0.1 & 0.0 & 0.0 & 0.0 & 0.1 \\
\hline \multicolumn{7}{|l|}{ Type of attendance at first delivery ${ }^{1}$} \\
\hline Doctor/ANM/nurse/LHV & 33.1 & 30.5 & 55.0 & 61.1 & 31.1 & 28.5 \\
\hline Midwife (trained) & 6.9 & 2.2 & 10.0 & 1.1 & 6.7 & 2.2 \\
\hline Other health personnel & 2.0 & 2.2 & 1.7 & 1.1 & 2.0 & 2.3 \\
\hline Dai/traditional birth attendant & 44.9 & 52.6 & 26.7 & 27.4 & 46.5 & 54.2 \\
\hline Friend/relative & 11.4 & 11.6 & 5.0 & 8.4 & 12.0 & 11.8 \\
\hline Other person ${ }^{2}$ & 0.4 & 0.3 & 0.0 & 0.0 & 0.5 & 0.3 \\
\hline None & 1.0 & 0.1 & 1.7 & 0.0 & 0.9 & 0.1 \\
\hline $\begin{array}{l}\text { Number whose first pregnancy outcome was a live or } \\
\text { still birth }\end{array}$ & 717 & 1,547 & 356 & 765 & 361 & 782 \\
\hline
\end{tabular}

Note: All Ns are unweighted. Column totals may not equal 100\% due to missing cases or "don't know" responses. ANM: Auxiliary nurse midwife; LHV: Lady health visitor. ${ }^{1}$ If the respondent reported that the delivery had occurred in a health institution, then it was assumed that a doctor/ANM/nurse/LHV had attended the birth. ${ }^{2}$ If the delivery was reported in transit, attendance at delivery was categorised as "other person". 
Institutional delivery was extremely limited for the first—and most risky-delivery. Indeed, just 23-25\% of youth reported that the first delivery took place in a health institution. Rural-urban differences were pronounced: $40 \%$ of young men and $51 \%$ of young women in urban areas reported an institutional delivery, compared to just $22-23 \%$ of youth in rural areas.

Skilled attendance at delivery was also limited; over two-fifths of young men $(42 \%)$ and one-third of young women $(35 \%)$ reported skilled attendance at first delivery. Rural-urban differences were pronounced; 63-67\% of urban youth reported skilled attendance at delivery, compared to $33-40 \%$ of rural youth. The fact that skilled attendance at birth exceeded institutional delivery may reflect the practice of home deliveries by ANMs and LHVs on the one hand and the tendency to refer to RMPs as doctors among many on the other (see for example, Barnes 2007).

\subsubsection{Children ever born and surviving}

Findings, revealed in Table 10.11, show that youth had experienced an average of about 1.7 pregnancies and just over one live birth. Differences by sex and place of residence of respondents were negligible.

Table 10.11: Reproductive history

Mean number of pregnancies experienced, percent distribution by children ever born and children surviving, and mean number of child deaths, stillbirths, miscarriages and abortions among married youth, according to residence, Bihar, 2007

\begin{tabular}{|c|c|c|c|c|c|c|}
\hline \multirow[t]{2}{*}{ Pregnancy outcomes (\%) } & $\begin{array}{c}\text { MM } \\
15-29\end{array}$ & $\begin{array}{l}\text { MW } \\
15-24\end{array}$ & $\begin{array}{c}\text { MM } \\
15-29\end{array}$ & $\begin{array}{c}\text { MW } \\
15-24\end{array}$ & $\begin{array}{c}\text { MM } \\
15-29\end{array}$ & $\begin{array}{c}\text { MW } \\
15-24\end{array}$ \\
\hline & \multicolumn{2}{|c|}{ Combined } & \multicolumn{2}{|c|}{ Urban } & \multicolumn{2}{|c|}{ Rural } \\
\hline Mean number of lifetime pregnancies & 1.7 & 1.7 & 1.6 & 1.8 & 1.7 & 1.7 \\
\hline \multicolumn{7}{|l|}{ Number of children ever born } \\
\hline 0 & 30.4 & 28.4 & 30.3 & 26.6 & 30.4 & 28.5 \\
\hline 1 & 28.0 & 26.4 & 28.1 & 25.9 & 27.9 & 26.4 \\
\hline 2 & 22.4 & 25.4 & 24.7 & 28.8 & 22.3 & 25.1 \\
\hline 3 & 12.6 & 12.9 & 11.2 & 12.2 & 12.7 & 12.9 \\
\hline 4 or more & 6.6 & 7.0 & 5.6 & 6.5 & 6.7 & 7.1 \\
\hline Mean number of children ever born & 1.3 & 1.4 & 1.3 & 1.4 & 1.3 & 1.4 \\
\hline \multicolumn{7}{|l|}{ Number of children surviving } \\
\hline 0 & 32.4 & 29.2 & 31.5 & 27.7 & 32.5 & 29.3 \\
\hline 1 & 28.9 & 29.2 & 29.2 & 28.5 & 28.8 & 29.2 \\
\hline 2 & 21.8 & 25.3 & 24.7 & 29.2 & 21.6 & 25.1 \\
\hline 3 & 11.6 & 11.3 & 10.1 & 10.9 & 11.8 & 11.3 \\
\hline 4 or more & 5.2 & 5.0 & 4.5 & 3.6 & 5.3 & 5.0 \\
\hline Mean number of children surviving & 1.2 & 1.3 & 1.2 & 1.3 & 1.2 & 1.3 \\
\hline Mean number of sons surviving & 0.6 & 0.6 & 0.6 & 0.7 & 0.6 & 0.6 \\
\hline Mean number of daughters surviving & 0.6 & 0.6 & 0.6 & 0.6 & 0.6 & 0.6 \\
\hline Mean number of children dead & 0.1 & 0.1 & 0.1 & 0.1 & 0.1 & 0.1 \\
\hline Reported one or more still births & 4.2 & 4.8 & 2.2 & 3.6 & 4.4 & 4.9 \\
\hline Reported one or more miscarriages & 9.3 & 8.9 & 9.0 & 11.6 & 9.5 & 8.7 \\
\hline Reported one or more induced abortions & 0.3 & 1.0 & 1.1 & 2.2 & 0.2 & 0.9 \\
\hline Number who had begun cohabiting & 1,072 & 2,236 & 525 & 1,102 & 547 & 1,134 \\
\hline
\end{tabular}

Note: All Ns are unweighted. Column totals may not equal 100\% due to missing cases or "don't know" responses. 
Few youth reported an infant or child death. The distribution of respondents by number of surviving children resembles that of children ever born, reported above. Youth typically reported about as many daughters as sons.

Somewhat more youth reported the experience of pregnancy loss. For example, stillbirths were reported by about $4-5 \%$ of youth. At least one miscarriage was reported by $9 \%$ of youth, and induced abortion was reported by $0.3 \%$ of young men and $1 \%$ of young women. Rural-urban differences in the experience of pregnancy loss were negligible.

Table 10.12 reports mean numbers of children ever born and surviving by respondents' socio-demographic characteristics. As expected, age was positively associated with both fertility indicators, irrespective of rural-urban residence. Religion- and caste-specific differences were narrow; however in urban areas, young

Table 10.12: Children ever born and surviving by selected background characteristics

Mean number of children ever born and children surviving among married youth by selected background characteristics, according to residence, Bihar, 2007

\begin{tabular}{|c|c|c|c|c|c|c|c|c|c|c|c|c|}
\hline \multirow{3}{*}{$\begin{array}{l}\text { Background } \\
\text { characteristics } \\
\text { (mean number) }\end{array}$} & \multicolumn{2}{|c|}{$\begin{array}{c}\text { MM } \\
15-29\end{array}$} & \multicolumn{2}{|c|}{$\begin{array}{c}\text { MW } \\
15-24\end{array}$} & \multicolumn{2}{|c|}{$\begin{array}{c}\text { MM } \\
15-29\end{array}$} & \multicolumn{2}{|c|}{$\begin{array}{c}\text { MW } \\
15-24\end{array}$} & \multicolumn{2}{|c|}{$\begin{array}{c}\text { MM } \\
15-29\end{array}$} & \multicolumn{2}{|c|}{$\begin{array}{c}\text { MW } \\
15-24\end{array}$} \\
\hline & \multicolumn{4}{|c|}{ Combined } & \multicolumn{4}{|c|}{ Urban } & \multicolumn{4}{|c|}{ Rural } \\
\hline & CEB & CS & CEB & CS & CEB & CS & CEB & CS & CEB & CS & CEB & CS \\
\hline Age (years) & $(02)$ & ( & 06 & 06 & * & * & 06 & 06 & $(0)$, & $(0,2)$ & 06 & \\
\hline $20-24$ & 0.8 & 0.8 & 2.0 & 1.8 & 0.9 & 0.9 & 1.7 & 1.6 & 0.8 & 0.8 & 2.0 & 1.9 \\
\hline $25-29$ & 1.8 & 1.7 & NA & NA & 1.6 & 1.5 & NA & NA & 1.9 & 1.7 & NA & NA \\
\hline Religion & & & & & & & & & & & & \\
\hline Hindu & 1.4 & 1.3 & 1.4 & 1.3 & 1.4 & 1.3 & 1.4 & 1.3 & 1.4 & 1.3 & 1.4 & 1.3 \\
\hline Muslim & 1.2 & 1.2 & 1.6 & 1.5 & 1.3 & 1.2 & 1.6 & 1.4 & 1.2 & 1.2 & 1.6 & 1.5 \\
\hline Caste & & & & & & & & & & & & \\
\hline $\mathrm{SC}$ & 1.4 & 1.2 & 1.4 & 1.3 & 1.7 & 1.5 & 1.6 & 1.4 & 1.4 & 1.2 & 1.4 & 1.3 \\
\hline $\mathrm{OBC}$ & 1.4 & 1.3 & 1.5 & 1.4 & 1.3 & 1.3 & 1.5 & 1.4 & 1.4 & 1.3 & 1.5 & 1.4 \\
\hline General $^{1}$ & 1.4 & 1.4 & 1.5 & 1.4 & 1.1 & 1.0 & 1.3 & 1.3 & 1.5 & 1.4 & 1.5 & 1.4 \\
\hline $\begin{array}{l}\text { Educational } \\
\text { level (years) }\end{array}$ & & & & & & & & & & & & \\
\hline None $^{2}$ & 1.5 & 1.3 & 1.5 & 1.4 & 1.6 & 1.5 & 1.7 & 1.5 & 1.5 & 1.3 & 1.5 & 1.4 \\
\hline $1-7$ & 1.3 & 1.2 & 1.4 & 1.3 & 1.4 & 1.4 & 1.6 & 1.5 & 1.2 & 1.2 & 1.3 & 1.3 \\
\hline $8-11$ & 1.5 & 1.4 & 1.3 & 1.3 & 1.4 & 1.3 & 1.3 & 1.3 & 1.5 & 1.4 & 1.3 & 1.3 \\
\hline 12 and above & 1.3 & 1.2 & 1.0 & 1.0 & 0.9 & 0.9 & 0.9 & 0.9 & 1.3 & 1.3 & * & * \\
\hline $\begin{array}{l}\text { Worked in last } \\
12 \text { months }\end{array}$ & & & & & & & & & & & & \\
\hline Yes & 1.4 & 1.3 & 1.6 & 1.5 & 1.4 & 1.3 & 1.6 & 1.4 & 1.4 & 1.3 & 1.6 & 1.5 \\
\hline No & $(0.7)$ & $(0.7)$ & 1.4 & 1.3 & * & * & 1.4 & 1.4 & * & * & 1.4 & 1.3 \\
\hline Wealth quintile & & & & & & & & & & & & \\
\hline First & 1.5 & 1.4 & 1.5 & 1.4 & * & * & (1.3) & $(1.2)$ & 1.5 & 1.4 & 1.5 & 1.4 \\
\hline Second & 1.6 & 1.4 & 1.5 & 1.4 & (1.6) & (1.5) & 1.6 & 1.5 & 1.6 & 1.4 & 1.5 & 1.4 \\
\hline Third & 1.3 & 1.3 & 1.4 & 1.3 & (1.5) & $(1.5)$ & 1.7 & 1.5 & 1.3 & 1.3 & 1.4 & 1.3 \\
\hline Fourth & 1.3 & 1.3 & 1.4 & 1.3 & 1.5 & 1.4 & 1.6 & 1.5 & 1.3 & 1.3 & 1.4 & 1.3 \\
\hline Fifth & 1.3 & 1.2 & 1.5 & 1.4 & 1.2 & 1.2 & 1.4 & 1.3 & 1.3 & 1.2 & 1.5 & 1.4 \\
\hline Total & 1.4 & 1.3 & 1.5 & 1.3 & 1.3 & 1.3 & 1.5 & 1.4 & 1.4 & 1.3 & 1.5 & 1.3 \\
\hline
\end{tabular}

Note: ( ) Based on 25-49 unweighted cases. ${ }^{*}$ Mean not shown, based on fewer than 25 unweighted cases. CEB: Children ever born. CS: Children surviving. NA: Not applicable. OBC: Other backward caste. SC: Scheduled caste. ST: Scheduled tribe. ${ }^{1}$ Includes all those not belonging to SC, ST or OBC. ${ }^{2}$ Includes non-literate and literate with no formal schooling. 
men belonging to scheduled castes were more likely than others to report more children ever born and surviving. Educational attainment level was, by and large, inversely associated with both children ever born and children surviving among young women, an association observed however in urban but not in rural areas. Among young men, a similar association was observed only in urban settings. Findings also show that young men (but not young women) who had worked in the year preceding the survey reported more children ever born and surviving than those who had not worked. Differentials in children ever born by household economic status were narrow.

\subsubsection{Wantedness of recent pregnancies}

All youth who reported one or more pregnancies were asked about the wantedness of their current (for those currently pregnant) or last pregnancies. Findings, presented in Table 10.13, suggest high levels of unplanned pregnancy. Gender differences were notable, with young women consistently more likely than young men to report a mistimed or unwanted pregnancy. For example, among young men whose wives were not pregnant and young women who were not pregnant at the time of interview, $23 \%$ of young men and $33 \%$ of young women reported that the last pregnancy was mistimed or unwanted. Rural-urban differences were muted among young men, but wide among young women, with larger percentages of urban young women than the rural reporting that the last pregnancy was mistimed or unwanted (45\% versus $32 \%)$. A similar pattern emerged with regard to the wantedness of the current pregnancy among those pregnant at the time of interview or whose wives were pregnant at the time of interview: of those young men who reported that their wives were pregnant at the time of the interview, 19\% reported that the pregnancy was either unwanted or wanted at a later time. In contrast, $39 \%$ of young women pregnant at the time of interview reported that the pregnancy was unwanted or mistimed. Somewhat more urban than rural youth reported that the current pregnancy was mistimed or unwanted ( $29 \%$ and $44 \%$ of urban young men and women, respectively, compared to $19 \%$ and $39 \%$ of rural counterparts, respectively).

\section{Table 10.13: Wantedness of most recent pregnancy}

Percentage of married youth by wantedness of most recent pregnancy in the three years preceding the interview, according to residence, Bihar, 2007

\begin{tabular}{|c|c|c|c|c|c|c|}
\hline \multirow[t]{2}{*}{ Status (\%) } & $\begin{array}{c}\text { MM } \\
15-29\end{array}$ & $\begin{array}{c}\text { MW } \\
15-24\end{array}$ & $\begin{array}{c}\text { MM } \\
15-29\end{array}$ & $\begin{array}{c}\text { MW } \\
15-24\end{array}$ & $\begin{array}{c}\text { MM } \\
15-29\end{array}$ & $\begin{array}{c}\text { MW } \\
15-24\end{array}$ \\
\hline & \multicolumn{2}{|c|}{ Combined } & \multicolumn{2}{|c|}{ Urban } & \multicolumn{2}{|c|}{ Rural } \\
\hline \multicolumn{7}{|l|}{ Wantedness status of last pregnancy ${ }^{1}$} \\
\hline Planned & 76.5 & 66.5 & 74.2 & 55.2 & 76.6 & 67.3 \\
\hline Mistimed & 20.5 & 27.0 & 22.7 & 37.1 & 20.4 & 26.3 \\
\hline Unwanted & 2.7 & 5.9 & 1.5 & 7.6 & 2.8 & 5.8 \\
\hline Number who had experienced at least one pregnancy & 784 & 1,680 & 390 & 839 & 394 & 841 \\
\hline \multicolumn{7}{|l|}{ Wantedness status of current pregnancy } \\
\hline Planned & 80.9 & 60.5 & 71.4 & 56.0 & 81.5 & 60.8 \\
\hline Mistimed & 15.1 & 31.2 & 28.6 & 36.0 & 14.7 & 30.9 \\
\hline Unwanted & 4.0 & 7.7 & 0.0 & 8.0 & 3.8 & 7.7 \\
\hline Number currently pregnant & 189 & 393 & 87 & 205 & 102 & 188 \\
\hline
\end{tabular}

Note: All Ns are unweighted. Column totals may not equal 100\% due to missing cases or "don't know" responses. ${ }^{1}$ Excludes respondents/respondents' wives currently pregnant for the first time or never been pregnant. 


\subsection{Ideal family size}

All respondents were asked to report the number of children they considered ideal, and among these the number of sons and daughters considered ideal. As several respondents reported that they were unconcerned about the sex of children, a third response "children of either sex" was also recorded.

As seen in Table 10.14, young men and women reported an ideal family size of 2.7 children. Ideal number of children expressed by rural youth (2.7-2.8) slightly exceeded the number expressed by urban youth (2.4) and considerably larger percentages of rural than urban youth reported three or more children as ideal (51-52\% versus $32-35 \%)$.

Table 10.14: Ideal family size

Percentage of married youth by their reported ideal number of children, according to residence, Bihar, 2007

\begin{tabular}{|c|c|c|c|c|c|c|}
\hline \multirow[t]{2}{*}{ Ideal family size (\%) } & $\begin{array}{c}\mathrm{MM} \\
15-29\end{array}$ & $\begin{array}{c}\text { MW } \\
15-24\end{array}$ & $\begin{array}{c}\mathrm{MM} \\
15-29\end{array}$ & $\begin{array}{c}\text { MW } \\
15-24\end{array}$ & $\begin{array}{c}\mathrm{MM} \\
15-29\end{array}$ & $\begin{array}{c}\text { MW } \\
15-24\end{array}$ \\
\hline & \multicolumn{2}{|c|}{ Combined } & \multicolumn{2}{|c|}{ Urban } & \multicolumn{2}{|c|}{ Rural } \\
\hline \multicolumn{7}{|l|}{ Ideal number of children: } \\
\hline 1 & 1.7 & 1.8 & 4.5 & 5.1 & 1.4 & 1.6 \\
\hline 2 & 41.9 & 41.5 & 55.1 & 58.0 & 40.7 & 40.4 \\
\hline 3 or more & 50.7 & 50.1 & 34.8 & 31.9 & 52.1 & 51.3 \\
\hline Other $^{1}$ & 5.7 & 6.6 & 5.6 & 5.1 & 5.8 & 6.7 \\
\hline Mean ideal number of children ${ }^{2}$ & 2.7 & 2.7 & 2.4 & 2.4 & 2.8 & 2.7 \\
\hline Number who had begun cohabiting & 1,072 & 2,236 & 525 & 1,102 & 547 & 1,134 \\
\hline
\end{tabular}

Note: All Ns are unweighted. Column totals may not equal 100\% due to missing cases or "don't know" responses. Includes "it's up to God," "difficult to say," etc. ${ }^{2}$ Includes only respondents who gave numeric responses.

Tables $10.15 \mathrm{a}$ and $10.15 \mathrm{~b}$ present the ideal number of sons and daughters reported by young men and women by a number of socio-demographic characteristics. Son preference was evident, with young men and women reporting a preference for 1.3-1.4 sons compared to $0.9-1.0$ daughters. Indeed, $37 \%$ of young men and $43 \%$ of young women reported a preference for more sons than daughters. Son preference was more common among rural than urban youth; $38-44 \%$ of rural youth compared to $27-28 \%$ of urban youth reported a preference for more sons than daughters. In contrast, no more than a handful (2-4\%) reported wanting more daughters than sons. The preference for sons was evident among all respondents, irrespective of the socio-demographic characteristics under consideration; however, socio-demographic differences in patterns were evident and these varied somewhat between young men and women. Among young men, son preference was more common among older than younger men; $40 \%$ of those aged 25-29 years, compared to 33-34\% of those aged 20-24 and 15-19 years respectively, reported a preference for more sons than daughters. In contrast, a reverse pattern was evident among young women (46\% of 15-19 year-olds versus $41 \%$ of $20-24$ year-olds). While religion-wise differences were narrow, it would appear that Hindus were somewhat more likely than Muslims to prefer more sons than daughters (43\% versus 37\% among young women; $37 \%$ and $33 \%$ among young men). Caste-wise differences suggest that among both young men and women, those of general castes were least likely to express son preference and those belonging to scheduled castes were most likely to so report. Differences by educational attainment levels were inverse, with those with 12 or more years of schooling considerably less likely than others to report son preference. Among both young men and women, son preference was consistently more common among the economically active than others. Finally, youth from households belonging to the wealthiest quintile were less likely than others to report son preference. 
Table 10.15a: Married young men's preferences for sons and daughters by selected background characteristics

Mean ideal number of sons, daughters and children of either sex and some indicators of sex preference by selected background characteristics of married young men, according to residence, Bihar, 2007

\begin{tabular}{|c|c|c|c|c|c|c|c|}
\hline \multirow[t]{3}{*}{ Background characteristics } & \multirow{2}{*}{\multicolumn{3}{|c|}{ Mean ideal number of: }} & \multirow{2}{*}{\multicolumn{4}{|c|}{$\begin{array}{c}\text { Indicators of sex preference } \\
\text { Percent who wanted: }\end{array}$}} \\
\hline & & & & & & & \\
\hline & Sons & Daughters & $\begin{array}{l}\text { Children } \\
\text { of either } \\
\text { sex }\end{array}$ & $\begin{array}{c}\text { More } \\
\text { sons than } \\
\text { daughters }\end{array}$ & $\begin{array}{c}\text { More } \\
\text { daughters } \\
\text { than sons }\end{array}$ & $\begin{array}{c}\text { At least } \\
\text { one } \\
\text { son }\end{array}$ & $\begin{array}{c}\text { At least } \\
\text { one } \\
\text { daughter }\end{array}$ \\
\hline \multicolumn{8}{|l|}{ Residence } \\
\hline Urban & 1.0 & 0.7 & 0.7 & 27.4 & 3.6 & 72.3 & 65.1 \\
\hline Rural & 1.4 & 1.0 & 0.4 & 37.6 & 4.2 & 84.1 & 79.7 \\
\hline \multicolumn{8}{|l|}{ Age (years) } \\
\hline $15-19$ & (1.4) & $(1.1)$ & $(0.3)$ & $(33.9)$ & (3.4) & $(88.1)$ & $(88.1)$ \\
\hline $20-24$ & 1.2 & 0.9 & 0.5 & 32.8 & 4.6 & 79.9 & 75.9 \\
\hline $25-29$ & 1.4 & 1.0 & 0.4 & 39.6 & 4.1 & 84.6 & 79.1 \\
\hline \multicolumn{8}{|l|}{ Religion } \\
\hline Hindu & 1.3 & 1.0 & 0.4 & 37.4 & 4.0 & 84.6 & 79.7 \\
\hline Muslim & 1.1 & 0.9 & 0.8 & 32.5 & 5.3 & 71.9 & 69.6 \\
\hline \multicolumn{8}{|l|}{ Caste } \\
\hline SC & 1.5 & 1.0 & 0.4 & 43.9 & 5.3 & 85.9 & 82.1 \\
\hline OBC & 1.3 & 0.9 & 0.5 & 35.2 & 3.0 & 81.8 & 76.8 \\
\hline General $^{1}$ & 1.1 & 0.9 & 0.6 & 29.8 & 7.0 & 81.6 & 77.2 \\
\hline \multicolumn{8}{|l|}{ Educational level (years) } \\
\hline None $^{2}$ & 1.4 & 0.9 & 0.6 & 43.3 & 3.3 & 81.1 & 73.4 \\
\hline $1-7$ & 1.3 & 1.0 & 0.4 & 36.0 & 6.1 & 84.2 & 81.1 \\
\hline $8-11$ & 1.4 & 0.9 & 0.3 & 38.5 & 2.5 & 86.3 & 83.5 \\
\hline 12 and above & 1.0 & 0.8 & 0.5 & 20.5 & 6.6 & 76.9 & 71.1 \\
\hline \multicolumn{8}{|l|}{ Worked in last 12 months } \\
\hline Yes & 1.3 & 0.9 & 0.5 & 36.9 & 4.4 & 83.1 & 78.5 \\
\hline No & $(1.1)$ & $(0.8)$ & $(0.3)$ & $(25.9)$ & $(0.0)$ & $(81.5)$ & $(75.0)$ \\
\hline \multicolumn{8}{|l|}{ Wealth quintile } \\
\hline First & 1.5 & 1.0 & 0.6 & 45.3 & 4.4 & 82.5 & 77.4 \\
\hline Second & 1.4 & 0.9 & 0.5 & 44.1 & 3.5 & 84.0 & 75.9 \\
\hline Third & 1.4 & 1.0 & 0.4 & 44.7 & 4.8 & 86.4 & 84.5 \\
\hline Fourth & 1.3 & 1.0 & 0.4 & 32.3 & 5.3 & 85.8 & 82.7 \\
\hline Fifth & 1.1 & 0.8 & 0.5 & 26.1 & 3.3 & 78.3 & 72.7 \\
\hline Total & 1.3 & 0.9 & 0.5 & 36.8 & 4.3 & 83.2 & 78.5 \\
\hline
\end{tabular}

Note: ( ) Based on 25-49 unweighted cases. OBC: Other backward caste. SC: Scheduled caste. ST: Scheduled tribe. ${ }^{1}$ Includes all those not belonging to SC, ST or OBC. ${ }^{2}$ Includes non-literate and literate with no formal schooling. 
Table 10.15b: Married young women's preferences for sons and daughters by selected background characteristics

Mean ideal number of sons, daughters and children of either sex and some indicators of sex preference by selected background characteristics of married young women, according to residence, Bihar, 2007

\begin{tabular}{|c|c|c|c|c|c|c|c|}
\hline \multirow[t]{3}{*}{ Background characteristics } & \multirow{2}{*}{\multicolumn{3}{|c|}{ Mean ideal number of: }} & \multirow{2}{*}{\multicolumn{4}{|c|}{$\begin{array}{c}\text { Indicators of sex preference } \\
\text { Percent who wanted: }\end{array}$}} \\
\hline & & & & & & & \\
\hline & Sons & Daughters & $\begin{array}{l}\text { Children } \\
\text { of either } \\
\text { sex }\end{array}$ & $\begin{array}{c}\text { More } \\
\text { sons than } \\
\text { daughters }\end{array}$ & $\begin{array}{c}\text { More } \\
\text { daughters } \\
\text { than sons }\end{array}$ & $\begin{array}{c}\text { At least } \\
\text { one } \\
\text { son }\end{array}$ & $\begin{array}{c}\text { At least } \\
\text { one } \\
\text { daughter }\end{array}$ \\
\hline \multicolumn{8}{|l|}{ Residence } \\
\hline Urban & 1.2 & 0.9 & 0.3 & 28.2 & 2.3 & 86.9 & 83.1 \\
\hline Rural & 1.5 & 1.0 & 0.3 & 43.6 & 1.8 & 89.9 & 85.7 \\
\hline \multicolumn{8}{|l|}{ Age (years) } \\
\hline 15-19 & 1.5 & 1.0 & 0.2 & 46.0 & 2.4 & 90.0 & 86.6 \\
\hline $20-24$ & 1.4 & 1.0 & 0.3 & 40.5 & 1.5 & 89.5 & 84.8 \\
\hline \multicolumn{8}{|l|}{ Religion } \\
\hline Hindu & 1.4 & 1.0 & 0.2 & 43.3 & 1.7 & 90.5 & 86.2 \\
\hline Muslim & 1.5 & 1.1 & 0.5 & 36.8 & 3.3 & 83.5 & 80.1 \\
\hline \multicolumn{8}{|l|}{ Caste } \\
\hline SC & 1.6 & 1.0 & 0.2 & 48.9 & 1.9 & 90.8 & 87.3 \\
\hline OBC & 1.5 & 1.0 & 0.2 & 42.8 & 2.0 & 90.7 & 86.5 \\
\hline General $^{1}$ & 1.1 & 0.8 & 0.5 & 24.8 & 1.0 & 80.7 & 75.2 \\
\hline \multicolumn{8}{|l|}{ Educational level (years) } \\
\hline None $^{2}$ & 1.6 & 1.0 & 0.2 & 49.4 & 1.8 & 91.5 & 86.3 \\
\hline $1-7$ & 1.4 & 1.0 & 0.3 & 39.8 & 1.4 & 90.3 & 87.7 \\
\hline $8-11$ & 1.1 & 0.9 & 0.3 & 23.3 & 2.8 & 84.4 & 81.6 \\
\hline 12 and above & 0.8 & 0.8 & 0.5 & 6.7 & 3.4 & 74.6 & 72.9 \\
\hline \multicolumn{8}{|l|}{ Worked in last 12 months } \\
\hline Yes & 1.6 & 1.0 & 0.2 & 51.0 & 2.3 & 92.2 & 87.1 \\
\hline No & 1.4 & 0.9 & 0.3 & 37.9 & 1.7 & 88.3 & 84.7 \\
\hline \multicolumn{8}{|l|}{ Wealth quintile } \\
\hline First & 1.6 & 1.0 & 0.4 & 49.9 & 1.3 & 88.0 & 83.8 \\
\hline Second & 1.6 & 1.1 & 0.1 & 47.5 & 2.1 & 93.3 & 89.3 \\
\hline Third & 1.5 & 1.0 & 0.2 & 43.0 & 2.3 & 91.7 & 84.5 \\
\hline Fourth & 1.4 & 1.0 & 0.2 & 44.5 & 2.4 & 90.7 & 87.8 \\
\hline Fifth & 1.1 & 0.9 & 0.3 & 26.0 & 0.8 & 84.6 & 82.0 \\
\hline Total & 1.4 & 1.0 & 0.3 & 42.6 & 1.9 & 89.7 & 85.6 \\
\hline
\end{tabular}

Note: OBC: Other backward caste. SC: Scheduled caste. ST: Scheduled tribe. ${ }^{1}$ Includes all those not belonging to SC, ST or $O B C .{ }^{2}$ Includes non-literate and literate with no formal schooling. 


\subsection{Summary}

Findings indicate that while most young men preferred to marry in young adulthood, almost one-quarter of young women preferred to marry before 18 years and as many as three-fifths preferred to marry before age 20, indicating an adherence to social norms around early marriage even by youth. Reiterating the fact that early marriage continues to characterise the lives of many young women and to certain extent the lives of young men as well, findings show that among young women aged 20-24 years as many as 46\% were married before age $15,77 \%$ before age 18 and $87 \%$ before age 20 . Even though early marriage was less prevalent among young men, $13 \%$ of those aged 20-24 years were married before age 18 and $31 \%$ before age 20 .

Not only did marriage occur at young ages but it was also often arranged without the participation of young people themselves, particularly young women. Almost all youth reported arranged marriages. As many as one in ten young men and over two in five young women reported that their parents did not seek their approval while determining their marriage partners. Hence, not surprisingly, reported pre-marital acquaintance was extremely limited. Just $4-7 \%$ of married youth reported that they had ever had a chance to meet and interact alone with their spouse-to-be prior to marriage. Over $90 \%$ of married youth reported that they met their spouses for the first time on the wedding day. Compounding the lack of pre-marital acquaintance was the lack of awareness of what to expect of married life, reported by over three-fifths of young men and four-fifths of young women.

Despite the existence of laws against dowry, dowry characterised the marriages of two-thirds of young men and women. Findings also show that families of urban youth appeared as likely to conform to traditional practices, such as payment of dowry, as their rural counterparts.

Reports of marital life suggest that although spousal communication was reported on several issues, it was far from universal. At the same time, marital life was marked by considerable violence. For example, couple communication on most topics was reported by over three in four young men and women, yet communication on contraceptive use was reported by somewhat fewer $(72 \%)$ of women and almost two-fifths of young men, clearly undermining married young people's ability to adopt protective actions. Physical violence and forced sex within marriage were reported by considerable proportions of youth. For example, $30 \%$ of young women reported that they had ever faced violence perpetrated by the husband and a similar percentage of young men reported perpetrating violence on their wives. Recent violence was likewise reported by one-quarter of young women and one-fifth of young men. Sexual violence was also reported. Indeed, half of young women reported that the first sexual experience within marriage had been forced. Overall, over half of young women reported ever being forced to engage in sex with their husbands; one in four young men reported forcing their wives to engage in sex. Recent sexual violence was reported by $27 \%$ of young women and $11 \%$ of young men.

While the Youth Study did not explore extra-marital sexual experiences in detail, the available data indicate that $4 \%$ of young men reported an extra-marital sexual encounter. In contrast, hardly any young women reported an extra-marital sexual encounter.

Contraceptive use at any time within marriage was limited, reported by $23 \%$ of young men and $20 \%$ of young women. Moreover, just $12 \%$ of young men and women reported current use of contraception. Among contraceptive methods currently used, condoms and female sterilisation were most likely to be reported. Few young people practised contraception to delay the first birth-just $8 \%$ of young men and $4 \%$ of young women. Not surprisingly, pregnancy typically occurred within a year of marriage for almost two-fifths of young women and half of young men who reported that they or their wives had been pregnant at least once. 
Moreover, large proportions of youth reported experiencing unintended pregnancy. For example, of those women who were not pregnant at the time of interview and those men whose wives were not pregnant at the time of interview, $33 \%$ of young women and $23 \%$ of young men reported that the last pregnancy was mistimed or unwanted.

Circumstances of the first birth suggest that institutional delivery and skilled attendance at delivery were extremely limited: only $23-25 \%$ of first births were delivered institutionally and $35-42 \%$ reported delivery by a skilled attendant.

Findings also show that although most respondents wanted one child of each sex, son preference was evident. Over one-third of young men and over two-fifths of young women preferred to have more sons than daughters. In contrast, just $2-4 \%$ preferred to have more daughters than sons. 


\section{Health and health seeking} behaviour

This chapter focuses on young people's patterns of substance use, health status and treatment-seeking for health problems experienced. The Youth Study probed alcohol, drug and tobacco use as well as, among those who reported substance use, consumption characteristics, including recent use and extent of use. It also included several questions relating to the experience of a number of health problems in the areas of general, sexual and reproductive health and mental health. This chapter also describes young people's care seeking practices for general and sexual and reproductive health problems as well as young people's attitudes towards pre-marital HIV testing for boys and girls and the extent to which youth had undergone an HIV test. Where numbers are small, we present combined findings for rural and urban respondents.

\subsection{Substance use}

Research has shown that substance use can directly compromise young people's health. For example, evidence suggests that use of alcohol and drugs among youth is associated with physical fights, risky sexual activity, depression and suicide as well as irregular school or work attendance and other negative outcomes (DiClemente, 1992; Ellickson, Saner and McGuigan, 1997; Gruber et al., 1996; Lowry et al., 1994; Mohan, Sankara Sarma and Thankappan, 2005; Singh and Saini, 2007).

Youth Study findings on the extent of substance use among young people themselves suggest that while less than $2 \%$ of young men (and no women) reported drug use (including, for example, ganja, charas, brown sugar, cocaine and bhang), a substantial proportion of young men and a small minority of young women reported consumption of tobacco and alcohol (Table 11.1). For example, $39 \%$ of young men and $2 \%$ of young women had ever consumed tobacco products. Most of those young men and women who had ever consumed tobacco products reported that they had done so once a week or more frequently in the month prior to interview. Married young men were considerably more likely than the unmarried to report that they had ever used tobacco products (69\% and 32\%, respectively) and tobacco use in the month prior to interview ( $62 \%$ and $25 \%$, respectively); this difference may be attributable to the fact that tobacco use is observed in general to increase with age and married men in our sample are considerably older than the unmarried. Rural-urban differences were negligible.

Fewer youth reported alcohol consumption. In contrast to young women, among whom almost no one reported ever or current consumption of alcohol, larger percentages of young men reported ever consuming $(17 \%)$ or recently consuming (5\%) alcohol. As in the case of tobacco use, married young men were far more likely to have ever consumed alcohol than the unmarried (36\% and $11 \%$, respectively). Recent alcohol use-once a week or more frequently in the month prior to interview was reported by many fewer: $12 \%$ and $3 \%$ of married and unmarried young men, respectively. Rural-urban differences were negligible, except that somewhat more married young men in urban than rural settings reported having ever consumed alcohol ( $42 \%$ versus $35 \%$ ). The large majority of young men who reported having ever consumed alcohol reported that they usually consumed alcohol with peers ( $81 \%$ and $83 \%$ of the married and unmarried, respectively) and $34 \%$ and $19 \%$ of married and unmarried men reported that they sometimes or often became drunk (not shown in tabular form). 
Table 11.1: Substance use

Percentage of youth reporting lifetime and recent substance use, according to residence, Bihar, 2007

\begin{tabular}{|c|c|c|c|c|c|c|}
\hline Substance use (\%) & $\begin{array}{c}\mathrm{M} \\
15-24\end{array}$ & $\begin{array}{c}\text { W } \\
15-24\end{array}$ & $\begin{array}{c}\text { MM } \\
15-29\end{array}$ & $\begin{array}{c}\text { MW } \\
15-24\end{array}$ & $\begin{array}{c}\text { UM } \\
15-24\end{array}$ & $\begin{array}{c}\text { UW } \\
\text { 15-24 }\end{array}$ \\
\hline \multicolumn{7}{|c|}{ Combined } \\
\hline \multicolumn{7}{|l|}{ Ever consumed } \\
\hline Tobacco and its products & 39.2 & 1.8 & 69.3 & 2.0 & 31.8 & 1.4 \\
\hline Alcohol & 16.7 & 0.1 & 35.7 & 0.1 & 10.8 & 0.1 \\
\hline Drugs $^{1}$ & 1.8 & 0.0 & 4.5 & 0.0 & 0.8 & 0.0 \\
\hline \multicolumn{7}{|c|}{ Consumed once a week or more frequently in last month } \\
\hline Alcohol & 5.1 & 0.1 & 12.3 & 0.1 & $\begin{array}{r}24.0 \\
2.7\end{array}$ & 0.1 \\
\hline Drugs $^{1}$ & 0.8 & 0.0 & 1.4 & 0.0 & 0.7 & 0.0 \\
\hline Number of respondents & 1,942 & 5,529 & 1,115 & 2,341 & 1,492 & 3,188 \\
\hline \multicolumn{7}{|c|}{ Urban } \\
\hline \multicolumn{7}{|l|}{ Ever consumed } \\
\hline Tobacco and its products & 35.8 & 1.1 & 70.7 & 1.4 & 31.4 & 0.6 \\
\hline Alcohol & 15.8 & 0.2 & 42.4 & 0.0 & 12.7 & 0.2 \\
\hline Drugs $^{1}$ & 1.8 & 0.0 & 3.3 & 0.0 & 1.2 & 0.0 \\
\hline \multicolumn{7}{|c|}{ Consumed once a week or more frequently in last month } \\
\hline Tobacco and its products & 30.5 & 0.8 & 67.4 & 1.4 & 25.7 & 0.2 \\
\hline Alcohol & 3.2 & 0.0 & 12.0 & 0.0 & 2.5 & 0.0 \\
\hline Drugs $^{1}$ & 0.7 & 0.0 & 1.1 & 0.0 & 0.8 & 0.0 \\
\hline Number of respondents & 1,039 & 2,581 & 547 & 1,136 & 833 & 1,445 \\
\hline \multicolumn{7}{|c|}{ Rural } \\
\hline \multicolumn{7}{|l|}{ Ever consumed } \\
\hline Tobacco and its products & 39.8 & 1.9 & 69.2 & 2.0 & 31.8 & 1.6 \\
\hline Alcohol & 16.9 & 0.1 & 35.1 & 0.1 & 10.4 & 0.1 \\
\hline Drugs $^{1}$ & 1.8 & 0.0 & 4.6 & 0.0 & 0.7 & 0.0 \\
\hline \multicolumn{7}{|c|}{ Consumed once a week or more frequently in last month } \\
\hline Tobacco and its products & 32.2 & 1.3 & 61.8 & 1.5 & 24.3 & 0.7 \\
\hline Alcohol & 5.5 & 0.1 & 12.3 & 0.1 & 2.7 & 0.1 \\
\hline Drugs $^{1}$ & 0.8 & 0.0 & 1.5 & 0.0 & 0.7 & 0.0 \\
\hline Number of respondents & 903 & 2,948 & 568 & 1,205 & 659 & 1,743 \\
\hline
\end{tabular}

Note: All Ns are unweighted. ${ }^{1}$ Includes ganja, charas, brown sugar, cocaine, bhang, etc.

\subsection{General and sexual and reproductive health problems}

General health problems about which youth were questioned included high fever and injury. Sexual and reproductive health problems included symptoms of genital infection (burning during urination, genital ulcers, itching, and swelling in the groin, genital discharge, for example), anxiety about nocturnal emission or swapnadosh (for young men) and menstrual problems (for young women). Findings related to recent experiences of various general, and sexual and reproductive health problems are presented in Table 11.2.

\subsubsection{General health problems}

Findings show that $26 \%$ of young men and $28 \%$ of young women had experienced high fever in the three months preceding the survey. We note the fact that the survey period covered the peak infection summer and monsoon months, which may partially explain the prevalence of high fever. While differences by marital status 
were negligible among young men, somewhat more married than unmarried young women had experienced high fever. Rural-urban differences were negligible.

Injuries were experienced by a minority of respondents in the three months preceding the survey, specifically, $12 \%$ of young men and $2 \%$ of young women. Differences by marital status and rural-urban residence were narrow; however, somewhat more unmarried young men in rural than urban areas reported having experienced injuries (13\% versus $7 \%)$.

\subsubsection{Sexual and reproductive health problems}

Table 11.2 presents young people's reported experiences of symptoms of genital infection in the three months preceding the survey. We note that these findings are based on self-reports and not on clinical examination or laboratory testing and therefore must be interpreted cautiously. Young women were more likely than young men to report these symptoms ( $22 \%$ versus $8 \%$ ). This difference is largely attributed to the finding that young women's experience related, for the most part (17\%) to vaginal discharge. Differences by marital status suggest that while married young men were slightly more likely than unmarried young men to have experienced

Table 11.2: Self-reported health problems

Percentage of youth reporting recent experiences of selected general and sexual and reproductive health problems, according to residence, Bihar, 2007

\begin{tabular}{|c|c|c|c|c|c|c|}
\hline $\begin{array}{l}\text { General/sexual and reproductive health } \\
\text { problems experienced }(\%)\end{array}$ & $\begin{array}{c}M \\
15-24\end{array}$ & $\begin{array}{c}W \\
15-24\end{array}$ & $\begin{array}{c}\mathrm{MM} \\
15-29\end{array}$ & $\begin{array}{c}\text { MW } \\
15-24\end{array}$ & $\begin{array}{c}\text { UM } \\
15-24\end{array}$ & $\begin{array}{l}\text { UW } \\
15-24\end{array}$ \\
\hline \multicolumn{7}{|c|}{ Combined } \\
\hline High fever in last 3 months & 25.6 & 28.3 & 24.2 & 30.8 & 25.6 & 23.7 \\
\hline Injury in last 3 months & 11.7 & 2.2 & 9.1 & 2.1 & 11.9 & 2.6 \\
\hline Symptoms of genital infection in last 3 months ${ }^{1}$ & 8.3 & 21.6 & 10.6 & 25.8 & 7.0 & 13.9 \\
\hline $\begin{array}{l}\text { Anxiety about swapnadosh/nocturnal emission in last } \\
12 \text { months }\end{array}$ & 18.6 & NA & 10.9 & NA & 20.8 & NA \\
\hline Menstrual problems in last 3 months & NA & 11.2 & NA & 12.3 & NA & 9.3 \\
\hline Number of respondents & 1,942 & 5,529 & 1,115 & 2,341 & 1,492 & 3,188 \\
\hline \multicolumn{7}{|c|}{ Urban } \\
\hline High fever in last 3 months & 25.0 & 27.9 & 22.8 & 31.0 & 24.9 & 25.7 \\
\hline Injury in last 3 months & 7.5 & 2.8 & 6.5 & 2.1 & 7.4 & 3.6 \\
\hline Symptoms of genital infection in last 3 months $^{1}$ & 8.6 & 16.2 & 9.8 & 23.1 & 8.2 & 11.3 \\
\hline $\begin{array}{l}\text { Anxiety about swapnadosh/nocturnal emission in last } \\
12 \text { months }\end{array}$ & 18.6 & NA & 8.7 & NA & 19.7 & NA \\
\hline Menstrual problems in last 3 months & NA & 8.9 & NA & 7.7 & NA & 9.9 \\
\hline Number of respondents & 1,039 & 2,581 & 547 & 1,136 & 833 & 1,445 \\
\hline \multicolumn{7}{|c|}{ Rural } \\
\hline High fever in last 3 months & 25.7 & 28.4 & 24.2 & 30.7 & 25.7 & 23.3 \\
\hline Injury in last 3 months & 12.3 & 2.1 & 9.4 & 2.0 & 12.8 & 2.4 \\
\hline Symptoms of genital infection in last 3 months ${ }^{1}$ & 8.2 & 22.3 & 10.8 & 26.0 & 6.8 & 14.3 \\
\hline $\begin{array}{l}\text { Anxiety about swapnadosh/nocturnal emission in last } \\
12 \text { months }\end{array}$ & 18.6 & NA & 11.1 & NA & 21.1 & NA \\
\hline Menstrual problems in last 3 months & NA & 11.5 & NA & 12.6 & NA & 9.2 \\
\hline Number of respondents & 903 & 2,948 & 568 & 1,205 & 659 & 1,743 \\
\hline
\end{tabular}

Note: All Ns are unweighted. NA: Not applicable. ${ }^{1}$ Includes genital ulcers, genital itching, swelling in the groin, discharge, burning during urination, etc. 
symptoms of genital infection ( $11 \%$ and $7 \%$ respectively), married young women were considerably more likely than the unmarried to report as such (26\% versus $14 \%$ ). Rural-urban differences were not evident among young men; however among young women, somewhat more rural than urban young women reported symptoms of genital infection (22\% versus $16 \%)$.

Previous research has documented the extent to which semen loss is associated with anxiety regarding masculine weakness and ill-health in South Asian cultures (Bhatia and Choudhary, 1998; Bhatia and Malik, 1991; Bhende, 1995; Collumbien et al., 2004; Khan et al., 2006; Pelto, 1999; Verma et al., 2003). Youth Study findings suggest that almost one-fifth of young men had indeed experienced anxiety about swapnadosh or nocturnal emission in the 12 months preceding the survey. Differences were observed by marital status: $11 \%$ of married men reported anxiety about nocturnal emission compared to $21 \%$ of unmarried young men. Differences by rural-urban residence were muted.

With regard to young women's experience of other reproductive health problems in the three months preceding the interview, findings suggest that $11 \%$ of young women experienced menstrual problems with little variation by marital status or rural-urban residence.

\subsection{Mental health disorders}

The mental health status of young people was assessed using their responses to the General Health Questionnaire (GHQ-12) (Goldberg, 1992; Patel and Andrew, 2001). This questionnaire, designed to detect possible mental disorders, is based on 12 questions that assess the respondent's general level of happiness, depression, anxiety and sleep disturbance in the one month preceding the interview. Threshold scores of 2, 3, 4 or more have been variously used to identify the possible presence of common mental disorders (Bashir et al., 1996; Donath, 2001; Jacob, Bhugra and Mann, 1997). Table 11.3 presents responses on each item of the GHQ-12, and a summary measure indicating the percentage who gave three or more responses suggestive of mental disorders.

Gender disparities in response patterns were evident. Young men reported responses suggestive of mental disorders on several more items than young women. Moreover, on most questions, larger percentages of young men than women indicated responses suggestive of mental disorders Among young men, 10\% or more indicated responses suggestive of mental disorders to several questions: feeling that they were not playing a useful role (24\%), feeling incapable of making decisions (19\%), feeling constantly under strain (14\%), losing sleep due to worry (14\%), feeling unhappy and depressed (11\%) and feeling incapable of overcoming their difficulties (10\%). In contrast, questions that elicited responses suggestive of mental disorder by one-tenth or more of young women included feeling incapable of making decisions (20\%), feeling incapable of overcoming their difficulties $(13 \%)$ and feeling incapable of facing up to one's problems $(10 \%)$.

Differences by marital status were more evident among young men than among young women. Married young men were more likely than unmarried young men to report that they lost sleep over worry (20\% versus $14 \%)$ and felt constantly under strain (21\% versus $13 \%)$. Conversely, unmarried young men were more likely than married young men to report that they felt that they were not playing a useful role (28\% versus $11 \%$ ) and felt incapable of making decisions (22\% versus $12 \%)$. Differences were negligible among young women, except that somewhat more married than unmarried young women reported that they had been thinking themselves as a worthless person (9\% versus $4 \%$ ). 
Table 11.3: Reported symptoms or behaviours suggestive of mental health disorders

Percentage of youth reporting symptoms or behaviours suggestive of mental health disorders experienced in the month preceding the interview, according to residence, Bihar, 2007

\begin{tabular}{|c|c|c|c|c|c|c|}
\hline Symptoms/behaviours (\%) & $\begin{array}{c}\mathrm{M} \\
15-24\end{array}$ & $\begin{array}{c}\text { W } \\
15-24\end{array}$ & $\begin{array}{c}\text { MM } \\
15-29\end{array}$ & $\begin{array}{c}\text { MW } \\
15-24\end{array}$ & $\begin{array}{c}\text { UM } \\
\text { 15-24 }\end{array}$ & $\begin{array}{c}\text { UW } \\
\text { 15-24 }\end{array}$ \\
\hline \multicolumn{7}{|c|}{ Combined } \\
\hline Unable to concentrate on whatever he/she was doing & 6.7 & 1.8 & 7.1 & 1.6 & 6.7 & 2.0 \\
\hline Lost much sleep over worry & 13.6 & 6.7 & 20.3 & 7.9 & 13.5 & 4.5 \\
\hline Felt that he/she was not playing a useful role & 23.5 & 3.6 & 10.7 & 3.2 & 27.9 & 4.4 \\
\hline Felt incapable of making decisions & 19.2 & 19.9 & 11.7 & 20.9 & 21.6 & 18.3 \\
\hline Felt constantly under strain & 13.8 & 6.9 & 20.5 & 7.6 & 12.7 & 5.7 \\
\hline Felt that he/she could not overcome his/her difficulties & 10.1 & 12.8 & 8.7 & 12.2 & 10.7 & 14.3 \\
\hline Unable to enjoy normal day-to-day activities & 5.3 & 2.0 & 5.4 & 2.1 & 5.7 & 1.7 \\
\hline Unable to face up to his/her problems & 6.4 & 9.6 & 5.6 & 9.3 & 6.8 & 10.4 \\
\hline Been feeling unhappy and depressed & 10.9 & 5.4 & 15.1 & 5.7 & 10.7 & 4.9 \\
\hline Been losing confidence in himself/herself & 7.9 & 4.1 & 10.0 & 4.1 & 7.6 & 4.3 \\
\hline Been thinking of himself/herself as a worthless person & 6.4 & 7.1 & 6.7 & 8.6 & 6.0 & 4.2 \\
\hline Not feeling reasonably happy, all things considered & 3.7 & 3.0 & 4.4 & 3.2 & 3.8 & 2.6 \\
\hline Three or more symptoms/behaviours & 16.2 & 8.7 & 17.3 & 9.0 & 16.8 & 8.3 \\
\hline Number of respondents & 1,942 & 5,529 & 1,115 & 2,341 & 1,492 & 3,188 \\
\hline \multicolumn{7}{|c|}{ Urban } \\
\hline Unable to concentrate on whatever he/she was doing & 5.4 & 2.0 & 5.4 & 2.1 & 4.9 & 2.2 \\
\hline Lost much sleep over worry & 13.6 & 6.1 & 20.7 & 7.0 & 12.7 & 5.5 \\
\hline Felt that he/she was not playing a useful role & 24.4 & 2.7 & 9.8 & 2.8 & 25.8 & 2.8 \\
\hline Felt incapable of making decisions & 16.5 & 16.3 & 7.6 & 18.3 & 17.6 & 15.2 \\
\hline Felt constantly under strain & 16.8 & 7.8 & 24.7 & 8.5 & 15.6 & 7.3 \\
\hline Felt that he/she could not overcome his/her difficulties & 10.0 & 10.4 & 9.8 & 10.6 & 9.8 & 10.1 \\
\hline Unable to enjoy normal day-to-day activities & 3.6 & 2.0 & 4.3 & 2.8 & 3.3 & 1.8 \\
\hline Unable to face up to his/her problems & 4.6 & 6.6 & 3.3 & 6.3 & 4.5 & 6.7 \\
\hline Been feeling unhappy and depressed & 13.3 & 6.0 & 16.3 & 6.3 & 13.5 & 5.7 \\
\hline Been losing confidence in himself/herself & 7.5 & 3.6 & 8.7 & 4.2 & 7.4 & 3.0 \\
\hline Been thinking of himself/herself as a worthless person & 5.4 & 6.1 & 5.4 & 8.5 & 5.7 & 4.3 \\
\hline Not feeling reasonably happy, all things considered & 5.0 & 2.4 & 7.5 & 2.8 & 4.9 & 2.0 \\
\hline Three or more symptoms/behaviours & 16.5 & 8.0 & 17.4 & 9.1 & 16.4 & 7.5 \\
\hline Number of respondents & 1,039 & 2,581 & 547 & 1,136 & 833 & 1,445 \\
\hline \multicolumn{7}{|c|}{ Rural } \\
\hline Unable to concentrate on whatever he/she was doing & 6.9 & 1.7 & 7.2 & 1.6 & 7.0 & 2.0 \\
\hline Lost much sleep over worry & 13.6 & 6.8 & 20.2 & 8.0 & 13.6 & 4.3 \\
\hline Felt that he/she was not playing a useful role & 23.4 & 3.7 & 10.7 & 3.3 & 28.4 & 4.7 \\
\hline Felt incapable of making decisions & 19.7 & 20.4 & 12.1 & 21.1 & 22.4 & 18.9 \\
\hline Felt constantly under strain & 13.3 & 6.8 & 20.1 & 7.5 & 12.2 & 5.4 \\
\hline Felt that he/she could not overcome his/her difficulties & 10.1 & 13.2 & 8.6 & 12.3 & 10.8 & 15.1 \\
\hline Unable to enjoy normal day-to-day activities & 5.6 & 1.9 & 5.4 & 2.0 & 6.1 & 1.7 \\
\hline Unable to face up to his/her problems & 6.8 & 10.0 & 5.8 & 9.5 & 7.3 & 11.1 \\
\hline Been feeling unhappy and depressed & 10.5 & 5.4 & 15.0 & 5.6 & 10.2 & 4.7 \\
\hline Been losing confidence in himself/herself & 8.0 & 4.2 & 10.1 & 4.0 & 7.6 & 4.6 \\
\hline Been thinking of himself/herself as a worthless person & 6.5 & 7.2 & 6.8 & 8.6 & 6.2 & 4.2 \\
\hline Not feeling reasonably happy, all things considered & 3.4 & 3.1 & 4.1 & 3.3 & 3.6 & 2.7 \\
\hline Three or more symptoms/behaviours & 16.1 & 8.8 & 17.3 & 9.0 & 16.9 & 8.5 \\
\hline Number of respondents & 903 & 2,948 & 568 & 1,205 & 659 & 1,743 \\
\hline
\end{tabular}

Note: All Ns are unweighted. 
Rural-urban differences were narrow on most items, but some differences were observed. For example, married young men in urban areas were somewhat more likely than their rural counterparts to report that they felt constantly under strain $(25 \%$ versus $20 \%)$. Similarly, unmarried young women in rural areas were somewhat more likely than their urban counterparts to report that they could not overcome their difficulties (15\% versus $10 \%)$.

Overall, $16 \%$ of young men and $9 \%$ young women reported three or more of the 12 symptoms/behaviours probed in the GHQ-12, indicative of mental disorders. Differences by marital status and rural-urban residence were negligible.

Figure 11.1: Percentage of youth reporting symptoms/behaviours suggestive of mental health disorders in the month preceding the interview, according to residence, Bihar, 2007
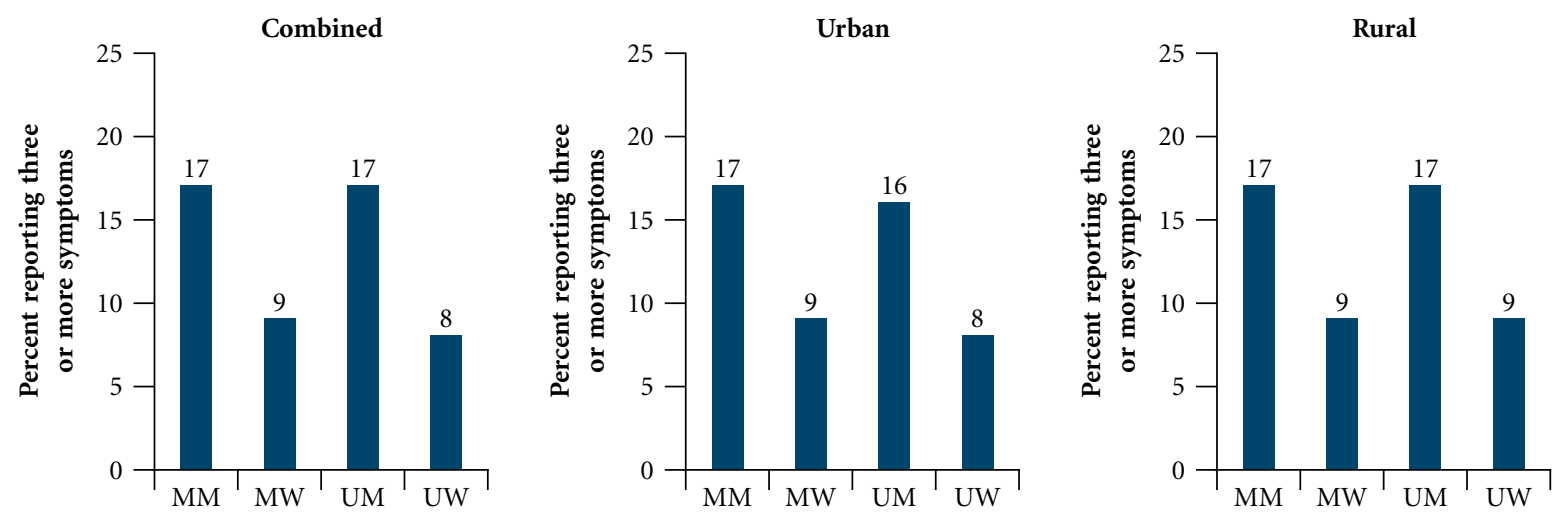

\subsection{Care and advice seeking}

Young people who reported physical or sexual and reproductive health problems were probed about whether they had sought care or advice for the problem and the source of this care or advice. Findings are presented in Table 11.4 and suggest that care and advice seeking differed by kind of problem experienced as well as, in several instances, sex and marital status of the respondent. Young women were less likely than young men to seek care for both general and sexual and reproductive health problems.

\subsubsection{General health problems}

According to findings presented in Table 11.4, over $85 \%$ of young people experiencing high fever had sought treatment. Somewhat more young men than women reported having sought treatment ( $92 \%$ versus $87 \%$ ). Differences by marital status were negligible. Treatment was sought from a government health care facility or provider by a relatively small percentage of those who sought care: $14 \%$ of young men and $9 \%$ of young women. The majority of respondents reportedly sought care from private sector providers $(66 \%$ of young men and $54 \%$ of young women), reflecting the pattern in health care seeking behaviour in India more generally. One-fifth of young men $(21 \%)$ and almost two-fifths of young women $(38 \%)$ sought treatment for high fever from sources other than government and private facilities or doctors, such as, for example, traditional health care providers or home remedies. Differences by marital status were modest; even so somewhat more unmarried than married young men sought care from private sector providers (64\% versus 58\%). 
Table 11.4: Care and advice seeking for reported health problems

Percentage of youth who experienced selected health problems by reported care and advice seeking and place of treatment, Bihar, 2007

\begin{tabular}{|c|c|c|c|c|c|c|}
\hline Care and advice seeking (\%) & $\underset{15-24}{M}$ & $\begin{array}{c}\text { W } \\
15-24\end{array}$ & $\begin{array}{c}\text { MM } \\
15-29\end{array}$ & $\begin{array}{l}\text { MW } \\
15-24\end{array}$ & $\begin{array}{c}\text { UM } \\
15-24\end{array}$ & $\begin{array}{l}\text { UW } \\
15-24\end{array}$ \\
\hline $\begin{array}{l}\text { Sought treatment for high fever } \\
\text { Number reporting high fever }\end{array}$ & $\begin{array}{r}92.4 \\
490\end{array}$ & $\begin{array}{r}86.7 \\
1,506\end{array}$ & $\begin{array}{r}93.0 \\
266\end{array}$ & $\begin{array}{r}86.9 \\
728\end{array}$ & $\begin{array}{r}93.2 \\
376\end{array}$ & $\begin{array}{r}85.8 \\
778\end{array}$ \\
\hline $\begin{array}{l}\text { Place treatment sought for high fever } \\
\text { Government facility/doctor } \\
\text { Private facility/doctor } \\
\text { Other }{ }^{2} \\
\text { Number who sought treatment for high fever }\end{array}$ & $\begin{array}{r}13.6 \\
65.7 \\
20.7 \\
\mathbf{4 4 7}\end{array}$ & $\begin{array}{r}8.6 \\
53.7 \\
37.5 \\
\mathbf{1 , 3 2 3}\end{array}$ & $\begin{array}{r}16.5 \\
58.0 \\
24.7 \\
242\end{array}$ & $\begin{array}{r}8.0 \\
52.6 \\
39.1 \\
638\end{array}$ & $\begin{array}{r}14.0 \\
63.8 \\
22.2 \\
343\end{array}$ & $\begin{array}{r}9.9 \\
55.6 \\
34.5 \\
685\end{array}$ \\
\hline $\begin{array}{l}\text { Sought treatment for injury } \\
\text { Number reporting injury }\end{array}$ & $\begin{array}{r}67.7 \\
191\end{array}$ & $\begin{array}{r}45.1 \\
144\end{array}$ & $\begin{array}{r}70.3 \\
85\end{array}$ & $\begin{array}{r}(41.5) \\
49\end{array}$ & $\begin{array}{r}66.7 \\
150\end{array}$ & $\begin{array}{r}50.6 \\
95\end{array}$ \\
\hline $\begin{array}{l}\text { Place treatment sought for injury } \\
\text { Government facility/doctor } \\
\text { Private facility/doctor } \\
\text { Other } \\
\text { Number who sought treatment for injury } \\
\text { Sought treatment for symptoms of genital infection }{ }^{3}\end{array}$ & $\begin{array}{r}14.2 \\
47.1 \\
38.7 \\
133 \\
50.3\end{array}$ & $\begin{array}{r}14.9 \\
43.2 \\
20.3 \\
82 \\
25.6\end{array}$ & $\begin{array}{r}14.1 \\
49.3 \\
36.6 \\
62 \\
60.2\end{array}$ & $\begin{array}{r}(13.3) \\
(33.3) \\
(20.0) \\
26 \\
27.1\end{array}$ & $\begin{array}{r}16.8 \\
49.6 \\
33.6 \\
103 \\
46.7\end{array}$ & $\begin{array}{r}18.6 \\
60.5 \\
18.6 \\
56 \\
19.5\end{array}$ \\
\hline $\begin{array}{l}\text { Number reporting symptoms of genital infection } \\
\text { Place treatment sought for symptoms of genital } \\
\text { infection } 1,3,4\end{array}$ & 167 & 992 & 113 & 575 & 115 & 417 \\
\hline $\begin{array}{l}\text { Government facility/doctor } \\
\text { Private facility/doctor } \\
\text { Other }{ }^{2}\end{array}$ & $\begin{array}{l}22.0 \\
53.7 \\
25.3\end{array}$ & $\begin{array}{l}12.8 \\
62.0 \\
25.2\end{array}$ & $\begin{array}{l}22.5 \\
29.6 \\
47.9\end{array}$ & $\begin{array}{l}12.8 \\
64.6 \\
22.6\end{array}$ & $\begin{array}{l}12.2 \\
67.3 \\
20.4\end{array}$ & $\begin{array}{l}11.6 \\
48.8 \\
39.5\end{array}$ \\
\hline $\begin{array}{l}\text { Number who sought treatment for symptoms of } \\
\text { genital infection }\end{array}$ & 84 & 272 & 63 & 186 & 56 & 86 \\
\hline $\begin{array}{l}\text { Sought advice on swapnadosh/nocturnal emission } \\
\text { Number reporting anxiety over swapnadosh/nocturnal } \\
\text { emission }\end{array}$ & 35.9 & NA & 32.8 & NA & 36.9 & NA \\
\hline $\begin{array}{l}\text { Person from whom advice was sought on swapnadosh/ } \\
\text { nocturnal emission } \\
\text { Friend } \\
\text { Parent } \\
\text { Relative } \\
\text { Traditional healer } \\
\text { Medical professional }\end{array}$ & $\begin{array}{r}64.1 \\
6.1 \\
2.3 \\
1.5 \\
24.4\end{array}$ & $\begin{array}{l}\text { NA } \\
\text { NA } \\
\text { NA } \\
\text { NA } \\
\text { NA }\end{array}$ & $\begin{array}{r}(60.0) \\
(0.0) \\
(10.0) \\
(0.0) \\
(15.0)\end{array}$ & $\begin{array}{l}\text { NA } \\
\text { NA } \\
\text { NA } \\
\text { NA } \\
\text { NA }\end{array}$ & $\begin{array}{r}66.4 \\
6.2 \\
0.0 \\
1.8 \\
25.7\end{array}$ & $\begin{array}{l}\text { NA } \\
\text { NA } \\
\text { NA } \\
\text { NA } \\
\text { NA }\end{array}$ \\
\hline $\begin{array}{l}\text { Number who sought advice for swapnadosh/nocturnal } \\
\text { emission } \\
\text { Sought treatment for menstrual problems } \\
\text { Number reporting menstrual problems }\end{array}$ & $\begin{array}{l}137 \\
\text { NA } \\
\text { NA }\end{array}$ & $\begin{array}{r}\text { NA } \\
35.6 \\
540\end{array}$ & $\begin{array}{r}41 \\
\mathrm{NA} \\
\mathrm{NA}\end{array}$ & $\begin{array}{r}\text { NA } \\
39.5 \\
236\end{array}$ & $\begin{array}{l}118 \\
\text { NA } \\
\text { NA }\end{array}$ & $\begin{array}{r}\text { NA } \\
24.8 \\
304\end{array}$ \\
\hline $\begin{array}{l}\text { Place treatment sought for menstrual problems }{ }^{1} \\
\text { Government facility/doctor } \\
\text { Private facility/doctor } \\
\text { Other }\end{array}$ & $\begin{array}{l}\text { NA } \\
\text { NA } \\
\text { NA }\end{array}$ & $\begin{array}{r}7.5 \\
62.6 \\
26.9\end{array}$ & $\begin{array}{l}\text { NA } \\
\text { NA } \\
\text { NA }\end{array}$ & $\begin{array}{r}6.8 \\
63.2 \\
26.5\end{array}$ & $\begin{array}{l}\text { NA } \\
\text { NA } \\
\text { NA }\end{array}$ & $\begin{array}{r}7.8 \\
58.4 \\
29.9\end{array}$ \\
\hline Number who sought treatment for menstrual problems & NA & 203 & NA & 104 & NA & 99 \\
\hline
\end{tabular}

Note: All Ns are unweighted. Column totals may not equal 100\% due to missing cases or "don't know" responses. ( ) Based on 25-49 unweighted cases. NA: Not applicable. ${ }^{1}$ Refers to the last time the respondent sought treatment. ${ }^{2}$ Includes registered medical practitioner, unregistered medical practitioner, vaid/traditional healer and home remedies. ${ }^{3}$ Includes genital ulcers, genital itching, swelling in the groin, genital discharge, burning during urination, etc. ${ }^{4}$ Multiple responses were given. 
Fewer youth had sought care for their reported injuries (68\% of young men and $45 \%$ of young women). Larger percentages of unmarried than married young women sought care for injuries (51\% versus $42 \%)$; no such difference was observed among young men. Among young men and women who sought treatment, largest proportions sought treatment from private facilities or providers ( $47 \%$ and $43 \%$ of young men and women, respectively), followed by other sources, including traditional health care providers and home remedies (39\% and 20\%, respectively). Differences by marital status were negligible, except that more unmarried than married young women reported that they sought care from private sector facilities (61\% versus $33 \%)$. We note that respondents may not always have been able to discern the extent to which the private sector provider from whom they sought care was indeed trained and licensed to provide such care.

\subsubsection{Sexual and reproductive health problems}

Responses regarding treatment seeking for sexual and reproductive health problems depict a somewhat different picture than that for general health ailments. In general, fewer young people had sought care for these problems than for general health problems. As in the case of general health problems, however, the majority who sought care did so from a private sector provider.

Of those young men who experienced symptoms of genital infection, 50\% had sought care. The married were considerably more likely than the unmarried to have sought treatment for symptoms of genital infection $(60 \%$ versus $47 \%)$. Of those who sought care, over half did so from a private sector provider $(54 \%)$, and one-quarter relied on traditional health care providers or home remedies $(25 \%)$. The married were more likely than the unmarried to rely on government facilities (23\% versus $12 \%)$ and others, including traditional health care providers and home remedies ( $48 \%$ versus $20 \%$ ) and less likely to seek care from private facilities (30\% versus $67 \%)$.

Young men who experienced anxiety about swapnadosh or nocturnal emission were asked if they had sought advice for this anxiety. Over one-third of all young men (36\%) had done so. The most common source was friends, from whom $64 \%$ of young men reported seeking advice. One-quarter sought advice from a medical professional (24\%); very few reported that they had sought advice from a traditional health care provider generally known to "treat" such symptoms (2\%). While the unmarried were more likely than the married to have sought advice from friends $(66 \%$ versus $60 \%)$ or parents $(6 \%$ versus $0 \%)$ or medical professionals ( $26 \%$ versus $15 \%)$, the married were more likely to have sought advice from others, specifically relatives $(10 \%$ versus $0 \%)$.

Seeking treatment for sexual and reproductive health problems was even more limited among young women than young men. For example, $36 \%$ of women experiencing menstrual problems had sought care for this problem, as did $26 \%$ of those who had experienced symptoms of genital infection. That even fewer young women had sought care for symptoms of genital infection than menstrual problems clearly highlights the fact that problems perceived to be associated with sex or sexual health matters were likely to go untreated by many. The married were considerably more likely than the unmarried to have sought treatment for symptoms of genital infection (27\% versus $20 \%$ ) and menstrual problems ( $40 \%$ versus $25 \%$ ), a finding attributable perhaps to the greater embarrassment that sexual and reproductive conditions may evoke among unmarried and their families than their married counterparts.

As in the case of general health problems, care was most likely to be sought from a private sector provider: $62-63 \%$ of those seeking care for symptoms of genital infection and menstrual problems. Considerable proportions (25-27\%) relied on traditional health care providers or home remedies for these problems. Larger percentages of married than unmarried young women relied on private sector providers for both 
problems, particularly for treating symptoms of genital infection (65\% versus $49 \%$ ) and conversely, more unmarried than married young women relied on traditional providers or home remedies ( $40 \%$ versus $23 \%$ for symptoms of infections, for example).

\subsection{Hesitation to access contraceptive supplies}

In order to capture the extent to which young people perceived that they could approach health care professionals for sexual and reproductive health services, the Youth Study posed two questions relating to accessing contraceptives, namely, whether the respondent would feel shy to approach a health care provider and a pharmacist, respectively, for contraceptives. Findings are presented in Table 11.5 and confirm that substantial proportions of young people would indeed feel shy to approach a health care provider or pharmacy/ medical shop for contraceptive supplies. Young men were somewhat less likely than young women to report discomfort in approaching a health care provider (40\% versus $45 \%)$ and considerably less likely to report discomfort in approaching a pharmacy or medical shop (37\% versus $53 \%)$.

Marital status differences suggest that the unmarried were more likely than the married to report discomfort in approaching a health care provider (43\% versus $25 \%$ among young men and $51 \%$ versus $42 \%$ among young women) and a pharmacy or medical shop ( $41 \%$ versus $22 \%$ among young men and $58 \%$ versus $51 \%$ among young women). Likewise, urban respondents were much less likely than rural respondents to feel hesitation in these circumstances. Findings confirm that many youth would indeed find it difficult to seek appropriate care for sexual and reproductive matters.

Table 11.5: Hesitation to access contraceptive supplies

Percentage of youth reporting hesitation to access contraceptive supplies from a health care provider or medical shop, according to residence, Bihar, 2007

\begin{tabular}{|c|c|c|c|c|c|c|}
\hline Indicators (\%) & $\begin{array}{c}M \\
15-24\end{array}$ & $\begin{array}{c}\text { W } \\
15-24\end{array}$ & $\begin{array}{c}\text { MM } \\
15-29\end{array}$ & $\begin{array}{c}\text { MW } \\
15-24\end{array}$ & $\begin{array}{c}\text { UM } \\
15-24\end{array}$ & $\begin{array}{c}\text { UW } \\
15-24\end{array}$ \\
\hline \multicolumn{7}{|c|}{ Combined } \\
\hline Would feel shy to approach an HCP for contraceptives & 39.7 & 44.6 & 24.8 & 41.7 & 43.4 & 50.8 \\
\hline $\begin{array}{l}\text { Would feel shy to approach a pharmacy/medical shop for } \\
\text { contraceptives }\end{array}$ & 36.9 & 53.4 & 22.2 & 51.4 & 40.8 & 57.7 \\
\hline Number of respondents & 1,942 & 5,529 & 1,115 & 2,341 & 1,492 & 3,188 \\
\hline \multicolumn{7}{|c|}{ Urban } \\
\hline Would feel shy to approach an HCP for contraceptives & 34.4 & 34.7 & 22.8 & 31.5 & 35.2 & 37.0 \\
\hline $\begin{array}{l}\text { Would feel shy to approach a pharmacy/medical shop for } \\
\text { contraceptives }\end{array}$ & 31.9 & 46.2 & 18.3 & 44.4 & 32.8 & 47.6 \\
\hline Number of respondents & 1,039 & 2,581 & 547 & 1,136 & 833 & 1,445 \\
\hline \multicolumn{7}{|c|}{ Rural } \\
\hline Would feel shy to approach an HCP for contraceptives & 40.6 & 45.9 & 24.9 & 42.4 & 45.0 & 53.3 \\
\hline $\begin{array}{l}\text { Would feel shy to approach a pharmacy/medical shop for } \\
\text { contraceptives }\end{array}$ & 37.7 & 54.3 & 22.5 & 51.9 & 42.4 & 59.5 \\
\hline Number of respondents & 903 & 2,948 & 568 & 1,205 & 659 & 1,743 \\
\hline
\end{tabular}

Note: All Ns are unweighted. HCP: Health care provider. 


\subsection{Attitudes towards pre-marital HIV testing and extent of HIV testing}

Youth who were aware of HIV/AIDS were asked whether they approved of pre-marital HIV testing for boys and girls, and whether they had ever undergone an HIV test. Findings, presented in Table 11.6, suggest that the large majority of youth agreed that boys and girls should be tested for HIV before marriage. More young men than women reported as such $(82-84 \%$ versus $71-75 \%)$. While differences by marital status were narrow among young men, somewhat more unmarried than married young women expressed favourable attitudes towards HIV testing (75-78\% versus 68-72\%). Likewise, while differences by place of residence were muted among young men, more urban than rural young women reported that boys and girls should be tested for HIV before marriage (83-85\% versus $69-72 \%)$.

Table 11.6: Attitudes towards pre-marital HIV testing and extent of HIV testing

Percentage of youth aware of HIV/AIDS who believe that boys/girls should be tested for HIV before marriage and percentage who have ever had an HIV test, according to residence, Bihar, 2007

\begin{tabular}{|c|c|c|c|c|c|c|}
\hline Attitudes/experiences (\%) & $\underset{15-24}{\mathrm{M}}$ & $\begin{array}{c}\text { W } \\
15-24\end{array}$ & $\begin{array}{c}\text { MM } \\
15-29\end{array}$ & $\begin{array}{c}\text { MW } \\
15-24\end{array}$ & $\begin{array}{c}\text { UM } \\
15-24\end{array}$ & $\begin{array}{c}\text { UW } \\
15-24\end{array}$ \\
\hline \multicolumn{7}{|c|}{ Combined } \\
\hline \multicolumn{7}{|c|}{ Boys should be tested for HIV before marriage } \\
\hline Yes & 83.8 & 74.6 & 83.7 & 72.0 & 83.9 & 77.5 \\
\hline No & 9.3 & 17.1 & 10.6 & 20.4 & 8.6 & 13.0 \\
\hline \multicolumn{7}{|c|}{ Girls should be tested for HIV before marriage } \\
\hline Yes & 82.1 & 71.3 & 80.9 & 68.4 & 82.4 & 74.5 \\
\hline No & 10.0 & 20.4 & 12.6 & 23.8 & 9.3 & 16.2 \\
\hline Youth who underwent an HIV test & 1.3 & 1.6 & 5.2 & 2.3 & 1.3 & 0.4 \\
\hline Number aware of HIV/AIDS & 1,768 & 3,334 & 1,004 & 1,299 & 1,366 & 2,035 \\
\hline \multicolumn{7}{|c|}{ Urban } \\
\hline \multicolumn{7}{|c|}{ Boys should be tested for HIV before marriage } \\
\hline Yes & 85.8 & 85.0 & 84.1 & 83.0 & 85.1 & 86.3 \\
\hline No & 9.0 & 9.7 & 10.2 & 12.0 & 9.4 & 8.1 \\
\hline \multicolumn{7}{|c|}{ Girls should be tested for HIV before marriage } \\
\hline Yes & 84.3 & 83.1 & 81.8 & 79.2 & 83.9 & 85.3 \\
\hline No & 9.7 & 11.5 & 12.5 & 15.8 & 9.7 & 9.0 \\
\hline Youth who underwent an HIV test & 2.6 & 2.0 & 6.9 & 5.0 & 2.1 & 0.2 \\
\hline Number aware of HIV/AIDS & 993 & 2,032 & 519 & 805 & 799 & 1,227 \\
\hline \multicolumn{7}{|c|}{ Rural } \\
\hline \multicolumn{7}{|c|}{ Boys should be tested for HIV before marriage } \\
\hline Yes & 83.5 & 72.2 & 83.7 & 70.8 & 83.7 & 74.6 \\
\hline No & 9.4 & 18.9 & 10.6 & 21.3 & 8.4 & 14.6 \\
\hline \multicolumn{7}{|c|}{ Girls should be tested for HIV before marriage } \\
\hline Yes & 81.8 & 68.5 & 80.8 & 67.0 & 82.1 & 70.9 \\
\hline No & 10.1 & 22.6 & 12.6 & 24.8 & 9.2 & 18.6 \\
\hline Youth who underwent an HIV test & 1.1 & 1.5 & 4.9 & 2.0 & 1.1 & 0.5 \\
\hline Number aware of HIV/AIDS & 775 & 1,302 & 485 & 494 & 567 & 808 \\
\hline
\end{tabular}

Note: All Ns are unweighted. Column totals may not equal 100\% due to missing cases or "don't know" responses. 
Despite favourable attitudes towards HIV testing, only a small minority of youth had ever undergone an HIV test: $1 \%$ of young men and $2 \%$ of young women. Differences by marital status and rural-urban residence were negligible. Even so, of all the categories of young people, it was married young men who were most likely to have undergone an HIV test (5\%).

\subsection{Summary}

Findings show that substantial proportions of young men reported the consumption of tobacco and alcohol; two-fifths of young men reported tobacco consumption and one-sixth reported alcohol consumption. Drug use was reported by less than $2 \%$. As expected, few young women reported that they consumed any of these substances.

Although youth is a generally healthy period of life, significant minorities reported experiencing general, mental, and sexual and reproductive health problems in the period preceding the interview. For example, $26 \%$ of young men and $28 \%$ of young women had experienced high fever, and $8 \%$ of young men and $22 \%$ of young women reported the experience of symptoms of genital infection. Moreover, $11 \%$ of young women reported menstrual problems and one-fifth of young men reported anxiety about nocturnal emission. Finally, responses indicative of mental disorders were reported by some $16 \%$ of young men and $9 \%$ of young women.

As far as care seeking for general and sexual and reproductive health problems was concerned, patterns varied by type of problem. While the large majority of those experiencing high fever, for example, sought care, many fewer sought care for sexual and reproductive health problems. Of those who sought treatment, the majority sought advice or treatment from a private facility or provider, irrespective of the type of problem. However, it is notable that one-quarter of youth who sought care for genital infections or menstrual problems used home remedies or the services of traditional or untrained providers. In the case of anxiety about nocturnal emission, moreover, the majority of young men preferred to seek advice from peers.

Findings suggest that youth were uncomfortable about seeking sexual and reproductive health services. For example, many youth would indeed find it difficult to approach a health care provider or a pharmacy/medical shop for contraceptives.

Finally, small minorities (1-2\%) reported that they had undergone HIV testing. Youth were, however, overwhelmingly in favour of pre-marital HIV testing. 


\section{Participation in civil society} and political life

The National Youth Policy 2003 has underscored the role of India's youth in political decision-making and argued for greater representation of youth in appropriate bodies and more extensive youth participation in the design and implementation of programmes (Ministry of Youth Affairs and Sports, 2003). Indeed, there is a recognition that today's youth, who have better access to skills and information than those of earlier generations, can play an important role in influencing political processes and the socio-economic development of the country.

This chapter presents a profile of youth involvement in government- and NGO-sponsored programmes, community activities and political processes. It also explores young people's behaviours and attitudes towards individuals of different religions and caste groups, violence within their community and their own participation in such violence, and their perceptions about the most important problem facing youth in India.

\subsection{Awareness of and participation in government- and NGO-sponsored programmes}

Youth were asked whether they were aware of programmes that had taken place in their village or urban neighbourhood in the three years preceding the interview, whether they had participated in these programmes and whether these programmes had been organised by government agencies or NGOs. Findings are presented in Table 12.1.

Awareness of programmes that addressed youth needs was extremely limited; just $15 \%$ of young men and $8 \%$ of young women reported awareness of one or more programmes that addressed youth needs organised in the three years prior to interview (see also Figure 12.1). Differences by marital status and rural-urban residence were negligible. No differences in the level of awareness were evident by the focus of programmes conducted.

Over three-fifths of young men (63\%) and almost three-quarters (72\%) of young women who were familiar with programmes reported that these were organised by government agencies. Moreover, $25 \%$ of young men and $8 \%$ of young women also reported awareness of programmes organised by the NGO sector. One-fifth of young women reported that they were unsure about the agency that organised these programmes. While somewhat more married than unmarried young men reported awareness of government-sponsored programmes (69\% versus 61\%), the reverse was true for young women (67\% of the married compared to $79 \%$ of the unmarried). Urban youth were far more likely than rural youth to report awareness of NGO-sponsored programmes ( $43 \%$ and $23 \%$ of young men in urban and rural areas, respectively; $14 \%$ and $7 \%$ of young women, respectively). Conversely, somewhat more rural than urban young men (but not women) reported awareness of programmes sponsored by government (64\% versus $58 \%$ ). 
Table 12.1: Awareness of and participation in government-and NGO-sponsored programmes

Percentage of youth reporting awareness of and participation in government- and NGO-sponsored programmes conducted in the village/neighbourhood in the three years preceding the interview, according to residence, Bihar, 2007

\begin{tabular}{|c|c|c|c|c|c|c|}
\hline $\begin{array}{l}\text { Awareness of and participation in } \\
\text { programmes }(\%)\end{array}$ & $\underset{15-24}{M}$ & $\begin{array}{c}\text { W } \\
15-24\end{array}$ & $\begin{array}{c}\text { MM } \\
15-29\end{array}$ & $\begin{array}{l}\text { MW } \\
15-24\end{array}$ & $\begin{array}{c}\text { UM } \\
15-24\end{array}$ & $\begin{array}{l}\text { UW } \\
15-24\end{array}$ \\
\hline \multicolumn{7}{|c|}{ Combined } \\
\hline Aware of programme(s) held & 14.7 & 7.5 & 13.2 & 7.0 & 14.9 & 8.6 \\
\hline Focus of programmes held & & & & & & \\
\hline Health promotion & 3.1 & 1.4 & 2.4 & 1.3 & 3.2 & 1.7 \\
\hline Awareness/leadership/vocational/life skills & 3.3 & 2.1 & 2.7 & 2.3 & 3.6 & 1.9 \\
\hline Employment $^{1}$ & 3.0 & 0.3 & 4.1 & 0.2 & 2.1 & 0.4 \\
\hline Self-help group & 0.5 & 0.7 & 0.3 & 0.9 & 0.6 & 0.4 \\
\hline Literacy & 4.4 & 3.7 & 3.8 & 3.3 & 4.5 & 4.5 \\
\hline Sports and recreation & 3.3 & 0.4 & 2.2 & 0.2 & 3.4 & 0.6 \\
\hline Number of respondents & 1,942 & 5,529 & 1,115 & 2,341 & 1,492 & 3,188 \\
\hline \multicolumn{7}{|l|}{ Organising agency } \\
\hline Government & 63.3 & 71.6 & 69.4 & 67.1 & 60.5 & 78.8 \\
\hline NGO & 25.3 & 7.5 & 19.7 & 6.1 & 25.4 & 9.2 \\
\hline Don't know & 6.9 & 20.7 & 4.1 & 26.8 & 9.4 & 12.1 \\
\hline Number aware of any programme(s) & 263 & 407 & 136 & 129 & 207 & 278 \\
\hline Participated in programme(s) held & 7.2 & 1.9 & 6.8 & 1.6 & 6.9 & 2.4 \\
\hline Number of respondents & 1,942 & 5,529 & 1,115 & 2,341 & 1,492 & 3,188 \\
\hline \multicolumn{7}{|l|}{ Participation in specific programmes } \\
\hline Health promotion & 16.1 & 10.5 & 6.7 & $(10.5)$ & 20.4 & 10.5 \\
\hline Awareness/leadership/vocational/life skills & 27.3 & 38.5 & 17.3 & $(43.6)$ & 30.1 & 32.9 \\
\hline Employment $^{1}$ & 16.2 & 4.8 & 32.9 & $(5.3)$ & 7.8 & 2.7 \\
\hline Self-help group & 2.8 & 4.8 & 0.0 & $(5.3)$ & 4.9 & 3.9 \\
\hline Literacy & 16.8 & 41.3 & 25.0 & $(42.1)$ & 17.5 & 41.3 \\
\hline Sports and recreation & 32.4 & 5.7 & 22.4 & $(0.0)$ & 34.0 & 12.0 \\
\hline Number who participated in any programme(s) & 125 & 112 & 65 & 31 & 94 & 81 \\
\hline \multicolumn{7}{|c|}{ Urban } \\
\hline Aware of programme(s) held & 12.5 & 6.8 & 10.9 & 3.5 & 13.1 & 9.1 \\
\hline \multicolumn{7}{|l|}{ Focus of programmes held } \\
\hline Health promotion & 5.0 & 1.6 & 3.3 & 1.4 & 5.3 & 1.8 \\
\hline Awareness/leadership/vocational/life skills & 4.7 & 1.6 & 3.3 & 0.7 & 5.3 & 2.2 \\
\hline Employment $^{1}$ & 1.8 & 0.5 & 2.2 & 0.0 & 1.6 & 0.6 \\
\hline Self-help group & 0.4 & 0.0 & 0.0 & 0.0 & 0.4 & 0.0 \\
\hline Literacy & 2.9 & 3.1 & 2.2 & 2.1 & 2.9 & 4.0 \\
\hline Sports and recreation & 3.2 & 0.5 & 2.2 & 0.0 & 3.3 & 0.8 \\
\hline Number of respondents & 1,039 & 2,581 & 547 & 1,136 & 833 & 1,445 \\
\hline \multicolumn{7}{|l|}{ Organising agency } \\
\hline Government & 58.3 & 74.4 & 60.0 & $(80.0)$ & 57.6 & 75.0 \\
\hline NGO & 42.9 & 13.6 & 33.3 & $(0.0)$ & 43.8 & 15.6 \\
\hline Don't know & 2.9 & 11.6 & 10.0 & $(20.0)$ & 3.1 & 11.1 \\
\hline
\end{tabular}


Table 12.1: (Cont'd)

\begin{tabular}{|c|c|c|c|c|c|c|}
\hline $\begin{array}{l}\text { Awareness of and participation in } \\
\text { programmes }(\%)\end{array}$ & $\underset{15-24}{M}$ & $\begin{array}{c}\text { W } \\
15-24\end{array}$ & $\begin{array}{c}\text { MM } \\
15-29\end{array}$ & $\begin{array}{c}\text { MW } \\
15-24\end{array}$ & $\begin{array}{c}\text { UM } \\
15-24\end{array}$ & $\begin{array}{c}\text { UW } \\
\text { 15-24 }\end{array}$ \\
\hline \multicolumn{7}{|c|}{ Urban } \\
\hline Number aware of any programme(s) & 128 & 174 & 58 & 42 & 108 & 132 \\
\hline Participated in programme(s) held & 6.1 & 2.0 & 4.3 & 0.7 & 6.1 & 2.8 \\
\hline Number of respondents & 1,039 & 2,581 & 547 & 1,136 & 833 & 1,445 \\
\hline \multicolumn{7}{|l|}{ Participation in specific programmes } \\
\hline Health promotion & 35.3 & 7.7 & $(50.0)$ & * & 40.0 & $(7.1)$ \\
\hline Awareness/leadership/vocational/life skills & 41.2 & 30.8 & $(50.0)$ & * & 40.0 & $(28.6)$ \\
\hline Employment $^{1}$ & 11.8 & 7.7 & $(25.0)$ & * & 12.5 & $(7.1)$ \\
\hline Self-help group & 0.0 & 0.0 & $(0.0)$ & * & 0.0 & $(0.0)$ \\
\hline Literacy & 17.6 & 46.2 & $(25.0)$ & * & 20.0 & $(40.0)$ \\
\hline Sports and recreation & 23.5 & 7.7 & $(0.0)$ & * & 20.0 & $(13.3)$ \\
\hline Number who participated in any programme(s) & 60 & 53 & 25 & 11 & 50 & 42 \\
\hline \multicolumn{7}{|c|}{ Rural } \\
\hline Aware of programme(s) held & 15.1 & 7.6 & 13.4 & 7.2 & 15.3 & 8.5 \\
\hline \multicolumn{7}{|l|}{ Focus of programmes held } \\
\hline Health promotion & 2.8 & 1.4 & 2.3 & 1.3 & 2.6 & 1.7 \\
\hline Awareness/leadership/vocational/life skills & 3.1 & 2.2 & 2.6 & 2.4 & 3.3 & 1.8 \\
\hline Employment $^{1}$ & 3.1 & 0.2 & 4.3 & 0.2 & 2.2 & 0.3 \\
\hline Self-help group & 0.5 & 0.8 & 0.3 & 1.0 & 0.6 & 0.5 \\
\hline Literacy & 4.6 & 3.8 & 3.9 & 3.5 & 4.8 & 4.6 \\
\hline Sports and recreation & 3.3 & 0.3 & 2.3 & 0.2 & 3.4 & 0.6 \\
\hline Number of respondents & 903 & 2,948 & 568 & 1,205 & 659 & 1,743 \\
\hline \multicolumn{7}{|l|}{ Organising agency } \\
\hline Government & 64.0 & 71.2 & 70.1 & 67.1 & 60.7 & 79.5 \\
\hline NGO & 22.8 & 7.0 & 18.8 & 6.3 & 22.0 & 7.9 \\
\hline Don't know & 7.5 & 21.8 & 3.6 & 27.2 & 10.5 & 12.7 \\
\hline Number aware of any programme(s) & 135 & 233 & 78 & 87 & 99 & 146 \\
\hline Participated in programme(s) held & 7.5 & 1.9 & 6.9 & 1.7 & 7.1 & 2.3 \\
\hline Number of respondents & 903 & 2,948 & 568 & 1,205 & 659 & 1,743 \\
\hline \multicolumn{7}{|l|}{ Participation in specific programmes } \\
\hline Health promotion & 13.5 & 10.9 & $(5.6)$ & * & $(18.2)$ & $(11.3)$ \\
\hline Awareness/leadership/vocational/life skills & 25.4 & 39.6 & $(16.7)$ & * & $(28.4)$ & $(32.8)$ \\
\hline Employment $^{1}$ & 16.8 & 4.4 & $(33.3)$ & * & $(6.8)$ & $(1.6)$ \\
\hline Self-help group & 3.2 & 5.5 & $(0.0)$ & * & $(4.6)$ & $(4.9)$ \\
\hline Literacy & 16.7 & 40.7 & $(25.4)$ & * & $(17.2)$ & $(41.0)$ \\
\hline Sports and recreation & 34.1 & 4.4 & $(22.5)$ & * & $(36.4)$ & $(12.9)$ \\
\hline Number who participated in any programme(s) & 65 & 59 & 40 & 20 & 44 & 39 \\
\hline
\end{tabular}

Note: All Ns are unweighted. ( ) Based on 25-49 unweighted cases. ${ }^{\star}$ Percentage not shown, based on fewer than 25 unweighted cases. Column totals may exceed $100 \%$ due to multiple responses. ${ }^{1}$ Includes Employment Guarantee Scheme (EGS), Jawahar Rozgar Yojana (JRY), National Rural Employment Programme (NREP), Pradhan Mantri Rozgar Yojana (PMRY), Training for Rural Youth for Self Employment (TRYSEM), etc. 
A small minority of youth reported participation in a programme in the preceding three years $-7 \%$ of young men and $2 \%$ of young women. Differences by marital status and rural-urban residence were negligible. Programmes in which youth participated differed between young men and women. Among young men who reported participation in any programme, the largest percentage participated in sports and recreation activities (32\%), followed by leadership and life skills programmes (27\%). One in six young men each reported participation in programmes that focus on health promotion, employment and literacy. In contrast, leading programmes in which young women participated included those that focus on literacy (41\%), leadership and life skills (39\%) and health promotion (11\%). Patterns differed between the married and the unmarried. While the programme in which the largest percentage of married young men participated was employment related programmes, it was sports and recreation programmes among the unmarried. Moreover, findings show that the unmarried were more likely than the married to report participation in programmes that focus on health promotion (20\% versus $7 \%$ ), leadership and life skills (30\% versus $17 \%$ ) and sports and recreation ( $34 \%$ versus $22 \%$ ) and less likely to participate in programmes that focus on employment ( $8 \%$ versus $33 \%$ ) and literacy (18\% versus $25 \%)$. Among young women, the unmarried were more likely than the married to participate in sports and recreation activities (12\% versus $0 \%)$. Conversely, they were less likely to participate in leadership and life skills programmes (33\% versus $44 \%)$.

Rural-urban differences were notable and varied across young men and women. Among young men, those in urban areas were more likely than their rural counterparts to report participation in health promotion (35\% versus $14 \%)$ and leadership and life skills development programmes (41\% versus $25 \%)$ and less likely to report participation in employment programmes (12\% versus $17 \%)$ and sports and recreation activities (24\% versus $34 \%)$. Among young women, those in rural areas were more likely than urban women to report participation in leadership and life skills programmes (40\% versus $31 \%)$ and self-help group activities (6\% versus $0 \%$ ). They were somewhat less likely, however, than their urban counterparts to have participated in literacy programmes $(41 \%$ versus $46 \%)$.

Figure 12.1: Percentage of youth reporting awareness of and participation in government- and NGO-sponsored programmes in the three years preceding the interview, according to residence, Bihar, 2007

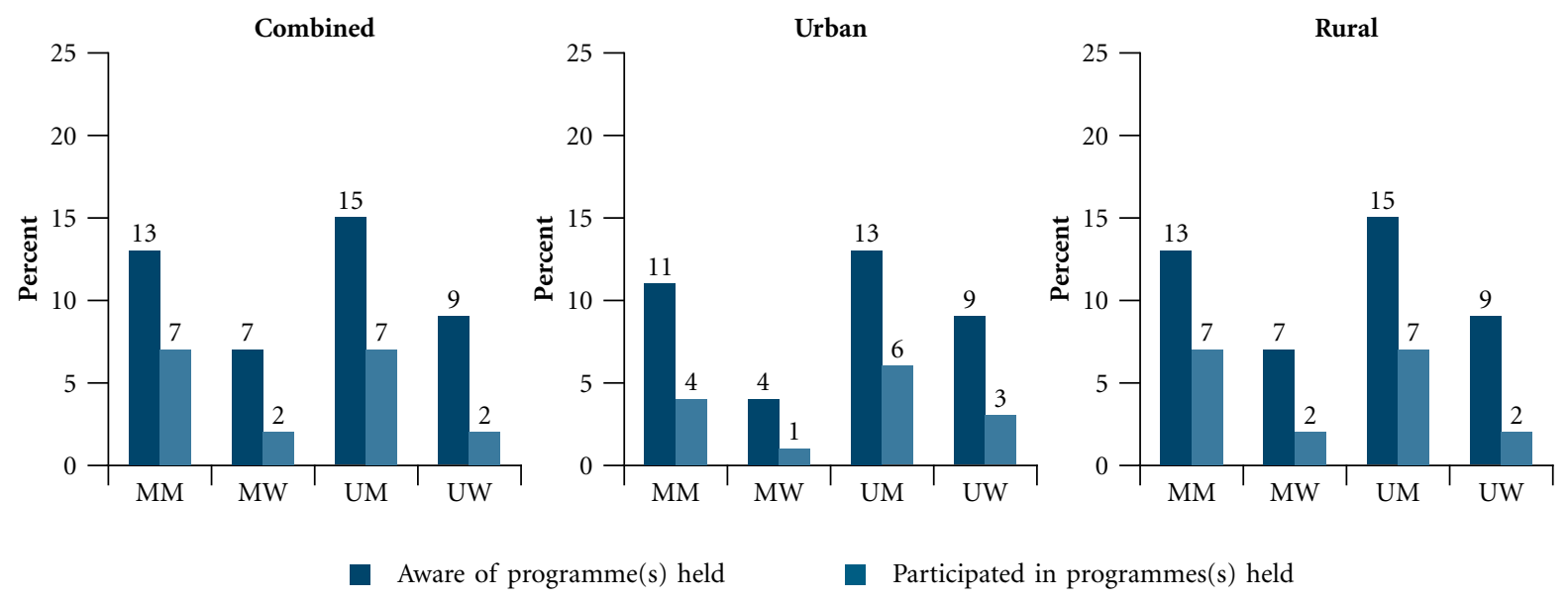




\subsection{Participation in community- or panchayat-sponsored programmes}

In many villages and urban neighbourhoods, community-led activities include, for example, cleanliness drives, health promotion activities, celebration of festivals and national days, and so on. As part of the Youth Study, youth were asked whether they had participated in any community-led activities organised by the panchayat/ community leaders in the 12 months prior to interview. Findings, reported in Table 12.2, suggest that youth participation in these activities was limited and that young women were far less likely than young men to have participated in these activities. Almost one-quarter of young men, compared to $4 \%$ of young women, reported having participated in a community-led programme in the last year. Participation was more likely to be reported by unmarried than married youth (24\% versus $17 \%$ among young men and $7 \%$ versus $2 \%$ among young women). Rural-urban differences were negligible.

Table 12.2: Participation in community-led programmes

Percentage of youth who attended community-led programmes in the village/urban neighbourhood and types of programmes attended in the 12 months preceding the interview, according to residence, Bihar, 2007

\begin{tabular}{|c|c|c|c|c|c|c|}
\hline Participation in community-led programmes (\%) & $\begin{array}{c}\mathrm{M} \\
15-24\end{array}$ & $\begin{array}{c}\text { W } \\
15-24\end{array}$ & $\begin{array}{c}\text { MM } \\
15-29\end{array}$ & $\begin{array}{c}\text { MW } \\
15-24\end{array}$ & $\begin{array}{c}\text { UM } \\
15-24\end{array}$ & $\begin{array}{c}\text { UW } \\
\text { 15-24 }\end{array}$ \\
\hline \multicolumn{7}{|c|}{ Combined } \\
\hline Attended any programme(s) organised & 22.6 & 3.9 & 17.1 & 2.1 & 24.2 & 7.4 \\
\hline Number of respondents & 1,942 & 5,529 & 1,115 & 2,341 & 1,492 & 3,188 \\
\hline Specific programmes attended & & & & & & \\
\hline Cleanliness/sanitation & 8.3 & 6.0 & 11.5 & $(8.0)$ & 6.6 & 5.5 \\
\hline Health promotion & 2.7 & 3.2 & 2.6 & $(4.0)$ & 3.6 & 3.0 \\
\hline Festival celebration & 42.6 & 43.1 & 60.4 & $(60.0)$ & 38.8 & 34.6 \\
\hline National day celebration & 69.5 & 64.7 & 53.9 & $(52.0)$ & 74.8 & 71.6 \\
\hline Number who attended above programmes & 446 & 259 & 179 & 41 & 369 & 218 \\
\hline \multicolumn{7}{|c|}{ Urban } \\
\hline Attended any programme(s) organised & 23.7 & 4.1 & 15.2 & 1.4 & 24.9 & 6.1 \\
\hline Number of respondents & 1,039 & 2,581 & 547 & 1,136 & 833 & 1,445 \\
\hline Specific programmes attended & & & & & & \\
\hline Cleanliness/sanitation & 10.6 & 3.8 & 14.3 & * & 9.8 & 6.7 \\
\hline Health promotion & 4.5 & 3.8 & 7.1 & * & 4.9 & 3.3 \\
\hline Festival celebration & 40.9 & 34.6 & 57.1 & * & 37.7 & 26.7 \\
\hline National day celebration & 74.2 & 61.5 & 50.0 & * & 77.0 & 73.3 \\
\hline Number who attended above programmes & 243 & 102 & 82 & 14 & 209 & 88 \\
\hline \multicolumn{7}{|c|}{ Rural } \\
\hline Attended any programme(s) organised & 22.4 & 3.9 & 17.3 & 2.2 & 24.1 & 7.6 \\
\hline Number of respondents & 903 & 2,948 & 568 & 1,205 & 659 & 1,743 \\
\hline Specific programmes attended & & & & & & \\
\hline Cleanliness/sanitation & 7.9 & 6.2 & 11.3 & $(8.3)$ & 6.0 & 5.3 \\
\hline Health promotion & 2.6 & 3.1 & 2.2 & $(4.2)$ & 3.3 & 2.9 \\
\hline Festival celebration & 43.0 & 44.0 & 60.5 & $(58.3)$ & 39.0 & 35.6 \\
\hline National day celebration & 68.7 & 65.1 & 53.9 & $(54.2)$ & 74.3 & 71.4 \\
\hline Number who attended above programmes & 203 & 157 & 97 & 27 & 160 & 130 \\
\hline
\end{tabular}

Note: All Ns are unweighted. ( ) Based on 25-49 unweighted cases. ${ }^{\star P e r c e n t a g e ~ n o t ~ s h o w n, ~ b a s e d ~ o n ~ f e w e r ~ t h a n ~} 25$ unweighted cases. Column totals may exceed $100 \%$ due to multiple responses. 
Findings suggest that among those who participated in community led programmes, the activity in which the largest percentage of youth participated was celebration of national days ( $70 \%$ of young men and $65 \%$ of young women), followed by celebration of festivals (43\%). More married than unmarried youth participated in celebrations of festivals (60\% versus 39\% among young men and $60 \%$ versus $35 \%$ among young women). In contrast, more unmarried than married youth participated in celebrations of national days $(75 \%$ versus $54 \%$ among young men and $72 \%$ versus $52 \%$ among young women). Hardly any young people participated, in community cleanliness or sanitation activities or health promotion activities (2-8\%).

\subsection{Membership in organised groups}

Youth were asked whether they belonged to any organised group, ranging from self-help groups to youth groups to sports and social clubs. Findings, reported in Table 12.3, suggest that relatively small proportions of youth were members of any group. Young men were somewhat more likely than young women to report such membership (8\% compared to 2\%). Marital status differences and rural-urban differences were narrow. The types of groups in which youth reported membership did not differ by marital status or residence of the respondent; however, while young men were most likely to report membership in a sports club or social group (6\%), percentages reporting membership did not exceed $1 \%$ among young women, irrespective of the type of group.

Among married young men who reported group membership, over four-fifths (82\%) had become members prior to marriage, including $84 \%$ in rural areas and $71 \%$ in urban areas. The opposite was true among married young women, of whom $67 \%$ had joined an organised group after marriage, confirming the limited duration of exposure, prior to marriage, of young women to organised groups.

Table 12.3: Membership in organised groups

Percentage of youth reporting membership in organised groups, according to residence, Bihar, 2007

\begin{tabular}{|c|c|c|c|c|c|c|}
\hline Membership in organised groups (\%) & $\underset{15-24}{\mathrm{M}}$ & $\begin{array}{c}\mathrm{W} \\
15-24\end{array}$ & $\begin{array}{c}\mathrm{MM} \\
15-29\end{array}$ & $\begin{array}{c}\text { MW } \\
15-24\end{array}$ & $\begin{array}{c}\text { UM } \\
15-24\end{array}$ & $\begin{array}{c}\text { UW } \\
15-24\end{array}$ \\
\hline \multicolumn{7}{|c|}{ Combined } \\
\hline Member of an organised group & 8.2 & 1.5 & 5.4 & 1.4 & 9.2 & 1.6 \\
\hline Self-help group & 0.4 & 0.1 & 0.7 & 0.1 & 0.3 & 0.0 \\
\hline Mahila mandal & NA & 0.7 & NA & 0.9 & NA & 0.1 \\
\hline Social or sports club & 6.1 & 0.4 & 3.5 & 0.2 & 7.0 & 0.8 \\
\hline Youth group/yuva/tarun/kishor/kishori mandal & 1.6 & 0.4 & 1.2 & 0.3 & 1.7 & 0.6 \\
\hline Other & 0.3 & 0.0 & 0.2 & 0.0 & 0.3 & 0.1 \\
\hline Number of respondents & 1,942 & 5,529 & 1,115 & 2,341 & 1,492 & 3,188 \\
\hline \multicolumn{7}{|l|}{ Became member of an organised group ${ }^{1}$} \\
\hline Before marriage & NA & NA & 82.3 & $(33.3)$ & NA & $\mathrm{NA}$ \\
\hline After marriage & NA & NA & 17.7 & $(66.7)$ & NA & NA \\
\hline Number reporting membership in an organised group & NA & NA & 73 & 36 & NA & NA \\
\hline \multicolumn{7}{|c|}{ Urban } \\
\hline Member of an organised group & 7.9 & 2.5 & 7.6 & 1.4 & 8.2 & 3.2 \\
\hline Self-help group & 0.7 & 0.3 & 0.0 & 0.0 & 0.4 & 0.2 \\
\hline Mahila mandal & NA & 0.3 & NA & 0.7 & NA & 0.2 \\
\hline
\end{tabular}

Cont'd on next page... 
Table 12.3: (Cont'd)

\begin{tabular}{|c|c|c|c|c|c|c|}
\hline Membership in organised groups (\%) & $\underset{15-24}{M}$ & $\begin{array}{c}\text { W } \\
15-24\end{array}$ & $\begin{array}{c}\text { MM } \\
15-29\end{array}$ & $\begin{array}{c}\text { MW } \\
15-24\end{array}$ & $\begin{array}{c}\text { UM } \\
15-24\end{array}$ & $\begin{array}{l}\text { UW } \\
\text { 15-24 }\end{array}$ \\
\hline \multicolumn{7}{|c|}{ Urban } \\
\hline Social or sports club & 5.0 & 1.6 & 4.3 & 0.7 & 5.3 & 2.0 \\
\hline Youth group/yuva/tarun/kishor/kishori mandal & 1.8 & 0.5 & 2.2 & 0.0 & 1.6 & 0.8 \\
\hline Other & 0.4 & 0.2 & 1.1 & 0.0 & 0.4 & 0.2 \\
\hline Number of respondents & 1,039 & 2,581 & 547 & 1,136 & 833 & 1,445 \\
\hline \multicolumn{7}{|l|}{ Became member of an organised group ${ }^{1}$} \\
\hline Before marriage & NA & NA & $(71.4)$ & * & NA & NA \\
\hline After marriage & NA & NA & $(28.6)$ & * & NA & NA \\
\hline Number reporting membership in an organised group & NA & NA & 43 & 18 & NA & NA \\
\hline \multicolumn{7}{|c|}{ Rural } \\
\hline Member of an organised group & 8.2 & 1.3 & 5.2 & 1.4 & 9.4 & 1.3 \\
\hline Self-help group & 0.4 & 0.1 & 0.8 & 0.1 & 0.3 & 0.0 \\
\hline Mahila mandal & NA & 0.7 & NA & 1.0 & NA & 0.1 \\
\hline Social or sports club & 6.2 & 0.3 & 3.4 & 0.1 & 7.2 & 0.6 \\
\hline Youth group/yuva/tarun/kishor/kishori mandal & 1.6 & 0.4 & 1.1 & 0.3 & 1.7 & 0.6 \\
\hline Other & 0.2 & 0.0 & 0.2 & 0.0 & 0.3 & 0.1 \\
\hline Number of respondents & 903 & 2,948 & 568 & 1,205 & 659 & 1,743 \\
\hline \multicolumn{7}{|l|}{ Became member of an organised group ${ }^{1}$} \\
\hline Before marriage & NA & NA & $(83.6)$ & * & NA & NA \\
\hline After marriage & NA & NA & $(16.4)$ & * & NA & NA \\
\hline Number reporting membership in an organised group & NA & NA & 30 & 18 & NA & NA \\
\hline
\end{tabular}

Note: All Ns are unweighted. ( ) Based on 25-49 unweighted cases. ${ }^{\star}$ Percentage not shown, based on fewer than 25 unweighted cases. NA: Not applicable. ${ }^{1}$ Column total may not equal $100 \%$ due to missing cases.

\subsection{Perceptions about action taken by rural panchayats in addressing defiance of social norms}

In the course of pre-survey qualitative investigations, researchers noted that in several rural areas, village panchayats took action in various situations in which youth did not adhere to social norms. Hence, youth in the rural areas were asked whether they believed that panchayats in their villages had ever taken action if someone was reported to have teased a girl or woman, if parents refused to permit their sons or daughters to marry someone of their choice, if youth were found to have engaged in pre- or extra-marital sex or if an unmarried girl became pregnant. Responses are reported in Table 12.4.

Considerable proportions of youth perceived that their village panchayats would punish those accused of teasing a girl or woman (58\% and $49 \%$ of young men and women, respectively) and would fine youth who had engaged in pre- or extra-marital sex (36\% and 39\% of young men and women, respectively). They were far less likely to report that the local panchayat would arrange the marriage of youth whose parents had refused to permit them to marry someone of their choice ( $21 \%$ and $25 \%$, respectively). One in ten young men and one in seven young women reported that the panchayat had ever forced a boy to marry a girl whom he made pregnant. As seen above, gender and marital status differences were typically modest. 
Table 12.4: Perceptions about actions taken by the panchayat in case of defiance of social norms

Percent distribution of youth by perceptions about actions taken by the panchayat in case of defiance of social norms in selected situations, Bihar (rural), 2007

\begin{tabular}{|c|c|c|c|c|c|c|}
\hline Perceptions (\%) & $\begin{array}{c}\mathrm{M} \\
15-24\end{array}$ & $\begin{array}{c}\text { W } \\
15-24\end{array}$ & $\begin{array}{c}\text { MM } \\
15-29\end{array}$ & $\begin{array}{l}\text { MW } \\
15-24\end{array}$ & $\begin{array}{l}\text { UM } \\
15-24\end{array}$ & $\begin{array}{l}\text { UW } \\
15-24\end{array}$ \\
\hline \multicolumn{7}{|c|}{$\begin{array}{l}\text { Panchayat would punish anyone who teases a } \\
\text { girl/woman }\end{array}$} \\
\hline Yes & 57.8 & 49.0 & 59.6 & 47.3 & 56.4 & 52.6 \\
\hline No & 33.0 & 32.9 & 34.6 & 32.6 & 32.6 & 33.7 \\
\hline Can't say & 9.2 & 18.1 & 5.8 & 20.1 & 11.0 & 13.7 \\
\hline \multicolumn{7}{|c|}{$\begin{array}{l}\text { Panchayat would fine a boy/girl who had } \\
\text { engaged in pre-/extra-marital sexual relations }\end{array}$} \\
\hline Yes & 36.3 & 38.7 & 33.3 & 39.2 & 36.9 & 37.7 \\
\hline No & 47.1 & 33.9 & 53.0 & 32.4 & 45.5 & 37.2 \\
\hline Can't say & 16.6 & 27.4 & 13.7 & 28.4 & 17.6 & 25.2 \\
\hline \multicolumn{7}{|c|}{$\begin{array}{l}\text { Panchayat would arrange the marriage of youth } \\
\text { if parents refused to let them marry }\end{array}$} \\
\hline Yes & 20.6 & 24.8 & 19.3 & 24.1 & 20.6 & 26.2 \\
\hline No & 64.6 & 57.1 & 69.0 & 55.8 & 63.6 & 59.8 \\
\hline Can't say & 14.8 & 18.1 & 11.7 & 20.1 & 15.8 & 14.0 \\
\hline \multicolumn{7}{|c|}{$\begin{array}{l}\text { Panchayat had ever forced a boy to marry a } \\
\text { girl who he had made pregnant }\end{array}$} \\
\hline Yes & 10.5 & 15.0 & 11.1 & 14.6 & 10.3 & 15.9 \\
\hline No & 64.5 & 47.1 & 70.6 & 45.9 & 63.1 & 49.8 \\
\hline Can't say & 25.0 & 37.8 & 18.3 & 39.5 & 26.7 & 34.4 \\
\hline Number of respondents & 903 & 2,948 & 568 & 1,205 & 659 & 1,743 \\
\hline
\end{tabular}

Note: All Ns are unweighted. Column totals may not equal 100\% due to missing cases. Questions were asked only of respondents in rural areas.

\subsection{Voting behaviour and perceptions of political matters}

Table 12.5 presents the percentage of eligible youth-that is, those at least 20 years of age at the time of interview who would have been eligible to vote prior to interview-who voted in the last election. Findings suggest that voting behaviour was far from universal and varied considerably by sex, marital status and ruralurban residence (see also Figure 12.2). Larger proportions of eligible young men (65\%) than women (51\%) and larger proportions of married than unmarried youth reported that they had voted in the last election held. Specifically, $79 \%$ of eligible married young men reported that they had voted in the last election, compared to $62 \%$ of unmarried young men. The corresponding percentages among young women were $53 \%$ and 34\%, respectively. As shown in Figure 12.2, rural-urban differences suggest that more rural than urban youth, particularly young women reported voting in the last election (65\% versus $61 \%$ among young men and $53 \%$ versus $37 \%$ among young women); the pattern differed in case of unmarried young women among whom more urban than rural women reported voting in the last election ( $40 \%$ versus $30 \%)$.

Table 12.5 also reports youth perceptions about political processes, notably the extent of disillusionment with the ability of any political party to achieve change at the community level and the extent to which respondents believed that people could vote freely and without fear, pressure or influence. 
Figure 12.2: Percentage of youth aged 20 or above who voted in the last election, according to residence, Bihar, 2007
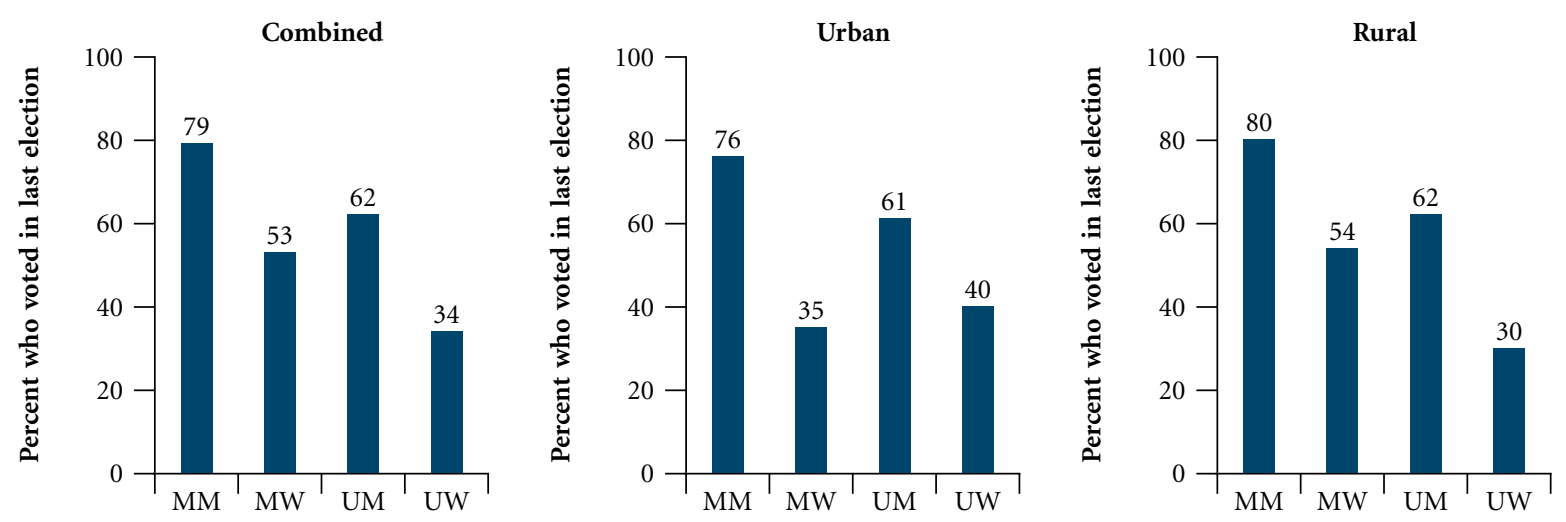

Findings indicate a gender divide in young people's perceptions about the commitment of political parties' to work for change at the community level. The majority of young men (69\%) reported disillusionment with political parties' commitment to improving local conditions. In contrast, the majority of young women (52\%) reported no such disillusionment and only one-third of young women agreed that there would be no improvement in their village/area no matter which political party was in control at the state level. Differences by marital status and rural-urban residence were negligible. At the same time, most young people- $82 \%$ and $80 \%$ of young men and women, respectively-felt that one could vote freely and without fear or pressure. Even so, it was notable that $13 \%$ of young men and $10 \%$ of young women felt that one could not vote freely. While differences by marital status were negligible, urban youth were more likely than rural youth to report that one could vote freely and without fear or pressure ( $86 \%$ versus $81 \%$ among young men and $88 \%$ versus $79 \%$ among young women).

\subsection{Expression of secular attitudes}

In order to gauge attitudes regarding social interaction with individuals of different castes and religions, the Youth Study inquired whether youth mixed freely with those of other castes and religions, whether they would eat together with a person from a different caste or religion, whether they would talk to someone who had an inter-caste marriage and whether they considered it acceptable to punish someone who showed disrespect to their religion. Findings, presented in Table 12.6, suggest that on balance, youth did not express secular attitudes; however, expressions of secular attitudes varied considerably by issue, sex of the respondent and rural-urban residence.

In response to specific issues, both young men and young women were most likely to report that they mixed freely with individuals of different castes (95\% and $87 \%$, respectively) and religions ( $92 \%$ and $82 \%$, respectively). Despite this relatively secular profile, many fewer reported that they would eat together with a person from a different caste or religion (64\% of young men and $38 \%$ of young women) or talk to someone who had an inter-caste marriage (35\% and $46 \%$, respectively). Likewise, $63 \%$ of young men and $57 \%$ of young women felt that it was acceptable to punish someone who showed disrespect to their religion. On all issues assessed, except on the issues of social interactions with a person who had an inter-caste marriage and tolerance in situations characterised by religious disharmony, young men were more likely than young women to report secular attitudes. 
Table 12.5: Voting behaviour of eligible youth and perceptions about political matters

Percentage of youth aged 20 or above who voted in the last election and percent distribution of all youth by their perceptions about political matters, according to residence, Bihar, 2007

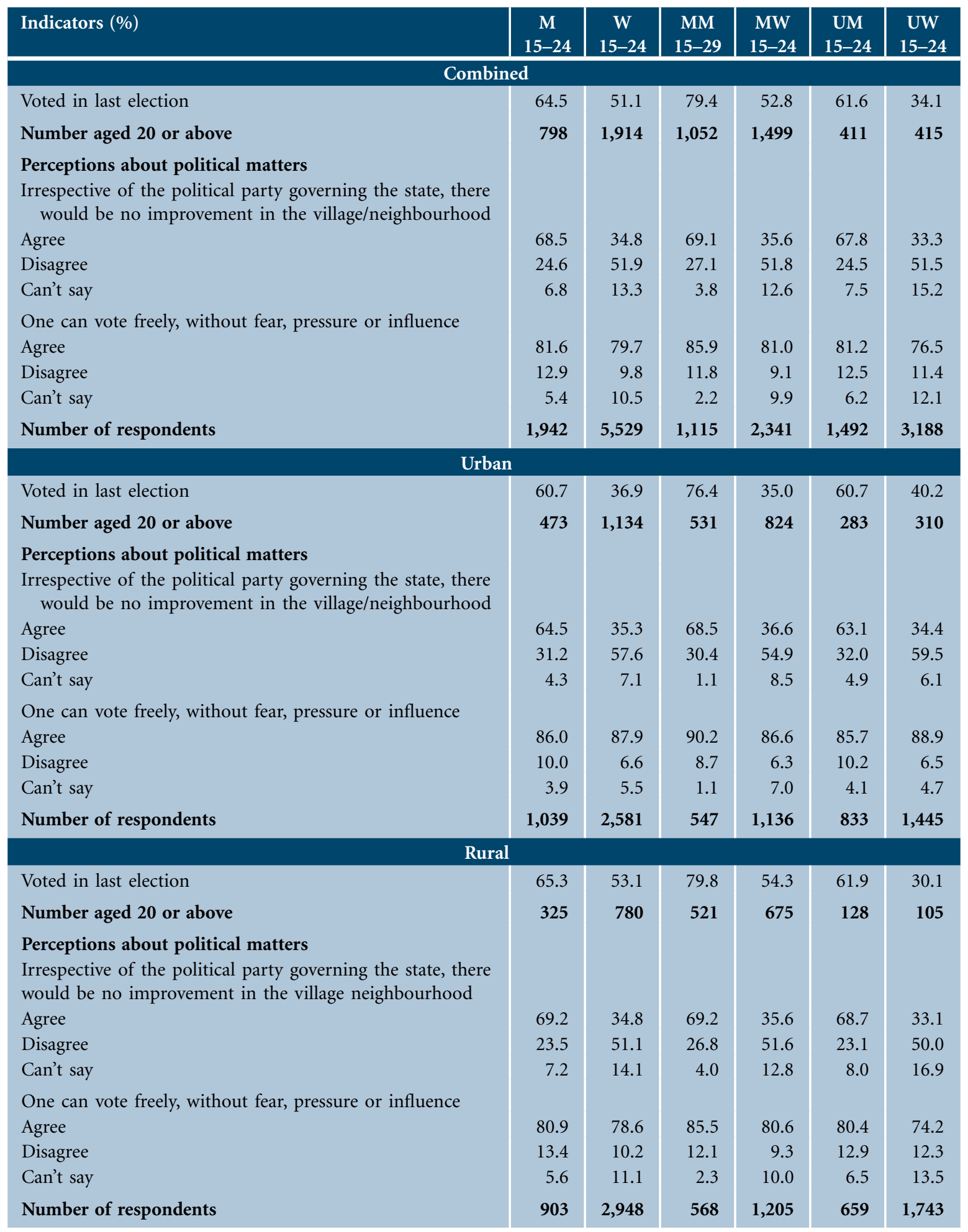

Note: All Ns are unweighted. Column totals may not equal 100\% due to missing cases. 
Table 12.6: Expression of secular attitudes

Percent distribution of youth by reported behaviours and attitudes towards interaction with people of different castes and religions, according to residence, Bihar, 2007

\begin{tabular}{|c|c|c|c|c|c|c|}
\hline Behaviours/attitudes (\%) & $\begin{array}{c}M \\
15-24\end{array}$ & $\begin{array}{c}\text { W } \\
15-24\end{array}$ & $\begin{array}{c}\text { MM } \\
15-29\end{array}$ & $\begin{array}{c}\text { MW } \\
15-24\end{array}$ & $\begin{array}{c}\text { UM } \\
15-24\end{array}$ & $\begin{array}{c}\text { UW } \\
\text { 15-24 }\end{array}$ \\
\hline \multicolumn{7}{|c|}{ Combined } \\
\hline \multicolumn{7}{|c|}{ Mixes freely with people of other castes } \\
\hline Yes & 95.3 & 87.2 & 97.6 & 87.1 & 95.2 & 86.9 \\
\hline No & 4.6 & 12.8 & 2.4 & 12.9 & 4.6 & 13.1 \\
\hline \multicolumn{7}{|c|}{ Mixes freely with people of other religions } \\
\hline Yes & 91.9 & 82.4 & 93.4 & 82.2 & 92.4 & 82.2 \\
\hline No & 7.9 & 17.5 & 6.5 & 17.6 & 7.4 & 17.6 \\
\hline \multicolumn{7}{|c|}{ Would eat together with a person of another caste/religion } \\
\hline Yes & 64.4 & 38.1 & 65.1 & 33.3 & 63.9 & 45.6 \\
\hline No & 35.0 & 61.6 & 34.6 & 66.3 & 35.3 & 54.2 \\
\hline \multicolumn{7}{|c|}{ Would talk to a person who has had an inter-caste marriage } \\
\hline Yes & 34.5 & 46.1 & 33.6 & 47.9 & 35.5 & 43.4 \\
\hline No & 64.2 & 53.5 & 65.1 & 51.8 & 63.3 & 55.9 \\
\hline \multicolumn{7}{|c|}{$\begin{array}{l}\text { Believes it is acceptable to punish someone who shows } \\
\text { disrespect to respondent's religion }\end{array}$} \\
\hline Yes & 62.6 & 56.8 & 62.0 & 58.8 & 62.1 & 53.5 \\
\hline No & 31.5 & 41.7 & 34.2 & 39.9 & 31.5 & 44.7 \\
\hline Number of respondents & 1,942 & 5,529 & 1,115 & 2,341 & 1,492 & 3,188 \\
\hline \multicolumn{7}{|c|}{ Urban } \\
\hline \multicolumn{7}{|c|}{ Mixes freely with people of other castes } \\
\hline Yes & 97.5 & 91.4 & 98.9 & 90.8 & 97.5 & 91.5 \\
\hline No & 2.5 & 8.6 & 1.1 & 9.2 & 2.5 & 8.5 \\
\hline \multicolumn{7}{|c|}{ Mixes freely with people of other religions } \\
\hline Yes & 96.1 & 89.3 & 97.8 & 88.0 & 96.3 & 90.1 \\
\hline No & 3.9 & 10.7 & 2.2 & 12.0 & 3.7 & 9.9 \\
\hline \multicolumn{7}{|c|}{ Would eat together with a person of another caste/religion } \\
\hline Yes & 77.1 & 61.1 & 74.7 & 50.7 & 77.5 & 68.8 \\
\hline No & 22.6 & 38.9 & 25.3 & 49.3 & 22.1 & 31.2 \\
\hline \multicolumn{7}{|c|}{ Would talk to a person who had an inter-caste marriage } \\
\hline Yes & 26.5 & 34.2 & 25.0 & 40.6 & 25.7 & 29.8 \\
\hline No & 72.4 & 65.3 & 75.0 & 59.4 & 72.7 & 69.6 \\
\hline \multicolumn{7}{|c|}{$\begin{array}{l}\text { Believes it is acceptable to punish someone who shows } \\
\text { disrespect to respondent's religion }\end{array}$} \\
\hline Yes & 53.4 & 49.8 & 55.4 & 52.8 & 53.5 & 48.0 \\
\hline No & 41.9 & 48.8 & 40.2 & 45.8 & 41.6 & 51.0 \\
\hline Number of respondents & 1,039 & 2,581 & 547 & 1,136 & 833 & 1,445 \\
\hline
\end{tabular}


Table 12.6: (Cont'd)

\begin{tabular}{|c|c|c|c|c|c|c|}
\hline Behaviours/attitudes (\%) & $\begin{array}{c}\text { M } \\
15-24\end{array}$ & $\begin{array}{c}\text { W } \\
15-24\end{array}$ & $\begin{array}{c}\text { MM } \\
15-29\end{array}$ & $\begin{array}{l}\text { MW } \\
15-24\end{array}$ & $\begin{array}{l}\text { UM } \\
15-24\end{array}$ & $\begin{array}{l}\text { UW } \\
15-24\end{array}$ \\
\hline \multicolumn{7}{|c|}{ Rural } \\
\hline \multicolumn{7}{|c|}{ Mixes freely with people of other castes } \\
\hline Yes & 95.0 & 86.6 & 97.5 & 86.9 & 94.8 & 86.0 \\
\hline No & 4.9 & 13.4 & 2.5 & 13.1 & 5.1 & 13.9 \\
\hline \multicolumn{7}{|c|}{ Mixes freely with people of other religions } \\
\hline Yes & 91.2 & 81.5 & 93.1 & 81.8 & 91.7 & 80.7 \\
\hline No & 8.6 & 18.3 & 6.8 & 18.0 & 8.2 & 19.1 \\
\hline \multicolumn{7}{|c|}{ Would eat together with a person of another caste/religion } \\
\hline Yes & 62.3 & 35.1 & 64.3 & 32.2 & 61.3 & 41.4 \\
\hline No & 37.1 & 64.5 & 35.4 & 67.4 & 37.9 & 58.4 \\
\hline \multicolumn{7}{|c|}{ Would talk to a person who had an inter-caste marriage } \\
\hline Yes & 35.8 & 47.6 & 34.4 & 48.4 & 37.3 & 46.0 \\
\hline No & 62.8 & 52.0 & 64.2 & 51.3 & 61.5 & 53.4 \\
\hline \multicolumn{7}{|c|}{$\begin{array}{l}\text { Believes it is acceptable to punish someone who shows } \\
\text { disrespect to respondent's religion }\end{array}$} \\
\hline Yes & 64.1 & 57.7 & 62.6 & 59.2 & 63.8 & 54.6 \\
\hline No & 29.8 & 40.8 & 33.6 & 39.5 & 29.5 & 43.6 \\
\hline Number of respondents & 903 & 2,948 & 568 & 1,205 & 659 & 1,743 \\
\hline
\end{tabular}

Note: All Ns are unweighted. Column totals may not equal 100\% due to missing cases or "don't know" responses.

Differences in the nature of behaviours and attitudes towards individuals of different castes and religions were negligible by marital status among young men. Among young women, however, the unmarried were more likely than the married to report that they would eat together with a person from a different caste or religion (46\% versus $33 \%$ ) and to tolerate without punishment someone who showed disrespect to their religion ( $45 \%$ versus $40 \%$ ). Conversely, the married were somewhat more likely than the unmarried to report that they would talk to someone who had an inter-caste marriage (48\% versus $43 \%$ ). Rural youth expressed considerably less secular attitudes than urban youth on such issues as mixing freely with individuals of different castes and religions and eating with those of other castes and religions. They were also considerably less likely than their urban counterparts to tolerate without punishment someone who showed disrespect to their religion (30-41\% versus $42-49 \%)$. Only on the issue of talking to someone who had an inter-caste marriage were urban youth less likely than their rural counterparts to express secular attitudes.

\subsection{Physical fights in the village or urban neighbourhood}

All respondents were asked whether physical fights—-more specifically, youth beating, slapping or pulling the hair of others-were common among young men and women, respectively, in their villages or neighbourhoods. Findings, presented in Table 12.7, suggest that physical fights were reported to be more common among young men than women. Four-fifths or more of youth- $79 \%$ of young men and $84 \%$ of young women-reported that young men engaged in physical fights sometimes or often and over one-third of young men and two-thirds of young women reported the same for young women. Differences by marital status in reports of physical fighting among young men and women were negligible. While rural-urban differences were negligible among young men, more rural than urban young women reported physical fighting among both young men and women. 
Table 12.7: Physical fights in village/neighbourhood

Percentage of youth reporting perceptions of youth involvement in physical fights in their village/neighbourhood and percentage of youth themselves involved in physical fights in the last 12 months, according to residence, Bihar, 2007

\begin{tabular}{|c|c|c|c|c|c|c|}
\hline Perceptions/experiences of physical fights (\%) & $\begin{array}{c}M \\
15-24\end{array}$ & $\begin{array}{c}\text { W } \\
15-24\end{array}$ & $\begin{array}{c}\mathrm{MM} \\
15-29\end{array}$ & $\begin{array}{c}\text { MW } \\
15-24\end{array}$ & $\begin{array}{c}\text { UM } \\
15-24\end{array}$ & $\begin{array}{c}\text { UW } \\
15-24\end{array}$ \\
\hline \multicolumn{7}{|c|}{ Combined } \\
\hline \multicolumn{7}{|l|}{$\begin{array}{l}\text { Respondents' perceptions of the extent to which: } \\
\text { Young men in the area engaged in physical fights }\end{array}$} \\
\hline Never & 20.9 & 15.6 & 22.0 & 16.6 & 21.8 & 13.5 \\
\hline Sometimes & 74.5 & 81.3 & 73.5 & 80.9 & 73.7 & 82.6 \\
\hline Often & 4.5 & 3.1 & 4.3 & 2.5 & 4.6 & 4.0 \\
\hline \multicolumn{7}{|l|}{ Young women in the area engaged in physical fights } \\
\hline Never & 63.2 & 34.3 & 62.6 & 34.1 & 64.7 & 34.0 \\
\hline Sometimes & 35.4 & 63.8 & 35.8 & 63.9 & 34.2 & 64.4 \\
\hline Often & 1.2 & 1.8 & 1.4 & 1.9 & 1.1 & 1.7 \\
\hline $\begin{array}{l}\text { Respondents themselves involved in physical fights in } \\
\text { last } 12 \text { months }\end{array}$ & 14.1 & 4.1 & 10.5 & 4.4 & 15.3 & 3.7 \\
\hline Number of respondents & 1,942 & 5,529 & 1,115 & 2,341 & 1,492 & 3,188 \\
\hline \multicolumn{7}{|c|}{ Urban } \\
\hline \multicolumn{7}{|l|}{$\begin{array}{l}\text { Respondents' perception of the extent to which: } \\
\text { Young men in the area engaged in physical fights }\end{array}$} \\
\hline Never & 21.1 & 19.8 & 23.9 & 19.0 & 20.5 & 20.0 \\
\hline Sometimes & 71.3 & 73.8 & 70.7 & 75.4 & 71.7 & 72.9 \\
\hline Often & 7.5 & 6.4 & 5.4 & 5.6 & 7.8 & 7.1 \\
\hline \multicolumn{7}{|l|}{ Young women in the area engaged in physical fights } \\
\hline Never & 65.4 & 44.3 & 64.1 & 43.0 & 65.7 & 45.3 \\
\hline Sometimes & 32.1 & 53.3 & 33.7 & 54.9 & 31.8 & 52.1 \\
\hline Often & 2.5 & 2.4 & 2.2 & 2.1 & 2.4 & 2.6 \\
\hline $\begin{array}{l}\text { Respondents themselves involved in physical fights in } \\
\text { last } 12 \text { months }\end{array}$ & 15.1 & 2.4 & 12.0 & 2.8 & 15.1 & 2.0 \\
\hline Number of respondents & 1,039 & 2,581 & 547 & 1,136 & 833 & 1,445 \\
\hline \multicolumn{7}{|c|}{ Rural } \\
\hline $\begin{array}{l}\text { Respondents' perception of the extent to which: } \\
\text { Young men in the area engaged in physical fights }\end{array}$ & & & & & & \\
\hline Never & 20.9 & 15.1 & 21.9 & 16.4 & 22.1 & 12.2 \\
\hline Sometimes & 75.0 & 82.3 & 73.7 & 81.4 & 74.1 & 84.3 \\
\hline Often & 4.0 & 2.6 & 4.2 & 2.3 & 3.8 & 3.4 \\
\hline Young women in the area engaged in physical fights & & & & & & \\
\hline Never & 62.9 & 33.0 & 62.6 & 33.6 & 64.4 & 31.9 \\
\hline Sometimes & 36.0 & 65.2 & 36.0 & 64.5 & 34.8 & 66.6 \\
\hline Often & 1.0 & 1.8 & 1.3 & 1.9 & 0.8 & 1.5 \\
\hline $\begin{array}{l}\text { Respondents themselves involved in physical fights in } \\
\text { last } 12 \text { months }\end{array}$ & 13.9 & 4.4 & 10.3 & 4.5 & 15.3 & 4.0 \\
\hline Number of respondents & 903 & 2,948 & 568 & 1,205 & 659 & 1,743 \\
\hline
\end{tabular}

Note: All Ns are unweighted. Column totals may not equal 100\% due to missing cases or "don't know" responses. 
Youth were also asked a direct question about their own involvement in physical fights with anyone within the village or urban neighbourhood in the 12 months preceding the interview. The question did not elaborate further and hence we acknowledge that responses may include fights among family members and others. One in seven young men and $4 \%$ of young women reported that they had been involved in physical fights. Rural-urban differences were negligible; marital status differences were negligible among young women, however, somewhat more unmarried than married young men reported the experience of violence $(15 \%$ and $11 \%$, respectively).

\subsection{Perceptions of leading problems facing youth}

Finally, youth were asked to give their opinion on the most important problem facing youth in their villages or urban areas. Findings presented in Table 12.8 clearly suggest that unemployment, poverty, lack of amenities and lack of educational opportunities were described as leading problems by both young men and women. Percentages reporting each of these problems varied enormously, however, by sex. Large proportions of young men, irrespective of marital status or rural-urban residence, reported difficulty in finding employment as the single most pressing problem (48\%), followed by poverty more generally $(21 \%)$, lack of educational opportunities $(16 \%)$ and concerns about lack of amenities or infrastructure-i.e., water and sanitation, roads and electricity-(7\%). Together, these four issues were expressed by $91 \%$ of young men. Young women, in contrast to young men, focused largely on lack of amenities and infrastructure $(31 \%)$ and, to a lesser extent, poverty more generally (20\%), lack of opportunities for education (14\%) and difficulty in finding employment $(13 \%)$. These four issues were together reported by $79 \%$ of young women.

Differences by marital status were modest; even so, compared to unmarried youth, the married were less likely to report lack of educational opportunities (9-10\% versus $18-25 \%)$ as leading problems facing youth. Married young men, in addition, were more likely than unmarried young men to report difficulty in finding a job (57\% versus $45 \%)$. Similarly, married young women were more likely than the unmarried to report poverty (23\% versus $16 \%)$ and concerns about lack of amenities or infrastructure (36\% versus $24 \%$ ).

Table 12.8: Perceptions about the leading problem facing youth

Percent distribution of youth by their perceptions of the leading problem facing youth, according to residence, Bihar, 2007

\begin{tabular}{|c|c|c|c|c|c|c|}
\hline Leading problem (\%) & $\begin{array}{c}M \\
15-24\end{array}$ & $\begin{array}{c}W \\
15-24 \\
\end{array}$ & $\begin{array}{c}\text { MM } \\
15-29\end{array}$ & $\begin{array}{c}\text { MW } \\
15-24 \\
\end{array}$ & $\begin{array}{c}\text { UM } \\
15-24 \\
\end{array}$ & $\begin{array}{c}\text { UW } \\
15-24\end{array}$ \\
\hline \multicolumn{7}{|c|}{ Combined } \\
\hline Finding a job/unemployment & 48.0 & 13.0 & 57.2 & 14.3 & 45.3 & 10.0 \\
\hline Poverty & 20.7 & 20.3 & 19.7 & 22.9 & 20.5 & 15.9 \\
\hline $\begin{array}{l}\text { Lack of amenities/infrastructure (water/toilets/ } \\
\text { roads/electricity) }\end{array}$ & 6.5 & 31.1 & 7.0 & 35.6 & 6.5 & 23.8 \\
\hline Health-/health service-related concerns & 0.2 & 0.5 & 0.0 & 0.6 & 0.3 & 0.4 \\
\hline Security of girls/law and order & 0.2 & 4.3 & 0.0 & 3.4 & 0.2 & 5.4 \\
\hline Finding a good spouse/dowry & 0.2 & 3.9 & 0.0 & 2.7 & 0.3 & 5.7 \\
\hline Lack of educational opportunities & 16.0 & 14.4 & 10.1 & 8.9 & 18.1 & 24.9 \\
\hline Lack of career counselling/vocational training & 0.5 & 6.7 & 0.4 & 5.8 & 0.5 & 8.3 \\
\hline Alcohol/drug abuse & 0.5 & 0.3 & 0.3 & 0.4 & 0.7 & 0.1 \\
\hline Lack of sex education & 0.1 & 0.1 & 0.2 & 0.1 & 0.1 & 0.1 \\
\hline Other $^{1}$ & 2.3 & 0.7 & 1.5 & 0.3 & 2.3 & 1.4 \\
\hline Don't know/can't say & 4.9 & 4.7 & 3.5 & 5.1 & 5.2 & 4.0 \\
\hline Number of respondents & 1,942 & 5,529 & 1,115 & 2,341 & 1,492 & 3,188 \\
\hline
\end{tabular}

Cont'd on next page... 


\begin{tabular}{|c|c|c|c|c|c|c|}
\hline Leading problem (\%) & $\begin{array}{c}M \\
15-24\end{array}$ & $\begin{array}{c}\text { W } \\
15-24\end{array}$ & $\begin{array}{c}\mathrm{MM} \\
15-29\end{array}$ & $\begin{array}{c}\text { MW } \\
15-24\end{array}$ & $\begin{array}{c}\text { UM } \\
15-24\end{array}$ & $\begin{array}{l}\text { UW } \\
15-24\end{array}$ \\
\hline \multicolumn{7}{|c|}{ Urban } \\
\hline Finding a job/unemployment & 59.6 & 23.1 & 69.9 & 28.2 & 58.8 & 19.2 \\
\hline Poverty & 16.1 & 13.1 & 16.1 & 16.2 & 15.9 & 10.9 \\
\hline $\begin{array}{l}\text { Lack of amenities/infrastructure (water/toilets/ } \\
\text { roads/electricity) }\end{array}$ & 4.6 & 15.1 & 4.3 & 19.0 & 4.5 & 11.9 \\
\hline Health-/health service-related concerns & 0.0 & 0.3 & 0.0 & 0.7 & 0.0 & 0.4 \\
\hline Security of girls/law and order & 0.4 & 9.9 & 0.0 & 4.9 & 0.4 & 13.6 \\
\hline Finding a good spouse/dowry & 0.0 & 8.8 & 0.0 & 6.3 & 0.0 & 10.7 \\
\hline Lack of educational opportunities & 10.7 & 13.2 & 6.5 & 7.7 & 11.4 & 17.0 \\
\hline Lack of career counselling/vocational training & 1.1 & 8.6 & 0.0 & 8.5 & 1.2 & 8.9 \\
\hline Alcohol/drug abuse & 0.7 & 0.3 & 1.1 & 0.7 & 0.4 & 0.2 \\
\hline Lack of sex education & 0.4 & 0.2 & 0.0 & 0.0 & 0.4 & 0.0 \\
\hline Other $^{1}$ & 1.8 & 2.2 & 1.1 & 0.7 & 2.0 & 3.2 \\
\hline Don't know/can't say & 4.6 & 5.2 & 1.1 & 7.0 & 4.9 & 3.8 \\
\hline Number of respondents & 1,039 & 2,581 & 547 & 1,136 & 833 & 1,445 \\
\hline \multicolumn{7}{|c|}{ Rural } \\
\hline Finding a job/unemployment & 46.0 & 11.7 & 56.1 & 13.4 & 42.7 & 8.2 \\
\hline Poverty & 21.5 & 21.3 & 20.1 & 23.3 & 21.4 & 16.8 \\
\hline $\begin{array}{l}\text { Lack of amenities/infrastructure (water/toilets/ } \\
\text { roads/electricity) }\end{array}$ & 6.8 & 33.2 & 7.2 & 36.7 & 6.9 & 26.0 \\
\hline Health-/health service-related concerns & 0.2 & 0.5 & 0.0 & 0.5 & 0.3 & 0.4 \\
\hline Security of girls/law and order & 0.1 & 3.5 & 0.0 & 3.3 & 0.2 & 3.9 \\
\hline Finding a good spouse/dowry & 0.2 & 3.2 & 0.0 & 2.5 & 0.3 & 4.8 \\
\hline Lack of educational opportunities & 16.8 & 14.5 & 10.5 & 9.0 & 19.4 & 26.3 \\
\hline Lack of career counselling/vocational training & 0.4 & 6.5 & 0.5 & 5.6 & 0.4 & 8.2 \\
\hline Alcohol/drug abuse & 0.5 & 0.3 & 0.2 & 0.4 & 0.6 & 0.1 \\
\hline Lack of sex education & 0.0 & 0.1 & 0.2 & 0.1 & 0.0 & 0.1 \\
\hline Other $^{1}$ & 2.4 & 0.5 & 1.6 & 0.2 & 2.4 & 1.0 \\
\hline Don't know/can't say & 5.0 & 4.7 & 3.7 & 5.0 & 5.3 & 4.1 \\
\hline Number of respondents & 903 & 2,948 & 568 & 1,205 & 659 & 1,743 \\
\hline
\end{tabular}

Note: All Ns are unweighted. Column totals may not equal $100 \%$ due to missing cases. ${ }^{1}$ Includes lack of recreational/sports facilities, lack of political participation, gambling, corruption, child marriage, lack of loan services, limited freedom for girls, social conflicts, generation gap, parents not allowing love marriage, caste differences, etc.

Urban youth were more likely than rural youth to feel that difficulty in finding employment was a leading problem facing youth (60\% versus $46 \%$ among young men and $23 \%$ versus $12 \%$ among young women). Conversely, rural youth were more likely than urban youth to feel that poverty was a leading problem facing youth $(22 \%$ and $21 \%$ of men and women in rural areas, compared to $16 \%$ and $13 \%$, respectively, in urban areas). Young men in rural areas were, in addition, more likely than urban young men to report lack of educational opportunities (17\% versus $11 \%)$ and young women in rural areas were more likely than those in urban areas to report lack of amenities/infrastructure (33\% versus 15\%). Conversely, young women in urban areas were more likely than those in rural areas to cite lack of safety for girls ( $10 \%$ versus $4 \%)$ and difficulty in findings a good spouse and dowry (9\% versus 3\%) as leading problems.

\subsection{Summary}

Findings highlight extremely limited participation of youth in civil society. Although a number of programmes are held to build youth skills, very few youth $(8-15 \%)$ reported familiarity with either government- or NGO-sponsored programmes organised at the community level in which youth could participate. Even fewer youth-7\% of young men $2 \%$ of young women-reported participating in any such programme. Almost 
one-quarter of young men (23\%) but hardly any young women (4\%) reported that they had participated in community-sponsored programmes such as cleanliness drives, celebration of festivals and national days, and so on. Finally, just $8 \%$ of young men and $2 \%$ of young women reported membership in organised groups.

Findings suggest that voting behaviour was far from universal. Among those eligible, 65\% of young men and $51 \%$ of young women had cast their votes in the most recent election for which they were eligible to vote. Four-fifths of youth perceived that one could vote freely and without fear and pressure. However, large proportions of youth, particularly young men-69\% of young men and 35\% of young women-reported disillusionment with the commitment of political parties to work for change at the community level.

Expressions of secular attitudes varied. Over $90 \%$ of young men and over $80 \%$ of young women reported that they mixed freely with individuals of different religions and castes. However, only $64 \%$ of young men and $38 \%$ of young women would eat together with a person of a different caste or religion, just $35 \%$ of young men and $46 \%$ of young women would talk to a person who has had an inter-caste marriage and only $32 \%$ of young men and $42 \%$ of young women agreed that it was best to tolerate rather than punish someone who insulted their religion.

Considerable proportions of young men and women acknowledged that physical fights among young men and also among young women did occur in their villages or urban neighbourhoods; one in seven young men and $4 \%$ of young women reported that they had been involved in a physical fight in the year preceding the interview.

The four leading problems facing youth expressed by both young men and women were unemployment, poverty, lack of amenities and lack of educational opportunities. However, young people's perceptions of the leading problems facing youth varied enormously by sex. Among young men, the majority reported difficulty in finding employment as the leading problem, followed by concerns about poverty more generally, lack of educational opportunities and lack of amenities or infrastructure. In contrast, the leading problem expressed by young women was lack of amenities and infrastructure, and to a lesser extent, poverty more generally, lack of opportunities for education and difficulties in finding employment. Marital status differences were also evident, with the unmarried far more concerned about lack of appropriate educational opportunities than the married, and the married, conversely, far more concerned than the unmarried about job opportunities. Rural youth too were far more concerned than urban youth about the lack of educational opportunities and far less concerned about finding a job. 


\section{Looking forward}

Findings of the Youth Study presented in earlier chapters highlight the situation of young men and women in Bihar. They underscore the fact that youth are a heterogeneous group with correspondingly diverse needs, and identify numerous challenges youth face in making the transition to adulthood. Findings suggest several priority programmatic areas for action as well as several themes requiring research attention, which are highlighted in this chapter.

\subsection{Recommendations for programmes}

The multiple socio-economic and sexual and reproductive health vulnerabilities faced by youth in Bihar call for programmatic attention at several levels, including at the youth, family and service delivery levels.

\section{Strengthen efforts to achieve universal school enrolment and at least primary school completion}

Youth Study findings that primary school enrolment is far from universal even among the youth cohort call for concerted efforts to achieve universal enrolment of children in school. Moreover, findings suggesting substantial declines in school attendance even at the primary level and relatively low rates of primary school completion emphasize in no uncertain terms that rigorous efforts are needed in order that the state meets the Millennium Development Goal of ensuring universal primary school completion.

While achieving universal enrolment and primary school attainment are key shorter-term goals, the importance of high school education in enabling youth to make a successful transition to adulthood underscores the need, at the same time, for efforts to overcome barriers to high school completion.

A number of factors have been identified in the Youth Study that inhibit school enrolment and primary school completion; leading among these are economic reasons, attitudes and perceptions of youth and their parents, as well as housework responsibilities among young women. Multiple activities are needed to address these barriers. Efforts must be made, for example, to address the economic pressures that dissuade parents from enrolling their children in school in the first place or from keeping them in school once enrolled. Conditional grants and targeted subsidies that encourage school enrolment and completion among disadvantaged groups need to be considered. At the same time, activities directed at parents are needed that promote positive attitudes towards education and school completion, raise aspirations for the education of their children and encourage greater parental involvement in their children's education.

Activities must also address school-level barriers, notably, distance to school, poor infrastructure and poor quality of education, significant motivating factors behind discontinuation particularly among young women. The state government has launched some schemes to address some of these barriers (see for example the bicycle scheme for girls); it is important that the effectiveness of such schemes is evaluated and promising lessons are assimilated and scaled up. 
There is also a need to incorporate livelihood skills building models within the school setting that provide opportunities for those in school to gain market-driven job skills and also expand young people's aspirations regarding their education and careers. Moreover, investments are needed that focus on providing better training and ensuring accountability for teachers and thereby improving the quality of the schooling experience. Finally, given the large proportions reporting that schooling had been interrupted because they were required for work on the family farm or business or for housework, and given the reality of young people's lives and the economic pressures on families, efforts need to be made to adjust school timings, including establishment of evening schools to enable children to accommodate work on the family farm or business without sacrificing their education.

Findings indicating transition to adult roles, particularly early marriage, as an important reason for school discontinuation — even as early as primary school—among girls, emphasise the fact that programmatic commitments outside the education sector are also critical to the achievement of universal school enrolment and completion. Specifically required are programmes that seek to critically examine norms and practices surrounding marriage and to eliminate the practice of early marriage. Explorations of subsidies and cash transfers that link school retention and delayed marriage among girls are needed.

The stark gender divide and rural-urban divide observed in school enrolment and attendance call for efforts that specifically target female children and rural children in general. Moreover, findings suggest that married young women remain considerably disadvantaged. Interventions are needed that give married young women a second chance to obtain a basic education.

\section{Invest in promoting youth employment}

Findings of the Youth Study that considerable proportions of youth had initiated work in childhood reiterate the recommendation highlighted above regarding the need to provide conditional grants and targeted subsidies to disadvantaged groups, which would encourage parents to opt for schooling over work for their children.

Findings have pointed to the effective unemployability of youth. For example, few youth had completed primary or high school and even fewer had attended a single vocational training programme. Moreover, considerable proportions of youth, particularly the educated, were unemployed. Clearly, the state must strengthen significantly its investments in programmes to enable youth to make successful transitions into work roles, including provision of soft loans for youth to set up their own enterprises. At the same time, efforts are needed that evaluate existing programmes, upscale successful models and raise awareness among youth about their availability. While enhancing employability will depend to a considerable degree on improvements in educational attainment discussed above, it will also require greater investment in enabling youth to acquire vocational skills. Formal mechanisms must be developed that provide opportunities to youth to acquire vocational skills for which there is an established market demand, and that link eligible youth to market opportunities. These efforts, through various livelihood schemes, must promote self-employment and entrepreneurship among young people.

Findings also suggest the need for a special focus on young women. Significant proportions of young women currently engaged in economic activities had done so only part-time and worked largely in agricultural activities. In addition, many young women were seeking employment at the time of the interview. These findings highlight the need for specially targeted programmes for young women. 
Promote youth agency and gender equitable norms among youth

Findings presented in this report highlight the persistence of gender double standards and extremely limited agency of young women. Stark gender differences were evident in school enrolment, attendance and completion, participation in the labour market, exposure to mass media, parental control of adolescent's mobility and interactions with peers, exercise of choice in matters affecting young people's lives, freedom of movement and access to resources. Gender equitable norms were not universally expressed; young women were more likely to express equitable gender role attitudes than young men, but were also more likely than young men to justify wife beating. These findings call for multi-pronged interventions to promote gender equitable norms and practices that are directed at young women, young men, their families, communities, and educational, labour and health systems.

A programme priority is to promote life skills education programmes for young women, both unmarried and married, that will not only raise their awareness of new ideas and the world around them but also enable them to put information into practice, encourage them to question gender stereotypes, develop self-esteem and strengthen their abilities in problems-solving, decision-making, communication and inter-personal relations and negotiations. Safe spaces should be identified in which young women can build social networks and find social support among peers.

Interventions intended to build life skills must also be inclusive of young men. Indeed, findings indicate that inegalitarian gender role attitudes were expressed by many young men. Moreover, while young men were clearly not as disadvantaged as young women, findings indicate that many young men were not able to exercise agency in their everyday lives. These findings call for life skills education programmes for young men that promote new concepts of masculinity and femininity among youth and at the same time, promote messages that build egalitarian relations between women and men.

Promoting gender equitable norms and practices requires active engagement with the community. It is essential that programmes for youth work with key community members, such as parents, political and religious leaders in the community, to critically examine prevailing gender norms and forces that perpetuate such norms.

An increasing number of intervention models to build agency and promote egalitarian gender role attitudes among young people have been tested in India. These models should be reviewed and replicated or scaled up as appropriate.

\section{Provide opportunities for formal saving, especially for young women}

Findings suggest that while young women were more likely than young men to report savings, few youth, irrespective of sex, owned a savings account. Among those who did own an account, young women were far less likely than young men to operate the account independently. Programmes are needed that inculcate a savings orientation among both young men and young women, that offer savings products that are attractive and appropriate to the small and erratic savings patterns of young people and that enable young women in particular to overcome obstacles related to owning and controlling savings products.

\section{Promote youth participation in civil society and political processes and reinforce secular attitudes}

Findings suggest that for many youth, opportunities to engage in civic and political life are limited and secular attitudes are not uniformly expressed. Programmes are needed—at the school, college and community levels, through national service programmes, sports and other non-formal mechanisms-that encourage civic 
participation, incorporate value building components and reinforce secular attitudes and values that espouse responsible citizenship.

Provide family life or sex education for those in school and out of school

Youth Study findings provide considerable evidence suggesting that family life or sex education is urgently needed among youth, both for those in school and those who have discontinued their education. Findings demonstrate extremely limited understanding of sexual and reproductive matters among young people, including the married. Misconceptions abound on most topics: sex and pregnancy, contraceptive methods including condoms, STIs and HIV/AIDS and the conditions under which abortion is legally available or restricted. And knowledge of STIs is far more limited than knowledge of HIV/AIDS. Where awareness of sexual and reproductive health matters exists, it is typically superficial.

Youth themselves have called for family life or sex education. Findings highlight that large proportions recognised the need for information on these issues, and indicated a preference for receiving this education from teachers, health care providers or parents. However, few young people had been exposed to family life or sex education. Substantial proportions of married young women and young men reported entering marriage completely unaware of what it entailed. At the same time, substantial minorities of young people had engaged in sexual risk taking.

There is clearly a pressing rationale for school-based family life or sex education for those in school and community-based expert-led education for those out of school. These programmes should be age-appropriate and provide information on sexual and reproductive matters and sexual and reproductive rights; about pregnancy as well as the causes, transmission routes and prevention of infection. However, they should be designed not only to raise awareness among youth but also to enable young people to correctly understand and assess the risks they face and to adopt appropriate protective actions.

In view of the finding that the media are a major source of sexual and reproductive health information for youth, efforts must be made, at the same time, for communications initiatives that inform while entertaining youth about sexual and reproductive matters.

In addition, special attention needs to be paid to the training of trainers. Considerable proportions of youth who reported having received formal family life or sex education reported feeling uncomfortable or embarrassed in the course of family life or sex education. These findings raise questions about the extent to which they were indeed able to participate freely and clarify their doubts and at the same time, about the ability of trainers to connect with youth to whom they provided this education. Such findings clearly highlight the need for improving the quality of training imparted to trainers. It is important that teachers, health care providers and other experts undergo training that enables them to overcome their reluctance about communicating with youth on sensitive sexual and reproductive matters, that dispels their misconceptions on these matters, and that enhances their technical knowledge on sexual and reproductive issues.

\section{Ensure that the transition to sexual life is safe and wanted}

While for the vast majority of young women and men sexual activity is initiated within the context of marriage, findings show that significant minorities of youth, particularly young men had engaged in sex before marriage. As documented in this report, many youth had initiated sexual activity uninformed, which reiterates the need for providing family life or sex education to young people. Moreover, findings that for many, pre-marital sexual experiences were unsafe or unwanted calls for programmes that focus on building sexual 
and reproductive health awareness among young people as well as developing their skills in negotiating safe sex and communicating with their partners. At the same time, programmes must make available appropriate family planning and infection prevention services for both married and unmarried young men and women in a manner acceptable to them.

\section{Intensify efforts to eliminate the practice of early marriage}

Findings indicate an adherence, even among youth, to the traditional social norms around child marriage and the practice of early marriage not only among young women but also, to a lesser extent, among young men in the state. These findings call for measures that go beyond information campaigns to address the social norms and economic factors driving early marriage and ensure the stricter enforcement of existing laws prohibiting early marriage in the state.

There is clearly a need for an intensified, multi-pronged approach to eliminate the practice of early marriage. Strategies are needed that mobilise communities to help parents resist pressures that foster the practice of early marriage. Strategies intended to evolve new norms and new practices should both actively engage influential persons in the community including religious and political leaders as well as implement campaigns highlighting the adverse consequences of early marriage, and how it is a violation of the rights of the child. Community mobilization efforts must involve youth themselves, their families, as well as influential persons in the community, including religious and political leaders.

Equally important is to ensure greater commitment on the part of law enforcement agencies to enforce existing laws on the minimum age at marriage and the registration of marriages, and to levy penalties for violators. Allowing anonymous reporting, working with the police and others to make clear that the practice of early marriage is not a minor violation, and making guidelines for penalties clear and transparent are some possible steps.

Efforts to delay marriage also require providing girls with meaningful and viable alternatives to early marriage. Advising families to send their daughters to school when schools are too far away, the classroom is hostile to girls, or education is of poor quality, will not work. Working with the education sector to make schooling for girls more accessible, and to make classrooms more gender-sensitive and responsive to the needs of young girls and the concerns of their parents is important. At the same time, it is necessary to make efforts to provide livelihoods training, within or outside the educational system.

Findings that marriages were often arranged without the participation of young people themselves and that few young people had an opportunity to meet their spouse-to-be prior to the wedding day call for actions to encourage parents to involve children in marriage-related decisions and enable them to interact with their prospective spouses prior to the wedding day. Parents must also be made aware of the physical and mental health dangers of early marriage and the adverse experiences of many young women (and some young men) who were married early or who were unprepared for marriage.

\section{Enable married young women to exercise greater control over their lives}

Findings on the multiple vulnerabilities faced by married young women underscore the need for programmes that support them, acknowledging that their situation and needs may differ from those of married adults. Married young women are notably isolated, have little decision-making authority and have few sources of support. They have limited communication with their husbands, and notable proportions have suffered physical and sexual violence perpetrated by their husbands. 
Efforts are needed that address the health and empowerment needs of married young women and enable them to have greater control over resources. Also needed are efforts to break down the social isolation of newly married young women, encourage couple communication and build negotiation and conflict management skills early in marriage. Intervention models that have attempted to address these needs exist in India; these should be reviewed and up-scaled as appropriate so that married young women have the opportunity to exercise control over their lives.

Support newly-weds to postpone the first pregnancy and promote pregnancy-related care among those who become pregnant

Findings show that the social pressure to bear children as soon as possible following marriage persists. Contraceptives were rarely used to postpone the first pregnancy and although the desire to have delayed the first pregnancy was expressed by large proportions of young men and women, many young women experienced their first pregnancy soon after marriage. It would appear that numerous forces work against delaying the first pregnancy-young people's lack of awareness of appropriate methods of contraception and access to supplies, their limited skills in countering social expectations and negotiating pregnancy postponement, overwhelming pressure from the family and community to bear children as soon as possible after marriage, and lack of attention from health care providers.

Programmes are needed that inform youth about their pregnancy postponement options and enable them to access appropriate contraception. At the same time, providers must be trained and charged with the responsibility of reaching married young women and men-including those who have not yet experienced pregnancy-with information regarding contraception and other reproductive health matters as well as contraceptive supplies. The limited mobility of married young women to seek health care underscores the need for health workers to seek these women—particularly those newly married and first time pregnant—in their homes.

Findings also underscore that access to maternal health services was limited, even at the time of the first-and often the most risky—pregnancy. Just one in four first births took place in a health facility; moreover, skilled attendance at first delivery was reported by just one in three young women and two in five young men. These findings highlight that reproductive and child health programmes in the state need to lay emphasis on increasing demand as well as improving the availability of such services to young people.

\section{Create a supportive family environment}

Findings highlight the limited interaction and social distance between parents and young people while growing up and the gendered nature of socialisation experiences. Efforts must be made to create a supportive environment for young people. While evidence on models that are effective in bridging the distance between parents and children or enabling parents to adopt gender-egalitarian socialisation practices is not currently available, findings presented in this report call for programmes that address parental inhibitions about discussing sexual matters with their children, encourage greater openness and interaction between parents and children, and enable the adoption of gender-egalitarian child-rearing practices.

Reorient service provision to address the unique needs of unmarried and married young women and men

Although the RCH Programme has advocated special services for youth, including the unmarried, these services have not reached youth. Few youth were aware of sources of sexual and reproductive health information or contraceptive supplies, few had sought care for symptoms of STI or gynaecological problems, and most 
of those who had sought care for the latter preferred private to public sector facilities. Moreover, findings suggest that many youth, including the married, would indeed find it difficult to seek appropriate care for sexual and reproductive matters.

The disconnect between the public health sector and youth underscores the need to sensitise health care providers about the special needs, heterogeneity and vulnerability of unmarried and married young women and men, and to orient them to the need for developing appropriate strategies to reach these diverse groups, including young newly-weds. Programmes must be inclusive of unmarried young people and recognise their need and right to sexual and reproductive health and related information and services. Counselling and contraceptive services must be made available to unmarried young people in a non-threatening, nonjudgmental and confidential environment. Indeed, these findings call for the implementation of strategies outlined under the National Rural Health Mission's RCH Programme.

Findings that very few youth sought care for health problems and that those who sought care preferred to seek care from the private sector and traditional providers rather than the public sector, suggest the need to explore the feasibility of implementing various demand side financing strategies, for example, health insurance, competitive voucher schemes and community financing schemes, in enabling youth to obtain quality care from a wider array of providers.

In addition, there is a great need for mental health issues to be addressed. Symptoms suggestive of mental health disorders were evident among sizeable proportions of youth. Efforts are needed to screen young people for mental health disorders when they avail of other primary health services, including, for example, sexual and reproductive health services, and to refer youth with such symptoms to appropriate health facilitates and providers.

\subsection{Directions for future research}

Findings presented in this report provide a broad picture of youth in Bihar. At the same time, however, they have raised a number of issues that require further investigation, particularly with regard to the determinants and consequences of youth behaviours and practices during their transition to adulthood. While the Youth Study is indeed a rich source of data that will enable investigators to fill many of the information gaps identified, there are several gaps in knowledge that will require additional research efforts.

A general research recommendation is the urgent need for prospective or panel study designs that follow a cohort of adolescents at regular intervals up to age 24. Thus far, research has relied on cross-sectional data. While these data are valuable in describing the levels and trends in key markers of transitions to adulthood, they rarely capture the ways in which the situation and experiences of youth in adolescence influence their life courses at later ages. Moreover, drawing causal inferences from cross-sectional surveys has several limitations.

\section{Non-enrolment and early school discontinuation}

While evidence presented in this report sheds some light on the reasons for non-enrolment in school and early discontinuation from school, further research is needed that profiles youth most at risk of never attending school or dropping out even before completing primary education. What are the obstacles faced by their families in retaining their children in school, and what approaches do parents and other gatekeepers recommend to mitigate these barriers? Moreover, even though large proportions of young women reported early transitions into marriage as a significant motivating factor behind school discontinuation, research is 
needed that explores whether it is early marriage that curtails schooling for young women or whether it is such factors as poverty and school-related factors including access and quality that lead to school discontinuation and thereby perpetuate early marriage. As suggested in the section on recommendations for programmes, a variety of interventions - that address school quality issues, enhance parental involvement in children's education, provide the out-of-school, and especially married young women, a second chance to continue their education and provide conditional grants and targeted subsidies for disadvantaged groups-need to be implemented and systematically documented. In addition, operations research is required that evaluates the effectiveness and feasibility of these programmes.

\section{Transition to work}

Several questions related to young people's transition to work remain unanswered. Large numbers of youth in Bihar make early transitions to work; yet, relatively little is known about the kinds of work they undertake, the time they spend in work activities and the extent to which their activities mark a significant labour contribution to their households. Further research is needed that explores these issues among those reporting early transitions into work.

With regard to vocational skills building, research is needed that explores the kinds of vocational training programmes that are available to youth, and the extent to which these are accessible in practice. Research is also needed that examines the factors underlying the findings that few young people received vocational skills training even though large proportions were in favour of receiving such training, and that despite the availability of a range of vocational skills training opportunities, many young women continued to opt for training in traditional vocational skills. Research must also assess the extent to which these programmes reflect market needs on the one hand and youth preferences on the other, and the extent to which vocational training enables youth-especially the relatively poorly educated-to secure employment in the field in which they have been trained. Finally, there is a need for operations research that will test models intended to enable youth to acquire skills for which there is an established market demand and link eligible youth to market opportunities.

\section{Socialisation experiences, interaction with parents}

Youth Study findings show that socialisation is highly gendered and parent-child interaction is limited, particularly on more sensitive issues such as those relating to sex and reproduction. Despite this, little is known about the factors that inhibit young people from confiding in parents on sensitive matters, or the ways in which limited interaction and hierarchical socialisation patterns may influence young people's lives, for example, their sexual behaviours, their aspirations for the future or their ability to exercise informed choices in their lives. Similarly, there is a dearth of research on parents' perspectives on the socialisation of sons and daughters, the extent to which parents perceive or justify different socialisation practices for sons and daughters, the extent to which and the ways in which parents communicate sensitive matters to their adolescent children, and the factors inhibiting parents from adopting gender-egalitarian socialisation practices and communicating with their adolescent children on sexual and reproductive matters. As mentioned earlier, there is a need to design and test interventions intended to involve parents more meaningfully in young people's transitions to adulthood in terms of educational attainment, work, marriage and initiation of sexual relations. 


\section{Sexual risk behaviours}

Research is needed that explores the correlates of behaviours that undermine healthy development among young people, for example, sexual risk behaviours, substance use and the linkages between them. At the same time, it would be useful to identify the characteristics of youth who make the transition in a healthy way, for example, practise consistent condom use, seek appropriate care and so on.

The Youth Study has raised serious methodological concerns that need to be addressed. For example, despite the fact that the study did employ such methods as gradual sequencing of questions to include progressively more sensitive questions (with regard to romantic and sexual relationships), anonymous third-party reporting and anonymous sealed envelope reporting, as in many studies, pre-marital sexual experience was far less likely to be reported among young women than young men. Moreover, sex worker, exchange, forced and same-sex relationships were rarely reported. Such findings emphasise the need to continue the search for appropriate methodologies to measure sensitive behaviours among youth; computer-assisted survey interviews are one such option. Indeed, methodological studies that compare estimates derived using different approaches could provide an insight into efforts to refine measures of reporting of sensitive behaviours among youth.

\section{Early marriage and childbearing}

Research is needed that explores the extent to which early marriage compromises young people's lives. For example, does early marriage impede young women's ability to exercise agency in the marital home? How prepared for marriage are those who marry early and how does preparedness or lack thereof influence married life?

Many youth reported that they had not used any contraceptives to delay the first pregnancy and consequently experienced pregnancy soon after marriage. Further research is needed that sheds light on the factors that undermine young people's ability to delay the first pregnancy.

Several promising interventions that are intended to address the social isolation experienced by married young women and/or their reproductive health needs, including delaying pregnancy or making pregnancy safe, have been implemented. However, few of these interventions have been rigorously evaluated and there is a need for research that assesses the feasibility and possibility of scaling up various such interventions.

\section{Partner violence}

Youth Study findings have documented domestic and sexual violence perpetrated by young men on their wives, as well as forced sex experienced in romantic and non-romantic situations by a small number of young women prior to marriage. Findings call for research that explores the factors underlying these experiences of violence, documents their health and social consequences for young women and men and their children, and tests interventions that enable youth to prevent such violence on one hand, and to overcome obstacles to seeking prompt and appropriate care on the other.

\section{Influence of family life or sex education on sexual relations}

Findings suggest that few youth are aware in-depth about sexual and reproductive matters, clearly posing an obstacle to their ability to make informed choices. Research is needed that explores the extent to which young people's awareness of sexual and reproductive matters and of sexual and reproductive rights varies according to the sources from which they derive their knowledge. Equally important are studies that examine 
the sexual and reproductive awareness and technical competence to communicate sensitive sexual matters to young people of those from whom information is sought-including, for example, teachers, health care providers and parents.

There has been some reticence in several states of the country to impart school-based family life or sex education to youth on the assumption-disproved in some settings-that such education will encourage youth to engage in risky sexual behaviours. Research is needed that explores the extent to which exposure to school-based family life or sex education does indeed enable youth to make informed decisions and adopt safe behaviours in the area of sexual and reproductive health. Research is also needed that explores whether the transition into married life is safer and healthier among those-particularly young women-who were exposed to such education.

\section{Agency and gender role attitudes}

While findings confirm the limited agency of youth, particularly young women, and gender inegalitarian attitudes held by youth, particularly young men, several gaps remain in our understanding of the ways in which these affect young people's transitions to adulthood. Further research is needed, for example, that identifies the factors underlying the espousal of unequal and equal gender role attitudes by young men, and that explores the ways in which inegalitarian gender role attitudes and limited agency compromise sexual and reproductive health among young men and women.

Methodological issues also arise. There is a need to refine measures of agency as applicable to young men and women. The Youth Study has obtained data on multiple dimensions of agency among young men and women, the married and the unmarried and those from rural and urban areas. These data lend themselves to methodological exercises that measure agency among youth, that assess the extent to which key components of agency may differ across different categories of youth and that explore whether a single summary measure of agency can be developed that are relevant to youth.

\section{Access to and control over resources}

Findings that very few youth, irrespective of sex, owned a savings account, and among those who did own an account, young women were far less likely than young men to operate the account independently call for efforts to map savings and credit options available to youth. At the same time, research is needed that assesses the financial literacy skills of youth, their savings and spending patterns, barriers faced in accessing existing financial products, and ways in which these barriers can be overcome

\section{Mental health disorders}

Findings suggest that many young people, particularly young men, experience symptoms suggestive of mental disorders. Research is needed that explores young people's mental health profiles in greater depth and that assesses the linkages between sexual and reproductive health on one hand, and mental health on the other; and that explores factors explaining why young men were more likely than young women to report mental health problems.

Health-seeking for sexual and reproductive health symptoms

Findings suggesting that health care seeking, particularly for sexual and reproductive matters, is limited, highlight the need for research that explores the factors inhibiting youth from seeking care. As a start, the 
Youth Study data will enable exploration of the factors distinguishing those who sought care from those who did not, in terms of both socio-economic factors as well as parental and peer interaction levels and youth inhibitions about seeking services relating to sexual matters. Other topics requiring research attention include in-depth explorations of ways articulated by youth to overcome these barriers to care seeking on the one hand and of reasons underlying youth preference for private and traditional rather than public sector health care on the other. At the same time, research is needed that assesses the perspective of providers with regard to the barriers they face in providing services to youth and ways in which to overcome these.

In brief, the Youth Study has documented, for the first time, the multi-faceted situation of youth in Bihar. The study alerts us to the many challenges confronting youth and their ability to make a successful transition to adulthood. It emphasises the heterogeneity of youth, not only in terms of their situation but also with regard to their stated needs and preferred mechanisms to address these needs. Programmes must recognise the heterogeneity of young people, and interventions and delivery mechanisms should be appropriately tailored to meet their needs. Evidence presented here provides not only a blue-print for the programming needs of youth in Bihar but also a base-line by which to measure the impact of programmes intended to address youth needs. 


\section{References}

Abraham, L. 2001. "Redrawing the lakshman rekha: Gender differences and cultural constructions in youth sexuality in urban India," South Asia 24:133-56.

Abraham, L. 2002. "Bhai-behen, true love, time pass: Friendships and sexual partnerships among youth in an Indian metropolis," Culture, Health, and Sexuality 4(3):337-53.

Abraham, L. and K.A. Kumar. 1999. "Sexual experiences and their correlates among college students in Mumbai city, India," International Family Planning Perspectives 25(3):139-46.

Alexander, M., L. Garda, S. Kanade et al. 2003. Formation of partnerships among young women and men in Pune district, Maharashtra, unpublished survey questionnaire. New Delhi: Population Council.

Alexander, M., L. Garda, S. Kanade et al. 2006a. Formation of Partnerships among Young Women and Men in Pune District, Maharashtra. New Delhi: Population Council.

Alexander, M., L. Garda, S. Kanade et al. 2006b. "Romance and sex: Pre-marital partnership formation among young women and men, Pune district, India," Reproductive Health Matters 14(28):144-55.

Andrew, G., V. Patel and J. Ramakrishna. 2003. "Sex, studies or strife? What to integrate in adolescent health services," Reproductive Health Matters 11(21):120-29.

Awasthi, S., M. Nichter and V.K. Pande. 2000. "Developing an interactive STD prevention programme for youth: Lessons from a north Indian slum," Studies in Family Planning 31(2):138-50.

Barnes, L. 2007. "Women's experience of childbirth in rural Jharkhand," Economic and Political Weekly 41(48): $62-70$.

Bashir, K., R. Blizard, R. Jenkins et al. 1996. "Validation of the 12-item General Health Questionnaire in British general practice," Primary Care Psychiatry 2:4-7.

Bhat, P.N.M. and A.J.F. Zavier. 2007. "Factors influencing the use of prenatal diagnostic techniques and sex ratio at birth in India," in Watering the Neighbour's Garden: The Growing Demographic Female Deficit in Asia, eds. I. Attane and C.Z. Guilmoto. Paris: Committee for International Cooperation in National Research in Demography, pp. 131-60.

Bhatia, M.S. and S. Choudhary. 1998. "Dhat symptoms: A culture-bound sex neurosis," Indian Journal of Medical Science 52:30-35. 
Bhatia, M.S. and S.C. Malik. 1991. "Dhat syndrome: A useful diagnostic entity in Indian culture," British Journal of Psychiatry 159:691-95.

Bhende, A.A. 1995. "Evolving a model for AIDS prevention education among underprivileged adolescent girls in urban India," Women and AIDS Research Program Report, Series No. 5. Washington, DC: International Centre for Research on Women.

Bhuiya, I., U. Rob, M.E. Khan et al. 2003. "Reproductive health services for adolescents: Recent experiences from a pilot project in Bangladesh," in Towards Adulthood: Exploring the Sexual and Reproductive Health of Adolescents in South Asia, eds. S. Bott, S.J. Jejeebhoy, I. Shah et al. Geneva: World Health Organisation, pp. 203-6.

Bihar State AIDS Control Society. 2004. Annual Report 2004, <www.bsacs.org/annual report/2004>

Chandrasekhar, C.P., J. Ghosh and A. Roychowdhury. 2006. “The 'demographic dividend' and young India's economic future," Economic and Political Weekly 41(49): 5055-63.

Cleland, J. 2001. "Illustrative questionnaire for interview-surveys with young people," in Asking Young People about Sexual and Reproductive Behaviours: Illustrative Core Instruments, eds. J. Cleland, R. Ingham and N. Stone. Geneva: World Health Organisation, pp. 5-16.

Collumbien, M., N. Bohidar, R. Das et al. 2004. "Etic and emic categories in male sexual health: A case study from Orissa," in Categories and Contexts: Anthropological and Historical Studies in Critical Demography, eds. S. Szreter, H. Sholkamy and A. Dharmalingam. New York: Oxford University Press, pp. 199-221.

Dagar, R. 2007. "Rethinking female foeticide: Perspective and issues," in Sex-selective Abortion in India: Gender, Society and New Reproductive Technologies, ed. T. Patel. New Delhi: Sage Publications, pp. 91-134.

Demographic Research and Development Foundation, Inc. (DRDF) and University of the Philippines Population Institute (UPPI). 2002. Young Adult Fertility and Sexuality Study [Married Female] Questionnaire. Quezon City, Philippines: DRDF and UPPI.

Department of Finance, Government of Bihar. 2008. Economic Survey 2007-2008, <http://finance.bih.nic.in/ Bud2008-09/Economic-Survey-2007-08.pdf>

Department of Health and Family Welfare, Government of Bihar. 2000. Bihar Population Policy 2000, $<$ www.shsbihar.org/pp2000/index.htm>

Department of Health and Family Welfare, Government of Bihar.(n.d). State Programme Implementation Plan, RCH-II, 2005-06 to 2009-10, <www.shrbihar.org.rch2/index.htm>

DiClemente, R.J. 1992. "Psychosocial determinants of condom use among adolescents," in Adolescents and AIDS: A Generation in Jeopardy, ed. R.J. DiClemente. Newbury Park, CA: Sage, pp. 34-51.

Donath, S. 2001. "The validity of the 12-item General Health Questionnaire in Australia: A comparison between three scoring methods," Australian and New Zealand Journal of Psychiatry 35(2):231-35.

Ellickson, P., H. Saner and K.A. McGuigan. 1997. "Profiles of violent youth: Substance use and other concurrent problems," American Journal of Public Health 87:985-91. 
Goldberg, D. 1992. General Health Questionnaire (GHQ-12). Windsor: NFER-Nelson.

Gruber, E., R.J. DiClemente, M.M. Anderson et al. 1996. "Early drinking onset and its association with alcohol use and problem behaviour in late adolescence," Preventive Medicine 25(3):293-300.

Guttmacher Institute. 2004a. 2004 Ghana National Survey of Adolescents: Survey Questionnaire, unpublished. New York: Guttmacher Institute.

Guttmacher Institute. 2004b. 2004 Malawi National Survey of Adolescents: Survey Questionnaire, unpublished. New York: Guttmacher Institute.

Guttmacher Institute. 2004c. 2004 Uganda National Survey of Adolescents: Survey Questionnaire, unpublished. New York: Guttmacher Institute.

International Institute for Population Sciences (IIPS) and Macro International. 2007a. National Family Health Survey (NFHS-3), 2005-06: India, Volume 1. Mumbai: IIPS.

International Institute for Population Sciences (IIPS) and Macro International. 2007b. National Family Health Survey (NFHS-3), 2005-06: India, Volume II. Mumbai: IIPS.

International Institute for Population Sciences and Macro International. 2008. National Family Health Survey (NFHS-3), India, 2005-06: Bihar, Mumbai: IIPS

International Institute for Population Sciences (IIPS) and Population Council. 2002. First-time Parents project: Baseline survey questionnaire, unpublished.

International Labour Organisation (ILO). 2006. Global Employment Trends for Youth. Geneva: ILO.

Jacob, K.S., D. Bhugra and A.H. Mann. 1997. "The validation of the 12-item General Health Questionnaire among ethnic Indian women living in the United Kingdom,” Psychological Medicine 27(5):1215-17.

Jejeebhoy, S.J. and M.P. Sebastian. 2003. "Actions that protect: Promoting sexual and reproductive health and choice among young people in India," Regional Working Papers, No. 18. New Delhi: Population Council.

Jejeebhoy, S.J. and M.P. Sebatian. 2004. "Young people's sexual and reproductive health," in Looking Back, Looking Forward: A Profile of Sexual and Reproductive Health in India, ed. S.J. Jejeebhoy. New Delhi: Population Council, pp. 138-68.

Khan, S.I., N. Hudson-Rodd, S. Saggers et al. 2006. “'Semen contains vitality and heredity, not germs': Seminal discourse in the AIDS era," Journal of Health, Population and Nutrition 24(4):426-37.

Laird, R.D., G.S. Pettit, K.A. Dodge et al. 2003. “Change in parents' monitoring knowledge: Links with parenting, relationship quality, adolescent beliefs, and antisocial behaviour," Social Development 12(3):401-19.

Lambert, H. and K. Wood. 2005. "A comparative analysis of communication about sex, health and sexual health in India and South Africa: Implications for HIV prevention," Culture, Health and Sexuality 7(6):527-41.

Lowry, R., D. Holtzman, B.I. Truman et al. 1994. "Substance use and HIV-related sexual behaviours among US high school students: Are they related?” American Journal of Public Health 84:1116-20. 
Marta, E. 1997. "Parent-adolescent interactions and psychosocial risk in adolescents: An analysis of communication, support and gender," Journal of Adolescence 20(5):473-87.

Mehra, S., R. Savithri and L. Coutinho. 2002. "Sexual behaviour among unmarried adolescents in Delhi, India: Opportunities despite parental controls," Paper presented at the IUSSP Regional Population Conference, Bangkok, June.

Mensch, B.S., D.N. Anh and W.H. Clark. 2000. Adolescents and Social Change in Vietnam: Preliminary Report. Hanoi: Population Council and Institute of Sociology.

Ministry of Finance. 2008. Economic Survey of India 2007-2008. New Delhi: Ministry of Finance, Government of India.

Ministry of Health and Family Welfare (MOHFW). 2000. National Population Policy 2000. New Delhi: Department of Family Welfare, MOHFW, Government of India.

Ministry of Health and Family Welfare (MOHFW). 2005. National Rural Health Mission: Meeting People's Health Needs, Framework for Implementation 2005-2012. New Delhi: MOHFW, Government of India. PDF file retrieved 1 January 2008 from <www.mohfw.nic.in/NRHM/Documents/NRHM-Framework for Implementation.pdf $>$.

Ministry of Health and Family Welfare (MOHFW). 2006. RCH Phase II: National Programme Implementation Plan. New Delhi: MOHFW, Government of India.

Ministry of Human Resource Development. 2007. Selected Educational Statistics 2004-2005. New Delhi: Ministry of Human Resource Development, Government of India.

Ministry of Statistics and Programme Implementation. 2008. New Series (1999-2000) Statement: Gross State Domestic Product at Current Prices, 28 February. New Delhi: Ministry of Statistics and Programme Implementation. Accessed 27 March 2008 at <www.mospi.nic.in/6_gsdp_cur_9394ser.htm>.

Ministry of Youth Affairs and Sports. 2003. National Youth Policy 2003. New Delhi: Government of India.

Mohan, S., P. Sankara Sarma and K.R. Thankappan. 2005. "Access to pocket money and low educational performance predict tobacco use among adolescent boys in Kerala, India," Preventive Medicine 41(2):685-92.

National Institute of Medical Statistics and National AIDS Control Organisation (NACO). 2008. National Behavioural Surveillance Survey (BSS), 2006; Youth (15-24 Years). New Delhi: NACO, Ministry of Health and Family Welfare, Government of India. Accessed 5 January 2009 at <http://www.nacoonline.org/Quick_Links/ Publication/ME_and_Research_Surveillance/Reports_and_Surveys/National_BSS_20062/>.

National Research Council and Institute of Medicine. 2005. Growing Up Global: The Changing Transition to Adulthood in Developing Countries. Washington, DC: National Academies Press.

National Sample Survey Organisation (NSSO). 2006. Employment and Unemployment Situation in India 2004-05, Part 1, NSS 61st Round. New Delhi: NSSO, Government of India. 
Office of the Registrar General and Census Commissioner. 2001a. Age Groups. Accessed on 27 November 2008 at $<$ http://www.censusindia.gov.in/Census_Data_2001/Census_Data_Online/Social_and_cultural/Age_Groups. aspx $>$.

Office of the Registrar General and Census Commissioner. 2001b. Primary Census Abstract, Total Population: Table A-5, Series 1. New Delhi: Office of the Registrar General and Census Commissioner.

Office of the Registrar General and Census Commissioner. 2001c. Religious Compositions. Accessed on 27 November 2008 at <http://www.censusindia.gov.in/Census_Data_2001/Census_Data_Online/Social_and_ cultural/Religion.aspx $>$.

Office of the Registrar General and Census Commissioner. 2001d. Inter-state in-migrants by last residence. Accessed on 27 November 2008 at <http://www.censusindia.gov.in/Census_Data_2001/Census_Data_Online/ Online_Migration/Inter_State_Migration_Top_Three_States.aspx.>.

Office of the Registrar General and Census Commissioner. 2001e. Literate. Accessed on 27 November 2008 at $<$ http://www.censusindia.gov.in/Census_Data_2001/Census_Data_Online/Social_and_cultural/Literate.aspx > .

Office of the Registrar General and Census Commissioner. 2001f. Educational Level by Age and Sex for Population Aged 7 and Above, Table C-8. New Delhi: Office of the Registrar General and Census Commissioner.

Office of the Registrar General and Census Commissioner. 2006. Population Projections for India and States 2001-2026 (Revised December 2006). New Delhi: Office of the Registrar General and Census Commissioner.

Patel, V. and G. Andrew. 2001. "Gender, sexual abuse and risk behaviours: A survey of school-based adolescents in Goa," National Medical Journal of India 14:263-67.

Pelto, P.J. 1999. "Sexuality and sexual behaviour: The current discourse," in Implementing a Reproductive Health Agenda in India: The Beginning, ed. S. Pachauri. New Delhi: Population Council, pp. 539-86.

Planning Commission. 2002. Tenth Five Year Plan (2002-2007), Volume II, Sectoral Policies and Programmes. New Delhi: Government of India.

Planning Commission. 2006. Towards Faster and More Inclusive Growth: An Approach to the 11th Five Year Plan (2007-2012). New Delhi: Government of India.

Planning Commission. 2007. Poverty Estimates for 2004-05, Press release, March. New Delhi: Government of India.

Ram, F., R.K. Sinha, S.K. Mohanty et al. 2006. Marriage and Motherhood: An Exploratory Study of the Social and Reproductive Health Status of Married Young Women in Gujarat and West Bengal, India. New Delhi: Population Council.

Rossier, C. 2003. "Estimating induced abortion rates: A review," Studies in Family Planning 34(2):87-102.

Sachdev, P. 1998. "Sex on campus: A preliminary study of knowledge, attitudes and behaviour of university students in Delhi, India," Journal of Biosocial Science 30:95-105. 
Santhya, K.G. and S.J. Jejeebhoy. 2006. "Young women's experience of forced sex within marriage: Evidence from India," in Sex without Consent: Young People in Developing Countries, eds. S.J. Jejeebhoy, I. Shah and S. Thapa. London: Zed Books, pp. 59-73.

Santhya, K.G., S.J. Jejeebhoy and S. Ghosh. 2008. Early Marriage and Sexual and Reproductive Health Risks: Experiences of Young Women and Men in Andhra Pradesh and Madhya Pradesh, India. New Delhi: Population Council.

Santhya, K.G., N. Haberland, A. Das et al. 2008. Empowering Married Girls and Improving their Sexual and Reproductive Health: Effects of the First-time Parents Project. New Delhi: Population Council.

Santhya, K.G., N. Haberland, F. Ram et al. 2007. "Consent and coercion: Examining unwanted sex among married young women in India," International Family Planning Perspectives 33(3):124-32.

Sathar, Z.A., M. Haque, A. Faizunnissa et al. 2003. Adolescents and Youth in Pakistan, 2001-02. Islamabad: Population Council.

Sebastian, M.P., M. Grant and B. Mensch. 2005. Integrating Adolescent Livelihood Activities Within a Reproductive Health Programme for Urban Slum Dwellers in India. New Delhi: Population Council.

Sebastian, M.P., M. Grant, B. Mensch et al. 2003. Integrating adolescent livelihood activities within a reproductive health programme for urban slum dwellers in India, unpublished survey questionnaire. New Delhi: Population Council.

Shekhar, M., S. Ghosh and P. Panda. 2007. Exploring safe sex awareness and sexual experiences of adolescents in Patna, Economic and Political Weekly, December 1: 48-55

Singh, S. and S. Saini. 2007. "HIV risk perception in relation to peer pressure and drug abuse behaviour among adolescents," Indian Journal of Sexually Transmitted Diseases 28:53-54.

Sroufe, J.W. 1991. "Assessment of parent-adolescent relationships: Implications for adolescent development," Journal of Family Psychology 5(1):21-45.

Ul Haque, M. and A. Faizunnisa. 2003. "Access to reproductive health information in Punjab and Sindh, Pakistan: The perspectives of adolescents and parents," in Towards Adulthood: Exploring the Sexual and Reproductive Health of Adolescents in South Asia, eds. S. Bott, S.J. Jejeebhoy, I. Shah et al. Geneva: World Health Organisation, pp. 153-55.

United Nations Development Programme (UNDP). 2000. Millennium Development Goals. United Nations website. Accessed 8 January 2008 at <http://www.un.org/millenniumgoals/goals.html>.

Verma, R.K., S. Sharma, R. Singh et al. 2003. "Beliefs concerning sexual health problems and treatment seeking among men in an Indian slum community," Culture, Health and Sexuality 5(3):265-76. 


\section{Appendix A}

\section{Principal investigators and authors}

Usha Ram, Associate Professor, Department of Public Health and Mortality Studies, International Institute for Population Sciences, Mumbai

S.K. Mohanty, Reader, Department of Fertility Studies, International Institute for Population Sciences, Mumbai

Abhishek Singh, Assistant Professor, Department of Public Health and Mortality Studies, International Institute for Population Sciences, Mumbai

F. Ram, Director and Senior Professor, International Institute for Population Sciences, Mumbai

Rajib Acharya, Senior Programme Officer, Population Council, New Delhi

Shireen J. Jejeebhoy, Senior Associate, Population Council, New Delhi

K.G. Santhya, Associate, Population Council, New Delhi 


\section{Appendix A \\ Bihar study staff}

Senior Research Officers

Shreeparna Ghosh

\section{Research Officers}

Shaikh Tayyaba Khatoon

Manas Ranjan Pradhan

Abhishek Kumar

Manoranjan Kumar

Rajesh Kumar

Sudhir Kumar Sharma

Lucky Singh

Prashant Kumar Singh

Santosh Kumar Singh

Finance Staff

Jeba Kumar

Anil Paul

Administrative Staff

Pranita H. Dalvi

Avadhesh Prajapati

Namrata Ghadge

Shyam V. Rathod

Seema Jadhav

Komal Saxena

M. A. Jose

Usha Sonavane

\section{Editorial Staff}

Shanti Menon

\section{Data Entry Operators}

Mohini Bisht

R. S. Sandhya

Amit Kumar

Vandana Sharma

Sunita Kumari

Vibha Trivedi

Shilpi Rampal

\section{Field Supervisor}

Shiv Chandra

Mithilesh Kumar

Binit Jha

Prabhat Ranjan

Ajay Kumar

Bhim Singh 
Field Editor

Anupa Kumari Burman

Veena Kumari

Sayda Nasrin

Prem Prakash

Office Editors

Satish Babu

Pankaj Bhuyar

Shrikant Gajbhiye

Binit Jha

Household Listers and Mappers

Md Parwez Alam

Uma Shankar Choudhary

Misbahul Iman

Abhay Kumar

Nagendra Kumar

Rakesh Kumar

Ravindra Kumar

Sanjeet Kumar

Srikant Kumar

Field Investigators

Shamim Ahmad

Badri Narayan Bhandary

Anupa Kumari Burman

Sunil Kumar Das

Devanand Devesh

Binod Giri

Misbahul Iman

Pushpa Kumari Karmah

Krishna Kumar

Nikesh Kumar

Prakesh Kumar

Sanjay Kumar

Shailendra Kumar

Shyam Kumar

Subodh Kumar

Sunil Kumar

Anita Kumari

Chhaya Kumari

Indra Kumar

Meena Kumari

Neetu Kumari
Imtiyaz Qaiyum

Sanjeev Ranjan

Bhagwat Singh

Rahul B Kamble

Jagdish Shinde

B. Srihari

Methar Chandra Mahto

Jaganath Prasad

Rajeeshwar Ram

Subodh Kumar Singh

Krishna Kumar Thakur

Jai Shankar Tiwari

Brijesh Kumar Upadhyay

Kapil Dev Prasad Yadav

Rajendra Prasad Yadav

Pratibha Kumari

Pusplata Kumari

Rakhi Kumari

Rani Kumari

Sarita Kumari

Veena Kumari

Methar Chandra Mehto

Sanjay Kumar Pandey

Bhola Prasad

Imtiyaz Qaiyum

Praven Ranjan

Sanjeev Ranjan

Ratna Roy

Dipu Kumar Sharma

Bhagwat Singh

Raju Kumar Singh

Virendra Singh

Kavita Sinha

Ruby Tabbassum

Rajendra Prasad Yadav

Radha Yadav 


\section{Appendix A}

\section{Advisory committees}

\section{Project Advisory Committee}

Additional Secretary

Ministry of Health and Family Welfare

Government of India

Chief Director

Ministry of Health and Family Welfare

Government of India

Deputy Secretary

Information, Education, Communication

Ministry of Health and Family Welfare

Government of India

Director

Directorate of Health Services

Government of Andhra Pradesh

Director

Directorate of Health Services

Government of Bihar

Director

Directorate of Health Services

Government of Jharkhand

Director

Directorate of Health Services

Government of Maharashtra

Director

Directorate of Health Services

Government of Rajasthan

Director

Directorate of Health Services

Government of Tamil Nadu
Director

Nehru Yuvak Kendra Sangatan

Ministry of Sports and Youth Affairs

Government of India

Director

National Institute of Health and Family Welfare,

New Delhi

Country Director (India)

John D. and Catherine T. Mac Arthur Foundation, New Delhi

Country Programme Advisor (India)

David and Lucile Packard Foundation,

New Delhi

Director

International Institute for Population Sciences,

Mumbai

Regional Director

Population Council, New Delhi

\section{NFHS Coordinator}

International Institute for Population Sciences, Mumbai

Shalini Bharat

Professor and Dean

School of Health Systems Studies

Tata Institute of Social Sciences, Mumbai

P.M. Kulkarni

Professor

Centre for the Study of Regional Development

Jawaharlal Nehru University, New Delhi 
Arvind Pandey

Director

Institute for Research in Medical Statistics,

New Delhi

G. Rama Rao

Former Officiating Director

International Institute for Population Sciences, Mumbai

Technical Advisory Committee

Shalini Bharat

Professor and Dean

School of Health Systems Studies

Tata Institute of Social Sciences, Mumbai

Director

International Institute for Population Sciences, Mumbai

P.M. Kulkarni

Professor

Centre for the Study of Regional Development Jawaharlal Nehru University, New Delhi

Arvind Pandey

Director

Institute for Research in Medical Statistics, New

Delhi
T.K. Roy

Former Director

International Institute for Population Sciences,

Mumbai

Leela Visaria

National Professor

Gujarat Institute of Development Research, Ahmedabad

Pertti J. Pelto

Professor Emeritus

Medical Anthropology

University of Connecticut, USA

T.K. Roy

Former Director

International Institute for Population Sciences, Mumbai

Leela Visaria

National Professor

Gujarat Institute of Development Research, Ahmedabad 


\section{Appendix B}

\section{Estimates of sampling errors}

As in the case of any sample survey, estimates from the Youth Study in Bihar, as presented in Chapters 1-12, are affected by two major sources of errors: non-sampling and sampling errors. Non-sampling errors are generally the result of procedural mistakes made during data collection and data processing, such as, the inability to locate and interview the correct household or individual, failure to conform to standard survey procedures laid out by the central office, misunderstanding of questions on the part of either the interviewer or the respondent, and data entry errors. At the same time, because of the inclusion of numerous sensitive issues, the Youth Study faced the risk of other non-sampling errors as well, such as, the deliberate skipping of sensitive questions by the interviewer or refusal to answer sensitive questions by the respondent. In order to minimise non-sampling errors, a number of precautions were taken during the implementation of the study, which are described in detail in Chapter 1 . However, we acknowledge that despite these efforts, non-sampling errors are impossible to avoid; they are, moreover, extremely difficult to evaluate statistically.

Sampling errors, on the other hand, can be evaluated statistically. These errors, as the name suggests, result from the choice of the particular sample selected. The sample of respondents selected in the Youth Study is only one of many possible samples that could have been selected from the population of Bihar, using the same design and expected sample size. Each of these samples would have yielded results that differed somewhat from the results of the sample selected. The sampling error is a measure of variability among all possible samples. Although the degree of variability may not be known exactly, it can be estimated from the survey results using standard statistical procedures.

A sampling error, usually measured in terms of the standard error for a particular statistic (mean, percentage, ratio, etc.), is the square root of the variance of that statistic. The standard error can be used to calculate confidence intervals within which the true value for the population can reasonably be assumed to fall. For example, for any given statistic calculated from the survey, the value of that statistic will fall within a range of plus or minus two times the standard error of that statistic in $95 \%$ of all possible samples of identical size and design.

If the sample of respondents had been selected as a simple random sample, it would have been possible to use straightforward formulae for calculating the variance of the statistic and consequently, sampling errors. However, the Youth Study sample is the result of a multi-stage stratified design, and consequently, it was necessary to use more complex formulae. The variance estimators that were used can be found in Cochran (1977) and Wolter (1985). The computer software used to calculate sampling errors for the Youth Study was programmed in STATA SE 8.2. This procedure uses the Taylor linearisation method for variance estimation for survey estimates that are means, proportions or ratios.

The Taylor linearisation method treats any percentage or average as a ratio estimate. Let $r=y / x$ be our sample estimate of the population ratio (mean or percentage) denoted by $\mathrm{R}=\mathrm{Y} / \mathrm{X}$, where $\mathrm{y}$ represents the total sample value for variable $\mathrm{Y}$, and $\mathrm{x}$ represents the total number of sample cases in the group or sub-group 
under consideration. Using first order Taylor expansion, it can be shown that the approximate variance of distribution of $r$ (square root of which is the standard error) is as below:

$$
\operatorname{Var}(r)=\frac{1-f}{x^{2}} \sum_{h=1}^{L}\left[\frac{n_{h}}{n_{h}-1}\left(\sum_{i=1}^{n_{h}} z_{h i}^{2}-\frac{z_{h}^{2}}{n_{h}}\right)\right]
$$

in which $\mathrm{z}_{h i}=\mathrm{y}_{h i}-\mathrm{rx}_{h i}$ and $\mathrm{z}_{h}=\mathrm{y}_{h}-\mathrm{rx}_{h}$

where $h \quad$ represents the sampling stratum which varies from 1 to $\mathrm{L}$,

$n_{h} \quad$ is the number of PSUs selected in the $\mathrm{h}^{\text {th }}$ stratum,

$y_{h i} \quad$ is the sum of the weighted values of variable $\mathrm{Y}$ in the $\mathrm{i}^{\text {th }}$ PSU in the $\mathrm{h}^{\text {th }}$ stratum,

$x_{h i} \quad$ is the sum of the weighted number of cases in the $\mathrm{i}^{\text {th }}$ PSU in the $\mathrm{h}^{\text {th }}$ stratum,

$f \quad$ is the overall sampling fraction, which is so small that it is ignored.

In addition to the standard error, the design effect (DEFT) for each estimate was also computed, which is defined as the ratio between the standard error using the given sample design and the standard error that would result if a simple random sample had been used (Kish, 1995) represented by the following simple formula:

$$
D E F T=\sqrt{\frac{\operatorname{Var}(r)}{\operatorname{Var}_{\text {srswr }}\left(r_{\text {srs }}\right)}}
$$

where $\operatorname{Var}(r)$ is a design-based estimate of variance for the parameter $r$,

$\operatorname{Var}_{\text {srswr }}\left(\mathrm{r}_{\text {srs }}\right)$ is an estimate of the variance for an estimator rsrs that would be obtained from a similar hypothetical survey conducted using simple random sampling (srs) with replacement (wr).

A DEFT value of 1.0 indicates that the sample design is as efficient as a simple random sample, while a value greater than 1.0 indicates the increase in the sampling error due to the use of a more complex and less statistically efficient design. Relative standard error (SE/R) and $95 \%$ confidence limits for each estimate were also computed.

Sampling errors for the Youth Study were calculated for selected variables and results are presented in this appendix for each sex and marital status sub-group of respondents for the state as a whole, and for those in urban and rural areas, respectively. For each variable, the type of statistic (mean, proportion or ratio) and the base population are given in Table B.1. Table B.2 presents the value of the statistic (R), its standard error $(\mathrm{SE})$, the number of unweighted $(\mathrm{N})$ and weighted $(\mathrm{WN})$ cases, the design effect (DEFT), the relative standard error $(\mathrm{SE} / \mathrm{R})$ and the $95 \%$ confidence limits, for each variable. 
Table B.1: List of selected variables for sampling errors, Bihar, 2007

\begin{tabular}{|c|c|c|}
\hline Variables & Estimates & Base Population \\
\hline Sex ratio (females per 1,000 males) & Ratio & De jure household population, all ages \\
\hline Sex ratio (females per 1,000 males) & Ratio & De jure household population, aged 0-6 \\
\hline $\begin{array}{l}\text { Currently married, including married but not } \\
\text { yet cohabiting }\end{array}$ & Proportion & De jure household population, aged $20-24$ \\
\hline No education & Proportion & De jure household population, aged 6 or above \\
\hline No education & Proportion & Young men and women \\
\hline Completed 12 or more years of education & Proportion & Young men and women \\
\hline Ever worked in last 12 months & Proportion & Young men and women \\
\hline Unemployed & Proportion & Young men and women in labour force \\
\hline Discussed friendships with father & Proportion & $\begin{array}{l}\text { Young men and women whose father was alive } \\
\text { at the time of interview }\end{array}$ \\
\hline Discussed friendships with mother & Proportion & $\begin{array}{l}\text { Young men and women whose mother was alive } \\
\text { at the time of interview }\end{array}$ \\
\hline $\begin{array}{l}\text { Independently makes decisions on choice of } \\
\text { friends, spending money and buying clothes for } \\
\text { oneself }\end{array}$ & Proportion & Young men and women \\
\hline $\begin{array}{l}\text { Can visit places outside village or } \\
\text { neighbourhood unescorted }\end{array}$ & Proportion & Young men and women \\
\hline Has savings of any amount & Proportion & Young men and women \\
\hline Justified wife beating in at least one situation & Proportion & Young men and women \\
\hline Awareness of sex- and pregnancy-related matters & Proportion & Young men and women \\
\hline $\begin{array}{l}\text { Correct specific knowledge of at least one } \\
\text { contraceptive method }\end{array}$ & Proportion & Young men and women \\
\hline Correct specific knowledge of condoms & Proportion & Young men and women \\
\hline Ever heard of HIV/AIDS & Proportion & Young men and women \\
\hline Comprehensive knowledge of HIV/AIDS & Proportion & Young men and women \\
\hline Ever heard of STIs other than HIV & Proportion & Young men and women \\
\hline $\begin{array}{l}\text { Correct knowledge of the conditions under } \\
\text { which abortion is legal }\end{array}$ & Proportion & Young men and women \\
\hline Ever received family life or sex education & Proportion & Young men and women \\
\hline Ever had an opposite-sex romantic partner & Proportion & Young men and women \\
\hline $\begin{array}{l}\text { Ever had sex with an opposite-sex romantic } \\
\text { partner }\end{array}$ & Proportion & Young men and women \\
\hline Ever had pre-marital sex & Proportion & Young men and women \\
\hline $\begin{array}{l}\text { Used condom consistently in pre-marital } \\
\text { relations }\end{array}$ & Proportion & $\begin{array}{l}\text { Young men and women who reported pre- } \\
\text { marital sex in face-to-face interview }\end{array}$ \\
\hline $\begin{array}{l}\text { Ever communicated with spouse on } \\
\text { contraception }\end{array}$ & Proportion & $\begin{array}{l}\text { Married young men and women who had } \\
\text { begun cohabiting }\end{array}$ \\
\hline Husband ever forced wife to have sex & Proportion & $\begin{array}{l}\text { Married young men and women who had } \\
\text { begun cohabiting }\end{array}$ \\
\hline $\begin{array}{l}\text { Husband ever perpetrated physical violence on } \\
\text { wife }\end{array}$ & Proportion & $\begin{array}{l}\text { Married young men and women who had } \\
\text { begun cohabiting }\end{array}$ \\
\hline $\begin{array}{l}\text { Husband ever perpetrated physical violence on } \\
\text { wife in last } 12 \text { months }\end{array}$ & Proportion & $\begin{array}{l}\text { Married young men and women who had } \\
\text { begun cohabiting }\end{array}$ \\
\hline $\begin{array}{l}\text { Currently using any modern contraceptive } \\
\text { method }\end{array}$ & Proportion & $\begin{array}{l}\text { Married young men and women who had } \\
\text { begun cohabiting }\end{array}$ \\
\hline
\end{tabular}




\section{Variables}

First delivery in a health institution

Mean number of children ever born

Mean number of children surviving

Mean ideal number of children

Experienced 3 or more symptoms or behaviours suggestive of mental health disorders in the month preceding the interview

Ever consumed alcohol

Participated in a government-/NGO-sponsored programme in the 3 years preceding the interview

Voted in last election
Base Population

Proportion

Married young men and women whose first pregnancy outcome was a live or still birth

Mean

Mean

Mean

Proportion

Married young men and women who had begun cohabiting

Married young men and women who had begun cohabiting

Married young men and women who had begun cohabiting and gave a numeric response

Young men and women

Proportion

Young men and women

Proportion

Young men and women

Proportion 
Table B.2: Sampling errors, Bihar, 2007

\begin{tabular}{|c|c|c|c|c|c|c|c|c|}
\hline \multirow{2}{*}{$\begin{array}{l}\text { Variable/ } \\
\text { respondent } \\
\text { category }\end{array}$} & \multirow{2}{*}{$\begin{array}{l}\text { Value } \\
(\mathbf{R})\end{array}$} & \multirow{2}{*}{$\begin{array}{c}\text { Standard } \\
\text { error } \\
(\mathrm{SE})\end{array}$} & \multicolumn{2}{|c|}{ Number of cases } & \multirow{2}{*}{$\begin{array}{c}\text { Design } \\
\text { effect } \\
\text { (DEFT) }\end{array}$} & \multirow{2}{*}{$\begin{array}{c}\text { Relative } \\
\text { standard } \\
\text { error } \\
(\mathrm{SE} / \mathrm{R})\end{array}$} & \multicolumn{2}{|c|}{ 95\% Confidence limit } \\
\hline & & & $\begin{array}{l}\text { Unweighted } \\
(\mathbf{N})\end{array}$ & $\begin{array}{l}\text { Weighted } \\
(\text { WN) }\end{array}$ & & & Lower & Upper \\
\hline \multicolumn{9}{|c|}{$\begin{array}{l}\text { Sex ratio } \\
\text { de jure household population, all ages) }\end{array}$} \\
\hline Combined & 1.0430 & 0.0086 & 78,553 & 75,347 & 2.0601 & 0.0083 & 1.0260 & 1.0599 \\
\hline Urban & 0.9265 & 0.0092 & 32,141 & 7,140 & 1.5658 & 0.0099 & 0.9085 & 0.9446 \\
\hline Rural & 1.0552 & 0.0096 & 46,412 & 68,211 & 1.7668 & 0.0091 & 1.0364 & 1.0740 \\
\hline \multicolumn{9}{|c|}{$\begin{array}{c}\text { Sex ratio } \\
\text { (females per 1,000 males, de jure household population, aged 0-6) }\end{array}$} \\
\hline Combined & 0.9351 & 0.0134 & 15,845 & 17,093 & 1.3105 & 0.0143 & 0.9088 & 0.9615 \\
\hline Urban & 0.9007 & 0.0194 & 5,257 & 1,169 & 1.0791 & 0.0215 & 0.8626 & 0.9388 \\
\hline Rural & 0.9377 & 0.0143 & 10,588 & 15,924 & 1.1061 & 0.0153 & 0.9095 & 0.9659 \\
\hline \multicolumn{9}{|c|}{$\begin{array}{l}\text { Currently married, including married but not yet cohabiting } \\
\text { (de jure household population, aged } 20-24)\end{array}$} \\
\hline \multicolumn{9}{|c|}{ Combined } \\
\hline Male & 0.4796 & 0.0150 & 5,364 & 4,251 & 2.1953 & 0.0312 & 0.4502 & 0.5091 \\
\hline Female & 0.9009 & 0.0077 & 7,083 & 6,868 & 2.1679 & 0.0085 & 0.8857 & 0.9161 \\
\hline \multicolumn{9}{|c|}{ Urban } \\
\hline Male & 0.2262 & 0.0125 & 2,737 & 605 & 1.5681 & 0.0554 & 0.2015 & 0.2509 \\
\hline Female & 0.6710 & 0.0169 & 2,888 & 639 & 1.9305 & 0.0252 & 0.6378 & 0.7043 \\
\hline \multicolumn{9}{|c|}{ Rural } \\
\hline Male & 0.5217 & 0.0172 & 2,627 & 3,646 & 1.7683 & 0.0330 & 0.4878 & 0.5557 \\
\hline Female & 0.9245 & 0.0083 & 4,195 & 6,229 & 2.0324 & 0.0090 & 0.9082 & 0.9408 \\
\hline
\end{tabular}

\begin{tabular}{|c|c|c|c|c|c|c|c|c|}
\hline & & (de j & touseho & $\begin{array}{l}\text { ducatio } \\
\text { pulatior }\end{array}$ & d 6 or & & & \\
\hline & & & & mbined & & & & \\
\hline Male & 0.3950 & 0.0087 & 65,297 & 60,944 & 4.5693 & 0.0221 & 0.3778 & 0.4122 \\
\hline Female & 0.6452 & 0.0082 & 65,392 & 65,199 & 4.3576 & 0.0126 & 0.6291 & 0.6612 \\
\hline & & & & Jrban & & & & \\
\hline Male & 0.2250 & 0.0106 & 27,775 & 6,167 & 4.2197 & 0.0470 & 0.2042 & 0.2458 \\
\hline Female & 0.3866 & 0.0139 & 25,760 & 5,731 & 4.5888 & 0.0360 & 0.3592 & 0.4140 \\
\hline & & & & Rural & & & & \\
\hline Male & 0.4141 & 0.0097 & 37,522 & 54,780 & 3.8127 & 0.0234 & 0.3950 & 0.4332 \\
\hline Female & 0.6701 & 0.0089 & 39,632 & 59,470 & 3.7543 & 0.0132 & 0.6527 & 0.6876 \\
\hline
\end{tabular}

Cont'd on next page... 
Table B.2: (Cont'd)

\begin{tabular}{|c|c|c|c|c|c|c|c|c|}
\hline \multirow{2}{*}{$\begin{array}{l}\text { Variable/ } \\
\text { respondent } \\
\text { category }\end{array}$} & \multirow{2}{*}{$\begin{array}{l}\text { Value } \\
\text { (R) }\end{array}$} & \multirow{2}{*}{$\begin{array}{l}\text { Standard } \\
\text { error } \\
\text { (SE) }\end{array}$} & \multicolumn{2}{|c|}{ Number of cases } & \multirow{2}{*}{$\begin{array}{l}\text { Design } \\
\text { effect } \\
\text { (DEFT) }\end{array}$} & \multirow{2}{*}{$\begin{array}{c}\text { Relative } \\
\text { standard } \\
\text { error } \\
(\mathrm{SE} / \mathrm{R})\end{array}$} & \multicolumn{2}{|c|}{ 95\% Confidence limit } \\
\hline & & & $\begin{array}{c}\text { Unweighted } \\
(\mathbf{N})\end{array}$ & $\begin{array}{l}\text { Weighted } \\
\text { (WN) }\end{array}$ & & & Lower & Upper \\
\hline \multicolumn{9}{|c|}{ No education (young men and women) } \\
\hline \multicolumn{9}{|c|}{ Combined } \\
\hline M (15-24) & 0.1608 & 0.0157 & 1,942 & 1,964 & 1.8811 & 0.0975 & 0.1299 & 0.1917 \\
\hline W (15-24) & 0.5101 & 0.0196 & 5,529 & 5,529 & 2.9093 & 0.0383 & 0.4716 & 0.5486 \\
\hline MM (15-29) & 0.2935 & 0.0244 & 1,115 & 1,115 & 1.7897 & 0.0832 & 0.2454 & 0.3416 \\
\hline MW (15-24) & 0.6408 & 0.0186 & 2,341 & 2,341 & 1.8711 & 0.0290 & 0.6042 & 0.6773 \\
\hline UM (15-24) & 0.1172 & 0.0157 & 1,492 & 1,492 & 1.8793 & 0.1336 & 0.0864 & 0.1480 \\
\hline UW (15-24) & 0.2816 & 0.0208 & 3,188 & 3,188 & 2.6113 & 0.0739 & 0.2406 & 0.3226 \\
\hline \multicolumn{9}{|c|}{ Urban } \\
\hline M (15-24) & 0.1044 & 0.0128 & 1,039 & 279 & 1.3444 & 0.1222 & 0.0793 & 0.1295 \\
\hline W (15-24) & 0.2226 & 0.0199 & 2,581 & 637 & 2.4354 & 0.0896 & 0.1833 & 0.2618 \\
\hline MM (15-29) & 0.2068 & 0.0226 & 547 & 92 & 1.3029 & 0.1092 & 0.1624 & 0.2513 \\
\hline MW (15-24) & 0.3619 & 0.0237 & 1,136 & 142 & 1.6589 & 0.0654 & 0.3153 & 0.4085 \\
\hline UM (15-24) & 0.0876 & 0.0125 & 833 & 245 & 1.2749 & 0.1426 & 0.0630 & 0.1122 \\
\hline UW (15-24) & 0.1221 & 0.0186 & 1,445 & 494 & 2.1587 & 0.1523 & 0.0855 & 0.1587 \\
\hline \multicolumn{9}{|c|}{ Rural } \\
\hline M (15-24) & 0.1702 & 0.0181 & 903 & 1,685 & 1.4488 & 0.1065 & 0.1345 & 0.2059 \\
\hline W (15-24) & 0.5475 & 0.0215 & 2,948 & 4,893 & 2.3445 & 0.0393 & 0.5052 & 0.5898 \\
\hline MM (15-29) & 0.3013 & 0.0264 & 568 & 1,023 & 1.3707 & 0.0877 & 0.2493 & 0.3533 \\
\hline MW (15-24) & 0.6588 & 0.0194 & 1,205 & 2,199 & 1.4200 & 0.0295 & 0.6206 & 0.6970 \\
\hline UM (15-24) & 0.1230 & 0.0186 & 659 & 1,247 & 1.4528 & 0.1513 & 0.0864 & 0.1596 \\
\hline UW (15-24) & 0.3109 & 0.0245 & 1,743 & 2,694 & 2.2121 & 0.0789 & 0.2626 & 0.3592 \\
\hline \multicolumn{9}{|c|}{ Completed 12 or more years of education (young men and women) } \\
\hline \multicolumn{9}{|c|}{ Combined } \\
\hline M (15-24) & 0.1218 & 0.0110 & 1,942 & 1,964 & 1.4759 & 0.0899 & 0.1003 & 0.1434 \\
\hline W (15-24) & 0.0453 & 0.0047 & 5,529 & 5,529 & 1.6810 & 0.1038 & 0.0360 & 0.0545 \\
\hline MM (15-29) & 0.1169 & 0.0177 & 1,115 & 1,115 & 1.8407 & 0.1516 & 0.0820 & 0.1517 \\
\hline MW (15-24) & 0.0256 & 0.0040 & 2,341 & 2,341 & 1.2144 & 0.1549 & 0.0178 & 0.0334 \\
\hline UM (15-24) & 0.1278 & 0.0118 & 1,492 & 1,492 & 1.3663 & 0.0925 & 0.1045 & 0.1510 \\
\hline UW (15-24) & 0.0715 & 0.0079 & 3,188 & 3,188 & 1.7287 & 0.1104 & 0.0560 & 0.0870 \\
\hline \multicolumn{9}{|c|}{ Urban } \\
\hline M (15-24) & 0.2497 & 0.0178 & 1,039 & 279 & 1.3225 & 0.0712 & 0.2147 & 0.2846 \\
\hline W (15-24) & 0.1973 & 0.0152 & 2,581 & 637 & 1.9425 & 0.0771 & 0.1673 & 0.2273 \\
\hline MM (15-29) & 0.2273 & 0.0199 & 547 & 92 & 1.1113 & 0.0877 & 0.1881 & 0.2666 \\
\hline MW (15-24) & 0.1200 & 0.0139 & 1,136 & 142 & 1.4399 & 0.1157 & 0.0927 & 0.1474 \\
\hline UM (15-24) & 0.2611 & 0.0191 & 833 & 245 & 1.2542 & 0.0731 & 0.2235 & 0.2987 \\
\hline UW (15-24) & 0.2530 & 0.0176 & 1,445 & 494 & 1.5353 & 0.0694 & 0.2184 & 0.2876 \\
\hline \multicolumn{9}{|c|}{ Rural } \\
\hline M (15-24) & 0.1006 & 0.0121 & 903 & 1,685 & 1.2067 & 0.1201 & 0.0768 & 0.1244 \\
\hline W (15-24) & 0.0255 & 0.0042 & 2,948 & 4,893 & 1.4383 & 0.1639 & 0.0173 & 0.0337 \\
\hline MM (15-29) & 0.1069 & 0.0191 & 568 & 1,023 & 1.4754 & 0.1791 & 0.0692 & 0.1446 \\
\hline MW (15-24) & 0.0195 & 0.0040 & 1,205 & 2,199 & 1.0042 & 0.2053 & 0.0116 & 0.0274 \\
\hline UM (15-24) & 0.1016 & 0.0134 & 659 & 1,247 & 1.1341 & 0.1314 & 0.0753 & 0.1279 \\
\hline UW (15-24) & 0.0382 & 0.0078 & 1,743 & 2,694 & 1.6898 & 0.2032 & 0.0229 & 0.0535 \\
\hline
\end{tabular}


Table B.2: (Cont'd)

\begin{tabular}{|c|c|c|c|c|c|c|c|c|}
\hline \multirow{2}{*}{$\begin{array}{l}\text { Variable/ } \\
\text { respondent } \\
\text { category }\end{array}$} & \multirow{2}{*}{$\begin{array}{l}\text { Value } \\
\text { (R) }\end{array}$} & \multirow{2}{*}{$\begin{array}{l}\text { Standard } \\
\text { error } \\
\text { (SE) }\end{array}$} & \multicolumn{2}{|c|}{ Number of cases } & \multirow{2}{*}{$\begin{array}{c}\text { Design } \\
\text { effect } \\
\text { (DEFT) }\end{array}$} & \multirow{2}{*}{$\begin{array}{c}\text { Relative } \\
\text { standard } \\
\text { error } \\
(\mathrm{SE} / \mathrm{R})\end{array}$} & \multicolumn{2}{|c|}{ 95\% Confidence limits } \\
\hline & & & $\begin{array}{c}\text { Unweighted } \\
\text { (N) }\end{array}$ & $\begin{array}{l}\text { Weighted } \\
\text { (WN) }\end{array}$ & & & Lower & Upper \\
\hline \multicolumn{9}{|c|}{ Ever worked in last 12 months (young men and women) } \\
\hline \multicolumn{9}{|c|}{ Combined } \\
\hline M (15-24) & 0.7269 & 0.0178 & 1,942 & 1,964 & 1.7565 & 0.0244 & 0.6919 & 0.7618 \\
\hline W (15-24) & 0.3680 & 0.0182 & 5,529 & 5,529 & 2.8094 & 0.0495 & 0.3321 & 0.4039 \\
\hline MM (15-29) & 0.9678 & 0.0064 & 1,115 & 1,115 & 1.2011 & 0.0066 & 0.9553 & 0.9803 \\
\hline MW (15-24) & 0.3803 & 0.0224 & 2,341 & 2,341 & 2.2323 & 0.0589 & 0.3362 & 0.4244 \\
\hline UM (15-24) & 0.6435 & 0.0214 & 1,492 & 1,492 & 1.7231 & 0.0332 & 0.6014 & 0.6856 \\
\hline UW (15-24) & 0.3601 & 0.0231 & 3,188 & 3,188 & 2.7129 & 0.0641 & 0.3147 & 0.4055 \\
\hline \multicolumn{9}{|c|}{ Urban } \\
\hline M (15-24) & 0.5866 & 0.0212 & 1,039 & 279 & 1.3867 & 0.0361 & 0.5449 & 0.6283 \\
\hline W (15-24) & 0.1509 & 0.0131 & 2,581 & 637 & 1.8554 & 0.0867 & 0.1251 & 0.1766 \\
\hline MM (15-29) & 0.9449 & 0.0108 & 547 & 92 & 1.1053 & 0.0114 & 0.9236 & 0.9661 \\
\hline MW (15-24) & 0.1236 & 0.0126 & 1,136 & 142 & 1.2936 & 0.1022 & 0.0988 & 0.1485 \\
\hline UM (15-24) & 0.5374 & 0.0228 & 833 & 245 & 1.3165 & 0.0423 & 0.4926 & 0.5822 \\
\hline UW (15-24) & 0.1705 & 0.0184 & 1,445 & 494 & 1.8643 & 0.1082 & 0.1342 & 0.2068 \\
\hline \multicolumn{9}{|c|}{ Rural } \\
\hline M (15-24) & 0.7501 & 0.0200 & 903 & 1,685 & 1.3868 & 0.0267 & 0.7108 & 0.7895 \\
\hline W (15-24) & 0.3963 & 0.0202 & 2,948 & 4,893 & 2.2401 & 0.0509 & 0.3565 & 0.4360 \\
\hline MM (15-29) & 0.9698 & 0.0068 & 568 & 1,023 & 0.9513 & 0.0070 & 0.9564 & 0.9833 \\
\hline MW (15-24) & 0.3970 & 0.0236 & 1,205 & 2,199 & 1.6724 & 0.0594 & 0.3505 & 0.4434 \\
\hline UM (15-24) & 0.6643 & 0.0249 & 659 & 1,247 & 1.3551 & 0.0376 & 0.6152 & 0.7134 \\
\hline UW (15-24) & 0.3949 & 0.0267 & 1,743 & 2,694 & 2.2830 & 0.0677 & 0.3422 & 0.4475 \\
\hline \multicolumn{9}{|c|}{ Unemployed (young men and women in labour force) } \\
\hline \multicolumn{9}{|c|}{ Combined } \\
\hline M (15-24) & 0.2246 & 0.0221 & 1,126 & 1,137 & 1.7765 & 0.0984 & 0.1811 & 0.2682 \\
\hline W (15-24) & 0.3573 & 0.0328 & 1,029 & 1,224 & 2.1928 & 0.0917 & 0.2928 & 0.4219 \\
\hline MM (15-29) & 0.0974 & 0.0167 & 1,002 & 993 & 1.7825 & 0.1715 & 0.0645 & 0.1303 \\
\hline MW (15-24) & 0.3449 & 0.0368 & 487 & 583 & 1.7064 & 0.1067 & 0.2725 & 0.4174 \\
\hline UM (15-24) & 0.2877 & 0.0275 & 740 & 719 & 1.6523 & 0.0956 & 0.2335 & 0.3419 \\
\hline UW (15-24) & 0.3766 & 0.0392 & 542 & 550 & 1.8839 & 0.1042 & 0.2993 & 0.4539 \\
\hline \multicolumn{9}{|c|}{ Urban } \\
\hline M (15-24) & 0.2143 & 0.0190 & 611 & 157 & 1.1428 & 0.0886 & 0.1770 & 0.2517 \\
\hline W (15-24) & 0.5816 & 0.0392 & 419 & 103 & 1.6243 & 0.0674 & 0.5044 & 0.6587 \\
\hline MM (15-29) & 0.0688 & 0.0151 & 497 & 84 & 1.3282 & 0.2193 & 0.0391 & 0.0986 \\
\hline MW (15-24) & 0.6770 & 0.0400 & 181 & 22 & 1.1477 & 0.0591 & 0.5982 & 0.7557 \\
\hline UM (15-24) & 0.2483 & 0.0215 & 432 & 126 & 1.0353 & 0.0868 & 0.2059 & 0.2907 \\
\hline UW (15-24) & 0.5167 & 0.0485 & 238 & 82 & 1.4950 & 0.0939 & 0.4211 & 0.6122 \\
\hline \multicolumn{9}{|c|}{ Rural } \\
\hline M (15-24) & 0.2263 & 0.0255 & 515 & 980 & 1.3804 & 0.1126 & 0.1761 & 0.2764 \\
\hline W (15-24) & 0.3367 & 0.0353 & 610 & 1,120 & 1.8429 & 0.1048 & 0.2672 & 0.4061 \\
\hline MM (15-29) & 0.1000 & 0.0182 & 505 & 910 & 1.3609 & 0.1819 & 0.0642 & 0.1358 \\
\hline MW (15-24) & 0.3317 & 0.0379 & 306 & 561 & 1.4053 & 0.1142 & 0.2571 & 0.4063 \\
\hline UM (15-24) & 0.2961 & 0.0330 & 308 & 592 & 1.2679 & 0.1116 & 0.2311 & 0.3612 \\
\hline UW (15-24) & 0.3520 & 0.0453 & 304 & 468 & 1.6503 & 0.1286 & 0.2629 & 0.4412 \\
\hline
\end{tabular}


Table B.2: (Cont'd)

\begin{tabular}{|c|c|c|c|c|c|c|c|c|}
\hline \multirow{2}{*}{$\begin{array}{l}\text { Variable/ } \\
\text { respondent } \\
\text { category }\end{array}$} & \multirow{2}{*}{$\begin{array}{l}\text { Value } \\
\text { (R) }\end{array}$} & \multirow{2}{*}{$\begin{array}{l}\text { Standard } \\
\text { error } \\
\text { (SE) }\end{array}$} & \multicolumn{2}{|c|}{ Number of cases } & \multirow{2}{*}{$\begin{array}{l}\text { Design } \\
\text { effect } \\
\text { (DEFT) }\end{array}$} & \multirow{2}{*}{$\begin{array}{c}\text { Relative } \\
\text { standard } \\
\text { error } \\
\text { (SE/R) }\end{array}$} & \multicolumn{2}{|c|}{ 95\% Confidence limits } \\
\hline & & & \begin{tabular}{|} 
Unweighted \\
(N)
\end{tabular} & $\begin{array}{l}\text { Weighted } \\
\text { (WN) }\end{array}$ & & & Lower & Upper \\
\hline \multicolumn{9}{|c|}{ Discussed friendships with father (young men and women whose father was alive at the time of interview) } \\
\hline \multicolumn{9}{|c|}{ Combined } \\
\hline M (15-24) & 0.5498 & 0.0206 & 1,704 & 1,755 & 1.7104 & 0.0375 & 0.5092 & 0.5904 \\
\hline W (15-24) & 0.2329 & 0.0119 & 4,973 & 4,907 & 1.9797 & 0.0510 & 0.2095 & 0.2563 \\
\hline MM (15-29) & 0.5589 & 0.0267 & 878 & 895 & 1.5926 & 0.0478 & 0.5063 & 0.6114 \\
\hline MW (15-24) & 0.1885 & 0.0143 & 2,012 & 2,019 & 1.6352 & 0.0757 & 0.1604 & 0.2166 \\
\hline UM (15-24) & 0.5607 & 0.0216 & 1,332 & 1,351 & 1.5845 & 0.0384 & 0.5183 & 0.6031 \\
\hline UW (15-24) & 0.2992 & 0.0148 & 2,961 & 2,981 & 1.7604 & 0.0495 & 0.2700 & 0.3284 \\
\hline \multicolumn{9}{|c|}{ Urban } \\
\hline M (15-24) & 0.5897 & 0.0235 & 890 & 240 & 1.4234 & 0.0398 & 0.5434 & 0.6359 \\
\hline W (15-24) & 0.4009 & 0.0166 & 2,298 & 567 & 1.6206 & 0.0413 & 0.3683 & 0.4335 \\
\hline MM (15-29) & 0.5185 & 0.0281 & 421 & 71 & 1.1508 & 0.0541 & 0.4632 & 0.5737 \\
\hline MW (15-24) & 0.3017 & 0.0167 & 973 & 122 & 1.1311 & 0.0552 & 0.2689 & 0.3345 \\
\hline UM (15-24) & 0.6001 & 0.0243 & 728 & 213 & 1.3397 & 0.0406 & 0.5521 & 0.6480 \\
\hline UW (15-24) & 0.4678 & 0.0210 & 1,325 & 452 & 1.5348 & 0.0450 & 0.4264 & 0.5092 \\
\hline \multicolumn{9}{|c|}{ Rural } \\
\hline M (15-24) & 0.5434 & 0.0236 & 814 & 1,514 & 1.3502 & 0.0434 & 0.4970 & 0.5899 \\
\hline W (15-24) & 0.2110 & 0.0129 & 2,675 & 4,340 & 1.6404 & 0.0613 & 0.1855 & 0.2365 \\
\hline MM (15-29) & 0.5624 & 0.0289 & 457 & 824 & 1.2437 & 0.0514 & 0.5055 & 0.6192 \\
\hline MW (15-24) & 0.1812 & 0.0151 & 1,039 & 1,897 & 1.2630 & 0.0833 & 0.1515 & 0.2110 \\
\hline UM (15-24) & 0.5533 & 0.0252 & 604 & 1,138 & 1.2438 & 0.0455 & 0.5038 & 0.6029 \\
\hline UW (15-24) & 0.2691 & 0.0165 & 1,636 & 2,528 & 1.5068 & 0.0614 & 0.2365 & 0.3016 \\
\hline \multicolumn{9}{|c|}{ Discussed friendships with mother (young men and women whose mother was alive at the time of interview) } \\
\hline \multicolumn{9}{|c|}{ Combined } \\
\hline M (15-24) & 0.5541 & 0.0210 & 1,819 & 1,844 & 1.8001 & 0.0379 & 0.5128 & 0.5955 \\
\hline W (15-24) & 0.6790 & 0.0150 & 5,172 & 5,108 & 2.3056 & 0.0220 & 0.6495 & 0.7084 \\
\hline MM (15-29) & 0.5353 & 0.0258 & 968 & 959 & 1.6098 & 0.0482 & 0.4845 & 0.5862 \\
\hline MW (15-24) & 0.6366 & 0.0190 & 2,121 & 2,117 & 1.8177 & 0.0298 & 0.5992 & 0.6740 \\
\hline UM (15-24) & 0.5720 & 0.0233 & 1,417 & 1,424 & 1.7689 & 0.0407 & 0.5263 & 0.6178 \\
\hline UW (15-24) & 0.7464 & 0.0159 & 3,051 & 3,060 & 2.0140 & 0.0213 & 0.7152 & 0.7777 \\
\hline \multicolumn{9}{|c|}{ Urban } \\
\hline M (15-24) & 0.6051 & 0.0232 & 971 & 262 & 1.4754 & 0.0383 & 0.5595 & 0.6506 \\
\hline W (15-24) & 0.7997 & 0.0153 & 2,409 & 595 & 1.8801 & 0.0192 & 0.7695 & 0.8299 \\
\hline MM (15-29) & 0.5280 & 0.0247 & 482 & 81 & 1.0837 & 0.0467 & 0.4795 & 0.5766 \\
\hline MW (15-24) & 0.7348 & 0.0222 & 1,031 & 129 & 1.6128 & 0.0302 & 0.6911 & 0.7785 \\
\hline UM (15-24) & 0.6185 & 0.0244 & 787 & 231 & 1.4076 & 0.0394 & 0.5705 & 0.6665 \\
\hline UW (15-24) & 0.8443 & 0.0141 & 1,378 & 471 & 1.4463 & 0.0167 & 0.8164 & 0.8721 \\
\hline \multicolumn{9}{|c|}{ Rural } \\
\hline M (15-24) & 0.5457 & 0.0241 & 848 & 1,582 & 1.4077 & 0.0441 & 0.4983 & 0.5931 \\
\hline W (15-24) & 0.6630 & 0.0167 & 2,763 & 4,513 & 1.8518 & 0.0251 & 0.6303 & 0.6958 \\
\hline MM (15-29) & 0.5360 & 0.0281 & 486 & 878 & 1.2412 & 0.0524 & 0.4807 & 0.5913 \\
\hline MW (15-24) & 0.6303 & 0.0201 & 1,090 & 1,988 & 1.3749 & 0.0319 & 0.5907 & 0.6698 \\
\hline UM (15-24) & 0.5630 & 0.0273 & 630 & 1,193 & 1.3798 & 0.0485 & 0.5093 & 0.6168 \\
\hline UW (15-24) & 0.7286 & 0.0185 & 1,673 & 2,589 & 1.6986 & 0.0254 & 0.6922 & 0.7650 \\
\hline
\end{tabular}


Table B.2: (Cont'd)

\begin{tabular}{|c|c|c|c|c|c|c|c|c|}
\hline \multirow{2}{*}{$\begin{array}{l}\text { Variable/ } \\
\text { respondent } \\
\text { category }\end{array}$} & \multirow{2}{*}{$\begin{array}{c}\text { Value } \\
\text { (R) }\end{array}$} & \multirow{2}{*}{$\begin{array}{l}\text { Standard } \\
\text { error } \\
\text { (SE) }\end{array}$} & \multicolumn{2}{|c|}{ Number of cases } & \multirow{2}{*}{$\begin{array}{c}\text { Design } \\
\text { effect } \\
\text { (DEFT) }\end{array}$} & \multirow{2}{*}{$\begin{array}{c}\text { Relative } \\
\text { standard } \\
\text { error } \\
(\mathrm{SE} / \mathrm{R})\end{array}$} & \multicolumn{2}{|c|}{ 95\% Confidence limits } \\
\hline & & & $\begin{array}{l}\text { Unweighted } \\
\text { (N) }\end{array}$ & $\begin{array}{l}\text { Weighted } \\
\text { (WN) }\end{array}$ & & & Lower & Upper \\
\hline \multicolumn{9}{|c|}{ Independently makes decisions about choice of friends, spending money and buying clothes for oneself (young men and women) } \\
\hline \multicolumn{9}{|c|}{ Combined } \\
\hline M (15-24) & 0.4605 & 0.0138 & 1,942 & 1,964 & 1.2157 & 0.0299 & 0.4334 & 0.4876 \\
\hline $\mathrm{W}(15-24)$ & 0.2447 & 0.0092 & 5,529 & 5,529 & 1.5869 & 0.0375 & 0.2266 & 0.2627 \\
\hline MM (15-29) & 0.7334 & 0.0180 & 1,115 & 1,115 & 1.3583 & 0.0245 & 0.6980 & 0.7688 \\
\hline MW (15-24) & 0.2473 & 0.0129 & 2,341 & 2,341 & 1.4412 & 0.0520 & 0.2220 & 0.2726 \\
\hline UM (15-24) & 0.3825 & 0.0163 & 1,492 & 1,492 & 1.2989 & 0.0427 & 0.3503 & 0.4147 \\
\hline UW (15-24) & 0.2273 & 0.0108 & 3,188 & 3,188 & 1.4570 & 0.0476 & 0.2060 & 0.2486 \\
\hline \multicolumn{9}{|c|}{ Urban } \\
\hline M (15-24) & 0.5571 & 0.0168 & 1,039 & 279 & 1.0909 & 0.0302 & 0.5240 & 0.5902 \\
\hline W (15-24) & 0.4102 & 0.0172 & 2,581 & 637 & 1.7752 & 0.0419 & 0.3763 & 0.4440 \\
\hline MM (15-29) & 0.7367 & 0.0232 & 547 & 92 & 1.2328 & 0.0315 & 0.6909 & 0.7824 \\
\hline MW (15-24) & 0.3477 & 0.0198 & 1,136 & 142 & 1.3973 & 0.0568 & 0.3088 & 0.3866 \\
\hline UM (15-24) & 0.5328 & 0.0178 & 833 & 245 & 1.0301 & 0.0334 & 0.4977 & 0.5679 \\
\hline UW (15-24) & 0.4552 & 0.0182 & 1,445 & 494 & 1.3907 & 0.0400 & 0.4194 & 0.4911 \\
\hline \multicolumn{9}{|c|}{ Rural } \\
\hline M (15-24) & 0.4445 & 0.0156 & 903 & 1,685 & 0.9451 & 0.0352 & 0.4137 & 0.4753 \\
\hline W (15-24) & 0.2231 & 0.0097 & 2,948 & 4,893 & 1.2663 & 0.0435 & 0.2040 & 0.2423 \\
\hline MM (15-29) & 0.7331 & 0.0195 & 568 & 1,023 & 1.0498 & 0.0266 & 0.6947 & 0.7715 \\
\hline MW (15-24) & 0.2409 & 0.0136 & 1,205 & 2,199 & 1.1030 & 0.0564 & 0.2141 & 0.2676 \\
\hline UM (15-24) & 0.3531 & 0.0186 & 659 & 1,247 & 0.9987 & 0.0527 & 0.3164 & 0.3897 \\
\hline UW (15-24) & 0.1855 & 0.0113 & 1,743 & 2,694 & 1.2160 & 0.0610 & 0.1632 & 0.2078 \\
\hline \multicolumn{9}{|c|}{ Can visit any place outside village or neighbourhood unescorted (young men and women) } \\
\hline \multicolumn{9}{|c|}{ Combined } \\
\hline W (15-24) & 0.0954 & 0.0071 & 5,529 & 5,529 & 1.7901 & 0.0742 & 0.0814 & 0.1093 \\
\hline MW (15-24) & 0.0979 & 0.0089 & 2,341 & 2,341 & 1.4529 & 0.0912 & 0.0803 & 0.1154 \\
\hline UM (15-24) & 0.8583 & 0.0146 & 1,492 & 1,492 & 1.6147 & 0.0170 & 0.8296 & 0.8870 \\
\hline UW (15-24) & 0.0827 & 0.0085 & 3,188 & 3,188 & 1.7392 & 0.1026 & 0.0660 & 0.0994 \\
\hline \multicolumn{9}{|c|}{ Urban } \\
\hline W (15-24) & 0.2003 & 0.0143 & 2,581 & 637 & 1.8118 & 0.0713 & 0.1722 & 0.2284 \\
\hline MW (15-24) & 0.1331 & 0.0131 & 1,136 & 142 & 1.3017 & 0.0986 & 0.1073 & 0.1589 \\
\hline UM (15-24) & 0.8686 & 0.0138 & 833 & 245 & 1.1755 & 0.0158 & 0.8415 & 0.8957 \\
\hline UW (15-24) & 0.2488 & 0.0193 & 1,445 & 494 & 1.6953 & 0.0775 & 0.2108 & 0.2867 \\
\hline \multicolumn{9}{|c|}{ Rural } \\
\hline W (15-24) & 0.0817 & 0.0075 & 2,948 & 4,893 & 1.4946 & 0.0923 & 0.0669 & 0.0966 \\
\hline MW (15-24) & 0.0956 & 0.0095 & 1,205 & 2,199 & 1.1170 & 0.0990 & 0.0769 & 0.1142 \\
\hline UM (15-24) & 0.8563 & 0.0172 & 659 & 1,247 & 1.2608 & 0.0201 & 0.8223 & 0.8902 \\
\hline UW (15-24) & 0.0523 & 0.0085 & 1,743 & 2,694 & 1.5960 & 0.1628 & 0.0355 & 0.0690 \\
\hline
\end{tabular}

Cont'd on next page... 
Table B.2: (Cont'd)

\begin{tabular}{|c|c|c|c|c|c|c|c|c|}
\hline \multirow{2}{*}{$\begin{array}{l}\text { Variable/ } \\
\text { respondent } \\
\text { category }\end{array}$} & \multirow{2}{*}{$\begin{array}{l}\text { Value } \\
\text { (R) }\end{array}$} & \multirow{2}{*}{$\begin{array}{l}\text { Standard } \\
\text { error } \\
\text { (SE) }\end{array}$} & \multicolumn{2}{|c|}{ Number of cases } & \multirow{2}{*}{$\begin{array}{l}\text { Design } \\
\text { effect } \\
\text { (DEFT) }\end{array}$} & \multirow{2}{*}{$\begin{array}{c}\text { Relative } \\
\text { standard } \\
\text { error } \\
(\mathrm{SE} / \mathrm{R})\end{array}$} & \multicolumn{2}{|c|}{ 95\% Confidence limits } \\
\hline & & & \begin{tabular}{|} 
Unweighted \\
(N)
\end{tabular} & $\begin{array}{l}\text { Weighted } \\
\text { (WN) }\end{array}$ & & & Lower & Upper \\
\hline \multicolumn{9}{|c|}{ Has savings of any amount (young men and women) } \\
\hline \multicolumn{9}{|c|}{ Combined } \\
\hline$M(15-24)$ & 0.1948 & 0.0169 & 1,942 & 1,964 & 1.8818 & 0.0868 & 0.1615 & 0.2281 \\
\hline W (15-24) & 0.4434 & 0.0183 & 5,529 & 5,529 & 2.7462 & 0.0414 & 0.4072 & 0.4795 \\
\hline MM (15-29) & 0.3035 & 0.0270 & 1,115 & 1,115 & 1.9579 & 0.0889 & 0.2504 & 0.3566 \\
\hline MW (15-24) & 0.4519 & 0.0228 & 2,341 & 2,341 & 2.2173 & 0.0505 & 0.4070 & 0.4968 \\
\hline UM (15-24) & 0.1644 & 0.0152 & 1,492 & 1,492 & 1.5802 & 0.0922 & 0.1346 & 0.1943 \\
\hline UW (15-24) & 0.4183 & 0.0179 & 3,188 & 3,188 & 2.0471 & 0.0428 & 0.3831 & 0.4535 \\
\hline \multicolumn{9}{|c|}{ Urban } \\
\hline M (15-24) & 0.2359 & 0.0214 & 1,039 & 279 & 1.6241 & 0.0907 & 0.1938 & 0.2781 \\
\hline W (15-24) & 0.5638 & 0.0232 & 2,581 & 637 & 2.3782 & 0.0412 & 0.5181 & 0.6095 \\
\hline MM (15-29) & 0.3267 & 0.0279 & 547 & 92 & 1.3905 & 0.0854 & 0.2717 & 0.3816 \\
\hline MW (15-24) & 0.5599 & 0.0267 & 1,136 & 142 & 1.8139 & 0.0477 & 0.5073 & 0.6125 \\
\hline UM (15-24) & 0.2269 & 0.0227 & 833 & 245 & 1.5663 & 0.1002 & 0.1821 & 0.2716 \\
\hline UW (15-24) & 0.5666 & 0.0250 & 1,445 & 494 & 1.9194 & 0.0442 & 0.5173 & 0.6159 \\
\hline \multicolumn{9}{|c|}{ Rural } \\
\hline M (15-24) & 0.1880 & 0.0195 & 903 & 1,685 & 1.4970 & 0.1036 & 0.1496 & 0.2263 \\
\hline W (15-24) & 0.4277 & 0.0204 & 2,948 & 4,893 & 2.2374 & 0.0477 & 0.3876 & 0.4678 \\
\hline MM (15-29) & 0.3014 & 0.0293 & 568 & 1,023 & 1.5195 & 0.0972 & 0.2437 & 0.3590 \\
\hline MW (15-24) & 0.4449 & 0.0242 & 1,205 & 2,199 & 1.6868 & 0.0543 & 0.3974 & 0.4925 \\
\hline UM (15-24) & 0.1522 & 0.0177 & 659 & 1,247 & 1.2618 & 0.1161 & 0.1174 & 0.1870 \\
\hline UW (15-24) & 0.3911 & 0.0206 & 1,743 & 2,694 & 1.7659 & 0.0528 & 0.3505 & 0.4318 \\
\hline \multicolumn{9}{|c|}{ Justified wife beating in at least one situation (young men and women) } \\
\hline \multicolumn{9}{|c|}{ Combined } \\
\hline M (15-24) & 0.4427 & 0.0189 & 1,942 & 1,964 & 1.6806 & 0.0428 & 0.4054 & 0.4800 \\
\hline W (15-24) & 0.5774 & 0.0156 & 5,529 & 5,529 & 2.3433 & 0.0270 & 0.5467 & 0.6080 \\
\hline MM (15-29) & 0.4654 & 0.0232 & 1,115 & 1,115 & 1.5555 & 0.0500 & 0.4196 & 0.5112 \\
\hline MW (15-24) & 0.6050 & 0.0190 & 2,341 & 2,341 & 1.8850 & 0.0315 & 0.5675 & 0.6425 \\
\hline UM (15-24) & 0.4418 & 0.0217 & 1,492 & 1,492 & 1.6876 & 0.0491 & 0.3990 & 0.4845 \\
\hline UW (15-24) & 0.5334 & 0.0162 & 3,188 & 3,188 & 1.8365 & 0.0304 & 0.5014 & 0.5653 \\
\hline \multicolumn{9}{|c|}{ Urban } \\
\hline M (15-24) & 0.3875 & 0.0185 & 1,039 & 279 & 1.2215 & 0.0477 & 0.3511 & 0.4239 \\
\hline W (15-24) & 0.4610 & 0.0267 & 2,581 & 637 & 2.7208 & 0.0579 & 0.4084 & 0.5135 \\
\hline MM (15-29) & 0.4263 & 0.0262 & 547 & 92 & 1.2376 & 0.0614 & 0.3747 & 0.4779 \\
\hline MW (15-24) & 0.5197 & 0.0268 & 1,136 & 142 & 1.8059 & 0.0515 & 0.4670 & 0.5724 \\
\hline UM (15-24) & 0.3864 & 0.0195 & 833 & 245 & 1.1536 & 0.0504 & 0.3480 & 0.4247 \\
\hline UW (15-24) & 0.4186 & 0.0300 & 1,445 & 494 & 2.3098 & 0.0716 & 0.3596 & 0.4776 \\
\hline \multicolumn{9}{|c|}{ Rural } \\
\hline M (15-24) & 0.4519 & 0.0218 & 903 & 1,685 & 1.3168 & 0.0483 & 0.4089 & 0.4948 \\
\hline W (15-24) & 0.5925 & 0.0171 & 2,948 & 4,893 & 1.8931 & 0.0289 & 0.5588 & 0.6263 \\
\hline MM (15-29) & 0.4689 & 0.0253 & 568 & 1,023 & 1.2056 & 0.0539 & 0.4192 & 0.5187 \\
\hline MW (15-24) & 0.6105 & 0.0202 & 1,205 & 2,199 & 1.4371 & 0.0331 & 0.5707 & 0.6502 \\
\hline UM (15-24) & 0.4526 & 0.0256 & 659 & 1,247 & 1.3178 & 0.0565 & 0.4023 & 0.5030 \\
\hline UW (15-24) & 0.5544 & 0.0182 & 1,743 & 2,694 & 1.5321 & 0.0329 & 0.5185 & 0.5904 \\
\hline
\end{tabular}


Table B.2: (Cont'd)

\begin{tabular}{|c|c|c|c|c|c|c|c|c|}
\hline \multirow{2}{*}{$\begin{array}{l}\text { Variable/ } \\
\text { respondent } \\
\text { category }\end{array}$} & \multirow{2}{*}{$\begin{array}{l}\text { Value } \\
\text { (R) }\end{array}$} & \multirow{2}{*}{$\begin{array}{l}\text { Standard } \\
\text { error } \\
\text { (SE) }\end{array}$} & \multicolumn{2}{|c|}{ Number of cases } & \multirow{2}{*}{$\begin{array}{l}\text { Design } \\
\text { effect } \\
\text { (DEFT) }\end{array}$} & \multirow{2}{*}{$\begin{array}{l}\text { Relative } \\
\text { standard } \\
\text { error } \\
(\mathrm{SE} / \mathrm{R})\end{array}$} & \multicolumn{2}{|c|}{ 95\% Confidence limits } \\
\hline & & & $\begin{array}{c}\text { Unweighted } \\
\text { (N) }\end{array}$ & $\begin{array}{l}\text { Weighted } \\
\text { (WN) }\end{array}$ & & & Lower & Upper \\
\hline \multicolumn{9}{|c|}{ Awareness of sex- and pregnancy-related matters (young men and women) } \\
\hline \multicolumn{9}{|c|}{ Combined } \\
\hline M (15-24) & 0.0366 & 0.0050 & 1,942 & 1,964 & 1.1820 & 0.1376 & 0.0267 & 0.0465 \\
\hline W (15-24) & 0.0211 & 0.0030 & 5,529 & 5,529 & 1.5527 & 0.1421 & 0.0152 & 0.0271 \\
\hline MM (15-29) & 0.0667 & 0.0099 & 1,115 & 1,115 & 1.3191 & 0.1479 & 0.0473 & 0.0861 \\
\hline MW (15-24) & 0.0270 & 0.0046 & 2,341 & 2,341 & 1.3622 & 0.1691 & 0.0180 & 0.0360 \\
\hline UM (15-24) & 0.0282 & 0.0045 & 1,492 & 1,492 & 1.0495 & 0.1595 & 0.0194 & 0.0371 \\
\hline UW (15-24) & 0.0082 & 0.0018 & 3,188 & 3,188 & 1.1007 & 0.2150 & 0.0047 & 0.0116 \\
\hline \multicolumn{9}{|c|}{ Urban } \\
\hline M (15-24) & 0.0613 & 0.0096 & 1,039 & 279 & 1.2849 & 0.1561 & 0.0424 & 0.0801 \\
\hline W (15-24) & 0.0485 & 0.0052 & 2,581 & 637 & 1.2261 & 0.1069 & 0.0383 & 0.0587 \\
\hline MM (15-29) & 0.1343 & 0.0154 & 547 & 92 & 1.0528 & 0.1144 & 0.1041 & 0.1646 \\
\hline MW (15-24) & 0.0808 & 0.0098 & 1,136 & 142 & 1.2165 & 0.1218 & 0.0614 & 0.1002 \\
\hline UM (15-24) & 0.0551 & 0.0102 & 833 & 245 & 1.2892 & 0.1851 & 0.0350 & 0.0751 \\
\hline UW (15-24) & 0.0253 & 0.0050 & 1,445 & 494 & 1.2161 & 0.1987 & 0.0154 & 0.0352 \\
\hline \multicolumn{9}{|c|}{ Rural } \\
\hline M (15-24) & 0.0325 & 0.0056 & 903 & 1,685 & 0.9498 & 0.1725 & 0.0215 & 0.0436 \\
\hline W (15-24) & 0.0176 & 0.0033 & 2,948 & 4,893 & 1.3689 & 0.1885 & 0.0111 & 0.0241 \\
\hline MM (15-29) & 0.0606 & 0.0106 & 568 & 1,023 & 1.0586 & 0.1751 & 0.0397 & 0.0815 \\
\hline MW (15-24) & 0.0235 & 0.0048 & 1,205 & 2,199 & 1.0988 & 0.2041 & 0.0141 & 0.0329 \\
\hline UM (15-24) & 0.0230 & 0.0049 & 659 & 1,247 & 0.8448 & 0.2148 & 0.0133 & 0.0327 \\
\hline UW (15-24) & 0.0050 & 0.0018 & 1,743 & 2,694 & 1.0582 & 0.3570 & 0.0015 & 0.0085 \\
\hline \multicolumn{9}{|c|}{ Correct specific knowledge of at least one contraceptive method (young men and women) } \\
\hline \multicolumn{9}{|c|}{ Combined } \\
\hline M (15-24) & 0.6507 & 0.0232 & 1,942 & 1,964 & 2.1437 & 0.0356 & 0.6051 & 0.6964 \\
\hline W (15-24) & 0.6221 & 0.0128 & 5,529 & 5,529 & 1.9680 & 0.0206 & 0.5969 & 0.6474 \\
\hline MM (15-29) & 0.7711 & 0.0237 & 1,115 & 1,115 & 1.8836 & 0.0308 & 0.7244 & 0.8177 \\
\hline MW (15-24) & 0.7156 & 0.0166 & 2,341 & 2,341 & 1.7834 & 0.0232 & 0.6828 & 0.7483 \\
\hline UM (15-24) & 0.6161 & 0.0253 & 1,492 & 1,492 & 2.0105 & 0.0411 & 0.5663 & 0.6660 \\
\hline UW (15-24) & 0.4375 & 0.0166 & 3,188 & 3,188 & 1.8850 & 0.0379 & 0.4049 & 0.4701 \\
\hline \multicolumn{9}{|c|}{ Urban } \\
\hline M (15-24) & 0.7167 & 0.0205 & 1,039 & 279 & 1.4631 & 0.0286 & 0.6764 & 0.7570 \\
\hline W (15-24) & 0.7136 & 0.0131 & 2,581 & 637 & 1.4698 & 0.0183 & 0.6878 & 0.7393 \\
\hline MM (15-29) & 0.8741 & 0.0165 & 547 & 92 & 1.1635 & 0.0189 & 0.8415 & 0.9066 \\
\hline MW (15-24) & 0.8527 & 0.0149 & 1,136 & 142 & 1.4205 & 0.0175 & 0.8232 & 0.8821 \\
\hline UM (15-24) & 0.6942 & 0.0215 & 833 & 245 & 1.3472 & 0.0310 & 0.6519 & 0.7366 \\
\hline UW (15-24) & 0.6133 & 0.0205 & 1,445 & 494 & 1.6013 & 0.0335 & 0.5729 & 0.6537 \\
\hline \multicolumn{9}{|c|}{ Rural } \\
\hline M (15-24) & 0.6398 & 0.0268 & 903 & 1,685 & 1.6789 & 0.0419 & 0.5870 & 0.6926 \\
\hline W (15-24) & 0.6102 & 0.0143 & 2,948 & 4,893 & 1.5915 & 0.0234 & 0.5821 & 0.6384 \\
\hline MM (15-29) & 0.7618 & 0.0258 & 568 & 1,023 & 1.4397 & 0.0338 & 0.7111 & 0.8125 \\
\hline MW (15-24) & 0.7067 & 0.0175 & 1,205 & 2,199 & 1.3356 & 0.0248 & 0.6722 & 0.7412 \\
\hline UM (15-24) & 0.6008 & 0.0301 & 659 & 1,247 & 1.5741 & 0.0500 & 0.5417 & 0.6600 \\
\hline UW (15-24) & 0.4052 & 0.0190 & 1,743 & 2,694 & 1.6145 & 0.0469 & 0.3678 & 0.4426 \\
\hline
\end{tabular}

Cont'd on next page... 
Table B.2: (Cont'd)

\begin{tabular}{|c|c|c|c|c|c|c|c|c|}
\hline \multirow{2}{*}{$\begin{array}{l}\text { Variable/ } \\
\text { respondent } \\
\text { category }\end{array}$} & \multirow{2}{*}{$\begin{array}{l}\text { Value } \\
\text { (R) }\end{array}$} & \multirow{2}{*}{$\begin{array}{c}\text { Standard } \\
\text { error } \\
\text { (SE) }\end{array}$} & \multicolumn{2}{|c|}{ Number of cases } & \multirow{2}{*}{$\begin{array}{c}\text { Design } \\
\text { effect } \\
\text { (DEFT) }\end{array}$} & \multirow{2}{*}{$\begin{array}{c}\text { Relative } \\
\text { standard } \\
\text { error } \\
(\mathrm{SE} / \mathrm{R})\end{array}$} & \multicolumn{2}{|c|}{ 95\% Confidence limit } \\
\hline & & & $\begin{array}{c}\text { Unweighted } \\
(\mathbf{N})\end{array}$ & $\begin{array}{l}\text { Weighted } \\
\text { (WN) }\end{array}$ & & & Lower & Upper \\
\hline \multicolumn{9}{|c|}{ Correct specific knowledge of condoms (young men and women) } \\
\hline \multicolumn{9}{|c|}{ Combined } \\
\hline M (15-24) & 0.6208 & 0.0240 & 1,942 & 1,964 & 2.1808 & 0.0387 & 0.5735 & 0.6681 \\
\hline W (15-24) & 0.2988 & 0.0143 & 5,529 & 5,529 & 2.3172 & 0.0477 & 0.2707 & 0.3269 \\
\hline MM (15-29) & 0.7351 & 0.0246 & 1,115 & 1,115 & 1.8643 & 0.0335 & 0.6866 & 0.7836 \\
\hline MW (15-24) & 0.3635 & 0.0201 & 2,341 & 2,341 & 2.0244 & 0.0554 & 0.3239 & 0.4032 \\
\hline UM (15-24) & 0.5870 & 0.0257 & 1,492 & 1,492 & 2.0193 & 0.0439 & 0.5363 & 0.6377 \\
\hline UW (15-24) & 0.1670 & 0.0126 & 3,188 & 3,188 & 1.9041 & 0.0753 & 0.1423 & 0.1918 \\
\hline \multicolumn{9}{|c|}{ Urban } \\
\hline M (15-24) & 0.7019 & 0.0211 & 1,039 & 279 & 1.4844 & 0.0300 & 0.6604 & 0.7433 \\
\hline W (15-24) & 0.4203 & 0.0179 & 2,581 & 637 & 1.8410 & 0.0426 & 0.3851 & 0.4556 \\
\hline MM (15-29) & 0.8641 & 0.0174 & 547 & 92 & 1.1839 & 0.0201 & 0.8299 & 0.8983 \\
\hline MW (15-24) & 0.5883 & 0.0235 & 1,136 & 142 & 1.6121 & 0.0400 & 0.5420 & 0.6347 \\
\hline UM (15-24) & 0.6796 & 0.0221 & 833 & 245 & 1.3635 & 0.0325 & 0.6362 & 0.7230 \\
\hline UW (15-24) & 0.2992 & 0.0219 & 1,445 & 494 & 1.8179 & 0.0732 & 0.2561 & 0.3423 \\
\hline \multicolumn{9}{|c|}{ Rural } \\
\hline M (15-24) & 0.6073 & 0.0278 & 903 & 1,685 & 1.7089 & 0.0458 & 0.5526 & 0.6621 \\
\hline W (15-24) & 0.2830 & 0.0158 & 2,948 & 4,893 & 1.9094 & 0.0560 & 0.2518 & 0.3142 \\
\hline MM (15-29) & 0.7235 & 0.0268 & 568 & 1,023 & 1.4251 & 0.0370 & 0.6708 & 0.7762 \\
\hline MW (15-24) & 0.3490 & 0.0212 & 1,205 & 2,199 & 1.5405 & 0.0606 & 0.3073 & 0.3906 \\
\hline UM (15-24) & 0.5689 & 0.0305 & 659 & 1,247 & 1.5821 & 0.0537 & 0.5087 & 0.6290 \\
\hline UW (15-24) & 0.1428 & 0.0142 & 1,743 & 2,694 & 1.6984 & 0.0997 & 0.1147 & 0.1708 \\
\hline \multicolumn{9}{|c|}{ Ever heard of HIV/AIDS (young men and women) } \\
\hline \multicolumn{9}{|c|}{ Combined } \\
\hline M (15-24) & 0.8732 & 0.0137 & 1,942 & 1,964 & 1.8184 & 0.0157 & 0.8461 & 0.9002 \\
\hline W (15-24) & 0.4694 & 0.0171 & 5,529 & 5,529 & 2.5524 & 0.0365 & 0.4356 & 0.5031 \\
\hline MM (15-29) & 0.8647 & 0.0175 & 1,115 & 1,115 & 1.7105 & 0.0203 & 0.8302 & 0.8992 \\
\hline MW (15-24) & 0.4265 & 0.0192 & 2,341 & 2,341 & 1.8747 & 0.0449 & 0.3888 & 0.4643 \\
\hline UM (15-24) & 0.8767 & 0.0154 & 1,492 & 1,492 & 1.8055 & 0.0175 & 0.8464 & 0.9070 \\
\hline UW (15-24) & 0.5275 & 0.0207 & 3,188 & 3,188 & 2.3454 & 0.0393 & 0.4866 & 0.5683 \\
\hline \multicolumn{9}{|c|}{ Urban } \\
\hline M (15-24) & 0.9584 & 0.0090 & 1,039 & 279 & 1.4473 & 0.0094 & 0.9407 & 0.9760 \\
\hline W (15-24) & 0.7928 & 0.0208 & 2,581 & 637 & 2.6082 & 0.0262 & 0.7519 & 0.8338 \\
\hline MM (15-29) & 0.9516 & 0.0114 & 547 & 92 & 1.2461 & 0.0120 & 0.9291 & 0.9742 \\
\hline MW (15-24) & 0.7093 & 0.0259 & 1,136 & 142 & 1.9232 & 0.0365 & 0.6583 & 0.7604 \\
\hline UM (15-24) & 0.9606 & 0.0087 & 833 & 245 & 1.2908 & 0.0091 & 0.9434 & 0.9777 \\
\hline UW (15-24) & 0.8530 & 0.0193 & 1,445 & 494 & 2.0660 & 0.0226 & 0.8151 & 0.8909 \\
\hline \multicolumn{9}{|c|}{ Rural } \\
\hline M (15-24) & 0.8591 & 0.0160 & 903 & 1,685 & 1.3788 & 0.0186 & 0.8276 & 0.8905 \\
\hline W (15-24) & 0.4273 & 0.0183 & 2,948 & 4,893 & 2.0125 & 0.0429 & 0.3912 & 0.4634 \\
\hline MM (15-29) & 0.8569 & 0.0192 & 568 & 1,023 & 1.3032 & 0.0224 & 0.8192 & 0.8946 \\
\hline MW (15-24) & 0.4082 & 0.0200 & 1,205 & 2,199 & 1.4126 & 0.0490 & 0.3688 & 0.4476 \\
\hline UM (15-24) & 0.8603 & 0.0183 & 659 & 1,247 & 1.3565 & 0.0213 & 0.8242 & 0.8964 \\
\hline UW (15-24) & 0.4678 & 0.0238 & 1,743 & 2,694 & 1.9898 & 0.0509 & 0.4209 & 0.5146 \\
\hline
\end{tabular}


Table B.2: (Cont'd)

\begin{tabular}{|c|c|c|c|c|c|c|c|c|}
\hline \multirow{2}{*}{$\begin{array}{l}\text { Variable/ } \\
\text { respondent } \\
\text { category }\end{array}$} & \multirow{2}{*}{$\begin{array}{l}\text { Value } \\
\text { (R) }\end{array}$} & \multirow{2}{*}{$\begin{array}{l}\text { Standard } \\
\text { error } \\
\text { (SE) }\end{array}$} & \multicolumn{2}{|c|}{ Number of cases } & \multirow{2}{*}{$\begin{array}{l}\text { Design } \\
\text { effect } \\
\text { (DEFT) }\end{array}$} & \multirow{2}{*}{$\begin{array}{c}\text { Relative } \\
\text { standard } \\
\text { error } \\
(\mathrm{SE} / \mathrm{R})\end{array}$} & \multicolumn{2}{|c|}{ 95\% Confidence limits } \\
\hline & & & \begin{tabular}{|} 
Unweighted \\
(N)
\end{tabular} & $\begin{array}{l}\text { Weighted } \\
\text { (WN) }\end{array}$ & & & Lower & Upper \\
\hline \multicolumn{9}{|c|}{ Comprehensive knowledge of HIV/AIDS (young men and women) } \\
\hline \multicolumn{9}{|c|}{ Combined } \\
\hline M (15-24) & 0.2778 & 0.0149 & 1,942 & 1,964 & 1.4607 & 0.0535 & 0.2486 & 0.3070 \\
\hline W (15-24) & 0.1538 & 0.0094 & 5,529 & 5,529 & 1.9299 & 0.0609 & 0.1353 & 0.1722 \\
\hline MM (15-29) & 0.2700 & 0.0196 & 1,115 & 1,115 & 1.4749 & 0.0727 & 0.2314 & 0.3086 \\
\hline MW (15-24) & 0.1318 & 0.0101 & 2,341 & 2,341 & 1.4417 & 0.0765 & 0.1120 & 0.1517 \\
\hline UM (15-24) & 0.2759 & 0.0172 & 1,492 & 1,492 & 1.4856 & 0.0623 & 0.2420 & 0.3097 \\
\hline UW (15-24) & 0.1800 & 0.0124 & 3,188 & 3,188 & 1.8238 & 0.0690 & 0.1555 & 0.2044 \\
\hline \multicolumn{9}{|c|}{ Urban } \\
\hline M (15-24) & 0.4192 & 0.0191 & 1,039 & 279 & 1.2488 & 0.0456 & 0.3816 & 0.4569 \\
\hline W (15-24) & 0.3686 & 0.0202 & 2,581 & 637 & 2.1263 & 0.0548 & 0.3288 & 0.4083 \\
\hline MM (15-29) & 0.4068 & 0.0240 & 547 & 92 & 1.1407 & 0.0589 & 0.3596 & 0.4540 \\
\hline MW (15-24) & 0.3231 & 0.0241 & 1,136 & 142 & 1.7365 & 0.0746 & 0.2757 & 0.3706 \\
\hline UM (15-24) & 0.4213 & 0.0210 & 833 & 245 & 1.2239 & 0.0497 & 0.3800 & 0.4625 \\
\hline UW (15-24) & 0.4014 & 0.0225 & 1,445 & 494 & 1.7476 & 0.0562 & 0.3570 & 0.4458 \\
\hline \multicolumn{9}{|c|}{ Rural } \\
\hline M (15-24) & 0.2543 & 0.0168 & 903 & 1,685 & 1.1584 & 0.0660 & 0.2213 & 0.2874 \\
\hline W (15-24) & 0.1258 & 0.0096 & 2,948 & 4,893 & 1.5739 & 0.0764 & 0.1069 & 0.1447 \\
\hline MM (15-29) & 0.2577 & 0.0212 & 568 & 1,023 & 1.1543 & 0.0823 & 0.2159 & 0.2994 \\
\hline MW (15-24) & 0.1194 & 0.0104 & 1,205 & 2,199 & 1.1120 & 0.0870 & 0.0990 & 0.1399 \\
\hline UM (15-24) & 0.2474 & 0.0198 & 659 & 1,247 & 1.1770 & 0.0800 & 0.2084 & 0.2864 \\
\hline UW (15-24) & 0.1393 & 0.0134 & 1,743 & 2,694 & 1.6152 & 0.0962 & 0.1130 & 0.1657 \\
\hline \multicolumn{9}{|c|}{ Ever heard of STIs other than HIV (young men and women) } \\
\hline \multicolumn{9}{|c|}{ Combined } \\
\hline M (15-24) & 0.1140 & 0.0118 & 1,942 & 1,964 & 1.6367 & 0.1036 & 0.0908 & 0.1373 \\
\hline W (15-24) & 0.1157 & 0.0110 & 5,529 & 5,529 & 2.5580 & 0.0951 & 0.0940 & 0.1373 \\
\hline MM (15-29) & 0.1894 & 0.0210 & 1,115 & 1,115 & 1.7914 & 0.1110 & 0.1480 & 0.2308 \\
\hline MW (15-24) & 0.1576 & 0.0163 & 2,341 & 2,341 & 2.1608 & 0.1033 & 0.1256 & 0.1897 \\
\hline UM (15-24) & 0.1057 & 0.0120 & 1,492 & 1,492 & 1.5073 & 0.1136 & 0.0821 & 0.1293 \\
\hline UW (15-24) & 0.0367 & 0.0043 & 3,188 & 3,188 & 1.2988 & 0.1178 & 0.0282 & 0.0452 \\
\hline \multicolumn{9}{|c|}{ Urban } \\
\hline M (15-24) & 0.1392 & 0.0117 & 1,039 & 279 & 1.0864 & 0.0839 & 0.1162 & 0.1622 \\
\hline W (15-24) & 0.1004 & 0.0079 & 2,581 & 637 & 1.3307 & 0.0784 & 0.0849 & 0.1159 \\
\hline MM (15-29) & 0.2421 & 0.0218 & 547 & 92 & 1.1873 & 0.0899 & 0.1993 & 0.2850 \\
\hline MW (15-24) & 0.1436 & 0.0136 & 1,136 & 142 & 1.3078 & 0.0948 & 0.1168 & 0.1704 \\
\hline UM (15-24) & 0.1283 & 0.0122 & 833 & 245 & 1.0495 & 0.0948 & 0.1043 & 0.1523 \\
\hline UW (15-24) & 0.0692 & 0.0072 & 1,445 & 494 & 1.0784 & 0.1041 & 0.0550 & 0.0834 \\
\hline \multicolumn{9}{|c|}{ Rural } \\
\hline M (15-24) & 0.1098 & 0.0136 & 903 & 1,685 & 1.3080 & 0.1240 & 0.0830 & 0.1367 \\
\hline W (15-24) & 0.1176 & 0.0124 & 2,948 & 4,893 & 2.0882 & 0.1053 & 0.0932 & 0.1420 \\
\hline MM (15-29) & 0.1847 & 0.0229 & 568 & 1,023 & 1.4024 & 0.1238 & 0.1397 & 0.2297 \\
\hline MW (15-24) & 0.1585 & 0.0173 & 1,205 & 2,199 & 1.6444 & 0.1092 & 0.1245 & 0.1926 \\
\hline UM (15-24) & 0.1012 & 0.0141 & 659 & 1,247 & 1.2022 & 0.1396 & 0.0734 & 0.1291 \\
\hline UW (15-24) & 0.0308 & 0.0049 & 1,743 & 2,694 & 1.1905 & 0.1601 & 0.0211 & 0.0405 \\
\hline
\end{tabular}


Table B.2: (Cont'd)

\begin{tabular}{|c|c|c|c|c|c|c|c|c|}
\hline \multirow{2}{*}{$\begin{array}{l}\text { Variable/ } \\
\text { respondent } \\
\text { category }\end{array}$} & \multirow{2}{*}{$\begin{array}{l}\text { Value } \\
\text { (R) }\end{array}$} & \multirow{2}{*}{$\begin{array}{l}\text { Standard } \\
\text { error } \\
\text { (SE) }\end{array}$} & \multicolumn{2}{|c|}{ Number of cases } & \multirow{2}{*}{$\begin{array}{c}\text { Design } \\
\text { effect } \\
\text { (DEFT) }\end{array}$} & \multirow{2}{*}{$\begin{array}{c}\text { Relative } \\
\text { standard } \\
\text { error } \\
(\mathrm{SE} / \mathrm{R})\end{array}$} & \multicolumn{2}{|c|}{ 95\% Confidence limits } \\
\hline & & & $\begin{array}{l}\text { Unweighted } \\
\text { (N) }\end{array}$ & $\begin{array}{l}\text { Weighted } \\
\text { (WN) }\end{array}$ & & & Lower & Upper \\
\hline \multicolumn{9}{|c|}{ Correct knowledge of the conditions under which abortion is legal (young men and women) } \\
\hline \multicolumn{9}{|c|}{ Combined } \\
\hline M (15-24) & 0.0636 & 0.0076 & 1,942 & 1,964 & 1.3765 & 0.1199 & 0.0486 & 0.0786 \\
\hline W (15-24) & 0.0276 & 0.0035 & 5,529 & 5,529 & 1.5919 & 0.1270 & 0.0207 & 0.0346 \\
\hline MM (15-29) & 0.0656 & 0.0100 & 1,115 & 1,115 & 1.3465 & 0.1523 & 0.0459 & 0.0853 \\
\hline MW (15-24) & 0.0261 & 0.0049 & 2,341 & 2,341 & 1.4709 & 0.1856 & 0.0166 & 0.0357 \\
\hline UM (15-24) & 0.0587 & 0.0081 & 1,492 & 1,492 & 1.3252 & 0.1375 & 0.0428 & 0.0745 \\
\hline UW (15-24) & 0.0297 & 0.0043 & 3,188 & 3,188 & 1.4414 & 0.1459 & 0.0212 & 0.0382 \\
\hline \multicolumn{9}{|c|}{ Urban } \\
\hline M (15-24) & 0.0734 & 0.0119 & 1,039 & 279 & 1.4688 & 0.1620 & 0.0500 & 0.0968 \\
\hline W (15-24) & 0.0384 & 0.0047 & 2,581 & 637 & 1.2476 & 0.1229 & 0.0291 & 0.0477 \\
\hline MM (15-29) & 0.0692 & 0.0135 & 547 & 92 & 1.2436 & 0.1951 & 0.0426 & 0.0958 \\
\hline MW (15-24) & 0.0373 & 0.0058 & 1,136 & 142 & 1.0316 & 0.1556 & 0.0258 & 0.0487 \\
\hline UM (15-24) & 0.0727 & 0.0123 & 833 & 245 & 1.3672 & 0.1692 & 0.0485 & 0.0970 \\
\hline UW (15-24) & 0.0392 & 0.0063 & 1,445 & 494 & 1.2417 & 0.1618 & 0.0267 & 0.0517 \\
\hline \multicolumn{9}{|c|}{ Rural } \\
\hline M (15-24) & 0.0620 & 0.0087 & 903 & 1,685 & 1.0788 & 0.1398 & 0.0449 & 0.0790 \\
\hline W (15-24) & 0.0262 & 0.0039 & 2,948 & 4,893 & 1.3268 & 0.1489 & 0.0186 & 0.0339 \\
\hline MM (15-29) & 0.0653 & 0.0108 & 568 & 1,023 & 1.0433 & 0.1658 & 0.0440 & 0.0866 \\
\hline MW (15-24) & 0.0254 & 0.0051 & 1,205 & 2,199 & 1.1349 & 0.2025 & 0.0153 & 0.0356 \\
\hline UM (15-24) & 0.0559 & 0.0093 & 659 & 1,247 & 1.0432 & 0.1672 & 0.0375 & 0.0743 \\
\hline UW (15-24) & 0.0280 & 0.0050 & 1,743 & 2,694 & 1.2628 & 0.1784 & 0.0182 & 0.0378 \\
\hline \multicolumn{9}{|c|}{ Ever received family life or sex education (young men and women) } \\
\hline \multicolumn{9}{|c|}{ Combined } \\
\hline$M(15-24)$ & 0.0717 & 0.0073 & 1,942 & 1,964 & 1.2481 & 0.1019 & 0.0573 & 0.0861 \\
\hline W (15-24) & 0.0250 & 0.0030 & 5,529 & 5,529 & 1.4329 & 0.1203 & 0.0191 & 0.0309 \\
\hline MM (15-29) & 0.0386 & 0.0074 & 1,115 & 1,115 & 1.2834 & 0.1919 & 0.0240 & 0.0532 \\
\hline MW (15-24) & 0.0092 & 0.0027 & 2,341 & 2,341 & 1.3597 & 0.2921 & 0.0039 & 0.0144 \\
\hline UM (15-24) & 0.0829 & 0.0088 & 1,492 & 1,492 & 1.2319 & 0.1061 & 0.0655 & 0.1002 \\
\hline UW (15-24) & 0.0508 & 0.0056 & 3,188 & 3,188 & 1.4439 & 0.1106 & 0.0397 & 0.0618 \\
\hline \multicolumn{9}{|c|}{ Urban } \\
\hline M (15-24) & 0.1065 & 0.0115 & 1,039 & 279 & 1.2033 & 0.1082 & 0.0838 & 0.1292 \\
\hline W (15-24) & 0.0843 & 0.0098 & 2,581 & 637 & 1.7900 & 0.1161 & 0.0650 & 0.1036 \\
\hline MM (15-29) & 0.0567 & 0.0105 & 547 & 92 & 1.0632 & 0.1855 & 0.0360 & 0.0775 \\
\hline MW (15-24) & 0.0307 & 0.0063 & 1,136 & 142 & 1.2330 & 0.2058 & 0.0182 & 0.0431 \\
\hline UM (15-24) & 0.1135 & 0.0128 & 833 & 245 & 1.1618 & 0.1126 & 0.0883 & 0.1386 \\
\hline UW (15-24) & 0.1230 & 0.0138 & 1,445 & 494 & 1.6003 & 0.1125 & 0.0958 & 0.1502 \\
\hline \multicolumn{9}{|c|}{ Rural } \\
\hline M (15-24) & 0.0659 & 0.0082 & 903 & 1,685 & 0.9922 & 0.1243 & 0.0498 & 0.0821 \\
\hline W (15-24) & 0.0173 & 0.0030 & 2,948 & 4,893 & 1.2585 & 0.1747 & 0.0113 & 0.0232 \\
\hline MM (15-29) & 0.0370 & 0.0080 & 568 & 1,023 & 1.0103 & 0.2166 & 0.0212 & 0.0527 \\
\hline MW (15-24) & 0.0078 & 0.0028 & 1,205 & 2,199 & 1.1122 & 0.3620 & 0.0022 & 0.0133 \\
\hline UM (15-24) & 0.0768 & 0.0101 & 659 & 1,247 & 0.9729 & 0.1315 & 0.0570 & 0.0967 \\
\hline UW (15-24) & 0.0375 & 0.0059 & 1,743 & 2,694 & 1.3033 & 0.1581 & 0.0258 & 0.0492 \\
\hline
\end{tabular}


Table B.2: (Cont'd)

\begin{tabular}{|c|c|c|c|c|c|c|c|c|}
\hline \multirow{2}{*}{$\begin{array}{l}\text { Variable/ } \\
\text { respondent } \\
\text { category }\end{array}$} & \multirow{2}{*}{$\begin{array}{l}\text { Value } \\
\text { (R) }\end{array}$} & \multirow{2}{*}{$\begin{array}{c}\text { Standard } \\
\text { error } \\
\text { (SE) }\end{array}$} & \multicolumn{2}{|c|}{ Number of cases } & \multirow{2}{*}{$\begin{array}{l}\text { Design } \\
\text { effect } \\
\text { (DEFT) }\end{array}$} & \multirow{2}{*}{$\begin{array}{c}\text { Relative } \\
\text { standard } \\
\text { error } \\
(\mathrm{SE} / \mathrm{R})\end{array}$} & \multicolumn{2}{|c|}{ 95\% Confidence limits } \\
\hline & & & \begin{tabular}{|} 
Unweighted \\
(N)
\end{tabular} & $\begin{array}{l}\text { Weighted } \\
\text { (WN) }\end{array}$ & & & Lower & Upper \\
\hline \multicolumn{9}{|c|}{ Ever had an opposite-sex romantic partner (young men and women) } \\
\hline \multicolumn{9}{|c|}{ Combined } \\
\hline M (15-24) & 0.1700 & 0.0119 & 1,942 & 1,964 & 1.4008 & 0.0703 & 0.1465 & 0.1935 \\
\hline W (15-24) & 0.0493 & 0.0047 & 5,529 & 5,529 & 1.6176 & 0.0955 & 0.0401 & 0.0586 \\
\hline MM (15-29) & 0.1677 & 0.0120 & 1,115 & 1,115 & 1.0727 & 0.0716 & 0.1441 & 0.1913 \\
\hline MW (15-24) & 0.0363 & 0.0055 & 2,341 & 2,341 & 1.4241 & 0.1517 & 0.0254 & 0.0471 \\
\hline UM (15-24) & 0.1596 & 0.0122 & 1,492 & 1,492 & 1.2848 & 0.0763 & 0.1357 & 0.1836 \\
\hline UW (15-24) & 0.0712 & 0.0061 & 3,188 & 3,188 & 1.3353 & 0.0854 & 0.0592 & 0.0832 \\
\hline \multicolumn{9}{|c|}{ Urban } \\
\hline M (15-24) & 0.2107 & 0.0141 & 1,039 & 279 & 1.1125 & 0.0668 & 0.1830 & 0.2384 \\
\hline W (15-24) & 0.0925 & 0.0065 & 2,581 & 637 & 1.1460 & 0.0707 & 0.0796 & 0.1054 \\
\hline MM (15-29) & 0.1954 & 0.0165 & 547 & 92 & 0.9743 & 0.0846 & 0.1629 & 0.2280 \\
\hline MW (15-24) & 0.0898 & 0.0089 & 1,136 & 142 & 1.0447 & 0.0987 & 0.0723 & 0.1072 \\
\hline UM (15-24) & 0.2061 & 0.0147 & 833 & 245 & 1.0461 & 0.0712 & 0.1772 & 0.2350 \\
\hline UW (15-24) & 0.0945 & 0.0082 & 1,445 & 494 & 1.0666 & 0.0869 & 0.0783 & 0.1106 \\
\hline \multicolumn{9}{|c|}{ Rural } \\
\hline M (15-24) & 0.1633 & 0.0137 & 903 & 1,685 & 1.1130 & 0.0839 & 0.1363 & 0.1902 \\
\hline W (15-24) & 0.0437 & 0.0052 & 2,948 & 4,893 & 1.3717 & 0.1182 & 0.0336 & 0.0539 \\
\hline MM (15-29) & 0.1652 & 0.0130 & 568 & 1,023 & 0.8335 & 0.0787 & 0.1396 & 0.1908 \\
\hline MW (15-24) & 0.0328 & 0.0058 & 1,205 & 2,199 & 1.1242 & 0.1759 & 0.0215 & 0.0442 \\
\hline UM (15-24) & 0.1505 & 0.0143 & 659 & 1,247 & 1.0223 & 0.0947 & 0.1225 & 0.1786 \\
\hline UW (15-24) & 0.0669 & 0.0070 & 1,743 & 2,694 & 1.1772 & 0.1053 & 0.0530 & 0.0808 \\
\hline \multicolumn{9}{|c|}{ Ever had sex with an opposite-sex romantic partner (young men and women) } \\
\hline \multicolumn{9}{|c|}{ Combined } \\
\hline M (15-24) & 0.0669 & 0.0083 & 1,942 & 1,964 & 1.4697 & 0.1245 & 0.0505 & 0.0834 \\
\hline W (15-24) & 0.0139 & 0.0020 & 5,529 & 5,529 & 1.2482 & 0.1415 & 0.0100 & 0.0178 \\
\hline MM (15-29) & 0.0808 & 0.0106 & 1,115 & 1,115 & 1.2936 & 0.1308 & 0.0600 & 0.1016 \\
\hline MW (15-24) & 0.0116 & 0.0025 & 2,341 & 2,341 & 1.1284 & 0.2151 & 0.0067 & 0.0165 \\
\hline UM (15-24) & 0.0617 & 0.0094 & 1,492 & 1,492 & 1.5117 & 0.1526 & 0.0432 & 0.0803 \\
\hline UW (15-24) & 0.0183 & 0.0026 & 3,188 & 3,188 & 1.1034 & 0.1432 & 0.0131 & 0.0235 \\
\hline \multicolumn{9}{|c|}{ Urban } \\
\hline M (15-24) & 0.0397 & 0.0074 & 1,039 & 279 & 1.2285 & 0.1875 & 0.0251 & 0.0544 \\
\hline W (15-24) & 0.0133 & 0.0026 & 2,581 & 637 & 1.1734 & 0.1992 & 0.0081 & 0.0185 \\
\hline MM (15-29) & 0.0485 & 0.0095 & 547 & 92 & 1.0341 & 0.1961 & 0.0298 & 0.0672 \\
\hline MW (15-24) & 0.0203 & 0.0044 & 1,136 & 142 & 1.0593 & 0.2183 & 0.0116 & 0.0291 \\
\hline UM (15-24) & 0.0368 & 0.0076 & 833 & 245 & 1.1632 & 0.2064 & 0.0218 & 0.0517 \\
\hline UW (15-24) & 0.0082 & 0.0026 & 1,445 & 494 & 1.0786 & 0.3123 & 0.0032 & 0.0132 \\
\hline \multicolumn{9}{|c|}{ Rural } \\
\hline M (15-24) & 0.0715 & 0.0096 & 903 & 1,685 & 1.1231 & 0.1348 & 0.0525 & 0.0904 \\
\hline W (15-24) & 0.0140 & 0.0022 & 2,948 & 4,893 & 1.0148 & 0.1570 & 0.0097 & 0.0183 \\
\hline MM (15-29) & 0.0837 & 0.0115 & 568 & 1,023 & 0.9862 & 0.1371 & 0.0611 & 0.1062 \\
\hline MW (15-24) & 0.0111 & 0.0026 & 1,205 & 2,199 & 0.8764 & 0.2388 & 0.0059 & 0.0163 \\
\hline UM (15-24) & 0.0666 & 0.0112 & 659 & 1,247 & 1.1479 & 0.1675 & 0.0447 & 0.0886 \\
\hline UW (15-24) & 0.0201 & 0.0031 & 1,743 & 2,694 & 0.9078 & 0.1517 & 0.0141 & 0.0262 \\
\hline
\end{tabular}


Table B.2: (Cont'd)

\begin{tabular}{|c|c|c|c|c|c|c|c|c|}
\hline \multirow{2}{*}{$\begin{array}{l}\text { Variable/ } \\
\text { respondent } \\
\text { category }\end{array}$} & \multirow{2}{*}{$\begin{array}{l}\text { Value } \\
(\mathbf{R})\end{array}$} & \multirow{2}{*}{$\begin{array}{c}\text { Standard } \\
\text { error } \\
\text { (SE) }\end{array}$} & \multicolumn{2}{|c|}{ Number of cases } & \multirow{2}{*}{$\begin{array}{c}\text { Design } \\
\text { effect } \\
\text { (DEFT) }\end{array}$} & \multirow{2}{*}{$\begin{array}{c}\text { Relative } \\
\text { standard } \\
\text { error } \\
(\mathrm{SE} / \mathrm{R})\end{array}$} & \multicolumn{2}{|c|}{ 95\% Confidence limit } \\
\hline & & & $\begin{array}{l}\text { Unweighted } \\
\text { (N) }\end{array}$ & $\begin{array}{l}\text { Weighted } \\
\text { (WN) }\end{array}$ & & & Lower & Upper \\
\hline \multicolumn{9}{|c|}{ Ever had pre-marital sex (young men and women) } \\
\hline \multicolumn{9}{|c|}{ Combined } \\
\hline M (15-24) & 0.1393 & 0.0118 & 1,942 & 1,964 & 1.5062 & 0.0850 & 0.1160 & 0.1626 \\
\hline W (15-24) & 0.0262 & 0.0034 & 5,529 & 5,529 & 1.5939 & 0.1308 & 0.0194 & 0.0329 \\
\hline MM (15-29) & 0.1805 & 0.0154 & 1,115 & 1,115 & 1.3349 & 0.0852 & 0.1502 & 0.2108 \\
\hline MW (15-24) & 0.0243 & 0.0042 & 2,341 & 2,341 & 1.3272 & 0.1739 & 0.0160 & 0.0326 \\
\hline UM (15-24) & 0.1075 & 0.0107 & 1,492 & 1,492 & 1.3379 & 0.0998 & 0.0864 & 0.1286 \\
\hline UW (15-24) & 0.0302 & 0.0043 & 3,188 & 3,188 & 1.4208 & 0.1427 & 0.0217 & 0.0386 \\
\hline \multicolumn{9}{|c|}{ Urban } \\
\hline M (15-24) & 0.1022 & 0.0118 & 1,039 & 279 & 1.2511 & 0.1151 & 0.0790 & 0.1253 \\
\hline W (15-24) & 0.0211 & 0.0034 & 2,581 & 637 & 1.1923 & 0.1599 & 0.0145 & 0.0277 \\
\hline MM (15-29) & 0.1626 & 0.0182 & 547 & 92 & 1.1549 & 0.1122 & 0.1267 & 0.1985 \\
\hline MW (15-24) & 0.0282 & 0.0050 & 1,136 & 142 & 1.0230 & 0.1783 & 0.0183 & 0.0381 \\
\hline UM (15-24) & 0.0804 & 0.0105 & 833 & 245 & 1.1136 & 0.1305 & 0.0598 & 0.1011 \\
\hline UW (15-24) & 0.0160 & 0.0036 & 1,445 & 494 & 1.0886 & 0.2248 & 0.0089 & 0.0231 \\
\hline \multicolumn{9}{|c|}{ Rural } \\
\hline M (15-24) & 0.1455 & 0.0136 & 903 & 1,685 & 1.1602 & 0.0936 & 0.1187 & 0.1723 \\
\hline W (15-24) & 0.0268 & 0.0038 & 2,948 & 4,893 & 1.2914 & 0.1433 & 0.0193 & 0.0344 \\
\hline MM (15-29) & 0.1821 & 0.0167 & 568 & 1,023 & 1.0285 & 0.0915 & 0.1493 & 0.2149 \\
\hline MW (15-24) & 0.0240 & 0.0045 & 1,205 & 2,199 & 1.0158 & 0.1865 & 0.0152 & 0.0329 \\
\hline UM (15-24) & 0.1128 & 0.0127 & 659 & 1,247 & 1.0263 & 0.1122 & 0.0879 & 0.1377 \\
\hline UW (15-24) & 0.0328 & 0.0050 & 1,743 & 2,694 & 1.1763 & 0.1532 & 0.0229 & 0.0426 \\
\hline \multicolumn{9}{|c|}{$\begin{array}{l}\text { Used condoms consistently in pre-marital relations } \\
\text { and women who reported pre-marital sex in face-to-face interview) }\end{array}$} \\
\hline \multicolumn{9}{|c|}{ Combined } \\
\hline M (15-24) & 0.0648 & 0.0193 & 206 & 238 & 1.1197 & 0.2971 & 0.0267 & 0.1028 \\
\hline W (15-24) & 0.0194 & 0.0123 & 99 & 109 & 0.8811 & 0.6328 & 0.0000 & 0.0437 \\
\hline MM (15-29) & 0.0439 & 0.0209 & 159 & 178 & 1.2848 & 0.4771 & 0.0025 & 0.0852 \\
\hline UM (15-24) & 0.0983 & 0.0331 & 113 & 135 & 1.1748 & 0.3362 & 0.0330 & 0.1636 \\
\hline \multicolumn{9}{|c|}{$\begin{array}{l}\text { Ever communicated with spouse on contraception } \\
\text { (married young men and women who had begun cohabiting) }\end{array}$} \\
\hline \multicolumn{9}{|c|}{ Combined } \\
\hline MM (15-29) & 0.3750 & 0.0220 & 1,072 & 1,072 & 1.4885 & 0.0587 & 0.3317 & 0.4184 \\
\hline MW (15-24) & 0.7157 & 0.0164 & 2,237 & 2,202 & 1.7167 & 0.0229 & 0.6834 & 0.7479 \\
\hline \multicolumn{9}{|c|}{ Urban } \\
\hline MM (15-29) & 0.4603 & 0.0266 & 525 & 88 & 1.2231 & 0.0579 & 0.4079 & 0.5127 \\
\hline MW (15-24) & 0.7976 & 0.0173 & 1,103 & 138 & 1.4280 & 0.0217 & 0.7635 & 0.8316 \\
\hline \multicolumn{9}{|c|}{ Rural } \\
\hline MM (15-29) & 0.3674 & 0.0239 & 547 & 984 & 1.1603 & 0.0652 & 0.3203 & 0.4145 \\
\hline MW (15-24) & 0.7102 & 0.0174 & 1,134 & 2,064 & 1.2914 & 0.0245 & 0.6759 & 0.7445 \\
\hline
\end{tabular}

Cont'd on next page... 
Table B.2: (Cont'd)

\begin{tabular}{|c|c|c|c|c|c|c|c|c|}
\hline \multirow{2}{*}{$\begin{array}{l}\text { Variable/ } \\
\text { respondent } \\
\text { category }\end{array}$} & \multirow{2}{*}{$\begin{array}{l}\text { Value } \\
(\mathbf{R})\end{array}$} & \multirow{2}{*}{$\begin{array}{c}\text { Standard } \\
\text { error } \\
\text { (SE) }\end{array}$} & \multicolumn{2}{|c|}{ Number of cases } & \multirow{2}{*}{$\begin{array}{c}\text { Design } \\
\text { effect } \\
\text { (DEFT) }\end{array}$} & \multirow{2}{*}{$\begin{array}{c}\text { Relative } \\
\text { standard } \\
\text { error } \\
\text { (SE/R) }\end{array}$} & \multicolumn{2}{|c|}{ 95\% Confidence limits } \\
\hline & & & $\begin{array}{l}\text { Unweighted } \\
\text { (N) }\end{array}$ & $\begin{array}{l}\text { Weighted } \\
\text { (WN) }\end{array}$ & & & Lower & Upper \\
\hline \multicolumn{9}{|c|}{$\begin{array}{l}\text { Husband ever forced wife to have sex } \\
\text { roung men and women who had begun cohabiting) }\end{array}$} \\
\hline \multicolumn{9}{|c|}{ Combined } \\
\hline MM (15-29) & 0.2481 & 0.0160 & 1,072 & 1,072 & 1.2104 & 0.0644 & 0.2167 & 0.2796 \\
\hline MW (15-24) & 0.5374 & 0.0208 & 2,237 & 2,203 & 1.9704 & 0.0387 & 0.4965 & 0.5783 \\
\hline \multicolumn{9}{|c|}{ Urban } \\
\hline MM (15-29) & 0.2107 & 0.0184 & 525 & 88 & 1.0340 & 0.0874 & 0.1744 & 0.2470 \\
\hline MW (15-24) & 0.4981 & 0.0212 & 1,102 & 138 & 1.4102 & 0.0427 & 0.4562 & 0.5399 \\
\hline \multicolumn{9}{|c|}{ Rural } \\
\hline MM (15-29) & 0.2515 & 0.0173 & 547 & 984 & 0.9336 & 0.0689 & 0.2174 & 0.2856 \\
\hline MW (15-24) & 0.5400 & 0.0221 & 1,135 & 2,065 & 1.4931 & 0.0409 & 0.4965 & 0.5835 \\
\hline \multicolumn{9}{|c|}{$\begin{array}{l}\text { Husband ever perpetrated physical violence on wife } \\
\text { (married young men and women who had begun cohabiting) }\end{array}$} \\
\hline \multicolumn{9}{|c|}{ Combined } \\
\hline MM (15-29) & 0.2965 & 0.0230 & 1,072 & 1,072 & 1.6457 & 0.0774 & 0.2513 & 0.3418 \\
\hline MW (15-24) & 0.2974 & 0.0155 & 2,236 & 2,202 & 1.6040 & 0.0522 & 0.2668 & 0.3279 \\
\hline \multicolumn{9}{|c|}{ Urban } \\
\hline MM (15-29) & 0.2497 & 0.0240 & 525 & 88 & 1.2684 & 0.0960 & 0.2025 & 0.2970 \\
\hline MW (15-24) & 0.2417 & 0.0161 & 1,102 & 138 & 1.2466 & 0.0666 & 0.2100 & 0.2733 \\
\hline \multicolumn{9}{|c|}{ Rural } \\
\hline MM (15-29) & 0.3007 & 0.0249 & 547 & 984 & 1.2684 & 0.0828 & 0.2517 & 0.3498 \\
\hline MW (15-24) & 0.3011 & 0.0165 & 1,134 & 2,064 & 1.2094 & 0.0547 & 0.2686 & 0.3335 \\
\hline \multicolumn{9}{|c|}{$\begin{array}{l}\text { Husband ever perpetrated physical violence on wife in last } 12 \text { months } \\
\text { (married young men and women who had begun cohabiting) }\end{array}$} \\
\hline \multicolumn{9}{|c|}{ Combined } \\
\hline MM (15-29) & 0.1865 & 0.0181 & 1,072 & 1,072 & 1.5231 & 0.0972 & 0.1508 & 0.2222 \\
\hline MW (15-24) & 0.2362 & 0.0131 & 2,236 & 2,202 & 1.4538 & 0.0553 & 0.2105 & 0.2619 \\
\hline \multicolumn{9}{|c|}{ Urban } \\
\hline MM (15-29) & 0.1682 & 0.0199 & 525 & 88 & 1.2187 & 0.1184 & 0.1290 & 0.2074 \\
\hline MW (15-24) & 0.1967 & 0.0159 & 1,102 & 138 & 1.3269 & 0.0808 & 0.1654 & 0.2280 \\
\hline \multicolumn{9}{|c|}{ Rural } \\
\hline MM (15-29) & 0.1882 & 0.0197 & 547 & 984 & 1.1759 & 0.1045 & 0.1495 & 0.2269 \\
\hline MW (15-24) & 0.2388 & 0.0139 & 1,134 & 2,064 & 1.0954 & 0.0581 & 0.2115 & 0.2661 \\
\hline \multicolumn{9}{|c|}{$\begin{array}{l}\text { Currently using any modern contraceptive method } \\
\text { (married young men and women who had begun cohabiting) }\end{array}$} \\
\hline \multicolumn{9}{|c|}{ Combined } \\
\hline MM (15-29) & 0.1093 & 0.0150 & 1,072 & 1,072 & 1.5706 & 0.1370 & 0.0799 & 0.1388 \\
\hline MW (15-24) & 0.0869 & 0.0086 & 2,237 & 2,202 & 1.4476 & 0.0993 & 0.0699 & 0.1038 \\
\hline \multicolumn{9}{|c|}{ Urban } \\
\hline MM (15-29) & 0.1978 & 0.0193 & 525 & 88 & 1.1091 & 0.0976 & 0.1598 & 0.2358 \\
\hline MW (15-24) & 0.1753 & 0.0124 & 1,103 & 138 & 1.0865 & 0.0710 & 0.1508 & 0.1998 \\
\hline \multicolumn{9}{|c|}{ Rural } \\
\hline MM (15-29) & 0.1014 & 0.0162 & 547 & 984 & 1.2574 & 0.1602 & 0.0694 & 0.1334 \\
\hline MW (15-24) & 0.0810 & 0.0091 & 1,134 & 2,064 & 1.1245 & 0.1126 & 0.0630 & 0.0989 \\
\hline
\end{tabular}

Cont'd on next page... 
Table B.2: (Cont'd)

\begin{tabular}{|c|c|c|c|c|c|c|c|c|}
\hline \multirow{2}{*}{$\begin{array}{l}\text { Variable/ } \\
\text { respondent } \\
\text { category }\end{array}$} & \multirow{2}{*}{$\begin{array}{l}\text { Value } \\
(\mathbf{R})\end{array}$} & \multirow{2}{*}{$\begin{array}{c}\text { Standard } \\
\text { error } \\
\text { (SE) }\end{array}$} & \multicolumn{2}{|c|}{ Number of cases } & \multirow{2}{*}{$\begin{array}{l}\text { Design } \\
\text { effect } \\
\text { (DEFT) }\end{array}$} & \multirow{2}{*}{$\begin{array}{c}\text { Relative } \\
\text { standard } \\
\text { error } \\
(\mathrm{SE} / \mathrm{R})\end{array}$} & \multicolumn{2}{|c|}{ 95\% Confidence limits } \\
\hline & & & $\begin{array}{l}\text { Unweighted } \\
\text { (N) }\end{array}$ & $\begin{array}{l}\text { Weighted } \\
\text { (WN) }\end{array}$ & & & Lower & Upper \\
\hline \multicolumn{9}{|c|}{$\begin{array}{l}\text { First delivery in a health institution } \\
\text { women whose first pregnancy outcome was a live or still birth) }\end{array}$} \\
\hline \multicolumn{9}{|c|}{ Combined } \\
\hline MM (15-29) & 0.2296 & 0.0247 & 717 & 710 & 1.5742 & 0.1077 & 0.1809 & 0.2784 \\
\hline MW (15-24) & 0.2485 & 0.0195 & 1,547 & 1,521 & 1.7788 & 0.0786 & 0.2100 & 0.2870 \\
\hline \multicolumn{9}{|c|}{ Urban } \\
\hline MM (15-29) & 0.3944 & 0.0331 & 356 & 60 & 1.2743 & 0.0838 & 0.3293 & 0.4595 \\
\hline MW (15-24) & 0.5044 & 0.0290 & 765 & 95 & 1.6015 & 0.0574 & 0.4474 & 0.5614 \\
\hline \multicolumn{9}{|c|}{ Rural } \\
\hline MM (15-29) & 0.2145 & 0.0270 & 361 & 651 & 1.2490 & 0.1260 & 0.1613 & 0.2677 \\
\hline MW (15-24) & 0.2314 & 0.0206 & 782 & 1,426 & 1.3643 & 0.0889 & 0.1909 & 0.2719 \\
\hline \multicolumn{9}{|c|}{$\begin{array}{l}\text { Mean number of children ever born } \\
\text { arried young men and women who had begun cohabiting) }\end{array}$} \\
\hline \multicolumn{9}{|c|}{ Combined } \\
\hline MM (15-29) & 1.3321 & 0.0488 & 1,115 & 1,115 & 1.2907 & 0.0366 & 1.2360 & 1.4282 \\
\hline MW (15-24) & 1.3676 & 0.0404 & 2,341 & 2,341 & 1.5355 & 0.0295 & 1.2881 & 1.4471 \\
\hline \multicolumn{9}{|c|}{ Urban } \\
\hline MM (15-29) & 1.2882 & 0.0585 & 547 & 92 & 1.1326 & 0.0454 & 1.1731 & 1.4033 \\
\hline MW (15-24) & 1.4130 & 0.0411 & 1,136 & 142 & 1.1356 & 0.0291 & 1.3320 & 1.4939 \\
\hline \multicolumn{9}{|c|}{ Rural } \\
\hline MM (15-29) & 1.3361 & 0.0530 & 568 & 1,023 & 0.9950 & 0.0396 & 1.2319 & 1.4403 \\
\hline MW (15-24) & 1.3647 & 0.0429 & 1,205 & 2,199 & 1.1672 & 0.0314 & 1.2802 & 1.4492 \\
\hline \multicolumn{9}{|c|}{$\begin{array}{l}\text { Mean number of children surviving } \\
\text { young men and women who had begun cohabiting) }\end{array}$} \\
\hline \multicolumn{9}{|c|}{ Combined } \\
\hline MM (15-29) & 1.2421 & 0.0453 & 1,115 & 1,115 & 1.2499 & 0.0364 & 1.1531 & 1.3312 \\
\hline MW (15-24) & 1.2639 & 0.0352 & 2,341 & 2,341 & 1.4396 & 0.0279 & 1.1946 & 1.3333 \\
\hline \multicolumn{9}{|c|}{ Urban } \\
\hline MM (15-29) & 1.2222 & 0.0562 & 547 & 92 & 1.1363 & 0.0460 & 1.1116 & 1.3328 \\
\hline MW (15-24) & 1.3128 & 0.0362 & 1,136 & 142 & 1.0794 & 0.0276 & 1.2415 & 1.3840 \\
\hline \multicolumn{9}{|c|}{ Rural } \\
\hline MM (15-29) & 1.2439 & 0.0491 & 568 & 1,023 & 0.9631 & 0.0394 & 1.1474 & 1.3405 \\
\hline MW (15-24) & 1.2608 & 0.0374 & 1,205 & 2,199 & 1.0938 & 0.0297 & 1.1871 & 1.3345 \\
\hline \multicolumn{9}{|c|}{$\begin{array}{l}\text { Mean ideal number of children } \\
\text { omen who had begun cohabiting and gave a numeric response) }\end{array}$} \\
\hline \multicolumn{9}{|c|}{ Combined } \\
\hline MM (15-29) & 2.7199 & 0.0367 & 1,013 & 1,011 & 1.2649 & 0.0135 & 2.6476 & 2.7923 \\
\hline MW (15-24) & 2.6821 & 0.0327 & 2,103 & 2,058 & 1.8581 & 0.0122 & 2.6177 & 2.7465 \\
\hline \multicolumn{9}{|c|}{ Urban } \\
\hline MM (15-29) & 2.4333 & 0.0395 & 497 & 84 & 1.1842 & 0.0162 & 2.3556 & 2.5109 \\
\hline MW (15-24) & 2.3689 & 0.0343 & 1,045 & 131 & 1.5213 & 0.0145 & 2.3013 & 2.4364 \\
\hline \multicolumn{9}{|c|}{ Rural } \\
\hline MM (15-29) & 2.7458 & 0.0396 & 516 & 927 & 0.9619 & 0.0144 & 2.6678 & 2.8238 \\
\hline MW (15-24) & 2.7033 & 0.0348 & 1,058 & 1,928 & & 0.0129 & 2.6349 & 2.7717 \\
\hline
\end{tabular}

Cont'd on next page... 
Table B.2: (Cont'd)

\begin{tabular}{|c|c|c|c|c|c|c|c|c|}
\hline \multirow{2}{*}{$\begin{array}{l}\text { Variable/ } \\
\text { respondent } \\
\text { category }\end{array}$} & \multirow{2}{*}{$\begin{array}{l}\text { Value } \\
(\mathbf{R})\end{array}$} & \multirow{2}{*}{$\begin{array}{c}\text { Standard } \\
\text { error } \\
\text { (SE) }\end{array}$} & \multicolumn{2}{|c|}{ Number of cases } & \multirow{2}{*}{$\begin{array}{c}\text { Design } \\
\text { effect } \\
\text { (DEFT) }\end{array}$} & \multirow{2}{*}{$\begin{array}{c}\text { Relative } \\
\text { standard } \\
\text { error } \\
\text { (SE/R) }\end{array}$} & \multicolumn{2}{|c|}{ 95\% Confidence limit } \\
\hline & & & $\begin{array}{l}\text { Unweighted } \\
\text { (N) }\end{array}$ & $\begin{array}{l}\text { Weighted } \\
\text { (WN) }\end{array}$ & & & Lower & Upper \\
\hline \multicolumn{9}{|c|}{$\begin{array}{c}\text { Experienced } 3 \text { or more symptoms or behaviours suggestive of mental health disorders in the month } \\
\text { preceding the interview (young men and women) }\end{array}$} \\
\hline \multicolumn{9}{|c|}{ Combined } \\
\hline M (15-24) & 0.1622 & 0.0145 & 1,942 & 1,964 & 1.7270 & 0.0891 & 0.1337 & 0.1906 \\
\hline $\mathrm{W}(15-24)$ & 0.0872 & 0.0079 & 5,529 & 5,529 & 2.0818 & 0.0906 & 0.0717 & 0.1028 \\
\hline MM (15-29) & 0.1735 & 0.0178 & 1,115 & 1,115 & 1.5679 & 0.1025 & 0.1384 & 0.2085 \\
\hline MW (15-24) & 0.0897 & 0.0086 & 2,341 & 2,341 & 1.4485 & 0.0954 & 0.0729 & 0.1066 \\
\hline UM (15-24) & 0.1685 & 0.0148 & 1,492 & 1,492 & 1.5219 & 0.0875 & 0.1395 & 0.1976 \\
\hline UW (15-24) & 0.0830 & 0.0106 & 3,188 & 3,188 & 2.1638 & 0.1274 & 0.0622 & 0.1038 \\
\hline \multicolumn{9}{|c|}{ Urban } \\
\hline M (15-24) & 0.1665 & 0.0131 & 1,039 & 279 & 1.1371 & 0.0790 & 0.1406 & 0.1924 \\
\hline W (15-24) & 0.0804 & 0.0079 & 2,581 & 637 & 1.4684 & 0.0977 & 0.0650 & 0.0959 \\
\hline MM (15-29) & 0.1762 & 0.0176 & 547 & 92 & 1.0785 & 0.0998 & 0.1416 & 0.2108 \\
\hline MW (15-24) & 0.0887 & 0.0111 & 1,136 & 142 & 1.3205 & 0.1256 & 0.0668 & 0.1106 \\
\hline UM (15-24) & 0.1641 & 0.0139 & 833 & 245 & 1.0809 & 0.0846 & 0.1368 & 0.1914 \\
\hline UW (15-24) & 0.0745 & 0.0085 & 1,445 & 494 & 1.2285 & 0.1140 & 0.0578 & 0.0912 \\
\hline \multicolumn{9}{|c|}{ Rural } \\
\hline M (15-24) & 0.1615 & 0.0167 & 903 & 1,685 & 1.3632 & 0.1034 & 0.1286 & 0.1944 \\
\hline W (15-24) & 0.0881 & 0.0089 & 2,948 & 4,893 & 1.6989 & 0.1007 & 0.0707 & 0.1056 \\
\hline MM (15-29) & 0.1732 & 0.0193 & 568 & 1,023 & 1.2158 & 0.1116 & 0.1352 & 0.2113 \\
\hline MW (15-24) & 0.0898 & 0.0091 & 1,205 & 2,199 & 1.1025 & 0.1011 & 0.0719 & 0.1077 \\
\hline UM (15-24) & 0.1694 & 0.0174 & 659 & 1,247 & 1.1922 & 0.1029 & 0.1351 & 0.2037 \\
\hline UW (15-24) & 0.0846 & 0.0124 & 1,743 & 2,694 & 1.8647 & 0.1470 & 0.0601 & 0.1090 \\
\hline \multicolumn{9}{|c|}{ Ever consumed alcohol (young men and women) } \\
\hline \multicolumn{9}{|c|}{ Combined } \\
\hline M (15-24) & 0.1671 & 0.0149 & 1,942 & 1,964 & 1.7635 & 0.0894 & 0.1377 & 0.1965 \\
\hline W (15-24) & 0.0010 & 0.0006 & 5,529 & 5,529 & 1.2971 & 0.5493 & 0.0000 & 0.0021 \\
\hline MM (15-29) & 0.3568 & 0.0231 & 1,115 & 1,115 & 1.6071 & 0.0646 & 0.3114 & 0.4022 \\
\hline MW (15-24) & 0.0009 & 0.0008 & 2,341 & 2,341 & 1.2618 & 0.8732 & 0.0000 & 0.0024 \\
\hline UM (15-24) & 0.1079 & 0.0127 & 1,492 & 1,492 & 1.5862 & 0.1181 & 0.0828 & 0.1329 \\
\hline UW (15-24) & 0.0012 & 0.0007 & 3,188 & 3,188 & 1.1832 & 0.6061 & 0.0000 & 0.0026 \\
\hline \multicolumn{9}{|c|}{ Urban } \\
\hline M (15-24) & 0.1567 & 0.0148 & 1,039 & 279 & 1.3095 & 0.0943 & 0.1276 & 0.1857 \\
\hline W (15-24) & 0.0015 & 0.0008 & 2,581 & 637 & 0.9773 & 0.4923 & 0.0000 & 0.0030 \\
\hline MM (15-29) & 0.4223 & 0.0266 & 547 & 92 & 1.2589 & 0.0630 & 0.3699 & 0.4747 \\
\hline MW (15-24) & 0.0018 & 0.0013 & 1,136 & 142 & 1.0221 & 0.7068 & 0.0000 & 0.0044 \\
\hline UM (15-24) & 0.1250 & 0.0150 & 833 & 245 & 1.3061 & 0.1198 & 0.0955 & 0.1544 \\
\hline UW (15-24) & 0.0013 & 0.0009 & 1,445 & 494 & 0.9650 & 0.7043 & 0.0000 & 0.0031 \\
\hline \multicolumn{9}{|c|}{ Rural } \\
\hline M (15-24) & 0.1688 & 0.0172 & 903 & 1,685 & 1.3813 & 0.1021 & 0.1349 & 0.2027 \\
\hline W (15-24) & 0.0009 & 0.0006 & 2,948 & 4,893 & 1.0942 & 0.6569 & 0.0000 & 0.0022 \\
\hline MM (15-29) & 0.3509 & 0.0251 & 568 & 1,023 & 1.2499 & 0.0714 & 0.3016 & 0.4002 \\
\hline MW (15-24) & 0.0008 & 0.0008 & 1,205 & 2,199 & 0.9935 & 0.9934 & 0.0000 & 0.0025 \\
\hline UM (15-24) & 0.1045 & 0.0150 & 659 & 1,247 & 1.2536 & 0.1431 & 0.0751 & 0.1339 \\
\hline UW (15-24) & 0.0012 & 0.0008 & 1,743 & 2,694 & 1.0230 & 0.7146 & 0.0000 & 0.0028 \\
\hline
\end{tabular}


Table B.2: (Cont'd)

\begin{tabular}{|c|c|c|c|c|c|c|c|c|}
\hline \multirow{2}{*}{$\begin{array}{l}\text { Variable/ } \\
\text { respondent } \\
\text { category }\end{array}$} & \multirow{2}{*}{$\begin{array}{c}\text { Value } \\
\text { (R) }\end{array}$} & \multirow{2}{*}{$\begin{array}{c}\text { Standard } \\
\text { error } \\
\text { (SE) }\end{array}$} & \multicolumn{2}{|c|}{ Number of cases } & \multirow{2}{*}{$\begin{array}{c}\text { Design } \\
\text { effect } \\
\text { (DEFT) }\end{array}$} & \multirow{2}{*}{$\begin{array}{c}\text { Relative } \\
\text { standard } \\
\text { error } \\
\text { (SE/R) }\end{array}$} & \multicolumn{2}{|c|}{ 95\% Confidence limits } \\
\hline & & & $\begin{array}{l}\text { Unweighted } \\
(\mathbf{N})\end{array}$ & $\begin{array}{l}\text { Weighted } \\
\text { (WN) }\end{array}$ & & & Lower & Upper \\
\hline
\end{tabular}

Participated in a government-/NGO- sponsored programme in the 3 years preceding the interview (young men and women)

\begin{tabular}{|c|c|c|c|c|c|c|c|c|}
\hline \multicolumn{9}{|c|}{ Combined } \\
\hline$M(15-24)$ & 0.0725 & 0.0089 & 1,942 & 1,964 & 1.5060 & 0.1222 & 0.0551 & 0.0900 \\
\hline $\mathrm{W}(15-24)$ & 0.0189 & 0.0039 & 5,529 & 5,529 & 2.1167 & 0.2051 & 0.0113 & 0.0265 \\
\hline MM (15-29) & 0.0677 & 0.0094 & 1,115 & 1,115 & 1.2475 & 0.1387 & 0.0492 & 0.0862 \\
\hline MW (15-24) & 0.0163 & 0.0045 & 2,341 & 2,341 & 1.7150 & 0.2757 & 0.0074 & 0.0251 \\
\hline UM (15-24) & 0.0689 & 0.0093 & 1,492 & 1,492 & 1.4197 & 0.1352 & 0.0505 & 0.0872 \\
\hline UW (15-24) & 0.0237 & 0.0047 & 3,188 & 3,188 & 1.7497 & 0.1988 & 0.0144 & 0.0330 \\
\hline \multicolumn{9}{|c|}{ Urban } \\
\hline$M(15-24)$ & 0.0595 & 0.0095 & 1,039 & 279 & 1.2995 & 0.1603 & 0.0407 & 0.0783 \\
\hline $\mathrm{W}(15-24)$ & 0.0211 & 0.0032 & 2,581 & 637 & 1.1225 & 0.1506 & 0.0148 & 0.0273 \\
\hline MM (15-29) & 0.0448 & 0.0097 & 547 & 92 & 1.0984 & 0.2171 & 0.0256 & 0.0639 \\
\hline MW (15-24) & 0.0098 & 0.0030 & 1,136 & 142 & 1.0249 & 0.3056 & 0.0039 & 0.0157 \\
\hline UM (15-24) & 0.0614 & 0.0105 & 833 & 245 & 1.2565 & 0.1703 & 0.0408 & 0.0820 \\
\hline UW (15-24) & 0.0292 & 0.0048 & 1,445 & 494 & 1.0759 & 0.1633 & 0.0198 & 0.0386 \\
\hline \multicolumn{9}{|c|}{ Rural } \\
\hline$M(15-24)$ & 0.0747 & 0.0102 & 903 & 1,685 & 1.1668 & 0.1367 & 0.0546 & 0.0948 \\
\hline $\mathrm{W}(15-24)$ & 0.0186 & 0.0044 & 2,948 & 4,893 & 1.7514 & 0.2342 & 0.0100 & 0.0272 \\
\hline MM (15-29) & 0.0698 & 0.0102 & 568 & 1,023 & 0.9522 & 0.1460 & 0.0497 & 0.0898 \\
\hline MW (15-24) & 0.0167 & 0.0048 & 1,205 & 2,199 & 1.2926 & 0.2860 & 0.0073 & 0.0261 \\
\hline UM (15-24) & 0.0703 & 0.0109 & 659 & 1,247 & 1.0973 & 0.1555 & 0.0488 & 0.0919 \\
\hline UW (15-24) & 0.0227 & 0.0055 & 1,743 & 2,694 & 1.5412 & 0.2421 & 0.0119 & 0.0336 \\
\hline \multicolumn{9}{|c|}{ Voted in last election (young men and women, aged 20 and above) } \\
\hline \multicolumn{9}{|c|}{ Combined } \\
\hline$M(15-24)$ & 0.6461 & 0.0229 & 798 & 747 & 1.3521 & 0.0354 & 0.6010 & 0.6912 \\
\hline W (15-24) & 0.5108 & 0.0235 & 1,914 & 2,232 & 2.0592 & 0.0461 & 0.4645 & 0.5571 \\
\hline MM (15-29) & 0.7943 & 0.0167 & 1,052 & 1,029 & 1.3428 & 0.0211 & 0.7614 & 0.8273 \\
\hline MW (15-24) & 0.5282 & 0.0259 & 1,499 & 1,334 & 2.0062 & 0.0490 & 0.4772 & 0.5791 \\
\hline UM (15-24) & 0.6164 & 0.0335 & 411 & 331 & 1.3942 & 0.0543 & 0.5505 & 0.6823 \\
\hline UW (15-24) & 0.3393 & 0.0329 & 415 & 273 & 1.4149 & 0.0970 & 0.2745 & 0.4041 \\
\hline \multicolumn{9}{|c|}{ Urban } \\
\hline$M(15-24)$ & 0.6100 & 0.0282 & 473 & 117 & 1.2556 & 0.0462 & 0.5545 & 0.6655 \\
\hline $\mathrm{W}(15-24)$ & 0.3670 & 0.0206 & 1,134 & 274 & 1.4421 & 0.0563 & 0.3264 & 0.4077 \\
\hline MM (15-29) & 0.7663 & 0.0201 & 531 & 89 & 1.0917 & 0.0262 & 0.7268 & 0.8058 \\
\hline MW (15-24) & 0.3524 & 0.0238 & 824 & 103 & 1.4301 & 0.0676 & 0.3055 & 0.3993 \\
\hline UM (15-24) & 0.6069 & 0.0335 & 283 & 84 & 1.1504 & 0.0551 & 0.5411 & 0.6728 \\
\hline UW (15-24) & 0.4025 & 0.0306 & 310 & 107 & 1.0961 & 0.0760 & 0.3423 & 0.4627 \\
\hline \multicolumn{9}{|c|}{ Rural } \\
\hline$M(15-24)$ & 0.6528 & 0.0266 & 325 & 628 & 1.0061 & 0.0408 & 0.6004 & 0.7052 \\
\hline $\mathrm{W}(15-24)$ & 0.5309 & 0.0265 & 780 & 1,959 & 1.4839 & 0.0500 & 0.4787 & 0.5831 \\
\hline MM (15-29) & 0.7970 & 0.0182 & 521 & 939 & 1.0319 & 0.0228 & 0.7612 & 0.8328 \\
\hline MW (15-24) & 0.5429 & 0.0279 & 675 & 1,231 & 1.4547 & 0.0514 & 0.4880 & 0.5979 \\
\hline UM (15-24) & 0.6196 & 0.0434 & 128 & 247 & 1.0066 & 0.0700 & 0.5342 & 0.7049 \\
\hline UW (15-24) & 0.2986 & 0.0508 & 105 & 166 & 1.1309 & 0.1700 & 0.1987 & 0.3985 \\
\hline
\end{tabular}

Note: M: Men, W: Women, MM: Married men, MW: Married women, UM: Unmarried men, UW: Unmarried women. 
Appendix C

\section{Data quality tables}

Table C.1: Household age distribution

Single-year age distribution of the de jure household population by sex (weighted), Bihar, 2007

\begin{tabular}{|c|c|c|c|c|c|c|c|c|c|}
\hline \multirow{2}{*}{$\begin{array}{l}\text { Age } \\
\text { (year) }\end{array}$} & \multicolumn{2}{|c|}{ Women } & \multicolumn{2}{|c|}{ Men } & \multirow{2}{*}{$\begin{array}{l}\text { Age } \\
\text { (year) }\end{array}$} & \multicolumn{2}{|c|}{ Women } & \multicolumn{2}{|c|}{ Men } \\
\hline & $\begin{array}{l}\text { Unweighted } \\
\text { Number }\end{array}$ & Percent & $\begin{array}{l}\text { Unweighted } \\
\text { Number }\end{array}$ & Percent & & $\begin{array}{l}\text { Unweighted } \\
\text { Number }\end{array}$ & Percent & $\begin{array}{c}\text { Unweighted } \\
\text { Number }\end{array}$ & Percent \\
\hline 0 & 1,809 & 2.6 & 1,922 & 2.8 & 36 & 684 & 0.8 & 717 & 0.9 \\
\hline 1 & 1,684 & 2.3 & 1,847 & 2.6 & 37 & 462 & 0.5 & 403 & 0.5 \\
\hline 2 & 1,931 & 2.6 & 2,113 & 3.1 & 38 & 972 & 1.2 & 848 & 1.0 \\
\hline 3 & 2,378 & 3.3 & 2,454 & 3.5 & 39 & 247 & 0.3 & 208 & 0.2 \\
\hline 4 & 2,095 & 2.9 & 2,189 & 3.1 & 40 & 2,355 & 2.9 & 2,392 & 2.9 \\
\hline 5 & 2,350 & 3.3 & 2,731 & 4.0 & 41 & 197 & 0.2 & 162 & 0.2 \\
\hline 6 & 2,381 & 3.3 & 2,589 & 3.6 & 42 & 763 & 0.9 & 804 & 0.9 \\
\hline 7 & 2,136 & 3.0 & 2,199 & 3.1 & 43 & 242 & 0.3 & 241 & 0.3 \\
\hline 8 & 2,520 & 3.4 & 2,755 & 3.8 & 44 & 243 & 0.3 & 188 & 0.2 \\
\hline 9 & 1,533 & 2.1 & 1,614 & 2.2 & 45 & 2,026 & 2.7 & 2,148 & 2.7 \\
\hline 10 & 2,715 & 3.6 & 3,091 & 4.3 & 46 & 357 & 0.4 & 349 & 0.4 \\
\hline 11 & 1,322 & 1.6 & 1,379 & 1.8 & 47 & 227 & 0.3 & 237 & 0.3 \\
\hline 12 & 2,412 & 3.0 & 2,870 & 3.9 & 48 & 598 & 0.7 & 561 & 0.7 \\
\hline 13 & 1,773 & 2.2 & 1,833 & 2.5 & 49 & 133 & 0.2 & 137 & 0.2 \\
\hline 14 & 1,296 & 1.7 & 1,628 & 1.8 & 50 & 1,890 & 2.4 & 1,989 & 2.6 \\
\hline 15 & 2,096 & 2.6 & 1,852 & 2.1 & 51 & 107 & 0.1 & 139 & 0.2 \\
\hline 16 & 1,757 & 2.0 & 1,708 & 2.0 & 52 & 370 & 0.5 & 399 & 0.5 \\
\hline 17 & 1,243 & 1.4 & 1,289 & 1.4 & 53 & 117 & 0.1 & 132 & 0.2 \\
\hline 18 & 2,078 & 2.6 & 2,002 & 2.3 & 54 & 108 & 0.1 & 158 & 0.2 \\
\hline 19 & 1,046 & 1.2 & 888 & 0.9 & 55 & 1,509 & 2.0 & 1,490 & 1.9 \\
\hline 20 & 2,140 & 2.7 & 1,612 & 1.7 & 56 & 185 & 0.2 & 209 & 0.2 \\
\hline 21 & 879 & 1.0 & 750 & 0.8 & 57 & 93 & 0.1 & 132 & 0.1 \\
\hline 22 & 1,797 & 2.3 & 1,448 & 1.6 & 58 & 290 & 0.4 & 275 & 0.3 \\
\hline 23 & 998 & 1.2 & 728 & 0.8 & 59 & 45 & 0.1 & 102 & 0.1 \\
\hline 24 & 1,269 & 1.5 & 826 & 0.9 & 60 & 1,665 & 2.2 & 1,641 & 2.3 \\
\hline 25 & 1,418 & 2.1 & 1,708 & 2.0 & 61 & 59 & 0.1 & 112 & 0.1 \\
\hline 26 & 1,127 & 1.4 & 914 & 1.0 & 62 & 234 & 0.2 & 297 & 0.4 \\
\hline 27 & 872 & 1.1 & 694 & 0.9 & 63 & 56 & 0.1 & 87 & 0.1 \\
\hline 28 & 1,468 & 1.8 & 1,103 & 1.1 & 64 & 62 & 0.1 & 88 & 0.1 \\
\hline 29 & 511 & 0.6 & 391 & 0.5 & 65 & 1,058 & 1.4 & 1,140 & 1.6 \\
\hline 30 & 2,541 & 3.4 & 1,823 & 1.9 & 66 & 75 & 0.1 & 123 & 0.1 \\
\hline 31 & 337 & 0.4 & 390 & 0.5 & 67 & 45 & 0.1 & 82 & 0.1 \\
\hline 32 & 1,268 & 1.6 & 1,280 & 1.5 & 68 & 129 & 0.1 & 149 & 0.2 \\
\hline 33 & 502 & 0.6 & 509 & 0.8 & 69 & 22 & 0.0 & 50 & 0.1 \\
\hline 34 & 480 & 0.6 & 463 & 0.6 & $70+$ & 1,618 & 2.0 & 2,069 & 2.7 \\
\hline 35 & 2,226 & 2.7 & 2,696 & 3.4 & Total & 77,639 & 100.0 & 78,553 & 100.0 \\
\hline
\end{tabular}

Note: The de jure population includes usual residents of the household. 
Table C.2: Single-year age distribution of eligible, selected and interviewed young men

Number and percentage of eligible, selected and interviewed young men and percentage of selected young men who were interviewed by single-year age (unweighted), Bihar, 2007

\begin{tabular}{|c|c|c|c|c|c|c|c|}
\hline \multirow[t]{2}{*}{ Age (years) } & \multicolumn{2}{|c|}{ Eligible } & \multicolumn{2}{|c|}{ Selected for interview } & \multicolumn{2}{|c|}{ Interviewed } & \multirow{2}{*}{$\begin{array}{l}\% \text { selected } \\
\text { respondents } \\
\text { interviewed }\end{array}$} \\
\hline & No. & $\%$ & No. & $\%$ & No. & $\%$ & \\
\hline \multicolumn{8}{|c|}{ MM (15-29) } \\
\hline 15 & 3 & 0.2 & 3 & 0.2 & 3 & 0.3 & 100.0 \\
\hline 16 & 10 & 0.6 & 9 & 0.6 & 8 & 0.7 & 88.9 \\
\hline 17 & 9 & 0.6 & 8 & 0.6 & 7 & 0.6 & 87.5 \\
\hline 18 & 39 & 2.4 & 33 & 2.3 & 22 & 2.0 & 66.7 \\
\hline 19 & 34 & 2.1 & 29 & 2.0 & 23 & 2.1 & 79.3 \\
\hline 20 & 79 & 4.9 & 62 & 4.3 & 43 & 3.9 & 69.4 \\
\hline 21 & 58 & 3.6 & 54 & 3.8 & 42 & 3.8 & 77.8 \\
\hline 22 & 162 & 10.1 & 145 & 10.1 & 103 & 9.2 & 71.0 \\
\hline 23 & 121 & 7.6 & 111 & 7.8 & 94 & 8.4 & 84.7 \\
\hline 24 & 133 & 8.3 & 123 & 8.6 & 105 & 9.4 & 85.4 \\
\hline 25 & 272 & 17.0 & 243 & 17.0 & 174 & 15.6 & 71.6 \\
\hline 26 & 171 & 10.7 & 149 & 10.4 & 119 & 10.7 & 79.9 \\
\hline 27 & 174 & 10.9 & 160 & 11.2 & 134 & 12.0 & 83.8 \\
\hline 28 & 206 & 12.9 & 186 & 13.0 & 142 & 12.7 & 76.3 \\
\hline 29 & 127 & 7.9 & 116 & 8.1 & 96 & 8.6 & 82.8 \\
\hline Total & 1,598 & 100.0 & 1,431 & 100.0 & 1,115 & 100.0 & 77.9 \\
\hline \multicolumn{8}{|c|}{ UM (15-24) } \\
\hline 15 & 493 & 15.3 & 314 & 16.9 & 263 & 17.6 & 83.8 \\
\hline 16 & 518 & 16.1 & 310 & 16.7 & 251 & 16.8 & 81.0 \\
\hline 17 & 400 & 12.4 & 262 & 14.1 & 218 & 14.6 & 83.2 \\
\hline 18 & 514 & 16.0 & 309 & 16.6 & 235 & 15.8 & 76.1 \\
\hline 19 & 231 & 7.2 & 133 & 7.2 & 114 & 7.6 & 85.7 \\
\hline 20 & 336 & 10.5 & 178 & 9.6 & 129 & 8.6 & 72.5 \\
\hline 21 & 190 & 5.9 & 104 & 5.6 & 83 & 5.6 & 79.8 \\
\hline 22 & 281 & 8.7 & 123 & 6.6 & 94 & 6.3 & 76.4 \\
\hline 23 & 123 & 3.8 & 64 & 3.4 & 52 & 3.5 & 81.3 \\
\hline 24 & 128 & 4.0 & 61 & 3.3 & 53 & 3.6 & 86.9 \\
\hline Total & 3,214 & 100.0 & 1,858 & 100.0 & 1,492 & 100.0 & 80.3 \\
\hline
\end{tabular}

Note: The difference between the number of respondents eligible for interview and the number who were selected for interview is due to the sampling design adopted in the Youth Study. Please refer to Chapter 1 for details. 
Table C.3: Single-year age distribution of eligible, selected and interviewed young women

Number and percentages of eligible, selected and interviewed female respondents and percentage of selected respondents who were interviewed by single-year age (unweighted), Bihar, 2007

\begin{tabular}{|c|c|c|c|c|c|c|c|}
\hline \multirow[t]{2}{*}{ Age (Years) } & \multicolumn{2}{|c|}{ Eligible } & \multicolumn{2}{|c|}{ Selected for interview } & \multicolumn{2}{|c|}{ Interviewed } & \multirow{2}{*}{$\begin{array}{l}\text { \% selected } \\
\text { respondents } \\
\text { interviewed }\end{array}$} \\
\hline & No. & $\%$ & No. & $\%$ & No. & $\%$ & \\
\hline \multicolumn{8}{|c|}{ MW(15-24) } \\
\hline 15 & 207 & 3.3 & 107 & 3.7 & 97 & 4.1 & 90.7 \\
\hline 16 & 294 & 4.7 & 151 & 5.3 & 136 & 5.8 & 90.1 \\
\hline 17 & 348 & 5.6 & 181 & 6.3 & 165 & 7.0 & 91.2 \\
\hline 18 & 801 & 12.9 & 281 & 9.8 & 226 & 9.7 & 80.4 \\
\hline 19 & 510 & 8.2 & 253 & 8.9 & 218 & 9.3 & 86.2 \\
\hline 20 & 1,131 & 18.3 & 435 & 15.2 & 308 & 13.2 & 70.8 \\
\hline 21 & 536 & 8.7 & 302 & 10.6 & 262 & 11.2 & 86.8 \\
\hline 22 & 919 & 14.8 & 401 & 14.0 & 306 & 13.1 & 76.3 \\
\hline 23 & 628 & 10.1 & 320 & 11.2 & 272 & 11.6 & 85.0 \\
\hline 24 & 821 & 13.3 & 424 & 14.9 & 351 & 15.0 & 82.8 \\
\hline Total & 6,195 & 100.0 & 2,855 & 100.0 & 2,341 & 100.0 & 82.0 \\
\hline \multicolumn{8}{|c|}{ UW (15-24) } \\
\hline 15 & 1,386 & 32.3 & 1,245 & 35.2 & 1,129 & 35.4 & 90.7 \\
\hline 16 & 897 & 20.9 & 782 & 22.1 & 711 & 22.3 & 90.9 \\
\hline 17 & 546 & 12.7 & 453 & 12.8 & 401 & 12.6 & 88.5 \\
\hline 18 & 504 & 11.8 & 364 & 10.3 & 326 & 10.2 & 89.6 \\
\hline 19 & 297 & 6.9 & 226 & 6.4 & 206 & 6.5 & 91.2 \\
\hline 20 & 250 & 5.8 & 178 & 5.0 & 149 & 4.7 & 83.7 \\
\hline 21 & 135 & 3.2 & 103 & 2.9 & 96 & 3.0 & 93.2 \\
\hline 22 & 142 & 3.3 & 100 & 2.8 & 90 & 2.8 & 90.0 \\
\hline 23 & 75 & 1.8 & 54 & 1.5 & 50 & 1.6 & 92.6 \\
\hline 24 & 53 & 1.2 & 35 & 1.0 & 30 & 0.9 & 85.7 \\
\hline Total & 4,285 & 100.0 & 3,540 & 100.0 & 3,188 & 100.0 & 90.1 \\
\hline
\end{tabular}

Note: The difference between the number of respondents eligible for interview and the number who were selected for interview is due to the sampling design adopted in the Youth Study. Please refer to Chapter 1 for details. 


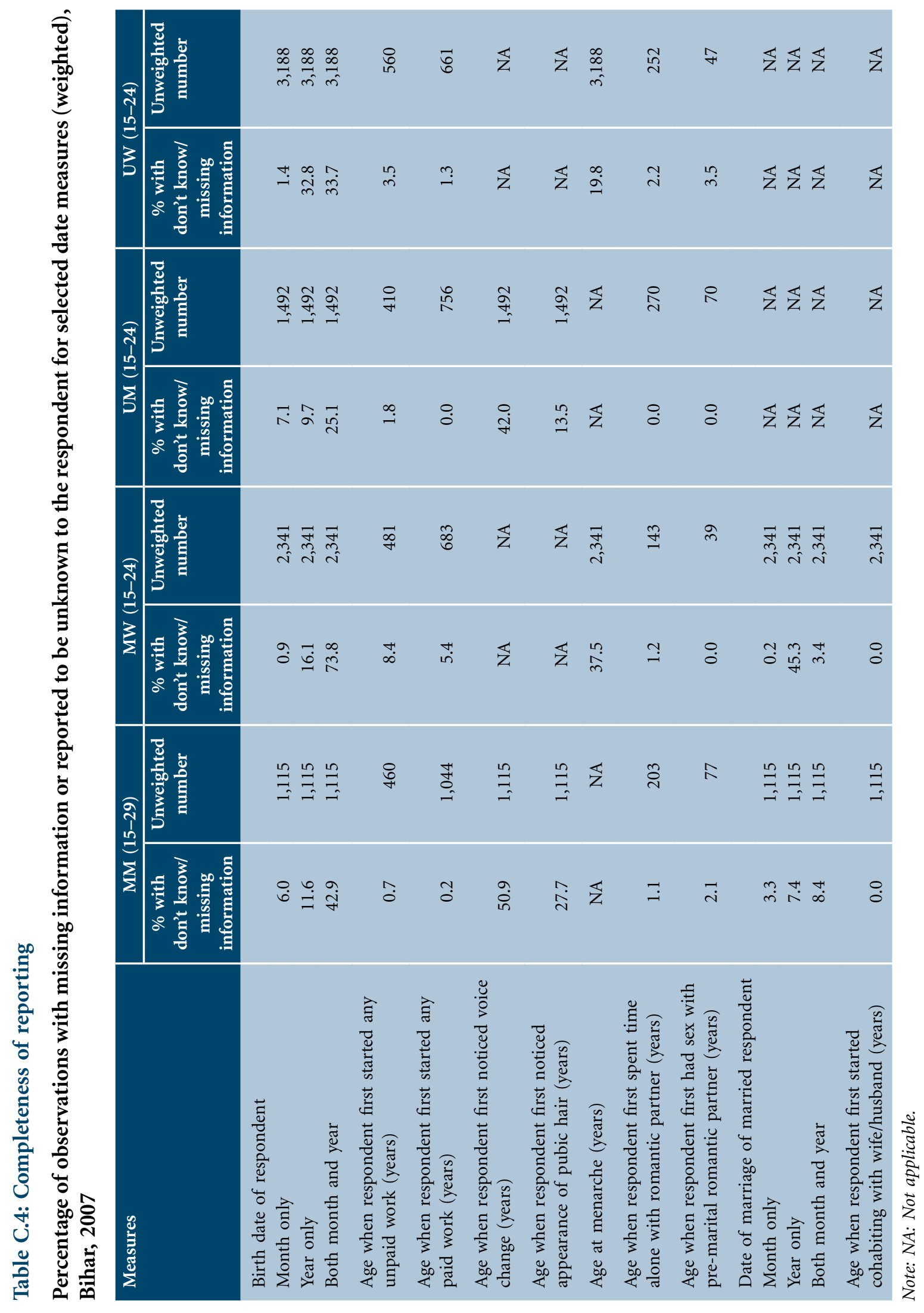




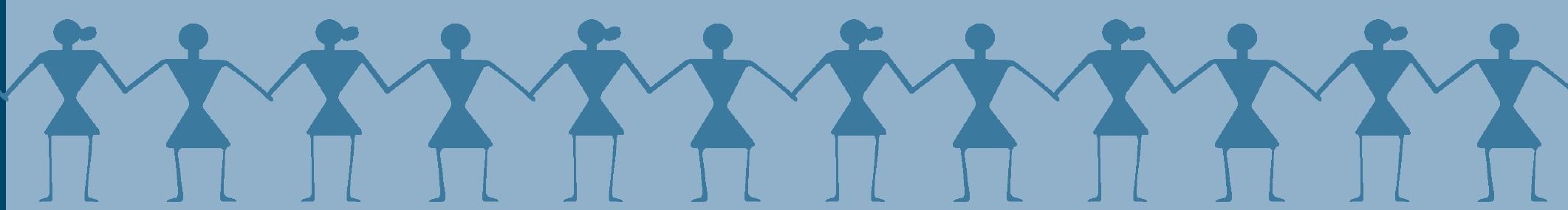


Supported by:

the David

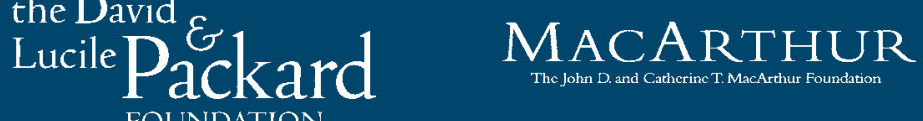

FOUNDATION 